\title{
Electrocatalytic Investigation of High Temperature PEM Fuel Cells
}

A Dissertation by

\section{Alin Orfanidi}

For the award of the degree of

DOCTOR OF PHILOSOPHY

DEPARTMENT OF CHEMICAL ENGINEERING

UNIVERSITY OF PATRAS

DECEMBER 2014 



\section{Dedicated to Dr.Stylianos Neophytides}

'Don't only practice your art, but force your way into its secrets, for it and knowledge can raise men to the divine' 


\section{Acknowledgements}

The present thesis was conducted in the laboratory of Catalysis and Hydrogen Technology at ICE-HT/FORTH during the period 2011-2014 in collaboration with the Department of Chemical Engineering of the University of Patras. It was funded by the European Commission, DEMMEA Project (2010-1012) 'Understanding the degradation mechanisms of membrane electrode assembly for high temperature PEMFCs and optimization of the individual components'.

I would like to thank my supervisory committee, .Prof. C.G Vayenas, Prof. S.Bebelis and Dr. S.Neophytides for all their helpful comments thrpought out the course of my studies,especially Dr. S.Neophytides for giving me the opportunity to complite the experiments in his laboratory and for his support and patience during my thesis.

I would also like to thank Dr Maria Daletou and Dr.Dimitris Niakolas, for their valuable input and help during my thesis. As well as Dr.Elina Siokou, Dr L.Sygellou, Dr. V. Drakopoulos ,Dr F.Paloukis and Dr. M. Kollia for their valuable help as it concerns XPS, SEM, EDX and TEM data acquisition and interpretation.

Last but not least, i would like to thank, the members of the laboratory for their support. 


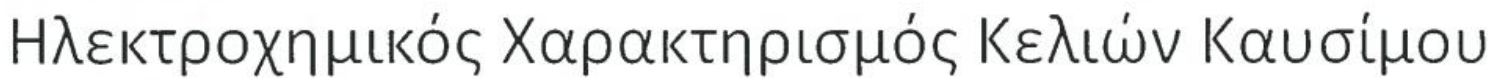

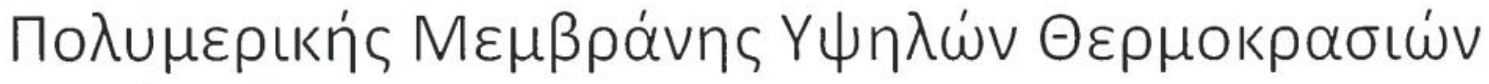

\author{
ҮП०

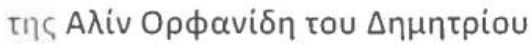

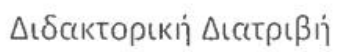

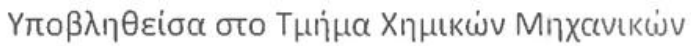

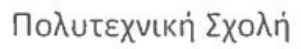

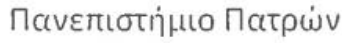

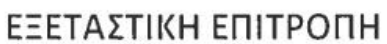

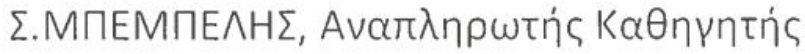

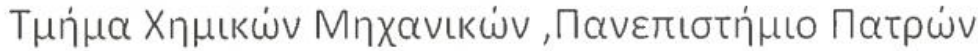

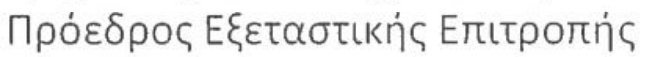

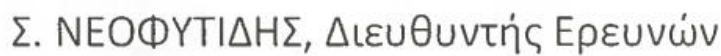

IEXMH/ITE

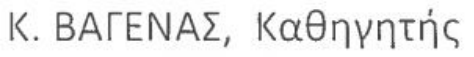

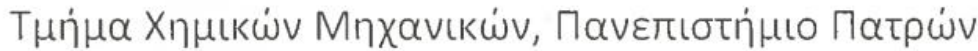

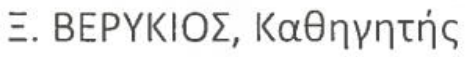

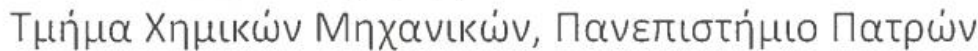

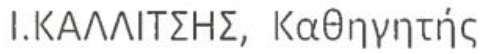

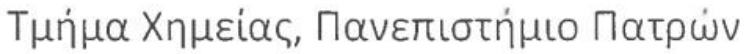

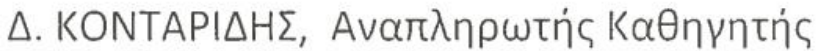

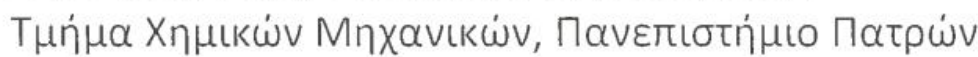

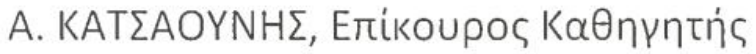

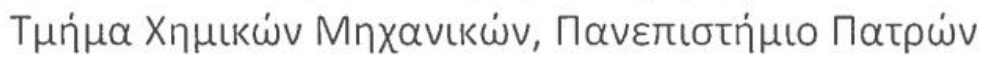

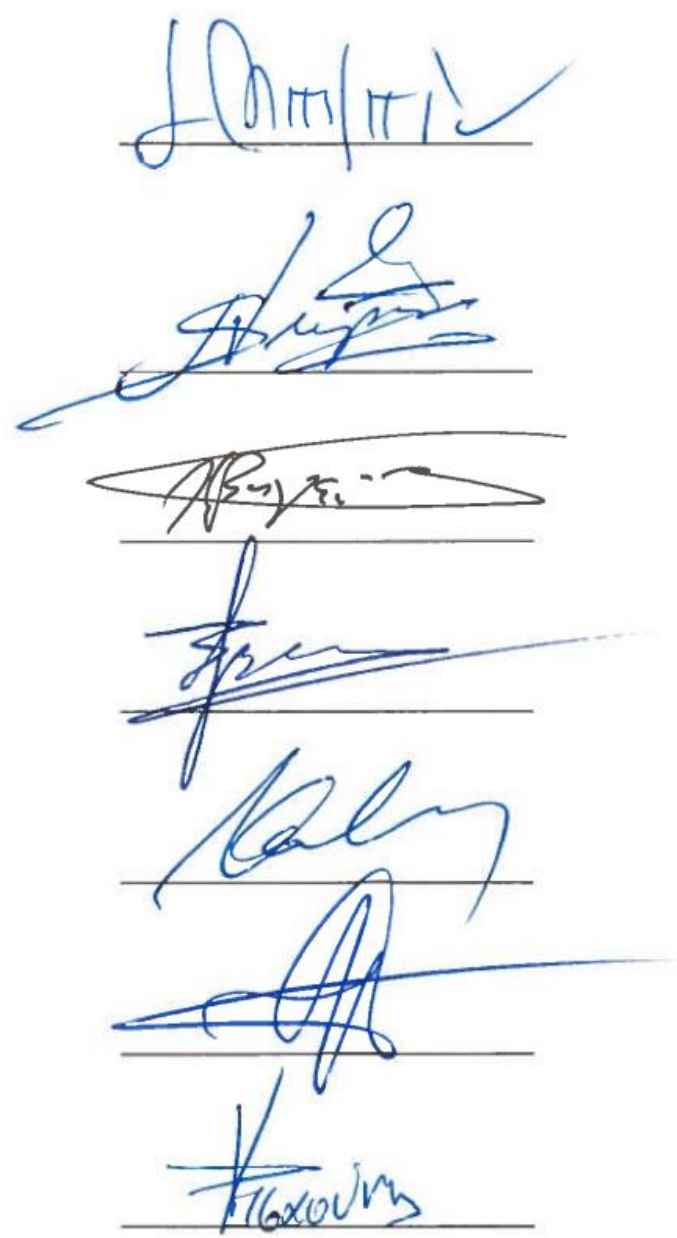




\begin{abstract}
The objective of this study is to shed more light on the electrochemical interface of HTPEM fuel cell. More specifically, to understand and improve the electrochemical interface of both the anodic and cathodic electrode in HTPEM fuel cells, as well as optimize the catalyst layer structure for operation under various challenging conditions. For that reason the effect of the PA amount in the catalyst layer and the effect of the catalyst's substrate on the fuel cell's performance were investigated.

Initially the poisoning effect of PA on the anodic electrode was investigated. The PA amount was altered in the anodic catalyst layer and its effect on the ECSA and the anode's performance were evaluated. It was observed that the reversible performance loss of the anodic electrode was a function of the PA amount in the catalyst layer. More specifically, under low PA loading $(<3 \mathrm{gPA} / \mathrm{gPt})$ on the anodic electrode, $<10 \%$ of the Pt active surface is electrochemically active under fuel cell operating conditions. This was attributed to the blockage of the Pt surface by pyrophosphoric acid or poly-phosphates, $\mathrm{H}_{2}$ reduced polyphosphoric acid species and the shrinkage of the interface due to the displacement of the $\mathrm{H}_{3} \mathrm{PO}_{4}$ by the adsorbed $\mathrm{H}_{2}$ species. High PA loadings reduced the poisoning effect of these reduced PA species ( $>3 \mathrm{gPA} / \mathrm{gPt}$ ). It was found that the controlled and increased PA content within the catalytic layer can result even up to the tenfold decrease in the Pt loading when the anode operates under $\mathrm{H}_{2}$ rich conditions.
\end{abstract}

In order to increase the fuel cell performance and increase the three phase boundary, a newly synthesized electrocatalyst was evaluated, and compared to the commercial $30 \mathrm{wt} \% \mathrm{Pt} / \mathrm{C}$. The new catalyst is based on pyridine functionalized carbon nanotubes ,30wt\%Pt/oxMWCNT-Py. Pyridine groups are known to interact with PA and thus it is expected to increase the TPB and lower the Pt loading.

CL employing the new catalyst were formulated and tested at the anodes. It was found that the presence of pyridine groups homogeneously distributed PA in the catalyst layer, resulting in high ECSA values, $40 \mathrm{~m}^{2} / \mathrm{gPt}$. As a result the MEA employing $30 \mathrm{wt} \% \mathrm{Pt}$ oxMWCNT-Py showed the same performance as the $30 \% \mathrm{Pt} / \mathrm{C}$ (having $1.3 \mathrm{mgPt} / \mathrm{cm}^{2}$ ), for Pt loading loadings as low as $0.2 \mathrm{mgPt} / \mathrm{cm}^{2}$. The performance of the anodic electrode was also found to be largely depended on the PA amount imbedded in the CL, when low Pt loading were used. The latter was an effect of the shrinkage of the ECSA as a result of the formation of PA poisoning species, as also mentioned in the previous paragraph.

Since 30wt\% Pt/oxMWCNT-Py exhibited very promising results and high ECSA values, its performance under harsh synthetic reformate gas was also evaluated. The synthetic 
reformate gas that was used comprised of $50.7 \mathrm{kPa}$ of $\mathrm{H}_{2}, 2 \mathrm{kPa}$ of $\mathrm{CO}$ and $33.5 \mathrm{kPa}$ of $\mathrm{H}_{2} \mathrm{O}$ balanced with Ar. It was found that the 30wt\%Pt/oxMWCNT-Py electrocatalyst are ideal candidates for operation under those harsh reformates conditions, as they exhibited smaller voltage losses and higher stability under these conditions. The interaction of pyridine groups with phosphoric acid not only promotes its uniform distribution on the CL but also stabilizes the EI under high partial pressure of water. Additionally, the use of pyridine functionalized MWCNT based electrocatalyst gives the opportunity of lowering the Pt loading in the electrodes without sacrificing the overall cell's performance under reformate conditions. The observed voltage loss under synthetic reformate gas rich in $\mathrm{CO}$ and steam was found to be a multi-step process and a function of the hydrophobicity of catalyst substrate, the PA loading in the CL as well as the water and CO molar fraction in the reformate gas.

In order to optimize the cathodic catalyst layer, CL were formulated using the newly synthesized electrocatalyst (30wt\%Pt/oxMWCNT-Py) and compared to the commercial $30 \mathrm{wt} \% \mathrm{Pt} / \mathrm{C}$. A full parametric analysis with respect to catalyst type, PA loading and $\mathrm{Pt}$ loading was conducted. It was found that the presence of pyridine groups homogeneously distributes PA in the catalyst layer minimizing the blockage of the pores of the catalyst layer and increase the three phase boundary. As a result the MEA employing 30wt $\% \mathrm{Pt}$ oxMWCNT-Py showed the same performance as the $30 \% \mathrm{Pt} / \mathrm{C}$ for half the Pt loading.

Despite the hard operating conditions the Pt particles attached to the ox.MWCNT-Py substrate exhibit the same stability as the commercial catalyst and the pyridine groups were found to be stable, at least for short term operation at the cathodic and anodic electrode. Also optimization of the ECSA evaluation procedure at the cathodic electrode, using CO as a probe molecule, without damaging the Pt distribution was found.

It is clear that the use of this newly synthesized electrocatalyts 30wt\%Pt/oxMWCNT-Py, at both electrodes, has major advantages as it increase the catalyst utilization and there is no need to use a polymer-binder inside the catalytic layer. Thus avoiding problems of inhomogeneous binder distribution and/or electronic insulation of catalyst nanoparticles. Using 30wt\%Pt/oxMWCNT-Py electrocatalyst opens the possibility of significant reduction of the amount of $\mathrm{Pt}$ on both electrodes, under various operation conditions, without sacrificing the performance and stability of the fuel cell. 


\section{Table of Content}

Chapter 1- Introduction to Hydrogen technology, Fuel Cells and theory 22

1.1 Introduction to $\mathrm{H} 2$ technology 22

1.2 Purpose of this thesis 23

1.3 Basic Components of High temperature PEMFC and the electrocatalytic

reactions at each electrode $\quad 24$

1.3.1 Fuel Cell Description $\quad 24$

1.3.2. Electrodes- Catalyst Layer $\quad 27$

1.3.3 Gas Diffusion Layer 28

1.3.4 Polymer Electrolyte Membrane 31

1.3.5 Current collectors and flow field/bipollar plates 33

1.3.6 Other components $\quad 35$

1.3.7 Mass transport in PEM fuel cells using single serpentine flow fields $\quad 36$

$\begin{array}{ll}1.4 \text { Thermodynamics } & 37\end{array}$

1.4.1 Basic Reactions

1.4.1.1 Oxygen Reduction Reaction $\quad 37$

1.4.1.2 Hydrogen Oxidation Reaction $\quad 38$

1.4.2 Heat of Reaction $\quad 39$

1.4.3 Gibbs Free Energy $\quad 39$

1.4.4 Reversible Fuel Cell Potential $\quad 40$

1.4.5 Effect of Operation Conditions on Reversible Fuel Cell Potential 40

1.4.6 Theoretical efficiency 41

1.4.7 Energy Conversion Efficiency for Fuel Cells $\quad 42$

1.5 Reaction Kinetics $\quad 42$

1.5.1 Current density and Exchange current density 42

1.5.2 Butler-Volmer Equation $\quad 43$

1.5.3 Linear Polarization $\quad 44$

1.5.4 Tafel Equation -Simplified Activation Kinetics 44

Chapter 2- Physicochemical and electrochemical characterization techniques used during the fulfillment of this thesis $\quad 50$

2.1 Introduction $\quad 50$

2.2 Electrochemical Characterization Techniques $\quad 50$

2.2.1 Basic Principle of operation of a Potentiostat-Galvanostat 51

2.2.2 Cyclic voltammetry $\quad 52$

2.2.3 Electrochemical Impedance Spectroscopy 53

2.2.4 Polarization Curves $\quad 55$

2.3 Physicochemical Characterization Techniques 56

2.3.1 X-Ray Photoelectron Spectroscopy (XPS) 56

2.3.2 X-Ray Diffraction (XRD) $\quad 58$

2.3.3 Transmission Electron Microscopy (TEM) 60

2.3.4 Scanning Electron Microscope (SEM) 61

2.3.5 Physisorption of $\mathrm{N}_{2}$-BET $\quad 62$

2.3.6 Measurement of Catalytic Active Surface Area with H2 and CO Chemisorption 64

2.4 Mass spectrometer (MS) 65 
Chapter 3- Experimental: Materials, Catalyst Synthesis, MEA assembly and Instrumentation

$\begin{array}{ll}3.1 \text { Introduction } & 68\end{array}$

$\begin{array}{ll}3.2 \text { Materials } & 68\end{array}$

3.3 Synthesis of MWNT supported electrocatalysts $\quad 69$

3.3.1 Functionalization of the MWNT substrate 69

3.3.2 Pt deposition on functionalized MWCNT substrate $\quad 70$

$\begin{array}{ll}3.4 \text { Test bench and fuel cell reactor } & 71\end{array}$

3.5 Electrode fabrication and Membrane Electrode Assembly $\quad 72$

3.5.1 Electrodes' fabrication $\quad 72$

$\begin{array}{ll}\text { 3.5.2 Membrane electrode Assembly } & 73\end{array}$

$\begin{array}{ll}3.6 \text { Instrumentation } & 73\end{array}$

Chapter 4 - The role of phosphoric acid in the anodic electrocatalytic layer of HT$\begin{array}{ll}\text { PEM fuel cells } & 76\end{array}$

4.1 Introduction $\quad 76$

$\begin{array}{ll}4.2 \text { Experimental } & 78\end{array}$

$\begin{array}{ll}\text { 4.2.1 Materials } & 78\end{array}$

$\begin{array}{ll}\text { 4.2.2 Instrumentation } & 78\end{array}$

4.2.3 Electrode Preparation and MEA assembly $\quad 79$

4.2.4 Electrochemical characterization $\quad 80$

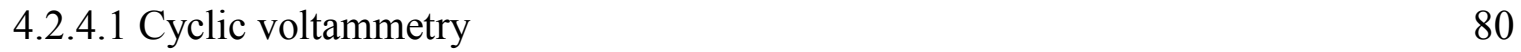

4.2.4.2 CO stripping voltammetry 81

$\begin{array}{ll}4.2 .5 \text { TEM analysis } & 82\end{array}$

4.2.6 XPS analysis $\quad 83$

4.2.6.1 Pt film preparation on polymer electrolyte for XPS experiments and SEM characterization $\quad 84$

4.2.6.2 Treatment of the Pt film surface in XPS chamber 85

4.3 Results and Discussion $\quad 86$

4.3.1 Determination of the electrochemically active surface area (ECSA) 86

4.3.2 Effect of H3PO4 loading on the anode 90

4.3.3 XPS measurements of the Pt/PA interface 102

4.3.4 The proposed mechanism 106

$\begin{array}{ll}4.4 \text { Conclusion } & 107\end{array}$

Chapter 5- Electrochemical characterization of the anodic catalyst layer employing Pt nanoparticles decorated on functionalized MWCNT in HTPEM fuel cells 112

$\begin{array}{ll}5.1 \text { Introduction } & 112\end{array}$

5.2 Experimental 114

$\begin{array}{ll}\text { 5.2.1 Materials } & 114\end{array}$

$\begin{array}{ll}\text { 5.2.2 Instrumentation } & 114\end{array}$

5.2.3 Catalyst film preparation on H3PO4 doped polymer electrolyte for XPS $\begin{array}{ll}\text { experiments } & 115\end{array}$

5.2.4 Electrode Preparation and MEA assembly 115

5.2.5 Electrochemical Characterization and Catalyst layer characterization $\quad 118$ 
5.2.5.2 Electrochemical Impedance Spectroscopy measurements 118

$\begin{array}{ll}\text { 5.2.5.3 CO stripping voltammetry } & 118\end{array}$

$\begin{array}{ll}\text { 5.2.6 TEM analysis } & 119\end{array}$

5.3 Results and discussion $\quad 119$

5.3.1 The effect of catalyst substrate on the anodic electrode's performance 119

5.3.2 Evaluation of ECSA and Pt utilization 131

5.3.3 Investigation of the interaction of PA with the pyridine groups in the CL by means of XPS

5.3.4 Kinetic of HOR

5.3 .5 Charge tras er resistance of the anodic electrode 136

5.3.5 Charge transfer resistance of the anodic electrode 139

5.3.6 Durability of electrocatalyst after short term operation 142

5.3.6.1 XPS measurements $\quad 142$

5.3.6.2. The effect of short term operation of the Pt particle size 143

5.3.6.3 Effect of PA on the Pt particle size during normal fuel cell operation $\quad 146$

5.3.6.4 Agglomeration mechanism of Pt particles on the ox.MWCNT-Py substrate 148

$\begin{array}{ll}5.4 \text { Conclusions } & 150\end{array}$

Chapter 6- The effect of H3PO4 loading and the electrocatalyst's substrate on the anode's performance under different reformate compositions

6.1 Introduction $\quad 155$

6.2 Experimental 157

$\begin{array}{ll}\text { 6.2.1 Materials } & 157\end{array}$

6.2.2 Instrumentation 157

6.2.3 Electrode Preparation and MEA assembly 157

6.2.4 Electrochemical Characterization $\quad 158$

6.3 Results 159

6.3.1 Fuel cell performance under various synthetic reformate conditions $\quad 160$

6.3.2. Effect of the PA loading in the catalyst layer 164

6.3.3 Voltage loss in the anodic electrodes under different fuel composition $\quad 170$

$\begin{array}{ll}\text { 6.3.4 AC Impedance Measurements } & 174\end{array}$

6.3.5 Stability test 180

6.3.6 Potential Oscillations under galvanostatic electrooxidation of $\mathrm{H} 2$ in reformate gas. $\quad 183$

6.4 Discussion 184

$\begin{array}{ll}6.5 \text { Conclusions } & 189\end{array}$

Chapter 7- Determination of the cathodic electrode's ECSA using CO as a probe molecule

$\begin{array}{ll}7.1 \text { Introduction } & 194\end{array}$

$\begin{array}{ll}7.2 \text { Experimental } & 195\end{array}$

$\begin{array}{ll}7.2 .1 \text { Materials } & 195\end{array}$

7.2.2 Instrumentation 195

7.2.3 Electrode Preparation and MEA assembly 196

$\begin{array}{ll}\text { 7.2.4 Electrochemical Characterization } & 196\end{array}$

$\begin{array}{ll}\text { 7.2.4.1 Cyclic Voltammetry } & 197\end{array}$ 
7.2.4.2 CO stripping voltammetry

7.2.4.3 CO bulk electroxidation (CO annealing) 197

$\begin{array}{ll}\text { 7.2.4.4 TEM } & 198\end{array}$

$\begin{array}{ll}7.3 \text { Results and discussion } & 198\end{array}$

$\begin{array}{ll}7.4 \text { Conclusion } & 209\end{array}$

Chapter 8 - Parametric Analysis: Optimization of cathodic catalyst layer of HTPEM fuel cells employing Pt nanoparticles decorated on functionalized MWCNT 212

$\begin{array}{ll}8.1 \text { Introduction } & 212\end{array}$

$\begin{array}{ll}8.2 \text { Experimental } & 214\end{array}$

$\begin{array}{ll}\text { 8.2.1 Materials } & 214\end{array}$

8.2.2 Instrumentation $\quad 215$

8.2.3 Electrode Preparation and MEA assembly 215

8.2.4 Electrochemical Characterization and Catalyst layer characterization $\quad 216$

8.2.4.1 Activation of MEA 216

8.2.4.2 Electrochemical Impedance Spectroscopy measurements 216

$\begin{array}{ll}\text { 8.2.4.3 CO stripping voltammetry } & 218\end{array}$

$\begin{array}{ll}\text { 8.2.4.4 TEM analysis } & 218\end{array}$

$\begin{array}{ll}8.3 \text { Results and discussion } & 219\end{array}$

8.3.1 Influence of PA amount in the CL on the fuel cell's performance 219

8.3.2 Influence of PA amount on the ECSA 229

8.3.3 Catalyst loading effect on MEA performance 231

8.3.4 Kinetics of $\mathrm{O} 2$ reduction reaction $\quad 235$

8.3.5 XPS post mortem analysis 241

8.3.6 Investigation of Pt particle size and distribution via TEM Imaging after short term operation 242

$\begin{array}{ll}8.4 \text { Conclusions } & 244\end{array}$

Chapter 9 - Summary and Future Work 249

9.1 Summary 249

9.2 Proposal for future work 251

\section{Appendix}

A1. Activation process of TPS $®$ based MEAs 253

B1. Polarizations curves under different fuel compositions -supporting information 258

$\begin{array}{ll}\text { C1 Greek abstract } & 264\end{array}$

D1. Curriculum Vitae 281 


\section{List of Figures}

Figure 1-1.Schematic model of a. the interaction of pyridine with $\mathrm{H}_{3} \mathrm{PO}_{4}$ and 24 b. the Pt/pyridine modified MWCNT

Figure 1-2. Schematic of basic principle of fuel cell operation

Figure 1-3.Cross section schematic of a cathodic electrode/membrane 27 interphase during operation.

Figure 1-4. Schematic and SEM micrographs showing gas diffusion layer, 30 (Toray TGP-H) graphite-fiber and polytetrafluoroethylene (PTFE) microstructure spanning approximately two orders of magnitude of length scale. Each scale plays a critical role in optimized gas and liquid transport.

Figure 1-5. PBI structure

Figure 1-6. General structure of pyridine based aromatic polyethers.

Figure 1-7. A simple flow field plate with a serpentine flow channel. Gas inlet 34 and outlet are located on the opposite face of the plate.

Figure 1-8. An exploded view of a unit cell. 1) MEA 2) PTL 3 ) Gasket 4) Flow field plates 5) Current collectors 6) Insulator 7) End plate.

Figure 1-9. Flow network model of GC with under rib convection effects: 37 definition of GC geometry and explanation of pressure drop.

Figure 1-10. Oxygen reduction mechanism on noble catalysts (Reprinted from Journal of Power Sources, 152(2), Wang Bin, Recent development of nonplatinum catalysts for oxygen reduction reaction, 1-15)

Figure 2-1. Basic diagram of a potentiostat/galvanostat.

Figure 2-2. An example of a cyclic voltamogram of a redox reversible system.

Figure 2-3. Equivalent circuit of a typical fuel cell at its simplest form.

Figure 2-4. A typical polarization curve of a fuel

Figure 2-5. Photoemission and the Auger process. Left: An incident X-ray photon is absorbed and a photoelectron emitted. Measurement of its kinetic energy allows the binding energy of the photoelectron to be calculated. The atom stays behind as an unstable ion with a hole in one of the core levels. Right: The excited ion relaxes by filling the core hole with an electron from a higher shell. The energy released by this transition is taken up by another electron, the Auger electron, which leaves the sample with an element-specific kinetic energy. In Auger spectroscopy a beam of energetic (2-5 keV) electrons creates the initial core holes.

Figure 2-6. X-rays scattered by atoms in an ordered lattice interfere constructively in directions given by Bragg's law. The angles of maximum intensity enable one to calculate the spacings between the lattice planes and allow furthermore for phase identification. Diffractograms are measured as a function of the angle $2 \theta$. When the sample is a polycrystalline powder, the diffraction pattern is formed by a small fraction of the particles only. Rotation of the sample during measurement enhances the number of particles that contribute to diffraction.

Figure 2-7. Schematic representation of typical MS operation

Figure 3-1. Schematic representation of the functionalized MWCNT substrates 
Figure 3-2. TEM micrographs a. 30wt\%Pt/oxMWCNT-Py and b. 71 30wt\%Pt/oxMWCNT

Figure 3-3. Schematic representation of the in-house test bench.

Figure 4-1. Schematic representation of the sample mounted on the heated 84 holder.

Figure 4-2. SEM image of Pt surface of the sample before use

Figure 4-3. (a) CO stripping voltammograms at various cell temperatures for constant water partial pressure of $10 \mathrm{kPa}(\mathrm{b})$ effect of temperature on $\mathrm{CO}$ uptake.

Figure 4-4. ECSA determination with increasing Pt loading based on CO stripping voltammetry measurements at $140^{\circ} \mathrm{C}$ (MEAs $1-5,7,12$ in Table 4-1).

Figure 4-5. CO stripping voltamograms, obtained after $\mathrm{CO}$ adsorption at open circuit potential and under polarization of the working electrode at $0.075 \mathrm{~V}$. Tcell $=140^{\circ} \mathrm{C}$.

Figure 4-6. Cyclic voltammograms (a) first cycle and (b) second cycle at $140^{\circ} \mathrm{C}$ after fuel cell operation at $180^{\circ} \mathrm{C}$ for $24 \mathrm{~h}$ at $0.2 \mathrm{~A} / \mathrm{cm}^{2}$ under pure $\mathrm{H}_{2} / \mathrm{O}_{2}$ flows $\left(\lambda_{\mathrm{H} 2}=1.2, \lambda_{\mathrm{O} 2}=2\right)$ for MEAs 1, 4, 6 and 7. Variation of the Pt loading with constant amount of PA on the catalytic layer.

Figure 4-7. Cyclic voltammograms, first cycle, at $140 \mathrm{oC}$ after fuel cell operation at $180 \mathrm{oC}$ for $24 \mathrm{~h}$ at $0.2 \mathrm{~A} / \mathrm{cm} 2$ under pure $\mathrm{H} 2 / \mathrm{O} 2$ flows $(\lambda \mathrm{H} 2=1.2$, $\lambda \mathrm{O} 2=2)$ for MEAs $13(2 \mathrm{gPA} / \mathrm{gPt})$ and MEA $15(6 \mathrm{gPA} / \mathrm{gPt})$.

Figure 4-8. Cyclic voltammograms, first (a) and second (b) cycle, at 140oC after fuel cell operation at $180 \mathrm{oC}$ for $24 \mathrm{~h}$ at $0.2 \mathrm{~A} / \mathrm{cm} 2$ under pure $\mathrm{H} 2 / \mathrm{O} 2$ flows $(\lambda \mathrm{H} 2=1.2, \lambda \mathrm{O} 2=2)$ for MEAs $10-12$, where the Pt loading per electrode varied and $\mathrm{gPA} / \mathrm{gPt}=2$.

Figure 4-9. Cyclic voltammograms (first cycle) of MEA 13 employing the commercial $30 \% \mathrm{wt} \mathrm{Pt} / \mathrm{C}$ catalyst at $140 \mathrm{oC}$ after continuous fuel cell operation at $180 \mathrm{oC}$ at $0.2 \mathrm{~A} / \mathrm{cm} 2$ under pure $\mathrm{H} 2 / \mathrm{O} 2$ flows $(\lambda \mathrm{H} 2=1.2, \lambda \mathrm{O} 2=2)$ for 2,3 and 8 days.

Figure 4-10. Cyclic voltammograms (first and second cycle) at $140^{\circ} \mathrm{C}$ and the corresponding $\mathrm{CO}_{2}$ signal as recorded from the on-line mass spectrometer at the outlet of the working electrode's compartment of (a) MEA 7 (1.71 gPA/gPt) and MEA $1(17.62 \mathrm{gPA} / \mathrm{gPt})$, after fuel cell operation at $180^{\circ} \mathrm{C}$ for $24 \mathrm{~h}$ at $0.2 \mathrm{~A} / \mathrm{cm}^{2}$ under pure $\mathrm{H}_{2} / \mathrm{O}_{2}$ flows $\left(\lambda_{\mathrm{H} 2}=1.2, \lambda_{\mathrm{O} 2}=2\right)$.

Figure 4-11. Polarization curves, IR drop corrected, after the break in period (24 $\mathrm{h}$ in operation at $0.2 \mathrm{~A} / \mathrm{cm} 2$ ) at $180 \mathrm{oC}$ before and after the cyclic voltammogram measurements for MEAs with different amount of sprayed PA at the anodic electrode under $\mathrm{H} 2$ and $\mathrm{O} 2$ flows $(200 \mathrm{cc} / \mathrm{min}$ respectively): (a) MEA 3 (5.42 gPA/gPt), (b) MEA 12 (2.00 gPA/gPt) and (c) MEA 8 (0 gPA/gPt). Black squares: before the $\mathrm{CV}$ and open circles: after the $\mathrm{CV}$.

Figure 4-12. Voltammograms of adsorbed $\mathrm{CO}$ electrooxidation (first and second cycle) at $140^{\circ} \mathrm{C}$ and the corresponding $\mathrm{CO}_{2}$ signal as recorded from the on-line mass spectrometer at the outlet of the working electrode's compartment, (a) before $\mathrm{CV}$ and (b) after $\mathrm{CV}$ for the anodic electrode of MEA 9 after fuel cell operation at $180^{\circ} \mathrm{C}$ for $24 \mathrm{~h}$ at $0.2 \mathrm{~A} / \mathrm{cm}^{2}$ under pure $\mathrm{H}_{2} / \mathrm{O}_{2}$ flows $\left(\lambda_{\mathrm{H} 2}=1.2, \lambda_{\mathrm{O} 2}\right.$ $=2)$. 
Figure 4-13. Polarization curves recorded after $24 \mathrm{~h}$ of operation at $0.2 \mathrm{~A} / \mathrm{cm} 2$, 100 IR drop corrected, at $180^{\circ} \mathrm{C}$ for MEAs with different Pt loading and amount of sprayed PA at the anodic electrode under $\mathrm{H}_{2}$ and $\mathrm{O}_{2}$ flows $(200 \mathrm{cc} / \mathrm{min}$ respectively). MEA 1: $0.2 \mathrm{mgPt} / \mathrm{cm}^{2}$ and $17.62 \mathrm{gPA} / \mathrm{gPt}$ and MEA 7: 2.06 $\mathrm{mgPt} / \mathrm{cm}^{2}$ and $1.71 \mathrm{gPA} / \mathrm{gPt}$.

Figure 4-14. $\mathrm{CO}$ stripping experiments at $140^{\circ} \mathrm{C}$ of MEA 14 employing $30 \% \mathrm{wt}$ $\mathrm{Pt} / \mathrm{C}$. Before each measurement the working electrode was pre-exposed at OCP and $180^{\circ} \mathrm{C}$ for 18 hours under dry $\mathrm{H}_{2}$, dry $\mathrm{Ar}, 10.5 \mathrm{kPa} \mathrm{H} \mathrm{H}_{2} \mathrm{O} / \mathrm{Ar}$ and $10.5 \mathrm{kPa}$ $\mathrm{H}_{2} \mathrm{O} / \mathrm{H}_{2}$ flows, while the counter electrode was fed with $\mathrm{Ar} / \mathrm{H}_{2} \mathrm{O}$.

Figure 4-15.XP spectra of $\mathrm{Pt} 4 \mathrm{f}$ (a) and $\mathrm{P} 2 \mathrm{p}$ (b), after heating at $200^{\circ} \mathrm{C}$ for $4 \mathrm{~h}$ in $\mathrm{H}_{2}$ flow (1), after heating at $180^{\circ} \mathrm{C}$ for $1.5 \mathrm{~h}$ in $4 \% \mathrm{H}_{2} \mathrm{O} / \mathrm{He}$ flow (2), after heating at $200^{\circ} \mathrm{C}$ for $4 \mathrm{~h}$ in UHV (3) and after heating at $200^{\circ} \mathrm{C}$ for $4 \mathrm{~h}$ in $\mathrm{CO}$ flow (4).

Figure 4-16. XP spectra of $\mathrm{O} 1 \mathrm{~s}$ peaks after heating at $200^{\circ} \mathrm{C}$ for $4 \mathrm{~h}$ in $\mathrm{H}_{2}$ flow (1), at $180^{\circ} \mathrm{C}$ for $1.5 \mathrm{~h}$ in $4 \% \mathrm{H}_{2} \mathrm{O} / \mathrm{He}$ flow (2), at $200^{\circ} \mathrm{C}$ for $4 \mathrm{~h}$ in $\mathrm{UHV}$ (3) and at $200^{\circ} \mathrm{C}$ for $4 \mathrm{~h}$ in $\mathrm{CO}$ flow (4).

Figure 4-17. Intensity ratio $\mathrm{P} 2 \mathrm{p} / \mathrm{Pt} 4 \mathrm{f}$ and $\mathrm{O} 1 \mathrm{~s} / \mathrm{Pt} 4 \mathrm{f}$ after annealing at $200^{\circ} \mathrm{C}$ for 4h in $\mathrm{H}_{2}$ flow, at $180^{\circ} \mathrm{C}$ for $1.5 \mathrm{~h}$ in $4 \% \mathrm{H}_{2} \mathrm{O} / \mathrm{He}$ flow, at $200^{\circ} \mathrm{C}$ for $4 \mathrm{~h}$ in UHV and at $200^{\circ} \mathrm{C}$ for $4 \mathrm{~h}$ in $\mathrm{CO}$ flow.

Figure 5-1. The effect of catalyst substrate on the fuel cell performance. Polarization curves, IR drop corrected, were acquired under pure $\mathrm{H}_{2}$ and $\mathrm{O}_{2}$ with a gas stoichiometry of $\lambda_{\mathrm{H} 2}=1.2$ and $\lambda_{\mathrm{O} 2}=2$ at $180^{\circ} \mathrm{C}$. MEA $4: 1.26 \mathrm{mgPt} / \mathrm{cm}^{2}$ and $2 \mathrm{gPA} / \mathrm{gPt}$ employing 30wt\%Pt/oxMWCNT-Py and MEA 22: $1.3 \mathrm{mgPt} / \mathrm{cm}^{2}$ and $2 \mathrm{gPA} / \mathrm{gPt}$ employing $30 \mathrm{wt} \% \mathrm{Pt} / \mathrm{C}$.

Figure 5-2. AC impedance spectra of MEA $4: 1.26 \mathrm{mgPt} / \mathrm{cm} 2$ and $2 \mathrm{gPA} / \mathrm{gPt}$ employing 30wt $\% \mathrm{Pt} / \mathrm{oxMWCNT}$-Py and MEA $22: 1.3 \mathrm{mgPt} / \mathrm{cm} 2$ and $2 \mathrm{gPA} / \mathrm{gPt}$ employing $30 \mathrm{wt} \% \mathrm{Pt} / \mathrm{C}$. The data were obtained at $0.2 \mathrm{~A} / \mathrm{cm}^{2}$ and at $180^{\circ} \mathrm{C}$ under constant high gas flow of pure $\mathrm{H}_{2}$ and $\mathrm{O}_{2}(200 \mathrm{cc} / \mathrm{min}$ respectively). a. Nyquist plot and $\mathrm{b}$. Bode plot

Figure 5-3. The effect of the anodic electrode's specification (Pt loading varies whereas the PA amount per electrode is kept constant) on the overall fuel cell performance. Polarization curves of MEA 7,8,11 and 13 employing $30 \mathrm{wt} \% \mathrm{Pt} / \mathrm{oxMWCNT}-\mathrm{Py}$, IR drop corrected, acquired under pure $\mathrm{H}_{2}$ and $\mathrm{O}_{2}$ and gas stoichiometry of $\lambda_{\mathrm{H} 2}=1.2$ and $\lambda_{\mathrm{O} 2}=2$ at $180^{\circ} \mathrm{C}$.

Figure 5-4. AC impedance spectra of MEAs employing 30wt\% $\mathrm{Pt} / \mathrm{oxMWCNT}$ Py at the anodic electrode, where the Pt and PA loadings differentiate. MEA 7: $0.2 \mathrm{mgPt} / \mathrm{cm}^{2}$ and $17.62 \mathrm{gPA} / \mathrm{gPt}$ and MEA $13: 2.06 \mathrm{mgPt} / \mathrm{cm}^{2}$ and $1.71 \mathrm{gPA} / \mathrm{gPt}$. The data were obtained at $0.2 \mathrm{~A} / \mathrm{cm}^{2}$ and at $180^{\circ} \mathrm{C}$ under constant gas flow of pure $\mathrm{H}_{2}$ and $\mathrm{O}_{2}(200 \mathrm{cc} / \mathrm{min}$ respectively). a. Nyquist plot and b. Bode plot

Figure 5-5. The effect of the gas stoichiometry on the AC impedance spectra of MEA employing 30wt\%Pt/oxMWCNT-Py at the anodic electrode, where Pt and PA loadings (gPA/gPt) differentiate. MEA 7: $0.2 \mathrm{mgPt} / \mathrm{cm} 2$ and $17.6 \mathrm{gPA} / \mathrm{gPt}$ and MEA $11: 1.25 \mathrm{mgPt} / \mathrm{cm} 2$ and $2 \mathrm{gPA} / \mathrm{gPt}$. The data were obtained at $0.2 \mathrm{~A} / \mathrm{cm}^{2}$ and at $180^{\circ} \mathrm{C}$. a. Nyquist plot and b. Bode plot

Figure 5-6. The effect of gas stoichiometry on the overall fuel cell performance of MEAs where Pt loading varied whereas the PA amount per electrode is kept constant at the anodic electrode. Polarization curves of MEA 7 and 13 
employing 30wt\%Pt/oxMWCNT-Py, IR drop corrected, acquired under pure $\mathrm{H}_{2}$ and $\mathrm{O}_{2}$ and gas stoichiometry of $\lambda_{\mathrm{H} 2}=1.2$ and $\lambda_{\mathrm{O} 2}=2$ and under constant $200 \mathrm{cc} / \mathrm{min} \mathrm{H}_{2} / \mathrm{O}_{2}$ at $180^{\circ} \mathrm{C}\left(\lambda_{\mathrm{H} 2}>15\right)$.

Figure 5-7. Voltage loss with respect to the applied current density due to dominating mass transport resistance as a result of different catalyst layer thickness for anodic electrodes employing 30wt\%Pt/oxMWCNT-Py (MEA 7 and 13 ) and $30 \mathrm{wt} \% \mathrm{Pt} / \mathrm{C}$ (MEA 22).

Figure 5-8. The effect of $\mathrm{PA}$ on anodic electrode with low Pt loadings. Polarization curves, IR drop corrected obtained at $\mathrm{T}_{\text {cell }}=180^{\circ} \mathrm{C}$ and under low gas stoichiometry $\lambda_{\mathrm{H} 2}=1.2$ and $\lambda_{\mathrm{O}_{2}}=2$. The MEAs employed 30wt\%Pt/oxMWCNT-Py, MEA 1: 0.29 and 2gPA/gPt, MEA 7: 0.2mgPt/cm and $17.6 \mathrm{gPA} / \mathrm{gPt}$. For comparison MEA 23 employing $30 \mathrm{wt} \% \mathrm{Pt} / \mathrm{C}$ is used: $1.55 \mathrm{mgPt} / \mathrm{cm} 2$ and $2 \mathrm{gPA} / \mathrm{gPt}$.

Figure 5-9. ECSA $\left(\mathrm{m}^{2} / \mathrm{gPt}\right)$ determination via CO stripping voltammetry measurements at $140^{\circ} \mathrm{C}$ with respect to the Pt loading of the anodic electrode employing different electrocatalysts : MEAs 30\% Pt/(ox.MWCNT)-Py, MEAs $30 \% \mathrm{Pt} / \mathrm{ox} . \mathrm{MWCNT}$ and MEAs $30 \% \mathrm{Pt} / \mathrm{C}$ (all listed in Table 5-1).

Figure 5-10. ECSA determination with increasing $\mathrm{Pt}$ loading based on $\mathrm{CO}$ stripping voltammetry measurements at $140^{\circ} \mathrm{C}$, for different $\mathrm{Pt}$ and $\mathrm{PA}$ loadings (MEAs 1-6 and MEA 7-13 in Table 5-1).

Figure 5-11. XPS spectra of 5wt\%Pt/oxMWCNT-Py in the presence and absent of PA in the catalyst layer.

Figure 5-12. The effect of $\mathrm{H}_{2}$ partial pressure on the fuel cell's performance of MEA 4. The polarization curves were IR drop corrected and were acquired under constant total flow of $200 \mathrm{cc} / \mathrm{min}$ of $\mathrm{H}_{2}$ balanced with $\mathrm{Ar}$ and $200 \mathrm{cc} / \mathrm{min}$ $\mathrm{O}_{2}$ at $180^{\circ} \mathrm{C}$. MEA 4 : employed 30wt\%Pt/oxMWCNT-Py, $1.26 \mathrm{mgPt} / \mathrm{cm} 2$ and $2 \mathrm{gPA} / \mathrm{gPt}$.

Figure 5-13. Impedance spectra of MEA 4 obtained at a current density of $0.2 \mathrm{~A} / \mathrm{cm}^{2}$ at $180^{\circ} \mathrm{C}$, under various $\mathrm{H}_{2}$ partial pressure (total flow $200 \mathrm{cc} / \mathrm{min}$ of $\mathrm{H}_{2}$ in $\left.\mathrm{Ar}\right)$. The cathodic electrode was fed with a redundant $\mathrm{O}_{2}$ flow $(200 \mathrm{cc} / \mathrm{min})$. a. Niquist plots and b. Bode plots

Figure 5-14. Polarization resistance of the anodic and cathodic electrode as a function of the applied current. Both MEAs employed 30wt $\% \mathrm{Pt} / \mathrm{oxMWCNT}$ $\mathrm{Py}$ at the anodic electrode and 30wt\% Pt/C at the cathode (MEA 7 and MEA 13).

Figure 5-15. AC impedance spectra of MEA 13 obtained at different current densities at $180^{\circ} \mathrm{C}$, under constant flow $200 \mathrm{cc} / \mathrm{min}$ of $\mathrm{H}_{2}$. The cathodic electrode was fed with a redundant $\mathrm{O}_{2}$ flow $(200 \mathrm{cc} / \mathrm{min})$. a. Nyquist plots and b. Bode plots

Figure 5-16. AC impedance spectra of MEA13 obtained at different current densities at $180^{\circ} \mathrm{C}$, under constant flow 200cc/min of $\mathrm{H}_{2}$ and $\mathrm{O}_{2}$, respectively. Nyquist plots and $b$. Bode plots

Figure 5-17 : TEM images and the corresponding Pt size distribution histogram after 6 days of continuous operation. a. $30 \mathrm{wt} \% \mathrm{Pgt} / \mathrm{C}$ and b. $30 \% \mathrm{Pt} / \mathrm{oxMWNT}-$ Py 
Figure 5-18 . The effect of reducing atmosphere $5 \% \mathrm{H} 2 / \mathrm{Ar}$ on the Pt particle size as a function of time. The XRD diffractograms of 30wt $\% \mathrm{Pt} / \mathrm{C}$ and $30 \mathrm{wt} \%$ $\mathrm{Pt} /$ oxMWCNT-Py were acquired at $180^{\circ} \mathrm{C}$, with $20 \mathrm{~min}$ acquisition time.

Figure 5-19. The effect of $\mathrm{PA}$ on the Pt particle size after 8 days of continuous operation. The average Pt particle size was measured by TEM and XRD.

Figure 5-20. TEM images after 8 days of continuous operation. a. MEA 11: $0.2 \mathrm{mgPt} / \mathrm{cm}^{2}$ and $17.6 \mathrm{mgPt} / \mathrm{cm}^{2}$ and $\mathrm{b}$. MEA $7: 1.25 \mathrm{mgPt} / \mathrm{cm}^{2}$ and $2.8 \mathrm{gPA} / \mathrm{gPt}$ Figure 5-21. TEM micrographs and the corresponding $\mathrm{Pt}$ size distribution histogram after 8 days of continuous operation. a. MEA 11: $0.2 \mathrm{mgPt} / \mathrm{cm}^{2}$ and $17.6 \mathrm{mgPt} / \mathrm{cm}^{2}$ and b. MEA 7: $1.25 \mathrm{mgPt} / \mathrm{cm}^{2}$ and $2.8 \mathrm{gPA} / \mathrm{gPt}$

Figure 5-22. The effect of the PA amount present in the anodic electrode on $\mathrm{Pt}$ size distribution of $30 \mathrm{wt} \% \mathrm{Pt} / \mathrm{oxMWNT}-\mathrm{Py}$, after 8 days of continuous fuel cell operation.

Figure 6-1. The effect of the catalyst substrate on the anode's performance under synthetic reformate gas consisting of $50.7 \mathrm{kPa} \mathrm{H}, 2 \mathrm{kPa} \mathrm{CO}$ and $33.5 \mathrm{kPa}$ $\mathrm{H}_{2} \mathrm{O}$ balanced with Ar. The polarization curves were obtained using a stoichiometry of $\lambda_{\mathrm{H} 2}=1.2$ and $\lambda_{\mathrm{O} 2}=2$, pure $\mathrm{O}_{2}$ was used at the cathode. The PA loading at the anodic electrode was $2 \mathrm{gPA} / \mathrm{gPt}$ and cell's temperature was kept at $180^{\circ} \mathrm{C}: 30 \mathrm{wt} \% \mathrm{Pt} /(\mathrm{ox} . \mathrm{MWNT})-\mathrm{Py}$ (MEA 1), 30wt\%Pt/oxMWCNT (MEA 7) and $30 \mathrm{wt} \% \mathrm{Pt} / \mathrm{C}$ (MEA 11).

Figure 6-2. The effect of the noble metal loading on the anode's performance under synthetic reformate gas consisting of $50.7 \mathrm{kPa} \mathrm{H}, 2 \mathrm{kPa} \mathrm{CO}$ and $33.5 \mathrm{kPa}$ $\mathrm{H}_{2} \mathrm{O}$ balanced with Ar. The polarization curves were obtained using a stoichiometry of $\lambda_{\mathrm{H} 2}=1.2$ and $\lambda_{\mathrm{O} 2}=2$, pure $\mathrm{O}_{2}$ was used at the cathode. The PA loading at the anodic electrode was $2 \mathrm{gPA} / \mathrm{gPt}$ and cell's temperature was kept at $180^{\circ} \mathrm{C}$. The anodic electrode employed 30wt\% Pt/oxMWCNT-Py (MEA 1-3). Figure 6-3. The effect of the gas stoichiometry on the MEAs performance under synthetic reformate feed. Polarization curves of MEA 1 and 10 (PA loading $=2 \mathrm{gPA} / \mathrm{gPt}$ ) were acquired at $180^{\circ} \mathrm{C}$ under various total flow rates of synthetic reformate gas mixture, $50.7 \mathrm{kPa} \mathrm{H}, 33.5 \mathrm{kPa} \mathrm{H}_{2} \mathrm{O}$ and $2 \mathrm{kPa} \mathrm{CO}$ balanced with Ar. Normal flows: stoichiometry of $\lambda_{\mathrm{H} 2}=1.2$ and $\lambda_{\mathrm{O} 2}=2$ and high flows: constant $200 \mathrm{cc} / \mathrm{min}$ total flow of reformate gas and $\mathrm{O}_{2}$, which corresponds to a nominal limiting current density of $3.5 \mathrm{~A} / \mathrm{cm}^{2}$.

Figure 6-4. The effect of the steam's partial pressure in the $\mathrm{H}_{2}$ feed on the cell's voltage. The values were obtained at $180^{\circ} \mathrm{C}$ and $0.4 \mathrm{~A} / \mathrm{cm}^{2}$ from MEAs employing 30\% Pt/(ox.MWNT)-Py, 30\% Pt/oxMWCNT and 30\%Pt/C (MEAs 1,7 and 12), while the anode was fed with a $\mathrm{H}_{2} / \mathrm{H}_{2} \mathrm{O}$ mixture $\left(\lambda_{\mathrm{H} 2}>15\right)$ and cathode with pure $\mathrm{O}_{2}\left(\lambda_{\mathrm{O} 2}>30\right), 200 \mathrm{cc} / \mathrm{min}$ respectively.

Figure 6-5. Polarization curves obtained under synthetic reformate fed $(50.7 \mathrm{kPa}$ $\mathrm{H}_{2}, 33.5 \mathrm{kPa} \mathrm{H} \mathrm{H}_{2} \mathrm{O}$ and $2 \mathrm{kPa} \mathrm{CO}$ balanced with Ar.) with gas stoichiometry of $\lambda_{\mathrm{H} 2}=1.2$ and $\lambda_{\mathrm{O} 2}=2$. The PA loading at the anodic electrode was $0.5 \mathrm{gPA} / \mathrm{gPt}$ and cell's temperature was kept at $180^{\circ} \mathrm{C}$.T MEAs used: $30 \% \mathrm{Pt} /(\mathrm{ox} . \mathrm{MWNT})-\mathrm{Py}$ (MEA 6), 30\%Pt/oxMWCNT( MEA 9) and 30\%Pt/C( MEA 13 and 15)

Figure 6-6. The effect of the steam's partial pressure in the $\mathrm{H}_{2}$ feed on the cell's voltage. The values were obtained at $180^{\circ} \mathrm{C}$ and $0.4 \mathrm{~A} / \mathrm{cm}^{2}$ from MEAs employing 30\%Pt/(ox.MWNT)-Py, 30\%Pt/oxMWCNT and 30\% Pt/C (MEAs 
6,9 and 13), while the anode was fed with a $\mathrm{H}_{2} / \mathrm{H}_{2} \mathrm{O}$ mixture $\left(\lambda_{\mathrm{H} 2}>15\right)$ and cathode with pure $\mathrm{O}_{2}\left(\lambda_{\mathrm{O} 2}>30\right), 200 \mathrm{cc} / \mathrm{min}$ respectively. The PA loading at the anode was $0.5 \mathrm{gPA} / \mathrm{gPt}$ for all cases.

Figure 6-7. Polarization curves obtained at $180^{\circ} \mathrm{C}$ under $\mathrm{H}_{2}$ and $\mathrm{O}_{2}$ stoichiometry of 1.2 and 2 respectively. The composition of the gas mixture was $50.7 \mathrm{kPa} \mathrm{H} 2$ and $2 \mathrm{kPa} \mathrm{CO}$, while the steam partial pressure was altered from 0 to $33.5 \mathrm{kPa}$ and balanced with Ar. Both anodic electrodes had $0.5 \mathrm{gPA} / \mathrm{gPt}$. a. MEA 14: employing $0.7 \mathrm{mgPt} / \mathrm{cm}^{2} 30 \mathrm{wt} \% \mathrm{Pt} / \mathrm{C}$ and $\mathrm{b}$. MEA 5: employing $0.68 \mathrm{mgPt} / \mathrm{cm}^{2} 30 \mathrm{wt} \% \mathrm{Pt} / \mathrm{ox} . \mathrm{MWCNT}-\mathrm{Py}$.

Figure 6-8. Polarization curves obtained at $180^{\circ} \mathrm{C}$ under $\mathrm{H}_{2}$ and $\mathrm{O}_{2}$ stoichiometry of 1.2 and 2 respectively. The composition of the gas mixture was $50.7 \% \mathrm{H} 2$ and $2 \% \mathrm{CO}$, while the steam partial pressure was altered from 0 to $33.5 \mathrm{kPa}$ and balanced with Ar. The anodic electrode employed $30 \mathrm{wt} \%$ Pt/oxMWCNT-Py, MEA 4: $0.47 \mathrm{mgPt} / \mathrm{cm}^{2}$ and $0.5 \mathrm{gPA} / \mathrm{gPt}$.

Figure 6-9. Voltage loss, IR drop corrected, at $180^{\circ} \mathrm{C}$ for MEAs employing a. 30wt \% Pt/ox.MWCNT MEA 7: $0.77 \mathrm{mgPt} / \mathrm{cm}^{2}$ and b. 30wt \% Pt/ox.MWCNTPy MEA 1: $0.77 \mathrm{mgPt} / \mathrm{cm}^{2}$, under various fuel composition and pure $\mathrm{O}_{2}$ flows (200cc/min respectively): Black square: $50.7 \mathrm{kPaH}_{2}$ balanced with $\mathrm{Ar}$, open circle: $50.7 \mathrm{kPa} \mathrm{H} 2$ and $2 \mathrm{kPa} \mathrm{CO}$ balanced with $\mathrm{Ar}$, and black triangle : $50.7 \mathrm{kPa}$ $\mathrm{H} 2,2 \mathrm{kPa} \mathrm{CO}$ and $33.5 \mathrm{kPa} \mathrm{H}_{2} \mathrm{O}$ balanced with $\mathrm{Ar}$. The PA loading was 2 $\mathrm{gPA} / \mathrm{gPt}$ at the anodic electrode.

Figure 6-10. Voltage loss, IR drop corrected, at $180^{\circ} \mathrm{C}$ for MEAs employing a. 30wt\% Pt/oxMWNT, MEA 9: $0.79 \mathrm{mgPt} / \mathrm{cm}^{2}$ and b. 30wt\% Pt/(ox.MWCNT)Py MEA 6: $0.82 \mathrm{mgPt} / \mathrm{cm}^{2}$, under various fuel composition and pure $\mathrm{O}_{2}$ flows (200cc/min respectively): Black square: $50.7 \mathrm{kPaH}_{2}$ balanced with $\mathrm{Ar}$, open circle: $50.7 \mathrm{kPa} \mathrm{H} 2$ and $2 \mathrm{kPa} \mathrm{CO}$ balanced with $\mathrm{Ar}$, and black triangle : $50.7 \mathrm{kPa}$ $\mathrm{H} 2,2 \mathrm{kPa} \mathrm{CO}$ and $33.5 \mathrm{kPa} \mathrm{H} \mathrm{H}_{2} \mathrm{O}$ balanced with $\mathrm{Ar}$. The PA loading was 0.5 $\mathrm{gPA} / \mathrm{gPt}$ at the anodic electrode.

Figure 6-11. AC impedance plots a. Nyquist and b. Bode, obtained at a current of $0.2 \mathrm{~A} / \mathrm{cm}^{2}$ at $180^{\circ} \mathrm{C}$, under various fuel compositions and pure $\mathrm{O}_{2}$ flows (200cc/min fuel mixture $/ \mathrm{O}_{2}$ ). Black square: $50.7 \mathrm{kPa} \mathrm{H}$ balanced with Ar, open circle: $50.7 \mathrm{kPaH}_{2}$ and $2 \mathrm{kPa} \mathrm{CO}$ balanced with $\mathrm{Ar}$, and black triangle: $50.7 \mathrm{kPa}$ $\mathrm{H}_{2}, 2 \mathrm{kPa} \mathrm{CO}$ and $33.5 \mathrm{kPa} \mathrm{H} \mathrm{H}_{2} \mathrm{O}$ balanced with Ar. MEA 1 employing $30 \mathrm{wt} \%$ $\mathrm{Pt} /\left(\right.$ ox.MWCNT)-Py: 0.77mgPt/ $\mathrm{cm}^{2}$ and $2 \mathrm{gPA} / \mathrm{gPt}$.

Figure 6-12. AC impedance plots a. Nyquist and b. Bode, obtained at a current of $0.2 \mathrm{~A} / \mathrm{cm}^{2}$ at $180^{\circ} \mathrm{C}$, under various fuel compositions and pure $\mathrm{O}_{2}$ flows (200cc/min fuel mixture $/ \mathrm{O}_{2}$ ). Black square: $50.7 \mathrm{kPa} \mathrm{H}$ balanced with Ar, open circle: $50.7 \mathrm{kPaH}_{2}$ and $2 \mathrm{kPa} \mathrm{CO}$ balanced with $\mathrm{Ar}$, and black triangle: $50.7 \mathrm{kPa}$ $\mathrm{H}_{2}, 2 \mathrm{kPa} \mathrm{CO}$ and $33.5 \mathrm{kPa} \mathrm{H}_{2} \mathrm{O}$ balanced with Ar. MEA 7 employing30wt\% Pt/oxMWNT: $0.77 \mathrm{mgPt} / \mathrm{cm}^{2}$ and $2 \mathrm{gPA} / \mathrm{gPt}$.

Figure 6-13. AC impedance plots a. Nyquist and b. Bode, obtained at a current of $0.2 \mathrm{~A} / \mathrm{cm}^{2}$ at $180^{\circ} \mathrm{C}$, under various fuel compositions and pure $\mathrm{O}_{2}$ flows (200cc/min fuel mixture $/ \mathrm{O}_{2}$ ). Black square: $50.7 \mathrm{kPa} \mathrm{H}$ balanced with Ar, open circle: $50.7 \mathrm{kPaH}_{2}$ and $2 \mathrm{kPa} \mathrm{CO}$ balanced with $\mathrm{Ar}$, and black triangle : $50.7 \mathrm{kPa}$ $\mathrm{H}_{2}, 2 \mathrm{kPa} \mathrm{CO}$ and $33.5 \mathrm{kPa} \mathrm{H}_{2} \mathrm{O}$ balanced with Ar. MEA 6 employing30wt\% Pt/(ox.MWCNT)-Py: $0.82 \mathrm{mgPt} / \mathrm{cm}^{2}$ and $0.5 \mathrm{gPA} / \mathrm{gPt}$. 
Figure 6-14. AC impedance plots a. Nyquist and b. Bode, obtained at a current of $0.2 \mathrm{~A} / \mathrm{cm}^{2}$ at $180^{\circ} \mathrm{C}$, under various fuel compositions and pure $\mathrm{O}_{2}$ flows (200cc/min fuel mixture $/ \mathrm{O}_{2}$ ). Black square: $50.7 \mathrm{kPa} \mathrm{H}_{2}$ balanced with Ar, open circle: $50.7 \mathrm{kPa} \mathrm{H}$ and $2 \mathrm{kPa} \mathrm{CO}$ balanced with $\mathrm{Ar}$, and black triangle: $50.7 \mathrm{kPa}$ $\mathrm{H}_{2}, 2 \mathrm{kPa} \mathrm{CO}$ and $33.5 \mathrm{kPa} \mathrm{H}_{2} \mathrm{O}$ balanced with Ar. MEA9 employing $30 \mathrm{wt} \% \mathrm{Pt} / \mathrm{oxMWNT}$ : $0.79 \mathrm{mgPt} / \mathrm{cm}^{2}$ and $0.5 \mathrm{gPA} / \mathrm{gPt}$.

Figure 6-15. Short term stability experiment using synthetic reformate gas at $0.2 \mathrm{~A} / \mathrm{cm}^{2}\left(\lambda_{\mathrm{H} 2}=3\right.$ and $\left.\lambda_{\mathrm{O} 2}=5\right)$ and $180^{\circ} \mathrm{C}$. The amount of PA in the CL was $2 \mathrm{gPA} / \mathrm{gPt}$ and the MEA that were tested: MEA 2: 30wt\%Pt/oxMWNT-Py and $0.87 \mathrm{mgPt} / \mathrm{cm}^{2}$, MEA 8: 30wt $\% \mathrm{Pt} / \mathrm{oxMNWT}$ and $0.875 \mathrm{mgPt} / \mathrm{cm}^{2}$ and MEA 10: $30 \mathrm{wt} \% \mathrm{Pt} / \mathrm{C}$ and $1.42 \mathrm{mgPt} / \mathrm{cm}^{2}$.

Figure 6-16. Short term stability experiment using synthetic reformate gas, consisting of $50.7 \mathrm{kPa} \mathrm{H}, 33.5 \mathrm{kPa} \mathrm{H}_{2} \mathrm{O}$ and $2 \mathrm{kPa} \mathrm{CO}$ balanced with Ar, at $0.4 \mathrm{~A} / \mathrm{cm}^{2}\left(\lambda_{\mathrm{H} 2}=1.5\right.$ and $\left.\lambda_{\mathrm{O} 2}=2.5\right)$ and $180^{\circ} \mathrm{C}$. The amount of PA in the CL was $0.5 \mathrm{gPA} / \mathrm{gPt}$ and the MEA that were tested: MEA 6: 30wt\%Pt/oxMWNT-Py and $0.82 \mathrm{mgPt} / \mathrm{cm} 2$, MEA 9: 30wt\%Pt/oxMNWT and $0.79 \mathrm{mgPt} / \mathrm{cm} 2$ and MEA 13 : $30 \mathrm{wt} \% \mathrm{Pt} / \mathrm{C}$ and $1.6 \mathrm{mgPt} / \mathrm{cm}^{2}$.

Figure 6-17. Short term stability experiment using synthetic reformate gas (consisting of $50.7 \mathrm{kPa}$ of $\mathrm{H} 2,2 \mathrm{kPa}$ of $\mathrm{CO}$ and $14.1 \mathrm{kPa}$ of $\mathrm{H}_{2} \mathrm{O}$ balanced with Ar) at $0.4 \mathrm{~A} / \mathrm{cm}^{2}\left(\lambda_{\mathrm{H} 2}=1.5\right.$ and $\left.\lambda_{\mathrm{O} 2}=2.5\right)$ and $180^{\circ} \mathrm{C}$. The amount of PA in the CL was $0.5 \mathrm{gPA} / \mathrm{gPt}$ and the MEAs that were tested: MEA $5: 30 \mathrm{wt} \% \mathrm{Pt} / \mathrm{oxMWNT}$ Py and $0.68 \mathrm{mgPt} / \mathrm{cm}^{2}$ and MEA $14: 30 \mathrm{wt} \% \mathrm{Pt} / \mathrm{C}$ and $0.7 \mathrm{mgPt} / \mathrm{cm}^{2}$.

Figure 6-18. Polarization curve (MEA 1) obtained under synthetic reformate gas consisting of $50.7 \mathrm{kPa}$ of $\mathrm{H}_{2}, 33.5 \mathrm{kPa}$ of $\mathrm{H}_{2} \mathrm{O}$ and $2 \mathrm{kPa} \mathrm{CO}$ balanced with $\mathrm{Ar}$, and a stoichiometry of $\lambda_{\mathrm{H} 2}=1.2$ and $\lambda_{\mathrm{O} 2}=2$.

Figure 6-19. The effect of the $\mathrm{H}_{2}$ partial pressure on the polarization resistance at $0.2 \mathrm{~A} / \mathrm{cm}^{2}$. AC impedance spectra obtained at $180^{\circ} \mathrm{C}$, under various fuel compositions $\left(100 \mathrm{kPaH}_{2}-20 \mathrm{kPa} \mathrm{H}_{2}\right.$ balanced with $\left.\mathrm{Ar}\right)$. The cathodic electrode was fed with a redundant $\mathrm{O}_{2}$ flow. a. Niquist plots and $b$. corresponding Bode plots

Figure 6-20. Schematic representation of the structure of the electrochemical interface for the case of $30 \mathrm{wt} \% \mathrm{Pt} / \mathrm{C}$, (a) under dry conditions (thin film formation), (b) under high humidity (ganglia formation).

Figure 6-21. Schematic representation of the structure of the electrochemical interface ,for the case of 30wt\%Pt/oxMWCNT-Py , (a) under dry conditions (thin film formation), (b) under high humidity (displacement of PA).

Figure 7-1. Cyclic voltamograms a. first and second $\mathrm{CO}$ adsorption under $0.1 \mathrm{~V}$ for $20 \mathrm{~min}$ and $\mathrm{b}$. the reference baseline where the electrode was polarized at $0.1 \mathrm{~V}$ for $20 \mathrm{~min}$ in the absence of CO.

Figure 7-2. TEM micrograph and the corresponding $\mathrm{Pt}$ size distribution histogram of $30 \mathrm{wt} \% \mathrm{Pt} / \mathrm{C}$ obtained after the activation period: a. before $\mathrm{CO}$ stripping and $\mathrm{b}$. after 2 sequential $\mathrm{CO}$ stripping experiments, where $\mathrm{CO}$ was adsorbed under polarization $0.1 \mathrm{~V}$. The Pt particle size distribution is included as an inset.

Figure 7-3. The effect of ECSA evaluation procedure on cathodic electrode performance. IR drop corrected polarization curve obtained at $180^{\circ} \mathrm{C}$ and under 
pure $\mathrm{H}_{2}$ and $\mathrm{O}_{2}$ (stoichiometry $\lambda_{\mathrm{H} 2}=1.2$ and $\lambda_{\mathrm{O} 2}=2$ ). MEA: $30 \mathrm{wt} \% \mathrm{Pt} / \mathrm{C}$ $0.6 \mathrm{mgPt} / \mathrm{cm}^{2}$ and $2 \mathrm{gPA} / \mathrm{gPt}$.

Figure 7-4. CO stripping voltammograms (first and second cycle) obtained after different $\mathrm{CO}$ adsorption procedure at $140^{\circ} \mathrm{C}$. The scan rate was $10 \mathrm{mV} / \mathrm{s}$. Both MEAs had a Pt loading of $1.4 \mathrm{mgPt} / \mathrm{cm} 2$ and $2 \mathrm{gPA} / \mathrm{gPt}$ was present in the CL.

Figure 7-5. TEM micrograph and the corresponding $\mathrm{Pt}$ size distribution histogram of $30 \mathrm{wt} \% \mathrm{Pt} / \mathrm{C}$ after exposure for $5 \mathrm{~min}$ in $\mathrm{H}_{2}$ and 2 sequential $\mathrm{CO}$ stripping experiments. The Pt particle size distribution is included as an inset and the average Pt particle size was estimated $4.7 \mathrm{~nm}$.

Figure 7-6. The effect of reducing atmosphere $5 \% \mathrm{H} 2 / \mathrm{Ar}$ on the Pt particle size as a function of time. The XRD diffractograms of $30 \mathrm{wt} \% \mathrm{Pt} / \mathrm{C}$ were acquired at $180^{\circ} \mathrm{C}$, with $20 \mathrm{~min}$ acquisition time.

Figure 7-7. First cycle of CO stripping voltamograms, obtained after several cycles of $\mathrm{CO}$ bulk electroxiadation. $\mathrm{CO}$ was adsorbed at open circuit potential for $20 \mathrm{~min}$ at Tcell $=140^{\circ} \mathrm{C}$.

Figure 7-8. TEM micrograph and the corresponding $\mathrm{Pt}$ size distribution histogram of $30 \mathrm{wt} \% \mathrm{Pt} / \mathrm{C}$ after 30 cycles of $\mathrm{CO}$ bulk electroxidation. The $\mathrm{Pt}$ particle size distribution is included as an inset and the average Pt particle size was estimated $5.5 \mathrm{~nm}$.

Figure 7-9. First cycle of sequential $\mathrm{CO}$ stripping experiments, obtained after normal fuel cell operation at the cathodic electrode. $\mathrm{CO}$ was adsorbed at open circuit potential for $20 \mathrm{~min}$ at $\mathrm{T}_{\text {cell }}=140^{\circ} \mathrm{C}$.

Figure 7-10. TEM micrograph and the corresponding $\mathrm{Pt}$ size distribution histogram of $30 \mathrm{wt} \% \mathrm{Pt} / \mathrm{C}$ after 5 sequential $\mathrm{CO}$ stripping experiments. The average $\mathrm{Pt}$ particle size was found to be $4.3 \mathrm{~nm}$.

Figure 8-1. The effect of PA amount imbedded in the cathodic electrode, on the overall fuel cell's performance. The polarzation curves, IR drop corrected, were obtained at $180^{\circ} \mathrm{C}$ and gas stoichiometry of 1.2 and 2 of pure $\mathrm{H}_{2}$ and $\mathrm{O}_{2}$, respectively. The PA amount varied between 2 and $8 \mathrm{gPA} / \mathrm{gPt}$. a. MEAs 2,9-12 employing 30wt\% Pt/oxMWCNT-Py and b. MEAs 17-20 employing 30wt $\%$ $\mathrm{Pt} / \mathrm{C}$.

Figure 8-2. The effect of PA amount on the fuel cell's voltage (IR drop corrected) under pure $\mathrm{H}_{2}$ and $\mathrm{O}_{2}$ (gas stoichiometry $\lambda_{\mathrm{H} 2}=1.2$ and $\lambda_{\mathrm{O} 2}=2$ ) at $180^{\circ} \mathrm{C}$ at a. $0.2 \mathrm{~A} / \mathrm{cm}^{2}$ and b. $0.6 \mathrm{~A} / \mathrm{cm}^{2}$

Figure 8-3. The effect of the PA amount imbedded in the cathodic catalyst layer on the charge transfer resistance a. Nyquist and b. bode plots. The data were obtained at $180^{\circ} \mathrm{C}$ and at a gas stoichiometry of 1.2 and 2 of pure $\mathrm{H}_{2}$ and $\mathrm{O}_{2}$, respectively. The current was set at $0.2 \mathrm{~A} / \mathrm{cm}^{2}$. The MEAs (2,9-12) employing 30wt\% Pt/oxMWCNT-Py MEA. The Pt loading was similar for all MEAs, whereas the amount of PA was altered from 2 to $8 \mathrm{gPA} / \mathrm{gPt}$.

Figure 8-4. The effect of the PA amount imbedded in the cathodic catalyst layer on the charge transfer resistance a. Nyquist and $b$. bode plots. The data were obtained at $180^{\circ} \mathrm{C}$ and at a gas stoichiometry of 1.2 and 2 of pure $\mathrm{H}_{2}$ and $\mathrm{O}_{2}$, respectively. The current was set at $0.2 \mathrm{~A} / \mathrm{cm}^{2}$. The MEAs (17-20) employed $30 \mathrm{wt} \% \mathrm{Pt} / \mathrm{C}$. The Pt loading was similar for all MEAs, whereas the amount of $\mathrm{PA}$ was altered from 2 to $8 \mathrm{gPA} / \mathrm{gPt}$. 
Figure 8-5. Polarization curves, IR drop corrected, at $180^{\circ} \mathrm{C}$ of MEAs 2 and 912 , employing $30 \mathrm{wt} \% \mathrm{Pt} / \mathrm{oxMWCNT}-\mathrm{Py}$ with different amount of sprayed PA at the cathodic electrode (2 to $8 \mathrm{gPA} / \mathrm{gPt}$ ) under high $\mathrm{H}_{2}$ and $\mathrm{O}_{2}$ flows (200 $\mathrm{cc} / \mathrm{min}$ respectively).

Figure 8-6 a. Nyquist and b. bode plots of MEA - employing 30wt $\%$ Pt/oxMWCNT-Py. The data were obtained at $0.2 \mathrm{~A} / \mathrm{cm}^{2}$ and at $180^{\circ} \mathrm{C}$ under constant high gas flow of pure $\mathrm{H}_{2}$ and $\mathrm{O}_{2}(200 \mathrm{cc} / \mathrm{min}$ respectively). The $\mathrm{Pt}$ loading was similar for all MEAs (2, 9-12), whereas the amount of PA was altered from 2 to $8 \mathrm{gPA} / \mathrm{gPt}$.

Figure 8-7. ECSA as measured via CO stripping, with respect to the PA amount in the CL a. after the activation of MEA employing 30wt\%Pt/oxMWCNT-Py and $30 \mathrm{wt} \% \mathrm{Pt} / \mathrm{C}$. b. before and after activation for MEAs employing $30 \mathrm{wt} \% \mathrm{Pt} / \mathrm{C}$. Figure 8-8. The effect of the Pt loading of the cathodic electrode on the MEA's performance. Polarization curves, IR drop corrected, at $180^{\circ} \mathrm{C}$ of MEAs employing 30wt\% Pt/oxMWCNT-Py. The gas stoichiometry was 1.2 and 2 for pure $\mathrm{H}_{2}$ and $\mathrm{O}_{2}$, respectively. The $\mathrm{Pt}$ loading varied between 0.43 and $2.2 \mathrm{mgPt} / \mathrm{cm}^{2}$ and the PA loading was a. $2 \mathrm{gPA} / \mathrm{gPt}$ (MEA 1-4) and b. $3 \mathrm{gPA} / \mathrm{gPt}$ (MEA 6,8 and 9). For comparison reasons MEA 16, employing 30wt\% $\mathrm{Pt} / \mathrm{C}$, was used.

Figure 8-9. The effect of low Pt loadings cathode on the fuel cell's performance. Polarization curves, IR drop corrected, at $180^{\circ} \mathrm{C}$ of MEAs employing $30 \mathrm{wt} \%$ $\mathrm{Pt} / \mathrm{oxMWCNT}-\mathrm{Py}$ and 30wt\% Pt/C . The gas stoichiometry was 1.2 and 2 for pure $\mathrm{H}_{2}$ and $\mathrm{O}_{2}$, respectively. The PA amount imbedded in the cathodic catalyst layer was $3 \mathrm{gPA} / \mathrm{gPt}$ and $2 \mathrm{gPA} / \mathrm{gPt}$ for the $30 \mathrm{wtPt} / \mathrm{oxMWCNT}$-py and $30 \mathrm{wt} \% \mathrm{Pt} / \mathrm{C}$, respectively. a. MEA:,8, 14 and 15 and b. MEA: 5,7,13 and 14 .

Figure 8-10. The effect of $\mathrm{O}_{2}$ partial pressure on the polarization resistance of the cathodic electrode of MEA 15. AC impedance spectra obtained at a current of $0.2 \mathrm{~A} / \mathrm{cm}^{2}$ at $180^{\circ} \mathrm{C}$, under various partial pressure of $\mathrm{O}_{2}$ balanced with Ar. The anodic electrode was fed with redundant $\mathrm{H}_{2}$ flow. a. Nyquist plots and $\mathrm{b}$. corresponding Bode plots

Figure 8-11. The effect of $\mathrm{O}_{2}$ partial pressure on the polarization resistance of the cathodic electrode of MEA 9. AC impedance spectra obtained at a current of $0.2 \mathrm{~A} / \mathrm{cm}^{2}$ at $180^{\circ} \mathrm{C}$, under various partial pressure of $\mathrm{O}_{2}$ balanced with Ar. The anodic electrode was fed with redundant $\mathrm{H}_{2}$ flow. a. Nyquist plots and $\mathrm{b}$. corresponding Bode plots

Figure 8-12. The effect of the catalyst support and the PA distribution on the Tafel slopes under various $\mathrm{O}_{2}$ partial pressures. The measurements were conducted under $200 \mathrm{cc} / \mathrm{min}$ of pure $\mathrm{H}_{2}$ and $\mathrm{O}_{2}$ balanced with $\mathrm{Ar}$, respectively, at $180^{\circ} \mathrm{C}$ : a. MEA 9 employing 30wt\%Pt/ox.MWCNT-Py and b. MEA 15 employing $30 \mathrm{wt} \% \mathrm{Pt} / \mathrm{C}$.

Figure 8-13. TEM micrographs after 144 hours of continuous operation of cathodic electrode employing a. 30wt \% Pt/C and b. 30\% Pt/oxMWCNT-Py. The $\mathrm{Pt}$ particle size distribution is included as an inset 


\section{List of Tables}

Table 4-1. Specifications of the MEAs tested and anodic electrodes. (noble metal loading and phosphoric acid, PA, sprayed on the electrodes prior to the MEA assembly)

Table 4-2. Mean $\mathrm{Pt}$ particle size and active $\mathrm{Pt}$ surface area for the $30 \mathrm{wt} \%$ $\mathrm{Pt} / \mathrm{C}$ catalysts, as measured via $\mathrm{H} 2$ chemisorbtion, $\mathrm{CO}$ chemisorbtion and TEM imaging.

Table 4-3. XPS binding energies with the corresponding FWHM in the braquets, intensity ratio of $\mathrm{O} 1 \mathrm{~s}$ of (peakII)/(peakI), as well as the atomic ratio $\mathrm{O} / \mathrm{P}$.

Table 5-1. Specifications of the anodic electrode of the tested MEAs tested (noble metal loading and phosphoric acid, PA, sprayed on the electrodes prior to the MEA assembly)

Table 5-2. Polarization resistance as calculated from Impedance spectra of MEAs employing 30wt $\% \mathrm{Pt} / \mathrm{C}$ and $30 \mathrm{wt} \% \mathrm{Pt} / \mathrm{oxMWCNT}-\mathrm{Py}$ at the anodic electrode, and $30 \mathrm{wt} \% \mathrm{Pt} / \mathrm{C}$ at the cathodic for both cases. The spectra were acquired at $0.2 \mathrm{~A} / \mathrm{cm}^{2}$ and under $200 \mathrm{cc} / \mathrm{min} \mathrm{H}_{2}$ and $\mathrm{O}_{2}$, and were deconvoluted using FRA module of Autolab Software

Table 5-3. Polarization resistance as calculated from Impedance spectra of MEAs employing different Pt loadings of 30wt\%Pt/oxMWCNT-Py at the anodic electrode. $30 \mathrm{wt} \% \mathrm{Pt} / \mathrm{C}$ is employed at the cathodic electrode having the same $\mathrm{Pt}$ loading approximately $2 \mathrm{mgPt} / \mathrm{cm}^{2}$. The spectra were acquired at $0.2 \mathrm{~A} / \mathrm{cm}^{2}$ and under $200 \mathrm{cc} / \mathrm{min}_{2}$ and $\mathrm{O}_{2}$, and were deconvoluted using FRA module of Autolab Software.

Table 5-4. Average Pt particle size after 48 hours of continuous operation for different catalyst, as measured from TEM, and their corresponding catalyst electrochemical utilization, UPt-max.

Table 5-5. N/Pt atomic ratio after certain operation time of the cathodic electrode of an MEA employing 30\% Pt/(ox.MWCNT)-Py.

Table 5-6. Pt particle size average diameter $(\mathrm{nm})$ with respect to operation time as estimated via TEM imaging, of 30wt $\% \mathrm{Pt} / \mathrm{C}$ and $30 \mathrm{wt} \% \mathrm{Pt} / \mathrm{oxMWCNT}-\mathrm{Py}$.

Table 6-1. Specifications (Pt metal loading per electrode and catalyst type) of the anodic electrodes that operated under reformate gas.

Table 6-2. The effect of the fuel composition with respect to the PA loading, on the polarization resistance of the anodic electrodes.

Table 8-1. Specifications of the cathodic electrode of the tested MEAs (noble metal loading and phosphoric acid, PA, sprayed on the electrodes prior to the MEA assembly)

Table 8-2. The average Pt particle size of the $30 \mathrm{wt} \% \mathrm{Pt} / \mathrm{oxMWCNT}-\mathrm{Py}$ and $30 \mathrm{wt} \% \mathrm{Pt} / \mathrm{C}$ when employed at the anode and cathode. The measurement of the Pt sizes were conducted after the activation period.

Table 8-3. The as calculated Tafel slopes, exchange current densities and charge transfer resistance from MEA 9 and MEA 15 with respect to the partial pressure of $\mathrm{O}_{2}$. 
Table 8-4. N/Pt atomic ratio after certain operation time of the cathodic 241 electrode of an MEA employing 30wt\% Pt/(ox.MWCNT)-Py.

Table 8-5. Pt particle size average diameter $(\mathrm{nm})$ with respect to operation time 243

as estimated by TEM imaging for cathodic electrodes employing 30 $\mathrm{wt} \%$ Pt/ox.MWCNT-Py and 30wt\%Pt/C. 


\section{Chapter 1}

\section{Introduction to Hydrogen technology, Fuel Cells and theory}

\subsection{Introduction to $\mathrm{H}_{2}$ technology}

Energy demand globally has been increasing, at least since 1990 [1], mainly due to the energy consumption of developing countries. Currently, the main energy source is fossil fuel (coal, crude oil and petroleum products), which are not sustainable and are expected to be depleted. A byproduct of fossil fuel consumption is $\mathrm{CO}_{2}$, which has been accounted as a primary source that increases the Earth's average temperature (IPCC, 2013), the socalled greenhouse effect. One method to address the problems of fossil fuel consumption is replacement by new alternative and sustainable energy sources.

The last decade alternative and renewable energy sources have been used to supplement energy requirements reaching 16\% in 2012 globally. Wind turbines, hydroelectric plants, photovoltaic panels, biomass are few of the renewable energy technologies being used. 
Hydrogen technology is considered a clean technology because hydrogen is high in energy and when oxidized (burned), its products cause almost no pollution. Thus it is an excellent candidate for energy supply and storage. Renewable power plants exploiting the sun use molten salts as energy storage that is in turn used to produce a constant power output even during nightime. Hydrogen can be used to replace molten salts and has the advantage that it can be transported, if needed from the source location without losses of its thermal capacity, unlike molten salts which require thermal insulation.

Fuel cells have attracted scientific interest due to their wide range of applications. They are considered a novel clean and highly promising energy production technology. Similar to a battery that turns a chemical reaction into electricity, a fuel cell combines hydrogen and oxygen to produce electricity, heat, and water. However, the benefit of using fuel cells is that they will produce electricity as long as fuel (hydrogen) is supplied, never losing its charge. Additionally, because fuel cells can generate power from a fraction of a watt to hundreds of kilowatts, they can be used in almost any application, from stationary power to vehicles of all sizes down to mobile phones. In order for the fuel cell technology to be commercially available optimization is required.

\subsection{Purpose of this thesis}

The scope of this thesis is the understanding and improvement of the electrochemical interface of the anodic and cathodic electrode in HTPEM fuel cells, as well as the optimization of the catalyst layer structure under various operation conditions.

The porous electrode's structure consists of the catalyst, usually Pt dispersed on carbon, mixed with a binder-ionomer and an ionic carrier, in order to establish a 3-D ionic link between the Pt catalyst active sites and the polymer electrolyte. The presence of PA possess limitations concerning the slow kinetic of the oxygen reduction reaction [2] and the reduced oxygen solubility in hot phosphoric acid [3], restricting the performance of the cathodic electrode. In addition, due to the liquid state of PA and its mobility, maximization of the triple-phase boundary is difficult to achieve. Thus, a certain amount of ionomer is required, that will bind the free PA in the catalyst layer, providing adequate contact with the $\mathrm{Pt}$ particles, in order to increase the catalyst utilization. Therefore, a delicate balance must be achieved between the binder and the ionic carrier for the efficient transport of gases and conductance of electrons and protons to the electrochemical interface. Uniform mixing and distribution of these components is hard and is not guaranteed, as a result part of the electrocatalyst is not used. 
In an attempt to solve the above presented problem and to increase the electrochemical active surface area, a newly synthesized electrocatalyst 30wt \% Pt/oxMWCNT-Py is tested at the anodic and cathodic electrode. The newly synthesized electrocatalyst is based on functionalized MWCNT. On the outer wall of the MWCNT pyridine groups are covalent attached, which are known to interact with phosphoric acid. In this way it is expected that they will bind the acid molecules creating proton conductive pathways throughout the catalytic layer resulting in the increase of the three phase boundary and ultimately the catalyst utilization. Thus, the acid-base interaction of the $\mathrm{H}_{3} \mathrm{PO}_{4}$ with the pyridine moieties is expected to secure the uniform distribution of the acid throughout the catalytic layer so that a $3 \mathrm{D}$ proton ionic link will provide an active electrochemical interface with all deposited Pt particles, as shown in figure 1-1.
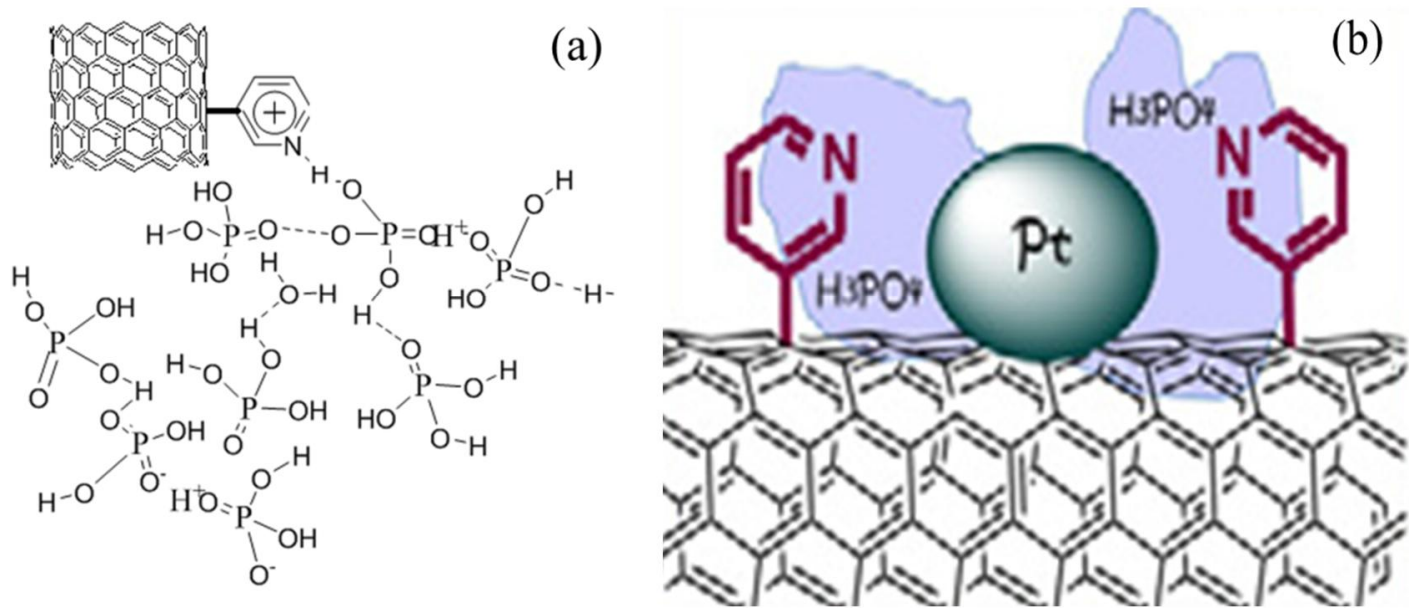

Figure 1-1 .Schematic model of a. the interaction of pyridine with $\mathrm{H}_{3} \mathrm{PO}_{4}$ and $\mathrm{b}$. the Pt/pyridine modified MWCNT

The advantage of this approach towards the increase of catalyst utilization lies in the lack of need for the use of a polymer-binder inside the catalytic layer, thus avoiding problems of inhomogeneous binder distribution and/or electronic insulation of catalyst nanoparticles. Using this newly synthesized electrocatalyst opens the possibility of significant reduction of the amount of Pt in the electrodes without sacrificing the performance and stability of the fuel cell.

\subsection{Basic Components of High temperature PEMFC and the electrocatalytic reactions at each electrode}

\subsubsection{Fuel Cell Description}

The heart of a PEM fuel cell is the membrane electrode assembly MEA. A MEA consists of two porous electrodes sandwiched with a membrane. The membrane separates the two gas compartments (Fig. 1-2). The membrane is designed as an electronic insulator material 
separating the reactants $\left(\mathrm{H}_{2}\right.$ and $\mathrm{O}_{2}$ /air) and allowing only protons to move towards the electrodes. The electrodes consists of a catalyst layer (CL), where the electrochemical reaction occurs, and a gas diffusion layer (GDL), providing access of the fuel and oxidant to the CLs.

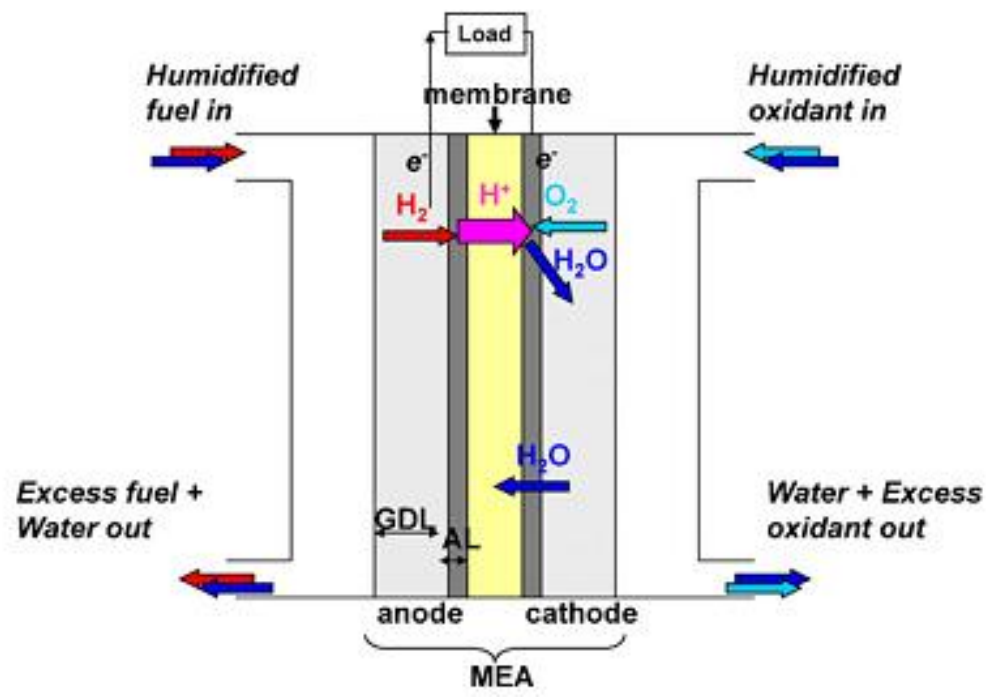

Figure 1-2. Schematic of basic principle of fuel cell operation

This MEA assembly is sandwiched between two current collectors, bipolar plates, in which gas distribution channels are integrated. The GDL typically consists of carbon cloth or carbon paper $(0.2-0.5 \mathrm{~mm}$ thickness $)$ with a layer of hydrophobic material that facilitates the product water drainage and prevents the gas paths from water accumulation. The CL, is placed on the GDL, and consists of catalyst particles supported on a carbon based substrate, ionomer and pore spaces which form a three-phase boundary where electrons, protons and reactant gases meet and electrochemical reaction takes place.

For the past years, the structure and composition of the MEAs have undergone a lot of changes. Up to now, platinum (pure or alloyed) is the catalyst material which gives the best electrocatalytic performances [4]. In the following sections, the individual components and the requirements they have to meet are being discussed. As shown in Fig 1-2, there are two oxido-reduction couples reactions involved in fuel cell's operation. At the anode, the negative electrode, the hydrogen is oxidized according to: 


$$
\left.\mathrm{H}_{2} \rightarrow 2 \mathrm{H}^{+}+2 \mathrm{e}^{-} \quad \text { (eq. } 1-1\right)
$$

At the cathode, the positive electrode, oxygen is reduced according to:

$$
\left.\mathrm{O}_{2}+4 \mathrm{H}^{+}+4 \mathrm{e}^{-} \rightarrow 2 \mathrm{H}_{2} \mathrm{O} \quad \text { (eq. } 1-2\right)
$$

Giving the overall reaction:

$$
2 \mathrm{H}_{2}+\mathrm{O}_{2} \rightarrow 2 \mathrm{H}_{2} \mathrm{O} \quad(\text { eq } 1-3)
$$

Besides water, the operation of a PEMFC releases electricity and heat. The following process takes place inside a fuel cell during normal operation conditions:

1. Gas flows through the channels, where some convective flow may be induced in the porous electrode

2. Gas diffusion through porous media

3. Electrochemical reaction

4. Proton transport through the membrane

5. Electron conduction through the electrically conductive cell components

6. Water transport through th9e polymer electrolyte membrane

7. Water transport through porous catalyst layer and gas diffusion layer

8. Heat transfer, including both conduction through solid components of the cell and convection to reactant gases and cooling medium. 


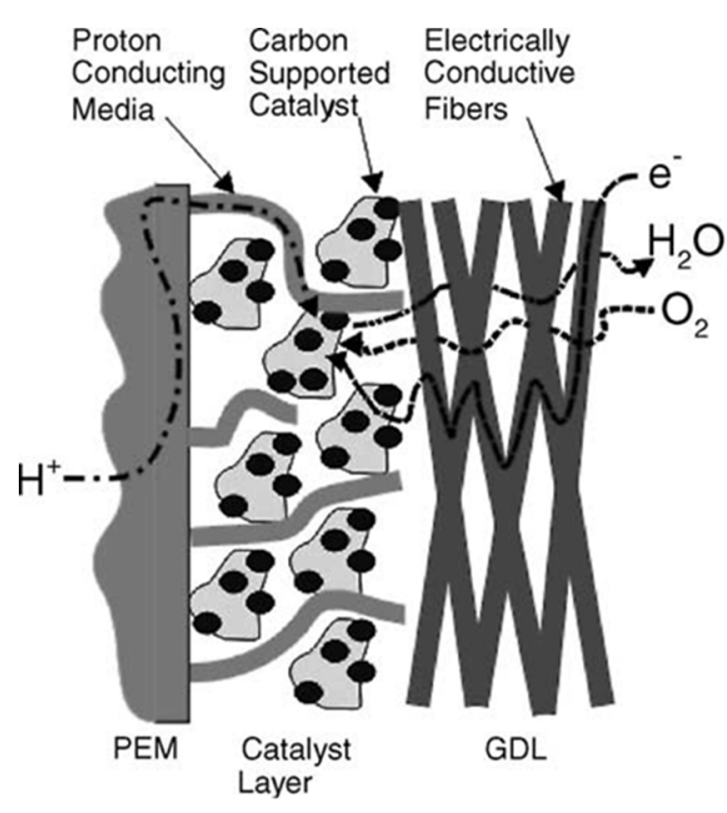

Figure 1- 3. Cross section schematic of a cathodic electrode/membrane interphase during operation

\subsubsection{Electrodes- Catalyst Layer}

Commercialization of PEMFCs is still significantly restricted by its high content in Pt or other noble metals. This has a significant impact on both the cost of the fuel cell and the quantitative availability of $\mathrm{Pt}$ as an abundant raw material for the preparation of the electrocatalytic layers. Reduced cost, resulting from increased catalyst utilization and/or catalyst stability, is highly desirable. The last decade great advances have been made towards the optimization and commercialization of low and high temperature PEMFC's. Many research studies have focused on the design and optimization of electrocatalytic layers in order to increase the triple-phase boundaries and the electrochemical interface $[4,5,6,7,8,9]$.

The structure of the catalytic layer usually comprises the catalyst, mainly Pt dispersed onto carbon black and a binder, aiming to the establishment of a 3-D ionic link between the Pt catalyst active sites and the polymer electrolyte. In this way the reaction interface is increased, given that only the Pt particles that are in direct contact with the proton 
conductor are electrochemically active. In such cases, the uniform mixing of the two components is hard to be guaranteed and part of the electrocatalyst is not used. Nevertheless, transport of gases and conductance of electrons and protons to the electrochemical interface must be optimized to provide efficient electrochemical reactions.

Porosity of the catalyst layer is an essential property for a high performance electrode. Pores allow transport of reactants onto three phase boundary sites and removal of reaction product water. The porous structure in the catalyst layer depends on the electrode's preparation method and can be controlled by adding a pore forming agent, which is later removed. The type of catalyst and substrate is also known to influence the pores distribution and size.

PEMFC electrodes are very thin, only ca. $10 \mu \mathrm{m}$, thinner than in other fuel cell types, and thus power density in PEMFC electrodes is higher compared to other fuel cells [10]. The most common used commercially available catalyst is based on carbon black particles (Pt/C catalyst) with Pt nanoparticles size ranging from $2-6 \mathrm{~nm}$ [11]. The role of the carbon substrates is not only to support the catalyst nanoparticles and stabilize them, in order to minimize their agglomeration, but also to ensure a good electronic conductivity in the catalyst layer.

Average catalyst loading on an electrode is usually between 0.1 and $0.4 \mathrm{mg} \mathrm{cm}^{-2}$ for anode and cathode, respectively, for low PEM fuel cells. Although it has to be mentioned that High temperature PEM fuel cells employing phosphoric acid embedded polymer electrolyte require higher catalyst loading compared to the perfluorosulfonic-acid-type membrane based PEM fuel cell. The low solubility of oxygen and slow diffusion in phosphoric acid [12] as well as phosphoric acid poisoning /adsorption [13] on the Pt surface are responsible for the higher demand in Pt loading. PBI based fuel cells are able to operate without significant loss in their performance utilizing Pt loading as low as $0.2 \mathrm{mgPt} / \mathrm{cm}^{2}$ at the anodic electrode and around $0.6 \mathrm{mgPt} / \mathrm{cm} 2$ for the cathodic electrode $[14,15]$.

\subsubsection{Gas Diffusion Layer [16]}

Gas diffusion layers (GDL) have many functions in a PEM fuel cell. Although it does not participate directly in the electrochemical reactions, the GDL is of great importance as: 
1. It provides mechanical support to the catalyst layer as well as to the MEA, preventing deformation when placed on top of the flow field channels

2. The porous nature of the materials provides the reactants access to the parts of the electrodes that are under the flow channel ridges, as well as a passage for reaction product water from the electrode to the flow channel. Reactant transport is usually driven by diffusion and in some cases by convection and water removal by capillary action.

3. It ensures a sufficient electric contact between the catalyst layer and the bipolar plate, allowing the electrons to travel from the anode to the cathode.

4. It conducts the heat, mainly via conduction, that is been generated by the electrochemical reactions in the catalyst layer.

In order to meet the above requirements the state of the art GDL consists of a complex porous composite network. It is composed by a bilayer structure, where the macroporous substrate faces the flow field and a microporous layer faces the catalyst layer. Due to the requirement of high gas permeability of reactants and reaction's products as well as sufficient water management, a delicate balance is required between the hydrophilic and hydrophobic pores. The pore size is also crucial, especially at the side facing the catalyst layer, as the catalyst particles are very small.

Due to the harsh operation conditions, high chemical and mechanical durability is highly desired. The GDL materials must have good electrical and thermal conductivity in-plane and through-plane, as well as low contact resistance in order to minimize ohmic losses inside the cell. The material should also be inexpensive in order to improve economic competitiveness of fuel cell systems.

Many of these properties are interconnected and thus best performance is often achieved by carefully balancing different properties instead of optimizing one. Barbir stated that 'One cannot change only one parameter in a fuel cell-change of one parameter causes a change in at least two other parameters, and at least one of them has an opposite effect of the one expected to be seen'[17]. 
Typically in PEMFC's GDL the macroporous layer are made of graphite-fiber substrate, such as carbon cloth, paper or felt. The diffusion media are usually treated with PTFE or similar fluorocarbon to increase hydrophobicity. A composite particle layer known as the microporous layer (MPL) is coated on one side of the macroporous layer and consists of a dispersion of carbon black and fluoropolymer. Most common PTFE loading used in the MPL are between $30 \%-50 \% \mathrm{wt}$. This configuration provides a porous structure with both hydrophobic and hydrophilic domains. It has to be noted that the hydrophobic properties of the GDL are rarely reported by the manufacturing companies as they are tailored for a specific cell design. Fig 1-4 presents a schematic and SEM micrograph of a typical GDL.

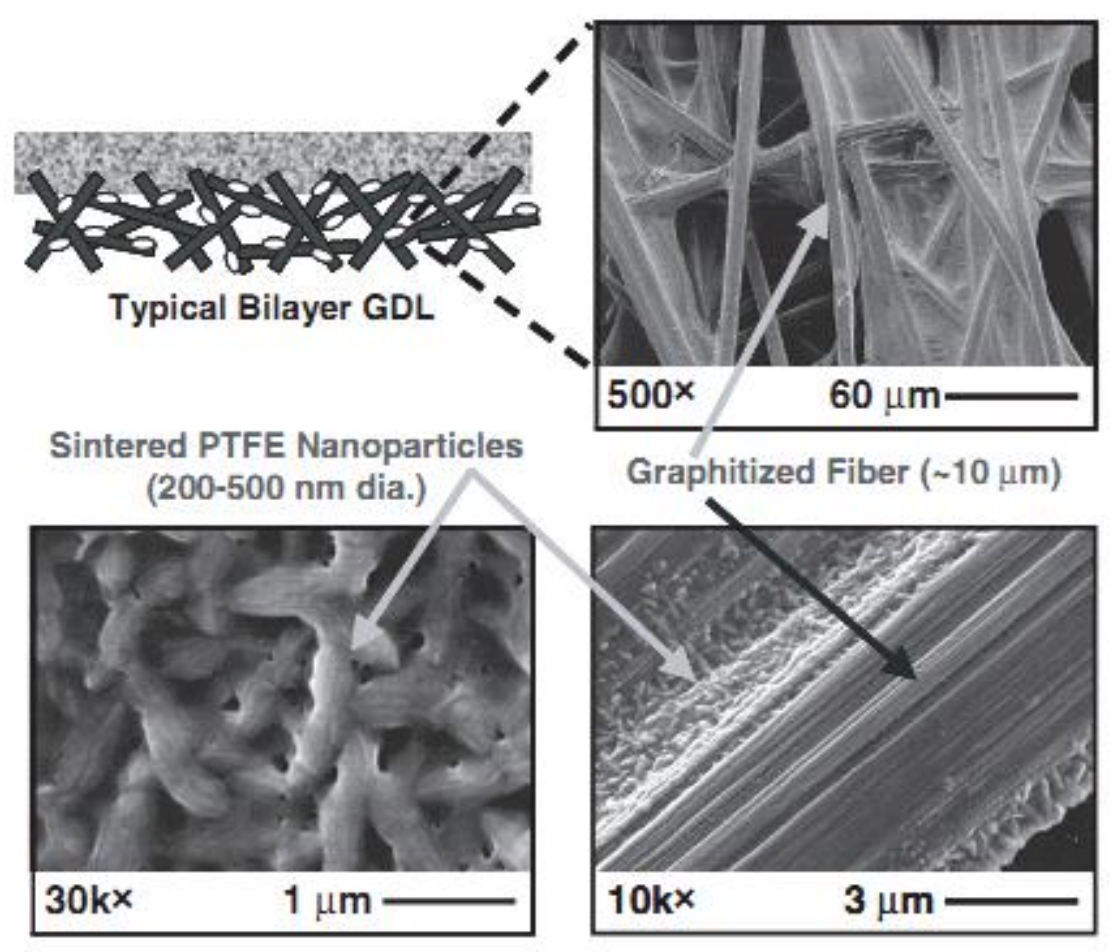

Figure 1- 4. Schematic and SEM micrographs showing gas diffusion layer, (Toray TGP$\mathrm{H})$ graphite-fiber and polytetrafluoroethylene (PTFE) microstructure spanning approximately two orders of magnitude of length scale. Each scale plays a critical role in optimized gas and liquid transport.[18]

The GDL plays a critical role in the overall performance of the PEM fuel cell, especially in the concentration overpotential region. Experiments and modeling have shown that majority of mass transfer losses occur due to limited oxygen diffusion through the cathode side GDL, especially when liquid water or $\mathrm{H}_{3} \mathrm{PO}_{4}$ is present, the later for the case of 
HTPEM. Mass transport of reactants and removal of excess water are greatly affected by GDL porosity, pore size distribution and ratio of hydrophobic and hydrophilic pores, gas permeability and material thickness.

\subsubsection{Polymer Electrolyte Membrane}

The heart of a PEM fuel cell is a polymeric membrane that presents specific capabilities. A good polymer electrolyte membrane must exhibit high proton conductivity, very low gas permeability of fuel and reactant gases and high chemical and mechanical stability in the demanding fuel cell environment. The polymer electrolyte membranes used for PEM fuel cell are divided into two categories: low (up to $100^{\circ} \mathrm{C}$ ) and high (up to $200^{\circ} \mathrm{C}$ ) temperature membranes.

The membranes employed in low-temperature PEM fuel cells consists of perfluorocarbon -sulfonic acid ionomers (PFSA)[19]. The best material known and most commonly used is Nafion [20] produced by DuPont, though similar materials have been developed for commercial or development purposes by other manufacturers such as Asahi Glass (Flemion) or Asahi Chemical (Aciplex).

Since this thesis is focused on HTPEM fuel cell, an extended discussion about the most common materials used and their properties is presented. As reported in the literature, most high temperature $\left(150-200^{\circ} \mathrm{C}\right)$ polymer electrolytes are based on acid-base systems. The polymer electrolyte membrane can be considered as a two component system. The polymeric membrane material consists of a backbone, side chains and any added components to enhance the properties, which corresponds to the first part of the solid electrolyte. The second one, is the proton carrier, which in HTPEM systems is usually an ionic medium such as $\mathrm{H}_{3} \mathrm{PO}_{4}$ or an ionic liquid. All membrane materials adsorb a desirable amount of the ionic carrier. If the adsorption is too high, it can lead in the weakening of the membrane structure, and if it's too less, in the severe reduction of the proton conductivity.

Due to the harsh chemical conditions existing in these types of fuel cells, very few polymeric materials can efficiently withstand [21]. Many approaches have been developed that include modified perfluorosulfonic acid polymer membranes, alternative sulfonated polymers and their composite membranes and acid-base polymer membranes. The most studied system for high temperature is PBI membrane doped with phosphoric acid [22]. 
In general, PBI materials are basic polymers $\left(\mathrm{pK}_{\mathrm{a}}=5.23\right.$ as protonated) containing benzimidazole units which bear both donor and acceptor hydrogen-bonding sites, as can be seen in figure 1-5. They can be synthesized by combining tetraamines and diacids. The proton acceptor sites of the imidazole ring react with $\mathrm{H}_{3} \mathrm{PO}_{4}$ or polyphosphoric acid as in a typical acid-base interaction. The basic polymer acts as a proton acceptor and an ion pair is formed.

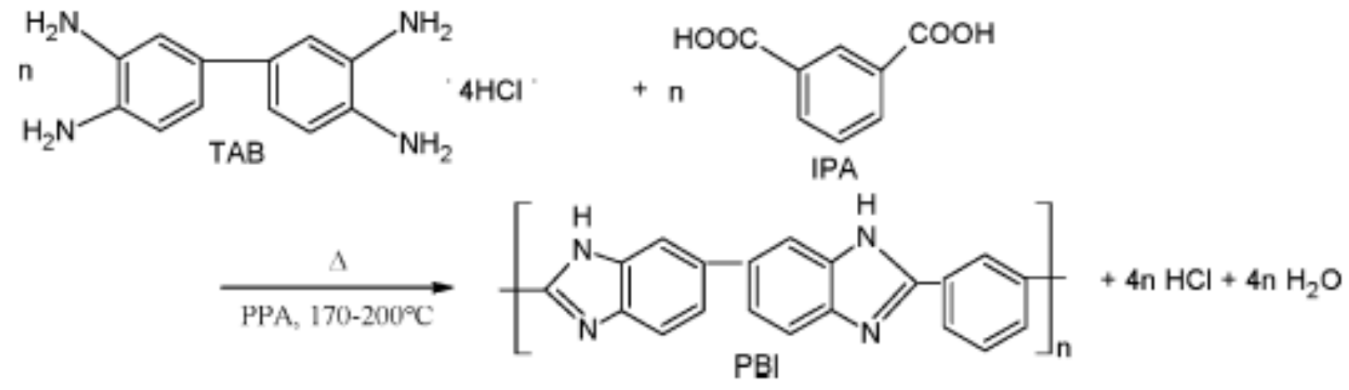

Figure 1-5. PBI structure

The challenge for the use of PBI membranes in HTPEMFCs is their long term stability. This is related to various factor comprising low molecular weights, mechanical integrity, chemical stability and phosphoric acid retention. In particular the incorporation of high amount of phosphoric acid in order to achieve high conductivity values, results in a highly plasticized membrane with $\mathrm{T}_{\mathrm{g}}$ values even below $200^{\circ} \mathrm{C}$. Improvements in the aforementioned properties are the focus of much research. Cross-linking of PBI material appears to be a promising method to overcome these issues. In addition the PBI polymer chains are vulnerable to oxidizing species (peroxide radicals), which are formed during fuel cell operation. Polymer oxidative degradation (attack by $\mathrm{H}_{2} \mathrm{O}_{2}$ and hydroxy and/or hyperoxy radicals) is likely the principal reason of the failure. Therefore, PBI presents serious drawbacks such as moderate mechanical properties and low oxidative stability.

Another very promising membrane for high temperature PEMFCs consists of pyridine based aromatic poly-ethers [23,24,25]. Pyridine based aromatic poly-ethers are novel polymer structures that contain polar pyridine groups as main chain linkage in aromatic copolymers. These polymer structures were designed and synthesized in order to provide functionalities such as high thermal and oxidative stability, mechanical integrity and the ability to form ionically conductive membranes after doping with phosphoric acid. The main or side chain pyridine units strongly bind phosphoric acid, which is retained in the membrane matrix [26]. Doping of these polyethers results in membranes with ionic 
conductivity values as high as $10^{-1} \mathrm{~S} / \mathrm{cm}$ at $180^{\circ} \mathrm{C}$. All the other functionalities can be met by the proper selection of the aromatic polyether structure of the copolymers. Figure 1-6 depicts a general structure of these polymers.

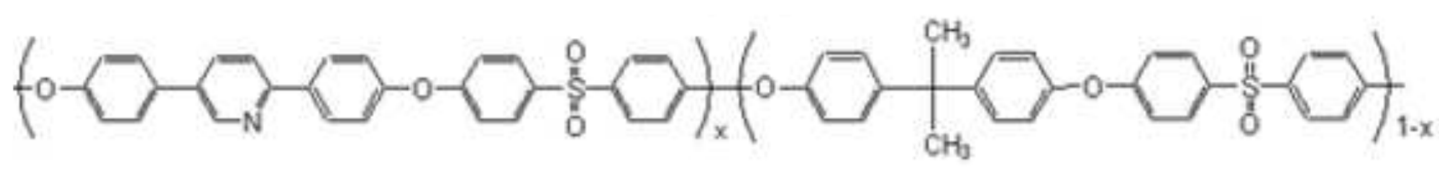

Figure 1-6. General structure of pyridine based aromatic polyethers.

This alternative type of polymeric materials has high thermal, mechanical and oxidative stability due to the aromatic ether bonds. These acid doped polymeric materials can be readily synthesized with tailor-made physicochemical properties due to the diversity of monomers that can be used. The oxidative stability of those membranes is exceptionally good and therefore are excellent candidates for high temperature PEMFCs application. ADVENT's TPS ${ }^{\circledR}$ polymer electrolyte MEA is a representative of this alternative polymer electrolyte membrane [27].

Both PBI and TPS polymers contain basic groups in their chemical structure (imidazole and pyridine groups respectively), which interact with PA thus facilitating the impregnation of PA into the polymer matrix. The PA network within the membrane provides the $\mathrm{H}^{+}$pathways, though the proton conductivity is affected by the polymer chemical structure $[28,29,30]$. The proton conductivity mechanism in phosphoric acid doped membranes is mainly controlled by structure diffusion (Grotthus mechanism) where proton transfer occurs between hydrogen bonds.

\subsubsection{Current collectors and flow field/bipollar plates [31]}

Flow field/bipollar plates bear flow channels for the uniform distribution of reactant gases over the active cell area. The bipolar plates provide an electrical interconnection between the electrodes and outer components of the fuel cell and serve to separate the individual electrochemical cells in stacks. Flow field plates in a fuel cell stack are referred as bipolar plates, as they act as an anode side plate for one electrochemical cell and cathode side plate for another, connecting electrically the anode of one unit cell with the cathode of another. In a single cell configuration, there are no bipolar plates, they are monopollar and can be considered to be halves of a bipolar plate at each side. 
The flow channels geometrical configurations have a variety of different designs, such as pin, straight or serpentine channels etc. which can affect the cell's performance [32]. A typical simple serpentine flow field plate is shown in Figure 1-7. Channel and ridge width, channel depth, surface properties and overall geometry is known to affect the reactant's distribution over the active surface.

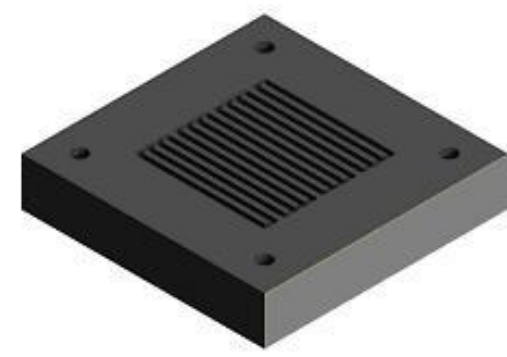

Figure 1-7. A simple flow field plate with a serpentine flow channel. Gas inlet and outlet are located on the opposite face of the plate.

Certain requirements have to be met in order to have the optimum performance. Beside the uniform gas distribution over the electrode and high values of electronic conductivity, high mechanical strength and corrosion resistance, as well as low gas permeability are highly required.

It is well known that the electrical and thermal properties have an effect on fuel cell operation and performance. Therefore in order to minimize ohmic losses in the flow field plate, the electrical conductivity of the bulk material should be high and the contact resistance between the flow field plate and adjacent components should be as low as possible $(<1 \mu \Omega \mathrm{cm} 2)$. Besides performance, the production cost has to be considered. It also have to be taken into consideration the fact that the bipolar plates consists of approximately $80 \%$ of the mass distribution in a stack, therefore low density materials are desired, in order to reduce the stack's weight. Flow field plates are manufactured mainly using graphite, graphite composites, and stainless steel and other metals. The most commonly used material is resin-impregnated graphite which exhibits low gas permeability and good electrical and thermal conductivity. A major drawback is that it is expensive and unsuitable for mass production since the flow channels have to be machined, which increases manufacturing costs. 


\subsubsection{Other components}

In addition to the core components described above, there are additional components required to make a functional unit cell or fuel cell stack. Figure 1-8 depicts the components of a simple single cell unit.

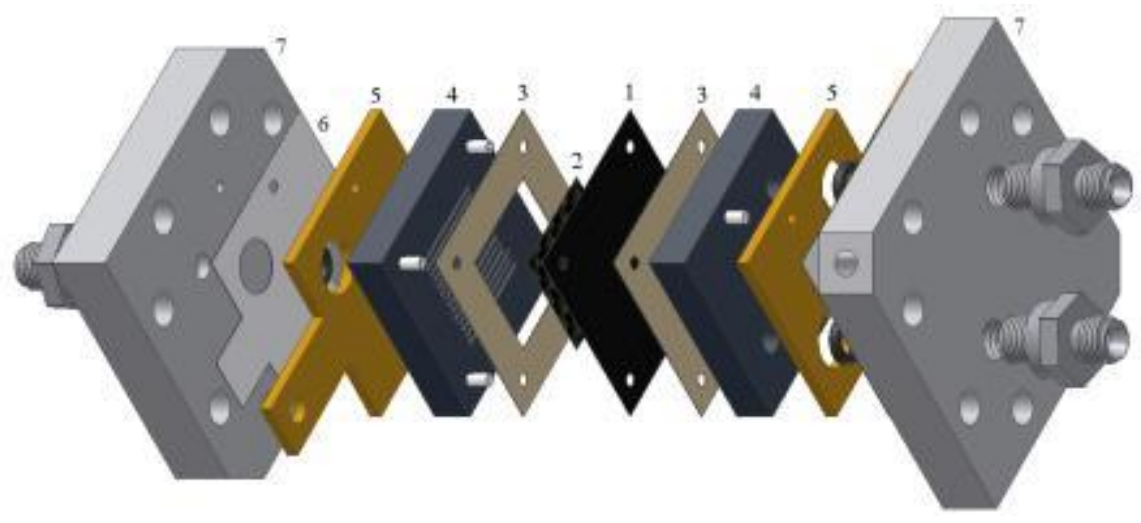

Figure 1-8. An exploded view of a unit cell. 1) MEA 2) PTL 3) Gasket 4) Flow field plates 5) Current collectors 6) Insulator 7) End plate.

Gaskets are necessary in order to prevent leaks and accidental mixing of reactants. The most common and frequently used gasket materials are PTFE, VDF, EPDM, FEP or other thermoplastic polymers that can endure the harsh conditions in a fuel cell.

Current collector plates are placed at both ends of the cell or stack and have terminals for current leads. Corrosion resistant and high conductivity are highly desired for the materials required for the manufacture of current collector plates.

Single cells and fuel cell stacks are held together by the end plates and clamping bolts, providing mechanical support and compression for adequate sealing as well as electrical and thermal contact between the components. The end plates consist of high conducting materials with high rigidity, which is required in order to ensure even compression between the different components. 
Fuel cell stacks require a cooling system for the facilitation of thermal management. For that reason cooling plates are often integrated into fuel cell stacks and are placed between bipolar plates that have been divided in two parts and feature channels for coolant

circulation. Alternatively, high thermal conductivity materials which feature cooling fins for heat removal outside the flow field area are used.

On a fully operation system level, more components are needed. The number and type depend on the needs of the system, but most common additional components are reactant lines and storage units, reactant humidification systems, compressors or blowers, valves, pressure and flow controllers and control electronics, heating and cooling systems, and power conditioning units.

\subsubsection{Mass transport in PEM fuel cells using single serpentine flow fields}

In PEM fuel cells, mass transport is a combination of two mechanisms: binary diffusion and convection. Binary diffusion is driven by gradient in concentration between the flow channel and the catalyst layer. In contrast, convection does not require a loss in concentration to drive reactants, rather a difference in pressure along the channel. The performance of a fuel cell, especially at high current densities, is affected by mass transport limitations originating by reactant deficiency in the under-rib regions.[33]

The thickness of the GDL contributes to diffusion-dominated mass transport and causes uneven distribution of current density due to different diffusion distances, especially under the ribs. Since convection does not require concentration gradient, higher reactant concentrations can be achieved in the catalyst layer under convection-dominated conditions. For that reason, under-rib convection is highly desired as it simultaneously increase reactant concentration and reduce product concentration in the catalyst layer, thus reducing the activation and mass-transport overpotentials [34]. By definition, under-rib convection is the flow of reactant gas through the GDLs porous structure and the under interconnected rib structure. The flow field design has a major impact on the mass transport limitations and influences the contribution of under-rib convection.

In single serpentine flow field, under-rib convection has been recognized as a major transport process in PEM fuel cells $[35,36,37,38]$. Along a single serpentine flow field a 
large pressure drop has to be generated in order to generate sufficient flow to satisfy the stoichiometry requirements. Due to the flow rate typically employed during operation of a PEM fuel cell, pressure difference occurs between adjacent channels due to viscous losses, figure 1-9. This pressure drop improves the performance of the electrode, due to increased contribution of the under-rib convection to the overall mass transport [39].Increasing the reactant flow rate, results in the increase the pressure drop between the channels, and consequently the under-rib convection dominates as the major contribution of mass transport.

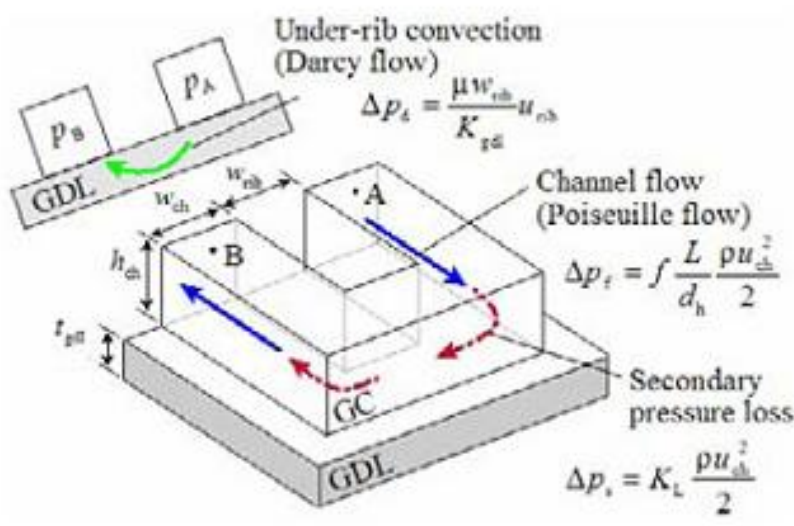

Figure 1-9. Flow network model of GC with under rib convection effects: definition of GC geometry and explanation of pressure drop [34].

\subsection{Thermodynamics}

\subsubsection{Basic Reactions}

Two electrochemical reactions take place during fuel cell operation as also mentioned in the previous section. At the anode, over which hydrogen gas passes, occurs the HOR. At the cathode, over which oxygen or air passes, the ORR occurs.

\subsubsection{Oxygen Reduction Reaction [40]}

The ORR is well recognized as a slow reaction in acid media. Due to the slow reaction kinetics the performance of a PEMFC suffers major voltage losses, as a result of high 
reduction overpotential. This is one of the most limiting factors in the energy conversion efficiency of the state-of-the-art PEMFC. For that reason, the ORR remains the focus of many studies. The ORR process on Pt catalysts includes several individual reaction steps, as shown in Figure 1-10. Two are the most examined processes, were each process contains discrete steps. One is the production of water through a four-electron pathway, and the other is production of hydrogen peroxide through a two-electron pathway.

The preferred route of ORR is the reduction of oxygen molecules to water through the fourelectron pathway. Incomplete reduction of oxygen to hydrogen peroxide leads to low energy conversion efficiency and to the production of reactive intermediate which are converted to free radical species.

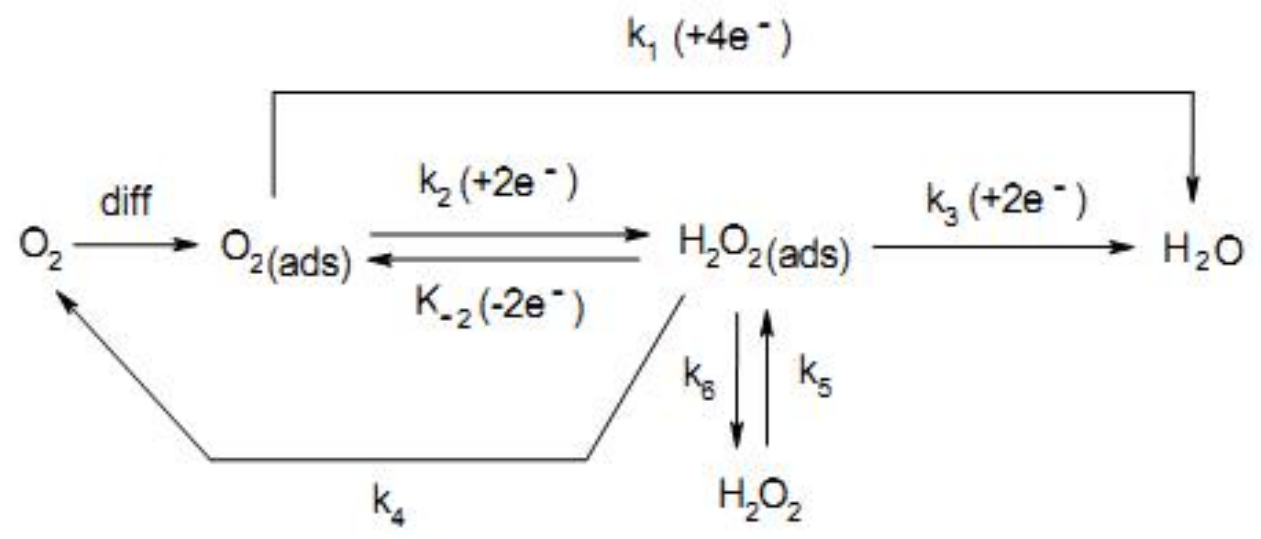

Figure 1-10. Oxygen reduction mechanism on noble catalysts (Reprinted from Journal of Power Sources, 152(2), Wang Bin, Recent development of non-platinum catalysts for oxygen reduction reaction, 1-15)

\subsubsection{Hydrogen Oxidation Reaction [41]}

At the anode, hydrogen is stripped of its electrons and becomes protons and electrons. For electrochemical reactions, even for the case of a simple one electron reaction, the reaction mechanism involves several steps. The overall reaction rate depends on the slowest elementary reaction, which is called the rate determining step. It is well known that $\mathrm{H}_{2}$ oxidation/evolution is described by the Tafel, Volmer and Heyrovski reaction steps according to the reacting scheme described by eqs 1-4 - 1-6. 


$$
\begin{array}{lll}
H_{2} \rightarrow 2 H_{a d} & \text { Tafel } & \text { (eq. 1-4) } \\
H_{a d} \rightarrow H^{+}+e^{-} & \text {Volmer } \\
H_{2} \rightarrow H_{a d}+H^{+}+e^{-} & \text {Heyrovski }
\end{array}
$$

Reaction 1-5 is fast and takes place at low overpotential being the primary charge transfer $\mathrm{H}_{2}$ electrooxidation reaction, while reaction 1-6 takes off at fairly higher overpotentials characterized by a larger Tafel slope [42]. Platinum (Pt)-based catalysts are widely used as the anodic electrode material for hydrogen oxidation as it presents lower oxidation overpotential and a higher kinetic rate. The apparent exchange current density of the HOR has been calculated to be five orders of magnitude higher compared to the ORR on Pt.

\subsubsection{Heat of Reaction}

The overall reaction of a hydrogen fuel cell (eq. 1-7) is an exothermic process.

$$
\mathrm{H}_{2}+1 / 2 \mathrm{O}_{2} \rightarrow \mathrm{H}_{2} \mathrm{O}+\text { Heat (eq. 1-7) }
$$

The heat or enthalpy of a chemical reaction is the difference between the heats of formation of products and reactants. For the above equation, the heat or enthalpy for the formation of liquid water is $-286 \mathrm{~kJ} / \mathrm{mol}$ at $25^{\circ}$. The negative sign of the enthalpy means that this is an exothermic reaction. The fuel cell reaction can now be written as

$$
\mathrm{H}_{2}+1 / 2 \mathrm{O}_{2} \rightarrow \mathrm{H}_{2} \mathrm{O}(\mathrm{l})+286 \mathrm{~kJ} / \mathrm{mol} \text { (eq. 1-8) }
$$

Equation 1-8 is only valid at $25^{\circ} \mathrm{C}$, meaning that both the reactant gases and the product water are at $25{ }^{\circ} \mathrm{C}$. The enthalpy of the hydrogen combustion reaction is also called the hydrogen's heating value. The value $286 \mathrm{~kJ} / \mathrm{mol}$ is known as hydrogen's higher heating value (HHV), which means that $1 \mathrm{~mol}$ of hydrogen is fully combusted with $1 / 2 \mathrm{~mol}$ of oxygen and cooled down to $25^{\circ} \mathrm{C}$. If hydrogen is combusted with sufficient excess oxygen and cooled down to $25^{\circ} \mathrm{C}$, the value will become $242 \mathrm{~kJ} / \mathrm{mol}$, which is known as hydrogen's lower heating value (LHV).

\subsubsection{Gibbs Free Energy}

The change in Gibbs free energy $\left(\Delta \mathrm{G}_{\mathrm{f}}\right)$ of a reaction is the difference between the Gibbs free energy of the product and the Gibbs free energy of the reactants. For the hydrogen/oxygen fuel cell reaction is given by the following equation: 


$$
\Delta G_{f}=G_{f, \text { products }}-G_{f, \text { reactants }} \quad \text { (eq. 1-8) }
$$

The change in Gibbs free energy depends on both temperature and pressure. $\Delta G_{f}^{o}$ is the change in Gibbs free energy at standard pressure, which varies with the temperature $\mathrm{T}$ of the fuel cell, in Kelvin.

$$
\Delta G_{f}=\Delta G_{f}^{o}-R T \ln \left[\frac{p_{H 2} p_{O 2}^{1 / 2}}{p_{H 2 O}}\right]
$$

$p_{H 2}, p_{O 2}$ and $p_{H 20}$ are the partial pressure of the hydrogen, oxygen, and vapor, respectively. $\mathrm{R}$ is the universal gas constant $\left(8.314 \mathrm{~J} / \mathrm{kg}^{*} \mathrm{~K}\right)$. A negative value of $\Delta \mathrm{G}_{\mathrm{f}}$ means that the reaction releases energy.

\subsubsection{Reversible Fuel Cell Potential}

The reversible voltage of the fuel cell is related to the change of Gibbs free energy of the reaction by the following equation:

$$
\Delta G_{f}=-n F E \quad(\text { eq. } 1-10)
$$

where F is Faraday constant (96485 Coulombs), E is the voltage of the fuel cell and $n$ is the number of electrons. For each mole of hydrogen two moles of electrons pass around the external circuit $(n=2)$. So the physical meaning of eq. 1-10 is the electrical work. $E$ is also known as the thermodynamic potential.

\subsubsection{Effect of Operation Conditions on Reversible Fuel Cell Potential}

The reversible voltage of the fuel cell, also known as Nernst voltage, can be expressed using the Nernst equation,

$$
E=\frac{-\Delta G_{f}}{2 F}=\frac{-\Delta G_{f}^{o}}{2 F}+\frac{R T}{2 F} \ln \left[\frac{p_{H 2} p_{O 2}^{1 / 2}}{p_{H 2 O}}\right]
$$

The reversible potenrial value at standard conditions $\left(25^{\circ} \mathrm{C}\right.$ and $\left.1 \mathrm{~atm}\right)$ is : 


$$
E=\frac{-\Delta G_{f}^{o}}{2 F}=\frac{237,200 \mathrm{~J} / \mathrm{mol}}{2 \times 96485 \mathrm{As} / \mathrm{mol}}=1.229 \mathrm{~V} \quad \text { (eq. 1-12) }
$$

The reversible potential of hydrogen/oxygen fuel cell depends on the operation temperature, as shown in equation 1-13:

$$
\frac{-\Delta G_{f}^{o}}{2 F}=1.229+\left(T-T^{o}\right)\left(\frac{\Delta S^{o}}{2 F}\right) \quad(\text { eq. } 1-13)
$$

Where $\mathrm{T}^{0}$ is the standard temperature $(298.15 \mathrm{~K})$ and $\Delta \mathrm{S}^{0}$ corresponds to the entropy change. It is known that the entropy change of a reaction is constant, therefore the above equation can be simplified to eq. 1-14 :

$$
\frac{-\Delta G_{f}^{o}}{2 F}=1.229-0.85 \times 10^{-3}(T-298.15)(\text { eq. } 1-14)
$$

At $200^{\circ} \mathrm{C}$ the theoretical reversible potential is equal to $1.14 \mathrm{~V}$.

The reversible cell potential affected by both the temperature and the pressure and is expressed in equation 1-15.

$$
E_{\text {Nernst }}=1.229-0.85 \times 10^{-3}(T-298.15)+4.3085 \times 10^{-5} T\left[\ln \left(p_{H_{2}}\right)+\frac{1}{2} \ln \left(p_{O 2}\right)\right](\text { eq. } 1-15)
$$

\subsubsection{Theoretical efficiency}

The efficiency of an energy conversion device is defined as the ratio between useful energy output and energy input. More specifically, for the case of a fuel cell, the useful energy output is the electrical energy produced, whereas the energy input is the enthalpy of hydrogen. If all the Gibbs free energy was converted into electrical energy the maximum possible (theoretical) efficiency of a fuel cell at $25^{\circ} \mathrm{C}$ can be calculated by equation $1-16$ :

$$
\left.n=\frac{\Delta G_{f}^{o}}{\Delta \mathrm{H}^{o}}=\frac{\frac{-\Delta G_{f}^{o}}{n F}}{\frac{-\Delta \mathrm{H}^{o}}{n F}}=\frac{1.23}{1.48}=83 \% \quad \text { (eq. } 1-16\right)
$$


where $1.23 \mathrm{~V}$ is the theoretical cell potential, and 1.48 is the potential corresponding to hydrogen's HHV, or the thermoneutral potential. The theoretical efficiency is sometimes also known as the thermodynamic efficiency or the maximum efficiency limit. The theoretical efficiency depends on both temperature and pressure.

\subsubsection{Energy Conversion Efficiency for Fuel Cells}

A fuel cell during normal operation conditions, the fuel fed to the anodic compartment is in excess with respect to the amount that is consumed. Therefore, the later has to be taken into account in order to calculate the cell efficiency. The fuel utilization coefficient, which is defined as

$$
\mu_{f}=\frac{\text { mass of fuel reacted in cell }}{\text { mass of fuel input in cell }}
$$

Therefore taking into consideration equation 1-17, the actual efficiency of the cell is defined as the actual voltage divided by the thermoneutral potential, based on HHV or LHV, equation 1-18 and 1-19 respectively:

$$
\begin{aligned}
& \text { Cell efficiency }=\mu_{f} \frac{V_{c e l l}}{1.48} \times 100 \% \\
& \text { Cell efficiency }=\mu_{f} \frac{V_{c e l l}}{1.23} \times 100 \%
\end{aligned}
$$

\subsection{Reaction Kinetics}

\subsubsection{Current density and Exchange current density}

The rate of an electrochemical reaction is determined by an activation energy barrier that the charge must overcome to move from electrolyte to a solid electrode and vice versa, and is described by the electrical current. Faraday's law relates the current density to the charge transferred the consumption of the reactant and the surface area.

$$
\mathrm{i}=\mathrm{nFv} \quad(\text { eq. } 1-20)
$$

where $\mathrm{F}$ is Faraday's constant $(96485 \mathrm{C} / \mathrm{mol})$, and the physical meaning of $\mathrm{nF}$ is the charge transferred and $\mathrm{v}$ is the reactant consumed with a unit of $\mathrm{mol} / \mathrm{cm}^{2} \mathrm{~s}$. It has to be noted that he reaction rate is easy to measure but it has to be kept in mind that the measured current 
density is a net current density, which is the difference between the forward and reverse current densities on the electrode.

\subsubsection{Butler-Volmer Equation}

The net current density of a redox reaction can be expressed by the following equation:

$$
i=n F\left[k_{0, f} C_{o x} \exp \left(\frac{-a_{R d} n F E}{R T}\right)-k_{0, b} C_{R d} \exp \left(\frac{\left(1-a_{R d}\right) n F E}{R T}\right)\right] \text { (eq. 1-21) }
$$

Where at equilibrium we have:

$$
i_{o}=n F k_{0, f} C_{0 x}^{b} \exp \left(\frac{-a_{R d} n F E_{e q}}{R T}\right)=k_{0, b} C_{R d}^{b} \exp \left(\frac{\left(1-a_{R d}\right) n F E_{e q}}{R T}\right)(\text { eq. 1-22) }
$$

Where $\mathrm{E}_{\mathrm{eq}}$ is the reversible potential and $C_{o x}^{b}$ and $C_{R d}^{b}$ are the bulk concentration of the reacting species $\left(\mathrm{mol} / \mathrm{cm}^{2}\right)$. According to equations (1-21) and (1-22), we can derive a simplified equation:

$$
i=i_{o}\left[\frac{C_{o x}}{C_{0 x}^{b}} \exp \left(\frac{-a_{R d} n F \eta}{R T}\right)-\frac{C_{R d}}{C_{R d}^{b}} \exp \left(\frac{\left(1-a_{R d}\right) n F \eta}{R T}\right)\right]
$$

Where $\eta$ is the overpotential (for the anodic reaction $\eta_{\alpha}=E_{\alpha}-E_{e q}>0$ and for the cathodic reaction $\left.\eta_{\mathrm{c}}=\mathrm{E}_{\mathrm{c}}-\mathrm{E}_{\mathrm{eq}}<0\right)$. Assuming that $\mathrm{C}_{\mathrm{ox}}=C_{o x}^{b}$ and $\mathrm{C}_{\mathrm{Rd}}=C_{R d}^{b}$ then:

$$
i=i_{o}\left[\exp \left(\frac{-a_{R d} n F \eta}{R T}\right)-\exp \left(\frac{\left(1-a_{R d}\right) n F \eta}{R T}\right)\right] \quad(\text { eq. 1-24) }
$$

The above equation is known as Butler-Volmer equation, and describes the current potential relationship. 


\subsubsection{Linear Polarization}

The Butler-Volmer equation for small overpotential $\left|\frac{\eta F n}{R T}\right| \square 1$ can be written as:

$$
i=i_{0}\left[1-\frac{a n F \eta}{R T}-\left(1+(1-a) \frac{n F \eta}{R T}\right)\right](\text { eq. } 1-25)
$$

which is further simplified to

$$
i=-i_{0} \frac{n F \eta}{R T}
$$

Therefore the charge transfer resistance, $\mathrm{R}_{\mathrm{ct}}$, which corresponds to the barrier electrons transfer from the electrode surface to the asorbed species and vice versa, can be calsulated by the following equation:

$$
R_{c t}=\frac{-\eta}{i}=\frac{R T}{n F i_{0}}
$$

As can be seen from equation 1-27, the resistance is related to the electrode potential, or the overpotential. $R_{\mathrm{ct}}$ is a very important kinetic parameter, as it describs the speed of the electrode reaction and can be measured by Electrochemical impedance spectroscopy. It has to be noted that the electron number refers only to the electrons involved in the rate determining step. For a multi-electron, multi-step reaction, $\mathrm{n}$ is not equal the total electrons involved in the whole reaction.

\subsubsection{Tafel Equation-Simplified Activation Kinetics}

At high overpotentials, one term of the Butler-Volmer equation can be ignored and the equation takes the following form. For the forward reaction: 


$$
i=i_{0} \exp \left(\frac{-a n F \eta}{R T}\right) \quad(\text { eq. } 1-28)
$$

And for the backward reaction:

$$
i=-i_{0} \exp \left(\frac{(1-a) n F \eta}{R T}\right) \quad(\text { eq. 1-29) }
$$

Rearrangement of the above two equations give the following:

$$
\eta=\frac{2.303 R T}{\alpha n F} \log i_{o}-\frac{2.303 R T}{\alpha n F} \log i \quad(\text { eq. 1-30) }
$$

Where if

$$
\alpha=\frac{2.303 R T}{\alpha n F} \log i_{o} \text { (eq. 1-31) and } \quad b=-\frac{2.303 R T}{\alpha n F}(\text { eq. } 1-32)
$$

the equation (1-30) is simplified in the following form:

$$
\eta=a-b \log i \quad(\text { eq. } 1-33)
$$

The above equation is known as Tafel equation, which shows us that in a certain current range, the overpotential has a linear dependence on the logarithm of the current density. Therefore the exchange current density can be obtained from the intercept at the current density axis. The slope of the line, which is known as Tafel slope, indicates the reaction kinetics. The higher the Tafel slope, the slower the reaction kinetics. 


\section{References}

[1] http://www.eia.gov/forecasts/ieo/

[2] Qinggang He,Xiaofang Yang, Wei Chen, Sanjeev Mukerjee,Bruce Koel and Shaowei, Influence of phosphate anion adsorption on the kinetics of oxygen electroreduction on low index Pt(hkl) single crystals, Chen, Phys. Chem. Chem. Phys. 12 (2010) 12544

[3] F. Gan, D.-T. Chin, Determination of diffusivity and solubility of oxygen in phosphoric acid using a transit time on a rotating ring-disc electrode, J. Applied Electrochemistry 23 (1993) 452

[4] K. Ruth,M. Vogt, R. Zuber, in:W. Vielstich, A. Lamm, H. Gasteiger (Eds.), Handbook of Fuel Cells: fundamentals technology and applications,Wiley, England,2003, p. 489-496.

[5] Song JM, Suzuki S, Uchida H, Watanabe M, Preparation of high catalyst utilization electrodes for polymer electrolyte fuel cells. Langmuir 22 (14) 6422

[6] Xu Z, Qi Z, Kaufman A, Superior catalysts for proton exchange membrane fuel cells. Electrochem Solid-State Lett 8(6) A313

[7] Kim H, Lee W, Yoo D, Functionalized carbon support with sulfonated polymer for direct methanol fuel cells. Electrochim Acta 52 (2006) 2620

[8] Du CY, Zhao TS, Liang ZX Sulfonation of carbon-nanotube supported platinum catalysts for polymer electroly,te fuel cells. J Power Sources 176 (2007) 9

[9] Liu Z, Wainright JS, Litt MH, Savinell RF, Study of the oxygen reduction reaction (ORR) at Pt interfaced with phosphoric acid doped polybenzimidazole at elevated temperature and low relative humidity. Electrochim Acta 51 (2006) 3914

[10] Li Q, Hjuler HA, Bjerrum NJ, Oxygen reduction on carbon supported platinum catalyst in high temperature polymer electrolytes. Electrochim Acta 45 (2000) 4219

[11] Li Q, Jensen JO, He R, Xiao G, Gao JA, Berg RW, et al. Acid doped polybenzimidazole membranes as electrolyte for fuel cells operating above $100 \mathrm{oC}$. In: Pandalai SG, editor. Recent research developments in electrochemistry, vol. 6. Kerala:Transworld Research Network; 2003. p. $1-26$.

[12] D. Thompsett, in: W. Vielstich, A. Lamm, H. Gasteiger (Eds.), Handbook of Fuel Cells: fundamentals technology and applications, Wiley, England, 2003,pp. 467-480.

[13] Qinggang He,Xiaofang Yang,Wei Chen, Sanjeev Mukerjee, Bruce Koel and Shaowei, Influence of phosphate anion adsorption on the kinetics of oxygen electroreduction on low index Pt(hkl) single crystals, Chen, Phys. Chem. Chem. Phys., 12 (2010) 12544 
[14] F. Gan, D.-T. Chin, Determination of diffusivity and solubility of oxygen in phosphoric acid using a transit time on a rotating ring-disc electrode, J. Applied Electrochemistry 23 (1993) 452

[15] Justo Lobato, Pablo Canizares, Manuel A. Rodrigo, Jose J. Linares, F. Javier Pinar, Study of the influence of the amount of PBI-H3PO4 in the catalytic layer of a high temperature PEMFC, International journal of Hydrogen Energy 35 (2010) 1347

[16] F. Seland, T. Berning 1, B. Børresen, R. Tunold, Improving the performance of hightemperature PEM fuel cells based on PBI electrolyte, Journal of Power Sources 160 (2006) 27

[17] Felix N. Büchi, Minoru Inaba, Thomas J. Schmidt, Polymer Electrolyte Fuel Cell Durabillity, Chapter GDL, Springer, ISBN: 978-0-387-85534-9 ,DOI: 10.1007/978-0-387-85536-3 ,159-198

[18] Frano Barbir, PEM Fuel Cell: Theory and Practice ,ISBN: 978-0-12-078142-3

[19] Felix N. Büchi, Minoru Inaba, Thomas J. Schmidt, Polymer Electrolyte Fuel Cell Durabillity, Springer, ISBN: 978-0-387-85534-9 ,DOI: 10.1007/978-0-387-85536-3 , p. 161

[20] R. Souzy, B. Ameduri, Functional fluoropolymers for fuel cell membranes, Prog. Polym. Sci. 30 (2005) 644.

[21] K. A. Mauritz and R. B. Moore, State of understanding of nafion, Chem. Rev. 104 (2004) 4535 .

[22] Li Qingfeng, Jensen Jens Oluf, Savinell, Robert F, Bjerrum Niels J, High temperature proton exchange membranes based on polybenzimidazoles for fuel cells., Progress in Polymer Science, 34 (2009) 449

[23] A Chandan, M Hattenberger, A El-Kharouf, S Du, A Dhir, V Self, BG Pollet, High temperature (HT) polymer electrolyte membrane fuel cells (PEMFC)-A review, Journal of Power Sources 231 (2013) 264

[24] E.K. Pefkianakis, Valadoula Deimede, Maria K. Daletou, Nora Gourdoupi andJoannis K. Kallitsis, Novel Polymer Electrolyte Membrane, Based on Pyridine Containing Poly(ether sulfone), for Application in High-Temperature Fuel Cells, Macromol. Rapid Commun. 26 (2005) 1724

[25] Ioannis Kalamaras, Maria K. Daletou, Stylianos G. Neophytides, Joannis K. Kallitsis Thermal crosslinking of aromatic polyethers bearing pyridine groups for use as high temperature polymer electrolytes, Journal of Membrane Science, 415-416 (2012) 42

[26] M. Geormezi, C.L. Chochos, N. Gourdoupi, S.G. Neophytides, J.K. Kallitsis, High performance polymer electrolytes based on main and side chain pyridine aromatic polyethers for high and medium temperature proton exchange membrane fuel cells, Journal of Power Sources,196 (22) 9382 
[27] Daletou, M. K.; Kallitsis, J. K.; Voyiatzis, G.; Neophytides, S. G., The interaction of water vapors with $\mathrm{H} 3 \mathrm{PO} 4$ imbibed electrolyte based on PBI/polysulfone copolymer blends, J. Membr. Sci. 326 (2009) 76

[28] M. Geormezi, C. Chochos, N. Gourdoupi, S.G. Neophytides, J.K. Kallitsis, High performance polymer electrolytes based on main and side chain pyridine aromatic polyethers for high and medium temperature proton exchange membrane fuel cells, J. Power Sources, 196 (2011) 9382.

[29] S.Yu, B.C. Benicewicz, Synthesis and properties of functionalized poly-benzimidazoles for high-temperature PEMFCs, Macromolecules 42 (2009) 8640.

[30] M.K. Daletou, J. Kallitsis, S.G. Neophytides, Proton conduction mechanism in H3PO4 imbibed PEMs: The effect of chemical structure and steam, ECS Trans. 33 (2010) 785.

[31] M.K. Daletou, M. Geormezi, E.K. Pefkianakis, C. Morfopoulou, J.K. Kallitsis, Fully aromatic copolyethers for high temperature Polymer Electrolyte Membrane Fuel Cells, Fuel Cells 10 (2010) 35.

[32] Felix N. Büchi, Minoru Inaba, Thomas J. Schmidt, Polymer Electrolyte Fuel Cell Durabillity, Springer, ISBN: 978-0-387-85534-9 ,DOI: 10.1007/978-0-387-85536-3 , 247

[33] Xianguo Li, Imran Sabir , Review of bipolar plates in PEM fuel cells: Flow-field designs, International, Journal of Hydrogen Energy, 30 (4) 359

[34] Seung Man Baek,DonpHyup Jeon, JinHyun Nam and Charn-Jung Kim, Pressure drop and flow distribution characteristics of single and parallel serpentine Flow fields for polymer electrolyte membrane fuel cells, Journal of Mechanical Science and Technology 26 (2012) 2995

[35] J.P. Feser, A.K. Prasad*, S.G. Advani, On the relative influence of convection in serpentine flow fields of PEM fuel cells, Journal of Power Sources 161 (2006) 404

[36] S.Dutta, S.Shimpalee, J.W.VanZee, Numerical prediction of mass exchange between cathode and anode channels in a PEM fuel cell, International Journal of Heat and Mass Transfer,44(2001) 2029

[37] M.V.Williams, H.R.Kunzand, J.M.Fenton, Influence of convection through gas diffusion layers on limiting current in PEM FCs using a serpentine flow field, Journal of the Electrochemical Society, 151(2004) A1617

[38] J.G.Pharoah, On the permeability of gas diffusion media used in PEM fuel cells, Journal of Power Sources, 144 (2005) 77

[39] P.H.Oosthuizen, L.Sunand, K.B.McAuley, The effect of Channel to channel gas crossover on the pressure and temperature distribution in PEM fuel cell flow plates, Applied Thermal Engineering, 25(2005)1083 
[40] C.XuandT.S.Zhao, A new flow field design for polymer electrolyte based fuel cells, Electrochemistry Communications, 9 (2007) 497

[41] Jiujun Zhang, PEM Fuel Cell Electrocatalysts and Catalyst Layers: Fundamentals and Applications , Springer 2008,ISBN 978-1-84800-935-6e, DOI 10.1007/978-1-84800-936-3, 89

[42] Jiujun Zhang, PEM Fuel Cell Electrocatalysts and Catalyst Layers :Fundamentals and Applications ,Springer 2008,ISBN 978-1-84800-935-6e, DOI 10.1007/978-1-84800-936-3, 135

[43] P.M. Quaino, J.L. Fernández, M.R. Gennero de Chialvo, A.C. Chialvo, Hydrogen oxidation reaction on microelectrodes: Analysis of the contribution of the kinetic routes, J. Mol. Catal. A: Chem. 252 (2006) 156. 


\section{Chapter 2}

\section{Physicochemical and electrochemical characterization techniques used during the fulfillment of this thesis}

\subsection{Introduction}

This chapter presents in detail all physicochemical and electrochemical techniques used during the fulfillment of this dissertation. A brief discussion of the basic principal of operation of each technique is also included. Concerning the physicochemical characterization of the electrocatalysts XPS, XRD, TEM and BET were employed. Whereas for the electrochemical characterization of the MEAs, polarization curves, cyclic voltammetry and AC impedance spectroscopy were empployed. Additionally, an online MS was used to monitor the composition of the gas in the outlet of the fuel cell.

\subsection{Electrochemical Characterization Techniques}

The electrochemical characterization of HTPEM fuel cell based on ADVENT's TPS 
membrane electrolyte, was conducted via cyclic voltammetry, polarization curves and electrochemical impedance spectroscopy. In the following section the basic principles of each technique is being briefly discussed.

\subsubsection{Basic Principle of operation of a Potentiostat-Galvanostat}

A potentiostat-galvanostat is a control and measuring device, and is a fundamental tool in modern electrochemistry for the investigations of reaction mechanisms related to redox chemistry. Potentiostats are designed to interface with a computer and operate through a automated software package, which allows the user to shift between experiments (cyclic voltammetry, electrochemical impedance spectroscopy etc). Up to date Hickling's principle on the three electrode potentiostat is in use [1].

\section{Principal of Operation [2]}

In potentiostatic mode, a potentiostat/galvanostat (PGSTAT) will accurately control the potential of the Counter Eletrode (CE) against the Working Electrode (WE) so that the potential difference between the working electrode (WE) and the Reference Electrode (RE) is well defined, and correspond to the value specified by the user.

In galvanostatic mode, the current flow between the $\mathrm{WE}$ and the $\mathrm{CE}$ is controlled. The potential difference between the RE and WE and the current flowing between the $\mathrm{CE}$ and WE are continuously monitored. By using a PGSTAT, the value specified by the user (i.e. applied potential or current) is accurately controlled, anytime during the measurement by using a negative feedback mechanism.

As can be seen from the diagram below, the CE is connected to the output of an electronic block which is called Control Amplifier (CA). The control amplifier forces current to flow through the cell. The value of the current is measured using a Current Follower (LowCF) or a Shunt (HighCR), for low and high currents, respectively. The potential difference is measured always between the RE and S with a Differential Amplifier (Diffamp).

Depending on the mode the instrument is used (potentiostatic or galvanostatic) the PSTAT/GSTAT switch is set accordingly. The signal is then fed into the Summation Point $(\Sigma)$ which, together with the waveform set by the digital-to-analog converter (Ein) will be used as an input for the control amplifier. 


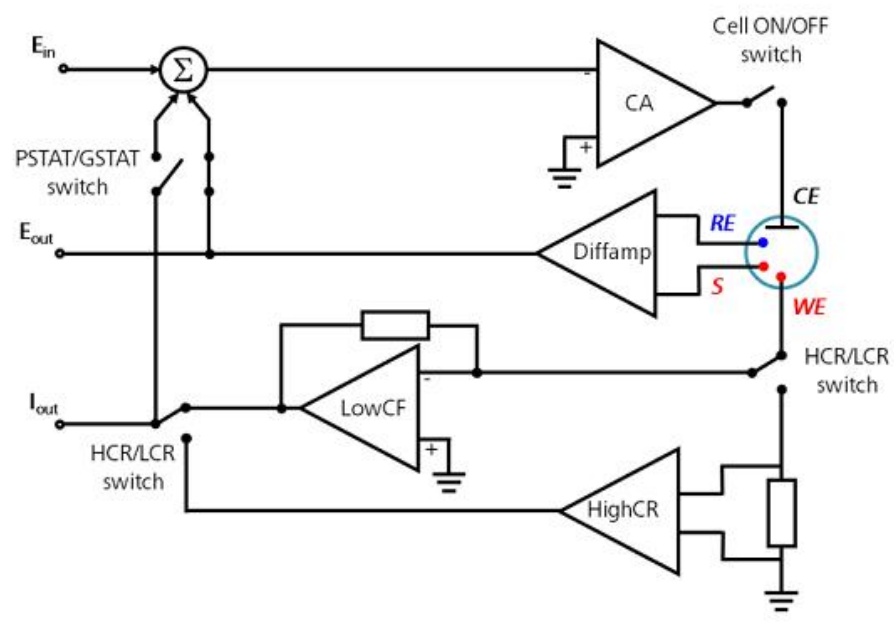

Figure 2-1. Basic diagram of a potentiostat/galvanostat.

\subsubsection{Cyclic voltammetry}

Cyclic voltammetry is one of the most common used techniques in electrochemistry, providing both quantitative and qualitative information, concerning electrochemical reactions occurring at the working electrode.

Cyclic voltammetry $(\mathrm{CV})$ is a potentiodynamic electrochemical measurement, where the working electrode potential is scanned linearly from an initial to a vertex value, and back to the initial point. This potential inversion can happen multiple times during an experiment and is referred as the number of scans. The acquired current of the working electrode is plotted versus the applied voltage, and corresponds to the cyclic voltammogram. This voltage ramping is known as the experiment's scan rate $(\mathrm{V} / \mathrm{s})$. During a typical cyclic voltammetry experiment, the potential is applied between the reference electrode and the working electrode and the current is measured between the working electrode and the counter electrode. A fuel cell use's typically a two electrode configuration; therefore the counter electrode will also act as a reference.

During the CV's measurement, the forward scan (usually towards positive values) produces current peaks due to the oxidation of species (or reduction depending on the scan direction). As the potential reaches the oxidation (or reduction) potential of the specie, the current reaches its maximum, and start decreasing as the concentration of the 


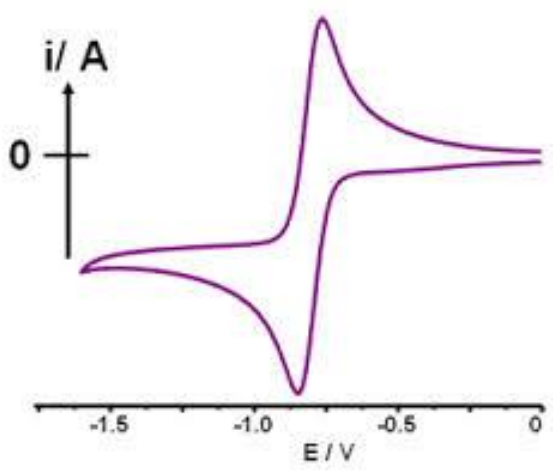

Figure 2-2. An example of a cyclic voltamogram of a redox reversible system.

later is depleted at the electrode surface. For the case of redox couples which is reversible, when the applied potential is reversed and will reach the potential that will reoxidize the product formed in the first reduction reaction, it will lead to the production of a current peak of reverse polarity from the forward scan. The oxidation peak usually has a similar shape to the reduction peak; an example is presented in Fig 2-2.

\subsubsection{Electrochemical Impedance Spectroscopy [3]}

The electrochemical impedance spectroscopy (EIS) is a characterization technique being utilized very frequently as a primary research tool in studies of proton exchange membrane fuel cells [4]. The EIS technique can provide information about the reaction kinetic and helps identifying the optimum operation conditions, as well as identifying different processes occurring at electrodes of the fuel cell, such as interfacial charge transfer, mass transfer phenomena etc. Also it allows the use of equivalent circuit to model the system, in order to obtain electrochemical parameters, both at open circuit potential and under load. Finally it can differentiate the contribution of each component of the fuel cell (membrane, electrode etc) to its total performance. A PEM fuel cell is a two electrode electrochemical system. For that reason, in-situ electrochemical impedance measurements are carried out using a two electrode connection, where both the counter and the reference are connected to one of the fuel cell's electrode. In order to measure the impedance a frequency response analyzer (FRA) and a load bank are required.

The FRA is connected to a DC load bank. During the measurement, a sinusoidal wave is generated by the FRA and applied to the cell through the electric load. This AC signal 
overlaps on the DC current and is send back to the FRA to generate the impedance spectra. The impedance spectra are generated by sweeping the frequencies usually from $10 \mathrm{kHz}$ to $10 \mathrm{mHz}$, using usually 50 points. The impedance spectra of the fuel cell can be measured in both galvanostatic and potentiostatic mode.

Potentiostatic Mode: In potentiostatic mode, during the AC impedance measurements, an $\mathrm{AC}$ potential perturbation is applied to the fuel cell, and the current response is measured. It is well known that the amplitude plays a critical role in obtaining good results. A usual AC signal amplitude, used in potentiostatic measurements of PEM fuel cell, is between 5$15 \mathrm{mV}$. Potentiostatic measurements have some disadvantages, as small changes in the potential could result in large changes in the current, which can affect significantly the accuracy of the information obtained from the impedance spectra.

Galvanostatic Mode: In galvanostatic mode, during the AC impedance measurements, an $\mathrm{AC}$ current perturbation is applied to the fuel cell and the potential response is measured. The use of this mode allows the current , AC and DC, to be controlled during the measurement, which is a great advantage. The amplitude, as for the potentiostatic mode, is very important and depends on the applied cell's load. Usually a 10 to $20 \mathrm{~mA}$ amplitude is used for the case of the fuel cell operating at $0.8 \mathrm{~A}$.

\section{$\underline{\text { Equivalent circuit }}$}

The electrochemical reactions that occurs at the electrodes of a fuel cell, consists of several processes. In order for the reactions to occur, several process are initiated, such as adsorption of electroactive species on the electrode surface, charge transfer on the electrode, diffusion of the electroactive species from the bulk of the electrolyte, the conduction of protons through the membrane and at the active sides etc.

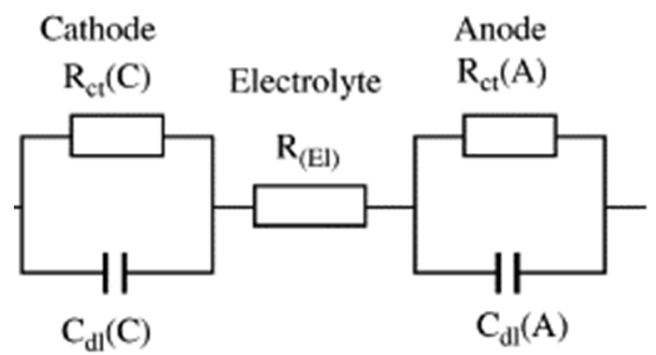

Figure 2-3. Equivalent circuit of a typical fuel cell at its simplest form. 
Each process can be represented as a simple electric component or circuit. Therefore, all the processes that occur during a fuel cell operation can be represented as an electrical circuit comprised of resistances, capacitors, inductors etc. Figure 2-3 shows a schematic representation of the equivalent circuit of a typical fuel cell at its simplest form.

\subsubsection{Polarization Curves}

Polarization curves represent the response of the current with the voltage change. The polarization curves provide useful data concerning the evaluation and characterization of a fuel cell system. Fuel cells exhibiting high current densities at high cell's potentials, which indicate high power output, are highly desired. Figure 2-4, shows a typical polarization curve of a fuel cell.

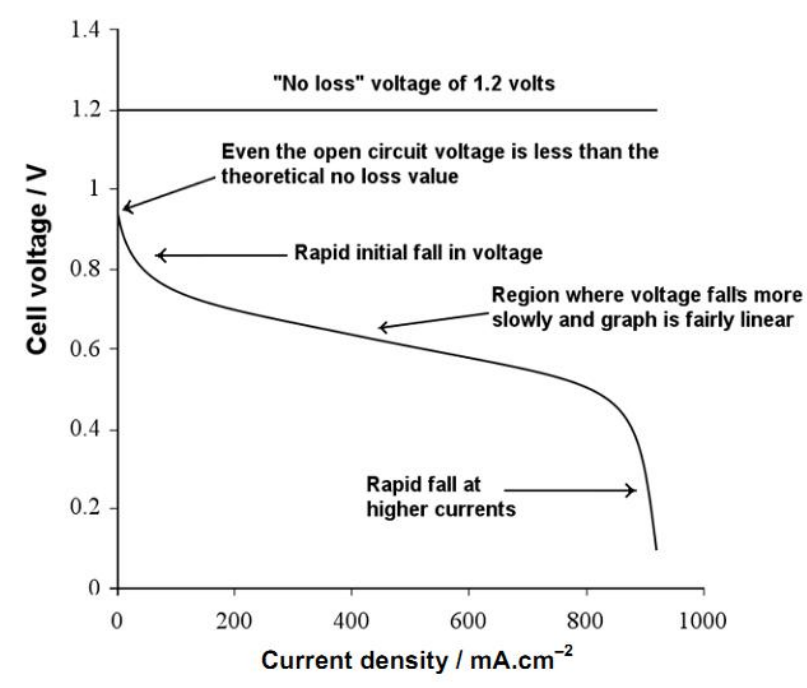

Figure 2-4. A typical polarization curve of a fuel cell [5].

Three different and distinct regions can be observed in the polarization curve, where the cell's voltage drop in each region is assigned to certain processes: kinetic, ohmic and mass transfer.

Kinetic Region: In the kinetic region, the cell's voltage drop is due to the charge transfer kinetic of the $\mathrm{H}_{2}$ oxidation and $\mathrm{O}_{2}$ reduction reaction. 
Ohmic Region: At the middle of the polarization curve, the observed voltage drop is a result of internal cell's resistance. The internal resistance of a fuel cell consists of the electric contact resistance between the various components, as well as the resistance of the proton conduction of the polymer electrolyte.

Mass transfer Region: The region at the end of the polarization curve is controlled my mass transfer phenomena and lead to further reduction of the fuel cell's voltage. At high current densities the reaction kinetics are very fast and the mass transfer of the reactant gases on the electrode's active sites is slow. As the reactant gas reaches the Pt surface, it is consumed immediately. The surface has insufficient reactant limiting the reaction rate. Therefore the limiting step in the whole reaction speed is the mass transfer.

\subsection{Physicochemical Characterization Techniques}

For the physicochemical characterization of the electrocatalysts XPS, XRD,SEM,BET and TEM were employed. As well as measurement of Catalytic Active Surface Area with $\mathrm{H}_{2}$ and $\mathrm{CO}$ Chemisorption were performed. This section presents the basic principles of operation of each technique.

\subsubsection{X-Ray Photoelectron Spectroscopy (XPS) [6]}

Today, XPS is among the most frequently used techniques in catalysis, as it provides information on the elemental composition, the oxidation state of the elements and, in favourable cases, on the dispersion of one phase over another. XPS is based on the photoelectric effect, whereby an atom absorbs a photon of energy, hv, after which a core or valence electron with binding energy $E_{b}$ is ejected with kinetic energy (Fig.2- 5):

$$
E_{k}=h v-E_{b}-\varphi \quad(\text { eq 2-1) }
$$

where $E_{k}$ is the kinetic energy of the photoelectron, $h$ is Planck's constant; $v$ is the frequency of the exciting radiation, $E_{b}$ is the binding energy of the photoelectron with respect to the Fermi level of the sample and $\varphi$ is the work function of the spectrometer. Routinely used X-ray sources are Mg Ka (1253.6 eV) and Al Ka (1486.3 eV). In XPS, one measures the intensity of photoelectrons $\mathrm{N}(\mathrm{E})$ as a function of their kinetic energy. The XPS spectrum, however, is usually a plot of $N(E)$ versus $E_{k}$, or, more often, versus the binding energy $\mathrm{E}_{\mathrm{b}}$. In addition to the expected photoelectron peaks, the spectrums 


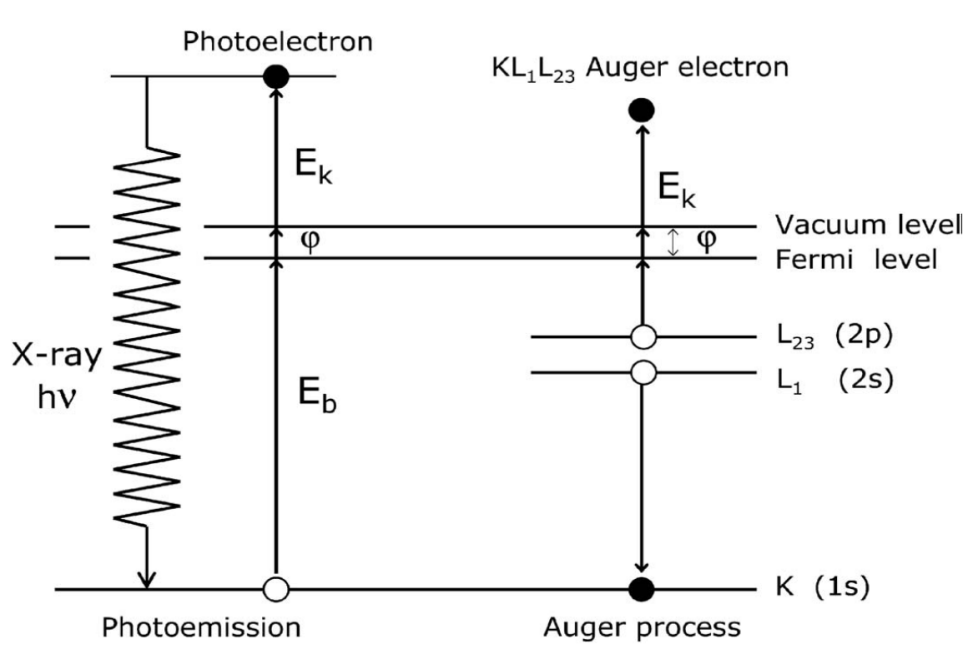

Figure 2-5. Photoemission and the Auger process. Left: An incident X-ray photon is absorbed and a photoelectron emitted. Measurement of its kinetic energy allows the binding energy of the photoelectron to be calculated. The atom stays behind as an unstable ion with a hole in one of the core levels. Right: The excited ion relaxes by filling the core hole with an electron from a higher shell. The energy released by this transition is taken up by another electron, the Auger electron, which leaves the sample with an element-specific kinetic energy. In Auger spectroscopy a beam of energetic (2-5 keV) electrons creates the initial core holes.

also contain peaks due to Auger electrons. The latter arise from the de-excitation of the photo ion by an Auger transition (Figure 2-5). Although Auger electrons have fixed kinetic energies, which are independent of the X-ray energy, Auger peaks are nevertheless plotted on the binding energy scale, which has of course no physical significance. Auger peaks can be recognized by recording the spectrum at two different X-ray energies: XPS peaks appear at the same binding energies, while Auger peaks will shift on the binding energy scale. This is the main reason why X-ray sources often contain a dual anode of $\mathrm{Mg}$ and $\mathrm{Al}$. By varying the X-ray energy Auger peaks are readily identified, and sometimes an overlap between XPS and Auger peaks can be avoided.

Photoelectron peaks are labeled according to the quantum numbers of the level from which the electron originates. An electron with orbital momentum $1(0 ; 1 ; 2 ; 3 ;$...indicated as $\mathrm{s}$; $\mathrm{p} ; \mathrm{d} ; \mathrm{f} ; \ldots$ ) and spin momentum $\mathrm{s}$ has a total momentum $\mathrm{j}=\mathrm{l}+\mathrm{s}$. As the spin may be either up $(s=+1 / 2)$ or down $(s=-1 / 2)$ each level with $1>=1$ has two sublevels, with an energy difference called the spin-orbit splitting. Spin-orbit splittings as well as binding energies of a particular electron level increase with increasing atomic number. 
The intensity ratio of the two peaks of a spin-orbit doublet is determined by the multiplicity of the corresponding levels. Thus, photoelectron peaks from core levels come in pairs (doublets) except for s levels, which give a single peak.

An XPS spectrometer contains an X-ray source and an analyzer which, in most commercial spectrometers, is hemispherical in design. In the entrance tube, the electrons are retarded or accelerated to a value called the "pass energy", at which they travel through the hemispherical filter. The lower the pass energy, the smaller the number of electrons that reaches the detector, but the more precisely is their energy determined. Behind the energy filter is the actual detector, which consists of an electron multiplier or a channeltron, which amplifies the incoming photoelectrons to measurable currents. Advanced hemispherical analyzers contain up to five multipliers. The resolution of XPS is determined by the line width of the X-ray source, the broadening due to the analyzer, and the natural line width of the level under study. These three factors are related as follows:

$$
(\Delta \mathrm{E})^{2}=\left(\Delta \mathrm{E}_{\mathrm{X}}\right)^{2}+\left(\Delta \mathrm{E}_{\text {an }}\right)^{2}+\left(\Delta \mathrm{E}_{\text {nat }}\right)^{2} \quad(\text { eq 2-2) }
$$

where $\Delta \mathrm{E}$ is the width of a photoemission peak at half-maximum, $\Delta \mathrm{E}_{\mathrm{X}}$ is the line width of the $\mathrm{X}$-ray source, $\Delta \mathrm{E}_{\mathrm{an}}$ is the broadening due to the analyser and $\Delta \mathrm{E}_{\text {nat }}$ is the natural line width.

\subsubsection{X-Ray Diffraction (XRD) [7]}

X-rays have wavelengths in the Angstrom range, and are sufficiently energetic not only to penetrate solids but also to probe their internal structure. XRD is used to identify bulk phases, to monitor the kinetics of bulk transformations, and to estimate particle sizes. An attractive feature is that the technique can be applied in situ. A conventional X-ray source consists of a target that is bombarded with high energy electrons. The emitted X-rays arise from two processes. Electrons slowed down by the target emit a continuous background spectrum of Bremsstrahlung. Superimposed on this are characteristic, narrow lines; the $\mathrm{Cu}$ Ka line, with an energy of $8.04 \mathrm{keV}$ and a wavelength of $0.154 \mathrm{~nm}$, arises because a primary electron creates a core hole in the K-shell, which is filled by an electron from the L-shell under emission of an X-ray quantum. $\mathrm{Kb}$ radiation is emitted when the $\mathrm{K}$-hole is filled from the M-shell, and so on. This process, which is called X-ray fluorescence, is the basis for X-ray sources and is also encountered in electron microscopy, EXAFS, and XPS. Xray diffraction is the elastic scattering of $\mathrm{X}$-ray photons by atoms in a periodic lattice. 
The scattered monochromatic X-rays that are in phase, produce constructive interference. Figure 2-6 illustrates how diffraction of X-rays by crystal planes allows one to derive lattice spacings by using the Bragg relationship:

$$
\mathrm{n} \lambda=2 \mathrm{~d} \sin \theta, \mathrm{n}=1,2, \ldots \quad(\text { eq. } 2-3)
$$

where $\lambda$ is the wavelength of the X-rays, $d$ is the distance between two lattice planes, $\theta$ is the angle between the incoming X-rays and the normal to the reflecting lattice plane and $n$ is the integer called the order of the reflection.
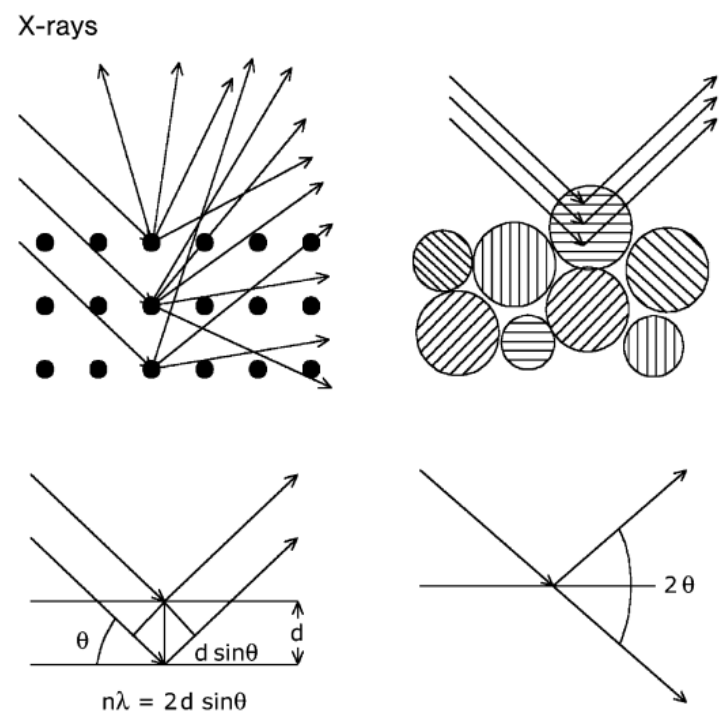

Figure 2-6. X-rays scattered by atoms in an ordered lattice interfere constructively in directions given by Bragg's law. The angles of maximum intensity enable one to calculate the spacings between the lattice planes and allow furthermore for phase identification. Diffractograms are measured as a function of the angle $2 \theta$. When the sample is a polycrystalline powder, the diffraction pattern is formed by a small fraction of the particles only. Rotation of the sample during measurement enhances the number of particles that contribute to diffraction.

If one measures the angles, $2 \theta$, under which constructively interfering X-rays leave the crystal, the Bragg relationship gives the corresponding lattice spacings, which are characteristic for a certain compound. The XRD pattern of a powdered sample is measured with a stationary $\mathrm{X}$-ray source (usually $\mathrm{Cu} \mathrm{Ka}$ ) and a movable detector, which 
scans the intensity of the diffracted radiation as a function of the angle $2 \theta$ between the incoming and the diffracted beams. When working with powdered samples, an image of diffraction lines occurs because a small fraction of the powder particles will be oriented such that by chance a certain crystal plane is at the correct angle y with the incident beam for constructive interference. Rotating powders during measurement enhances the fraction of particles that contributes to the diffraction pattern. In catalyst characterization, diffraction patterns are mainly used to identify the crystallographic phases that are present in the catalyst. XRD has one important limitation, namely that clear diffraction peaks are only observed when the sample possesses sufficient long-range order. The advantage of this limitation is that the width (or rather the shape) of diffraction peaks carries information on the dimensions of the reflecting planes. For crystallite sizes below $100 \mathrm{~nm}$, however, line broadening occurs due to incomplete destructive interference in scattering directions where the X-rays are out of phase. The Scherrer formula relates crystal size to line width:

$$
<L>=\frac{K \lambda}{\beta \cos \theta}
$$

Where $<\mathrm{L}>$ is a measure for the dimension of the particle in the direction perpendicular to the reflecting plane, $\lambda$ is the X-ray wavelength, $\beta$ is the peak width, $\theta$ is the angle between the beam and the normal on the reflecting plane and $\mathrm{K}$ is a constant (often taken as 1). Scherrer equation indicates that measuring at smaller wavelengths gives sharper peaks, not only because $\lambda$ becomes smaller but also because the diffraction lines shift to lower angles, which decreases the $1=\cos \theta$ term. Both effects help to reduce line broadening. Thus, by using Mo Ka X-rays, one can obtain diffraction patterns from smaller particles than with $\mathrm{Cu} \mathrm{Ka}$ radiation. X-ray line broadening provides a quick - but not always reliable estimate of the particle size. The technique provides clear and unequivocal information on particles that are sufficiently large, but it does not see particles that are either too small or amorphous. Hence, one can never be sure that no other phases are present than those detected with XRD.

\subsubsection{Transmission Electron Microscopy (TEM) [8]}

Transmission electron microscopy (TEM) is a microscopy technique whereby a high energy electron beam is transmitted through an ultra thin specimen, and interacts with the specimen as it passes through. As a result of the interaction of the electrons that were transmitted through the specimen, an image is formed. The image is magnified and 
focused onto a fluorescent screen, and is being acquired through a layer of photographic film, or a CCD camera. The high energy electrons are generated by a process known as thermionic emission from a filament, usually tungsten, or alternatively by field electron emission. The electrons are then accelerated by an electric potential (measured in volts) and focused by electrostatic and electromagnetic lenses onto the sample. The transmitted beam contains information about electron density, phase and periodicity; this beam is used to form an image. TEMs are capable of imaging at a significantly higher resolution than light microscopes, due to the small de Broglie wavelength of electrons. This enables the instrument's user to examine fine detail, as a single column of atoms.

At smaller magnifications TEM image contrast is due to absorption of electrons in the material, due to the thickness and composition of the material. At higher magnifications complex wave interactions modulate the intensity of the image, requiring expert analysis of observed images. Alternate modes of use allow for the TEM to observe modulations in chemical identity, crystal orientation, electronic structure and sample induced electron phase shift as well as the regular absorption based imaging.

\subsubsection{Scanning Electron Microscope (SEM) [9]}

A scanning electron microscope (SEM) is a type of electron microscope that images a sample by scanning it with a high-energy beam of electrons in a raster scan pattern. The electrons interact with the atoms producing signals that contain information about the sample's surface topography, composition, and other properties such as electrical conductivity. In a typical SEM, an electron beam is thermo-ionically emitted from an electron gun fitted with a tungsten filament cathode. Tungsten is normally used in thermionic electron guns because it has the highest melting point and lowest vapour pressure of all metals, thereby allowing it to be heated for electron emission, and because of its low cost.

The electron beam, which typically has an energy ranging from $0.5 \mathrm{keV}$ to $40 \mathrm{keV}$, is focused by one or two condenser lenses to a spot about $0.4 \mathrm{~nm}$ to $5 \mathrm{~nm}$ in diameter. The beam passes through pairs of scanning coils or pairs of deflector plates in the electron column, typically in the final lens, which deflect the beam in the $x$ and $y$ axes so that it scans in a raster fashion over a rectangular area of the sample surface. When the primary electron beam interacts with the sample, the electrons loses energy by repeated random scattering and absorption within a teardrop-shaped volume of the specimen known as the interaction volume, which extends from less than $100 \mathrm{~nm}$ to around $5 \mu \mathrm{m}$ into the surface. 
The size of the interaction volume depends on the electron's landing energy, the atomic number of the specimen and the specimen's density. The energy exchange between the electron beam and the sample results in the reflection of high-energy electrons by elastic scattering, emission of secondary electrons by inelastic scattering and the emission of electromagnetic radiation, each of which can be detected by specialized detectors. The beam current absorbed by the specimen can also be detected and used to create images of the distribution of specimen current. Electronic amplifiers of various types are used to amplify the signals which are displayed as variations in brightness on a cathode ray tube. The raster scanning of the CRT display is synchronised with that of the beam on the specimen in the microscope, and the resulting image is therefore a distribution map of the intensity of the signal being emitted from the scanned area of the specimen. The image is digitally captured and displayed on a computer monitor and saved to a computer's hard disk.

\subsubsection{Physisorption of $\mathrm{N}_{2}-\mathrm{BET}$}

BET is a very important analysis technique as it provides information about the specific surface area of materials. BET operation is based on the the physical adsorption of gas molecules on a solid surface. The specific surface area of a powder is determined by physical adsorption of a gas on the surface of the solid and by calculating the amount of adsorbate gas corresponding to a monomolecular layer on the surface.

In physical adsorption weak forces (van der Waals forces) are present between the adsorbate gas molecules and the adsorbent surface area of the test powder. The determination of the specific surface area is usually carried out at the temperature of liquid nitrogen. The amount of gas adsorbed can be measured by a volumetric or continuous flow procedure. The BET adsorption isotherm equation is used to interpret the data:

$$
\frac{1}{v[(P o / P)-1]}=\frac{c-1}{v_{m} c}\left(\frac{P}{P o}\right)+\frac{1}{v_{m} c}
$$

$P$ and $P_{0}$ are the equilibrium and the saturation pressure of adsorbates at the temperature of adsorption, $v$ is the adsorbed gas quantity (for example, in volume units), and $v_{m}$ is the monolayer adsorbed gas quantity. $c$ is the BET constant, which is expressed by (eq. 2-6): 


$$
c=\exp \left(\frac{E_{1}-E_{L}}{R T}\right) \quad(\text { eq. } 2-6)
$$

$E_{1}$ is the heat of adsorption for the first layer, and $E_{L}$ is that for the second and higher layers and is equal to the heat of liquefaction. Equation (2-5) is an adsorption isotherm and can be plotted as a straight line with $1 / v\left[\left(P_{0} / P\right)-1\right]$ on the y-axis and $\varphi=P / P_{0}$ on the $\mathrm{x}-$ axis according to experimental results. This plot is called a BET plot. The linear relationship of this equation is maintained only in the range of $0.05<P / P_{0}<0.35$. The value of the slope $A$ and the y-intercept $I$ of the line are used to calculate the monolayer adsorbed gas quantity $v_{m}$ and the BET constant $c$. The following equations can be used:

$$
\begin{aligned}
& v_{m}=\frac{1}{A+I} \\
& c=1+\frac{A}{I}
\end{aligned}
$$

The BET method is widely used in surface science for the calculation of surface areas of solids by physical adsorption of gas molecules. A total surface area $S_{\text {total }}$ and a specific surface area $S$ are given by the following equations:

$$
S_{B E T, t o t a l}=\frac{\left(v_{m} N s\right)}{V} \quad(\text { eq. } 2-9)
$$

where $v_{m}$ is in units of volume which are also the units of the molar volume of the adsorbate gas

$$
S_{B E T}=\frac{S_{\text {total }}}{a}
$$

Where $\mathrm{N}$ is the Avogadro's number, $\mathrm{s}$ is the adsorption cross section of the adsorbing species, $\mathrm{V}$ is the molar volume of the adsorbate gas and $\alpha$ is the mass of the adsorbent in gr. For the measurement of total surface area of a given sample $\mathrm{N}_{2}$ is been physisorped at $77.34 \mathrm{~K}$ and the range of the pressure is below $1 \mathrm{~atm}$. 
The system used for the measurement conducted in this thesis was a Quantachrome device. At first the sample is placed in the outgassing position prior to measurement, were the sample is being heated under vacuum condition to remove all traces of water or other solvents. The sample is then placed at the sample position, in order to begin the physisorption of $\mathrm{N}_{2}$.

\subsubsection{Measurement of Catalytic Active Surface Area with $\mathrm{H}_{2}$ and $\mathrm{CO}$ Chemisorption}

Chemisorption is an interaction between the surface, where the adsorption takes place, and the adsorbed substance in the presence of a catalyst. In ordinary circumstances, a solid surface would not react easily with most gases as there would not be enough kinetic energy between the two. But when a catalyst and a reactive gas are introduced at the point of contact, the catalyst raises the kinetic energy and sets the reaction rolling. But the quantity and characteristics of this catalyst have to be carefully controlled and monitored.

In the case of supported catalysts it is very useful to know the active metal surface area, which is usually smaller than the total area of the catalyst. Under certain experimental conditions, chemisorption of a specific gas such as Hydrogen or $\mathrm{CO}$ takes place only at the metal surface area and not at the substrates surface. The formation of an adsorbed monolayer that covers the whole metals surface takes place. For the case of Hydrogen is chemisorbed on a metal surface area, the stoichiometry number is 2 , since hydrogen is adsorbed dissociative. In that case one molecule of hydrogen corresponds to 2 atoms of metal. The adsorption isotherms can not only give us information about the total metal surface area of the catalyst but also about the dispersion of the metal particles and the particles average size. These isotherms resemble to the Langmuir isotherm with the difference that the adsorption is not being zero at high pressures. This is attributed due to the fact that hydrogen is weakly adsorbed on the catalyst substrate or due to formation of a physisorbed layer of hydrogen on top of the chemisorbed monolayer. The volume $\mathrm{Vm}$ that corresponds to a monolayer coverage of the surface is been calculated with the extension of the straight line to zero pressure. We can also calculate from these isotherms the mean particle size and the dispersion of the metal on the substrates surface. If we assume that the crystallites are spheres, then the mean diameter is given by the following equation:

$$
d=\frac{6 V}{S}
$$


Where Vis the specific volume of the metal, $\mathrm{cm}^{3} / \mathrm{gr}$ and $\mathrm{S}$ the area per gram of metal

$$
S=\frac{S_{m}}{X}
$$

Where $S_{m}$ is the metal surface area per gram of catalyst and $X$ is the percentage of metal on the catalyst, gr-met./gr-cat. The dispersion of the metal on the substrates is given by the following equation:

$$
D=\frac{\frac{V_{m}}{22415} \cdot n}{X}
$$

Where $\mathrm{n}$ is the stoichiometry of the chemisorption.

\subsection{Mass spectrometer (MS) [10]}

Mass spectrometry (MS) is an analytical technique that measures the mass-to-charge ratio and relative concentrations of charged atoms and molecules. It is used for determining masses of particles as well as elemental composition of a sample or molecule and other chemical compounds. The technique has both qualitative and quantitative uses. In a typical MS procedure the compounds of the samples are ionized, usually to cations by a loss of an electron.

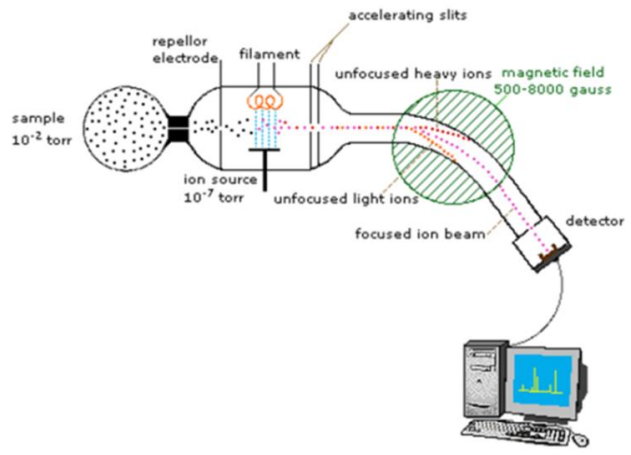

Figure 2-7. Schematic representation of typical MS operation 
Thereafter the ions are being separated according to their mass and charge ratio in an analyzer by electromagnetic field and are detected using a quantitative method, where the ion signal is converted into mass spectra. MS instruments consist of three modules: An ion source, which can convert gas phase sample molecules into ions (or, in the case of electrospray ionization, move ions that exist in solution into the gas phase); mass analyzer, which sorts the ions by their masses by applying electromagnetic fields ; and a detector, which measures the value of an indicator quantity and thus provides data for calculating the abundances of each ion present. Because ions are very reactive and short-lived, their formation and manipulation must be conducted in a vacuum. The pressure under which ions may be handled is roughly $10^{-5}$ to $10^{-8}$ torr (less than a billionth of an atmosphere). 


\section{$\underline{\text { References }}$}

[1] Hickling, A. (1942). "Studies in electrode polarisation. Part IV.-The automatic control of the potential of a working electrode". Transactions of the Faraday Society 38: 27-33.

[2] Metrohm, Autolab Application Note EC08

[3] Xiao-Zi Yuan ,Chaojie Song ,Haijiang Wang, Jiujun Zhang ,Electrochemical Impedance Spectroscopy in PEM Fuel Cells:Fundamentals and Applications, Springer 2010, ISBN 978-184882-845-2 .DOI 10.1007/978-1-84882-846-9

[4] Xiaozi Yuan et al, Review , International journal of hydrogen energy 32 (2007) 4365-4380

[5]Xiao-Zi Yuan, Chaojie Song, Haijiang Wang, Jiujun Zhang, Electrochemical Impedance Spectroscopy in PEM Fuel Cells: Fundamentals and Applications, Springer 2010, ISBN 978-184882-845-2 „DOI 10.1007/978-1-84882-846-9, Chapter 1 , p 32

[6] J. W. Niemantsverdriet ,Spectroscopy in Catalysis: An introduction, Third, Completely Revised and Enlarged Edition, 2007, Wiley, ISBN 978-3-527-31651-9, p 41

[7] J. W. Niemantsverdriet ,Spectroscopy in Catalysis: An introduction, Third, Completely Revised and Enlarged Edition, 2007, Wiley, ISBN 978-3-527-31651-9, p 148

[8] J. W. Niemantsverdriet, Spectroscopy in Catalysis: An introduction, Third, Completely Revised and Enlarged Edition, 2007, Wiley, ISBN 978-3-527-31651-9, p 182

[9] J. W. Niemantsverdriet ,Spectroscopy in Catalysis: An introduction, Third, Completely Revised and Enlarged Edition, 2007, Wiley, ISBN 978-3-527-31651-9, p 184

[10] Pfeiffer user manual 


\section{Chapter 3}

\section{Experimental: Materials, Catalyst Synthesis, MEA assembly and Instrumentation}

\subsection{Introduction}

This chapter presents in detail the materials that were used, the synthetic procedure of the under-study MWCNT based electrocatalysts, the manufacturing process of the electrodes and the membrane electrode assembly, as well as the infrastructure used for the electrochemical and physicochemical characterization of the samples.

\subsection{Materials}

High purity MWCNTs $97 \%$ of diameter ranging between 10 and $30 \mathrm{~nm}$ were purchased from Nanothinx S.A.. Chemical modifications of MWCNTs were carried out using the chemicals: isopentyl nitrate $\geq 97 \%$ supplied by Aldrich, 3-aminopyridine $99 \%$ and 2amino-3-hydroxypyridine 98\% purchased from Acros Organics. For catalyst preparation, 
dihydrogen hexachloroplatinate(IV) hexahydrate 99.95\% (metals basis), 37.5\% Pt basis and sodium borohydride 98\% were purchase from Alfa Aesar. The solvents N,Ndimethylformamide (99\%) and nitric acid 65\% were purchased from Aldrich. Ethylene glycol, (L) ascorbic acid, 2-propanol 99.8 $\leq$, and $\mathrm{H}_{3} \mathrm{PO}_{4}$ purum p.a. $\geq 85 \%$ were purchased from Merck or Sigma Aldrich. All chemicals were used as received unless otherwise noted.

\subsection{Synthesis of MWNT supported electrocatalysts}

Functionalized MWCNT were used as substrates for the newly Pt based electrocatalysts. The newly synthesized electroctalysts were thoroughly characterized, physicochemically and electrochemically (RDE), during the fulfillment of my Master thesis [1]. In continuance to this work, catalyst layers were formulated and their performance was evaluated under various conditions in HTPEM fuel cells. The carbon nanotubes were functionalized with oxygen containing groups and pyridine groups. Chemical oxidation of the carbon nanotube was used to insert carboxyl and hydroxyl groups on the side walls of the nanotube and a solvent free functionalization method in order to attach the pyridine to the outer surface of the nanotubes A brief description of the synthetic procedure is described below.

\subsubsection{Functionalization of the MWNT substrate}

This section presents a description of the synthetic procedure of each carbon nanotube based substrate. Figure 3-1 depicts the chemical structure of the functionalized MWCNT substrates.

ox.MWNT : $0.2 \mathrm{~g}$ of the as received MWCNTs were dispersed in $20 \mathrm{ml}$ of nitric acid (65 wt.\%) in a $50 \mathrm{ml}$ round bottom flask equipped with a condenser and the dispersion was refluxed under magnetic stirring for $48 \mathrm{~h}$. The resulting solid was washed up to neutral $\mathrm{pH}$ and was dried in vacuum at $100{ }^{\circ} \mathrm{C}$ overnight.

oxMWNT-Py: Functionalization of MWCNT or the as received MWCNTs took place using the solvent-free functionalization method. $0.2 \mathrm{~g}$ of the MWCNTs and $5.56 \mathrm{mmol}$ of 3-aminopyridine $(0.523 \mathrm{~g})$ were added in a $50 \mathrm{ml}$ round bottom flask equipped with a condenser. A slow addition of $1.14 \mathrm{ml}$ isoamyl nitrate $(8.4 \mathrm{mmol})$ followed and the system was left under inert argon atmosphere and vigorous stirring for $1 \mathrm{~d}$ at 65- 
$70{ }^{\circ} \mathrm{C}$. The paste was thereafter diluted in dimethylformamide (DMF) and filtered through a Nylon $(200 \mathrm{~nm})$ membrane.

The solid was washed with DMF until a colorless filtrate was obtained. The solid was dispersed in DMF, sonicated and filtrated again. Finally, it was washed with ether and dried at $80 \circ \mathrm{C}$ under vacuum.
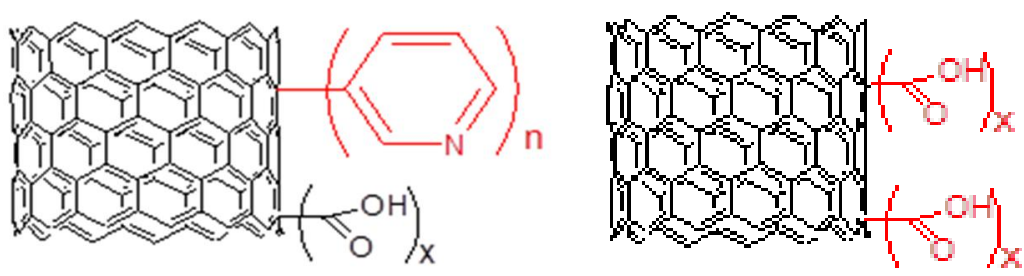

Figure 3-1. Schematic representation of the functionalized MWCNT substrates

All the pyridine functionalized substrates employed for the synthesis of the $\mathrm{Pt}$ electrocatalysts, used during the fulfilment of this thesis, had an atomic ratio of $\mathrm{C} / \mathrm{N}$ of 25 , as measured by XPS.

\subsubsection{Pt deposition on functionalized MWCNT substrate}

Platinum was introduced on the different MWCNT based supports. Dihydrogen hexachloroplatinate(IV) hexahydrate was dissolved in water resulting in a $0.25 \mathrm{M}$ solution and was used as the platinum precursor. The amount of the precursor was calculated in order to obtain a final $30 \mathrm{wt} \% \mathrm{Pt}$ catalyst loading with respect to the CNTs. The optimization of the reaction conditions were reported in an earlier work[1]. The same optimized experimental procedure was followed for all MWCNT functionalized substrates.

In a round bottom glass flask, 60mg of ox-MWCNT or ox.MWCNT-Py were dispersed in $100 \mathrm{ml}$ of an ethylene glycol $/ \mathrm{H}_{2} \mathrm{O} 2 / 1 \mathrm{v} / \mathrm{v}$ mixture. $0.22 \mathrm{ml}$ of the hexacloroplatinic acid solution was added and the solution was left stirring for $20 \mathrm{~h}$ at RT. The reduction reaction was performed under reflux conditions at $120^{\circ} \mathrm{C}$ for $2 \mathrm{~h}$. The catalyst was obtained by filtration, washed with deionized water and dried overnight at $90^{\circ} \mathrm{C}$ under vacuum. The as synthesized pyridine functionalized electrocatalyst, used during the fulfilment of this thesis, had a fine Pt distribution, as shown from TEM micrographs (figure 3-2). The average Pt particle size for the 30wt\%Pt/oxMWCNT-Py was $3.2 \mathrm{~nm}$, whereas for the case of $30 \mathrm{wt} \% \mathrm{Pt} / \mathrm{oxMWCNT}$ was $4.3 \mathrm{~nm}$. 


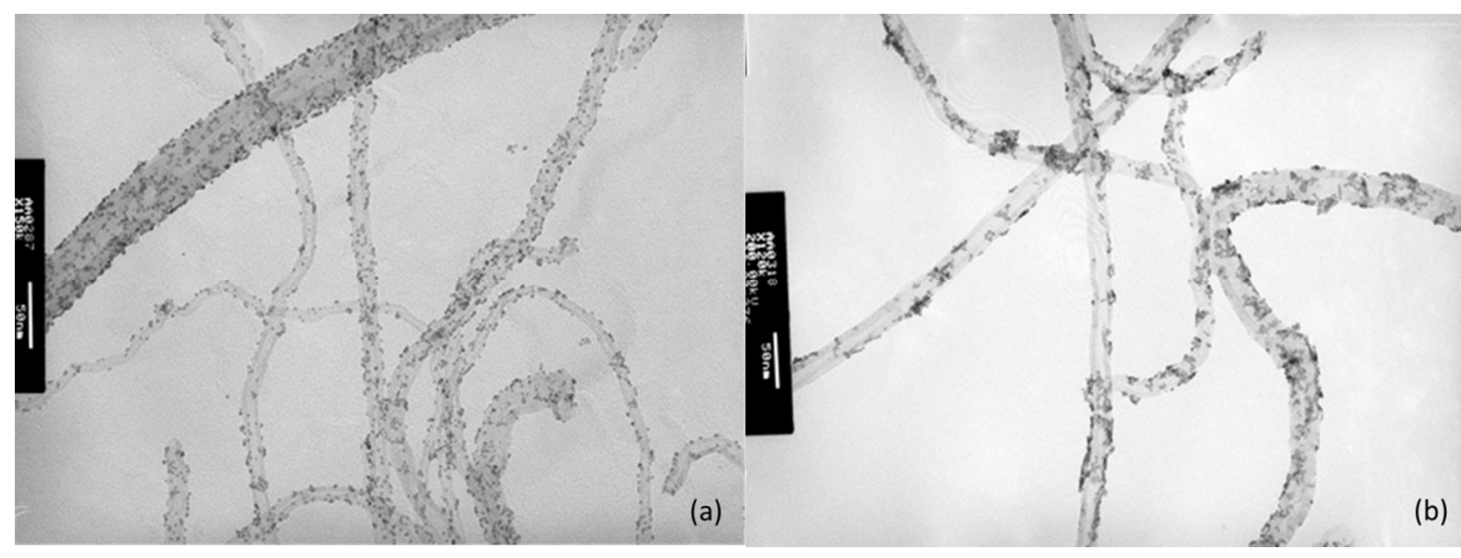

Figure 3-2. TEM micrographs a. 30wt\%Pt/oxMWCNT-Py and b. 30wt\%Pt/oxMWCNT

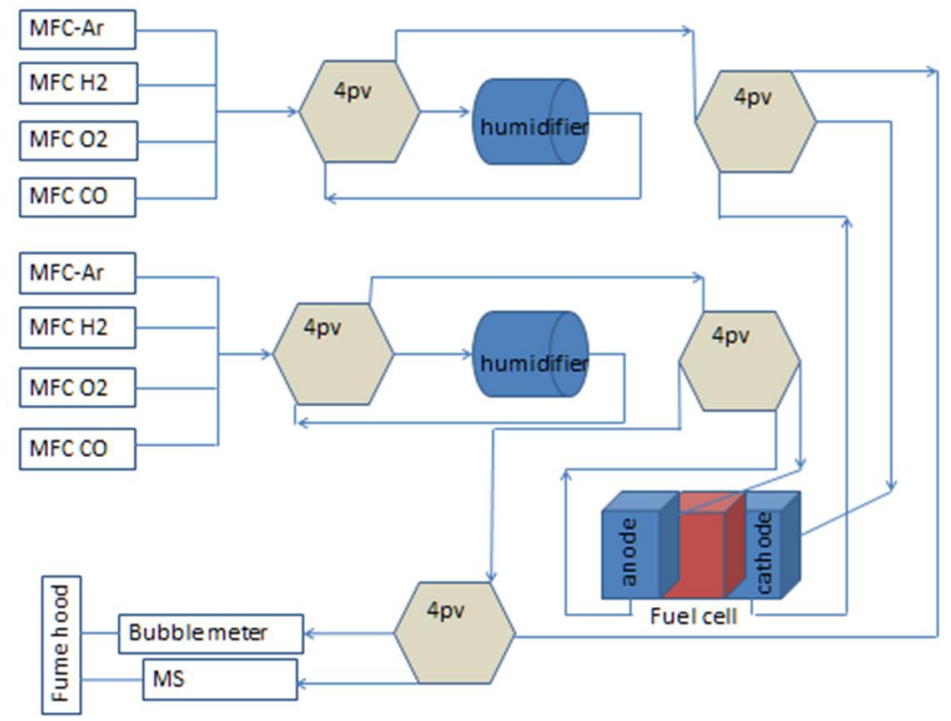

Figure 3-3. Schematic representation of the in-house test bench.

\subsection{Test bench and fuel cell reactor}

The electrochemical measurements were performed on a home-made test bench unit, which had provisions for controlling temperature, humidification, and flow of reacting 
gases, interfaced with an Autolab potentiostat-galvanostat PGSTAT-302. The potentiostatgalvanostat was equipped with the software and hardware being able to carry out steady state potentiostatic and potentiodynamic measurements like cyclic voltamograms and AC impedance measurements. The composition of the various reaction gas mixtures was controlled by several mass flow controllers (Bronkhost), where the flow rate of each gas was controlled separately. The mole fraction of water was adjusted by means of a humidifying system (Fuel cells technology Inc). Fuel Cell Technology Inc single cell was used with effective electrodes area $4 \mathrm{~cm} 2(2 \mathrm{~cm} \times 2 \mathrm{~cm})$. Both graphitic bipolar plates of the single fuel cell had the same single serpentine flow field. The measurements were made in a two-electrode arrangement and the outlet of the cell's anodic or cathodic compartment was monitored online by an OMNISTAR Balzers quadrapole mass spectrometer so that the transient and steady state gas outlet composition can be recorded.

\subsection{Electrode fabrication and Membrane Electrode Assembly}

\subsubsection{Electrodes' fabrication}

In general the preparation of the MEAs was based on the manufacturing procedures of ADVENT MEAs. For the construction of the electrodes, carbon cloth supporting layer was purchased from Fuel Cell Earth LLC, Vulcan XC-72R powder from RawChem Ltd and the PTFE dispersion in water from Sigma-Aldrich. The gas diffusion layer, GDL, used in this study was prepared by spraying a slurry made of carbon Vulcan XC-72R and PTFE solution $(50 \mathrm{wt} \% \mathrm{PTFE} / \mathrm{C})$ on carbon cloth, followed by heat treatment at $300^{\circ} \mathrm{C}$ under static air for $40 \mathrm{~min}$. The electrodes were prepared using two different optimized methods, depending on the catalyst type. Two types of electrocatalysts were used, Pt supported on carbon black (30wt \% Pt/C purchased from Tanaka Kikinzoku International) and $\mathrm{Pt}$ supported on MWCNTs (30wt \% Pt/(ox.MWCNT)-Py and 30wt\% Pt/oxMWCNT). The electrodes containing 30wt \% Pt/C were prepared by spraying 2-propanol-Pt/C ink on the GDL. The electrodes were then treated for 12 hours at $80^{\circ} \mathrm{C}$ and 3 days at $190^{\circ} \mathrm{C}$ under vacuum, in order to remove all traces of the organic solvent. The electrodes containing MWCNT based catalysts were prepared by dispersing the catalyst powder in a 2propanol/3-D $\mathrm{H}_{2} \mathrm{O} 2 / 1(\mathrm{v} / \mathrm{v})$ solution. The catalyst slurry was applied on the GDL by a spatula and the electrodes were left over night to dry at room temperature prior heating at $100^{\circ} \mathrm{C}$ in an oven for 1 hour. The MEA's anodic and the cathodic electrodes were modified in terms of catalyst type, noble metal loading and PA loading in the catalyst layer, according to the requirements of each experiment. Several MEAs were 
prepared by ADVENT technologies based on the TPS ${ }^{\circledR}$ polymer membrane imbibed with $200 \mathrm{wt} \%$ PA and were tested at ICE-HT/Laboratory of $\mathrm{H}_{2}$ technology for comparison reasons.

\subsubsection{Membrane electrode Assembly}

Before the assembly a certain amount of phosphoric acid (purum p.a. $\geq 85 \%$ purchased from Merck or Sigma Aldrich) was sprayed onto the catalytic layer. MEAs were fabricated by placing the electrodes on both sides of the phosphoric acid doped membrane TPS $^{\circledR}$ (doping level $\sim 200 \mathrm{wt} \%$ ). FEP gaskets were placed between the electrodes and the membrane in order to ensure gas isolation between the two compartments. The MEA was then placed between two metal plates with Teflon gaskets in order to ensure uniform pressure. The latter was placed into a pressing machine. The hot pressing procedure that was followed is described in detail below:

Initially the temperature is increased from $25^{\circ} \mathrm{C}$ to $60^{\circ} \mathrm{C}$ while the applied pressure on the metal plates is set to 10 bar. Isothermal at $60^{\circ} \mathrm{C}$ for 10 minutes and followed by increase of the temperature up to $75^{\circ} \mathrm{C}$. Isothermal for 10 minutes followed by temperature increase up to $90{ }^{\circ} \mathrm{C}$. Isothermal for 30 minutes. The temperature is further increased up to $110^{\circ} \mathrm{C}$. Isothermal for 30 minutes. Increase temperature up to $150{ }^{\circ} \mathrm{C}$. Isothermal for 5 minutes. The applied pressure was then increased to 20bar. Isothermal for 15 minutes to $150{ }^{\circ} \mathrm{C}$. Thereafter the cooling system was initiated and decreases the temperature to $50{ }^{\circ} \mathrm{C}$ and release pressure. No temperature ramp was used in any of the above described steps.

\subsection{Instrumentation}

The Autolab potentiostat-galvanostat PGSTAT-302 was equipped with the software being able to carry out steady state potentiostatic and potentiodynamic measurements like cyclic voltammograms and AC impedance measurements.

An OMNISTAR Balzers quadrapole mass spectrometer was employed for on-line monitoring of the transient and steady state gas outlet composition can be recorded. .

The crystalline structure of the catalysts was analyzed with an X-ray powder diffractometer (Bruker D8 Advance) using $\mathrm{Cu} K \alpha$ radiation $(\lambda=0.15418 \mathrm{~nm})$. The XRD measurements were carried out in the $2 \theta$ angle range of $20-90^{\circ}$. The mean particle 
diameter of Pt was calculated from the X-ray line broadening of the $\operatorname{Pt}(111)$ diffraction peak according to Scherrer's equation.

A Zeiss SUPRA 35VP scanning electron microscope (SEM) analyse the catalysts produced in this study. SEM measurements were carried out in collaboration with Dr.V.Drakopoulos at ICE-HT/FORTH .

X-ray Photoelectron spectroscopy (XPS), was incorporated for determination of the elemental composition and the chemical state of the elements that exist within the various CNT supports prepared in this work. The XPS experiments were performed in an UHV chamber (base pressure $8 \times 10^{-10}$ mbar), equipped with a hemispherical electron energy analyzer, a twin anode X-ray gun. The unmonochromatized $\mathrm{Mg} \mathrm{K} \alpha$ line at $1253.6 \mathrm{eV}$ and a constant pass energy mode for the analyzer were used in the XPS experiments. Pass energy of $97 \mathrm{eV}$ resulted in a full width at the half- maximum (FWHM) of $1.8 \mathrm{eV}$ for the $\mathrm{Au} 4 f_{7 / 2}$ peak of a reference foil. The energy scale of the spectrometer was calibrated with both the $\mathrm{Au} 4 f_{7 / 2}$ line at $84.0 \mathrm{eV}$ and the $\mathrm{Ni} 2 p_{3 / 2}$ line at $852.4 \mathrm{eV}$ of carefully cleaned polycrystalline $\mathrm{Au}$ and $\mathrm{Ni}$ foils. Curve fitting was performed with a least-squares curve fitting program based on a mixed Gaussian/Lorentzian function, which accounted for the band asymmetry at higher binding energies in the core level spectra of metallic species. XPS analysis were carried out in collaboration with Dr. L. Sygelou, from the laboratory of Surface Science in ICE-HT/FORTH .

The size of Pt nanoparticles supported on CNTs and carbon black were also characterized by transmission electron microscopy (TEM) on JEOL JEM2100 operating at $200 \mathrm{kV}$. Sample preparation for TEM examination involved the ultrasonic dispersion of the sample in water and placing a drop of the suspension on $3 \mathrm{~mm}$ carbon coated copper grids (Electron Microscopy Sciences). TEM imaging was carried out in collaboration with Dr. M.Kollia.

Equilibrium chemisorption of hydrogen and $\mathrm{CO}$ at $40^{\circ} \mathrm{C}$ was performed in an automated apparatus (Autosorb-1, Quantachrome) for the determination of the Pt surface by CO and $\mathrm{H}_{2}$ uptake. The Pt particles surface area and dispersion was calculated by assuming a 1:1 hydrogen:metal atomic stoichiometry. Each sample was pre-treated as follows: outgassing under vacuum at $120^{\circ} \mathrm{C}$ for $2 \mathrm{~h}$, thereafter it was exposed 4 times under hydrogen atmosphere in order to reduce the metal surface area for 30 min at $45,70,95$ and $120^{\circ} \mathrm{C}$ followed each time by outgassing under vacuum for $30 \mathrm{~min}$. 


\section{Reference}

[1] A.Orfanidi, M.K.Daletou and S.G.Neophytides Preparation and characterization of Pt on modified multi-wall carbon nanotubes to be used as electrocatalyst in high temperature fuel cells applications, Applied Catalysis B: Environmental, 106 (2011) 379-389 


\section{Chapter 4}

\section{The role of phosphoric acid in the anodic electrocatalytic layer of HT-PEM fuel cells}

\subsection{Introduction}

Commercialization of PEMFCs is still significantly restricted by its high content in Pt or other noble metals. This will have significant impact on both the cost of the fuel cell and the quantitative availability of $\mathrm{Pt}$ as an abundant raw material for the preparation of the electrocatalytic layers. Reduced cost, resulting from increased catalyst utilization and/or catalyst stability, is highly desirable. The last decade great advances have been made towards the optimization and commercialization of HT PEMFCs. Many research studies have focused on the design and optimization of electrocatalytic layers in order to increase the triple-phase boundaries at the electrochemical interface [2-5]. The structure of the catalytic layer usually comprises the catalyst, mainly Pt dispersed onto carbon black and a binder, aiming to the establishment of a 3-D ionic link between the Pt catalyst active sites and the polymer electrolyte. In this way the reaction interface is increased, given that only the Pt particles that are in direct contact with the proton conductor are electrochemically active. In such cases, the uniform mixing of the two components is hard to be guaranteed 
and part of the electrocatalyst is not used. Moreover, the phosphoric acid presence in the HT PEMFC system creates limitations concerning the slow reaction kinetics of the oxygen reduction reaction, ORR, and reduced oxygen solubility in phosphoric acid. Nevertheless, transport of gases and conductance of electrons and protons to the electrochemical interface must be optimized to provide efficient electrochemical reactions.

In a previous work, a new approach towards the development of Pt based electrocatalytic layers with increased catalyst utilization for use in high temperature PEMFCs was reported [6]. Surface modified multi-wall carbon nanotubes, MWCNTs, were used as the support. The aim was to achieve a uniform distribution of polar pyridine groups which can interact with phosphoric acid, on the surface of the carbon support. The polar groups are expected to bind the acid molecules creating proton conductive pathways throughout the catalytic layer resulting in the increase of the three phase boundary and ultimately the catalyst utilization. Stable and finely distributed Pt catalysts, Pt/(ox.MWCNT)-Py, with nanoparticle size ranging between 2-4nm were obtained.

As also mentioned above, the presence of phosphoric acid in the catalyst layer is critical for the creation of an ionic link between the Pt particles and the membrane. The electric fields at electrode/electrolyte interface that is being generated and controlled by the applied potential, plays a significant role as it does not only affect the double layer structure influencing the orientation of adsorbed species but also may modify the reactions' pathway and the electrochemical reactions' rate [14]. Phosphoric acid, in strong acidic solution ( $\mathrm{pH}$ lower than 1) is adsorbed on the Pt surface with the non protonated atom, but becomes ionized as the potential increases. Adsorbed dihydrogen phosphate, $\mathrm{H}_{2} \mathrm{PO}^{-}$, presents two coordinations, which are potential depended [15]. Also, PA coverage on Pt increases with potential and the maximum value is obtained at $0.8 \mathrm{~V}$ vs NHE, the later was studied in a phosphoric acid solution containing $1 \mathrm{~mol} / \mathrm{dm}^{3} \mathrm{HClO}_{4}[16]$. It has been reported that phosphoric acid adsorption compete with other molecules like water for the free Pt sites $[17,18]$. Anion adsorption, $\mathrm{H}_{2} \mathrm{PO}_{4}^{-}[19,20]$ amounts to 4 per cent of the surface coverage in dilute phosphoric acid at room temperature for an adsorption potential of $0.1 \mathrm{~V}$ (RHE). The adsorption of $\mathrm{H}_{2} \mathrm{PO}_{4}^{-}$is likely to be appreciably higher in 96 $\mathrm{wt} \%$ phosphoric acid where the mole ratios of acid and water are shifted to a large excess of $\mathrm{H}_{2} \mathrm{PO}_{4}^{-}$anions. Dehydration (or condensation) of orthosphosphoric acid, leads to its polymerization. In this way, a series of polyphosphoric acids can be obtained. The "backbone" chain of these types of molecules consists of alternating $\mathrm{P}$ and $\mathrm{O}$ atoms covalently bonded. Polyphosphoric acid molecules can have dozens of such phosphoric units bonded in a row. Phosphoric acids molecules can also be bounded together in rings or from the third $-\mathrm{OH}$ group on an orthophosphoric acid unit with other phosphoric groups to form branches in the polyphosphoric/polyphosphate chains. 
The hydrogen oxidation is very fast in the HTPEM fuel cells and requires less Pt loading in the electrodes, compared to the cathodic electrode. Nevertheless, the available active sites can be significantly reduced due to the adsorption of phosphoric acid species and therefore influence the performance of the anodic electrode [23]. The influence of the operating conditions on the performance of the anodic electrode especially when it operates under reformate conditions motivated us to study the influence of the amount of phosphoric acid in the anodic catalyst layer. In order to understand the performance of the anodic electrode in a high temperature acid doped PEM fuel cell the physical chemistry and electrochemistry of PA in the presence of $\mathrm{H}_{2}$ over the surface of platinum must be understood. As it will be explicitly discussed PA is catalytically reduced on the Pt surface by $\mathrm{H}_{2}$, thus resulting in the decrease of the electrochemical active surface area. The formation of PA species during normal fuel cell operation on the Pt surface, was evaluated via cyclic voltammetry and $\mathrm{CO}$ stripping voltammetry. Also the phosphoric acid species that poison the Pt active sites and the effect of $\mathrm{H}_{2} \mathrm{O}, \mathrm{CO}$ and $\mathrm{H}_{2}$ on the distribution of PA on the Pt surface have been thoroughly investigated by means of XPS. The catalyst layer consisted of the newly synthesized electrocatalyst 30\%wt Pt/(ox.MWNT)-Py [6], while the commercial 30\%wt $\mathrm{Pt} / \mathrm{C}$ was also employed for comparison.

\subsection{Experimental}

\subsubsection{Materials}

Phosphoric acid doped polymer electrolyte membranes were provided from Advent Technologies S.A.. The in-house synthesized 30wt\% Pt/(ox.MWCNT)-Py catalyst used in this study was synthesized according to literature [6]. Catalyst powder 30wt \% Pt/Vulcan $\mathrm{XC}-72 \mathrm{R}$ was purchased from Tanaka Kikinzoku International. For the construction of the electrodes, carbon cloth supporting layer was purchased from Fuel Cell Earth LLC, Vulcan $\mathrm{XC}-72 \mathrm{R}$ powder from RawChem Ltd and the PTFE dispersion in water from SigmaAldrich. For the preparation of $\mathrm{Pt}$ unsupported nanoparticles dihydrogen hexachloroplatinate(IV) hexahydrate $99.95 \%$ (metals basis), Pt 37.5\% min were purchased from Alfa Aesar and L(+) ascorbic acid 99.0\% from Carlo Erba. 2-propanol 99.8 $\leq$, dimethylacetamide (DMAc) and $\mathrm{H}_{3} \mathrm{PO}_{4}$ purum p.a. $\geq 85 \%$ were purchased from Merck or Sigma Aldrich. All chemicals were used as received unless otherwise noted.

\subsubsection{Instrumentation}

The electrochemical measurements were performed using a single cell purchased from Fuel Cell Technologies Inc. Both graphite bipolar plates of the single cell had the same single serpentine flow field for the distribution of reacting gases. The active area of the electrodes 
was $2 \mathrm{~cm} \times 2 \mathrm{~cm}$. The assembling torque applied for the single cell was $4.8 \mathrm{Nm}$. The cell was installed in a fuel cell test station which was built in-house and had provisions for controlling temperature, humidification, and flow of reacting gases. The measurements were made in a two-electrode arrangement using an Autolab potentiostat-galvanostat PGSTAT-302. The outlet of the cell's anodic compartment was connected to an OMNISTAR Balzers quadrapole mass spectrometer for the transient and steady state monitoring of the composition of the outlet gas. The size of the Pt nanoparticles of the MWCNT and carbon based electrocatalysts was characterized by transmission electron microscopy (TEM) on JEOL JEM2100 operating at $200 \mathrm{kV}$. Equilibrium chemisorption of hydrogen and $\mathrm{CO}$ at $40{ }^{\circ} \mathrm{C}$ was performed in an automated apparatus (Autosorb1, Quantachrome) for the determination of the $\mathrm{Pt}$ surface $\mathrm{H}_{2}$ and $\mathrm{CO}$ uptake. The $\mathrm{Pt}$ particles surface area and dispersion were calculated by assuming a 1:1 $\mathrm{H} / \mathrm{Pt}$ and $\mathrm{CO} / \mathrm{Pt}$ atomic stoichiometry. The photoemission experiments were carried out in a ultrahigh vacuum system equipped with a SPECS LHS-10 hemispherical electron analyzer.

\subsubsection{Electrode Preparation and MEA assembly}

Electrodes' fabrication: The gas diffusion layer, GDL, used in this study was prepared by spraying a slurry made of carbon Vulcan XC-72R and PTFE solution (50 wt\% PTFE/C) on carbon cloth, followed by heat treatment at $300^{\circ} \mathrm{C}$ under static air for $40 \mathrm{~min}$. The electrodes were prepared using two different optimized methods, depending on the catalyst type. Two types of electrocatalysts were used, Pt supported on carbon black (30wt \% Pt/C) and Pt supported on MWCNTs (30wt \%t Pt/(ox.MWCNT)-Py). The electrodes containing $30 \mathrm{wt} \% \mathrm{Pt} / \mathrm{C}$ were prepared by spraying 2-propanol-Pt/C ink on the GDL. The electrodes were then treated for 12 hours at $80^{\circ} \mathrm{C}$ and 3 days at $190^{\circ} \mathrm{C}$ under vacuum, in order to remove all traces of the organic solvent. The electrodes containing MWCNT based catalysts were prepared by dispersing the catalyst powder in a 2-propanol/3- $\mathrm{D} \mathrm{H}_{2} \mathrm{O} 2 / 1(\mathrm{v} / \mathrm{v}$ ) solution. The catalyst slurry was applied on the GDL by a spatula and the electrodes were left over night to dry at room temperature prior heating at $100^{\circ} \mathrm{C}$ in an oven for 1 hour.

The cathodic electrode's specifications were kept constant for all MEAs. The carbon black based electrocatalyst (30wt\% Pt/C) was used and the Pt loading was around $2 \mathrm{mgPt} / \mathrm{cm}^{2}$ in all cases. The electrocatalyst used for the construction of the anodic electrodes differed and the Pt loading varied from 0.20 to $2.15 \mathrm{mgPt} / \mathrm{cm}^{2}$.

Membrane Electrode Assembly, MEA: Before the MEA assembly, a certain amount of phosphoric acid, PA, was sprayed onto the electrodes. The cathodic electrodes were sprayed with $2 \mathrm{gPA} / \mathrm{gPt}$, whereas in the case of the anodic electrodes the ratio of $\mathrm{gPA} / \mathrm{gPt}$ varied from 0 to 17.8. The electrode active area was $2 \mathrm{~cm}$ x $2 \mathrm{~cm}$. Several MEAs with 
differences in the anodic electrode were fabricated. The specifications of the anodic electrodes employing either $30 \mathrm{wt} \% \mathrm{Pt} /(\mathrm{ox} . \mathrm{MWCNT})-\mathrm{Py}$ (MEAs $1-12$ ) or $30 \mathrm{wt} \% \mathrm{Pt} / \mathrm{C}$ (MEAs 13-15) are depicted in Table 4-1. In the case of MEAs 1-7 the Pt loading varied, while the amount of phosphoric acid was kept constant per electrode (at 0.0203 gPA/electrode). Whereas for MEAs 9-12 the Pt loading varied, while the amount of phosphoric acid sprayed on the catalyst layer was kept constant, at $2.00 \mathrm{gPA} / \mathrm{gPt}$. Differentiation of the Pt loading in the electrode, results in the alteration of the catalyst layer thickness. By keeping the PA per electrode constant, while at the same time varying the Pt loading (ergo catalyst thickness), the PA distribution per volume of catalyst layer will be altered at each case. More specifically, a thin catalyst layer will contain more PA per volume of catalyst layer, as compared to a thicker one, and therefore more PA will be available for the wetting of the of the Pt particles' surface ( $\mathrm{gPA} / \mathrm{gPt})$. On the other hand, for the second case, were the $\mathrm{gPA} / \mathrm{gPt}$ ratio is kept constant, the available PA for the wetting of the Pt particles' surface is expected to be the same for each Pt loading. The second set of MEAs were tested in order to verify that the phenomena that will be described in detail further in this paper are not related to the amount of $\mathrm{Pt}$ in the electrode, but are in a direct reliance to the amount of PA present within the catalyst layer.For comparison, a MEA with no PA sprayed at the electrode (MEA 8) was also prepared.The MEAs were fabricated by hot pressing, the phosphoric acid doped membranes (doping level $\sim 200 \mathrm{wt} \%$ ) between the two electrodes at $150^{\circ} \mathrm{C}$ for $5 \mathrm{~min}$. For comparison reasons, MEAs having similar specifications but employing the commercial 30wt \% Pt/C electrocatalyst at the anodic electrode were also prepared and studied in parallel.

\subsubsection{Electrochemical Characterization.}

\subsubsection{Cyclic Voltammetry.}

The pretreatment and investigation of the anodic electrodes, prior to the CO stripping voltammetry measurements, were conducted via linear sweep voltammetry. The potential was cycled between 0.075 and $0.9 \mathrm{~V}$ with a scan rate of $10 \mathrm{mV} / \mathrm{s}$. The cell temperature was $140^{\circ} \mathrm{C}$. One compartment was supplied with a constant flow of $200 \mathrm{cc} / \mathrm{min}$ of $10 \% \mathrm{H}_{2}$ in argon and the corresponding electrode acted as the reference electrode. The compartment with the electrode under investigation (working electrode) was fed with $200 \mathrm{cc} / \mathrm{min}$ of $\mathrm{Ar}$ flow, which was prior humidified by passing through a thermostated humidifier and the partial pressure of water was adjusted at $10.5 \mathrm{kPa}$ by controlling the operating temperature of the humidifier at $45^{\circ} \mathrm{C}$. 
Table 4-1. Specifications of the MEAs tested and anodic electrodes. (noble metal loading and phosphoric acid, PA, sprayed on the electrodes prior to the MEA assembly)

\begin{tabular}{|c|c|c|c|}
\hline Catalyst used & $\begin{array}{l}\text { MEA } \\
\text { No }\end{array}$ & $\begin{array}{l}\text { Pt loading } \\
\left(\mathrm{mgPt} / \mathrm{cm}^{2}\right)\end{array}$ & $\begin{array}{l}\text { PA amount } \\
\text { (gPA/gPt) }\end{array}$ \\
\hline \multirow{12}{*}{ 30\% Pt/(ox.MWCNT)-Py } & 1 & 0.20 & 17.62 \\
\hline & 2 & 0.40 & 8.81 \\
\hline & 3 & 0.65 & 5.42 \\
\hline & 4 & 1.02 & 3.45 \\
\hline & 5 & 1.25 & 2.82 \\
\hline & 6 & 1.49 & 2.36 \\
\hline & 7 & 2.06 & 1.71 \\
\hline & 8 & 2.03 & 0.00 \\
\hline & 9 & 0.86 & 2.00 \\
\hline & 10 & 0.29 & 2.00 \\
\hline & 11 & 0.77 & 2.00 \\
\hline & 12 & 1.77 & 2.00 \\
\hline \multirow{3}{*}{$30 \% \mathrm{Pt} / \mathrm{C}$} & 13 & 1.61 & 2.00 \\
\hline & 14 & 1.50 & 2.00 \\
\hline & 15 & 1.64 & 6.00 \\
\hline
\end{tabular}

\subsubsection{CO stripping voltammetry}

The electrochemically active surface area, ECSA, of the Pt based catalyst was determined via $\mathrm{CO}$ stripping voltammetry. $\mathrm{CO}$ stripping measurements were performed after a standard activation period of 48 hours of continuous operation. The cell temperature was kept constant at $140^{\circ} \mathrm{C}$. One compartment was supplied with a constant flow of $200 \mathrm{cc} / \mathrm{min}$ 
of $10 \% \mathrm{H}_{2}$ in argon and the corresponding electrode acted as the reference electrode. The compartment with the electrode under investigation (working electrode) was fed with 25\% $\mathrm{CO}$ in argon (air liquide) at a flow rate of $100 \mathrm{cc} / \mathrm{min}$ at open circuit potential. It was found that the maximum coverage with $\mathrm{CO}$ was achieved in approximately 8 minutes. Subsequently, the compartment was flushed with $200 \mathrm{cc} / \mathrm{min}$ argon in order to remove all the traces of $\mathrm{CO}$ in the gas phase. The argon flow was humidified by passing through a thermostated humifier kept at a constant temperature of $45^{\circ} \mathrm{C}$, prior to its introduction to the cell. The potential was then cycled between 0.075 and $0.9 \mathrm{~V}$ with a scan rate $10 \mathrm{mV} / \mathrm{s}$. To calculate the CO stripping charge, $\mathrm{Q}_{\mathrm{CO}}$ (in $\mathrm{C}$ ), the area under the peak that corresponds to the $\mathrm{CO}$ electrooxidation was integrated. The second cycle in the same experiment was used for baseline subtraction, considering that during the second cycle $\mathrm{CO}$ was absent from the Pt surface. The ECSA, in $\mathrm{m}^{2}$ was determined considering a charge of $420 \mu \mathrm{C} \mathrm{cm}$ for $\mathrm{CO}$ oxidation that corresponds to a monolayer of adsorbed $\mathrm{CO}$ on polycrystalline $\mathrm{Pt}$ as follows [21]:

$$
\operatorname{ECSA}\left(\mathrm{m}^{2}\right)=\mathrm{Q}_{\mathrm{CO}}(\mathrm{C}) / 4.20 \mathrm{C} \mathrm{m}^{-2}
$$

The $\mathrm{CO}_{2}$ produced during the $\mathrm{CO}$ stripping experiment was recorded at the exit of the working electrode by a quadrupole Mass Spectrometer (Buzzlers Omnistar).

\subsubsection{TEM analysis}

Post mortem analysis of the catalytic layer of used membrane electrode assemblies (MEA) was carried out by using transmission electron microscopy (TEM) . The electrodes were separated from the membrane after approximately 50 hours of continuous fuel cell operation for the evaluation of the $\mathrm{Pt}$ particle size distribution and morphology of the electrocatalyst. Adequate amounts of catalysts were scrapped off the used electrodes and were ultrasonically dispersed in water. A drop of the suspension was placed on a $3 \mathrm{~mm}$ carbon coated copper grids (Electron Microscopy Sciences). The Pt particle size distributions were obtained by manually measuring over $800 \mathrm{Pt}$ particles from the TEM pictures. The mean Pt particle size was then calculated using the following equation:

$$
\mathrm{D}_{\mathrm{APt}}=\Sigma \mathrm{D}_{\mathrm{Pt}} \mathrm{N}_{\mathrm{D}} / \mathrm{n} \quad(\mathrm{nm})
$$

where $\mathrm{N}_{\mathrm{D}}$ is the number of particles corresponding to a Pt particle size with diameter $\mathrm{D}_{\mathrm{Pt}}$ $(\mathrm{nm})$ and $\mathrm{n}$ is the total number of measured particles. Pt active surface area, is calculated by the use of eq. 4-3[6],

$$
\mathrm{CSA}_{\mathrm{TEM}}=6000 / \mathrm{d}_{\mathrm{Pt}} \mathrm{D}_{\mathrm{APt}} \quad\left(\mathrm{m}^{2} / \mathrm{gr}\right)
$$


where $d_{P t}$ is the surface density of bulk Pt $\left(21.4 \mathrm{~g} / \mathrm{cm}^{3}\right)$. Pt utilization $\left(U_{P t}\right)$ can be determined by comparing the results obtained for the ECSA by the CO stripping voltametry

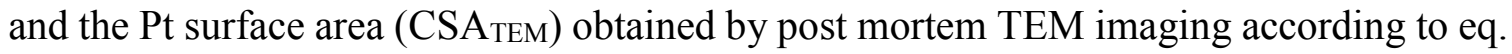
4-4.

$$
\mathrm{U}_{\mathrm{Pt}}=\mathrm{ECSA} / \mathrm{CSA}_{\mathrm{TEM}}
$$

The validity of the method was checked by the determination of the active Pt surface (CSA) by the chemisorption of $\mathrm{CO}$ or $\mathrm{H}_{2}$ on the $\mathrm{Pt}$ surface of $30 \mathrm{wt} \% \mathrm{Pt} / \mathrm{C}$ catalyst. Table $4-2$ shows the results obtained from $\mathrm{H}_{2}$ and $\mathrm{CO}$ chemisorption as well as data obtained from TEM imaging for the same catalyst. It is apparent that the results are in good correlation and the maximum deviation between the three methods does not exceed $10 \%$. Thus CO can be safely used as the probe molecule for the determination of the ECSA by the use of CO stripping voltammetry and can be compared with the Pt surface area determined using TEM post mortem measurements in order to derive the electrochemically active surface area utilization $\left(\mathrm{U}_{\mathrm{Pt}}\right)$.

\subsubsection{XPS measurements}

The photoemission experiments were carried out in a ultrahigh vacuum system equipped with a SPECS LHS-10 hemispherical electron analyzer, which additionally comprises a water cooled high pressure cell $\left(10^{-3} \mathrm{mbar}-2 \mathrm{bar}\right)$ connected with the vacuum chambers through a fast entry system, a sample preparation chamber (base pressure $5 \times 10^{-9}$ ) and an analysis chamber with a base pressure $\sim 10^{-10}$ mbar where the XPS measurements took place. The XPS measurements were performed at room temperature using unmonochromatized $\mathrm{AlKa}$ radiation under conditions optimized for maximum signal (constant $\Delta \mathrm{E}$ mode with pass energy of $97 \mathrm{eV}$ giving a full width at half maximum (FWHM) of $1.7 \mathrm{eV}$ for the $\mathrm{Au} 4 \mathrm{f} 7 / 2$ peak). The measurements were recorded at $0^{\circ}$ with respect to the sample surface normal. The analyzed area was a rectangle with dimensions $2.5 \times 4.5 \mathrm{~mm}^{2}$.

The sample was mounted on a heated stainless steel holder, figure 4-1. The under study electrode was a Pt nanoparticle film interfaced on PA imbibed TPS $®$ membrane. The Pt film was grounded in order to avoid any surface charging during data acquisition. 


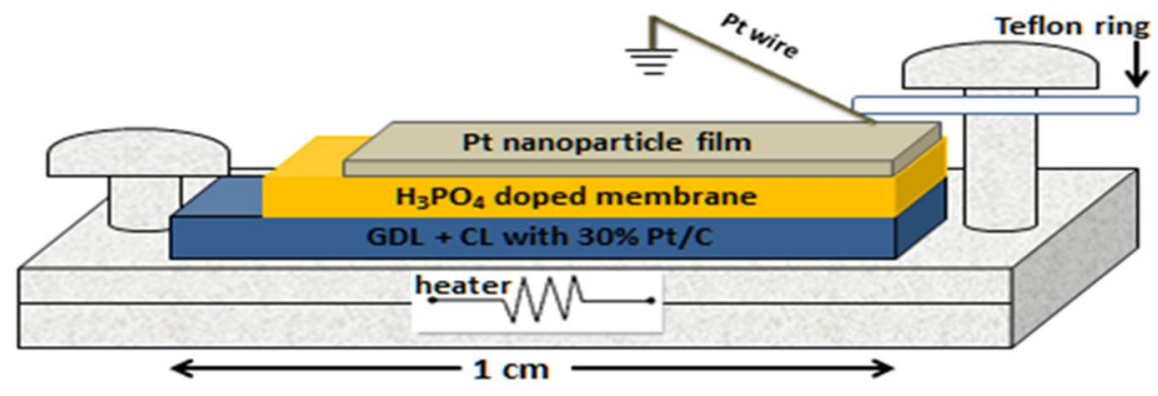

Figure 4- 1. Schematic representation of the sample mounted on the heated holder.

\subsubsection{Pt film preparation on polymer electrolyte for XPS experiments and SEM characterization}

Pt thin film fabrication: $0.3 \mathrm{ml}$ of $\mathrm{H}_{2} \mathrm{PtCl}_{6}\left(\mathrm{H}_{2} \mathrm{O}\right) 0.25 \mathrm{M}$ was added in $10 \mathrm{ml}$ of 3-distilled water. Ascorbic acid (AA) solution was used as reduction agent. $0.07 \mathrm{~g}$ of AA was diluted in $10 \mathrm{ml}$ of 3 -distilled water and then mixed with the Pt precursor solution. The solution was then heated at $140^{\circ} \mathrm{C}$ on a preheated hot plate for a few minutes until nucleation and crystal growth was observed. The solution prior to heating had a light clear orange color, during the heating process the color changed from light grey to black. As soon as the solution turned black it was removed from the hot plate. The black color of the solution indicates the formation of Pt crystallites. The solution was then left over night at room temperature for ripening. Thereafter it was poured in an $8 \mathrm{~cm}$ diameter glass disc and placed on a hot plate at $140^{\circ} \mathrm{C}$ until complete water evaporation. The Pt unsupported nanopaticles formed a thin conductive film. The Pt film was carefully washed several times with 3distilled water and heated again until complete water evaporation.

The pyridine based aromatic polyether membrane was immersed in a $85 \mathrm{wt} \% \mathrm{H} 3 \mathrm{PO} 4$ solution at $140 \mathrm{oC}$ for 20 hours in order to obtain the desired phosphoric acid doping level ( 200 wt \%). The weight gain was calculated by using the sample's weight before and after the immersion. The doped membrane was then pressed against the Pt film for a few minutes, while heating the glass disc on a hot plate at $140^{\circ} \mathrm{C}$. The membrane was carefully lifted from the glass disc surface. The procedure was repeated until desirable conductivity and film thickness were obtained. Figure 4-2 shows a SEM image of a freshly made Pt 
nanoparticle film. It consists of large aggregates of approximately $30 \mathrm{~nm}$ to $60 \mathrm{~nm}$, which are formed by Pt particles of average particle size of $5 \mathrm{~nm}$.

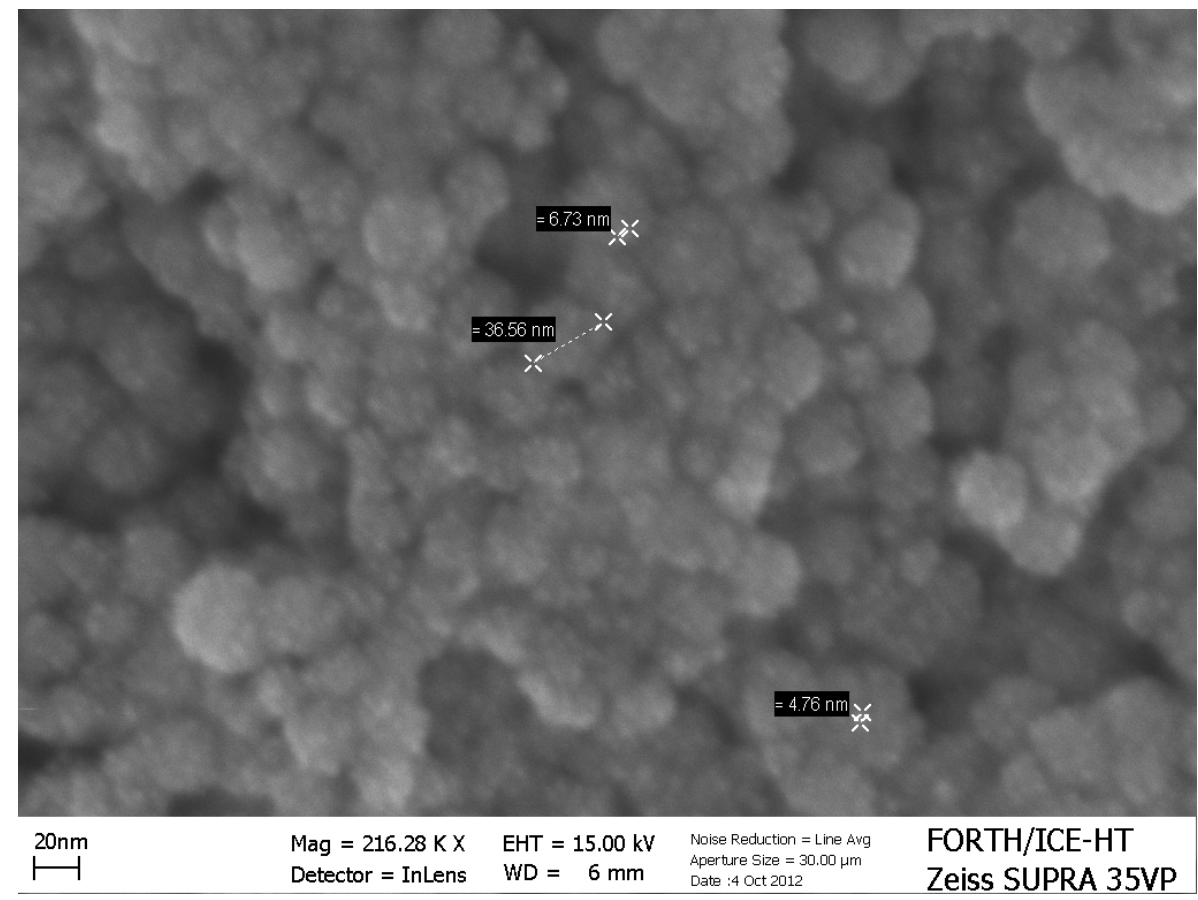

Figure 4-2. SEM image of Pt surface of the sample before use

\subsubsection{Treatment of the Pt film surface in XPS chamber}

A Pt nanoparticle film deposited on a PA doped membrane (section 4.2.6.1) was exposed to several gas atmospheres for several hours in the high pressure cell chamber of the UHV system. More specifically, the sample was inserted in the high pressure cell and was heated at $200^{\circ} \mathrm{C}$ or $180^{\circ} \mathrm{C}$ under $\mathrm{H}_{2}, \mathrm{He} / \mathrm{H}_{2} \mathrm{O}$ or $\mathrm{CO}$ flows. The $\mathrm{He}$ flow was humidified by the use of a water saturator at room temperature (water partial pressure $5 \mathrm{kPa}$ ). In the case of $\mathrm{He} / \mathrm{H}_{2} \mathrm{O}$ exposure, the sample temperature was set at $180^{\circ} \mathrm{C}$ for $90 \mathrm{~min}$. The gas flow was adjusted at $100 \mathrm{cc} / \mathrm{min}$ by the use of mass flow controller. Each gas exposure of the sample lasted for 4 hours. Thereafter the temperature was lowered to room temperature. As the high pressure chamber was attached on the UHV, the sample was subsequently inserted in the analysis chamber without being exposed to the ambient atmosphere.

After each measurement the sample was returned to its initial state by a two step procedure, before its subsequent exposure to another gas atmosphere. Initially the sample was placed in the high pressure cell under a constant flow of humidified $\mathrm{He}(100 \mathrm{cc} / \mathrm{min})$ for $90 \mathrm{~min}$ at $180^{\circ} \mathrm{C}$. The humidifier was set at room temperature. Subsequently the sample's 
temperature was lowered to R.T. and the sample was transferred in the preparation chamber.

\subsection{Results and Discussion}

MEAs were fabricated employing the 30wt $\% \mathrm{Pt} /($ ox.MWCNT)-Py electrocatalyst at the anodic electrode and the conventional $30 \mathrm{wt} \% \mathrm{Pt} / \mathrm{C}$ at the cathodic electrode. The specifications of the anodic electrodes, Table 4-1, altered as to differentiate the catalyst layer (CL) thickness and/or the amount of phosphoric acid (PA) sprayed on the electrode. The electrodes were prepared by changing the catalyst loading while keeping constant the sprayed amount of phosphoric acid per electrode or per Pt gram for the MEAs (1-7) and MEAs (9-12) (table 5-1), respectively. MEAs employing the commercial 30wt\% Pt/C at the anodic electrodes with similar specifications were also prepared and evaluated.

All MEAs under study require a break in period of approximately $48 \mathrm{~h}$ under the constant low current density of $0.2 \mathrm{~A} / \mathrm{cm}^{2}$ at $180^{\circ} \mathrm{C}$. During this galvanostatic break-in period, the cell performance improves gradually, with the cell voltage to increase by $30-35 \mathrm{mV}$ for the specific current. The voltage increase is attributed to the so called activation of the MEA, although its exact nature has not yet been determined. Plausible reasons causing the improvement in the cell's performance are the decrease of the ionic resistance of the membrane due to humidification and reorganization of the $\mathrm{PA} /$ polymer matrix during fuel cell operation, removal of impurities from the Pt active sites and redistribution of the phosphoric acid in the catalyst layer leading to the increase in the three phase boundaries. As discussed earlier, only the Pt particles that are in direct contact with the proton conductor are electrochemically active. The phosphoric acid amount in the catalytic layer is a very important and critical parameter for the achievement of a good proton conductive pathway throughout the catalyst layer.

\subsubsection{Determination of the electrochemically active surface area (ECSA)}

As has been described in the experimental section the calculation of ECSA, can be determined by the electrochemical oxidation of the pre-adsorbed $\mathrm{CO}$ on the Pt electrode. In the case of high temperature PEMFCs, the electroxidation conditions i.e. chemisorption temperature and steam partial pressure, can play a significant role on the reliability of the measurements [22]. For the determination of the optimum conditions of $\mathrm{CO}$ stripping voltammetry, $\mathrm{CO}$ was adsorbed and electroxidized at various cell temperatures (100$180^{\circ} \mathrm{C}$ ). As expected the voltage position of the $\mathrm{CO}$ electroxidation peak depends on cell's 
temperature (Fig 4-3a), while the integrated charge under the $\mathrm{CO}$ oxidation peaks is constant at temperatures below $140^{\circ} \mathrm{C}$. Thus, it is safe to consider that a full monolayer of adsorbed $\mathrm{CO}$ is developed at temperatures below $140^{\circ} \mathrm{C}$. As described in the experimental section, the outlet of the working electrode compartment was monitored by means of a mass spectrometer. It must be noted that there exists a direct correspondence between the calculated area from the $\mathrm{CO}$ titration peaks of the $\mathrm{CV}$ and the mass spectrometry recorded $\mathrm{CO}_{2}$ peaks. Fig. 4-4 depicts the ECSA values calculated from the $\mathrm{CO}$ uptake measurements (eq. 4-1) for electrodes with various Pt loadings (MEAs 1-5,7,12), The linear evolution with increasing Pt loading at the electrode confirms the validity of the method for the determination of ECSA. The ECSA was found to be $40 \mathrm{~m}^{2} \mathrm{Pt} / \mathrm{gPt}$, and the post mortem TEM derived average $\mathrm{Pt}$ particle size was found $6 \mathrm{~nm}$, resulting in $\mathrm{CSA}_{\mathrm{TEM}}=46.7 \mathrm{~m}^{2} \mathrm{Pt} / \mathrm{gPt}$. This corresponds to $\mathrm{UPt}_{\mathrm{Pt}}$ approximately $85 \%$ for all $\mathrm{Pt}$ loadings on the electrode.

Table 4-2. Mean Pt particle size and active $\mathrm{Pt}$ surface area for the $30 \% \mathrm{Pt} / \mathrm{C}$ catalysts, as measured via $\mathrm{H}_{2}$ chemisorbtion, $\mathrm{CO}$ chemisorbtion and TEM imaging.

\begin{tabular}{ccc}
\hline & Pt particle size (nm) & $\begin{array}{c}\text { Active Pt surface area } \\
\left(\mathbf{m}^{\mathbf{2}} \mathbf{P t} / \mathbf{g P t}\right)\end{array}$ \\
\hline H2 chemisorption & 3.5 & 80.3 \\
CO chemisorption & 3.7 & 75.8 \\
TEM & 3.2 & 87.6 \\
\hline
\end{tabular}

It has to be mentioned that $\mathrm{CO}$ was adsorbed at both open circuit potential and at $0.075 \mathrm{~V}$ at $140^{\circ} \mathrm{C}$. It was found that the $\mathrm{CO}$ uptake was exactly the same for both cases, as shown in figure 4-5. Therefore, $\mathrm{CO}$ adsorption was conducted at OCP conditions. T.Vidakovic et al [42] also observed no difference in the $\mathrm{CO}$ uptake under potential and OCP conditions. 

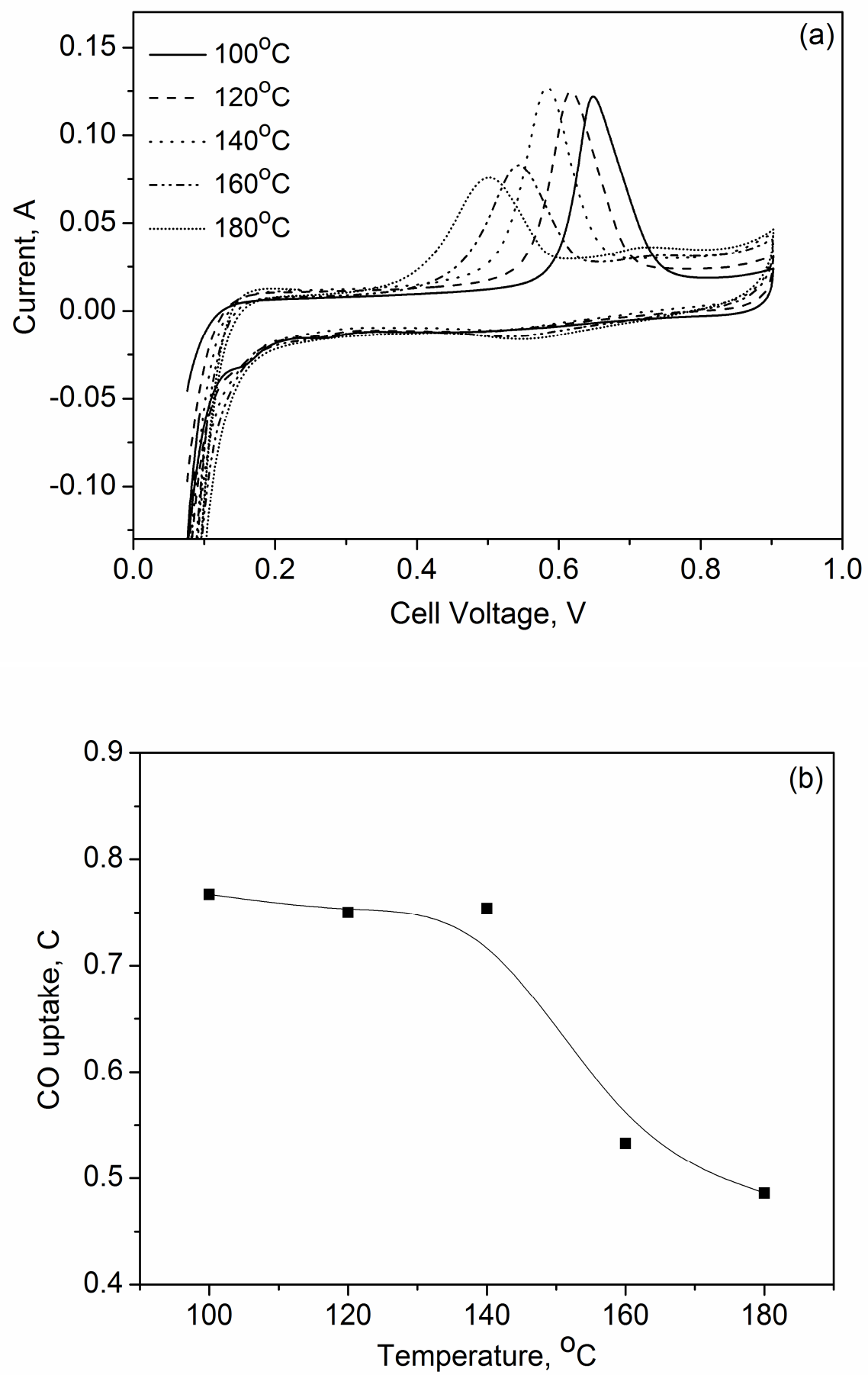

Figure 4-3. (a) CO stripping voltammograms at various cell temperatures for constant water partial pressure of $10 \mathrm{kPa}(\mathrm{b})$ effect of temperature on $\mathrm{CO}$ uptake. 


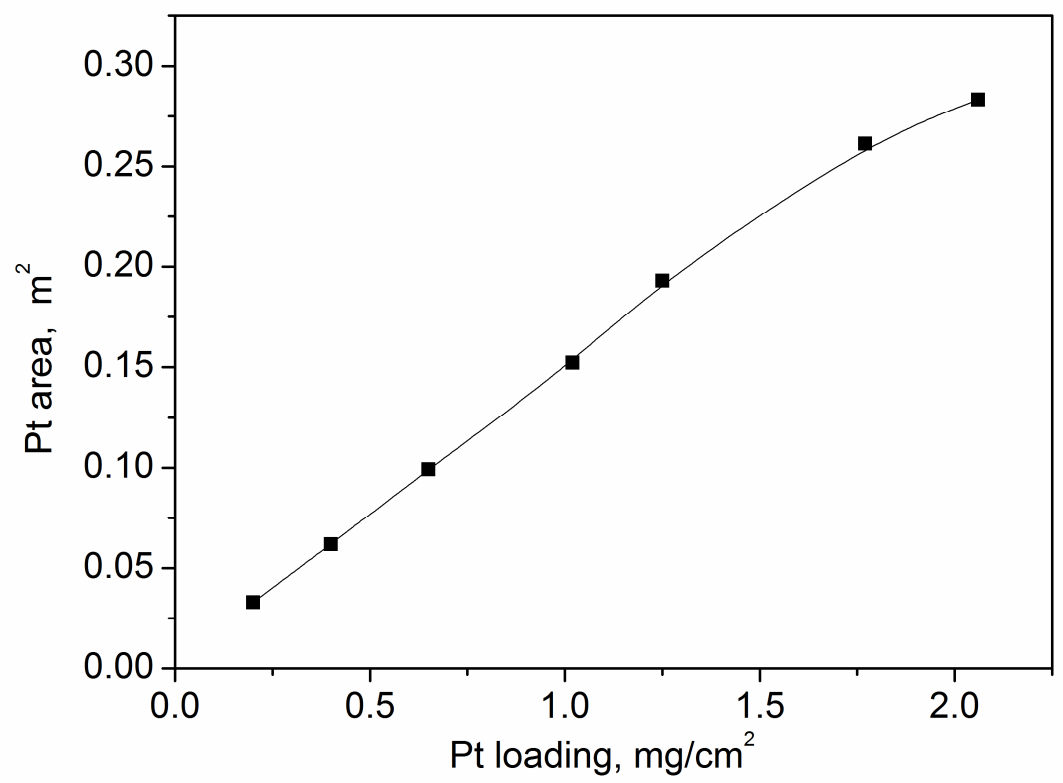

Figure 4-4. ECSA determination with increasing Pt loading based on $\mathrm{CO}$ stripping voltammetry measurements at $140^{\circ} \mathrm{C}$ (MEAs $1-5,7,12$ in Table 4-1).

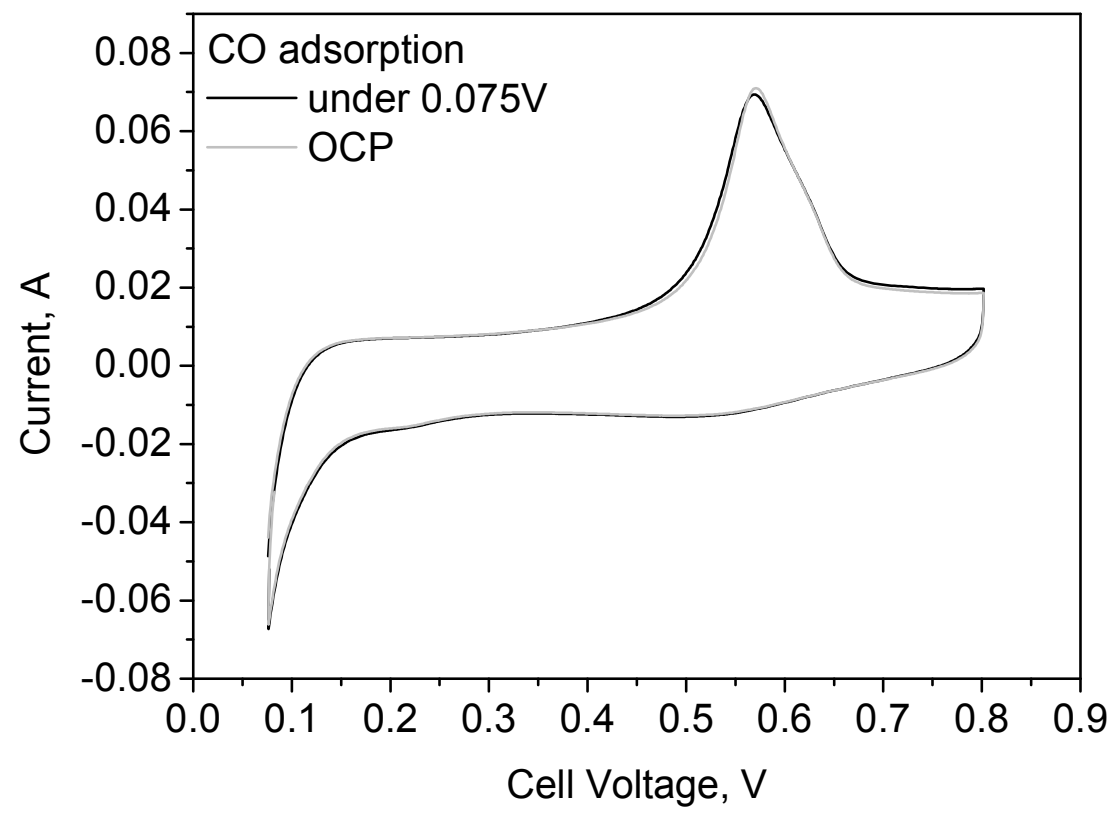

Figure 4-5. CO stripping voltamograms, obtained after $\mathrm{CO}$ adsorption at open circuit potential and under polarization of the working electrode at $0.075 \mathrm{~V}$. Tcell $=140^{\circ} \mathrm{C}$. 


\subsubsection{Effect of $\mathrm{H}_{3} \mathrm{PO}_{4}$ loading on the anode}

All MEAs, were left under continuous fuel cell operation at $180^{\circ} \mathrm{C}$ at $0.2 \mathrm{~A} / \mathrm{cm}^{2}$ under pure $\mathrm{H}_{2} / \mathrm{O}_{2}$ flows (gas stoichiometry of $\lambda_{\mathrm{H} 2}=1.2, \lambda_{\mathrm{O} 2}=2$ ) for at least 15 hours after the breakin period prior to any measurement. Thereafter cyclic voltammetric experiments were performed at the anodic electrodes. All cyclic voltammograms were normalized with respect to the Pt loading per electrode for comparison reasons. The first and second recorded cycles for the first set of MEAs 1-7 (Table 4-1) are presented in Figures 4-6a and $4-6 \mathrm{~b}$, respectively. During the first voltage sweep of the cyclic voltammetry measurements, two oxidation peaks were observed at approximately 500 and $750 \mathrm{mV}$ in the anodic scan. These peaks appear only when the sprayed PA amount on the electrode was below 3 gPA/gPt (Fig. 4-6a). Interestingly these peaks were not detectable during the second voltage sweep (Fig. 4-6b). Increasing the gPA/gPt ratio such peaks at 500 and $750 \mathrm{mV}$ do not appear, though a slight increase in the double layer charging is observed. The above mentioned behavior and the appearance of the peaks was also observed when electrodes employing the commercial 30wt $\% \mathrm{Pt} / \mathrm{C}$ were prepared with similar specifications (MEAs 13 and 15) and were used under the same conditions (Fig 4-7).

In order to clarify whether the aforementioned oxidation peaks are related to the amount of $\mathrm{Pt}$ and/or the double layer charging a set of MEAs was tested, where Pt loading was varied from 0.29 to $1.77 \mathrm{mgPt} / \mathrm{cm} 2$ and the ratio of $\mathrm{gPA} / \mathrm{gPt}$ was kept at constant $2 \mathrm{gPa} / \mathrm{gPt}$ for all cases (MEAs 10-12). The first and second recorded cycles are present in Fig 4-8. It is clear that the appearance of the two oxidation peaks at 500 and $750 \mathrm{mV}$ is not related to the $\mathrm{Pt}$ loading but instead to PA amount sprayed in the electrodes, which is within the region that the above observed anodic peaks appear. Also it has to be noted, that when similar experiments were conducted at the cathodic electrode for low PA amounts in the electrodes, no such peaks were observed thus showing that the appearance of these peaks is directly related to the anodic process. 

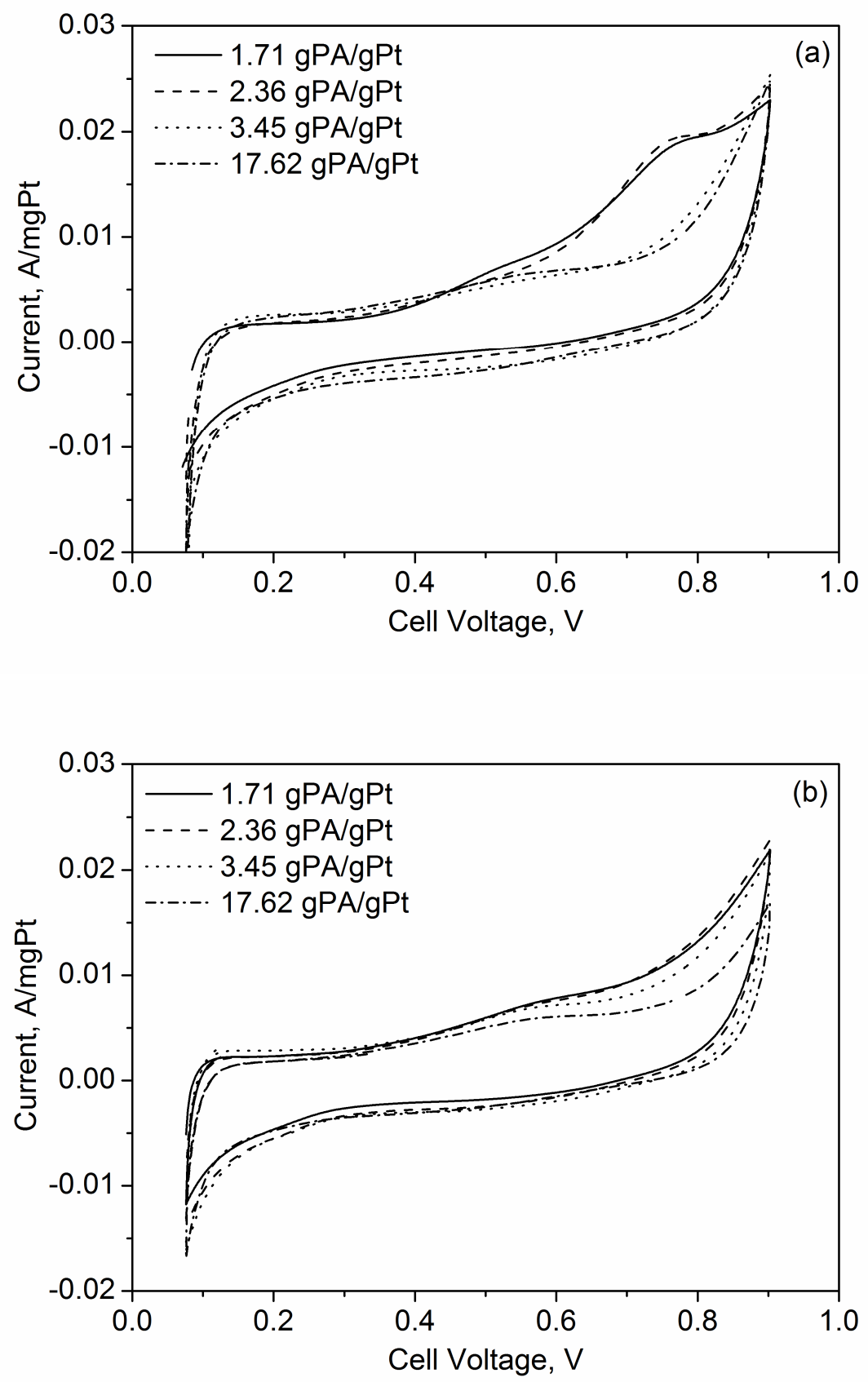

Figure 4-6. Cyclic voltammograms (a) first cycle and (b) second cycle at $140^{\circ} \mathrm{C}$ after fuel cell operation at $180^{\circ} \mathrm{C}$ for $24 \mathrm{~h}$ at $0.2 \mathrm{~A} / \mathrm{cm}^{2}$ under pure $\mathrm{H}_{2} / \mathrm{O}_{2}$ flows $\left(\lambda_{\mathrm{H} 2}=1.2, \lambda_{\mathrm{O} 2}=2\right)$ for MEAs 1, 4, 6 and 7. Variation of the Pt loading with constant amount of PA on the catalytic layer. 


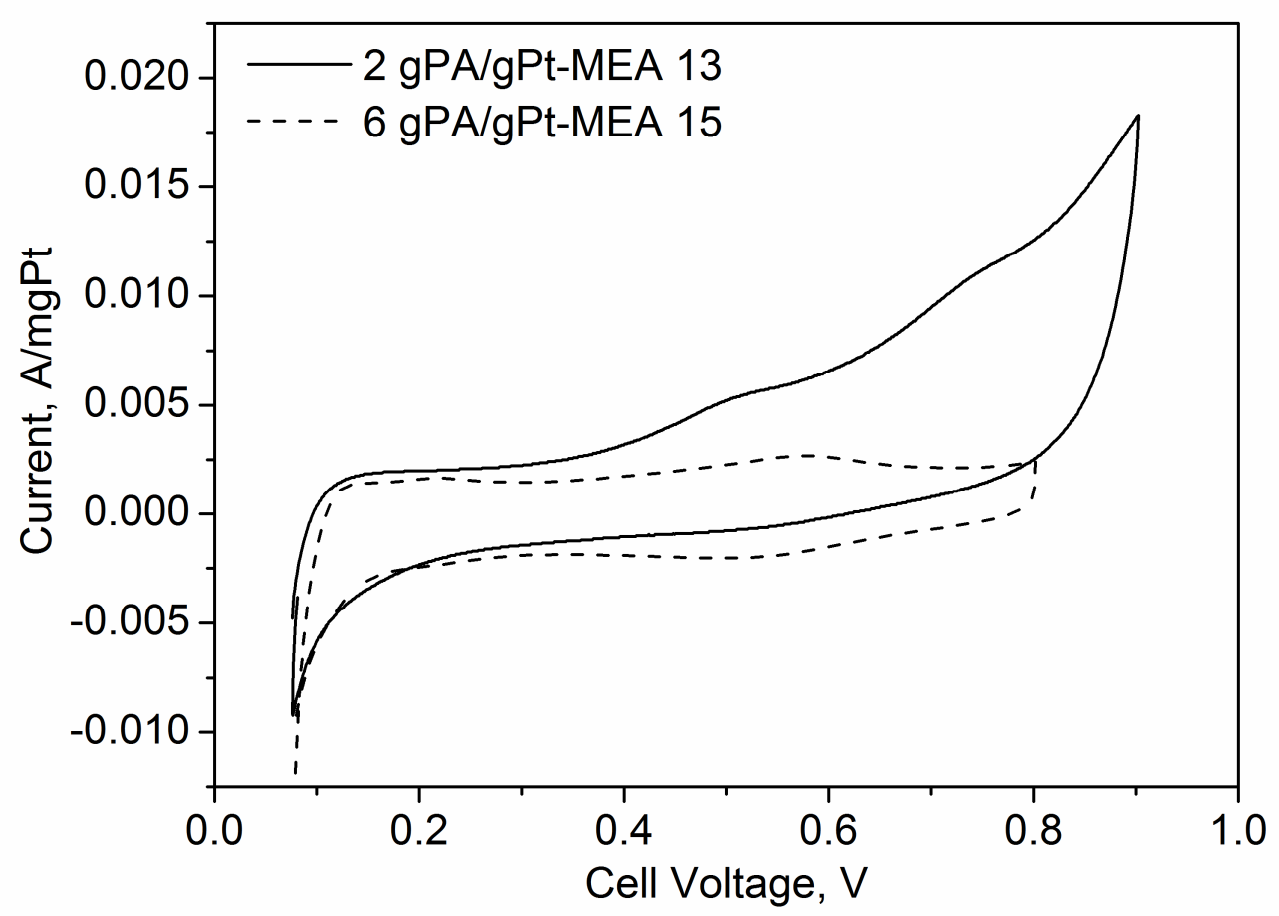

Figure 4-7. Cyclic voltammograms, first cycle, at $140^{\circ} \mathrm{C}$ after fuel cell operation at $180^{\circ} \mathrm{C}$ for $24 \mathrm{~h}$ at $0.2 \mathrm{~A} / \mathrm{cm}^{2}$ under pure $\mathrm{H}_{2} / \mathrm{O}_{2}$ flows $\left(\lambda_{\mathrm{H} 2}=1.2, \lambda_{\mathrm{O} 2}=2\right)$ for MEAs $13(2 \mathrm{gPA} / \mathrm{gPt})$ and MEA 15 ( $6 \mathrm{gPA} / \mathrm{gPt})$.

Similar peaks were reported by Buelte et al in a half cell apparatus using standard $\mathrm{Pt} / \mathrm{C}$ based electrode, especially at high phosphoric acid concentration, $115 \mathrm{wt} \%$ phosphoric acid ( $75 \mathrm{wt} \% \mathrm{P} 2 \mathrm{O} 5)$. They assumed, that those peaks during $\mathrm{CV}$ is the result of phosphoric acid species adsorption on the Pt surface and subsequent oxidation followed by polarization [23]. Several studies in literature report the appearance of a peak at around $0.7 \mathrm{~V}$ when performing cyclic voltammetry of Pt in different phosphoric acid based systems [18,2429]. The peak was attributed to the anodic oxidation of impurities formed electrochemically from the phosphoric acid in the hydrogen region; phosphine formation by direct reduction of $\mathrm{H}_{3} \mathrm{PO}_{4}$ or by a partial disproportionation of phosphorous with simultaneous formation of oxyacids such as $\mathrm{H}_{3} \mathrm{PO}_{3}$, adsorbed at low potentials.

However, Burke et al [30] gave another explanation for the peak's origin and concluded that it arises from the pre-monolayer oxidation processes and is independent of the electrolyte anion, therefore it is a property of the metal rather than the electrolyte. This 

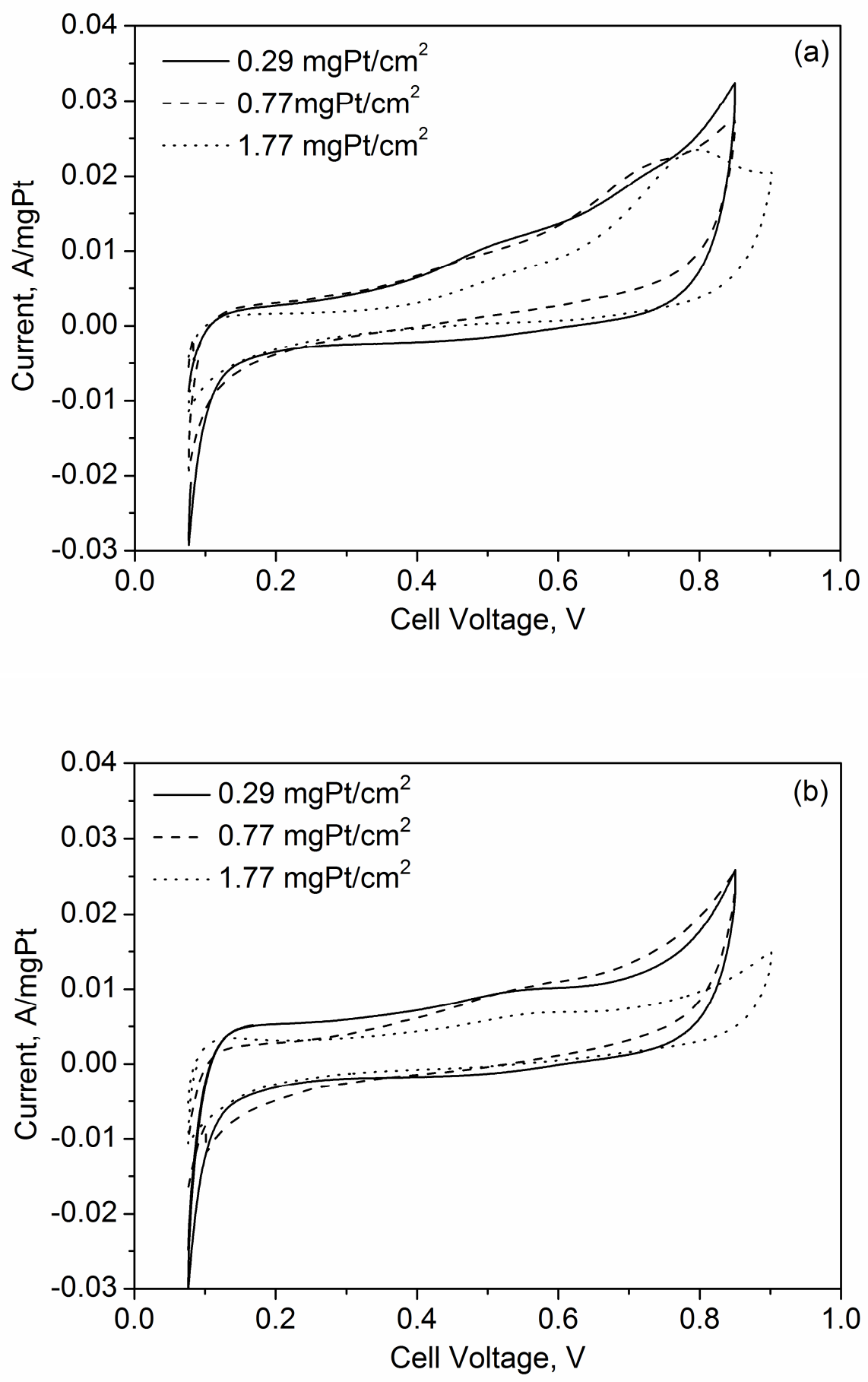

Figure 4-8. Cyclic voltammograms, first (a) and second (b) cycle, at $140^{\circ} \mathrm{C}$ after fuel cell operation at $180^{\circ} \mathrm{C}$ for $24 \mathrm{~h}$ at $0.2 \mathrm{~A} / \mathrm{cm}^{2}$ under pure $\mathrm{H}_{2} / \mathrm{O}_{2}$ flows $\left(\lambda_{\mathrm{H} 2}=1.2, \lambda_{\mathrm{O} 2}=2\right)$ for MEAs 10-12, where the $\mathrm{Pt}$ loading per electrode varied and $\mathrm{gPA} / \mathrm{gPt}=2$. 
hypothesis was supported by the observation of a similar peak in systems not involving phosphoric acid $[31,32]$. The reaction producing the peak under low water environments (dehydrated) was reported to be slow [32] and was described as the formation of submonolayer quantities of hydrous oxide films where the reduced state is a Pt adatom, rather than a normal surface or bulk lattice atoms [31].

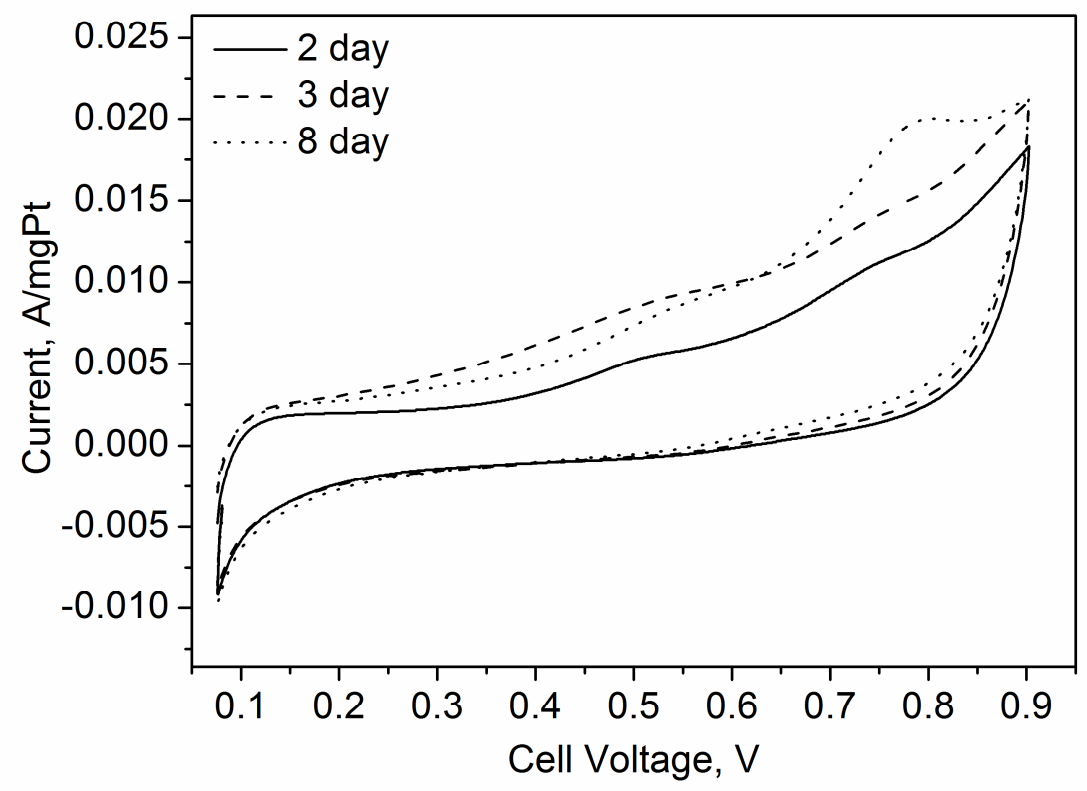

Figure 4-9. Cyclic voltammograms (first cycle) of MEA 13 employing the commercial $30 \%$ wt $\mathrm{Pt} / \mathrm{C}$ catalyst at $140^{\circ} \mathrm{C}$ after continuous fuel cell operation at $180^{\circ} \mathrm{C}$ at $0.2 \mathrm{~A} / \mathrm{cm}^{2}$ under pure $\mathrm{H}_{2} / \mathrm{O}_{2}$ flows $\left(\lambda_{\mathrm{H} 2}=1.2, \lambda_{\mathrm{O} 2}=2\right)$ for 2,3 and 8 days.

The cumulative built up of the of the species that appear in the CVs of Figs 4-6, 4-7 and 48 at 500-750mV is shown in Figure 4-9 for an MEA left under constant polarization mode at $0.2 \mathrm{~A} / \mathrm{cm}^{2}$ for longer operation intervals. As depicted in Fig. 4-9, the CVs were obtained after 2, 3 and 8 days of continuous fuel cell operation at $180^{\circ} \mathrm{C}$. The increase of the intensity of the peaks with time is apparent and denotes the accumulation of electro-active species on the Pt surface. For the anodic electrodes with PA loading higher than $3 \mathrm{gPA} / \mathrm{gPt}$, no peaks were noticed during the first $\mathrm{CV}$ cycle with respect to the operation time. On the other hand, for PA loadings lower than $3 \mathrm{gPA} / \mathrm{gPt}$, an increased variation of the peak intensity at $750 \mathrm{mV}$ on the duration of the cell's operation was observed in all cases.

During all the above voltage sweeps, the outlet of the working electrode compartment was monitored using an on-line mass spectrometer. In the course of the potentiodynamic scan of the electrode, production of $\mathrm{CO}_{2}$ was recorded. As depicted in Figure 4-10 the $\mathrm{CO}_{2}$ 
MS signal is plotted versus potential. The observed $\mathrm{CO}_{2}$ production is a result of the carbon substrate's electrooxidation during cyclic voltammetry and is not correlated with the anodic peak at $750 \mathrm{mV}$. Interestingly, the intensity of the $\mathrm{CO}_{2}$ formation followed a very specific pattern when relatively low amounts of PA (below $3 \mathrm{gPA} / \mathrm{gPt}$ ) were present in the catalyst layer. A striking difference between the first and second cycle of the cyclic voltammograms is the increase in $\mathrm{CO}_{2}$ electrooxidation rate during the second cycle. This is accompanied by the non appearance of the oxidation peak at $750 \mathrm{mV}$. The low $\mathrm{CO}_{2}$ electrooxidation rate observed during the first cycle in Fig.4-10 is indicating either a blockage of the electrochemically active surface area or a reconstruction of the electrochemical interface. The hysteresis observed in the $\mathrm{CO}_{2}$ signal during the first cycle can be attributed to the activation of the electrochemical surface after the appearance of the oxidation peak at $750 \mathrm{mV}$. Further potential cycling after the second cycle, did not result in any additional change either in the voltammogram or the amount of $\mathrm{CO}_{2}$ production. A totally different behavior is observed in the case of high $\mathrm{gPA} / \mathrm{gPt}>3$. As it is depicted in Fig. 4-10b there is no difference between the $1^{\text {st }}$ and $2^{\text {nd }}$ cycle either in the $\mathrm{CV}$ or the $\mathrm{CO}_{2}$ signal.

Polarization curves were obtained from various MEAs with different anodic electrode specifications for the evaluation of the effect depicted in Fig. 4-10 on the performance of the MEAs after short term operation. Figure 4-11 depicts three characteristic IV plots were the PA amount differs in the catalytic layer of the anodic electrode, while maintaining the cathodes specification constant for all cases.

Each diagram shows two IV curves, which were recorded under high excess of $\mathrm{H}_{2}$ and $\mathrm{O}_{2}$ (200cc/min $\mathrm{H}_{2}$ and $\mathrm{O}_{2}$, respectively). The first was recorded after the continuous fuel cell operation of the MEA for $48 \mathrm{~h}$ at $0.2 \mathrm{~A} / \mathrm{cm}^{2}$ under pure $\mathrm{H}_{2} / \mathrm{O}_{2}$ flows (gas stoichiometry $\lambda_{\mathrm{H} 2}$ $=1.2, \lambda_{\mathrm{O} 2}=2$ ). Subsequently a $\mathrm{CV}$ was carried out within the potential range of $0.07-0.9$ $\mathrm{V}$ and the polarization curve of the same MEA was recorded again. The electrode with 5.42 $\mathrm{gPA} / \mathrm{gPt}$, i.e. higher than $3 \mathrm{gPA} / \mathrm{gPt}$, did not exhibit any apparent changes in the performance (Fig. 4-11a). An improvement in the performance especially at high current densities is observed for the low $\mathrm{gPA} / \mathrm{gPt}$ ratios after the CVs were carried out (Figs 4-11b and 4-11c). The improvement is more significant or otherwise the long term degradation is larger upon decreasing the $\mathrm{gPA} / \mathrm{gPt}$ ratio. Although the MEAs had different Pt loadings the highest performance is observed for the MEA with the lower Pt loading $\left(0.65 \mathrm{mg} / \mathrm{cm}^{2}\right)$ and the highest ratio of $\mathrm{gPA} / \mathrm{gPt}$, while the lowest performance is observed for the anode with the highest Pt loading $\left(2 \mathrm{mg} / \mathrm{cm}^{2}\right)$ without PA sprayed on the anode. It must be noted that in the later case the electrochemical interface must be formed by the diffusion of part of PA from the membrane. 

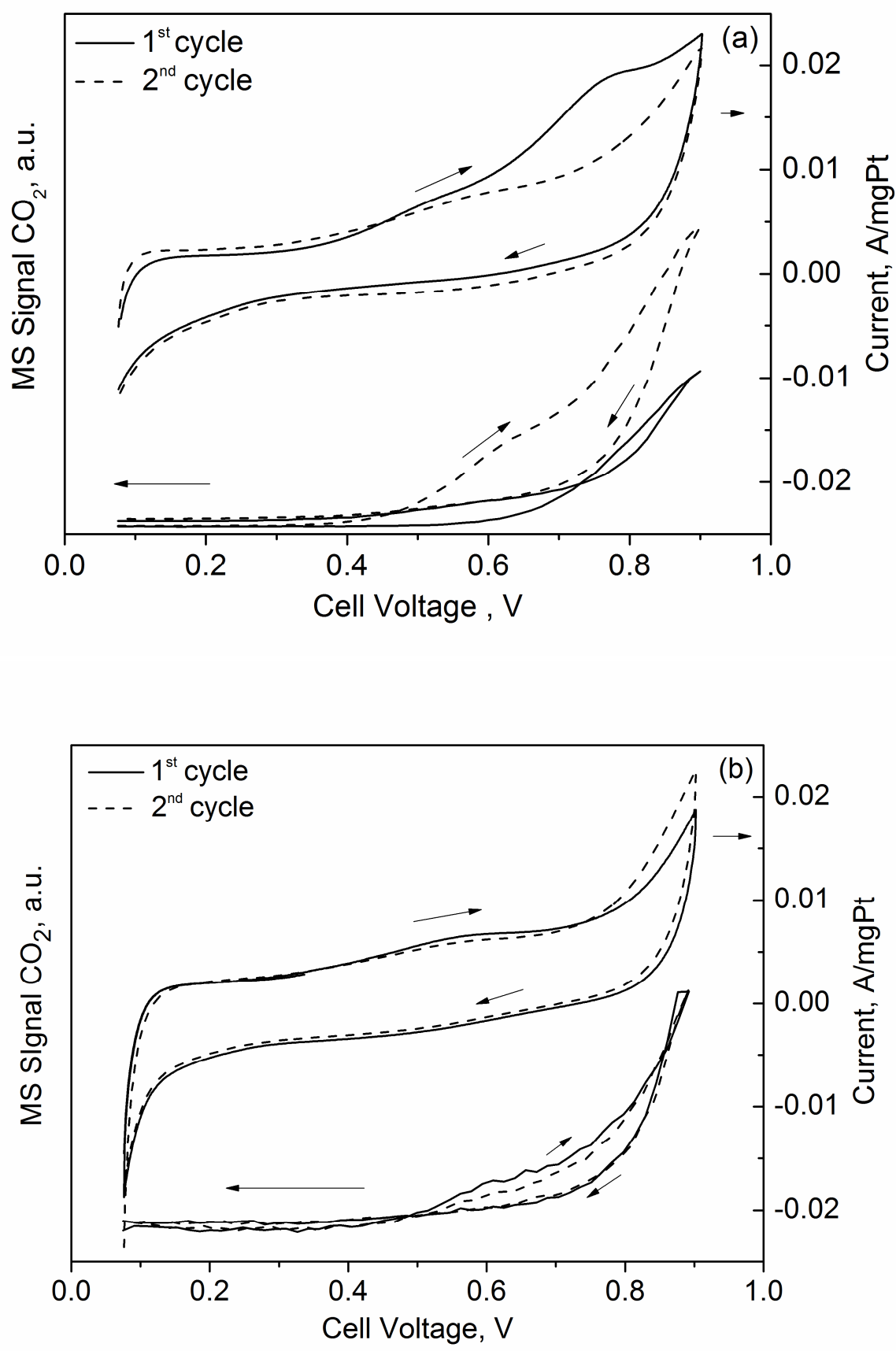

Figure 4-10. Cyclic voltammograms (first and second cycle) at $140^{\circ} \mathrm{C}$ and the corresponding $\mathrm{CO}_{2}$ signal as recorded from the on-line mass spectrometer at the outlet of the working electrode's compartment of (a) MEA 7 (1.71 gPA/gPt) and MEA 1 (17.62 $\mathrm{gPA} / \mathrm{gPt}$ ), after fuel cell operation at $180^{\circ} \mathrm{C}$ for $24 \mathrm{~h}$ at $0.2 \mathrm{~A} / \mathrm{cm}^{2}$ under pure $\mathrm{H}_{2} / \mathrm{O}_{2}$ flows $\left(\lambda_{\mathrm{H} 2}=1.2, \lambda_{\mathrm{O} 2}=2\right)$. 

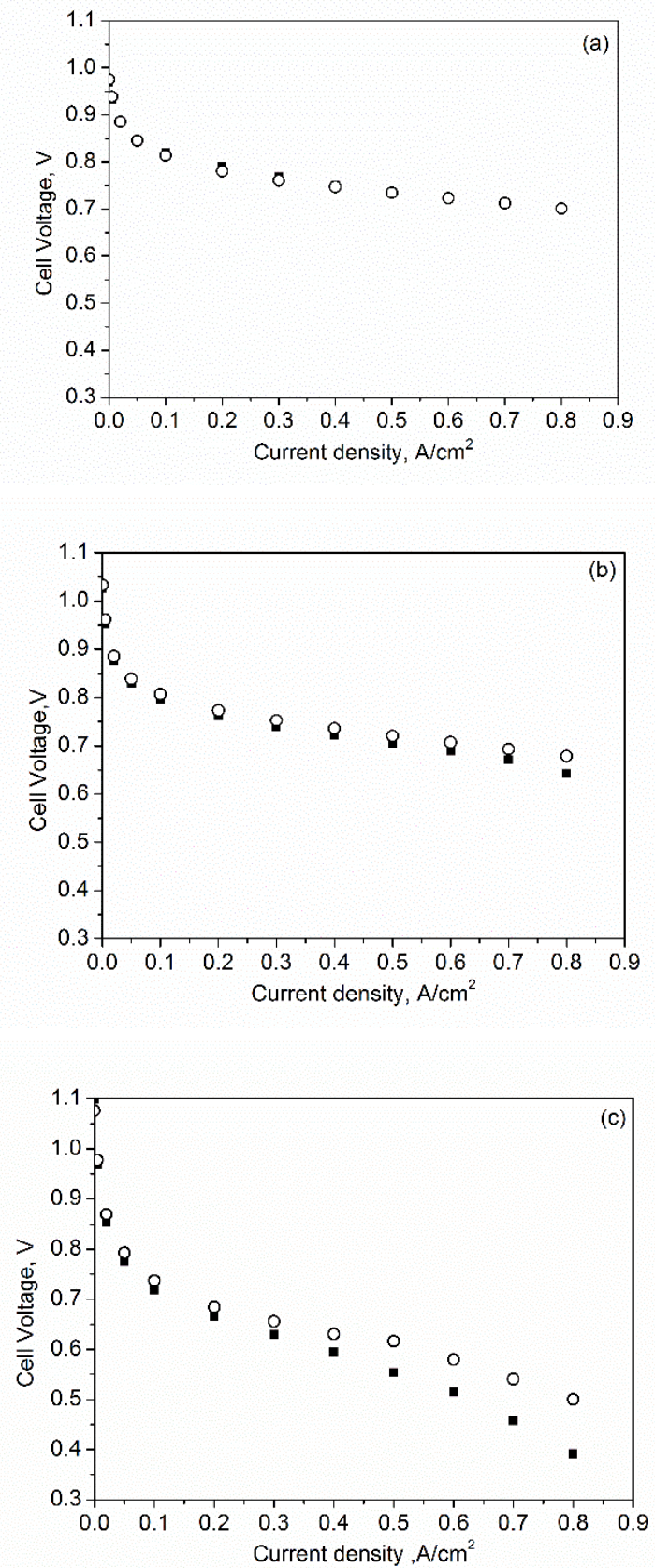

Figure 4-11. Polarization curves, IR drop corrected, after the break in period $(24 \mathrm{~h}$ in operation at $0.2 \mathrm{~A} / \mathrm{cm} 2$ ) at $180^{\circ} \mathrm{C}$ before and after the cyclic voltammogram measurements for MEAs with different amount of sprayed PA at the anodic electrode under $\mathrm{H}_{2}$ and $\mathrm{O}_{2}$ flows (200 cc/min respectively): (a) MEA 3 (5.42 gPA/gPt), (b) MEA 12 (2.00 gPA/gPt) and (c) MEA 8 (0 gPA/gPt). Black squares: before the $\mathrm{CV}$ and open circles: after the $\mathrm{CV}$.

97 
These results indicate two issues regarding the effect of the amount of PA on the electrode:

1. Increased amount of PA at the anode is beneficial for the efficient performance of the anode and more importantly with significantly lower degradation

2. Decreased amount of PA affects both the performance and induces higher degradation rate due to the formation of reduced species on the electroactive Pt surface.

The long term poisoning effect of the lower quantities of the PA on the electrode is observed at high current densities where the turnover of the electrochemical interface is higher.

In order to confirm that the cell performance improvement upon potential cycling is due to the 're-activation' of the Pt active sites, the ECSA of the different MEAs was measured after several hours of continuous fuel cell operation by means of $\mathrm{CO}$ stripping voltammetry. After the first CO stripping measurement, a second $\mathrm{CO}$ stripping experiment was sequentially carried out. The outlet gas of the working electrode was monitored with an on-line mass spectrometer. At high $\mathrm{gPA} / \mathrm{gPt}>3$ ratio on the electrode, the measured $\mathrm{CO}$ uptake and consequently the calculated ECSA in the first and second CO stripping experiments were exactly the same. On the other hand, for the MEAs with low PA loading in the anodic $\mathrm{CL}(<3 \mathrm{gPA} / \mathrm{gPt})$, the $\mathrm{CO}$ stripping experiment gave very different results before and after the CV. Fig. 4-12 shows an example for MEA 8.

In this figure, the two subsequent $\mathrm{CO}$ stripping measurements are depicted, along with the corresponding $\mathrm{MS}$ signal of $\mathrm{CO}_{2}$ at the electrode's outlet, produced during $\mathrm{CO}$ electrooxidation. As can be seen in Fig. 4-12a (first CO stripping), immediately after 15 hours of continuous fuel cell operation at $0.2 \mathrm{~A} / \mathrm{cm}^{2}$, the measured $\mathrm{CO}$ oxidation peak was very small, which is also confirmed by the $\mathrm{MS} \mathrm{CO}_{2}$ signals. As expected, in the second sequential $\mathrm{CO}$ adsorption and stripping voltammetry (Fig. 4-12b) the $\mathrm{CO}$ electrooxidation peak in the $\mathrm{CV}$ and the $\mathrm{MS} \mathrm{CO}_{2}$ signal are 11 times larger than the initial measurement (Fig. 4-12a). Therefore, it becomes obvious that at low PA loadings and after a certain time of operation the real ECSA decreases. It can be recovered after the CVs depicted in Figs 4-6 up to 4-10 and the oxidation of the electroactive species at $750 \mathrm{mV}$. This phenomenon can have a negative influence in the long term performance of the anodic electrode.

Based on the results of Fig. 4-12 it can be concluded that under low PA loading on the anodic electrode only $<10 \%$ of the Pt active surface is electrochemically active under fuel cell operating conditions. However, the image is drastically different at high $\mathrm{gPA} / \mathrm{gPt}>3$ loadings, from that depicted in Fig. 4-12. The initial CO adsorption coverage 

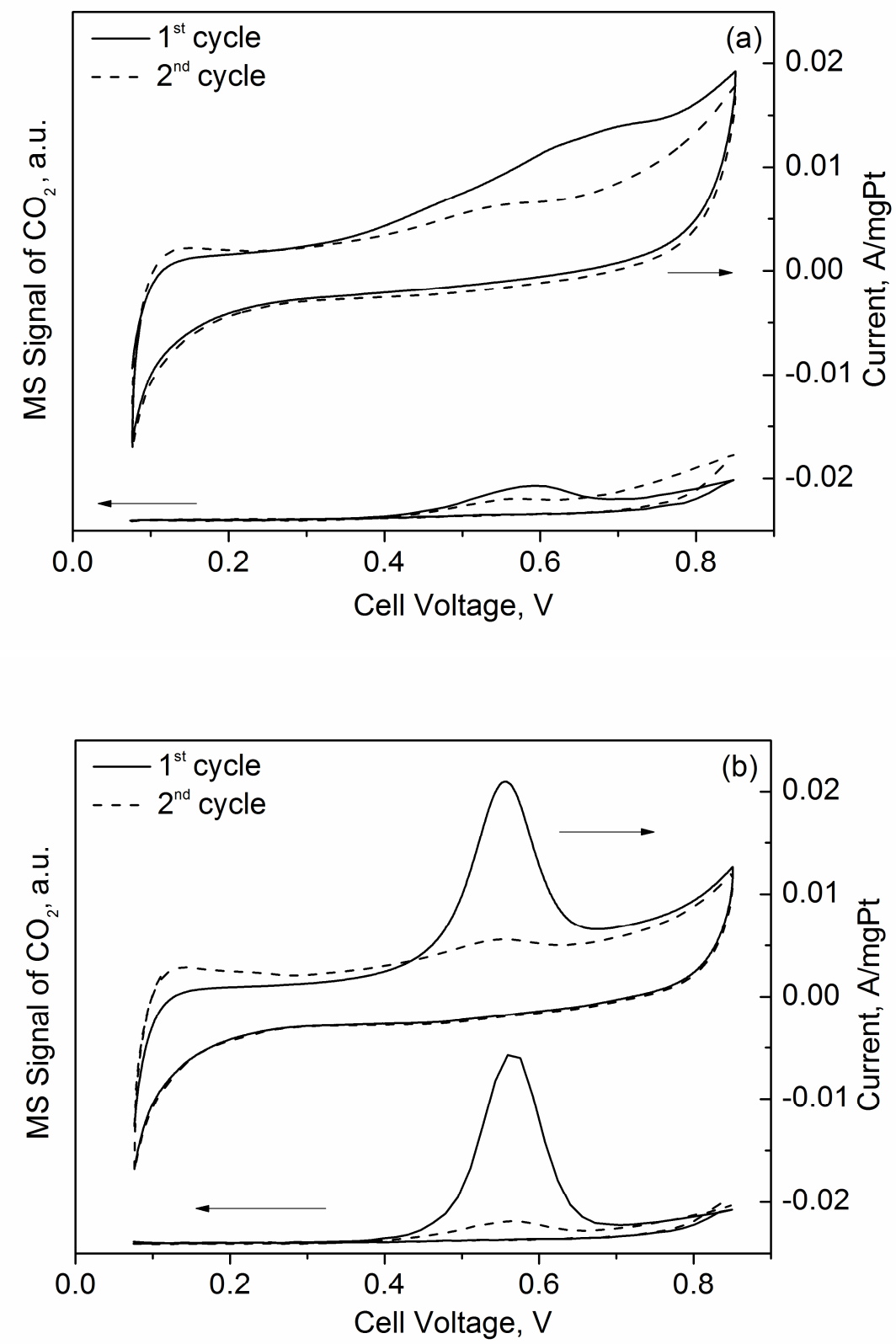

Figure 4-12. Voltammograms of adsorbed $\mathrm{CO}$ electrooxidation (first and second cycle) at $140^{\circ} \mathrm{C}$ and the corresponding $\mathrm{CO}_{2}$ signal as recorded from the on-line mass spectrometer at the outlet of the working electrode's compartment, (a) before CV and (b) after CV for the anodic electrode of MEA 9 after fuel cell operation at $180^{\circ} \mathrm{C}$ for $24 \mathrm{~h}$ at $0.2 \mathrm{~A} / \mathrm{cm}^{2}$ under pure $\mathrm{H}_{2} / \mathrm{O}_{2}$ flows $\left(\lambda_{\mathrm{H} 2}=1.2, \lambda_{\mathrm{O} 2}=2\right)$. 


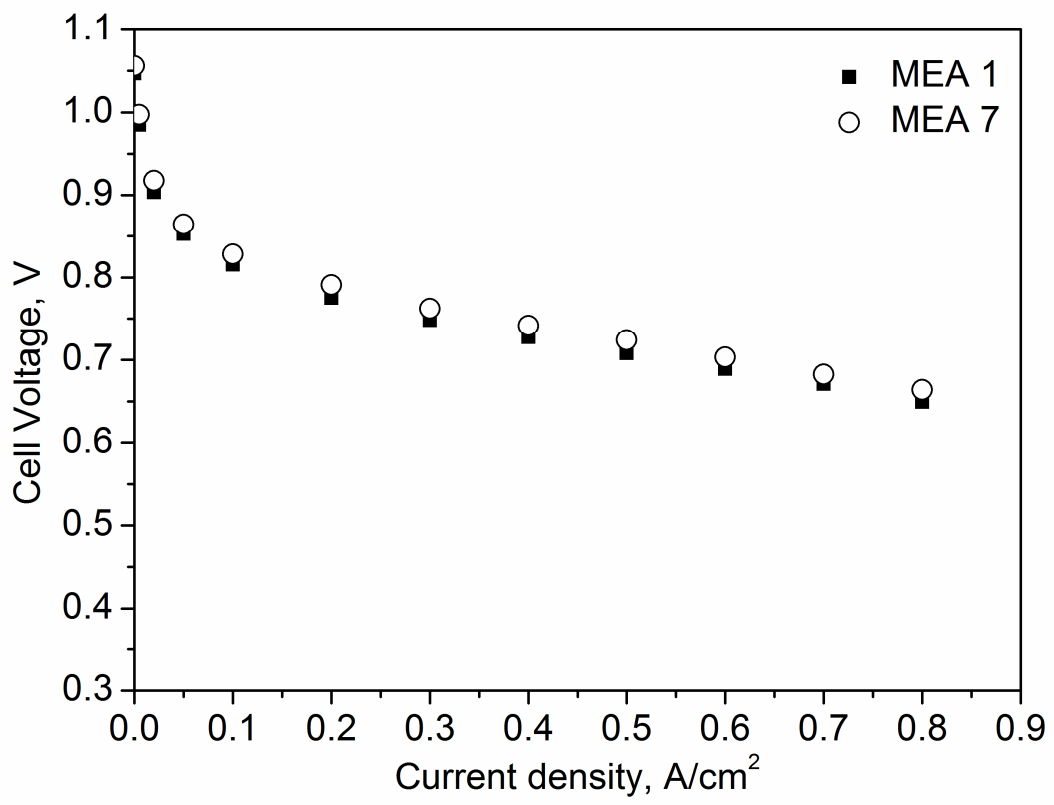

Figure 4-13. Polarization curves recorded after $24 \mathrm{~h}$ of operation at $0.2 \mathrm{~A} / \mathrm{cm} 2$, IR drop corrected, at $180^{\circ} \mathrm{C}$ for MEAs with different Pt loading and amount of sprayed PA at the anodic electrode under $\mathrm{H}_{2}$ and $\mathrm{O}_{2}$ flows (200cc/min respectively). MEA 1: $0.2 \mathrm{mgPt} / \mathrm{cm}^{2}$ and $17.62 \mathrm{gPA} / \mathrm{gPt}$ and MEA 7: $2.06 \mathrm{mgPt} / \mathrm{cm}^{2}$ and $1.71 \mathrm{gPA} / \mathrm{gPt}$.

is not inhibited but instead is identical to the subsequent adsorption and CO stripping experiment, thus implying a stable and high Pt surface utilization (>80\%), as the ECSA was found to be approximately $39 \mathrm{~m}^{2} \mathrm{Pt} / \mathrm{gPt}$. In this respect similar long term performance can be achieved at very low Pt loadings $\left(0.2 \mathrm{mg} / \mathrm{cm}^{2}\right)$ by adjusting the $\mathrm{gPA} / \mathrm{gPt}$ ratio at the anode. This is clearly shown in Fig. 4-13 where the performance of MEA 1 and MEA 7 after the break in period is almost identical though the Pt loading at the corresponding anodes differs by a factor of 10 . This can be attributed to the fact that in the case of MEA 7 the low PA loading may result in a 10 fold decrease in the ECSA according to the findings of Fig. 4-12. On the contrary, almost full exploitation is achieved in MEA 1 which has 10 times lower Pt loading due to its high $\mathrm{gPA} / \mathrm{gPt}$ ratio.

In order to shed more light in these phenomena $\mathrm{CO}$ stripping experiments were carried out after the electrode exposure in dry and humidified $\mathrm{H}_{2}$ and Ar atmospheres for $24 \mathrm{~h}$ at open circuit potential, where the cell's temperature was set at $180^{\circ} \mathrm{C}$. As depicted in Fig. $4-14$ a fourfold inhibition of $\mathrm{CO}$ oxidation peak is observed when the Pt electrode is exposed under dry Ar compared to the exposure of the same electrode under $\mathrm{Ar} / \mathrm{H}_{2} \mathrm{O}$ flow. This can 
be rationalized by considering that under dry Ar flow the formation of pyrophosphoric or triphosphoric acid is formed that blocks the Pt surface. This type of fuel cells usually operate at a temperature range of $160-180^{\circ} \mathrm{C}$. As has been mentioned in the introduction, within this temperature range phosphoric acid is known to dehydrate and polymerize, , creating poly-phosphoric acid species. When two molecules of ortho-phosphoric acid are dehydrated, they create pyro-phosphoric acid releasing a water molecule. A general formula for such poly-acid compounds is $\mathrm{HO}\left(\mathrm{PO}_{2} \mathrm{OH}\right)_{\mathrm{x}} \mathrm{H}$, where $\mathrm{x}=$ number of phosphoric units in the molecule. S.J.Buelte et al [23] reported that prolonged exposure of a Pt electrode in phosphoric acid using a half cell set up, resulted in the decrease of the electrocatalyst active area. The later was enhanced as the phosphoric acid concentration increased from $105 \mathrm{wt} \%$ to $115 \%$ wt phosphoric acid solution and it was attributed to the formation of polyphosphates on the Pt surface.

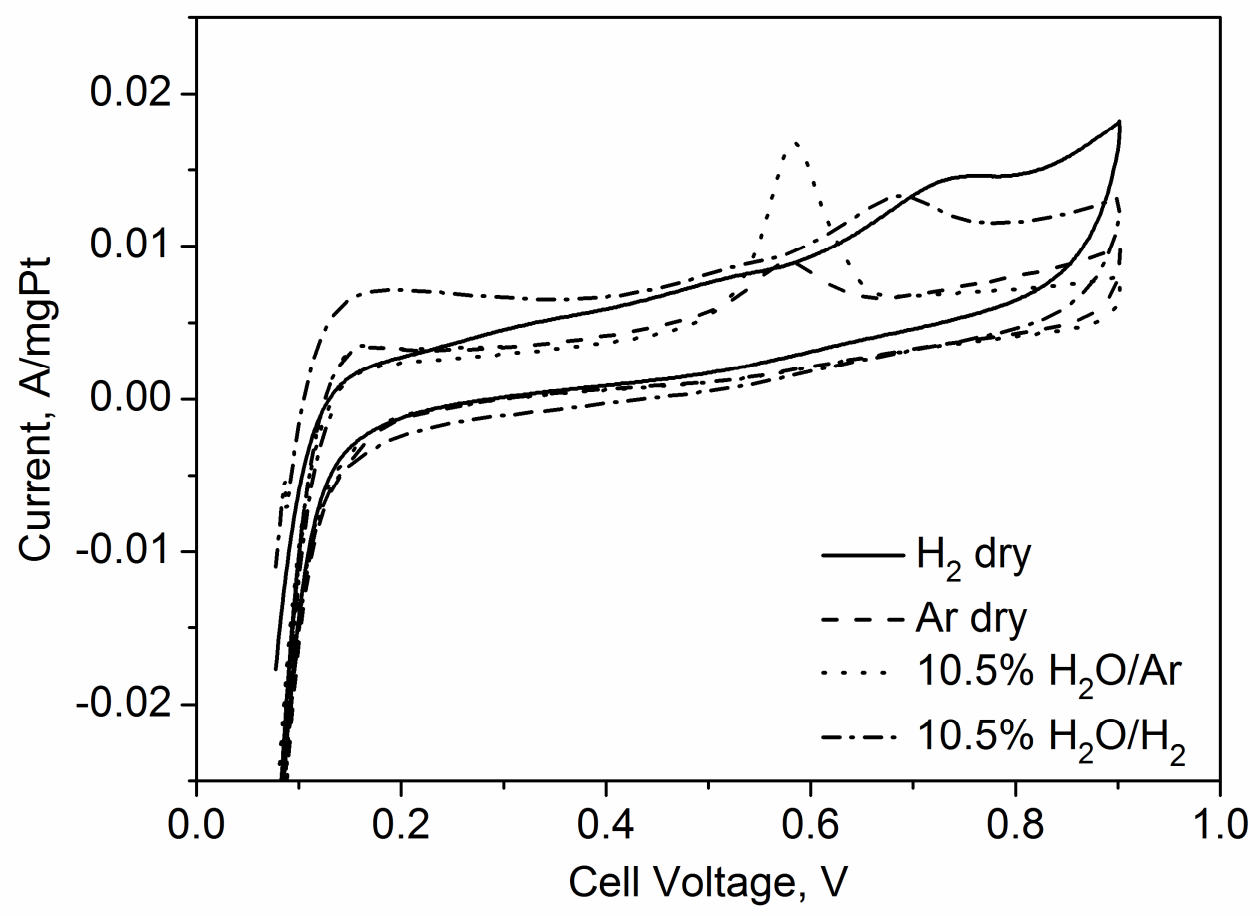

Figure 4-14. $\mathrm{CO}$ stripping experiments at $140^{\circ} \mathrm{C}$ of MEA 14 employing $30 \% \mathrm{wt} \mathrm{Pt} / \mathrm{C}$. Before each measurement the working electrode was pre-exposed at OCP and $180^{\circ} \mathrm{C}$ for 18 hours under dry $\mathrm{H}_{2}$, dry $\mathrm{Ar}, 10.5 \mathrm{kPa} \mathrm{H}_{2} \mathrm{O} / \mathrm{Ar}$ and $10.5 \mathrm{kPa} \mathrm{H}_{2} \mathrm{O} / \mathrm{H}_{2}$ flows, while the counter electrode was fed with $\mathrm{Ar} / \mathrm{H}_{2} \mathrm{O}$.

In both $\mathrm{H}_{2}$ flow conditions (i.e. dry or humidified) the $\mathrm{CO}$ stripping spectra show significant decrease in the $\mathrm{CO}$ oxidation peak. Thus beyond the formation of polymeric 
dehydrated forms of PA that can be adsorbed strongly on Pt, other specific interactions of $\mathrm{H}_{2}$ with PA can be the reason for the significant decrease of either the Pt $\mathrm{CO}$ uptake or the decrease of the $\mathrm{CO}$ oxidation peak. This can be chemical reduction of PA and formation of reduced species or even displacement of PA from catalyst surface, thus inducing shrinkage of the electrochemical interface.

\subsubsection{XPS measurements of the Pt/PA interface}

Taking into consideration all the aforementioned information, the reversible degradation observed at low PA loadings on the anodic electrode can possibly be a complex phenomenon that can be attributed either to oxidizable species, which is formed during fuel cell operation and its exposure to $\mathrm{H}_{2}$, thus blocking the Pt surface, or due to restructuring of the electrochemical interface because of the displacement of the PA from the catalytic layer. The clarification of the aforementioned processes was attempted by means of XPS.

A Pt film deposited on a PA doped membrane was exposed to several gas atmospheres for several hours in the atmospheric pressure chamber of the ultrahigh vacuum system equipped with XPS (see experimental). The BE values for the main detectable elemental states were obtained from the spectra of the $\mathrm{O} 1 \mathrm{~s}$, $\mathrm{Pt} 4 \mathrm{f}$ and $\mathrm{P} 2 \mathrm{p}$ regions, (Table 4-3, Figs 415 and 4-16). The binding energy of Pt4f spectra is shown in Fig. 5-15a, where the doublet with binding energies $71.4(\mathrm{Pt} \mathrm{4f7/2)}$ and $74.7 \mathrm{eV}(\mathrm{Pt} \mathrm{4f5/2)}$ is attributed to metallic $\mathrm{Pt}$ for all measurements [33]. It is obvious, that the different experimental conditions did not affect the binding energy or the oxidative state of Pt. The later confirms the validity of our measurements since no charging of the surface occurred during measurements.

Table 4-3. XPS binding energies with the corresponding FWHM in the braquets, intensity ratio of $\mathrm{O} 1 \mathrm{~s}$ of (peakII)/(peakI), as well as the atomic ratio $\mathrm{O} / \mathrm{P}$.

\begin{tabular}{|c|c|c|c|c|}
\hline $\begin{array}{l}\text { Experimental } \\
\text { Conditions }\end{array}$ & $\begin{array}{c}\text { B.E.(FWHM) } \\
\text { (eV) P2p }\end{array}$ & $\begin{array}{l}\text { B.E.(FWHM) } \\
\text { (eV) O1s }\end{array}$ & $\begin{array}{c}\text { O1s intensity } \\
\text { ratio } \\
\text { (peakII)/(peakI) }\end{array}$ & $\begin{array}{c}\mathrm{O} / \mathrm{P} \\
\text { atomic } \\
\text { ratio }\end{array}$ \\
\hline $200^{0} \mathrm{C} 4 \mathrm{~h} \mathrm{H}_{2}(1)$ & $\begin{array}{c}134.8,131.0 \\
(3.2)\end{array}$ & $\begin{array}{c}532.0,533.5 \\
(2.4)\end{array}$ & 1.44 & 2.48 \\
\hline $\begin{array}{c}180^{\circ} \mathrm{C} 1.5 \mathrm{~h} \mathrm{H}_{2} \mathrm{O} \\
\text { (2) }\end{array}$ & $133.9(3.0)$ & $\begin{array}{c}531.9,533.3 \\
(2.7)\end{array}$ & 0.57 & 3.60 \\
\hline $\begin{array}{c}200^{\circ} \mathrm{C} 4 \mathrm{~h} \mathrm{UHV} \\
\text { (3) }\end{array}$ & $134.8(3.0)$ & $\begin{array}{c}531.7,533.4 \\
(2.7)\end{array}$ & 1.28 & 3.18 \\
\hline $\begin{array}{c}200^{\circ} \mathrm{C} 4 \mathrm{~h} \mathrm{CO} \\
\text { (4) }\end{array}$ & $134.8(3.0)$ & $\begin{array}{c}532.0,533.5 \\
(2.4)\end{array}$ & 1.34 & 3.32 \\
\hline
\end{tabular}


The various recorded spectra of P2p are shown in Fig. 4-15b. As also shown in Table 43 , there is an apparent shift in the binding energies of the P2p peaks, which depends on the temperature and gas phase pretreatment of the sample. The peak at $133.9 \mathrm{eV}$ of spectrum 2 in Fig. 4-15b, is attributed to ortho-phosphate $\left(\mathrm{H}_{2} \mathrm{PO}_{4}{ }^{-}\right)$species [34]. Upon heating the sample to $200^{\circ} \mathrm{C}$ for 4 hours under vacuum the $\mathrm{P} 2 \mathrm{p}$ signal increases and shifts to higher binding energy positioned at $134.8 \mathrm{eV}$. This can be attributed to the dehydration of the ortho-phosphate species into pyrophosphates or even polyphosphates (pyro- or polyphosphoric acid). The P2p peak at $134.8 \mathrm{eV}$ (spectra 1, 3 and 4), is assigned in the literature to pyrophosphoric acid or poly-phosphate species [35,36]. After the exposure of the sample in humidified He at elevated temperature $\left(180^{\circ} \mathrm{C}\right)$ the ortho-phosphate peak at $133.9 \mathrm{eV}$ is recovered. The increase in the signal corresponds to the increase in the $\mathrm{P} 2 \mathrm{p} / \mathrm{Pt} 4 \mathrm{f}$ ratio (Fig. 4-17), thus showing a significant degree of restructuring of the $\mathrm{PA} / \mathrm{Pt}$ film interface. This observation in combination to the observed decrease in $\mathrm{CO}$ oxidation peak in Fig. 4-14, after the long exposure of the electrode in dry Ar flow, strongly indicates that pyrophosphoric acid may block the Pt surface. Whereas in all other cases the decreased total $\mathrm{P} 2 \mathrm{p} / \mathrm{Pt} 4 \mathrm{f}$ ratio indicates a displacement of the phosphoric or pyrophosphoric acid from the Pt surface by $\mathrm{H}_{2}, \mathrm{H}_{2} \mathrm{O}$ and $\mathrm{CO}$. It is worth noticing that long exposure of the sample under $\mathrm{CO}$ flow at elevated temperatures can even further displace pyrophosphoric acid as this is evidently shown in Fig.4-17. The same trend was observed for the case of $\mathrm{O} / \mathrm{Pt}$ ratio, showing that the measured $\mathrm{O} 1$ s oxygen species are chemically combined in the various $\mathrm{P}$ and $\mathrm{O}$ containing adsorbed species.

Under dry $\mathrm{H}_{2}$ flow for 4 hours at $200^{\circ} \mathrm{C}$ a second peak appears at significantly lower binding energy $(131 \mathrm{eV})$, which suggests the formation of a compound that originates from the catalytic reduction of the pyrophosphoric acid on the Pt surface. It is worth noticing that the species after $\mathrm{H}_{2}$ treatment contain the smallest amount oxygen thus showing the severe reduction of the pyro/poly-phosphate species.

The data acquired from O1s peaks, give further insight confirming the previous conclusions. Fig. 4-16 shows the XP Spectra of O1s peak. The peak is deconvoluted in all cases in two components with equal FWHM (Table 4-3). The binding energy difference is $1.5 \mathrm{eV}$. It has been reported that the $\mathrm{O} 1 \mathrm{~s}$ peak positions range between 531.6 and $532.6 \mathrm{eV}$ (peak I) for non-bridging oxygen, (NBO:PO- $\mathrm{POH}^{-} \mathrm{P}=\mathrm{O}$ ) and 533.1 and $534.1 \mathrm{eV}$ (peakII) for bridging oxygen (BO: P-O-P) [37]. The intensity ratio of these different chemical states, is shown in Table 4-3 and can be considered as an indication of the most pronounced chemical structure of the various phosphoric acid species on the surface. It is deduced that increased peak intensity of the BO prompts to the formation of pyrophosphoric or polyphodphoric acid with bridging oxygen in their structure. Using the total peak area ofPt4f, O1s, and $\mathrm{P} 2 \mathrm{p}$ peaks, in each experiment and the appropriate 

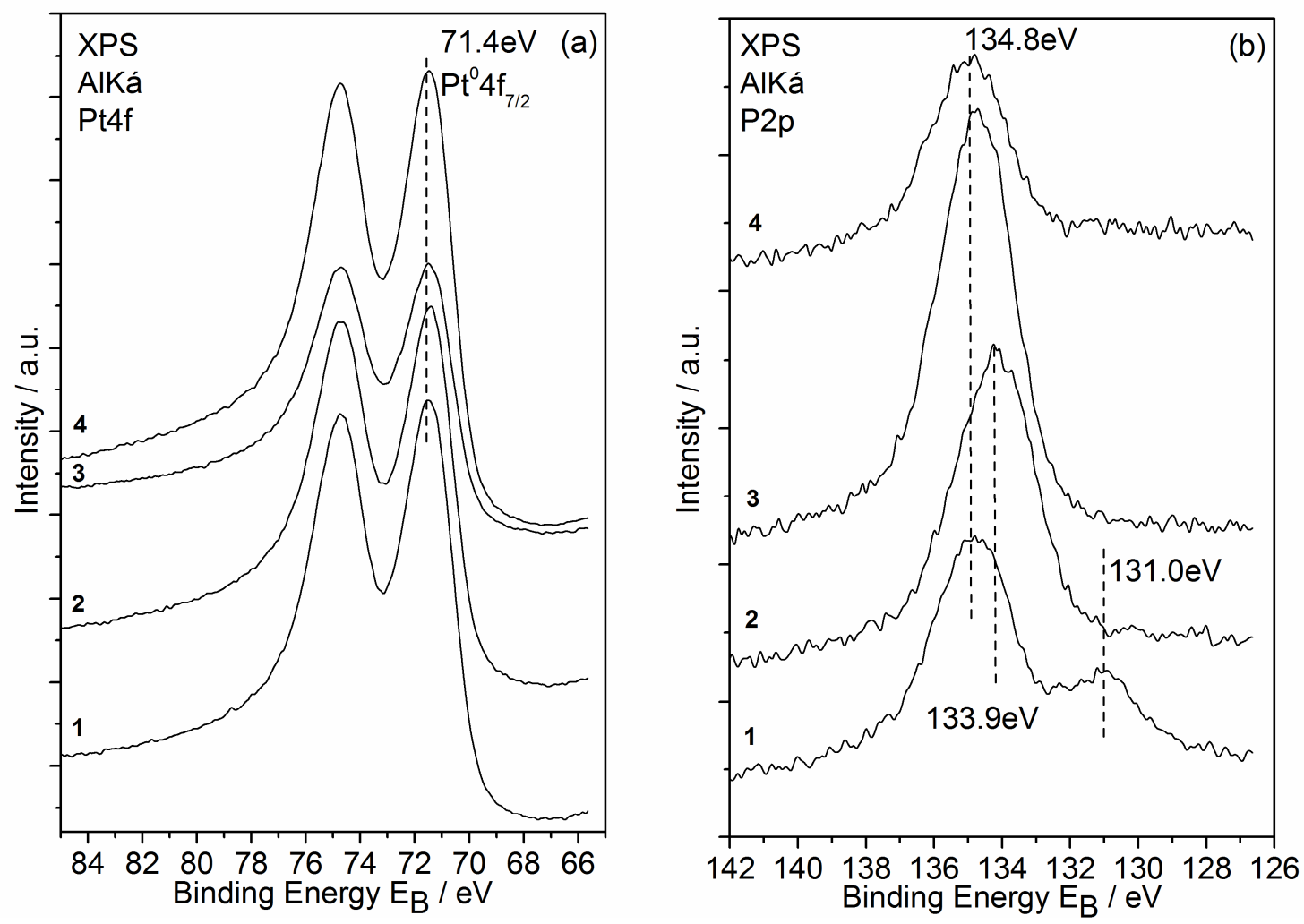

Figure 4-15. XP spectra of Pt4f(a) and $\mathrm{P} 2 \mathrm{p}(\mathrm{b})$, after heating at $200^{\circ} \mathrm{C}$ for $4 \mathrm{~h}$ in $\mathrm{H}_{2}$ flow(1), after heating at $180^{\circ} \mathrm{C}$ for $1.5 \mathrm{~h}$ in $4 \% \mathrm{H}_{2} \mathrm{O} / \mathrm{He}$ flow (2), after heating at $200^{\circ} \mathrm{C}$ for $4 \mathrm{~h}$ in $\mathrm{UHV}(3)$ and after heating at $200^{\circ} \mathrm{C}$ for $4 \mathrm{~h}$ in $\mathrm{CO}$ flow (4).

sensitivity factors (based on Wagner's collection and adjusted to the transmission characteristics of analyser EA10) and equations, the $\mathrm{O} / \mathrm{P}, \mathrm{O} / \mathrm{Pt}$ and $\mathrm{P} / \mathrm{Pt}$ atomic ratio is determined (Table 4-3). As itis shown in Table 4-3 both the $\mathrm{O} / \mathrm{P}$ and the peakII/peakI O1s ratios decrease after heating at $180^{\circ} \mathrm{C}$ under vacuum, thus indicating the formation of pyrophosphates or polyphosphates. The O/P nominal atomic ratio for phosphoric acid or ortho-phosphates is 4, whereas for the case of pyrophoshoric acid or pyrophosphates is 3.5 . Heating under $\mathrm{H}_{2}$ atmosphere, the $\mathrm{O} / \mathrm{P}$ ratio is even lower 2.48 (Table 4-3), thus implying further reduction of the phosphate or pyrophosphate species by abstraction of oxygen. The atomic ratio of $\mathrm{O} 1 \mathrm{~s}$ peak to $\mathrm{P} 2 \mathrm{p}$ at $134.8 \mathrm{eV}$ of spectrum 1 in Fig. 4-15 was found to be 3.3, which lies between the O/P ratios of spectra 2 and 3 in Figs 4-15, Table 4-3. According to the aforementioned considerations $\mathrm{P}$ species appearing at $\mathrm{P} 2 \mathrm{p}=131 \mathrm{eV}$ originates from the reduction of the pyrophosphate species containing significantly reduced oxygen amount. It must be noted that the peak at $131 \mathrm{eV}$ cannot be attributed to elemental $\mathrm{P}^{\mathrm{o}}$ as the latter appears according to the literature at $130 \mathrm{eV}[38,39]$. Thus it is proposed that the low binding energy peak originates from the catalytic reduction of 

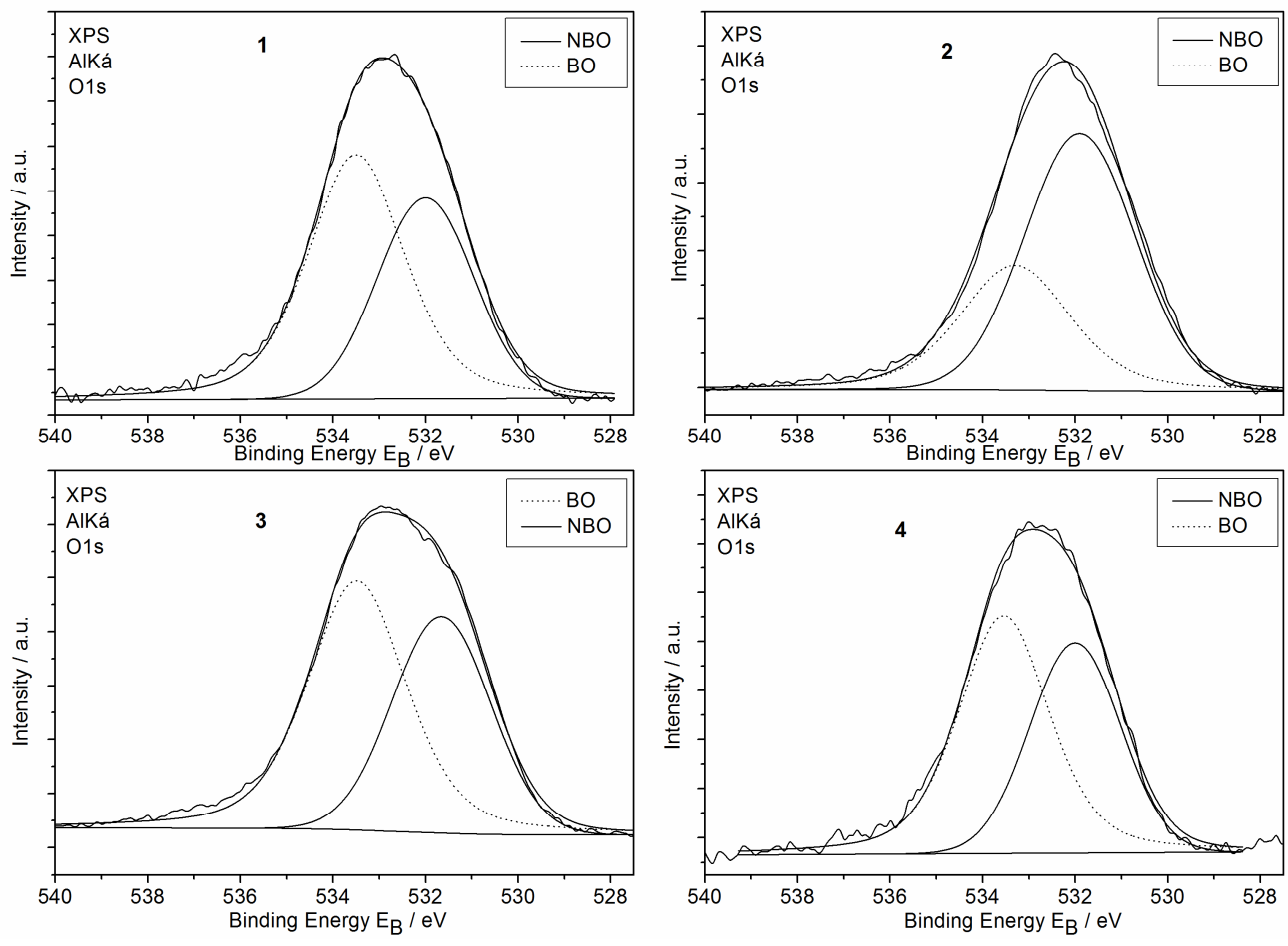

Figure 4-16. $\mathrm{XP}$ spectra of $\mathrm{O} 1 \mathrm{~s}$ peaks after heating at $200^{\circ} \mathrm{C}$ for $4 \mathrm{~h}$ in $\mathrm{H}_{2}$ flow (1), at $180^{\circ} \mathrm{C}$ for $1.5 \mathrm{~h}$ in $4 \% \mathrm{H}_{2} \mathrm{O} / \mathrm{He}$ flow (2), at $200^{\circ} \mathrm{C}$ for $4 \mathrm{~h}$ in UHV (3) and at $200^{\circ} \mathrm{C}$ for $4 \mathrm{~h}$ in $\mathrm{CO}$ flow (4).

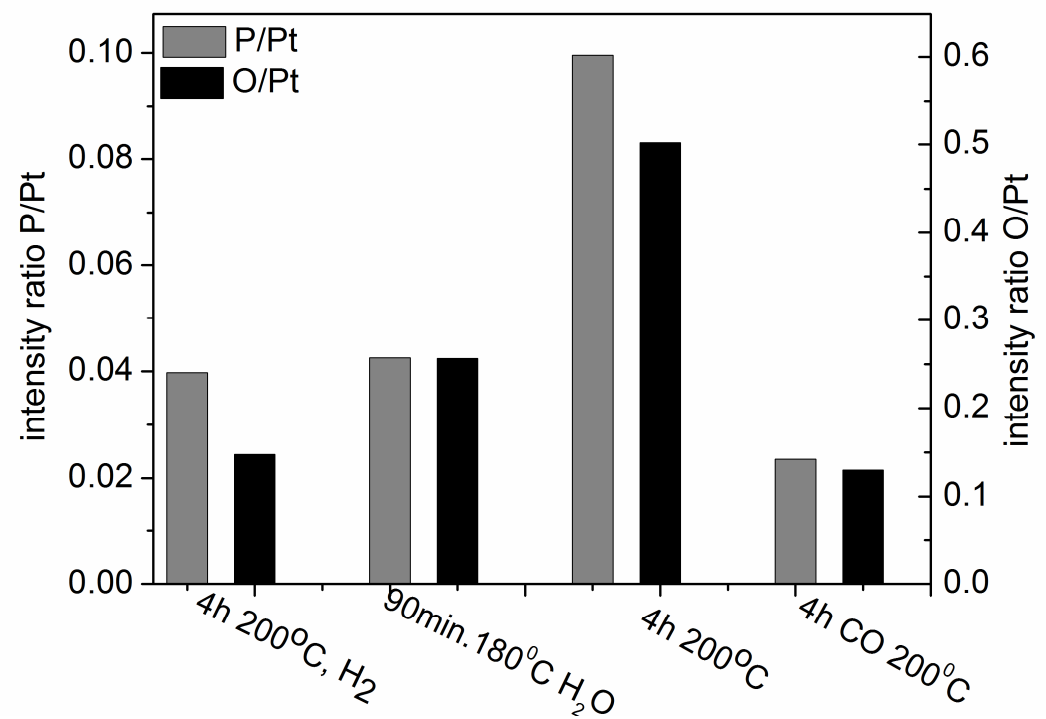

Figure 4-17. Intensity ratio $\mathrm{P} 2 \mathrm{p} / \mathrm{Pt} 4 \mathrm{f}$ and $\mathrm{O} 1 \mathrm{~s} / \mathrm{Pt} 4 \mathrm{f}$ after annealing at $200^{\circ} \mathrm{C}$ for $4 \mathrm{~h}$ in $\mathrm{H}_{2}$ flow, at $180^{\circ} \mathrm{C}$ for $1.5 \mathrm{~h}$ in $4 \% \mathrm{H}_{2} \mathrm{O} / \mathrm{He}$ flow, at $200^{\circ} \mathrm{C}$ for $4 \mathrm{~h}$ in $\mathrm{UHV}$ and at $200^{\circ} \mathrm{C}$ for $4 \mathrm{~h}$ in $\mathrm{CO}$ flow. 
pyro/poly-phosphate species by $\mathrm{H}_{2}$ on the Pt surface through probably the abstraction of hydroxyl groups, thus leaving a phosphorous-oxygen polymeric structure which can account for the lower binding energy of the P2p. The hydroxyl removal may result into a lower oxygen content chemical structure, thus resulting in the decrease of the valence of $\mathrm{P}$ and the increase of the electron density around the $\mathrm{P}$ atom. In this respect we can possibly rationalize the significant decrease in binding energy by $3.5 \mathrm{eV}$.

\subsubsection{The proposed mechanism}

To summarize, after careful evaluation of all of the above results, the following chemical mechanism can be responsible for Pt electrochemical active area loss, especially for the case of low PA amounts in the anodic catalyst layer $(<3 \mathrm{gPA} / \mathrm{gPt})$. During normal fuel cell operation, three processes occur at the anode electrode. The phosphoric acid is dehydrated, according to eq. 4-5, into pyrophosphoric or poly-phosphoric species which spreads and blocks the Pt surface.

\section{$2 \mathrm{H}_{3} \mathrm{PO}_{4} \rightarrow \mathrm{H}_{4} \mathrm{P}_{2} \mathrm{O}_{7}+\mathrm{H}_{2} \mathrm{O}$ or $\mathrm{nH}_{3} \mathrm{PO}_{4} \rightarrow \mathrm{H}_{n+2} \mathrm{P}_{\mathrm{n}} \mathrm{O}_{3 n+1}+\mathrm{n}-1 \mathrm{H}_{2} \mathrm{O}$ (eq. 4-5)}

Displacement of phosphoric acid and the formed poly-phosphoric species from the $\mathrm{Pt}$ surface is possible by $\mathrm{H}_{2}$ adsorption and spill-over. Concurrently, poly-phosphoric acid, is being catalytically reduced most probably into an oxide form of $\mathrm{P}$ with less oxygen. The exact chemical structure of this oxidic species cannot be defined within the context of the present study. It is considered that this species covers and poisons the Pt surface. Upon exposure of the sample in humidified He ortho-phosphate species is recovered (Fig. 4-15, spectrum 2), thus showing the reversibility of the processes. The electrochemical oxidation of these species can take place during the CVs of Figs 4-6 to4-10 at $750 \mathrm{mV}$ with the simultaneous recovery of the $\mathrm{Pt}$ surface. The electrochemical oxidation of reduced phosphoric acid species (hypophosphite $\mathrm{H}_{2} \mathrm{PO}_{2}^{-}$) on Ni surfaces, has been reported in the literature [40].

It has been clearly shown that the PA amount sprayed on the anodic electrode influences the anode performance. At least for the studied high temperature MEA system, an inadequate amount of PA at the anodic electrode can lead to the gradual shrinking of the electrochemical interface, as well as a gradual "poisoning" of the Pt electrocatalyst by species originating from dehydrated and especially $\mathrm{H}_{2}$ reduced chemical structures of PA.

Though one would expect that the poisoning effect of higher amount of PA on the anodic electrode would have been more severe, it turns out that the utilization of PA above a certain ratio 
( $>3 \mathrm{gPA} / \mathrm{gPt}$ ) to be beneficial, as the species that are oxidized at $750 \mathrm{mV}$ or the shrinkage of the ECSA were not observed. This controversial behavior strongly indicates that this is directly related and limited to the catalytic interference of the PA with the Pt surface under lean hydration conditions, while the active water molecules can be provided through the absorbed water in the PA. It can be assumed that increased amount of distributed PA within the catalytic layer will absorb and store higher amount of water. In addition the increased amount of PA and its fine distribution within the catalytic layer will result in a more extended Pt/PA interface, thus providing higher hydration degree onto the Pt surface. In this respect the poisoning and blocking species can be displaced from the Pt surface. Another significant reason for the beneficial role of the increased PA amount in the catalytic layer is to compensate its displacement either by $\mathrm{H}_{2}$ or $\mathrm{CO}$ as this is clearly shown in Fig. 4-17b, thus providing the necessary boundary length of the electrochemical interface to facilitate the $\mathrm{H}_{2}$ electrooxidation. As was formerly stated [41] the amount/concentration of water plays an autocatalytic role for the facilitation of $\mathrm{H}_{2}$ electrochemical oxidation on PA imbibed high temperature PEM electrochemical interface.

\subsection{Conclusions}

It was observed that the reversible performance loss of the anodic electrode was a function of the PA amount in the catalyst layer. More specifically, under low PA loading $(<3$ $\mathrm{gPA} / \mathrm{gPt}$ ) on the anodic electrode, $<10 \%$ of the Pt active surface is electrochemically active under fuel cell operating conditions. This was attributed to the blockage of the Pt surface by pyrophosphoric acid or poly-phosphates, $\mathrm{H}_{2}$ reduced polyphosphoric acid species and the shrinkage of the interface due to the displacement of the $\mathrm{H}_{3} \mathrm{PO}_{4}$ by the adsorbed $\mathrm{H}_{2}$ species. The results are dramatically different for high PA loadings, where it was found that the Pt surface utilization was very high $(>80 \%)$ and has been attributed to the enhanced degree of the hydration of the electrochemical interface that accompanies the more uniform distribution of the $\mathrm{H}_{3} \mathrm{PO}_{4}$ within the catalytic layer. The controlled and increased PA content within the catalytic layer can result even up to the tenfold decrease in the Pt loading when the anode operates under $\mathrm{H}_{2}$ rich conditions. 


\section{$\underline{\text { References }}$}

[2] Song JM, Suzuki S, Uchida H, Watanabe M (2006) Preparation of high catalyst utilization electrodes for polymer electrolyte fuel cells. Langmuir 22(14):6422-6428. doi: $10.1021 / \mathrm{la} 060671 \mathrm{w}$

[3] Xu Z, Qi Z, Kaufman A (2005) Superior catalysts for proton exchange membrane fuel cells. Electrochem Solid-State Lett 8(6):A313-315. doi: 10.1149/1.1912018

[4] Kim H, Lee W, Yoo D (2007) Functionalized carbon support with sulfonated polymer for direct methanol fuel cells. Electrochim Acta 52(7):2620-2624. Doi: 10.1016/j.electacta.2006.09.017

[5] Du CY, Zhao TS, Liang ZX (2008) Sulfonation of carbon-nanotube supported platinum catalysts for polymer electrolyte fuel cells. J Power Sources 176(1):9-15. doi: 10.1016/j.jpowsour.2007.10.016

[6] Orfanidi A, Daletou MK, Neophytides SG (2011) Preparation and characterization of Pt on modified multi-wall carbon nanotubes to be used as electrocatalysts for high temperature fuel cell applications. Appl Catal B-Environ 106:379-389. doi: 10.1016/j.apcatb.2011.05.043

[7] Pefkianakis EK, Deimede V, Daletou MK, Gourdoupi N, Kallitsis JK (2005) Novel polymer electrolyte membrane, based on pyridine containing poly(ether sulfone), for application in high-temperature fuel cells. Macromol. Rapid Commun. 26:1724-1728. doi: 10.1002/marc.200500540

[8] Daletou MK, Geormezi M, Pefkianakis EK, Morfopoulou C, Kallitsis JK, Fully aromatic copolyethers for high temperature polymer electrolyte membrane fuel cells. Fuel Cells 10(1):35-44. doi: 10.1002/fuce.200900032

[9] Kalamaras I, Daletou MK, Gregoriou VG, Kallitsis JK (2011) Sulfonated aromatic polyethers containing pyridine units as electrolytes for high temperature Fuel Cells. Fuel Cells 11:921-931. doi: 10.1002/fuce.201100024

[10] Geormezi M, Chochos CL, Gourdoupi N, Neophytides SG, Kallitsis JK (2011) High performance polymer electrolytes based on main and side chain pyridine aromatic polyethers for high and medium temperature proton exchange membrane fuel cells. J Power Sources 196:9382-9390. doi: 10.1016/j.jpowsour.2011.06.031 
[11] Kalamaras I, Daletou MK, Neophytides SG, Kallitsis JK (2012) Thermal crosslinking of aromatic polyethers bearing pyridine groups for use as high temperature polymer electrolytes. J Membr Sci 415-416:42-50. doi: 10.1016/j.memsci.2012.04.057

[12] Li Q, Jensen JO, Savinell RF, Bjerrum NJ (2009) High temperature proton exchange membranes based on polybenzimidazoles for fuel cells, Prog Polym Sci 2009, 34(5):449477. doi: 10.1016/j.progpolymsci.2008.12.003

[13] Daletou MK, Kallitsis JK, Voyiatzis G, Neophytides SG (2009) The interaction of water vapors with $\mathrm{H}_{3} \mathrm{PO}_{4}$ imbibed electrolyte based on $\mathrm{PBI} /$ polysulfone copolymer blends. J Membr Sci 326:76-83. doi: 10.1016/j.memsci.2008.09.040

[14] Norskov JK, Rossmeisl J, Logadottir A, Lindqvist L, Kitchin JR, Bligaard T, Jonsson H (2004) J Phys Chem B 108:17886-17892. doi: 10.1021/jp047349j

[15] Nart FC, Iwasita $\mathrm{T}$ (1992) On the adsorption of $\mathrm{H}_{2} \mathrm{PO}^{-}$and $\mathrm{H}_{3} \mathrm{PO}_{4}$ on platinum: an in situ FT-ir study. Electrochim Acta 37(3):385-391. doi:10.1016/0013-4686(92)87026-V

[16] Habib MA, Bockris J O'M (1985) Adsorption at the Solid/Solution Interface, An FTIR Study of Phosphoric Acid on Platinum and Gold. J Electrochem Soc 132(1): 108-114. doi: $10.1149 / 1.2113736$

[17] Das SK, Reis A, Berry KJ (2009) Experimental evaluation of CO poisoning on the performance of a high temperature proton exchange membrane fuel cell. J Power Sources 193:691-698. doi: 10.1016/j.jpowsour.2009.04.021

[18] Camargo APM, Previdello BAF, Varela H, Gonzalez ER (2010) Effect of temperature on the electro-oxidation of ethanol on platinum, Quim Nova 33:2143-2147. doi:10.1590/S0100-40422010001000026

[19] G. Kohlmayr, P. Stonehart (1973), Adsorption kinetics for carbon monoxide on platinum in hot phosphoric acid, j. Electrochimica Acta, Volume 18,Issue 2,pp 211-223, doi: 10.1016/0013-4686(73)80014-3

[20] V.S. Bagotzky,Yu.B. Vassilyev, J. Weber, J.N. Pirtskhalava (1970), Adsorption of anions on smooth platinum electrodes, Journal of Electroanalytical Chemistry and Interfacial Electrochemistry Volume 27, Issue 1,pp 31- 46 doi: 10.1016/S00220728(70)80200-5

[21] Vidaković T, Christov M, Sundmacher K (2007), The use of CO stripping for in situ fuel cell catalyst characterization. Electrochim Acta 52:5606-5613. doi:10.1016/j.electacta.2006.12.057 
[22] Ruichun Jiang, H. Russell Kunz, James M. Fenton (2006), Influence of temperature and relative humidity on performance and $\mathrm{CO}$ tolerance of PEM fuel cells with Nafion ${ }^{\circledR}$ Teflon $^{\circledR}-\mathrm{Zr}\left(\mathrm{HPO}_{4}\right)_{2}$ higher temperature composite membranes, Electrochimica Acta, Volume 51 ,Issue 26, pp5596-5605,doi: 10.1016/j.electacta.2006.02.033

[23] Buelte SJ, Lewis D, Eisman G (2011) Effects of Phosphoric Acid Concentration on Platinum Catalyst and Phosphoric Acid Hydrogen Pump Performance. ECS Trans 41(1):1955-1966. doi: 10.1149/1.3635725

[24] Vogel WM, Baris JM (1978) Changes in the surface of platinum in hot concentrated phosphoric acid at low potentials. J Electrochim Acta 23:463-466. doi:10.1016/00134686(78) $87047-9$

[25] Sugishima N, Hinatsu JT, Foulkes FR (1994) Phosphorous acid impurities in phosphoric acid fuel cell electrolytes II. Effects on the oxygen reduction reaction at platinum electrodes. J Electrochem Soc 141:3332-3335. doi: 10.1149/1.2059335

[26] Huang JC, Sen RK, Yeager E (1979) Oxygen reduction on platinum in $85 \%$ orthophosphoric acid. J Electrochem Soc 126:786-792. doi: 10.1149/1.2129139

[27] Clouser SJ, Huang JC, Yeager E (1993) Temperature dependence of the Tafel slope for oxygen reduction on platinum in concentrated phosphoric acid, J Appl Electrochem 23:597-605. doi: 10.1007/BF00721951

[28] Sugishima N, Hinatsu JT, Foulkes FR (1994) Phosphorous acid impurities in phosphoric acid Fuel Cell electrolytes I. Voltammetric study of impurity formation. J. Electrochem. Soc. 141:3325-3331. doi: 10.1149/1.2059334

[29] Camargo APM, Previdello BAF, Varela H, Gonzalez ER (2010) The impact of water concentration on the catalytic oxidation of ethanol on platinum electrode in concentrated phosphoric acid. Electrochem Commun 12:140-143. doi:10.1016/j.elecom.2009.11.008

[30] Burke LD, Morrissey JA (1994) Hydrous oxide formation on platinum in phosphoric acid solution. J Electrochem Soc 141:2361-2368. doi: 10.1149/1.2055126

[31] Burke LD, Casey JK (1992) The role of hydrous oxide species on platinum electrocatalysts in the methanol/air Fuel Cell. Electrochim Acta 37:1817-1829. doi: 10.1016/0013-4686(92)85086-Z

[32] Sleightholme AES, Kucernak A (2011) An anomalous peak observed in the electrochemistry of the platinum/perfluorosulfonic acid membrane interface. Electrochim Acta 56:4396-4402. doi:10.1016/j.electacta.2010.12.036 
[33] C.R. Parkinson, M. Walker, C.F. McConville, (2003)Reaction of atomic oxygen with a Pt(111) surface: chemical and structural determination using XPS, CAICISS and LEED, Surface Science, Volume 545 ,pp19-33 doi: 10.1016/j.susc.2003.08.029

[34] Heuberger, R., Rossi, A., Spencer, N.D. (2006), XPS study of the influence of temperature on ZnDTP tribofilm composition. Tribology Letters, Volume 25, Issue 3 pp 185-196, doi: 10.1007/s11249-006-9166-9

[35] Jingfa Deng, Jinhai Wang, Xinhua Xu, Hsing-Hua Huang, Guo-Qin Xu (1996), Oxidative dehydrogenation of glycol to glyoxal on a P-modified electrolytic silver catalyst ,Catalysis Letters, Volume 36, Issue 3-4, pp 207-214

[36] J. G. Zhou, J. Thompson, J. Cutler, R. Blyth, M. Kasrai, G. M. Bancroft, E. Yamaguchi(2010), Resolving the Chemical Variation of Phosphates in Thin ZDDP Tribofilms by X-ray Photoelectron Spectroscopy Using Synchrotron Radiation: Evidence for Ultraphosphates and Organic Phosphates , Tribology Letters, Volume 39, Issue 1 pp101-107, doi: 10.1007/s11249-010-9619-z

[37] M.Eglin, A.Rossi, N.D.Spencer (2003), X-ray photoelectron spectroscopy analysis of tribostressed samples in the presence of ZnDTP: a combinatorial approach, Tribology Letters, Vol. 15, Issue.3, (2003), pp 199-209, doi: 1023-8883/03/1000-0199/0

[38] Annett Thøgersen, Marie Syre, Birger Retterstol Olaisen,Spyros Diplas (2013), Studies of the oxidation states of phosphorus gettered silicon substrates using X-ray photoelectron spectroscopy and transmission electron microscopy,J. Appl. Phys. 113, 044307 , doi: $10.1063 / 1.4775818$

[39] H. Li, W. Wang, H. Chen, J. Deng, (2001), Surface morphology and electronic state characterization of Ni-P amorphous alloy films, J. Non-cryst. Solids, Volume 281, Issue 1-3 , pp 31-38, doi: 10.1016/S0022-3093(00)00430-0

[40] Y. Zeng, S. Zhou (1999), In situ UV-Vis spectroscopic study of the electrocatalytic oxidation of hypophosphite on a nickel electrode, Electrochemistry Communications, Volume 1, Issue 6, pp 217-222, doi: 10.1016/S1388-2481(99)00043-0

[41] Daletou MK, Kallitsis JK, Neophytides SG (2011) "Materials, proton conductivity and electrocatalysis in high temperature PEM fuel cells". In Vayenas SC (Eds.) Interfacial phenomena in Electrochemistry, Modern Aspects of Electrochemistry, Modern Springer NY, Volume 51, pp 301-368. doi: 10.1007/978-1-4419-5580-7_6.

[42] Vidaković T, Christov M, Sundmacher K (2007), The use of CO stripping for in situ fuel cell catalyst characterization. Electrochim Acta 52:5606-5613. doi: 10.1016/j.electacta.2006.12.057 


\section{Chapter 5}

\section{Electrochemical characterization of the anodic catalyst layer employing $\mathrm{Pt}$ nanoparticles decorated on functionalized MWCNT in HTPEM fuel cells}

\subsection{Introduction}

Up to date, PEMFCs commercialization is still significantly restricted by its high noble metal content and low cathode performance. Reducing cost via reduction of the Pt content, arising from high catalyst utilization and stability, is highly desired. The design and optimization of electrocatalytic layer, towards the increase of the electrocatalytic active surface area, has been the focus of many studies $[1,2,3,4]$.

The porous electrode's structure consists of the catalyst, usually Pt dispersed on carbon, mixed with a binder-ionomer and an ionic carrier, in order to establish a 3-D ionic link between the Pt catalyst active sites and the polymer electrolyte. In HTPEM fuel cell's, PBI is commonly used as the ionomer [5,6,7], and phosphoric acid as the ionic-proton carrier. Due to the liquid state of PA and its mobility, maximization of the triple-phase boundary is difficult to achieve. Thus, a certain amount of ionomer is required, that will bind the free 
PA in the catalyst layer, providing adequate contact with the Pt particles, in order to increase the catalyst utilization. A delicate balance must be achieved between the binder and the ionic carrier for the efficient transport of gases and conductance of electrons and protons to the electrochemical interface. Uniform mixing and distribution of these components is hard and is not guaranteed, as a result part of the electrocatalyst is not used. For that reason, many studies have been focused on the synthesis and characterization of carbon MWNT or nanofiber supported Pt catalyst, aiming to the increase of Pt utilization in fuel cells. To this respect, many groups have successfully functionalized MWCNT using polymers or monomers in an attempt to increase the three-phase boundary (TPB) $[8,9]$.

High temperature PEM fuel cell employing phosphoric acid embedded polymer electrolyte require higher catalyst loading at the anode, compared to the perfluorosulfonic-acid-type membrane based PEM fuel cell. Although, it has been reported that PBI based fuel cell are able to operate without significant loss in their performance utilizing Pt loading as low as $0.2 \mathrm{mgPt} / \mathrm{cm}^{2}$ for the anodic electrode, for specific PBI loading in the CL $[10,11]$. Phosphoric acid poisoning on the Pt surface at the anodic electrode is responsible for the higher demand in Pt loading, as described in detail in chapter 4. The available active sites can be significantly reduced due to the adsorption of reduced phosphoric acid species and therefore influence the performance of the anodic electrode.

During my master thesis, a new functionalized MWCNT Pt based electrocatalyst was developed in an attempt to increased catalyst utilization in high temperature PEMFCs [12]. Pyridine groups were covalently attached at the outer wall of the MWCNT and were used as the catalyst support. The aim was to achieve a uniform distribution of polar pyridine groups in the catalyst layer. The pyridine groups are known to bind phosphoric acid molecules and was expected that a good proton conductive pathways would be created throughout the catalytic layer, which would result in the increase of the three phase boundary and ultimately the catalyst utilization. The obtained Pt/(ox.MWCNT)-Py electrocatalyst had finely and homogeneously distributed Pt particles, with size ranging between $2-4 \mathrm{~nm}$.

In continuance to this work, CLs were fabricated employing this newly synthesized electrocatalyst and were tested as anodic electrocatalysts. In the present chapter, anodic catalyst layers employing the newly synthesized $30 \mathrm{wt} \%$ Pt/ox.MWNT-Py were optimized with respect to the Pt loading and PA loading and tested in HTPEM fuel cells. The electrochemical active surface area and the Pt utilization for the aforementioned electrocatalyst, as well as its durability after short term operation, are also presented. 


\subsection{Experimental}

\subsubsection{Materials}

Phosphoric acid doped polymer electrolyte membranes were provided from Advent Technologies S.A. The in-house synthesized 30wt\% Pt/(ox.MWCNT)-Py, 5wt $\%$ Pt/oxMWCNT-Py, and 30wt\% Pt/oxMWCNT catalysts used in this study were synthesized according to the results reported in my master thesis. Catalyst powder $30 \mathrm{wt} \%$ $\mathrm{Pt} /$ Vulcan XC-72R was purchased from Tanaka Kikinzoku International. For the construction of the electrodes, carbon cloth supporting layer was purchased from Fuel Cell Earth LLC, Vulcan XC-72R powder from RawChem Ltd and the PTFE dispersion in water from Sigma-Aldrich. 2-propanol $99.8 \leq$ and $\mathrm{H}_{3} \mathrm{PO}_{4}$ purum p.a. $\geq 85 \%$ were purchased from Merck or Sigma Aldrich. All chemicals were used as received unless otherwise noted.

\subsubsection{Instrumentation}

The electrochemical measurements were performed using a single cell purchased from Fuel Cell Technologies Inc. Both graphite bipolar plates of the single cell had the same single serpentine flow field for the distribution of reacting gases. The active area of the electrodes was $2 \mathrm{~cm} \times 2 \mathrm{~cm}$. The assembling torque applied for the single cell was $5 \mathrm{Nm}$. The cell was installed in a fuel cell test station which was built in-house and had provisions for controlling temperature, humidification, and flow of reacting gases. The measurements were made in a two-electrode arrangement using an Autolab potentiostat-galvanostat PGSTAT-302.

The size of the Pt nanoparticles of the MWCNT and carbon based electrocatalysts was characterized by transmission electron microscopy (TEM) on JEOL JEM2100 operating at $200 \mathrm{kV}$. The crystalline structure of the catalysts was analyzed with an X-ray powder diffractometer (Bruker D8 Advance) using $\mathrm{Cu} \mathrm{K} \alpha$ radiation $(\lambda=0.15418 \mathrm{~nm})$. The XRD measurements were carried out in the $2 \theta$ angle with the range of $20-90 \circ$. The mean particle diameter of Pt was calculated from the X-ray line broadening of the Pt(111) diffraction peak according to Scherrer's equation.

The photoemission experiments were carried out in a ultrahigh vacuum system equipped with a SPECS LHS-10 hemispherical electron analyzer, which additionally comprises a water cooled high pressure cell $\left(10^{-3} \mathrm{mbar}-2\right.$ bar $)$ connected with the vacuum chambers through a fast entry system, a sample preparation chamber (base pressure $5 \times 10^{-9}$ ) and an analysis chamber with a base pressure $\sim 10^{-10}$ mbar where the XPS measurements took place. The XPS measurements were performed at room temperature using unmonochromatized AlKa radiation under conditions optimized for maximum signal 
(constant $\Delta \mathrm{E}$ mode with pass energy of $97 \mathrm{eV}$ giving a full width at half maximum (FWHM) of $1.7 \mathrm{eV}$ for the $\mathrm{Au} 4 \mathrm{f}_{7 / 2}$ peak). The measurements were recorded at $0^{\circ}$ with respect to the sample surface normal. The analyzed area was a rectangle with dimensions $2.5 \times 4.5 \mathrm{~mm}^{2}$. The sample was mounted on a heated stainless steel holder. The under study electrode was a $5 \mathrm{wt} \% \mathrm{Pt} / \mathrm{oxMWCNT}$ powder/layer interfaced on PA imbibed TPS $^{\circledR}$ membrane. The catalyst powder/layer was grounded in order to avoid any surface charging during data acquisition.

\subsubsection{Catalyst film preparation on $\mathrm{H}_{3} \mathrm{PO}_{4}$ doped polymer electrolyte for XPS experiments}

The pyridine based aromatic polyether membrane was immersed in a $85 \mathrm{wt} \% \mathrm{H}_{3} \mathrm{PO}_{4}$ solution at $140^{\circ} \mathrm{C}$ for 20 hours in order to obtain the desired phosphoric acid doping level ( $200 \mathrm{wt} \%)$. The weight gain was calculated by using the sample's weight before and after the immersion. The doped membrane was then hot pressed against a thin layer of catalyst powder for 15 minutes at $150^{\circ} \mathrm{C}$. During the assembly process, free phosphoric acid is been redistributed from the membrane in the catalyst layer. The catalyst powder film consisting of $5 \mathrm{wt} \% \mathrm{Pt} / \mathrm{oxMWNT}$-Py was thick, insuring complete coverage of the membrane, as no peaks originating from the membrane were detected during the XPS measurement. For comparison reasons $5 \mathrm{wt} \% \mathrm{Pt} / \mathrm{oxMWNT}$-Py catalyst powder was also analysed, in the absence of PA, originating from the same batch as the one used above. Prior to each measurement, all samples were heated for 4 hours in the preparation chamber, at $180^{\circ} \mathrm{C}$, in order to precondition the catalyst surface resembling temperature of fuel cell operation. The sample was cooled down and transferred to the analysis chamber.

\subsubsection{Electrode Preparation and MEA assembly}

Electrodes' fabrication: The gas diffusion layer, GDL, used in this study was prepared by spraying a slurry made of carbon Vulcan XC-72R and PTFE solution (50 wt\% PTFE/C) on carbon cloth, followed by heat treatment at $300^{\circ} \mathrm{C}$ under static air for $40 \mathrm{~min}$. The electrodes were prepared using two different optimized methods, depending on the catalyst type. Two types of electrocatalysts were used, Pt supported on carbon black (30wt \% Pt/C) and Pt supported on MWCNTs (30wt \%t Pt/(ox.MWCNT)-Py and 30wt \%Pt/oxMWCNT).

The electrodes containing 30wt \% Pt/C were prepared by spraying 2-propanol-Pt/C ink on the GDL. The electrodes were then treated for 12 hours at $80^{\circ} \mathrm{C}$ and 3 days at $190^{\circ} \mathrm{C}$ under

vacuum, in order to remove all traces of the organic solvent. The electrodes containing MWCNT based catalysts were prepared by dispersing the catalyst powder in a 2- 
propanol/3-D $\mathrm{H}_{2} \mathrm{O} 2 / 1(\mathrm{v} / \mathrm{v})$ solution. The catalyst slurry was applied on the GDL by a spatula and the electrodes were left over night to dry at room temperature prior heating at $100^{\circ} \mathrm{C}$ in an oven for 1 hour.

The cathode's specifications were kept constant for all MEAs. The carbon black based electrocatalyst (30wt\% Pt/C) was used at counter electrode and the Pt loading was around $2 \mathrm{mgPt} / \mathrm{cm}^{2}$ in all cases. The electrocatalysts used for the construction of the anodic electrodes differed and the Pt loading varied from 0.20 to $2.15 \mathrm{mgPt} / \mathrm{cm}^{2}$.

Membrane Electrode Assembly, MEA: Before the MEA assembly, a certain amount of phosphoric acid, PA, was sprayed onto the electrodes. The cathodic electrodes were sprayed with $2 \mathrm{gPA} / \mathrm{gPt}$, whereas in the case of the anodic electrodes the ratio of $\mathrm{gPA} / \mathrm{gPt}$ varied from 1.71 to 17.6. The electrode active area was $2 \mathrm{~cm} \times 2 \mathrm{~cm}$. Several MEAs with differences in the anodic electrode were fabricated. The specifications of the anodic electrodes employing either 30wt \% Pt/(ox.MWCNT)-Py (MEAs 1-14), 30wt\% Pt/oxMWCNT (MEAs 15-19) or 30wt \% Pt/C (MEAs 20-25) are depicted in Table 5-1. In the case of MEAs 7-13 the Pt loading varied, while the amount of phosphoric acid was kept constant per electrode (at $0.0203 \mathrm{gPA} /$ electrode). Whereas for MEAs 1-6, MEA 15-19 and MEA 20-25 the Pt loading varied, while the amount of phosphoric acid sprayed on the catalyst layer was kept constant, at $2.00 \mathrm{gPA} / \mathrm{gPt}$. Differentiation of the Pt loading in the electrode, results in the alteration of the catalyst layer thickness. By keeping the PA per electrode constant, while at the same time varying the Pt loading (ergo catalyst thickness), the PA distribution per volume of catalyst layer will be altered at each case. More specifically, a thin catalyst layer will contain more PA per volume of catalyst layer, as compared to a thicker one, and therefore more PA will be available for the wetting of the of the Pt particles' surface $(\mathrm{gPA} / \mathrm{gPt})$. On the other hand, for the second case, were the $\mathrm{gPA} / \mathrm{gPt}$ ratio is kept constant, the available PA for the wetting of the Pt particles' surface is expected to be the same for each Pt loading.

The MEAs were fabricated by hot pressing, the phosphoric acid doped membranes (doping level $\sim 200 \mathrm{wt} \%$ ) between the two electrodes at $150^{\circ} \mathrm{C}$ for $5 \mathrm{~min}$. For comparison reasons, MEAs having similar specifications but employing the commercial $30 \mathrm{wt} \% \mathrm{Pt} / \mathrm{C}$ electrocatalyst at the anodic electrode were also prepared and studied in parallel. 
Table 5-1 . Specifications of the anodic electrode of the tested MEAs tested (noble metal loading and phosphoric acid, PA, sprayed on the electrodes prior to the MEA assembly)

\begin{tabular}{|c|c|c|c|}
\hline Catalyst used & MEA No & $\begin{array}{l}\text { Pt loading } \\
\left(\mathrm{mgPt} / \mathrm{cm}^{2}\right)\end{array}$ & $\begin{array}{l}\text { PA amount } \\
\text { (gPA/gPt) }\end{array}$ \\
\hline \multirow{14}{*}{$30 \%$ Pt/(oxMWCNT)-Py } & 1 & 0.29 & 2.00 \\
\hline & 2 & 0.77 & 2.00 \\
\hline & 3 & 0.87 & 2.00 \\
\hline & 4 & 1.26 & 2.00 \\
\hline & 5 & 1.77 & 2.00 \\
\hline & 6 & 2.15 & 2.00 \\
\hline & 7 & 0.20 & 17.62 \\
\hline & 8 & 0.40 & 8.81 \\
\hline & 9 & 0.65 & 5.42 \\
\hline & 10 & 1.02 & 3.45 \\
\hline & 11 & 1.25 & 2.82 \\
\hline & 12 & 1.49 & 2.36 \\
\hline & 13 & 2.06 & 1.71 \\
\hline & 14 & 1.55 & 1.40 \\
\hline \multirow{6}{*}{$30 \% \mathrm{Pt} / \mathrm{ox} . \mathrm{MWCNT}$} & 15 & 0.37 & 2.00 \\
\hline & 16 & 0.77 & 2.00 \\
\hline & 17 & 0.875 & 2.00 \\
\hline & 18 & 1.52 & 2.00 \\
\hline & 19 & 2.19 & 2.00 \\
\hline & 20 & 0.35 & 2.00 \\
\hline \multirow[t]{5}{*}{$30 \% \mathrm{Pt} / \mathrm{C}$} & 21 & 0.98 & 2.00 \\
\hline & 22 & 1.30 & 2.00 \\
\hline & 23 & 1.55 & 2.00 \\
\hline & 24 & 1.61 & 2.00 \\
\hline & 25 & 1.99 & 2.00 \\
\hline
\end{tabular}




\subsubsection{Electrochemical Characterization and Catalyst layer characterization}

\subsubsection{Activation of MEA}

All MEAs were activated under galvanostatic mode. Initially the cell's temperature was increased from room temperature to $100^{\circ} \mathrm{C}$ while $\mathrm{Ar}$ flushed both compartments. Subsequently, the gases were switched to pure $\mathrm{H}_{2}$ and $\mathrm{O}_{2}$ and the temperature was set at $140^{\circ} \mathrm{C}$. Once the temperature was reached, a current of $0.2 \mathrm{~A} / \mathrm{cm}^{2}$ was applied to the fuel cell using pure $\mathrm{H}_{2}$ and $\mathrm{O}_{2}$ (gas stoichiometry $\lambda_{\mathrm{H} 2}=1.2$ and $\lambda_{\mathrm{O} 2}=2$ ). The temperature was then set at $180^{\circ} \mathrm{C}$. The break in period was found to be approximately 48 hours. Details concerning the activation of the MEAs are provided in detail in the appendix A1.

\subsubsection{Electrochemical Impedance Spectroscopy measurements}

All electrochemical impedance spectroscopy measurements were conducted in galvanostatic mode using a sinusoidal current wave with an amplitude of $5 \mathrm{~mA} / \mathrm{cm}^{2}$ and sweeping the frequency from $50 \mathrm{kHz}$ up to $10 \mathrm{mHz}$ with a logarithmic distribution of 50 points. The measurements were conducted without interrupting the cell's operation.

\subsubsection{CO stripping voltammetry}

The electrochemically active surface area, ECSA, of the Pt based catalyst was determined via $\mathrm{CO}$ stripping voltammetry. $\mathrm{CO}$ stripping measurements were performed after a standard activation period of 48 hours of continuous operation. The cell temperature was kept constant at $140^{\circ} \mathrm{C}$. One compartment was supplied with a constant flow of $200 \mathrm{cc} / \mathrm{min}$ of $10 \% \mathrm{H}_{2}$ in argon and the corresponding electrode acted as the reference electrode. The compartment with the electrode under investigation (working electrode) was fed with 25\% $\mathrm{CO}$ in argon (air liquid) at a flow rate of $100 \mathrm{cc} / \mathrm{min}$ at open circuit potential. It was found that the maximum coverage of $\mathrm{CO}$ was achieved in approximately 8 minutes. Subsequently, the compartment was flushed with $200 \mathrm{cc} / \mathrm{min}$ argon in order to remove all the traces of $\mathrm{CO}$ in the gas phase. The argon flow was humidified by passing through a thermostated humidifier kept at a constant temperature of $45^{\circ} \mathrm{C}$, prior to its introduction to the cell. The potential was then cycled between 0.075 and $0.9 \mathrm{~V}$ with a scan rate of 10 $\mathrm{mV} / \mathrm{s}$. To calculate the $\mathrm{CO}$ stripping charge, $\mathrm{Q}_{\mathrm{CO}}$ (in $\mathrm{C}$ ), the area under the peak that corresponds to the $\mathrm{CO}$ electrooxidation was integrated. The second cycle in the same experiment was used for baseline subtraction, considering that during the second cycle $\mathrm{CO}$ was absent from the Pt surface. The ECSA, in $\mathrm{m}^{2}$ was determined considering a charge of 
$420 \mu \mathrm{C} \mathrm{cm}^{-2}$ for $\mathrm{CO}$ oxidation that corresponds to a monolayer of adsorbed $\mathrm{CO}$ on polycrystalline $\mathrm{Pt}$ as follows:

$$
\operatorname{ECSA}\left(\mathrm{m}^{2}\right)=\mathrm{Q}_{\mathrm{CO}}(\mathrm{C}) / 4.20 \mathrm{C} \mathrm{m}^{-2}
$$

The validity of the method was checked and presented in detail in Chapter 4.

\subsubsection{TEM analysis}

Post mortem analysis of the catalytic layers of used membrane electrode assemblies (MEA) was carried out by using transmission electron microscopy (TEM) . The electrodes were separated from the membrane after approximately 50 hours of continuous fuel cell operation for the evaluation of the Pt particle size distribution and morphology of the electrocatalyst. Adequate amounts of catalysts were scrapped off the used electrodes and were ultrasonically dispersed in water. A drop of the suspension was placed on a $3 \mathrm{~mm}$ carbon coated copper grids (Electron Microscopy Sciences). The Pt particle size distributions were obtained by manually measuring over $1000 \mathrm{Pt}$ particles from the TEM pictures. The mean Pt particle size was then calculated using the following equation:

$$
\mathrm{D}_{\mathrm{APt}}=\Sigma \mathrm{D}_{\mathrm{Pt}} \mathrm{N}_{\mathrm{D}} / \mathrm{n} \quad(\mathrm{nm})
$$

where $\mathrm{N}_{\mathrm{D}}$ is the number of particles corresponding to a Pt particle size with diameter $\mathrm{D}_{\mathrm{Pt}}$ $(\mathrm{nm})$ and $\mathrm{n}$ is the total number of measured particles. Pt active surface area, is calculated by the use of eq. 5-3[6],

$$
\mathrm{CSA}_{\mathrm{TEM}}=6000 / \mathrm{d}_{\mathrm{Pt}} \mathrm{D}_{\mathrm{APt}} \quad\left(\mathrm{m}^{2} / \mathrm{gr}\right)
$$

where $\mathrm{d}_{\mathrm{Pt}}$ is the density of bulk Pt $\left(21.4 \mathrm{~g} / \mathrm{cm}^{3}\right)$. Pt utilization ( $\left.\mathrm{UPt}_{\mathrm{Pt}}\right)$ can be determined by comparing the results obtained for the ECSA by the CO stripping voltammetry and the Pt surface area (CSA TEM) obtained by post mortem TEM imaging according to eq. 5-4.

$$
\mathrm{U}_{\mathrm{Pt}}=\mathrm{ECSA} / \mathrm{CSA}_{\mathrm{TEM}}
$$

\subsection{Results and discussion}

\subsubsection{The effect of catalyst substrate on the anodic electrode's performance}

Despite the fast reaction rate of HOR, higher Pt loadings are required compared to the Nafion-based fuel cells, due to phosphoric acid poisoning of the anodic electrode (chapter 4). Operation at low Pt loading without sacrificing the fuel cell performance is highly desired, therefore the newly synthesized electrocatalyst 30wt\% Pt/oxMWCNT-Py, was 
evaluated towards its performance and compared with the conventional $30 \mathrm{wt} \% \mathrm{Pt} / \mathrm{C}$, with respect to different PA and Pt loading. Initially the effect of the catalyst substrate on the anodic electrode's performance was evaluated. For that reason, MEAs were formulated employing 30wt\%Pt/C and 30wt\%Pt/oxMWCNT-Py at the anodic electrode, having the same $\mathrm{Pt}$ and PA loading, $1.3 \mathrm{mgPt} / \mathrm{cm}^{2}$ and $2 \mathrm{gPA} / \mathrm{gPt}$ respectively. Figure $5-1$ shows the polarization curves of MEA 4 and MEA 22, obtained at $180^{\circ} \mathrm{C}$ under pure $\mathrm{H}_{2}$ and $\mathrm{O}_{2}$ and gas stoichiometry of $\lambda_{\mathrm{H} 2}=1.2$ and $\lambda_{\mathrm{O} 2}=2$. The potential is IR drop corrected.

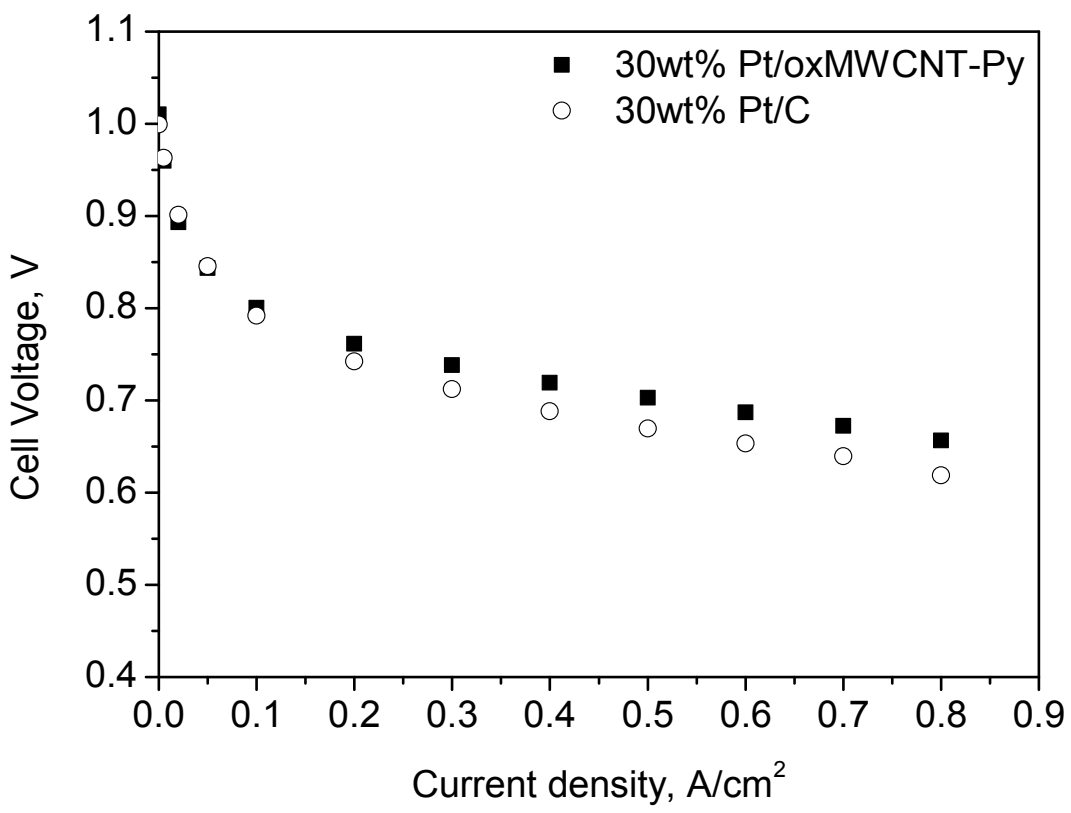

Figure 5-1. The effect of catalyst substrate on the fuel cell performance. Polarization curves, IR drop corrected, were acquired under pure $\mathrm{H}_{2}$ and $\mathrm{O}_{2}$ with a gas stoichiometry of $\lambda_{\mathrm{H} 2}=1.2$ and $\lambda_{\mathrm{O} 2}=2$ at $180^{\circ} \mathrm{C}$. MEA $4: 1.26 \mathrm{mgPt} / \mathrm{cm}^{2}$ and $2 \mathrm{gPA} / \mathrm{gPt}$ employing 30wt\%Pt/oxMWCNT-Py and MEA 22: $1.3 \mathrm{mgPt} / \mathrm{cm}^{2}$ and $2 \mathrm{gPA} / \mathrm{gPt}$ employing $30 \mathrm{wt} \%$ $\mathrm{Pt} / \mathrm{C}$.

As shown in figure 5-1, the performance of MEA 4 employing 30wt\%Pt/oxMWCNT-Py is higher compared to MEA 22. Despite the fact that the HOR is very fast, compared to the ORR, it is clear that changes at the anodic electrode influences the overall fuel cell performance, as also discussed in the previous chapter. In order to verify that the improvement of the performance originates from the anodic electrode and is due to the newly synthesized electrocatalyst, the contribution of the cathodic electrode had to be quantified. For that reason impedance spectra were acquired for both MEAs at $0.2 \mathrm{~A} / \mathrm{cm}^{2}$. 
The spectra were recorded at high excess of reacting gasses $\left(200 \mathrm{cc} / \mathrm{min}\right.$ of pure $\mathrm{H}_{2}$ and $\left.\mathrm{O}_{2}\right)$ to ensure their uniform pressure distribution over the electrode's surface and reduce the mass transport resistance, especially under the ribs of the flow field. The gas transfer resistance is associated with transfer of reactant gasses to the active sites of the electrodes, in terms of convection in the channels of the bipolar plate and diffusion in the GDL and catalyst layer (chapter 1, section 1.3.7). The gas transfer resistance is part of the polarization resistance of each electrode. Although when low gas stoichiometry is used, the polarization resistance increases due to contribution of mass transport to its value, and an additional separate semicircle or Warburg resistance (finite or infinite) is formed. However, when the cell is supplied with a sufficient flow of reactants or high gas stoichiometry the gas transfer resistance becomes negligible or at least constant if the flow remains unchanged [14]. This way by supplying $200 \mathrm{cc} / \mathrm{min}$ of pure $\mathrm{H}_{2}$ and $\mathrm{O}_{2}$, a more accurate picture concerning the effect of the catalyst substrate to the overall cells performance can be derived.

Two arcs are observed in figure 5-2. The first one, which appears at high frequencies, corresponds to the impedance of the $\mathrm{H}_{2}$ oxidation reaction at the anode side $[15,16]$, as also confirmed by experiments that will be presented in section 5.3.4. Whereas the second arc, appearing in the medium and low frequency region, originates from dominant cathode effects. As shown in figure 5-2, the polarization resistance of the anodic electrode for the MEA employing 30wt\% Pt/oxMWCNT-Py is smaller than the one for the commercial catalyst, indicating faster charge transfer and thus higher reaction yield. The FRA module of Autolab software was used to quantify the polarization resistance of both electrodes, by deconvolution of the spectra. The results are summarized in table 5-2. The polarization resistance values of the cathodic electrodes in both cases are similar, whereas for the case of the anodic electrode they exhibit a significant difference. When 30wt\%Pt/oxMWCNTPy is used the overall cell performance is enhanced, as it presents a polarization resistance of $87.5 \mathrm{mOhm} * \mathrm{~cm}^{2}$, as opposed to $125.8 \mathrm{mOhm} * \mathrm{~cm}^{2}$ for the case of $30 \mathrm{wt} \% \mathrm{Pt} / \mathrm{C}$. Therefore, it is clear that the anodic electrodes contribution is responsible for the performance difference observed in the polarization curves.

Table 5-2. Polarization resistance as calculated from Impedance spectra of MEAs employing $30 \mathrm{wt} \% \mathrm{Pt} / \mathrm{C}$ and $30 \mathrm{wt} \% \mathrm{Pt} / \mathrm{oxMWCNT}-\mathrm{Py}$ at the anodic electrode, and $30 \mathrm{wt} \% \mathrm{Pt} / \mathrm{C}$ at the cathodic for both cases. The spectra were acquired at $0.2 \mathrm{~A} / \mathrm{cm}^{2}$ and under $200 \mathrm{cc} / \mathrm{min} \mathrm{H}_{2}$ and $\mathrm{O}_{2}$, and were deconvoluted using FRA module of Autolab Sof.

\begin{tabular}{lcccccc}
\hline & \multicolumn{2}{c}{ Anode } & \multicolumn{3}{c}{ Cathode } \\
& $\mathrm{Rp} / \mathrm{Ohm}^{*} \mathrm{~cm}^{2}$ & $\mathrm{CPE} / \mathrm{F}$ & $\mathrm{n}$ & $\mathrm{Rp} / \mathrm{Ohm}^{*} \mathrm{~cm}^{2}$ & $\mathrm{CPE} / \mathrm{F}$ & $\mathrm{n}$ \\
\hline MEA 4 & 0.0875 & $4.231 \mathrm{e}-1$ & 0.557 & 0.2608 & $5.719 \mathrm{e}-1$ & 0.851 \\
MEA 22 & 0.1258 & $1.366 \mathrm{e}-1$ & 0.612 & 0.2456 & $4.748 \mathrm{e}-1$ & 0.874 \\
\hline
\end{tabular}



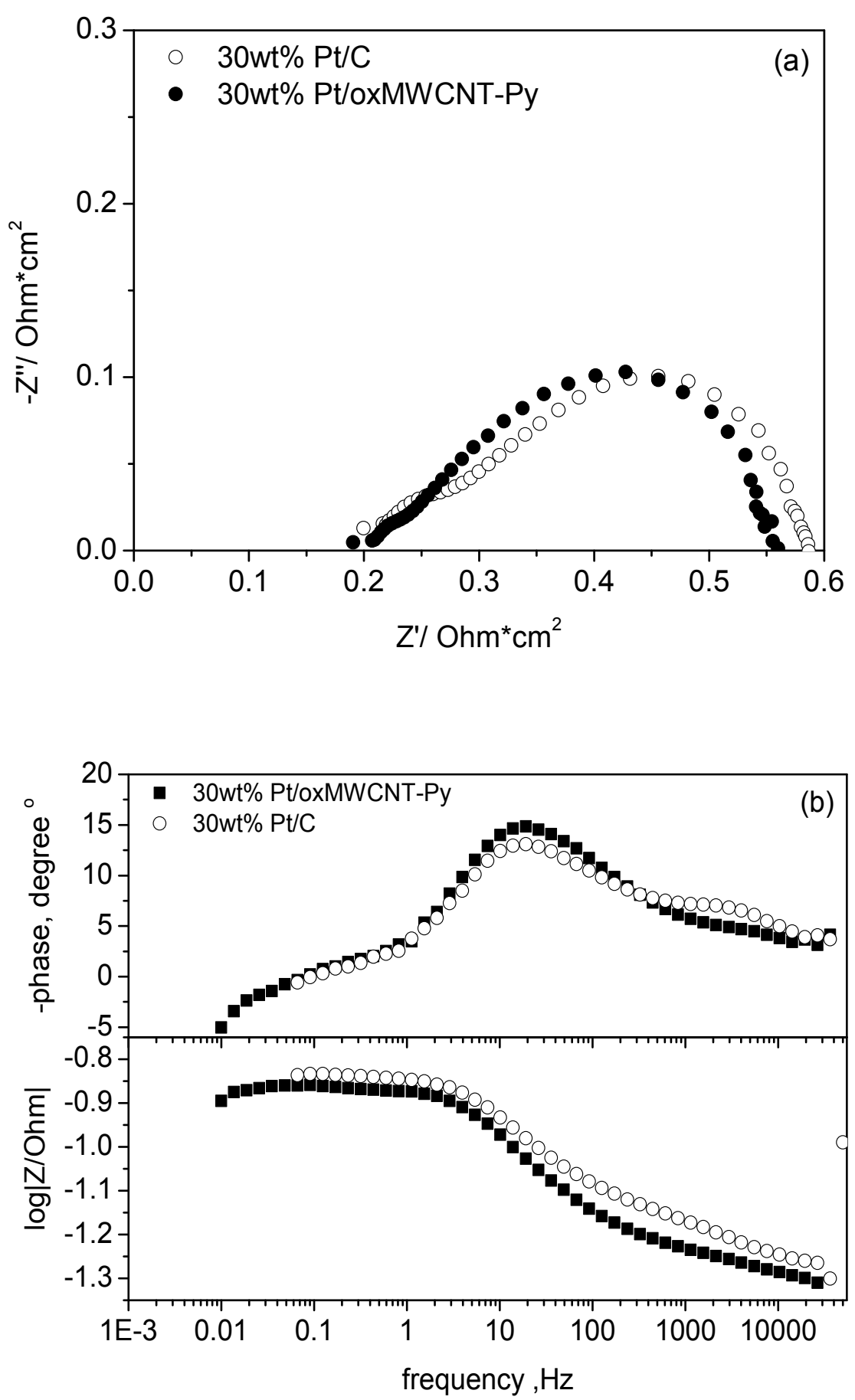

Figure 5-2. AC impedance spectra of MEA $4: 1.26 \mathrm{mgPt} / \mathrm{cm} 2$ and $2 \mathrm{gPA} / \mathrm{gPt}$ employing $30 \mathrm{wt} \% \mathrm{Pt} / \mathrm{oxMWCNT}-\mathrm{Py}$ and MEA 22: $1.3 \mathrm{mgPt} / \mathrm{cm} 2$ and $2 \mathrm{gPA} / \mathrm{gPt}$ employing $30 \mathrm{wt} \% \mathrm{Pt} / \mathrm{C}$. The data were obtained at $0.2 \mathrm{~A} / \mathrm{cm}^{2}$ and at $180^{\circ} \mathrm{C}$ under constant high gas flow of pure $\mathrm{H}_{2}$ and $\mathrm{O}_{2}(200 \mathrm{cc} / \mathrm{min}$ respectively). a. Nyquist plot and b. Bode plot 
It is clear from the above results that the performance of the anodic electrode can be enhanced when the Pt catalyst substrate is being replaced by oxMWCNT-Py. As thoroughly discussed in the previous chapter, PA loading plays a significant role in the performance of the anodic electrode, due to the formation of reduced PA species at low PA loadings, which results in the diminution of the ECSA. Therefore in continuance to the findings in chapter 4 , and in order to further maximize the performance of the anodic electrode, the catalyst layer specifications employing 30wt\%Pt/oxMWCNT-Py were altered with respect to PA and Pt loading. The specifications of the anodic electrodes, Table 5-1, were altered as to differentiate the catalyst layer (CL) thickness and the amount of phosphoric acid (PA) sprayed on the electrode. The electrodes were prepared by changing the catalyst loading between 0.2 and $2.06 \mathrm{mgPt} / \mathrm{cm} 2$ while keeping constant the sprayed amount of phosphoric acid per electrode $(0.0203 \mathrm{gPA} / \mathrm{electrode})$.

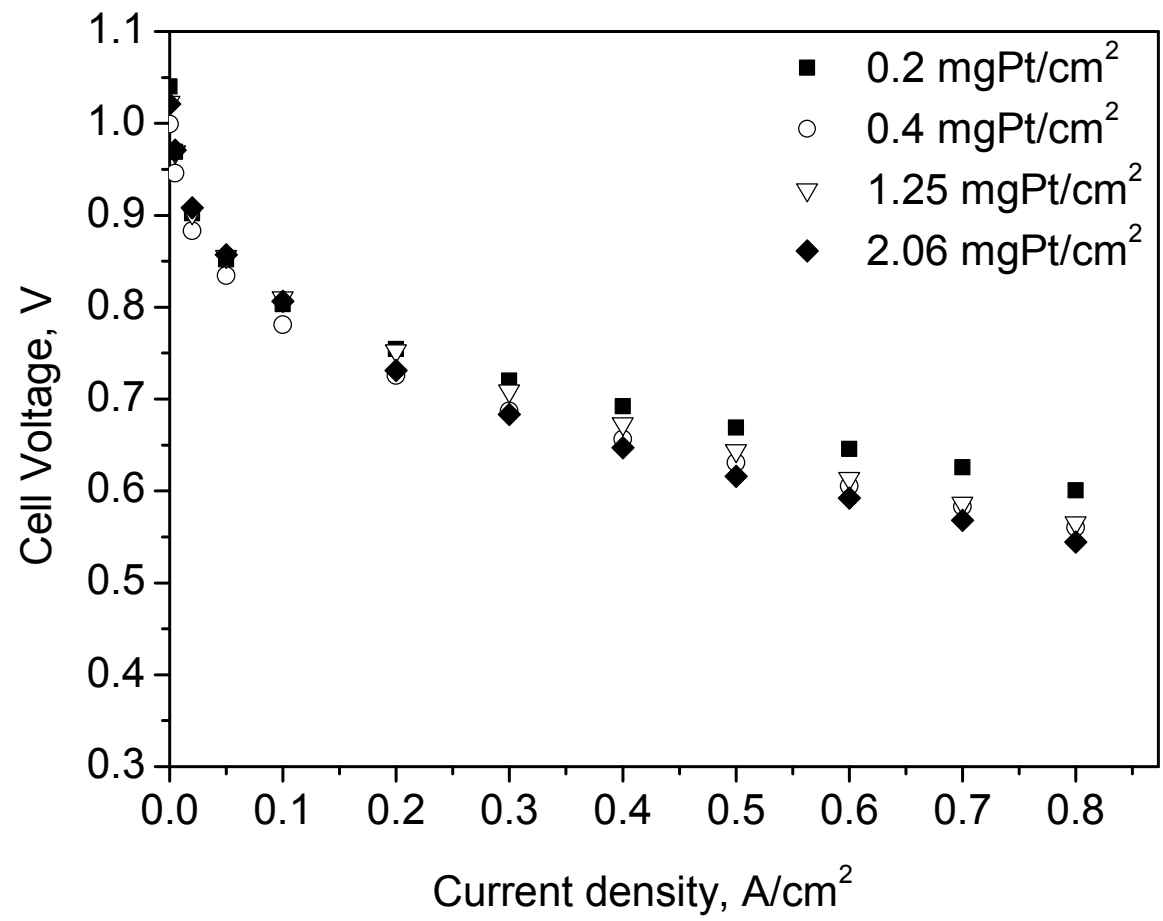

Figure 5-3. The effect of the anodic electrode's specification (Pt loading varies whereas the PA amount per electrode is kept constant) on the overall fuel cell performance. Polarization curves of MEA 7,8,11 and 13 employing 30wt\%Pt/oxMWCNT-Py, IR drop corrected, acquired under pure $\mathrm{H}_{2}$ and $\mathrm{O}_{2}$ and gas stoichiometry of $\lambda_{\mathrm{H} 2}=1.2$ and $\lambda_{\mathrm{O} 2}=2$ at $180^{\circ} \mathrm{C}$. 
Figure 5-3 presents the polarization curves of MEA 7,8,11 and 13, IR drop corrected, acquired using a gas stoichiometry of $\lambda_{\mathrm{H} 2}=1.2$ and $\lambda_{\mathrm{O} 2}=2.0$, at $180^{\circ} \mathrm{C}$. The MEA with the lower Pt loading $0.2 \mathrm{mgPt} / \mathrm{cm}^{2}$ and higher PA loading $17.6 \mathrm{gPA} / \mathrm{gPt}$, exhibit the higher performance. As the Pt loading increases and the ratio of $\mathrm{gPA} / \mathrm{gPt}$ decreases. As a result, the performance of the fuel cell diminishes. The reduction of the PA amount in the catalyst layer results in the formation of phosphoric acid poising species, as thoroughly discussed in the previous chapter 4, shrinking the ECSA and in turn affecting the anodic electrode's performance. Despite the high Pt loading for the case of $2.06 \mathrm{mgPt} / \mathrm{cm}^{2}$ the fuel cell exhibits the lower performance. To further support the above and ensure that the latter is not an effect of the cathodic electrode, impedance spectra were recorded at high excess of the reacting gasses, $200 \mathrm{cc} / \mathrm{min}$ of pure $\mathrm{H}_{2}$ and $\mathrm{O}_{2}$. The calculated polarization resistance, from deconvolution of the AC spectra of the aforementioned MEAs (7,8,11 and 13) are summarized in table 5-3. It is clear that the polarization resistance values of the cathodic electrodes are similar for all cases, whereas the anodic electrodes suffers major changes. The AC spectra of MEA 7 and MEA 13 are shown in figure 5-4 for immediate comparison.

Table 5-3. Polarization resistance as calculated from Impedance spectra of MEAs employing different $\mathrm{Pt}$ loadings of $30 \mathrm{wt} \% \mathrm{Pt} / \mathrm{oxMWCNT}-\mathrm{Py}$ at the anodic electrode. $30 \mathrm{wt} \% \mathrm{Pt} / \mathrm{C}$ is employed at the cathodic electrode having the same $\mathrm{Pt}$ loading approximately $2 \mathrm{mgPt} / \mathrm{cm}^{2}$. The spectra were acquired at $0.2 \mathrm{~A} / \mathrm{cm}^{2}$ and under $200 \mathrm{cc} / \mathrm{min}$ $\mathrm{H}_{2}$ and $\mathrm{O}_{2}$, and were deconvoluted using FRA module of Autolab Software.

\begin{tabular}{|c|c|c|c|c|c|c|c|}
\hline & \multirow{2}{*}{$\begin{array}{l}\text { Anodic Pt } \\
\text { loading } \\
\mathrm{mgPt} / \mathrm{cm}^{2}\end{array}$} & \multicolumn{3}{|c|}{ Anode } & \multicolumn{3}{|c|}{ Cathode } \\
\hline & & $\begin{array}{c}\mathrm{Rp} \\
\mathrm{Ohm}^{*} \mathrm{~cm}^{2}\end{array}$ & $\mathrm{CPE} / \mathrm{F}$ & $\mathrm{N}$ & $\begin{array}{c}\mathrm{Rp} \\
\mathrm{Ohm}^{*} \mathrm{~cm}^{2}\end{array}$ & $\mathrm{CPE} / \mathrm{F}$ & $\mathrm{n}$ \\
\hline MEA 7 & 0.2 & 0.07868 & $1.963 \mathrm{e}-1$ & 0.608 & 0.26488 & $5.858 \mathrm{e}-1$ & 0.835 \\
\hline MEA 8 & 0.4 & 0.06652 & $1.796 \mathrm{e}-1$ & 0.656 & 0.27952 & $4.321 \mathrm{e}-1$ & 0.804 \\
\hline MEA 11 & 1.25 & 0.1128 & $1.353 \mathrm{e}-1$ & 0.647 & 0.26116 & $6.072 \mathrm{e}-1$ & 0.8256 \\
\hline MEA 13 & 2.06 & 0.1084 & $1.516 \mathrm{e}-1$ & 0.663 & 0.2514 & $5.285 \mathrm{e}-1$ & 0.879 \\
\hline
\end{tabular}

Besides the PA poisoning which affects the anodic electrode's performance at low PA loadings (MEA 11 and 13), the high catalyst layer thickness has to be taken also into consideration, as it may affects mass transport. High Pt loadings or thick catalyst layers, may result in the presence of additional mass transport resistance in the anodic electrode that contributes to some extent to the performance loss observed for Pt loadings higher than $1.25 \mathrm{mgPt} / \mathrm{cm}^{2}$. A qualitative way to confirm the latter is through impedance spectra acquired under both low gas stoichiometry of pure $\mathrm{H}_{2}$ and $\mathrm{O}_{2}\left(\lambda_{\mathrm{H} 2}=1.2\right.$ and $\left.\lambda_{\mathrm{O} 2}=2\right)$ and high excess of reacting gasses $\left(200 \mathrm{cc} / \mathrm{min} \mathrm{H}_{2}\right.$ and $\left.\mathrm{O}_{2}\right)$. 

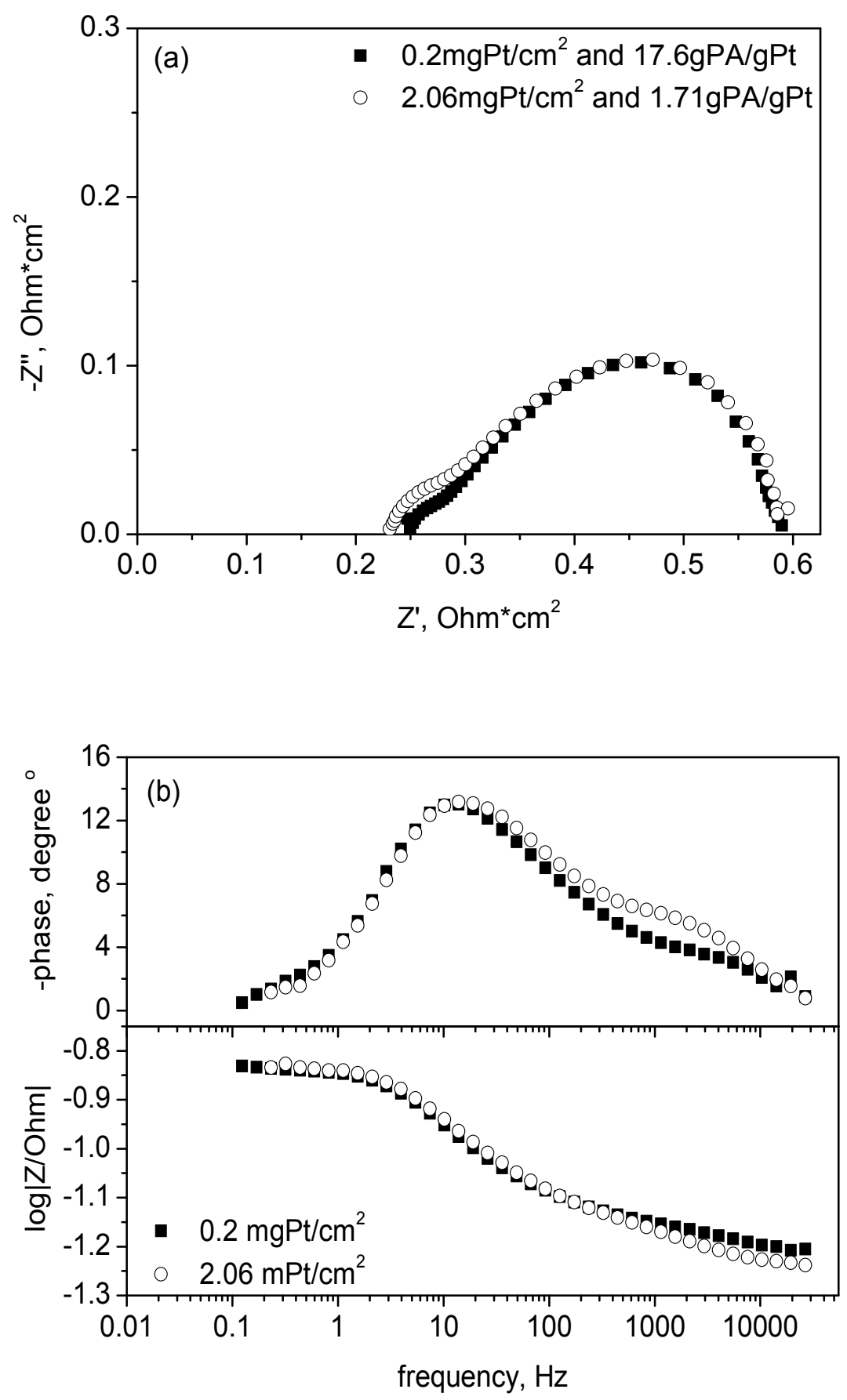

Figure 5-4. AC impedance spectra of MEAs employing 30wt\%Pt/oxMWCNT-Py at the anodic electrode, where the Pt and PA loadings differentiate. MEA 7: $0.2 \mathrm{mgPt} / \mathrm{cm}^{2}$ and 17.62 $\mathrm{gPA} / \mathrm{gPt}$ and MEA 13: $2.06 \mathrm{mgPt} / \mathrm{cm}^{2}$ and $1.71 \mathrm{gPA} / \mathrm{gPt}$. The data were obtained at $0.2 \mathrm{~A} / \mathrm{cm}^{2}$ and at $180^{\circ} \mathrm{C}$ under constant gas flow of pure $\mathrm{H}_{2}$ and $\mathrm{O}_{2}(200 \mathrm{cc} / \mathrm{min}$ respectively). a. Nyquist plot and b. Bode plot 
Figure 5-5, shows impedance spectra acquired under different gas stoichiometry at $0.2 \mathrm{~A} / \mathrm{cm}^{2}$ of MEA7 and 11 with Pt loading $0.2 \mathrm{mgPt} / \mathrm{cm}^{2}$ and $1.25 \mathrm{mgPt} / \mathrm{cm}^{2}$ respectively. As also mentioned earlier, under low gas stoichiometry, mass transfer resistance contributes to the acquired polarization resistance of each electrode [13]. This is clearly seen in figure 5-5, where the first semicircle decreases as the gas stoichiometry increases, whereas the cathode's polarization resistance remains almost unaffected. For the case of $1.25 \mathrm{mgPt} / \mathrm{cm}^{2}$ the decrease of the polarization resistance of the anodic electrode is higher when switch from low to high gas stoichiometry , as opposed for the case of $0.2 \mathrm{mgPt} / \mathrm{cm}^{2}$. This is an indication of the difference in the mass transport limitations as a function of the catalyst layer thickness. The cathodic electrodes polarization resistance doesn't suffer such big changes as the anodic electrode. The beginning of a third semicircle which occurs at low gas stoichiometry, according to the literature, corresponds to the mass transport resistance of the cathodic electrode [13,17]. Although according to I. A. Schneider et al, the low frequency arc is formed due to sinusoidal oxygen-concentration oscillations within the cathode gas along the flow field [18].

The ohmic resistance at high frequencies, which corresponds mainly to the proton conduction resistance shifts to higher values as the gas stoichiometry increases. This is to be expected; as the reactant flow increases the water's partial pressure in the cathodic compartment is being reduced. During operation at those elevated temperatures and low partial pressure of water, two processes take place: phosphoric acid is being dehydrated and pyrophosphoric acid or other dimerised products are being formed (eq.5-5 and 5-6), and hydration of the latter when exposed to water vapor and formation of phosphoric acid $[19,20]$. The equilibrium concentrations of these reversible reactions are temperature and relative humidity dependent.

$$
\begin{aligned}
& 2 \mathrm{H}_{3} \mathrm{PO}_{4} \leftrightarrow \mathrm{H}_{4} \mathrm{P}_{2} \mathrm{O}_{7}+\mathrm{H}_{2} \mathrm{O} \uparrow \\
& \mathrm{H}_{4} \mathrm{P}_{2} \mathrm{O}_{7}+\mathrm{H}_{3} \mathrm{PO}_{4} \leftrightarrow \mathrm{H}_{5} \mathrm{P}_{3} \mathrm{O}_{10}+\mathrm{H}_{2} \mathrm{O} \uparrow
\end{aligned}
$$

The dimerised products are less proton conductive than phosphoric acid [21,22] which explains the increase of the proton conduction resistance when high gas stoichiometry is used [23].

Unfortunately deconvolution of the impedance spectra at low gas stoichiometry was not possible due to the complexity of the equivalent circuit, which requires modeling of the data. Therefore only qualitative information was obtained from the impedance spectra concerning the effect of the catalyst layer thickness on the mass transport limitations of the anodic catalyst layer. In an attempt to quantify the voltage loss originating from mass transport limitations at the anodic electrode, polarization curves were obtained under 

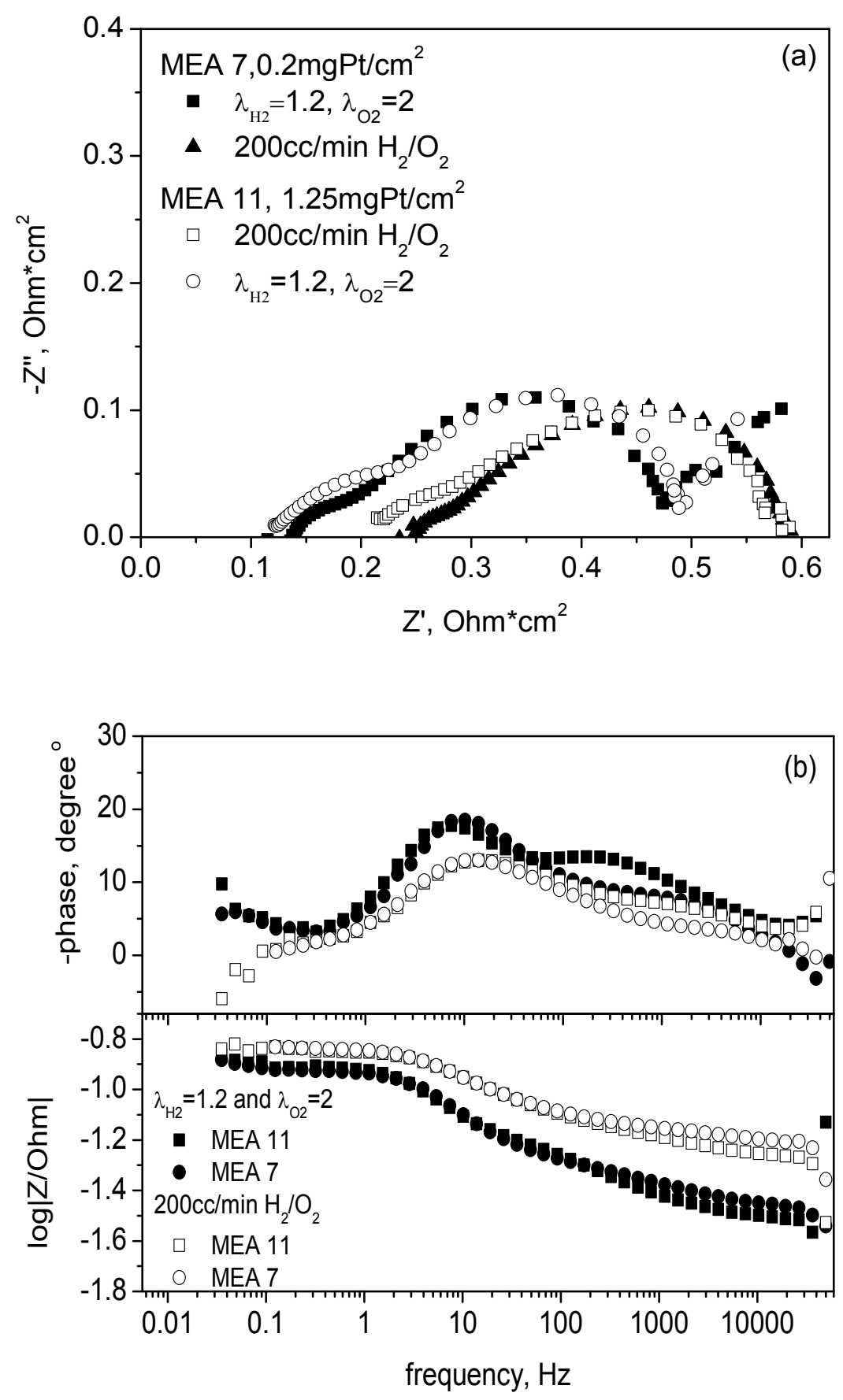

Figure 5-5. The effect of the gas stoichiometry on the AC impedance spectra of MEA employing 30wt\%Pt/oxMWCNT-Py at the anodic electrode, where Pt and PA loadings $(\mathrm{gPA} / \mathrm{gPt})$ differentiate. MEA $7: 0.2 \mathrm{mgPt} / \mathrm{cm} 2$ and $17.6 \mathrm{gPA} / \mathrm{gPt}$ and $\mathrm{MEA}$ $11: 1.25 \mathrm{mgPt} / \mathrm{cm} 2$ and $2 \mathrm{gPA} / \mathrm{gPt}$. The data were obtained at $0.2 \mathrm{~A} / \mathrm{cm}^{2}$ and at $180^{\circ} \mathrm{C}$. a. Nyquist plot and b. Bode plot 
different gas stoichiometry $\left(\lambda_{\mathrm{H} 2}=1.2\right.$ and $\lambda_{\mathrm{O} 2}=2$ and $200 \mathrm{cc} / \mathrm{min} \mathrm{H}_{2} / \mathrm{O}_{2}$ constant flow which corresponds to a nominal current density of $6.6 \mathrm{~A} / \mathrm{cm}^{2}$ ). Figure 5-2 shows the effect of the gas stoichiometry on the performance MEA 7 and 13, having a noble metal loading of $0.206 \mathrm{mgPt} / \mathrm{cm}^{2}$ and $2.06 \mathrm{mgPt} / \mathrm{cm}^{2}$ respectively. Both electrodes exhibit the same performance at high flow rates $\left(200 \mathrm{cc} / \mathrm{min}_{2} / \mathrm{O}_{2}\right)$, as opposed to when low stoichiometry is used. The voltage loss due to mass transport limitations was calculated using the following equation:

$$
\mathrm{V}_{\text {loss }}=\mathrm{V}_{\mathrm{HF}}-\mathrm{V}_{\mathrm{NF}} \quad(\text { eq } 5-7)
$$

Where $\mathrm{V}_{\mathrm{HF}}$ and $\mathrm{V}_{\mathrm{NF}}$, is the voltage which corresponds to high and low gas stoichiometry respectively and is IR drop corrected. Since the cathodic electrode had the same specifications and, as shown from the impedance spectra (figure 5-5), exhibited similar polarization resistance under different gas stoichiometry, it is safe to assume that the voltage loss originates mainly from the anodic electrode. The results are depicted in figure $5-7$.

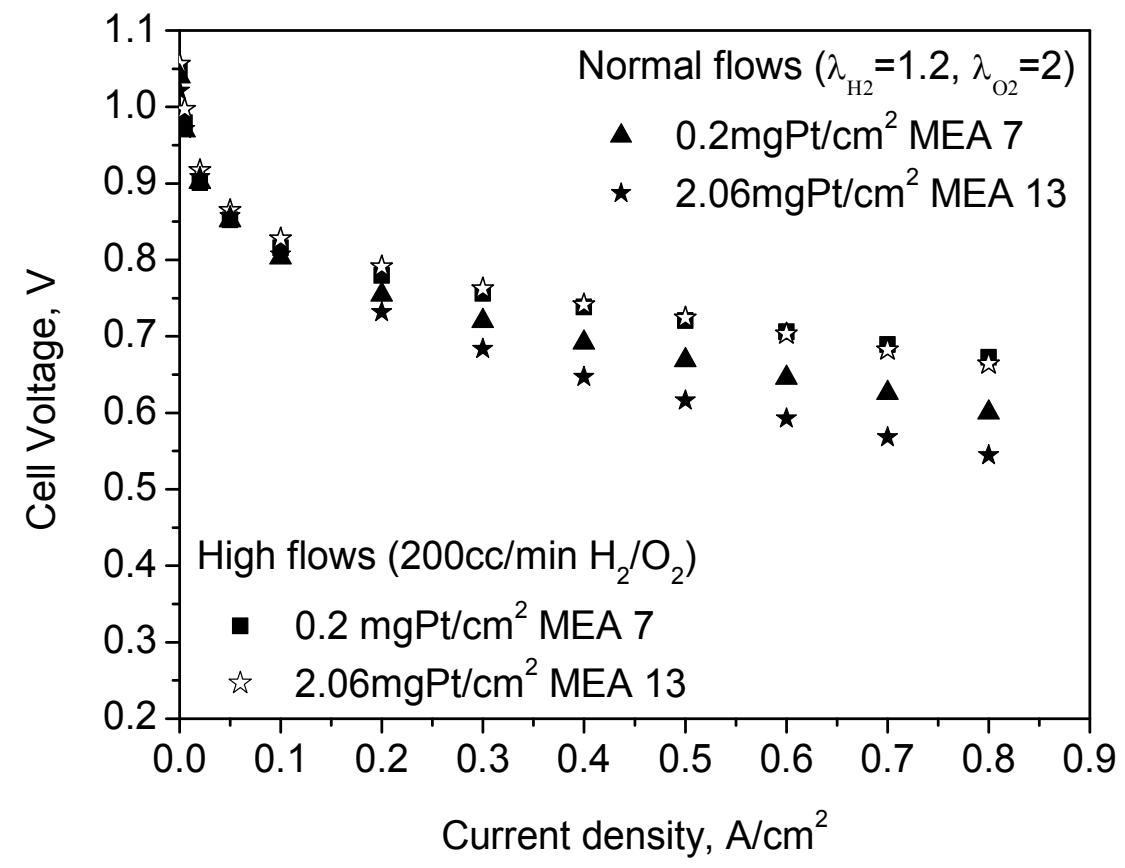

Figure 5-6. The effect of gas stoichiometry on the overall fuel cell performance of MEAs where Pt loading varied whereas the PA amount per electrode is kept constant at the anodic electrode. Polarization curves of MEA 7 and 13 employing 30wt\%Pt/oxMWCNT-Py, IR drop corrected, acquired under pure $\mathrm{H}_{2}$ and $\mathrm{O}_{2}$ and gas stoichiometry of $\lambda_{\mathrm{H} 2}=1.2$ and $\lambda_{\mathrm{O} 2}=2$ and under constant $200 \mathrm{cc} / \mathrm{min}_{2} / \mathrm{O}_{2}$ at $180^{\circ} \mathrm{C}\left(\lambda_{\mathrm{H} 2}>15\right)$. 
From the results depicted in figure 5-7 it is clear that the catalyst layer with a noble metal loading of $0.2 \mathrm{mgPt} / \mathrm{cm}^{2}$ exhibits smaller voltage loss as compared to the one with $2.06 \mathrm{mgPt} / \mathrm{cm}^{2}$, which was expected. By comparing under the same experimental conditions the voltage loss due to mass transport resistance of MEA 22 employing $1.3 \mathrm{mgPt} / \mathrm{cm}^{2} 30 \mathrm{wt} \% \mathrm{Pt} / \mathrm{C}$ at the anodic electrode, it was clear that the later exhibited the minimum voltage loss, even at such high catalyst layer thickness. This is believed to be due to the difference in the porous structure of the catalyst layer as well as due to the manufacturing process of the $\mathrm{CL}$.

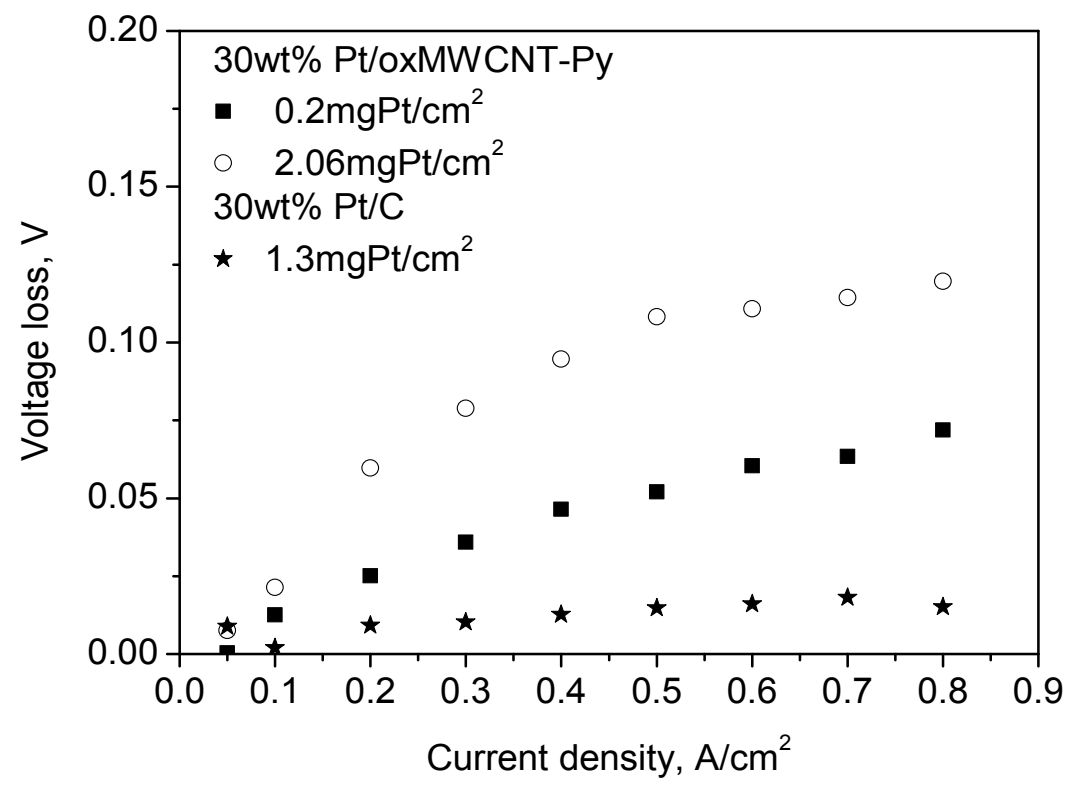

Figure 5-7. Voltage loss with respect to the applied current density due to dominating mass transport resistance as a result of different catalyst layer thickness for anodic electrodes employing 30wt\%Pt/oxMWCNT-Py (MEA 7 and 13) and 30wt\%Pt/C (MEA 22).

Therefore in order to minimize the mass transport limitations at the anodic electrode for the case of MWCNT based electrocatalyst, operation at low Pt loadings is necessary, as opposed to carbon black based catalyst. Nevertheless, it has to be noted that although MEA 22 exhibited the lowest mass transport resistance, its performance was less satisfactory than MEA 4, under the same electrode specifications and operation conditions, figure 5-1. This indicates that the pyridine functionalized MWCNT based catalyst creates a higher and well extended three phase boundary. The latter is fully discussed in the next section. 
The effect of PA loading for low Pt loadings on the fuel cell performance, where the mass transport resistance is the lowest for the MWCTN based CL, is being presented in figure 5-8. Both MEAs (MEA 1 and 7) had similar Pt loading around $0.2 \mathrm{mgPt} / \mathrm{cm}^{2}$ and the PA loading was $2 \mathrm{gPA} / \mathrm{gPt}$ and $17.6 \mathrm{gPA} / \mathrm{gPt}$, respectively. At low Pt loadings the PA poisoning effect at the anodic electrode severely affects the performance of the fuel cell, therefore in order to be able to operate without any performance losses, high PA loadings are required. This is clearly seen in figure 5-8. The anodic electrode with a PA loading of $17.6 \mathrm{gPA} / \mathrm{gPt}$ exhibits higher performance as expected, compared to the one with $2 \mathrm{gPA} / \mathrm{gPt}$. MEA 7 showed the same performance as MEA 23 employing the commercial catalyst with a noble metal loading of $1.55 \mathrm{mgPt} / \mathrm{cm} 2$ and $2 \mathrm{gPA} / \mathrm{gPt}$. These results are very encouraging. Using this newly synthesized electrocatalyst opens the possibility of significant reduction of the amount of $\mathrm{Pt}$ in the anodic electrodes without sacrificing the performance and stability of the fuel cell.

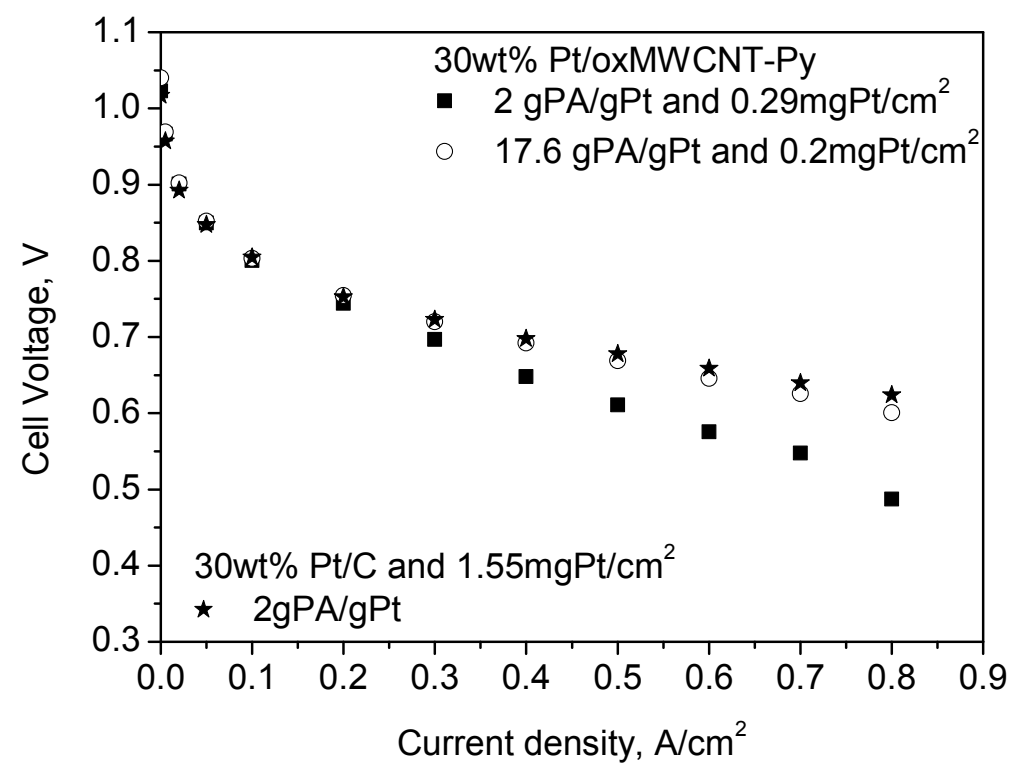

Figure 5-8. The effect of PA on anodic electrode with low Pt loadings. Polarization curves, IR drop corrected obtained at $\mathrm{T}_{\text {cell }}=180^{\circ} \mathrm{C}$ and under low gas stoichiometry $\lambda_{\mathrm{H} 2}=1.2$ and $\lambda_{\mathrm{O} 2}=2$. The MEAs employed 30wt $\%$ Pt/oxMWCNT-Py, MEA 1: 0.29 and $2 \mathrm{gPA} / \mathrm{gPt}$, MEA 7: $0.2 \mathrm{mgPt} / \mathrm{cm}^{2}$ and 17.6gPA/gPt. For comparison MEA 23 employing $30 \mathrm{wt} \% \mathrm{Pt} / \mathrm{C}$ is used: $1.55 \mathrm{mgPt} / \mathrm{cm} 2$ and $2 \mathrm{gPA} / \mathrm{gPt}$.

To summarize, taking all of the above into consideration and despite the higher mass transport limitations as opposed to the commercial $30 \mathrm{wt} \% \mathrm{Pt} / \mathrm{C}$, the higher performance of the $30 \mathrm{wt} \% \mathrm{Pt} / \mathrm{oxMWCNT}-\mathrm{Py}$ electrocatalyst is believed to be due to the presence of 
pyridine groups. The role and the contribution of the pyridine groups on the creation of high three-phase boundary is investigated and presented in the next section in detail.

\subsubsection{Evaluation of ECSA and Pt utilization}

The Pt surface area available for the electrochemical reaction is critical in order to sustain high fuel cell performance, especially under lean partial pressure of $\mathrm{H}_{2}$ and high mole fraction of $\mathrm{CO}$ in the fuel gas. It is widely known that the properties of the catalyst support can affect the wettability and the interaction with the acid electrolyte and thus influence the length of the reaction's TPB.

For that reason, the catalyst layer thickness was altered, in order to study the effectiveness of the phosphoric acid toward the creation of a 3-D ionic link between the catalyst nanoparticles and the ion conductor as a function of the catalyst support. The three catalyst supports were evaluated: the commercial $30 \mathrm{wt} \% \mathrm{Pt} / \mathrm{C}$, the newly synthesized $30 w t \% P y / o x M W C N T-P y$ and 30wt\%Pt/oxMWCNT. The latter was only measured for comparison reasons in order to distinguish the effect of the pyridine groups from the overall effect of using carbon nanotubes as a substrate, towards the creation of high three phase boundary. All three substrates have different morphologies and hydrophilic properties, and thus different interaction is expected with the acid electrolyte influencing the lengthiness of the reaction's TPB. Three series of MEAs were fabricated employing 30wt $\%$ Pt/(ox.MWCNT)-Py (MEA 1-6), 30wt\%Pt/oxMWNT (MEA 15-19) and a conventional $30 \mathrm{wt} \% \mathrm{Pt} / \mathrm{C}$ (MEA 20,21,24 and 25) electrocatalyst at the anodic electrode (table 5-1). The noble metal loading of the anodic electrode varied from 0.2 to $2.15 \mathrm{mgPt} / \mathrm{cm} 2$, while maintaining the PA amount constant at $2 \mathrm{gPA} / \mathrm{gPt}$.

The electrochemical active surface area (ECSA) of all the aforementioned anodic electrodes was measured via CO stripping voltammetry, as described in the experimental section and validated in chapter 4 . Since the formation of the reduced phosphoric acid species at the anodic electrode cannot be avoided during the activation period, for the chosen PA loading, preconditioning of the working electrode via cyclic voltammetry for the complete removal the PA 'poisoning' species was necessary. The obtained ECSA will be higher than the actual one during normal fuel cell operation, as also mentioned in chapter 4. Nonetheless, it will not influence the overall ECSA pattern for each electrocatalyst. The results with respect to the Pt loading in the electrocatalytic layer are depicted in Figure 59. For comparison reasons, the ECSA is normalized to the mass of the noble metal and therefore expressed as $\mathrm{m}^{2} / \mathrm{g}_{\mathrm{Pt}}$.

It is clearly shown that the ECSA is independent of the Pt loading on the electrodes for all three Pt supported electrocatalyst. The 30\%Pt/ox.MWCNT catalyst exhibits an ECSA 
value of approximately $25 \mathrm{~m}^{2} \mathrm{Pt} / \mathrm{grPt}$, whereas the $30 \% \mathrm{Pt} /(\mathrm{ox} . \mathrm{MWCNT})-\mathrm{Py}$ and the $30 \% \mathrm{Pt} / \mathrm{C}$ approaches $40 \mathrm{~m}^{2} / \mathrm{grPt}$ and $35 \mathrm{~m}^{2} / \mathrm{grPt}$, respectively. The thickness of the catalyst layer does not affect the ECSA, above a certain noble metal loading $(0.7 \mathrm{mgPt} / \mathrm{cm} 2)$. Nevertheless, it is worth mentioning that for the case of $30 \mathrm{wt} \% \mathrm{Pt} / \mathrm{oxMWCNT}-\mathrm{Py}$ and for a Pt loading as low as $0.2 \mathrm{mgPt} / \mathrm{cm}^{2}$, the ECSA is significantly higher as compared to the commercial catalyst, $30 \mathrm{wt} \% \mathrm{Pt} / \mathrm{C}$.

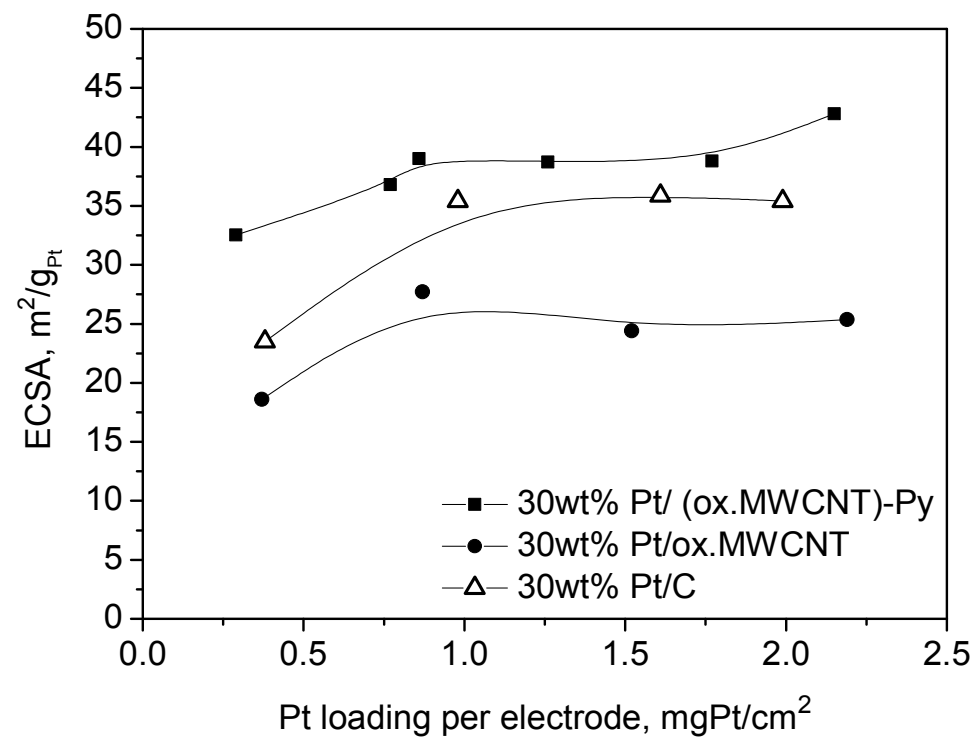

Figure 5-9. ECSA $\left(\mathrm{m}^{2} / \mathrm{gPt}\right)$ determination via CO stripping voltammetry measurements at $140^{\circ} \mathrm{C}$ with respect to the $\mathrm{Pt}$ loading of the anodic electrode employing different electrocatalysts : MEAs 30\% Pt/(ox.MWCNT)-Py, MEAs 30\% Pt/ox.MWCNT and MEAs 30\% Pt/C (all listed in Table 5-1).

In continuance, the specifications of the anodic electrodes, MEA 7-13, were altered as to differentiate the catalyst layer (CL) thickness and the amount of phosphoric acid (PA) sprayed on the electrode. The electrodes were prepared by changing the catalyst loading while keeping constant the sprayed amount of phosphoric acid per electrode. By keeping the PA per electrode constant, while at the same time varying the Pt loading (ergo catalyst thickness), the PA distribution per volume of catalyst layer will be altered at each case. More specifically, a thin catalyst layer will contain more PA per volume of catalyst layer, as compared to a thicker one, and therefore more PA will be available for the wetting of the Pt particles' surface (gPA/gPt). The phosphoric acid amount in the catalytic layer is a very important and critical parameter for the achievement of a good proton conductive pathway throughout the catalyst layer. In the case of MEAs 7-13 the Pt loading varied, 
while the amount of phosphoric acid was kept constant per electrode (at 0.0203 gPA/electrode). The measured ECSA was compared with the case of altering the $\mathrm{Pt}$ loading while maintaining the PA loading in the catalyst layer constant, $2 \mathrm{gPA} / \mathrm{gPt}$, figure 5-10. Overall, the 30wt\%Pt/oxMWCNT-Py exhibits a high ECSA value even at Pt loadings as low as $0.2 \mathrm{mgPt} / \mathrm{cm}^{2}$ and $17.6 \mathrm{gPA} / \mathrm{gPt}$. It is clear that for the aforementioned catalyst the ECSA is independent of the Pt loading and the PA amount present in the catalyst layer.

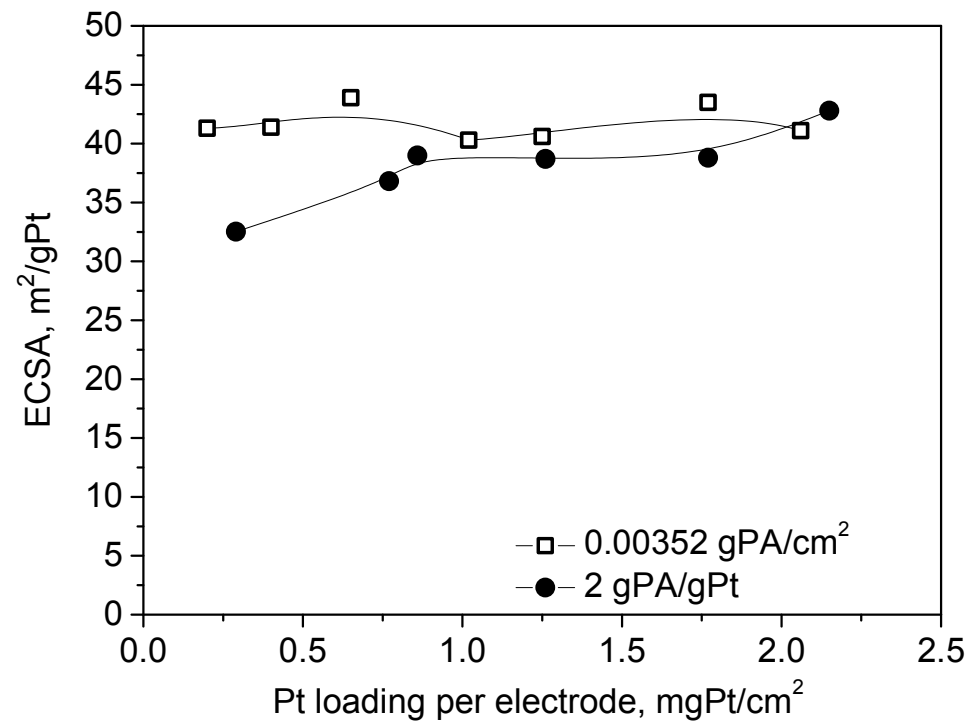

Figure 5-10. ECSA determination with increasing Pt loading based on CO stripping voltammetry measurements at $140^{\circ} \mathrm{C}$, for different Pt and PA loadings (MEAs 1-6 and MEA 7-13 in Table 5-1).

Accurate calculation of Pt utilization requires measurement of the average Pt particle size after 48 hours of continuous operation (activation period), since the ECSA is being evaluated after the activation period. TEM imaging was used for the evaluation of the $\mathrm{Pt}$ average Pt size, as described in detail in the experimental section. The measured Pt particle size of each catalyst and the corresponding Pt utilization are shown in Table 5-4.

Pt sintering and agglomeration can be affected by a numerous of factors such as operation time [23], temperature [24,25] etc. Nanoparticles have the tendency to agglomerate into bigger particles in order to reduce their high surface energy. As particles grow, their surface energy decreases and their growth process slows down [26]. Therefore, MEAs with the same specification were fabricated, as the ones used for the determination of the ECSA. The Pt particle sizes of the anodic electrodes were then measured via TEM imaging. The results revealed that $\mathrm{Pt}$ growth at the anode, meanly occurs during the activation period of the MEA. No significant change was observed when the operation time was increased by 
24 hours (will be discussed in detail in section 5.3.6.2). Although all three as prepared electrocatalysts had similar particle size, $3.2-4 \mathrm{~nm}$, they showed different Pt growth rate.

The growth of the Pt particles for the 30wt\% Pt/(ox-MWNT)-Py electrocatalyst was smaller than in the case of $30 \mathrm{wt} \% \mathrm{Pt} /$ (oxMWNT), table 5-4. Within 48 hours of continuous fuel cell operation the average Pt particle size for the pyridine functionalized MWNT based catalyst reached an average value of $5.6 \mathrm{~nm}$, whereas $7.9 \mathrm{~nm}$ for the one without. It is widely established, that the nature of the supporting substrate can strongly influence the stabilization of particles and determine the size of supported metals. It our case, it is very clear that the presence of pyridine groups has a positive effect in stabilizing the Pt particle size as compared to the ox.MWCNT based catalysts. There are contradictory results in the literature concerning the effect of oxidative pre-treatment and the role of oxygen groups of the carbon on platinum dispersion and sintering. Some groups [27,28] reported, that the presence of weak acid groups, strongly increases the interaction between the metal particle and the support, while others [29,30,31], instead, reported that the presence of oxygen surface groups inhibit the metal dispersion, and thus would be more prone to sintering. On the other hand, a small increase in the particle size was observed for the case of the $30 \mathrm{wt} \%$ $\mathrm{Pt} / \mathrm{C}$, which showed to be more sintering resistant under these operation conditions (table $5-4)$.

Table 5-4. Average Pt particle size after 48 hours of continuous operation for different catalyst, as measured from TEM, and their corresponding catalyst electrochemical utilization, UPt-max.

\begin{tabular}{ccc}
\hline Catalyst & Pt size (nm) & UPt-max (\%) \\
\hline 30wt\% Pt/C & 4.1 & 51.4 \\
30wt\% Pt/(ox.MWCNT) & 7.9 & 70.4 \\
30wt\% Pt/(ox.MWCNT)-Py & 5.6 & 80.5 \\
\hline
\end{tabular}

The Pt utilization was calculated as described in the experimental section. As shown in table 5-4, the highest Pt utilization was found to be for the 30wt\% Pt/(ox.MWNT)-Py electrocatalyst around $80.5 \%$, whereas $70.4 \%$ and $51.4 \%$ for $30 \mathrm{wt} \% \mathrm{Pt} / \mathrm{oxMWCNT}$ and $30 \mathrm{wt} \% \mathrm{Pt} / \mathrm{C}$,respectively. It is clear that the carbon nanotube supported catalyst exhibited higher Pt utilization as compared to the conventional carbon based, due to the difference in the wettability properties and interaction of the substrate with the PA. The high value of catalyst utilization in the case of $30 \mathrm{wt} \% \mathrm{Pt} /(\mathrm{ox} . \mathrm{MWCNT})-\mathrm{Py}$ is undeniably due to the homogeneous distribution of polar pyridine groups in the catalyst layer. Pyridine groups, which are covalently attached on the carbon support, interact and bind phosphoric acid 
promoting its uniform distribution and stabilizing it inside the catalytic layer. The created well extended 3D ionic network of PA also minimizes the local flooding in the electrodes.

\subsubsection{Investigation of the interaction of PA with the pyridine groups in the CL by means of XPS}

The PA interaction with the pyridine groups attached on the outer wall of the MWCNT was verified by means of XPS. The pyridine groups are identified by the XP spectra of N1s, as depicted in Figure 5-11.

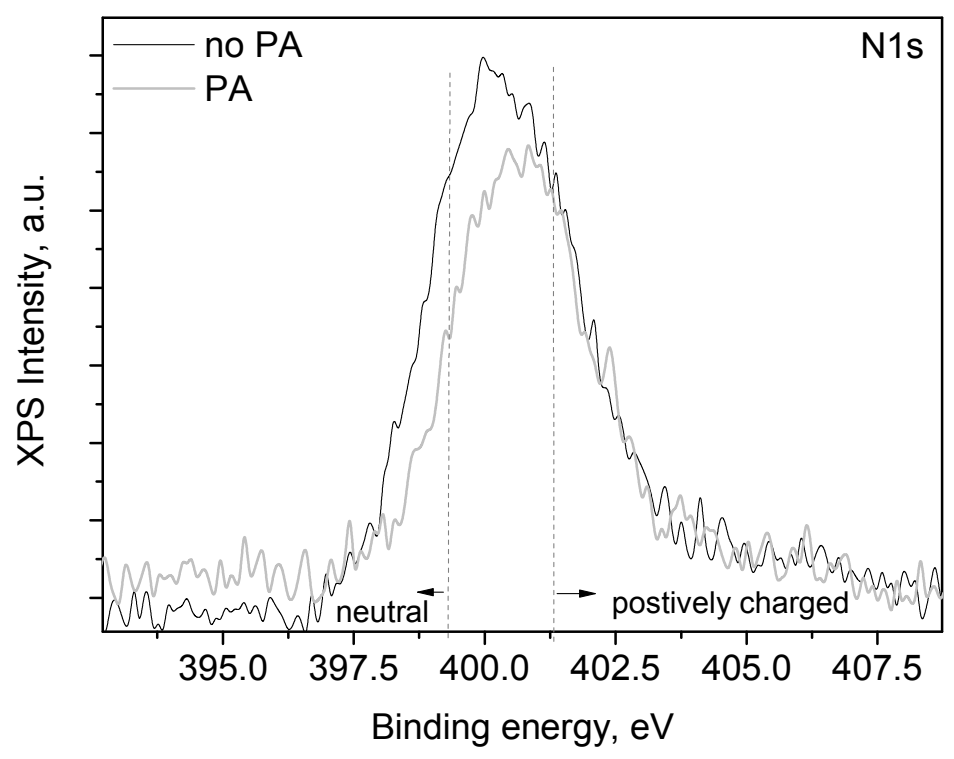

Figure 5-11. XPS spectra of 5wt $\% \mathrm{Pt} / \mathrm{oxMWCNT}-\mathrm{Py}$ in the presence and absent of PA in the catalyst layer.

As mentioned in the experimental section, the noble metal loading on the MWNT based substrate that was used for the XPS experiment was 5wt\% Pt per carbon support, despite the fact that the catalyst used in the electrochemical measurements had a noble metal loading of $30 \mathrm{wt} \% \mathrm{Pt}$ per carbon support. The pyridine groups are homogeneously spread on the outer wall of the carbon nanotube, as reported from earlier work, therefore during Pt deposition, the Pt particles will cover a large amount of the pyridine groups. Thus, lowering the Pt loading on the substrate reduces the surface coverage, leaving exposed a larger number of pyridine groups, resulting in a higher measured signal of the N1s peak ,which increases the accuracy of any observed change due to the presence of PA. For that reason, $5 \%$ wt Pt per carbon support was considered appropriate. 
Figure 5-11, presents the results of the N1s spectra of the catalyst powder in the absence and presence of phosphoric acid. These spectra are resolved into two peaks. The main feature is the peak at $398.8 \pm 0.3 \mathrm{eV}$ binding energy, which corresponds to the pyridinic nitrogen $[32,33]$ while the second peak, at $401+0.5 \mathrm{eV}$ binding energy, to quaternary $\mathrm{N}$ (positively charged) [34,32]. The $\mathrm{C} 1 \mathrm{~s}$ for both cases was identical and presented a binding energy of the main peak at $284.3 \mathrm{eV}$ which corresponds to the typical graphitic carbon peak, indicating that no charging of the surface occurred and that it was properly grounded. When PA is present in the catalyst layer, as indicated in the N1s spectra, an apparent decrease in the low binding energy peak is observed, where it is clear that the neutral's

pyridine contribution has decreased. This is a clear indication of the interaction and charge transfer between the phosphoric acid in the catalyst layer and the pyridine groups attached on the MWNT. Also, the decrease in the acquired signal might be due to the PA coverage of the MWNT surface.

\subsubsection{Kinetic of HOR}

The kinetics of HOR on both $30 \mathrm{wt} \% \mathrm{Pt} / \mathrm{C}$ and $30 \mathrm{wt} \% \mathrm{Pt} / \mathrm{oxMWCNT}-\mathrm{Py}$ was studied. The strong dependence of the reaction kinetics on the partial pressure of $\mathrm{H}_{2}$ is shown by the $\mathrm{AC}$ impedance spectra recorded at various partial pressures of $\mathrm{H}_{2}$ and at the same current density (Figure 5-13). The dependence of the reaction kinetics on the partial pressure of $\mathrm{H}_{2}$ was independent of the catalyst substrate, where the exact same response was observed for both catalyst. For that reason the only data obtained from MEA 4 were chosen to be presented. The spectra were recorded at high excess of the reacting gasses to ensure their uniform partial pressure distribution over the electrode's surface. The monitoring of the spectra at the same current density allows for the isolation of the effect of the partial pressure of $\mathrm{H}_{2}$ on certain features of the $\mathrm{AC}$ spectra that characterize the response of the anode's electrokinetic behavior. In this respect cathode remains unaffected. Thus, as it is clearly shown in Figure 5-13 the variation of the polarization resistance of the high frequency arc is definitely attributed to the anode. Eq. 5-8, is derived from the Tafel equation and is related the polarization resistance $R_{p}$ of the EI with the applied current I and the transfer coefficient $\alpha$ or otherwise the Tafel slope $\alpha \mathrm{F} / \mathrm{RT}$.

$$
\left.R_{p}=\frac{1}{I} \frac{R T}{\alpha F} \quad \text { (eq. } 5-8\right)
$$

The variation of the polarization resistance under constant current density implies variation in the transfer coefficient $\alpha$, which depends on the reaction mechanism and the limiting step of the electrocatalytic process [35]. In the present case its variation indicates the transition in the electrochemical reaction steps that drive the $\mathrm{H}_{2}$ electrooxidation reaction. 
It is well known that $\mathrm{H}_{2}$ oxidation/evolution is described by the Tafel, Volmer and Heyrovski reaction steps according to the reacting scheme described by Eqs 5-9 -10-11.

$$
\begin{array}{ll}
H_{2} \rightarrow 2 H_{a d} & \text { Tafel } \\
H_{a d} \rightarrow H^{+}+e^{-} & \text {Volmer } \\
H_{2} \rightarrow H_{a d}+H^{+}+e^{-} & \text {Heyrovski }
\end{array}
$$

Reaction 3 is fast and takes place at low overpotential being the primary charge transfer $\mathrm{H}_{2}$ electrooxidation reaction, while reaction 4 takes off at fairly higher overpotentials characterized by a larger Tafel slope [36]. Two factors corroborate for this kinetic transition. Either the low partial pressure of $\mathrm{H}_{2}$ thus affecting the rate of reaction (5-9) or the limited surface sites (phosphoric acid poisoning), thus posing restrictions on reaction (5-10) due to the low coverage of the adsorbed hydrogen $\mathrm{H}_{\mathrm{ad}}$. In this respect, the change in the slope of the I-V curves of Figure 5-12 is attributed to the transition from the Volmer reaction (5-10) to the activation of the Heyrovski reaction (5-11).

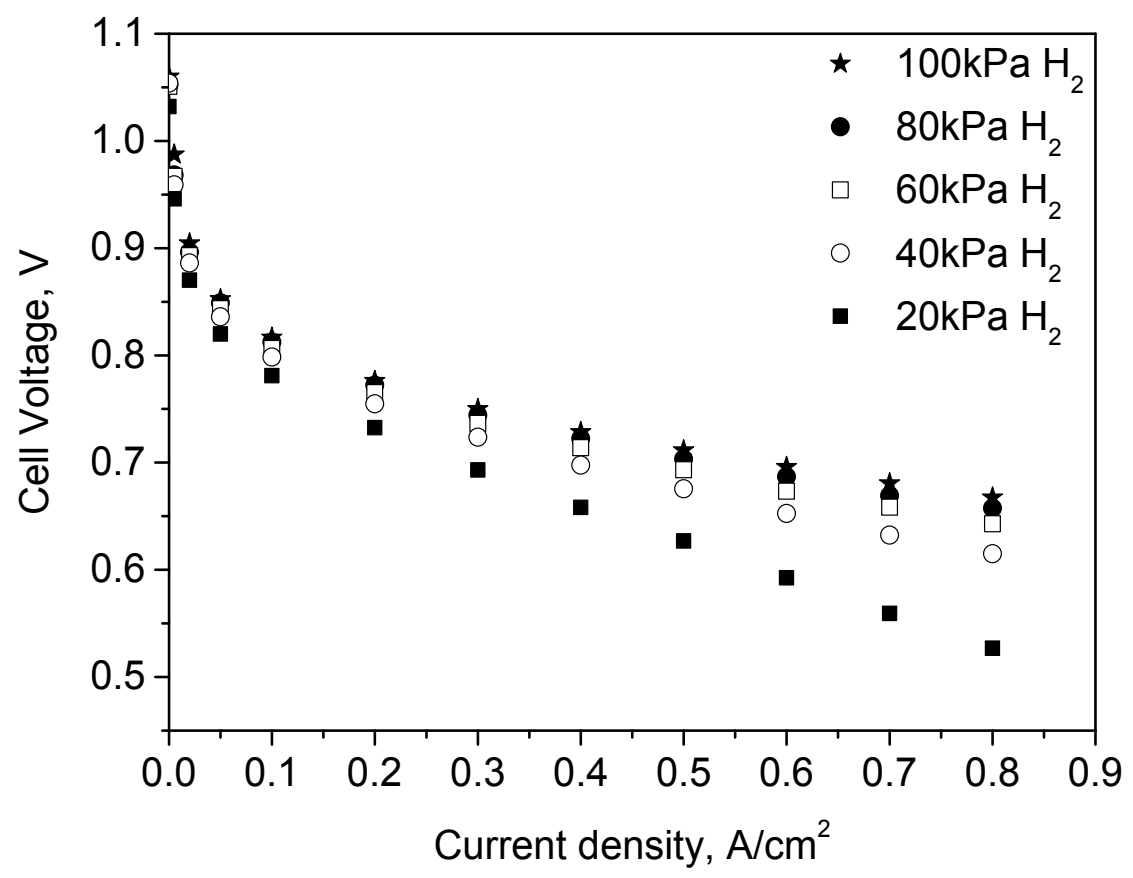

Figure 5-12. The effect of $\mathrm{H}_{2}$ partial pressure on the fuel cell's performance of MEA 4. The polarization curves were IR drop corrected and were acquired under constant total flow of $200 \mathrm{cc} / \mathrm{min}$ of $\mathrm{H}_{2}$ balanced with $\mathrm{Ar}$ and $200 \mathrm{cc} / \mathrm{min}_{2}$ at $180^{\circ} \mathrm{C}$. MEA 4 : employed $30 \mathrm{wt} \% \mathrm{Pt} / \mathrm{oxMWCNT}-\mathrm{Py}, 1.26 \mathrm{mgPt} / \mathrm{cm} 2$ and $2 \mathrm{gPA} / \mathrm{gPt}$. 

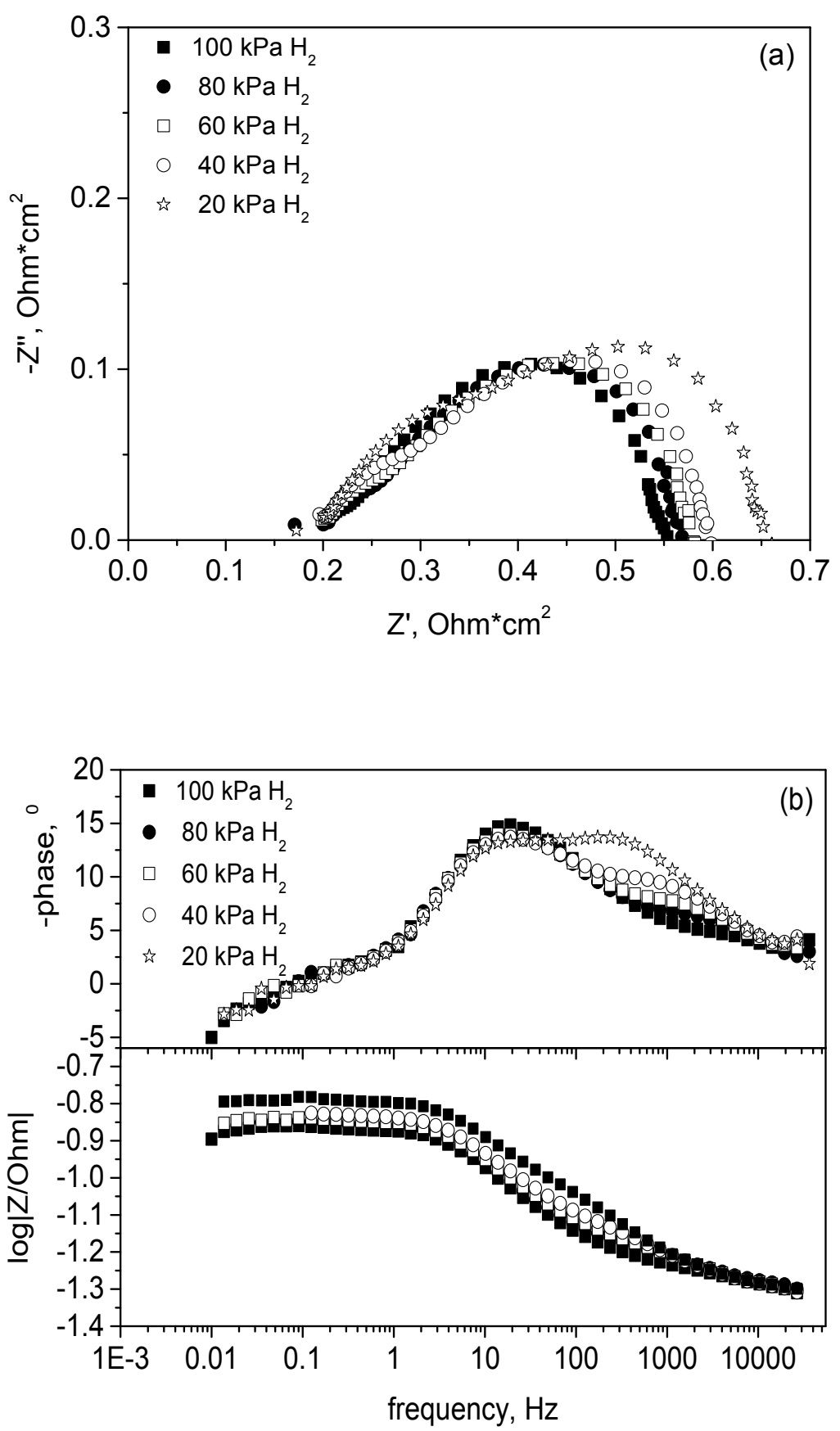

Figure 5-13. Impedance spectra of MEA 4 obtained at a current density of $0.2 \mathrm{~A} / \mathrm{cm}^{2}$ at $180^{\circ} \mathrm{C}$, under various $\mathrm{H}_{2}$ partial pressure (total flow $200 \mathrm{cc} / \mathrm{min}$ of $\mathrm{H}_{2}$ in $\mathrm{Ar}$ ). The cathodic electrode was fed with a redundant $\mathrm{O}_{2}$ flow $(200 \mathrm{cc} / \mathrm{min})$. a. Niquist plots and b. Bode plots 


\subsubsection{Charge transfer resistance of the anodic electrode}

The change of the charge transfer resistance with the applied current is being presented in this section. The change of the charge transfer resistant as a function of the applied current density was found to be independent of the catalyst substrate and the PA amount present in the catalyst layer (figure 5-14), as the exact same response was observed for all cases. Figure 5-14 presents the results obtained from MEA 7 and 13.

AC impedance spectra were acquired at different current densities under high constant gas flow $\left(200 \mathrm{cc} / \mathrm{min}_{2}\right.$ and $\left.\mathrm{O}_{2}\right)$ and are depicted in figure 5-15 and figure 5-16. It can be seen that the overall charge transfer resistance decreases with increasing current density. In the low current region, the oxygen reduction reaction (ORR) at the cathode dominates while the hydrogen oxidation reaction (HOR) at the anode is negligible. In the low current region the polarization resistance of the anodic electrode is significantly lower than the one assigned to the cathode electrode (figure 5-14). In the high current density region, the HOR becomes as important as the ORR, as the polarization resistance of the anodic electrode has similar values with the cathodic polarization resistance.

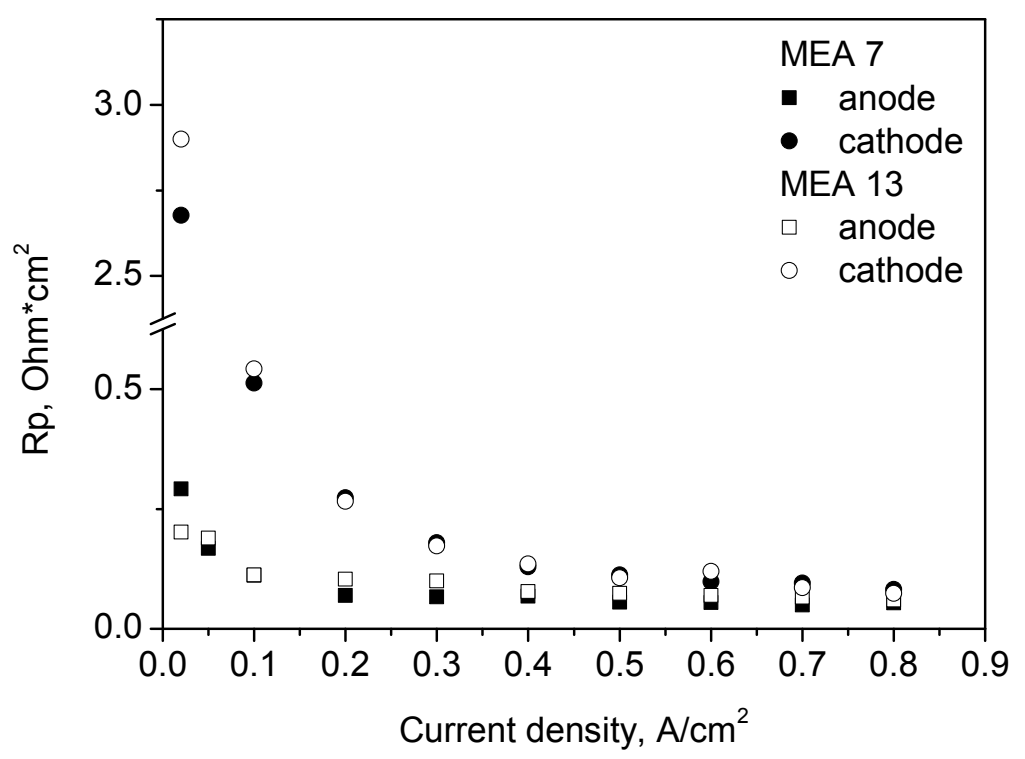

Figure 5-14. Polarization resistance of the anodic and cathodic electrode as a function of the applied current. Both MEAs employed 30wt\%Pt/oxMWCNT-Py at the anodic electrode and $30 \mathrm{wt} \% \mathrm{Pt} / \mathrm{C}$ at the cathode (MEA 7 and MEA 13). 

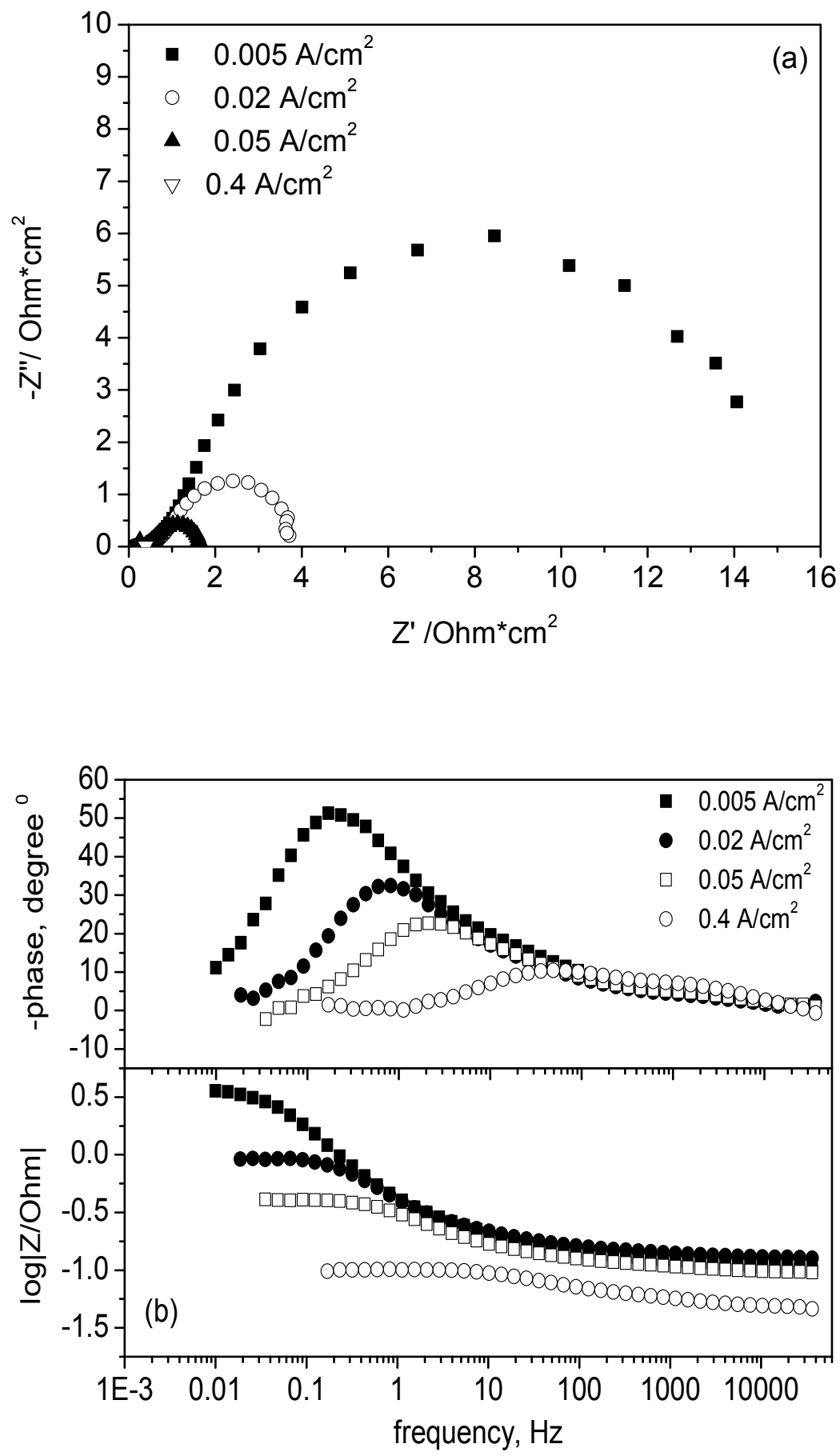

Figure 5-15. AC impedance spectra of MEA 13 obtained at different current densities at $180^{\circ} \mathrm{C}$, under constant flow $200 \mathrm{cc} / \mathrm{min}$ of $\mathrm{H}_{2}$. The cathodic electrode was fed with a redundant $\mathrm{O}_{2}$ flow $(200 \mathrm{cc} / \mathrm{min})$. a. Nyquist plots and b. Bode plots 

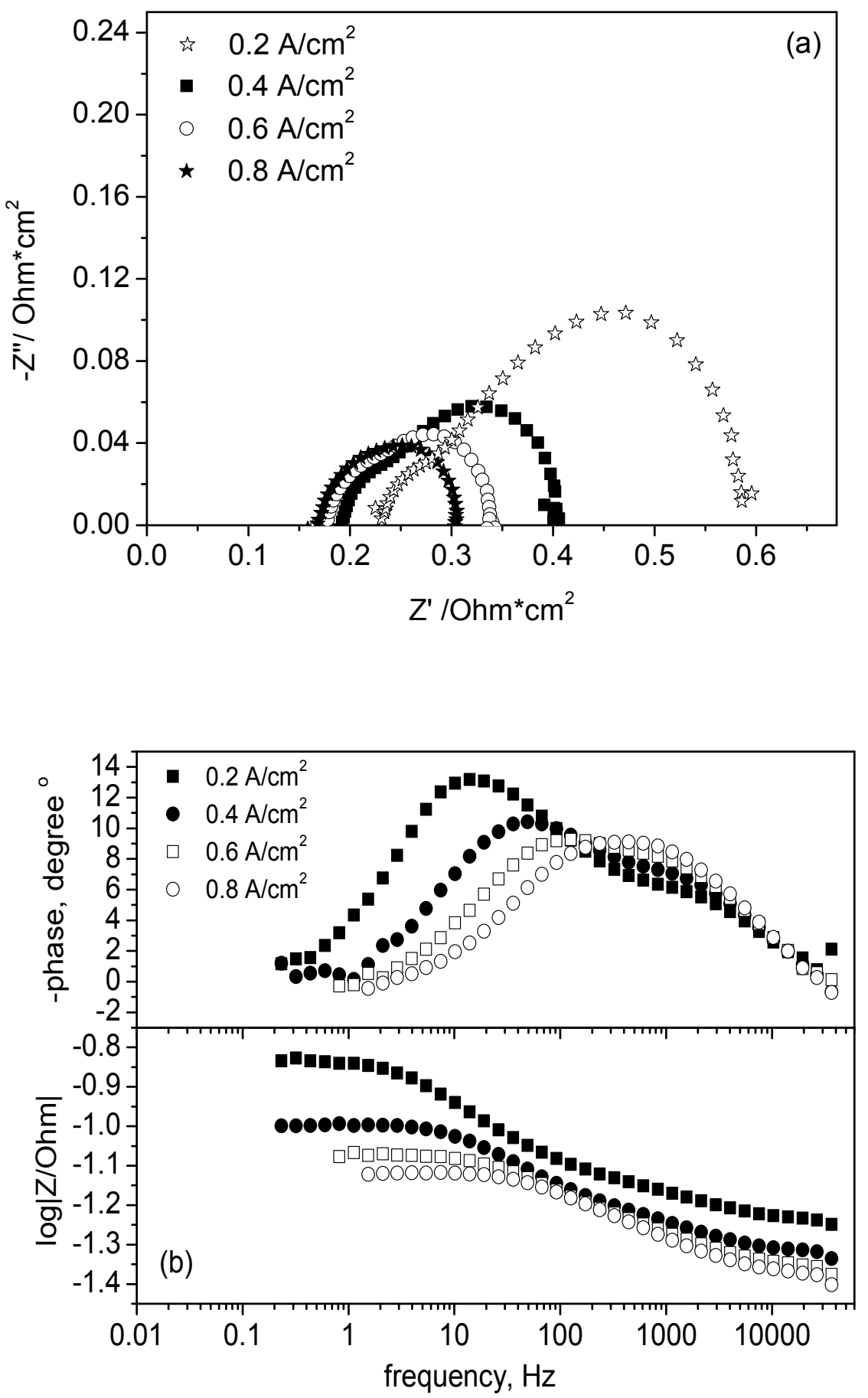

Figure 5-16. AC impedance spectra of MEA13 obtained at different current densities at $180^{\circ} \mathrm{C}$, under constant flow $200 \mathrm{cc} / \mathrm{min}$ of $\mathrm{H}_{2}$ and $\mathrm{O}_{2}$, respectively. Nyquist plots and $\mathrm{b}$. Bode plots 
This can be explained if we take into consideration the oxidative state of the Pt surface at the cathodic electrode. In the low current region, the ORR is controlled by pure electron transfer, where the catalyst surface is partially covered by PtO. However, the ORR in the high current region, is controlled by electron transfer and mass transfer, where the catalyst surface is mainly reduced Pt [37]. The HOR occurs on pure Pt, for all current densities. The dependence of the polarization resistance of the anodic and cathodic electrode with the applied current density has been reported in the literature for PBI based systems under similar conditions, exhibiting the same phenomena [38,39].

\subsubsection{Durability of electrocatalyst after short term operation}

\subsubsection{XPS measurements}

Pyridine groups are an essential part of the newly synthesized catalyst, as they interact with the free phosphoric acid, homogeneously distributing it in the catalyst layer. Although thermogravimetric analysis showed that the pyridine groups are thermally stable below $200^{\circ} \mathrm{C}$ [40], due to the harsh operating conditions of a HT-PEM fuel cell it was necessary to investigate the stability of the pyridine groups after various operation periods. For that reason, MEAs were formulated with the same specifications. The anodic electrode, which employed 30\%Pt/oxMWCNT-Py, had a Pt loading of approximately $1 \mathrm{mgPt} / \mathrm{cm}^{2}$ and an acid loading of $4 \mathrm{gPA} / \mathrm{gPt}$.

Table 5-5. N/Pt atomic ratio after certain operation time of the cathodic electrode of an MEA employing 30\% Pt/(ox.MWCNT)-Py.

\begin{tabular}{cc}
\hline Operations Conditions & $\mathrm{N} / \mathrm{Pt}$ atomic ratio \\
\hline Oven at $180^{\circ} \mathrm{C}$ & 0.444 \\
OCV for $24 \mathrm{~h}$ & 0.165 \\
$0.2 \mathrm{~A} / \mathrm{cm}^{2}$ for $24 \mathrm{~h}$ & 0.244 \\
$0.2 \mathrm{~A} / \mathrm{cm}^{2}$ for $50 \mathrm{~h}$ & 0.360 \\
$0.2 \mathrm{~A} / \mathrm{cm}^{2}$ for 8 days & 0.151 \\
\hline
\end{tabular}

The MEAs were left at $180^{\circ} \mathrm{C}$ to operate for various periods (24 hours to 8 days) at a constant current density of $0.2 \mathrm{~A} / \mathrm{cm}^{2}$ using pure $\mathrm{H}_{2}$ and $\mathrm{O}_{2}$ (gas stoichiometry $\lambda_{\mathrm{H} 2}=1.2$ and $\lambda_{\mathrm{O} 2}=2$ ). Moreover, an amount of the catalyst powder was placed in a furnace at $180^{\circ} \mathrm{C}$ and left under $\mathrm{H}_{2}$ atmosphere for almost 24 hours and an MEA was left at OCP conditions at 
$180^{\circ} \mathrm{C}$ for $24 \mathrm{~h}$ (both served as reference sample). Thereafter, the MWCNT based catalyst were characterized using X-ray photoelectron spectroscopy in order to determine and verify the functionalization degree of the carbon nanotube based catalyst.

Table 5-5 depicts the results of the XPS characterization. Due to the structure of the electrode, after fuel cell operation, it is very difficult to scratch off the catalytic layer without any trace of the gas diffusion layer. Therefore, the functionalization degree of the carbon nanotube could not be estimated through the atomic ratio of N/C. For that reason it was considered more appropriate to use the atomic ratio of N/Pt. Also due to the different degree of Pt sintering, which will be thoroughly discussed in the next section, as a result of the different operation times and conditions, and the surface sensitivity of the XPS, only estimation can be made about the amount of pyridine group on the outer wall of the MWNT. From the results shown in table 5-5, it was concluded that the pyridine groups are stable after short term operation.

\subsubsection{The effect of short term operation of the Pt particle size}

The reduction of the electrochemical active surface area during normal fuel cell operation is attributed to several factors, such as Pt sintering/agglomeration or Pt migration and loss. In order to investigate the effect of reducing atmosphere and operation time in the ECSA diminution of the newly synthesized electrocatalyst, used membrane electrode assemblies were thoroughly analyzed using transmission electron microscopy (TEM) and X-ray diffraction (XRD). The electrodes were detached from the membrane in order to investigate the Pt particle size after various operation times of the anodic electrodes by means of XRD analysis. TEM postmortem analysis was also performed on the same samples to investigate the Pt size distribution and morphology of the electrocatalyst. Adequate amount of catalysts were scrapped off the used electrodes and were ultrasonically dispersed in water and placed a drop of the suspension on a $3 \mathrm{~mm}$ carbon coated copper grids (Electron Microscopy Sciences). The Pt particle size distributions were obtained by manually measuring all the particles from the TEM pictures. The distribution and the average Pt particle size were calculated as thoroughly described in the experimental section.

As mentioned earlier, the Pt particles undergo an increase in their diameter, depending on the operation conditions and time. The relatively low temperature $\left(180^{\circ} \mathrm{C}\right)$ and the reducing atmosphere leading to the sintering of the platinum particles, points out some questions about the behavior of the electrocatalysts under Fuel Cell working conditions. The same MEAs as the one mentioned in section 5.3.6.1, were used for the determination of the $\mathrm{Pt}$ particle size at the anodic electrode with respect to operation time $\left(1 \mathrm{mgPt} / \mathrm{cm}^{2}\right.$ and $4 \mathrm{gPA} / \mathrm{gPt}$ ). The as synthesized 30\%Pt/oxMWNT-Py electrocatalyst has an average Pt 
particle size of $3.2 \mathrm{~nm}$ as measured by TEM imaging. The same experiment was conducted for MEAs employing 30wt $\% \mathrm{Pt} / \mathrm{C}$ for comparison reason. The as received $30 \mathrm{wt} \% \mathrm{Pt} / \mathrm{C}$ electrocatalyst has an initial average Pt particle size of $2.9 \mathrm{~nm}$ as measure by TEM.

Table 5-6. Pt particle size average diameter $(\mathrm{nm})$ with respect to operation time as estimated via TEM imaging, of 30wt\%Pt/C and 30wt\%Pt/oxMWCNT-Py.

\begin{tabular}{ccc}
\hline $\begin{array}{c}\text { Operation } \\
\text { Conditions }\end{array}$ & \multicolumn{2}{c}{ Pt particle size (nm) } \\
& 30wt\%Pt/C & 30wt\%Pt/oxMWCNT-Py \\
\hline 24 OCV & N.A & 4.8 \\
24 hours & 4.0 & 5.4 \\
50 hours & 4.1 & 5.6 \\
6 days & 5.1 & 5.75 \\
\hline
\end{tabular}

As shown in table 5-6, as the operation time increases, the growth of the Pt particles in the anodic electrode slows down. After only 50 hours of fuel cell operation the Pt particle size increases from to $3.2 \mathrm{~nm}$ and reaches $5.6 \mathrm{~nm}$, for the case of $30 \mathrm{wt} \% \mathrm{Pt} / \mathrm{oxMWCNT}-\mathrm{Py}$, whereas after 6 days of operation in reaches $5.75 \mathrm{~nm}$. During the first operation hours sintering of the smaller crystallites are expected, whereas as the operation time increases, sintering of bigger particles is likely involved and thus the sintering rate slows down. From the data obtained for different operation times it is clear that the mean Pt particle growth on the anode side occurs during the first hours of cell operation for both electrocatalyst. It has to be mentioned that the $30 \mathrm{wt} \% \mathrm{Pt} / \mathrm{C}$ is more sintering resistant than the carbon nanotube supported electrocatalyst.

TEM micrographs were obtained for the examination of the morphology of the catalyst after various operation time of the anodic electrodes. Figure 5-17 shows the obtained TEM migrographs and their corresponding Pt size distribution histograms for the case of 6 days of continuous operation for both electrocatalyst. It is clear that the Pt distribution is wider for the case of the carbon nanotube based catalyst. However both exhibit log-normal distribution, indicating the same Pt growth mechanism, which is thoroughly discussed in the next section.

The sintering process of both commercial and functionalized MWCNTs based catalysts was investigated ex-situ to clarify the effect of the reducing atmosphere. Both electrocatalyst were placed in an oven in a XRD apparatus. Initially a spectum was acquired at $25^{\circ} \mathrm{C}$ under $\mathrm{H}_{2}$ atmosphere and served as a benchmark. XRD diffractograms were then obtained at $180^{\circ} \mathrm{C}$ under constant $5 \% \mathrm{H} 2 /$ Ar flow with $1 \mathrm{~h}$ step and 20 min acquisition time at each step. X-ray diffraction measurements as a function time showed that sintering of

144

Department of Chemical Engineering, University of Patras 
the platinum crystallites takes place during the 1-2hours of $\mathrm{H}_{2}$ exposure and remains unchanged after even after 20 hours, figure 5-18.
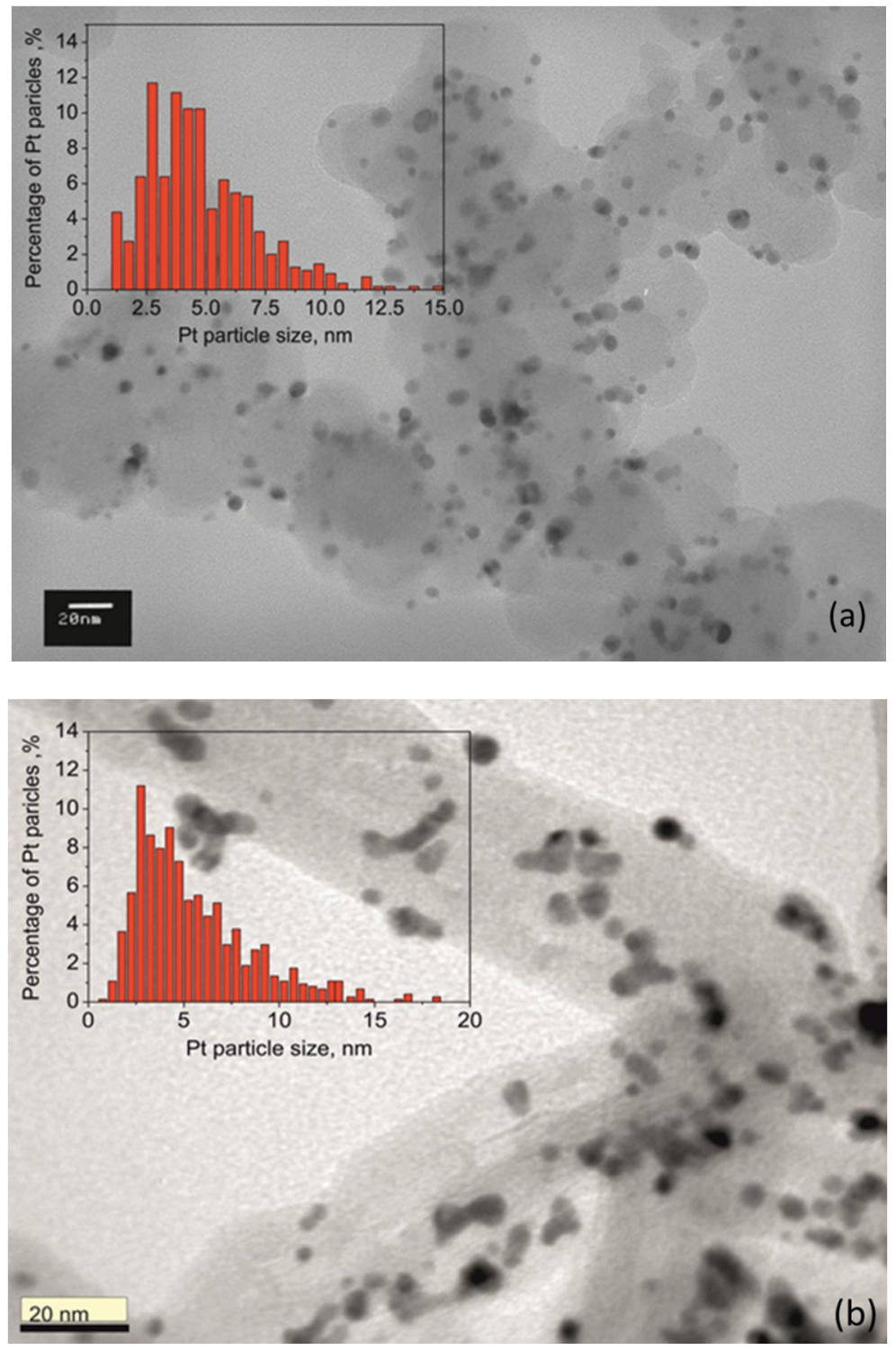

Figure 5-17 : TEM images and the corresponding Pt size distribution histogram after 6 days of continuous operation. a. 30wt\%Pgt/C and b. 30\%Pt/oxMWNT-Py

Due to the low operation temperature of $180^{\circ} \mathrm{C}$, probably sintering of the more energetic smaller particles takes place, however sintering of larger crystallites can also occur in this temperature region [41]. The final $\mathrm{Pt}$ particle size for both electrocatalysts as calculated from Scherrer's equation, is slightly smaller compared to the case when the catalysts where exposed in $\mathrm{H}_{2}$ during normal fuel cell operation. This result indicates that the presence of PA is not such an important factor in the sintering/agglomeration process during the first 
operation hours of a fuel cell. A full discussion concerning the latter is presented in the following section.

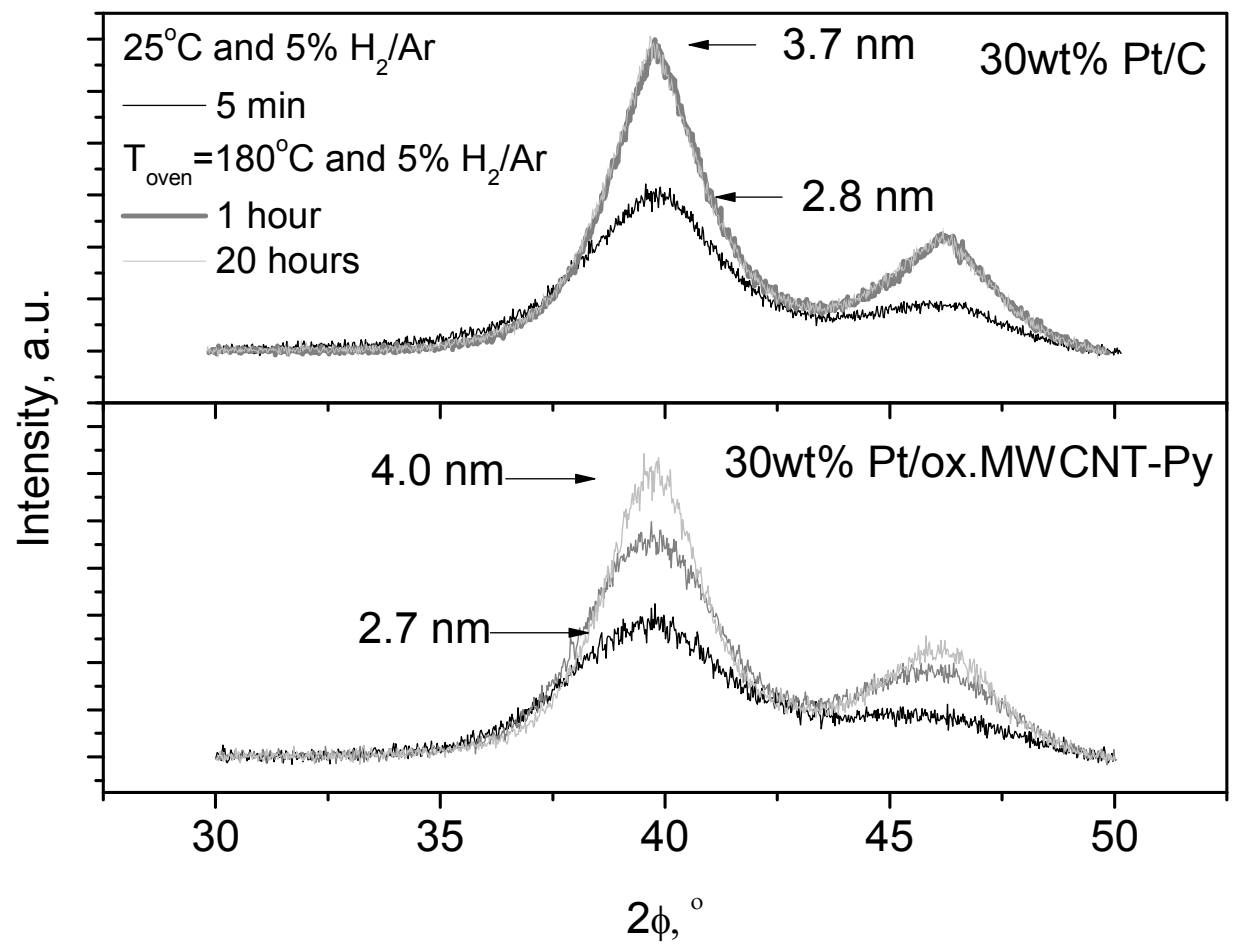

Figure 5-18 . The effect of reducing atmosphere 5\% $\mathrm{H} 2 / \mathrm{Ar}$ on the $\mathrm{Pt}$ particle size as a function of time. The XRD diffractograms of 30wt $\% \mathrm{Pt} / \mathrm{C}$ and 30wt\% Pt/oxMWCNT-Py were acquired at $180^{\circ} \mathrm{C}$, with $20 \mathrm{~min}$ acquisition time.

\subsubsection{Effect of PA on the Pt particle size during normal fuel cell operation}

The effect of the PA amount present in the catalyst layer towards the Pt sintering process after short term operation was also investigated for the anodic electrode. The average Pt particle size was measured by TEM and XRD. The PA amount in the catalyst layer varied from 1.4 to $17.6 \mathrm{gPA} / \mathrm{gPt}$ (MEA 7-14). The MEAs operated at $180^{\circ} \mathrm{C}$ under constant operation conditions for 8 days. From the results depicted in figure $5-19$, is that a fast sintering is observed at low PA loadings up to $3 \mathrm{grPA} / \mathrm{grPt}$ while thereafter the sintering procedure is lower. The amount of phosphoric acid seams to plays a major role in the $\mathrm{Pt}$ sintering/agglomeration of the nanoparticles for values as high as $17.6 \mathrm{gPA} / \mathrm{gPt}$. Both TEM and XRD results are in perfect agreement. 


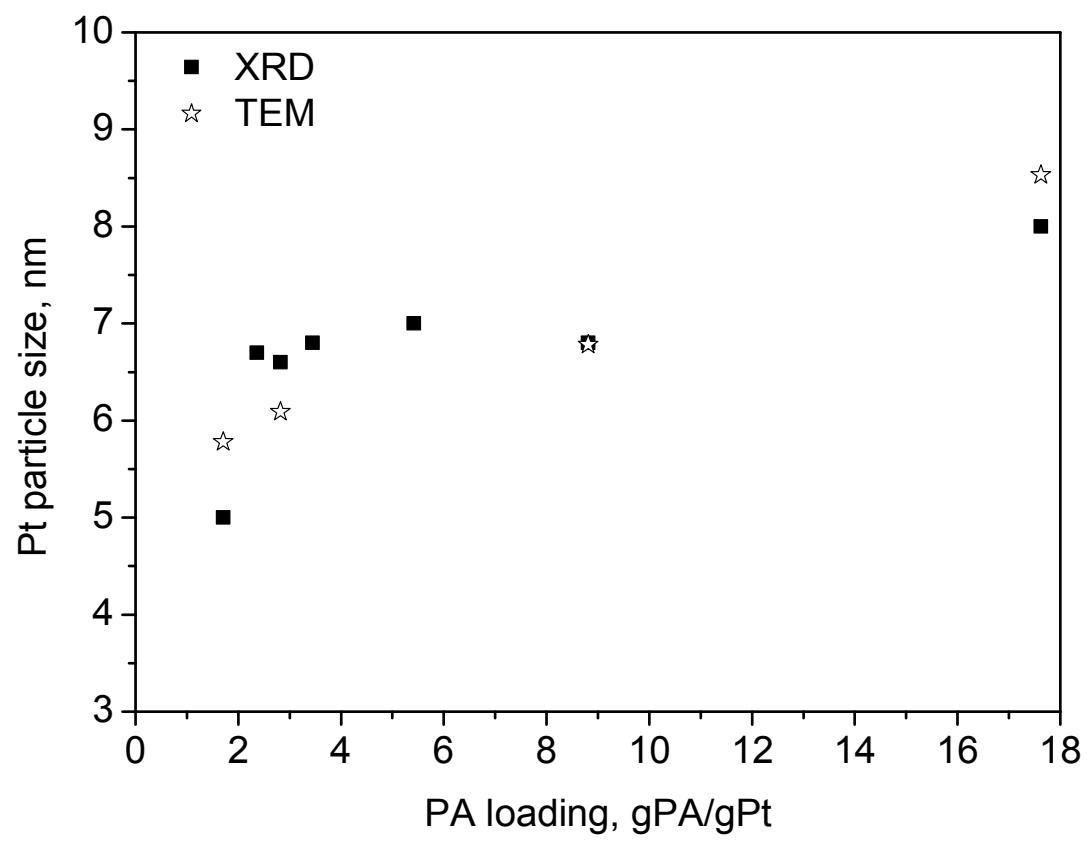

Figure 5-19. The effect of PA on the Pt particle size after 8 days of continuous operation. The average Pt particle size was measured by TEM and XRD.

TEM micrographs were obtained for the examination of the morphology of the catalyst with respect to PA loading in the catalyst layer, after 8 days of continuous operation. A significant sintering on the anode catalyst was observed for the ratio of 17.6 (figure 5- 20a and 5-21a), as compared to the lower ratio 2.8gPA/gPt (figure 5-20b and 5-21b). Migration of the Pt metal particles from the surface of the nanotube towards neighboring particles was observed and as a consequence particle size growth occurred. The higher the amount of PA, the average Pt particle size increases and the Pt size distribution became wider (as shown in the histograms, figure 5-21a), as the agglomeration and sintering process were enhanced. It was also observed that when the content of PA in the catalyst layer increased, the number of $\mathrm{Pt}$ particles that were detached from the substrate and were found unsupported on the TEM grid increased as well. This could be attributed due to corrosion of the outer wall of the carbon nanotube, leading to the detachment of Pt particles from the nanotube surface. 

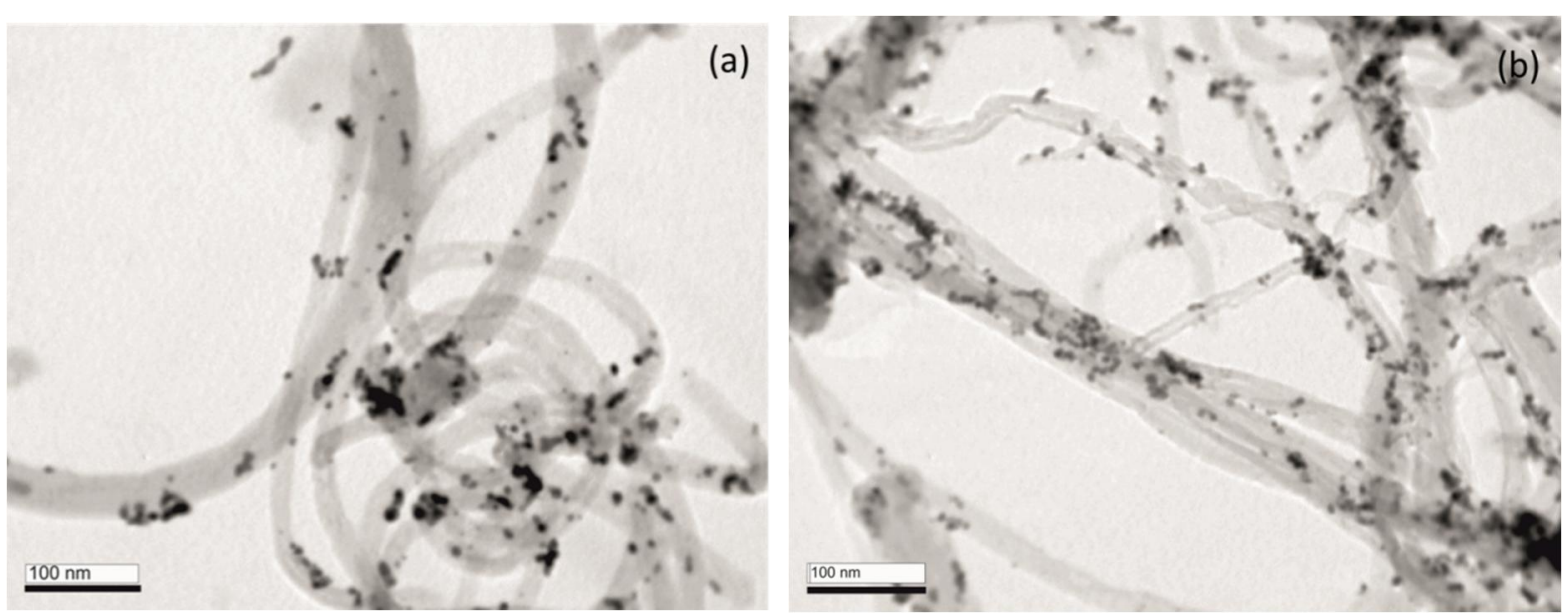

Figure 5-20. TEM images after 8 days of continuous operation. a. MEA 11: 0.2mgPt/ $\mathrm{cm}^{2}$ and $17.6 \mathrm{mgPt} / \mathrm{cm}^{2}$ and b. MEA 7: $1.25 \mathrm{mgPt} / \mathrm{cm}^{2}$ and $2.8 \mathrm{gPA} / \mathrm{gPt}$
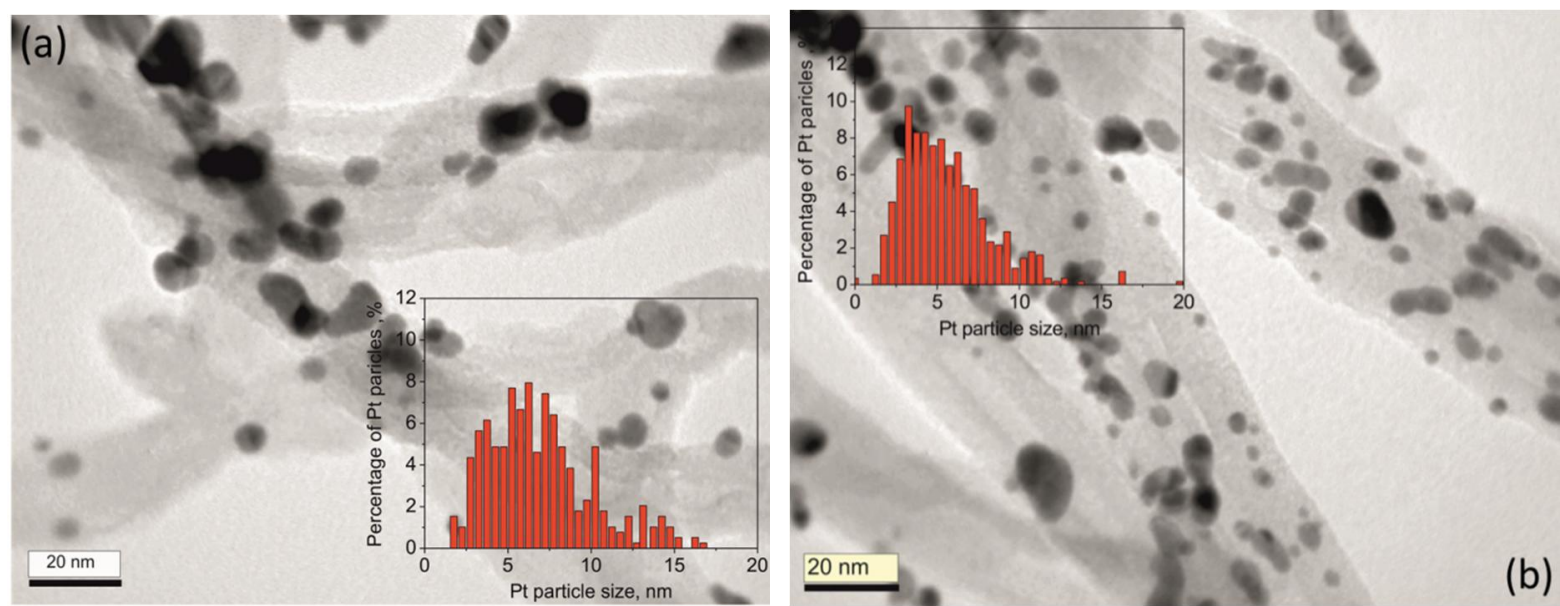

Figure 5-21. TEM micrographs and the corresponding Pt size distribution histogram after 8 days of continuous operation. a. MEA 11: $0.2 \mathrm{mgPt} / \mathrm{cm}^{2}$ and $17.6 \mathrm{mgPt} / \mathrm{cm}^{2}$ and $\mathrm{b}$. MEA 7: $1.25 \mathrm{mgPt} / \mathrm{cm}^{2}$ and $2.8 \mathrm{gPA} / \mathrm{gPt}$

\subsubsection{Agglomeration mechanism of Pt particles on the ox.MWCNT-Py substrate}

In principle particle growth on a substrate can occur through two different mechanism, the coalescence/sintering growth and the Ostwald ripening process [42]. In the first case, whole islands are moving over the substrate's surface, leading to cluster-cluster collisions accompanied by liquid-like coalescence of the nanoparticles. In the case of Ostwald ripening, atoms are migrating from small crystals to larger ones. The driving force for this particle growth process is the minimization of the clusters Gibbs free energy or a corrosion 
type mechanism due to nonuniform voltage distribution along the electrochemical interface within the catalytic layer.

It has been reported in the literature that the shape of the size distribution can be used to determine which particle growth mechanism is more dominant [43]. Ascarelli et al. discussed that their method for distinguishing those two growth mechanism, could also be used for Pt /C electrocatalyst with different size distributions in HTPEM fuel cells. In the case of a coalescence process, the particle size distribution is negligible below a certain finite size, has a maximum on the small particle size and a tail towards the larger particle size. Whereas, for the Ostwald ripening process, the particle size distribution is considered negligible above a certain finite size, has a maximum on the large particle sizes and a tail toward the small particle sizes.

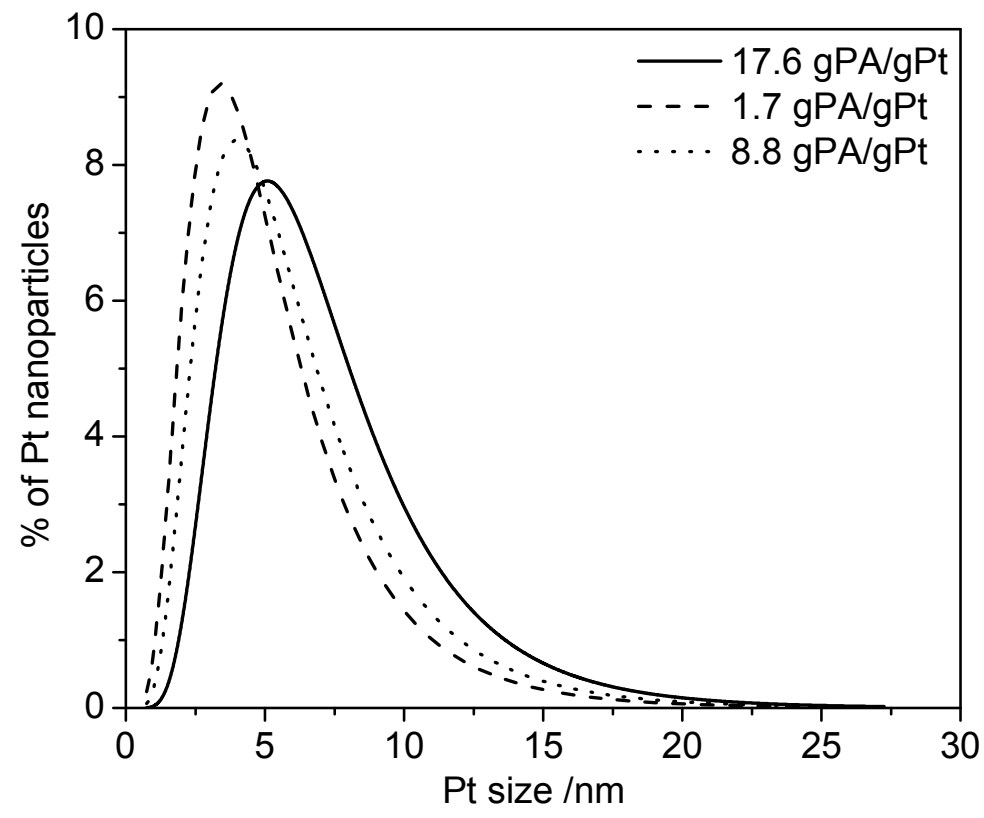

Figure 5-22. The effect of the PA amount present in the anodic electrode on Pt size distribution of $30 \mathrm{wt} \% \mathrm{Pt} / \mathrm{oxMWNT}-\mathrm{Py}$, after 8 days of continuous fuel cell operation.

Since the PA amount, as discussed in the above section, influence the Pt growth, we wanted to investigate whether the PA amount influenced also the growth mechanism. In figure 521 , the Pt particle size distribution is plotted with respect to the different amount of PA that was sprayed in the electrocatalytic layer. MATLAB was used for the fitting of the data obtained from the TEM histograms. Before the use of the electrocatalyst in a fuel cell, the 
Pt size distribution is a typical normal distribution. After 8 days of continuous operation, regardless the PA amount in the electrodes, all the Pt size distributions shows a typical lognormal distribution. As shown in figure 5-22, the distribution is negligible below a certain finite size, the maximum appears on the small particle size and the tail is towards the larger particle sizes. Increasing the PA amount, lead to an increase in the maximum position and to a longer tail towards the larger Pt sizes. Therefore, regardless the amount of PA in the anodic catalyst layer, the Pt growth mechanism is via coalescence.

\subsection{Conclusions}

The catalyst efficiency largely depends on the catalyst morphology and its interface with the proton conductor, thus this study opens new roads towards the creation of high efficiency catalyst layers employing very low Pt loadings. The newly synthesized electrocatalyst 30wt\% Pt/oxMWCNT-Py was evaluated as an anodic electrocatalyst in HTPEM fuel cell. The presence of pyridine groups was found to homogeneously distribute PA in the catalyst layer, resulting in high ECSA values, $40 \mathrm{~m}^{2} / \mathrm{gPt}$. As a result the MEA employing 30wt $\%$ Pt oxMWCNT-Py showed the same performance as the $30 \% \mathrm{Pt} / \mathrm{C}$ (having $1.3 \mathrm{mgPt} / \mathrm{cm}^{2}$ ), for Pt loading loadings as low as $0.2 \mathrm{mgPt} / \mathrm{cm}^{2}$. The performance of the anodic electrode was also found to be largely depended on the PA amount imbedded in the CL, when low Pt loading were used. Despite the hard operating conditions the Pt particles attached to the ox.MWCNT-Py substrate exhibit similar stability as the commercial catalyst and the pyridine groups were found to be stable as well, at least for short term operation. 


\section{$\underline{\text { References }}$}

[1] J.M. Song, S. Suzuki, H. Uchida, M. Watanabe, Preparation of High Catalyst Utilization Electrodes for Polymer Electrolyte Fuel Cells, Langmuir 22 (2006) 6422

[2] Z. Xu, Z. Qi, A. Kaufman, Superior Catalysts for Proton Exchange Membrane Fuel Cells: Sulfonation of Carbon-Supported Catalysts Using Sulfate Salts, J Electrochem. Solid-State Lett. 8 (2005) A313

[3] H. Kim, W. Lee, D. Yoo, Functionalized carbon support with sulfonated polymer for direct methanol fuel cells, J Electrochim. Acta 52 (2007) 2620

[4] C.Y. Du, T.S. Zhao, Z.X. Liang, Sulfonation of carbon-nanotube supported platinum catalysts for polymer electrolyte fuel cells, J. Power Sources 176 (2008) 9

[5] Li Q, Jensen JO, Pan C, Bandur V, Nilsson MS, Schonberger F, et al., Partially fluorinated aarylene polyethers and their ternary blends with PBI and H3PO4. Part II. Characterisation and fuel cell test of the ternary membranes. Fuel Cells 3-4 (2008) 188

[6] Kim J-H, Kim H-J, Lim H-T., Dependence of the performance of a high-temperature polymer electrolyte fuel cell on phosphoric acid-doped polybenzimidazole ionomer content in cathode catalyst layer, J Power Sources 170 (2007) 275

[7] Pan C, Li Q, Jensen JO, He R, Cleemann LN, Nilsson MS,Preparation and operation of gas diffusion electrodes for high-temperature proton exchange membrane fuel cells, J Power Sources 172 (2007) 278

[8] Hyung-Suk Oh, Kwanghyun Kim, Hansung Kim, Polypyrrole-modified hydrophobic carbon nanotubes as promising electrocatalyst supports in polymer electrolyte membrane fuel cells, International journal of Hydrogen energy 36 (2011) 11564

[9] Kazuya Matsumoto,Tsuyohiko Fujigaya, Kazunari Sasaki and Naotoshi Nakashima, Bottomup design of carbon nanotube-based electrocatalysts and their application in high temperature operating polymer electrolyte fuel cells, J. Mater. Chem. 21 (2011) 1187

[10] Justo Lobato, Pablo Canizares, Manuel A. Rodrigo, Jose J. Linares, F. Javier Pinar, Study of the influence of the amount of PBI-H3PO4 in the catalytic layer of a high temperature PEMFC, International journal of Hydrogen energy 35 (2010) 1347

[11] F. Seland, T. Berning, B. Børresen, R. Tunold, Improving the performance of high-temperature PEM fuel cells based on PBI electrolyte, Journal of Power Sources 160 (2006) 27

[12] A. Orfanidi, M.Daletou, S.G. Neophytides, Preparation and characterization of Pt on modified multi-wall carbon nanotubes to be used as electrocatalysts for high temperature fuel cell applications, J Applied Catalysis B: Environmental 106 (2011) 379 
[13] Jesper Lebæk Jespersen, Erik Schaltz, Søren Knudsen Kær, Electrochemical characterization of a polybenzimidazole-based high temperature proton exchange membrane unit cell, Journal of Power Sources 191 (2009) 289

[14] Andreasen SJ, Jespersen JL, Schaltz E, Kær SK., Characterisation and modelling of a high temperature PEM fuel cell stack using electrochemical impedance spectroscopy, Fuel Cells 9 (2009) 463

[15] ZhuWH, Payne RU, Tatarchuk BJ. PEM stack test and analysis in a power system at operational load via AC impedance, Journal of Power Sources 168 (2007) 211

[16] M. Ciureanu, R. Roberge, Electrochemical Impedance Study of PEM Fuel Cells. Experimental Diagnostics and Modeling of Air Cathodes, J. Phys. Chem. B , 105 (2001) 3531

[17] I. A. Schneider, S. A. Freunberger, D. Kramer, A. Wokaun, and G. G. Scherer, Oscillations in Gas Channels Part I. The Forgotten Player in Impedance Spectroscopy in PEFCs, Journal of the Electrochemical Society, 154 (2007) B383

[18] M.K. Daletou, M. Geormezi, E. Vogli, G.A. Voyiatzis, S.G. Neophytides, The interaction of H3PO4 and steam with PBI and TPS polymeric membranes. A TGA and Raman study, J. Mater. Chem. A, 2 (2014) 1117.

[19] M. K. Daletou, J. K. Kallitsis, G. Voyiatzis, S. G. Neophytides, The interaction of water vapors with H3PO4 imbibed electrolyte based on PBI/polysulfone copolymer blends, J. Membr. Sci. 326 (2009) 76.

[20] Y.L.Ma, $\mathrm{PhD}$ thesis, The fundamental studies of polybenzimidazole/phosphoric acid polymer electrolyte for fuel cells, CaseWestern Reserve University, Cleveland, 2004

[21] M. Mamlouk, K. Scott , Analysis of high temperature polymer electrolyte membrane fuel cell electrodes using electrochemical impedance spectroscopy, Electrochimica Acta 56 (2011) 5493

[22] K.Wippermann, C.Wannek, H.-F. Oetjen, J. Mergel,W. Lehnert, Cell resistances of poly(2,5benzimidazole)-based high temperature polymer membrane fuel cell membrane electrode assemblies: Time dependence and influence of operating parameters, Journal of Power Sources 195 (2010) 2806

[23] Yunfeng Zhai, Huamin Zhang, Danmin Xing, Zhi-Gang Shao, The stability of Pt/C catalyst in H3PO4/PBI PEMFC during high temperature life test, Journal of Power Sources, 164 (2007) 126

[24] R.L. Borup, J.R. Davey, F.H. Garzon, D.L. Wood, M.A. Inbody, PEM fuel cell electrocatalyst durability measurements, J. Power Sources, 163 (2006) 76

[25] T. Yoda, H. Uchida, M.Watanabe, Effects of operating potential and temperature on degradation of electrocatalyst layer for PEFCs, Electrochim. Acta 52 (2007) 5997 
[26] Shengsheng Zhang, Xiao-Zi Yuan, Jason Ng Cheng Hin, Haijiang Wang, K. Andreas

Friedrich, Mathias Schulze, A review of platinum-based catalyst layer degradation in proton exchange membrane fuel cells, Journal of Power Sources 194 (2009) 588

[27] Weimin Chen, Qin Xin, Gongquan Sun, Qi Wang, Qing Mao, Huidong Su, The effect of carbon support treatment on the stability of Pt/C electrocatalysts Journal of Power Sources 180 (2008) 199

[28] C. Prado-Burguete, A. Linares-Solano, F. Rodríguez-Reinoso, C.Salinas-Martínez de Lecea, The effect of oxygen surface groups of the support on platinum dispersion in Pt/carbon catalysts, Journal of Catalysis, 115 (1989) 98

[29] M. Roman-Martinez, D. Cazorla-Amoros, A. Linares-Solano, C. Lecea, Metal-support interaction in $\mathrm{Pt} / \mathrm{C}$ catalysts. Influence of the support surface chemistry and the metal precursor, Carbon 33 (1995) 3

[30] P. Ehrburger, O. Majahan, P. Walker, Carbon as a support for catalysts: I. Effect of surface heterogeneity of carbon on dispersion of platinum, J. Catal. 43 (1976) 61.

[31] A. Guerriero-Ruiz, P. Badenes, I. Rodriguez-Ramos, Study of some factors affecting the Ru and Pt dispersions over high surface area graphite-supported catalysts, Appl. Catal. A 173 (1998) 313.

[32] J.P. Boudou, Surface chemistry of a viscose-based activated carbon cloth modified by treatment with ammonia and steam, Carbon 41 (2003) 1955-1963

[33] Bayazit, MK ; Clarke, LS ; Coleman, KS ; Clarke, N, Pyridine-Functionalized Single-Walled Carbon Nanotubes as Gelators for Poly(acrylic acid) Hydrogels, J. Am Chem. Soc. 132 (2010) 15814

[34] E. T. Kang et al, Molecular Physics, 1990, VOL. 70, No. 6, 1057-1064

[35] J. O’M. Bockris, A.K.N. Reddy, M. Gamboa-Aldeco, Modern Electrochemistry, Vol. 2A, Fundamentals of electrodics, Kluwer Academic/Plenum Publishers, New York ISBN: 0-30646166-8 Z, 2nd ed., (2000), 1166.

[36] P.M. Quaino, J.L. Fernández, M.R. Gennero de Chialvo, A.C. Chialvo, Hydrogen oxidation reaction on microelectrodes: Analysis of the contribution of the kinetic routes, J. Mol. Catal. A: Chem. 252 (2006) 156.

[37] Chaojie Song, Yanghua Tang, Jian Lu Zhang, Jiujun Zhang, Haijiang Wang, Jun Shen, Scott McDermid, Jing Li , Paul Kozak, PEM fuel cell reaction kinetics in the temperature range of 23$120{ }^{\circ}$ C, Electrochimica Acta 52 (2007) 2552

[38] J. Zhang, Y. Tang, C. Song, J. Zhang, Polybenzimidazole-membrane-based PEM fuel cell in the temperature range of $120-200{ }^{\circ} \mathrm{C}$, J. Power Sources 172 (2007) 163 
[39] Chen-Yu Chen,Wei-Hsiang Lai, Effects of temperature and humidity on the cell performance and resistance of a phosphoric acid doped polybenzimidazole fuel cell, Journal of Power Sources 195 (2010) 7152

[40] Alin Orfanidi, Master thesis, Synthesis and characterization of Pt /modified MWNT as electrocatalysts for application in high temperature PEMFCs', University of Patras, http://nemertes.lis.upatras.gr/jspui/bitstream/10889/5072/3/Nimertis_Orfanidi

[41] Influence of surfactant removal by chemical or thermal methods on structure and electroactivity of $\mathrm{Pt} / \mathrm{C}$ catalysts prepared by water-in-oil microemulsion, S. Brimaud, C. Coutanceau, E. Garnier, J.-M. Leger ,F. Gerard, S. Pronier, M. Leoni, Journal of Electroanalytical Chemistry 602 (2007) 226

[42] C.G. Granqvist, R.A. Buhrman, Size distributions for supported metal catalysts: Coalescence growth versus ostwald ripening, Journal of Catalysis 42(1976) 477

[43] P.Ascarelli, Formation process of nanocrystalline materials from x-ray diffraction profile analysis: Application to platinum catalysts, J. Appl. Phys. 91 (2002) 4556 


\section{Chapter 6}

\section{The effect of $\mathrm{H}_{3} \mathrm{PO}_{4}$ loading and the electrocatalyst's substrate on the anode's performance under different reformate compositions}

\subsection{Introduction}

The highest performance of a fuel cell is achieved when pure hydrogen is employed as fuel, however the production of pure $\mathrm{H}_{2}$ gas presents some difficulties, such as high production cost. The most common and cost effective route for production of hydrogen is through steam reforming of hydrocarbons and water gas shift reactions for the production of the so called reformate gas. These methods produce carbon monoxide as a byproduct, the concentration of which has to be dramatically reduced in order to be suitable for PEM fuel cell applications. Typically, the composition of reformate gas is a mixture of approximately $50-60 \% \mathrm{H}_{2}, 1-2 \% \mathrm{CO}, 10-15 \% \mathrm{CO}_{2}$ and $20-33 \% \mathrm{H}_{2} \mathrm{O}$.

CO severely poisons platinum catalysts as it binds strongly on the catalyst sites, reducing the active sites available for hydrogen adsorption and oxidation [1,2].The proposed models of Pt-CO bonding include either linear bond between the carbon atom of $\mathrm{CO}$ and a single Pt active site or bridge bonding where the carbon atom of the $\mathrm{CO}$ bonds with two adjacent $\mathrm{Pt}$ active sites $[3,4]$. Desorption of carbon monoxide from the Pt sites can be easily achieved via oxidation. In order to oxidize electrochemically $\mathrm{CO}$ to $\mathrm{CO}_{2}$, water is required, as from 
its dissociation the necessary oxygen atoms are obtained. The formation of oxygen containing species at the surface of Pt occurs at an anodic potential of $0.5 \mathrm{~V}$ vs RHE. Since,

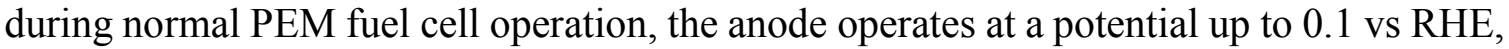
the inert $\mathrm{CO}$ electrooxidation is not favored. The performance losses in PEM fuel cells due to $\mathrm{CO}$ poisoning are strongly related to the concentration of $\mathrm{CO}$ in the fuel, the exposure time to $\mathrm{CO}$, the cell operation temperature and the anode catalyst type [5].

Many hydrogen purification techniques have been developed. CO removal increases the complexity the stability and the cost of the system's balance of plant, which among others comprises the fuel processor and the fuel cell stack. In order to minimize $\mathrm{H}_{2}$ purification cost, and increase CO tolerance in PEM fuel cells, several approaches have been proposed. The development of CO tolerant electrocatalyst has been one of them. Many studies demonstrated that the use of Pt based alloys [6,7,8], instead of pure Pt, exhibited higher $\mathrm{CO}$ tolerance [9]. The most promising, which have attracted a lot of scientific interest is Pt-Ru electrocatalyst [10,11]. An alternative to Pt based alloys, is the operation of fuel cells at elevated temperatures. New polymer electrolyte membranes have been developed capable of operating at temperatures as high as $200^{\circ} \mathrm{C}$. At those temperatures, the $\mathrm{CO}$ tolerance, increases significantly up to $30,000 \mathrm{ppm}$, without any significant loss in the MEA performance $[12,13,14]$.

Despite the fact that PA imbibed HT PEM fuel cells are highly tolerant towards CO poisoning, under certain reformate conditions the performance of the anode is severely affected. This negative effect appears in relation to the structure of the electrochemical interface, as this is specified by the amount of PA on the electrode, when high partial pressure of water is present in the reformate gas. It was concluded that a hydrophilic catalytic layer is highly preferred for the uniform distribution of PA on the electrocatalytic surface and its stability under humidified conditions.[15]

In continuance to the above mentioned work, this chapter focuses on the creation of an electrocatalytic layer that can operate in a HTPEM fuel cell using reformate gas consisting of high $\mathrm{CO}$ and steam mole fraction without sacrificing its performance. The selected electrocatalysts (30wt\% Pt/(ox.MWCNT)-Py and 30wt\% Pt/oxMWCNT) were formulated into Gas Diffusion Electrodes, GDEs. The fuel composition used in our experiments was similar with industrial reformate gas. A parametric analysis of each component that participates in the reformate gas composition and the effect of the different catalyst substrate, for its effect on the performance of High temperature PEM fuel cells is under investigation. The interaction of both $\mathrm{CO}$ and water with the catalyst surface, as well as the influence of PA and its distribution in the catalyst layer, appear to be the key in the optimization of the HTPEM system working under these harsh conditions. 


\subsection{Experimental}

\subsubsection{Materials}

Phosphoric acid doped polymer electrolyte membranes were provided from Advent Technologies S.A. The in-house synthesized 30wt\% Pt/(ox.MWCNT)-Py and 30wt $\%$ Pt/oxMWCNT catalyst used in this study was synthesized according to literature [16]. Catalyst powder $30 \mathrm{wt} \% \mathrm{Pt} / \mathrm{Vulcan} \mathrm{XC}-72 \mathrm{R}$ was purchased from Tanaka Kikinzoku International. For the construction of the electrodes, carbon cloth supporting layer was purchased from Fuel Cell Earth LLC, Vulcan XC-72R powder from RawChem Ltd and the PTFE dispersion in water from Sigma-Aldrich. 2-propanol 99.8 $\leq$, and $\mathrm{H}_{3} \mathrm{PO}_{4}$ purum p.a. $\geq 85 \%$ were purchased from Merck or Sigma Aldrich. All chemicals were used as received unless otherwise noted.

\subsubsection{Instrumentation}

The electrochemical measurements were performed using a single cell purchased from Fuel Cell Technologies Inc. Both graphite bipolar plates of the single cell had the same single serpentine flow field for the distribution of reacting gases. The active area of the electrodes was $2 \mathrm{~cm} \times 2 \mathrm{~cm}$. The assembling torque applied for the single cell was $4.8 \mathrm{Nm}$. The cell was installed in a fuel cell test station which was built in-house and had provisions for controlling temperature, humidification, and flow of reacting gases. The measurements were made in a two-electrode arrangement using an Autolab potentiostat-galvanostat PGSTAT-302. The gas composition of the various reaction mixtures including the reformate gas was set by several mass flow controllers (Bronkhost), by controlling the flow rate of $\mathrm{H}_{2}, \mathrm{CO}$ and Ar separately. The mole fraction of water was adjusted by means of a humidifying system (Fuel Cell Technology Inc). The outlet of the cell's anodic compartment was connected to an OMNISTAR Balzers quadrapole mass spectrometer for the transient and steady state monitoring of the composition of the outlet gas.

\subsubsection{Electrode Preparation and MEA assembly}

Electrode fabrication: The gas diffusion layer, GDL, used in this study was prepared by spraying a slurry made of carbon Vulcan XC-72R and PTFE solution (50wt \% PTFE/C) on carbon cloth, followed by heat treatment at $300^{\circ} \mathrm{C}$ under static air for $40 \mathrm{~min}$. The electrodes were prepared using two different optimized methods, depending on the catalyst type. Two types of electrocatalysts were used, Pt supported on carbon black (30wt \% Pt/C) and Pt supported on MWCNTs (30wt \%t Pt/(ox.MWNT)-Py and 30wt \% Pt/ox.MWCNT). 
The electrodes containing 30wt \% Pt/C were prepared by spraying 2-propanol-Pt/C ink on the GDL. The electrodes were then treated for 12 hours at $80^{\circ} \mathrm{C}$ and 3 days at $190^{\circ} \mathrm{C}$ under vacuum, in order to remove all traces of the organic solvent. The electrodes containing MWCNT based catalysts were prepared by dispersing the catalyst powder in a 2propanol/3-D $\mathrm{H}_{2} \mathrm{O} 2 / 1(\mathrm{v} / \mathrm{v})$ solution. The catalyst slurry was applied on the GDL by a spatula and the electrodes were left over night to dry at room temperature prior heating at $100^{\circ} \mathrm{C}$ in an oven for 1 hour. The noble metal loading of the anodic electrodes varied from 0.43 to $2.15 \mathrm{mgPt} / \mathrm{cm}^{2}$.

The cathodic electrode's specifications were kept constant for all MEAs. The carbon black based electrocatalyst (30w t $\% \mathrm{Pt} / \mathrm{C}$ ) was used and the Pt loading was around $1.6 \mathrm{mgPt} / \mathrm{cm}^{2}$ in all cases.

Membrane Electrode Assembly (MEA): Before the MEA assembly, a certain amount of phosphoric acid, PA, was sprayed onto the electrodes. The cathodic electrodes were sprayed with $2 \mathrm{gPA} / \mathrm{gPt}$, whereas in the case of the anodic electrodes the ratio of $\mathrm{gPA} / \mathrm{gPt}$ varied $(0.5 \mathrm{gPA} / \mathrm{gPt}$ and $2 \mathrm{gPAg} / \mathrm{Pt})$. The electrode active area was $2 \mathrm{~cm} \times 2 \mathrm{~cm}$. Several MEAs were fabricated, the specifications of which are depicted in Table 6-1. The MEAs were fabricated by hot pressing, the phosphoric acid doped membranes (doping level 200 wt \%) between the two electrodes at $150^{\circ} \mathrm{C}$ for $5 \mathrm{~min}$, as described in detail in Chapter 3 .

\subsubsection{Electrochemical Characterization}

All MEAs were activated before each measurement. Initially the cell's temperature was increased from room temperature to $100^{\circ} \mathrm{C}$ while $40 \mathrm{cc} / \mathrm{min}$ Ar flushed both compartments of the reactor. Subsequently, the gases were switched to pure $\mathrm{H}_{2}(15 \mathrm{cc} / \mathrm{min})$ and $\mathrm{O}_{2}$ $(15 \mathrm{cc} / \mathrm{min})$ and the temperature was set at $140^{\circ} \mathrm{C}$. Once the temperature was reached, a current of $0.2 \mathrm{~A} / \mathrm{cm}^{2}$ was applied to the fuel cell. The temperature was then set at $180^{\circ} \mathrm{C}$ and was kept for 48-62 hours under these conditions. Details concerning the activation of the MEAs are provided in detail in the appendix A1.

Galvanostatic measurements were taken under both high flows (constant 200cc/min $\mathrm{H}_{2} / \mathrm{O}_{2}$ ) and low gas stoichiometry of $\mathrm{H}_{2}\left(\lambda_{\mathrm{H} 2}=1.2,1.5\right.$ and 3) and $\mathrm{O}_{2}\left(\lambda_{\mathrm{O} 2}=2,2.5\right.$ and 5). Pure $\mathrm{O}_{2}$ was supplied at the cathode, whereas the anode was fed with pure $\mathrm{H}_{2}$ or various fuel composition $\left(50.7 \mathrm{kPa} \mathrm{H}\right.$ in $\mathrm{Ar}, 50.7 \mathrm{kPa} \mathrm{H}$ and $2 \mathrm{kPa} \mathrm{CO}$ in $\mathrm{Ar}, 100-66.5 \mathrm{kPa} \mathrm{H}_{2}$ and 0 $33.5 \mathrm{kPa} \mathrm{H} \mathrm{H}_{2} \mathrm{O}$ ), as well as synthetic reformate gas. The synthetic reformate gas that was used consisted of $50.7 \mathrm{kPa} \mathrm{H}, 2 \mathrm{kPa} \mathrm{CO}, 33.5 \mathrm{kPa} \mathrm{H}_{2} \mathrm{O}$ balanced with Ar. The cell's temperature was kept constant at $180^{\circ} \mathrm{C}$. Each catalyst was tested several times in MEAs to ensure reproducibility. 
All electrochemical impedance spectroscopy measurements were conducted in galvanostatic mode $\left(0.2 \mathrm{~A} / \mathrm{cm}^{2}\right)$ using a sinusoidal current wave with amplitude of $5 \mathrm{~mA} / \mathrm{cm}^{2}$. The frequency was swept from $50 \mathrm{kHz}$ to $10 \mathrm{mHz}$ with a logarithmic distribution of 50 points. The measurements were conducted without interrupting the cell's operation.

Table 6-1. Specifications (Pt metal loading per electrode and catalyst type) of the anodic electrodes that operated under reformate gas.

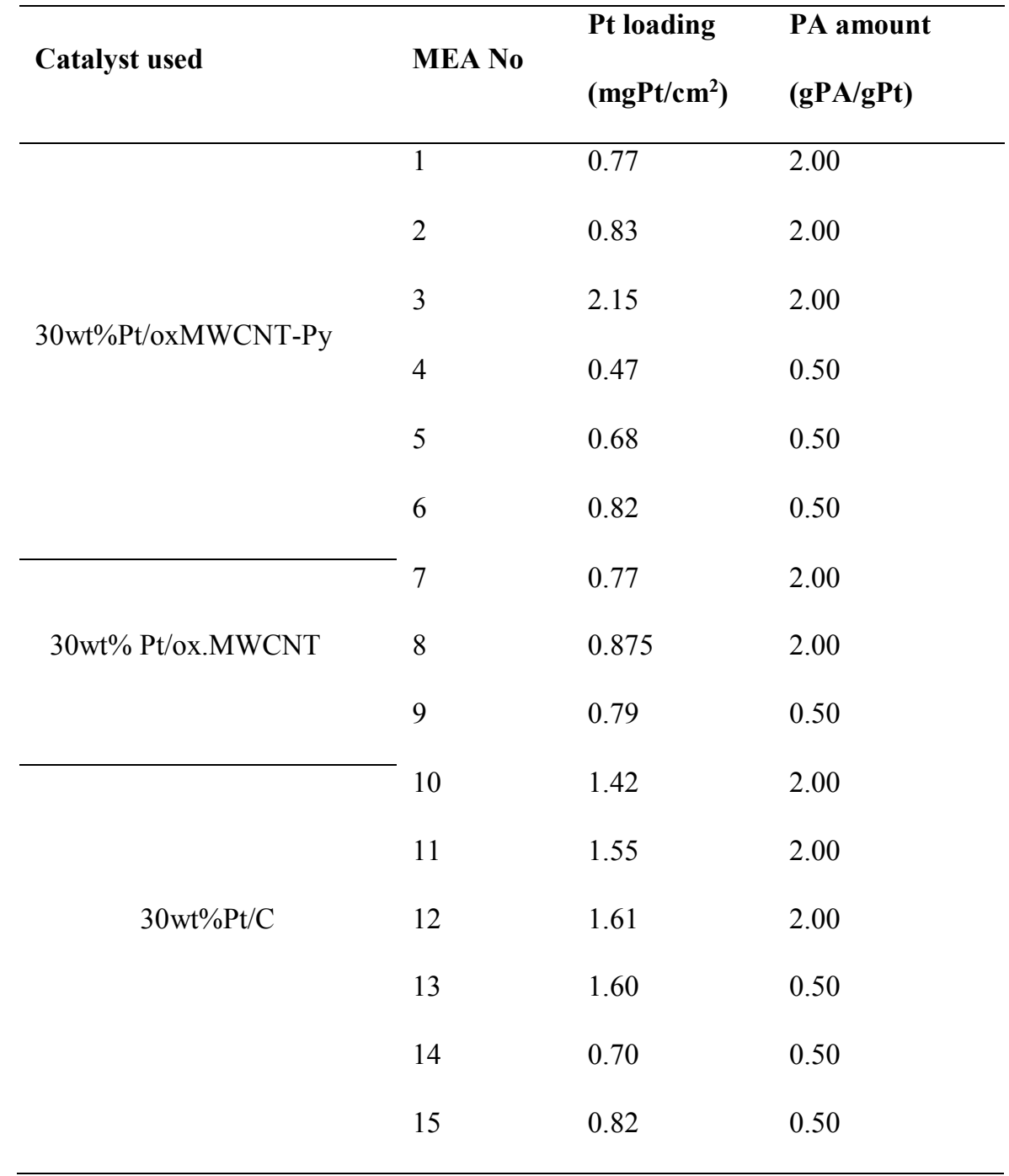

\subsection{Results}

This section presents a detailed analysis concerning the effect of the catalyst substrate, the noble metal loading as well as the PA loading in the CL, on the anode's performance under synthetic reformate gas in various compositions. 


\subsubsection{Fuel cell performance under various synthetic reformate conditions}

Initially the effect of the catalyst substrate on the fuel cell's performance was investigated. Figure 6-1 shows the effect of the catalyst substrate on the fuel cell's performance when synthetic reformate gas consisting of $50.7 \mathrm{kPa} \mathrm{H}, 2 \mathrm{kPa} \mathrm{CO}$ and $33.5 \mathrm{kPa} \mathrm{H}_{2} \mathrm{O}$ balanced with Ar, was fed at the anode. A stoichiometry of $\lambda_{\mathrm{H} 2}=1.2$ for $\mathrm{H}_{2}$ was used at the anode, whereas at the cathode side pure $\mathrm{O}_{2}$ was used with a stoichiometry of $\lambda_{\mathrm{O} 2}=2$. Polarization curves where acquired from MEA 1, 7 and 11, prepared with different Pt supports as Shown in Fig. 6-1. The fuel cell's temperature was kept at $180^{\circ} \mathrm{C}$ and the PA amount in the CL was set at $2 \mathrm{gPA} / \mathrm{gPt}$ (table $6-1)$.

Three regions in the polarization curves are observed when $2 \mathrm{gPA} / \mathrm{gPt}$ are imbedded in the anodic catalyst layer (figure 6-1). At low current densities there are adequate Pt sites free for the $\mathrm{H}_{2}$ electro-oxidation reaction, as the poisoning effect is negligible, and therefore the performance of the fuel cell is not severely affected. As the current density increases, the HOR is being constrained by the remaining free Pt sites due to blockage of the Pt surface by poisoning adsorbents.

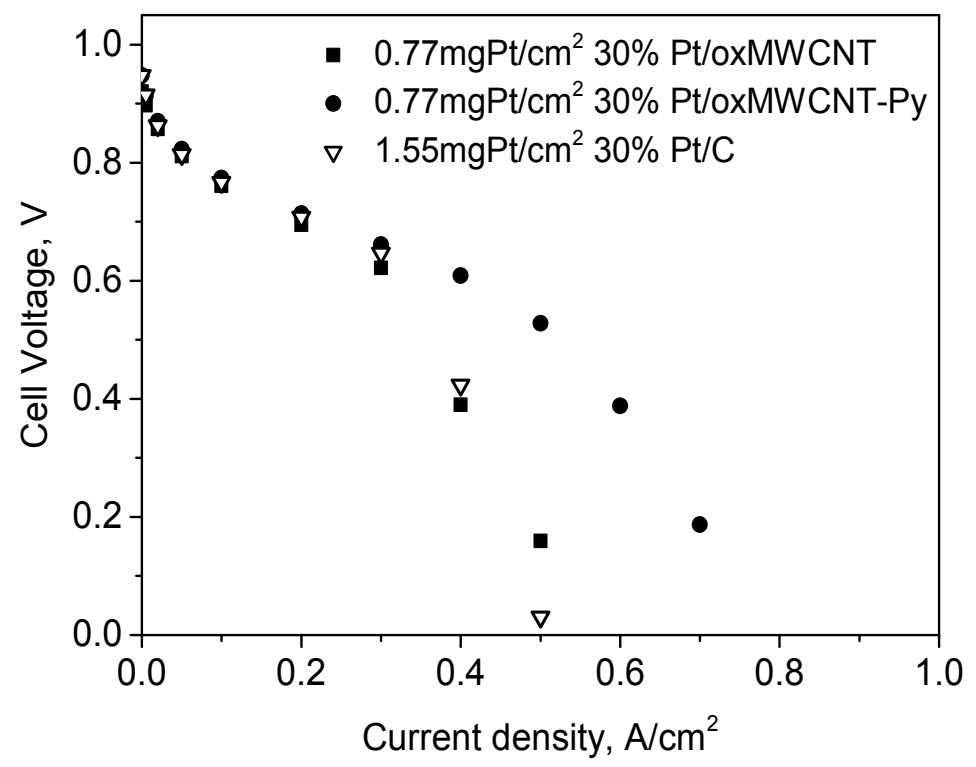

Figure 6-1. The effect of the catalyst substrate on the anode's performance under synthetic reformate gas consisting of $50.7 \mathrm{kPa} \mathrm{H}_{2}, 2 \mathrm{kPa} \mathrm{CO}$ and $33.5 \mathrm{kPa} \mathrm{H}_{2} \mathrm{O}$ balanced with Ar. The polarization curves were obtained using a stoichiometry of $\lambda_{\mathrm{H} 2}=1.2$ and $\lambda_{\mathrm{O} 2}=2$, pure $\mathrm{O}_{2}$ was used at the cathode. The PA loading at the anodic electrode was $2 \mathrm{gPA} / \mathrm{gPt}$ and cell's temperature was kept at $180^{\circ} \mathrm{C}: 30 \mathrm{wt} \% \mathrm{Pt} /(\mathrm{ox} . \mathrm{MWNT})-\mathrm{Py}$ (MEA 1), 30wt\%Pt/oxMWCNT (MEA 7) and 30wt\%Pt/C (MEA 11). 
At current densities higher than $0.4 \mathrm{~A} / \mathrm{cm}^{2}$, low performance region, there is a shortage of available free $\mathrm{Pt}$ active sites and cannot sustain the HOR, reaching limiting current at relatively low current densities. Note that the current density at 0 cell voltage is much lower than the limiting current corresponding to the $\mathrm{H}_{2}$ feed flow rate.

For current densities below $0.2 \mathrm{~A} / \mathrm{cm}^{2}$, no significant difference in the obtained voltage among the three different carbon based electrocatalyst supports was observed. MEA 11, employing the commercial catalyst $30 \% \mathrm{Pt} / \mathrm{C}\left(1.55 \mathrm{mgPt} / \mathrm{cm}^{2}\right)$, suffered a significant voltage loss reaching a short circuit current $\left(\mathrm{V}_{\text {cell }}=0 \mathrm{~V}\right)$ equal to $0.5 \mathrm{~A} / \mathrm{cm}^{2}$. Similar behavior was observed for MEA 7, employing 30\% Pt/ox.MWCNT, reaching the same short circuit current, despite having half the Pt loading in the CL $\left(0.77 \mathrm{mgPt} / \mathrm{cm}^{2}\right)$. A substantial improvement of the cell's performance was observed when 30\%Pt/oxMWNT-Py electrocatalyst was used (MEA 1) under the same conditions. The pyridine modified MWCNT based electrocatalyst reached a short circuit current value of approximately $0.7 \mathrm{~A} / \mathrm{cm}^{2}$ having a noble metal loading equal to $0.77 \mathrm{mgPt} / \mathrm{cm}^{2}$.

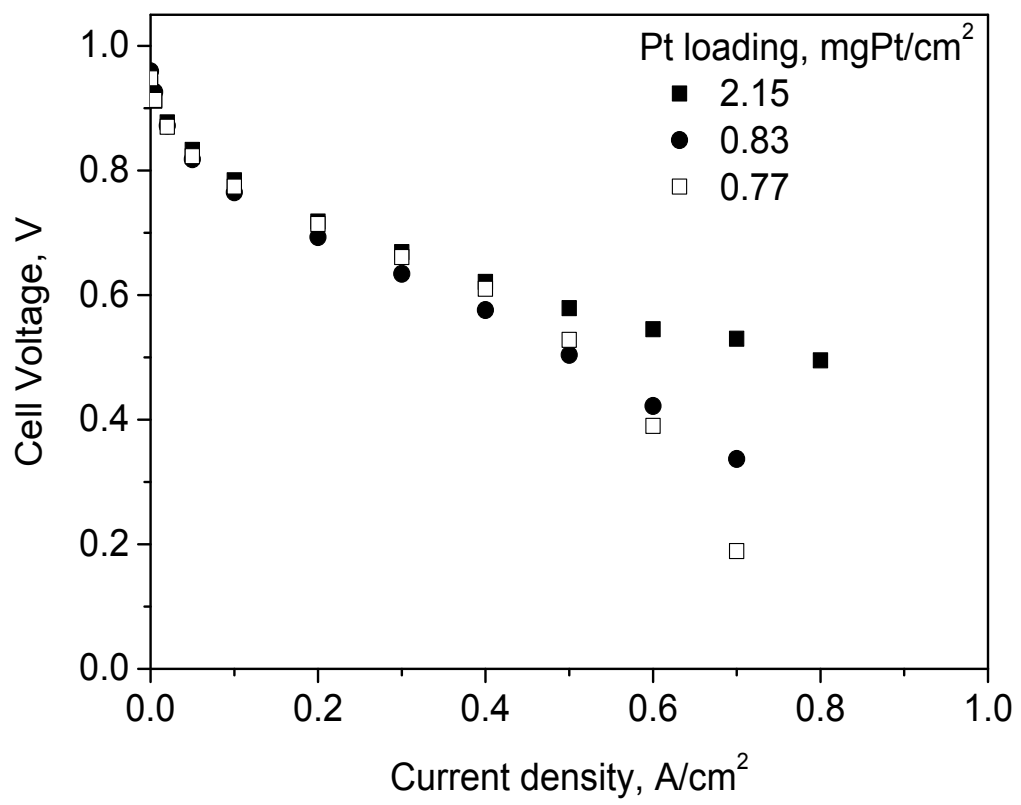

Figure 6-2. The effect of the noble metal loading on the anode's performance under synthetic reformate gas consisting of $50.7 \mathrm{kPa} \mathrm{H}, 2 \mathrm{kPa} \mathrm{CO}$ and $33.5 \mathrm{kPa} \mathrm{H}_{2} \mathrm{O}$ balanced with Ar. The polarization curves were obtained using a stoichiometry of $\lambda_{\mathrm{H} 2}=1.2$ and $\lambda_{\mathrm{O} 2}=2$, pure $\mathrm{O}_{2}$ was used at the cathode. The PA loading at the anodic electrode was $2 \mathrm{gPA} / \mathrm{gPt}$ and cell's temperature was kept at $180^{\circ} \mathrm{C}$. The anodic electrode employed $30 \mathrm{wt} \%$ Pt/oxMWCNT-Py (MEA 1-3). 
Increasing the noble metal loading, while keeping the PA loading constant $(2 \mathrm{gPA} / \mathrm{gPt})$, resulted in a significant increase of the fuel cell's performance, (figure 6-2) for the case of the ox.MWCNT-Py based catalyst, due to the higher ECSA utilization as thoroughly discussed in Chapter 5. On the other hand it was observed that the performance of the anodic electrodes employing 30\% $\mathrm{Pt} / \mathrm{C}$ (50\% Pt utilization), exhibit a stronger dependence on the noble metal loading, compared to the MWCNT based, especially at low Pt loadings.

In continuance, the gas stoichiometry was altered in order to shed more light to the origin of the overall voltage loss in the high current density region under these harsh operation conditions. In the case of normal flows, the gas stoichiometry was $\lambda_{\mathrm{H} 2}=1.2$ and $\lambda_{\mathrm{O} 2}=2$, whereas for the case of high flows the $\mathrm{H}_{2}$ flow $(50.7 \%$ ) was $101.4 \mathrm{cc} / \mathrm{min}$ corresponding to a nominal limiting current of approximately $3.5 \mathrm{~A} / \mathrm{cm}^{2}$. When the synthetic reformate gas flow at the anodic compartment was increased, it led to an improvement of the anode's performance, as shown in figure 6-3.

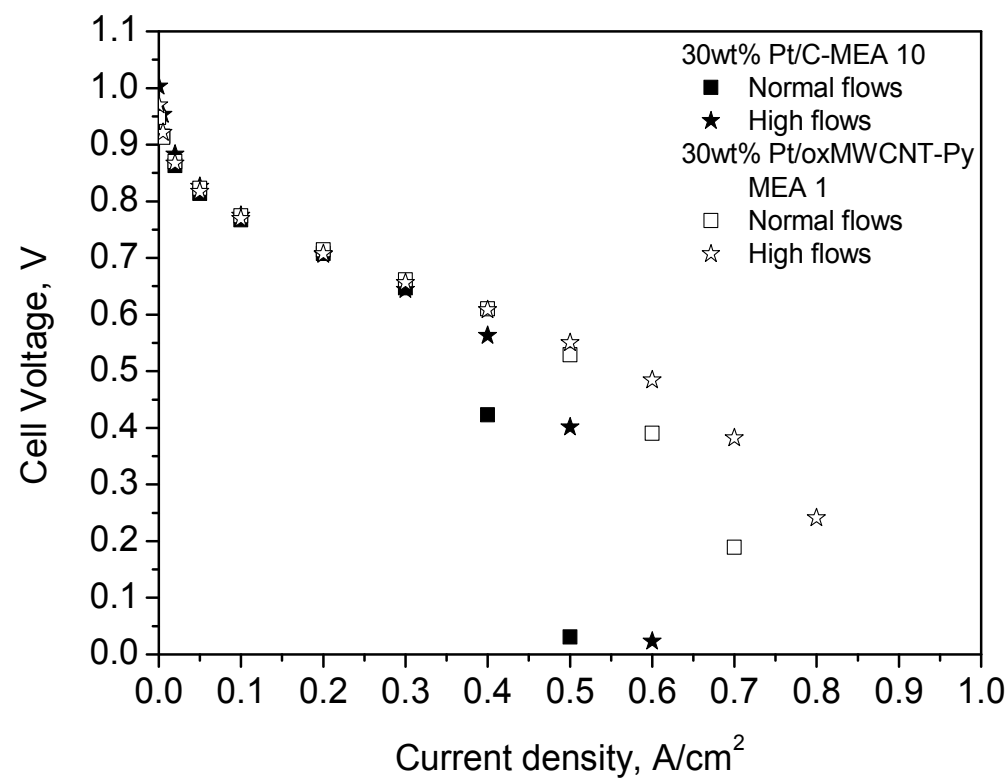

Figure 6-3. The effect of the gas stoichiometry on the MEAs performance under synthetic reformate feed. Polarization curves of MEA 1 and 10 (PA loading=2gPA/gPt) were acquired at $180^{\circ} \mathrm{C}$ under various total flow rates of synthetic reformate gas mixture, $50.7 \mathrm{kPa} \mathrm{H}_{2}, 33.5 \mathrm{kPa} \mathrm{H}_{2} \mathrm{O}$ and $2 \mathrm{kPa} \mathrm{CO}$ balanced with Ar. Normal flows: stoichiometry of $\lambda_{\mathrm{H} 2}=1.2$ and $\lambda_{\mathrm{O} 2}=2$ and high flows: constant $200 \mathrm{cc} / \mathrm{min}$ total flow of reformate gas and $\mathrm{O}_{2}$, which corresponds to a nominal limiting current density of $3.5 \mathrm{~A} / \mathrm{cm}^{2}$.

Interestingly the improvement was only restricted to the low performance region. A small change in the slope was observed as the gas stoichiometry increased, while the low 
performance region shifts to higher current densities, although never reaching the nominal limiting current density value. The later was found to be independent of the catalyst type, as clearly depicted in figure 6-3.

Since steam constitutes up to $33.5 \mathrm{kPa}$ of the reformate fuel's feed, its effect on the anode's performance was studied with respect to the catalyst type. The porosity and the hydrophobicity of the catalytic layer are expected to depend on the properties of the catalyst substrate. Hence, different behavior is anticipated under various partial pressures of water in the anodic feed gas. The PA imbedded in the CL is of great importance, therefore in that account the PA amount was kept at $2 \mathrm{gPA} / \mathrm{gPt}$. Pure $\mathrm{H}_{2}$ gas was humidified using a thermostated humidifier, where the temperature varied accordingly, prior its introduction to the cell. The measurements were taken under high reactant gas flow $\left(200 \mathrm{cc} / \mathrm{min} \mathrm{H}_{2} / \mathrm{O}_{2}\right.$ ) in order to ensure their uniform partial pressure distribution over the electrode's surface. The applied current density was set at $0.4 \mathrm{~A} / \mathrm{cm}^{2}$, whereas the temperature of the cell was kept constant at $180^{\circ} \mathrm{C}$.

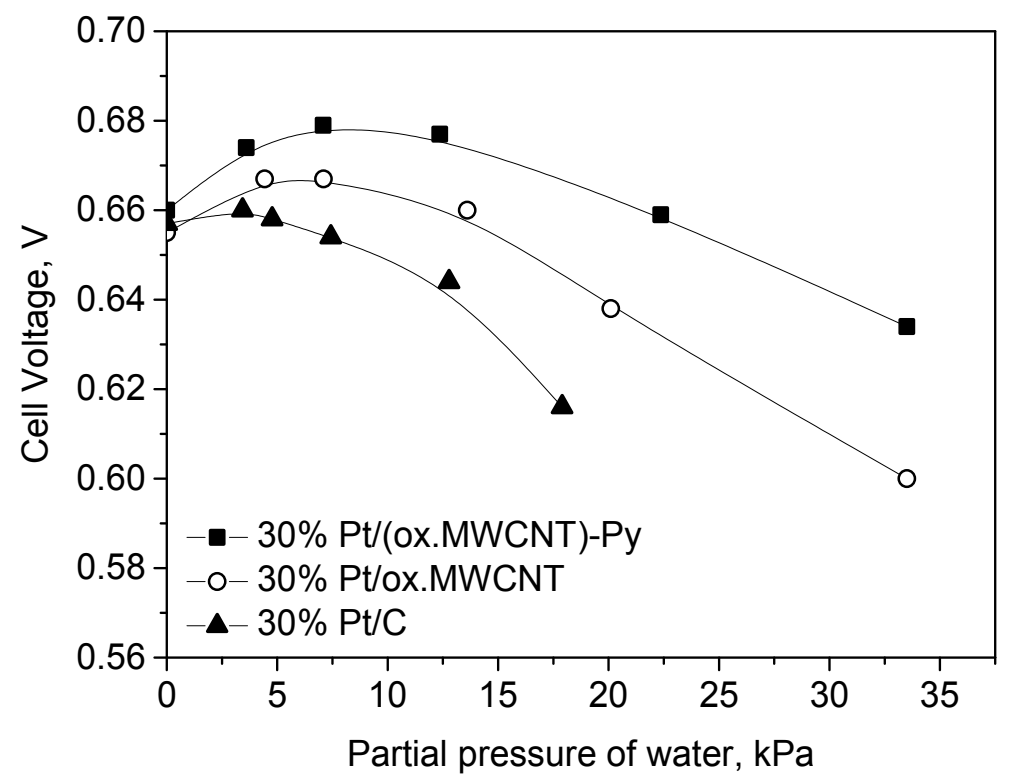

Figure 6-4. The effect of the steam's partial pressure in the $\mathrm{H}_{2}$ feed on the cell's voltage. The values were obtained at $180^{\circ} \mathrm{C}$ and $0.4 \mathrm{~A} / \mathrm{cm}^{2}$ from MEAs employing $30 \% \mathrm{Pt} /($ ox.MWNT)-Py, 30\%Pt/oxMWCNT and 30\%Pt/C (MEAs 1,7 and 12), while the anode was fed with a $\mathrm{H}_{2} / \mathrm{H}_{2} \mathrm{O}$ mixture $\left(\lambda_{\mathrm{H}_{2}}>15\right)$ and cathode with pure $\mathrm{O}_{2}\left(\lambda_{\mathrm{O} 2}>30\right)$, $200 \mathrm{cc} / \mathrm{min}$ respectively.

As shown in figure 6-4, for the carbon black based catalyst (MEA 12), increasing water's partial pressure in the anodic gas feed up to $10 \mathrm{kPa}$ led to a slow reduction in the 
performance of the single cell. Further increase of the steam mole fraction, above $10 \mathrm{kPa}$, led to a significant voltage loss of $50 \mathrm{mV}$. Though significantly improved a similar behavior is observed in the cases of MWCNT based catalysts (MEA 1 and MEA 7). For the case of low partial pressures of water in the hydrogen flow, up to $10 \mathrm{kPa}$, a small increase of the cell's performance was observed. Further increase, led to a gradual reduction of the cell performance.

It is clear that the MWCNT based electrocatalyst exhibit lower performance losses in all cases. This can be attributed to the difference in the hydrophobic/ hydrophilic properties of the catalyst layer. As reported in a previous work, hydrophilic substrates are highly desired for the uniform distribution of PA on the electrocatalytic surface and its stability under humidified conditions [15]. High steam content in the gas feed could displace or restructure the PA in the electrocatalytic layer, leading to a reduction of the three phase boundaries. As can be observed from Figure 6-4, the 30wt\% Pt/(ox.MWCNT)-Py catalyst shows higher and more stable behavior under high water partial pressures as compared to $30 \mathrm{wt} \%$ Pt/ox.MWCNT. This is attributed to the nature of the pyridine groups attached on the MWNT surface. Pyridine groups bind phosphoric acid through acid-base interactions, thus contributing into its homogeneous distribution and stabilization inside the catalytic layer. Therefore, under high water partial pressures the ionic conductive pathway in the electrodes is less affected compared to the $30 \mathrm{wt} \% \mathrm{Pt} / \mathrm{ox} . \mathrm{MWCNT}$ catalyst.

\subsubsection{Effect of the PA loading in the catalyst layer}

The amount of PA on the electrodes plays a significant role in the formation of the EI. In order to determine the effect of the PA amount on the performance of the anodic electrode several MEAs were prepared where the PA loading of the anodic catalyst layer was lowered and set at $0.5 \mathrm{gPA} / \mathrm{gPt}$ (Table 6-1). The anodic electrodes had similar Pt loading as the ones reported in the previous section 6.3.1. The performance was evaluated under synthetic reformate gas consisting of $50.7 \mathrm{kPa} \mathrm{H}_{2}, 2 \mathrm{kPa} \mathrm{CO}$ and $33.5 \mathrm{kPa} \mathrm{H}_{2} \mathrm{O}$ balanced with Ar. A stoichiometry of $\lambda_{\mathrm{H} 2}=1.2$ for $\mathrm{H}_{2}$ was used at the anode, whereas at the cathode side pure $\mathrm{O}_{2}$ was used with a stoichiometry of $\lambda_{\mathrm{O} 2}=2$.

Three anodic electrodes were formulated consisting of the three different electrocatalysts, having similar Pt loadings, approximately $0.8 \mathrm{mgPt} / \mathrm{cm}^{2}$ (MEA 6,9 and 15) and their performance is shown in figure 6-5. The electrode employing 30\% Pt/C exhibited high voltage loss even at low current densities, reaching a short circuit current of $0.5 \mathrm{~A} / \mathrm{cm}^{2}$. On the contrary, the MWCNT based electrocatalysts exhibited high endurance and performance, none of which reached short circuit current in the applied current range. 


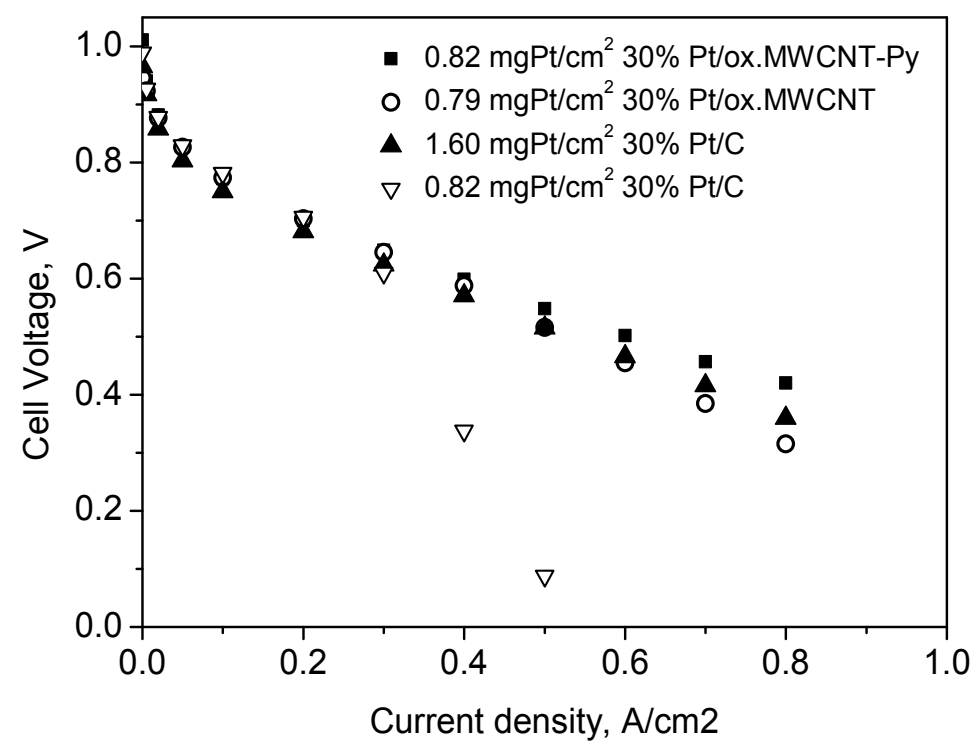

Figure 6-5. Polarization curves obtained under synthetic reformate fed $\left(50.7 \mathrm{kPa} \mathrm{H}_{2}\right.$, $33.5 \mathrm{kPa} \mathrm{H} \mathrm{H}_{2} \mathrm{O}$ and $2 \mathrm{kPa} \mathrm{CO}$ balanced with Ar.) with gas stoichiometry of $\lambda_{\mathrm{H} 2}=1.2$ and $\lambda_{\mathrm{O} 2}=2$. The PA loading at the anodic electrode was $0.5 \mathrm{gPA} / \mathrm{gPt}$ and cell's temperature was kept at $180^{\circ} \mathrm{C}$.T MEAs used: $30 \% \mathrm{Pt} /($ ox.MWNT)-Py (MEA 6), 30\%Pt/oxMWCNT( MEA 9) and $30 \% \mathrm{Pt} / \mathrm{C}($ MEA 13 and 15)

When the noble metal loading was increased to $1.60 \mathrm{mgPt} / \mathrm{cm}^{2}$, for the case of $30 \% \mathrm{Pt} / \mathrm{C}$, the MEA exhibited similar performance with the MWCNT based electrocatalyst under the same conditions (figure 6-5). From the results depicted in figure 6-5, it is clear that by lowering the $\mathrm{PA}$ amount to $0.5 \mathrm{gPA} / \mathrm{gPt}$, a significant increase in reformate tolerance is observed.

Since steam constitutes up to $33.5 \mathrm{kPa}$ of the reformate fuel's feed, its effect on the anode's performance was also studied with respect to the catalyst type and the PA amount. The same procedure was followed as the one described in section 6.3.1. Pure $\mathrm{H}_{2}$ gas was humidified using a thermostated humidifier, where the temperature varied accordingly, prior its introduction to the cell.

The measurements were taken under high reactant gas flow $\left(200 \mathrm{cc} / \mathrm{min}_{2} / \mathrm{O}_{2}\right)$ in order to ensure their uniform partial pressure distribution over the electrode's surface. The applied current density was set at $0.4 \mathrm{~A} / \mathrm{cm}^{2}$, whereas the temperature of the cell was kept constant at $180^{\circ} \mathrm{C}$. 


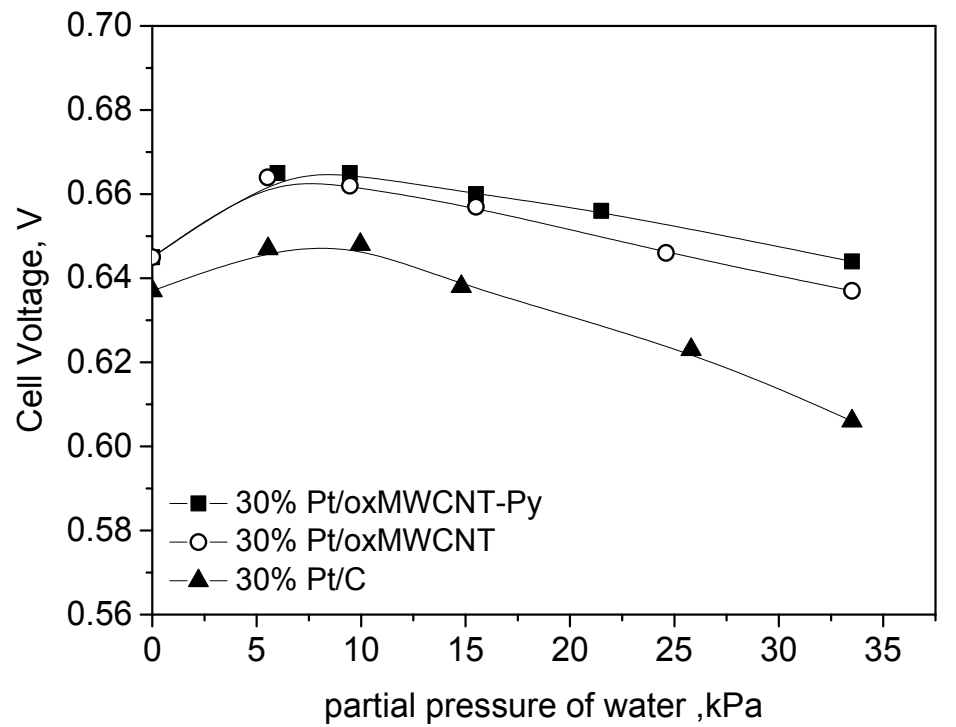

Figure 6-6. The effect of the steam's partial pressure in the $\mathrm{H}_{2}$ feed on the cell's voltage. The values were obtained at $180^{\circ} \mathrm{C}$ and $0.4 \mathrm{~A} / \mathrm{cm}^{2}$ from MEAs employing 30\%Pt/(ox.MWNT)-Py, 30\%Pt/oxMWCNT and 30\%Pt/C (MEAs 6,9 and 13), while the anode was fed with a $\mathrm{H}_{2} / \mathrm{H}_{2} \mathrm{O}$ mixture $\left(\lambda_{\mathrm{H}_{2}}>15\right)$ and cathode with pure $\mathrm{O}_{2}\left(\lambda_{\mathrm{O} 2}>30\right)$, $200 \mathrm{cc} / \mathrm{min}$ respectively. The PA loading at the anode was $0.5 \mathrm{gPA} / \mathrm{gPt}$ for all cases.

Compared to the data recorded for the case of $2 \mathrm{gPA} / \mathrm{gPt}$ (figure 6-4), the voltage loss with respect to the water partial pressure is reduced, for all catalysts (MEA 6,9 and 13), especially under high water mole fraction. It is evident that the PA amount, due to its hydrophilic nature, significantly affects the stability and performance of the fuel cell under high partial pressure of steam even at high $\mathrm{H}_{2}$ partial pressures.

It is clear from the above presented results that $0.5 \mathrm{gPA} / \mathrm{gPt}$ is the optimum amount of PA for all tested electrocatalyst, under these experimental conditions. All of the above presented results, revealed a clear reliance between the water partial pressure in the fuel mixture towards the fuel cell's voltage loss. Thus, the effect of water partial pressure in the reformate gas feed was investigated with respect to different catalyst layers, with a PA loading of $0.5 \mathrm{gPA} / \mathrm{gPt}$.

Figure 6-7 depicts the performance response as a function of water partial pressure in the reformate gas mixture of MEA 14 employing the commercial $30 \% \mathrm{Pt} / \mathrm{C}$ and MEA 5 employing 30\% Pt/oxMWNCT-Py. Both anodic electrodes had similar Pt $\left(0.7 \mathrm{gPt} / \mathrm{cm}^{2}\right)$ and PA $(0.5 \mathrm{gPA} / \mathrm{gPt})$ loadings (table 6-1). Polarization curves were obtained at $180^{\circ} \mathrm{C}$ and the stoichiometry of $\mathrm{H}_{2}$ and $\mathrm{O}_{2}$ was set at 1.2 and 2, respectively. The composition of the 
fuel mixture comprised of $50.7 \mathrm{kPa} \mathrm{H} 2$ and $2 \mathrm{kPa} \mathrm{CO}$, while the steam partial pressure was altered from 0 to $33.5 \mathrm{kPa}$ and was balanced with Ar.

Both electrocatalysts did not exhibit any significant voltage loss up to $9.46 \mathrm{kPa} \mathrm{H}_{2} \mathrm{O}$. Further increase in the partial pressure of water in the reformate mixture, resulted in gradual decrease in the fuel cell's potential at elevated current densities for the case of carbon black based electrocatalyst. For $14.1 \mathrm{kPa} \mathrm{H}_{2} \mathrm{O}$ the commercial catalyst reaches a short circuit current of $0.7 \mathrm{~A} / \mathrm{cm}^{2}$, as opposed to the $30 \%$ Pt/oxMWNT-Py. The anode employing $30 \% \mathrm{Pt} / \mathrm{oxMWCNT}$-Py catalyst was found to be unaffected by higher partial pressures of water. The later sustained the $\mathrm{HOR}$ even at $33.5 \mathrm{kPa} \mathrm{H} 2 \mathrm{O}$, without reaching limiting current behavior in the specific operation range. It has to be mentioned that lowering the partial pressure of water to $9.46 \mathrm{kPa}$ in the reformate gas mixture allowed us to reduce further the Pt loading to $0.47 \mathrm{mgPt} / \mathrm{cm}^{2}$ for the case of $30 \mathrm{wt} \% \mathrm{Pt} / \mathrm{oxMWCNT}$-Py without sever performance losses (figure 6-8). The later is a very promising result, as it allies for the 3 fold reduction of the $\mathrm{Pt}$ loading at the anode when operating under reformate conditions as compared to the commercial Pt/Celectrocatalyst. 

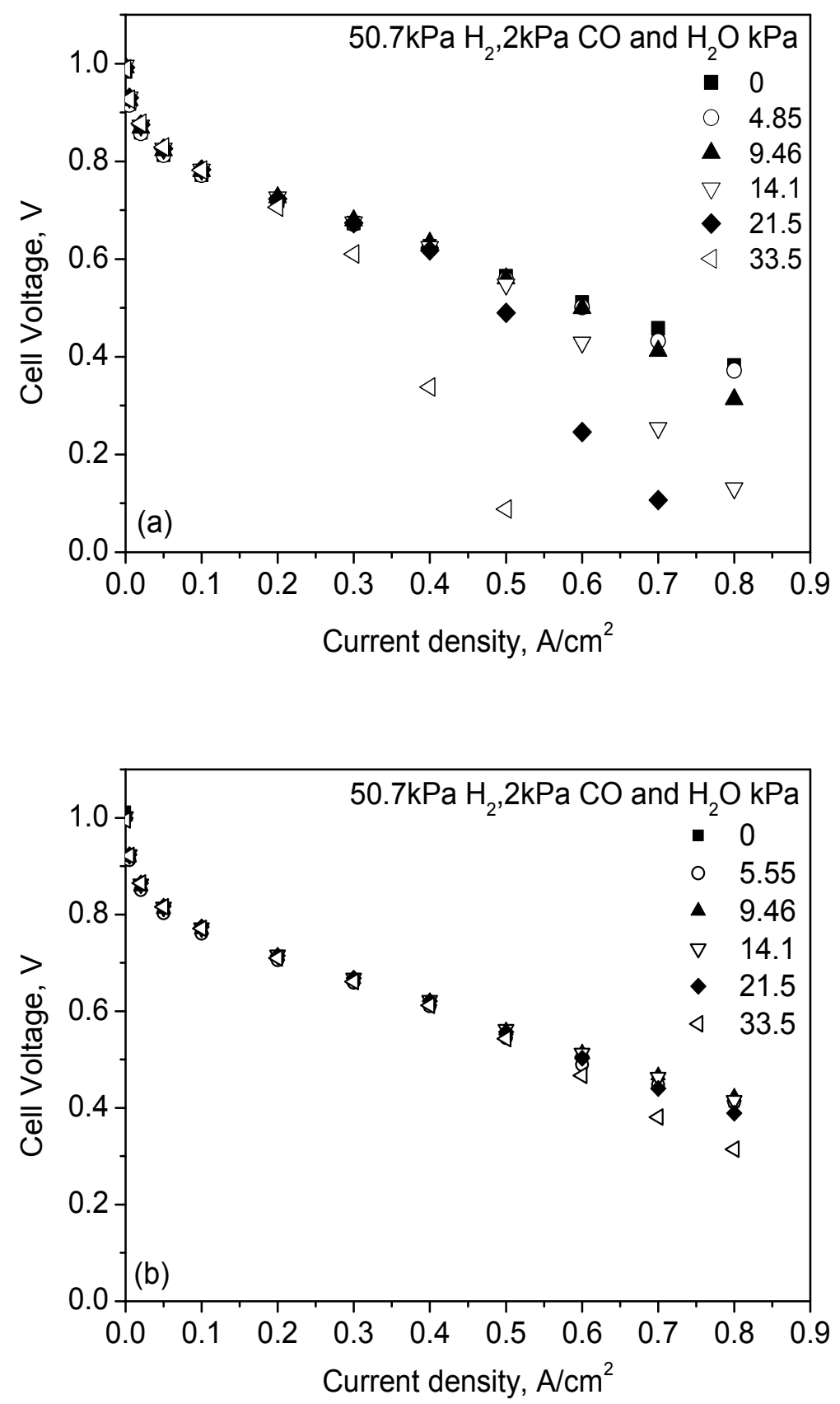

Figure 6-7. Polarization curves obtained at $180^{\circ} \mathrm{C}$ under $\mathrm{H}_{2}$ and $\mathrm{O}_{2}$ stoichiometry of 1.2 and 2 respectively. The composition of the gas mixture was $50.7 \mathrm{kPa} \mathrm{H} 2$ and $2 \mathrm{kPa} \mathrm{CO}$, while the steam partial pressure was altered from 0 to $33.5 \mathrm{kPa}$ and balanced with Ar. Both anodic electrodes had $0.5 \mathrm{gPA} / \mathrm{gPt}$. a. MEA 14: employing $0.7 \mathrm{mgPt} / \mathrm{cm}^{2} 30 \mathrm{wt} \% \mathrm{Pt} / \mathrm{C}$ and b. MEA 5: employing $0.68 \mathrm{mgPt} / \mathrm{cm}^{2} 30 \mathrm{wt} \% \mathrm{Pt} / \mathrm{ox}$.MWCNT-Py . 
Therefore it is clear that the steam partial pressure in the reformate gas influences the overall cell's performance and it was found to depend not only on the PA amount imbedded in the CL but also on the properties of the catalyst layer.

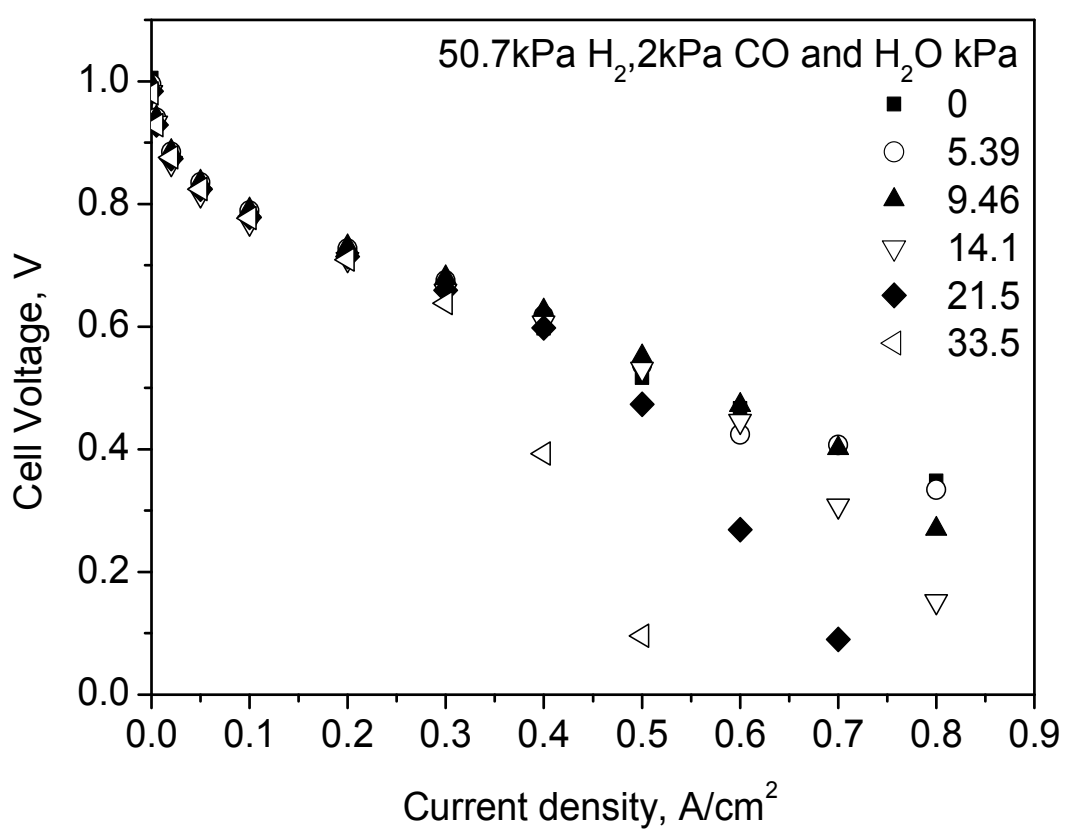

Figure 6-8. Polarization curves obtained at $180^{\circ} \mathrm{C}$ under $\mathrm{H}_{2}$ and $\mathrm{O}_{2}$ stoichiometry of 1.2 and 2 respectively. The composition of the gas mixture was $50.7 \% \mathrm{H} 2$ and $2 \% \mathrm{CO}$, while the steam partial pressure was altered from 0 to $33.5 \mathrm{kPa}$ and balanced with Ar. The anodic electrode employed 30wt\% Pt/oxMWCNT-Py, MEA 4: $0.47 \mathrm{mgPt} / \mathrm{cm}^{2}$ and $0.5 \mathrm{gPA} / \mathrm{gPt}$.

Linares et al. studied the performance of an ABPBI-based High Temperature $\mathrm{H}_{2} / \mathrm{O}_{2}$ PEMFC system under different experimental conditions. The platinum loading for both electrodes was $0.5 \mathrm{mg} / \mathrm{cm}^{2}$, with a PBI loading normalized with respect to the carbon catalyst loading (C/PBI ratio) of 20. Afterwards, the electrodes were cured in an oven at $190^{\circ} \mathrm{C}$ for $2 \mathrm{~h}$, and then impregnated with a $10 \% \mathrm{H} 3 \mathrm{PO} 4$ (loading of $20 \mathrm{mg} / \mathrm{cm}^{2}$ ), leaving them overnight in order to ensure the complete soaking of the electrodes. They claimed that their maximum power density at $180 \mathrm{C}$ was around $240 \mathrm{~mW} / \mathrm{cm}^{2}$, when the anode was supplied with $2 \mathrm{kPa}$ of $\mathrm{CO}$ and $20 \mathrm{kPa}$ of water in the $\mathrm{H}_{2}$ supply [17]. Comparing their results to ours under similar conditions the 30wt\%Pt/oxMWCNT-Py (figure 6-8) reaches approximately $250 \mathrm{~mW} / \mathrm{cm}^{2}$ under similar conditions $\left(2 \mathrm{kPa}\right.$ of $\mathrm{CO}, 50.7 \mathrm{kPa}$ of $\mathrm{H}_{2}$ and $21.5 \mathrm{kPa} \mathrm{H}_{2} \mathrm{O}$ ) and $\mathrm{Pt}$ loadings. 


\subsubsection{Voltage loss in the anodic electrodes under different fuel composition}

Generally the kinetic of HOR depends on electrolyte composition, $\mathrm{pH}$, temperature, $\mathrm{H}_{2}$ partial pressure, electrode physical and chemical nature, as well as the applied potential. It is well known that lean partial pressure of $\mathrm{H}_{2}$ in the fuel gas influences the reaction rate [18], increasing the anode's overpotential. Therefore, due to the composition of synthetic reformate gas, it was essential to understand and quantify each component's contribution to the voltage loss, with respect to the PA in the CL. Since a two electrode arrangement was used, the anode's voltage loss was estimated according to the literature $[19,20]$. At a constant current density, the difference in the polarization of the anode between, the cases

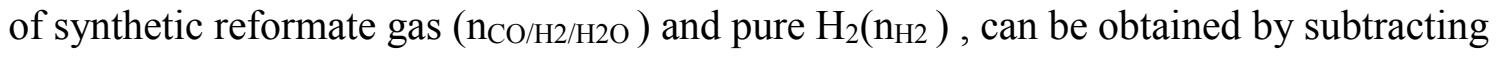
the cell voltage under identical experimental conditions:

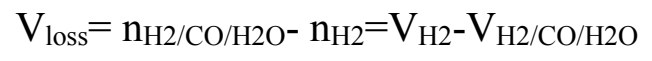

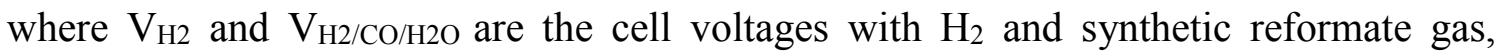
respectively. Thus, the anode voltage loss for the case of reformate gas is assumed to be the cell voltage difference between the two cases, i.e.

$$
\mathrm{V}_{\text {loss }}==\mathrm{V}_{\mathrm{H} 2}-\mathrm{V}_{\mathrm{H} 2 / \mathrm{CO} / \mathrm{H} 2 \mathrm{O}}
$$

The voltage loss of the anode was also calculated in the same manner for different fuel composition $\left(50.7 \mathrm{kPa} \mathrm{H}, 50.7 \mathrm{kPaH}_{2}\right.$ and $2 \mathrm{kPa} \mathrm{CO}$ balanced with Ar). The obtained voltage values for all cases were obtained under high flow of gas $(200 \mathrm{cc} / \mathrm{min}$ total flow $)$ in order to ensure uniform partial pressure distribution over the electrode's surface, where the supplied $\mathrm{H}_{2}$ corresponded to a nominal limiting current of $3.5 \mathrm{~A} / \mathrm{cm}^{2}$. The curves were IR drop corrected prior to any calculation. The IR drop corrected IV plots used for the above described calculations are presented as additional information in appendix B1. The calculated voltage losses are depicted in figure 6-9 (MEA 1 and 7) for the MWCNT based electrocatalysts, having a phosphoric acid loading of $2 \mathrm{gPA} / \mathrm{gPt}$.

As shown in figure 6-9, a small voltage loss is observed when pure $\mathrm{H}_{2}$ is switched to $50.7 \mathrm{kPa} \mathrm{H}_{2} / \mathrm{Ar}$ mixture. The obtained anodic voltage loss was the same for all electrocatalysts. At $0.8 \mathrm{~A} / \mathrm{cm}^{2}$ a value of approximately $35 \mathrm{mV}$ was calculated for all electrocatalysts. When $2 \mathrm{kPaCO}$ was added to the fuel gas mixture $(50.7 \mathrm{kPa} \mathrm{H}, 2 \mathrm{kPa} \mathrm{CO}$ balanced with Ar), its effect on the anode's performance was apparent for the case of ox.MWNT based electrocatalyst (figure 6-9a). The voltage loss due to $\mathrm{CO}$ poisoning, was $121 \mathrm{mV}$ at $0.8 \mathrm{~A} / \mathrm{cm}^{2}$. On the contrary, for the pyridine functionalized ox.MWNT-Py, no significant increase in the anode's overpotential/voltage loss was observed, which remained at $52 \mathrm{mV}$ (figure 6-9b). The ECSA of the ox.MWNT-Py supported electrocatalyst 
is higher compared to the ox.MWNT, as discussed in Chapter 5, therefore more active sites are available for the HOR, minimizing the $\mathrm{CO}$ poisoning effect.

Similar losses were reported by Li et al., where they observed small voltage losses of less than $10 \mathrm{mV}$ with current densities up to $1.3 \mathrm{~A} \cdot \mathrm{cm}^{-2}$ for $\mathrm{CO}$ levels $1 \%$ and $3 \%$ in pure undiluted $\mathrm{H}_{2}$ and dry conditions at temperatures $150{ }^{\circ} \mathrm{C}$ and $200{ }^{\circ} \mathrm{C}$, respectively. They used a fuel cell based on $\mathrm{PBI} / \mathrm{H}_{3} \mathrm{PO}_{4}$ with a Pt loading of $0.5 \mathrm{mgPt} / \mathrm{cm}^{2}$.Chien-Ping Wang et al. developed a transient on dimensional mathematic model predicting the influence of $\mathrm{H}_{2}$ dilution in the fuel gas containing $1 \% \mathrm{CO}$ on the performance and $\mathrm{H}_{2}$ coverage on the $\mathrm{Pt}$ surface. Their results on PBI based MEA were validated with experimental data. They claimed that for $40 \mathrm{kPa} \mathrm{H}_{2}$ in a gas mixture, the coverage $\theta$ had the lowest value, which obviously increased with hydrogen content in the gas feed [21]. As expected at low partial pressure of hydrogen, $\mathrm{CO}$ poisoning effect is more enhanced

Synthetic reformate gas $\left(50.7 \mathrm{kPa} \mathrm{H}, 2 \mathrm{kPaCO}\right.$ and $33.5 \mathrm{Kpa} \mathrm{H}_{2} \mathrm{O}$ balanced with $\left.\mathrm{Ar}\right)$ was supplied to the fuel cell. For all cases, an eminent increase of the voltage loss, after specific current densities, was observed. If we define reformate tolerance as a voltage loss less than $70 \mathrm{mV}$ at $180^{\circ} \mathrm{C}$, the $30 \% \mathrm{Pt} / \mathrm{oxMWNT}$-Py exhibited a tolerance at current densities up to $0.5 \mathrm{~A} / \mathrm{cm}^{2}$. Whereas for the case of the $30 \% \mathrm{Pt} / \mathrm{oxMWNT}$ the tolerance was at smaller current densities, below $0.2 \mathrm{~A} / \mathrm{cm}^{2}$. Similar values, with the later, were obtained for the $30 \% \mathrm{Pt} / \mathrm{C}$ electrocatalyst (MEA 11).

When the PA loading was lowered in the catalyst layer $(0.5 \mathrm{gPA} / \mathrm{gPt})$, figure $6-10$, the voltage loss pattern with respect to the fuel composition remained the same, as described above. However the voltage loss values where substantially lower and the reformate tolerance increased. The pyridine functionalized and the oxidized MWCNT based catalysts, presented a reformate tolerance to current densities up to $0.8 \mathrm{~A} / \mathrm{cm}^{2}$ and $0.5 \mathrm{~A} / \mathrm{cm}^{2}$, respectively, having a Pt loading of approximately $0.8 \mathrm{mgPt} / \mathrm{cm}^{2}$. Whereas for the case of MEA 13, employing $30 \mathrm{wt} \% \mathrm{Pt} / \mathrm{C}$ and $1.60 \mathrm{mgPt} / \mathrm{cm}^{2}$, the reformate tolerance was 0.6 $\mathrm{A} / \mathrm{cm}^{2}$, under the same conditions (supporting information -appendix B1). Lowering the Pt loading (MEA 15) to $0.8 \mathrm{mgPt} / \mathrm{cm}^{2}$ resulted in a significant decrease of the reformate tolerance to $0.2 \mathrm{~A} / \mathrm{cm}^{2}$.It is clear from the above results that the observed anodic voltage loss cannot be solely attributed to $\mathrm{CO}$ poisoning of the Pt active sites, as there is a clear reliance between the reformate composition and the phosphoric acid amount and the catalyst substrate under these operating conditions. 

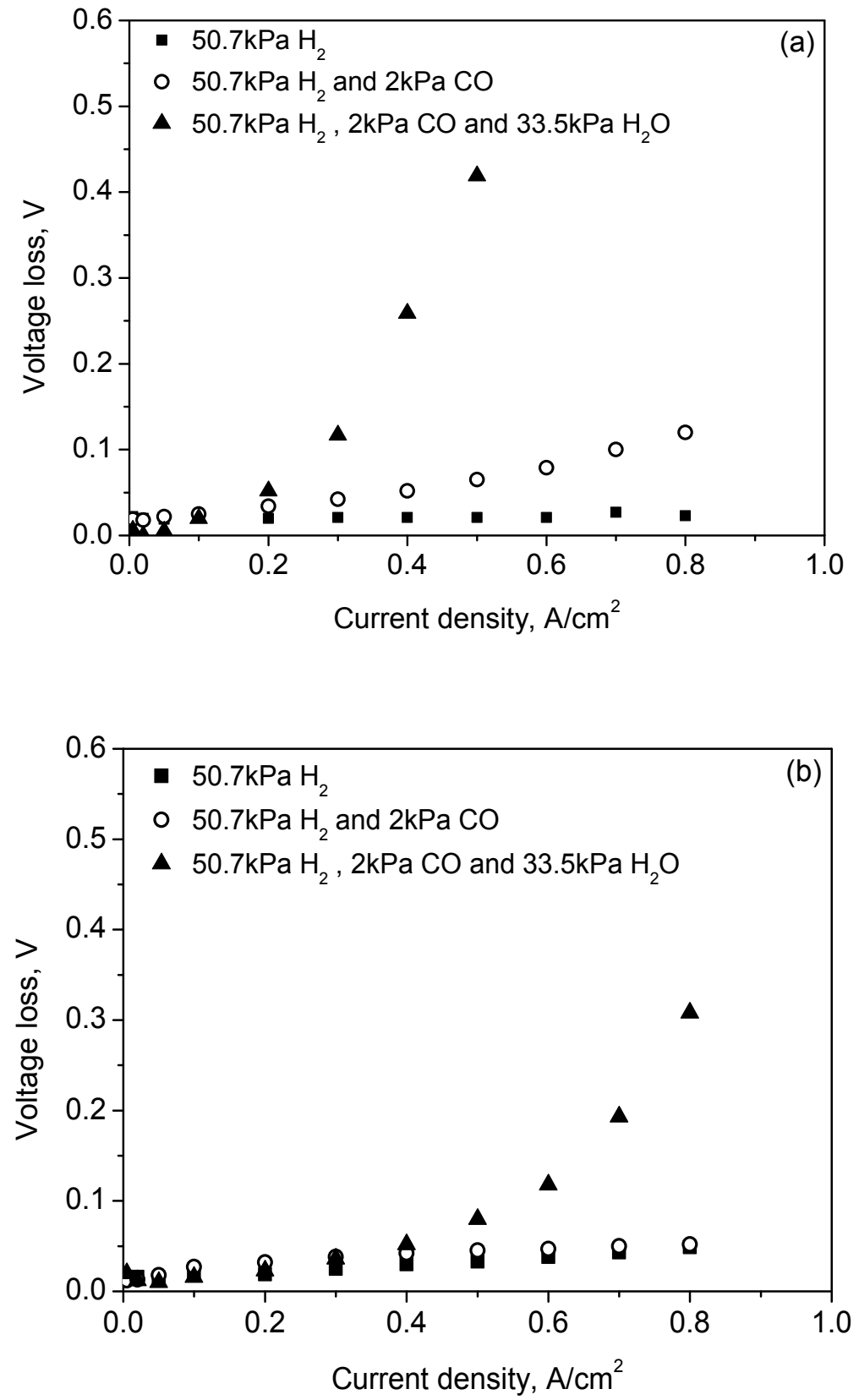

Figure 6-9. Voltage loss, IR drop corrected, at $180^{\circ} \mathrm{C}$ for MEAs employing a. $30 \mathrm{wt} \%$ Pt/ox.MWCNT MEA 7: $0.77 \mathrm{mgPt} / \mathrm{cm}^{2}$ and b. $30 \mathrm{wt} \%$ Pt/ox.MWCNT-Py MEA 1: 0.77 $\mathrm{mgPt} / \mathrm{cm}^{2}$, under various fuel composition and pure $\mathrm{O}_{2}$ flows $(200 \mathrm{cc} / \mathrm{min}$ respectively): Black square: $50.7 \mathrm{kPaH}_{2}$ balanced with $\mathrm{Ar}$, open circle: $50.7 \mathrm{kPa} \mathrm{H} 2$ and $2 \mathrm{kPa} \mathrm{CO}$ balanced with $\mathrm{Ar}$, and black triangle : $50.7 \mathrm{kPa} \mathrm{H} 2,2 \mathrm{kPa} \mathrm{CO}$ and $33.5 \mathrm{kPa} \mathrm{H}_{2} \mathrm{O}$ balanced with Ar. The PA loading was $2 \mathrm{gPA} / \mathrm{gPt}$ at the anodic electrode. 

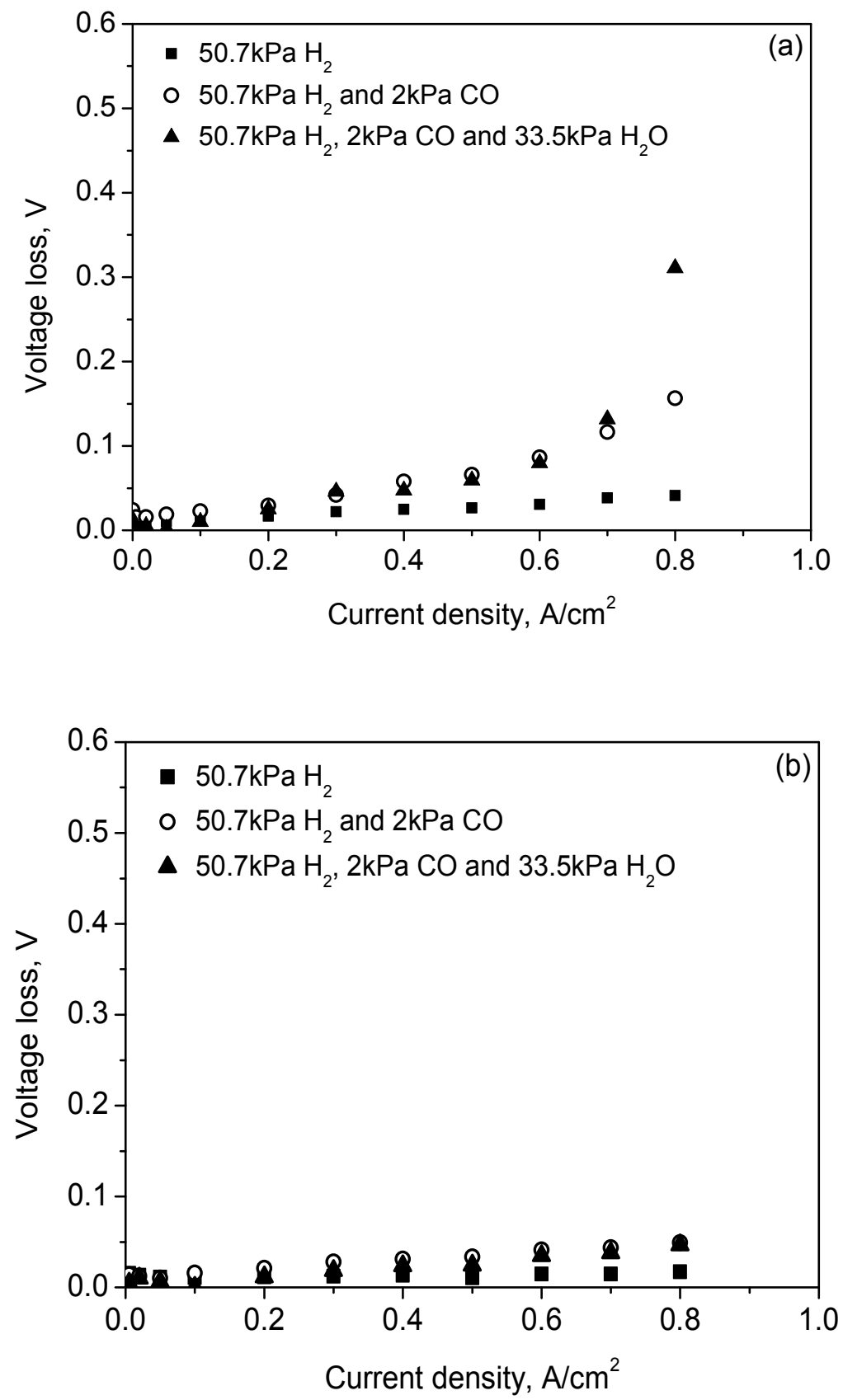

Figure 6-10. Voltage loss, IR drop corrected, at $180^{\circ} \mathrm{C}$ for MEAs employing a. $30 \mathrm{wt} \%$ Pt/oxMWNT, MEA 9: $0.79 \mathrm{mgPt} / \mathrm{cm}^{2}$ and b. 30wt\% Pt/(ox.MWCNT)-Py MEA 6: 0.82 $\mathrm{mgPt} / \mathrm{cm}^{2}$,under various fuel composition and pure $\mathrm{O}_{2}$ flows $(200 \mathrm{cc} / \mathrm{min}$ respectively): Black square: $50.7 \mathrm{kPaH}_{2}$ balanced with $\mathrm{Ar}$, open circle: $50.7 \mathrm{kPa} \mathrm{H} 2$ and $2 \mathrm{kPa} \mathrm{CO}$ balanced with $\mathrm{Ar}$, and black triangle : $50.7 \mathrm{kPa} \mathrm{H} 2,2 \mathrm{kPa} \mathrm{CO}$ and $33.5 \mathrm{kPa} \mathrm{H}_{2} \mathrm{O}$ balanced with $\mathrm{Ar}$. The PA loading was $0.5 \mathrm{gPA} / \mathrm{gPt}$ at the anodic electrode. 


\subsubsection{AC Impedance Measurements}

Figure 6-11 and 6-12 show the change in the polarization resistance with respect to the gas fed composition of the anodic electrode for the two modified carbon nanotube based electrocatalyst (MEA 1 and MEA 7) for a PA loading of $2 \mathrm{gPA} / \mathrm{gPt}$ in the CL. The AC impedance spectra were acquired under the same conditions as described in section 6.3.3 and at a current density of $0.2 \mathrm{~A} / \mathrm{cm}^{2}$.

Two arcs are observed. The first one, which appears at high frequencies corresponds to the impedance of the $\mathrm{H}_{2}$ oxidation reaction at the anode side [22,23], as thoroughly discussed in chapter 5. Although, others claim that the high frequency arc is related to the proton conduction in the catalyst layer [24]. Whereas for the second arc appearing in the intermediate and low frequency, the general consensus is that it originates from activation losses and from dominated cathode effects. Some research groups claim that it originates from the combination of anode and cathode activation $[25,26]$. The polarization resistance of the anodic electrode was calculated by fitting the acquired data to an appropriate equivalent circuit model, using software provided by Autolab. The polarization resistance values as calculated under various fuel compositions are summarized in table 6-2.

Table 6-2. The effect of the fuel composition with respect to the PA loading, on the polarization resistance of the anodic electrodes.

\begin{tabular}{|c|c|c|c|}
\hline \multirow[b]{2}{*}{$\begin{array}{l}\text { MEA/ catalyst type } \\
\text { and PA loading }\end{array}$} & \multicolumn{3}{|c|}{ Anode's polarization resistance, $\mathrm{Rp}\left(\mathrm{Ohm} * \mathrm{~cm}^{2}\right)$} \\
\hline & $50.7 \mathrm{kPa} \mathrm{H}$ & $\begin{array}{c}50.7 \mathrm{kPa} \mathrm{H} \text { and } \\
2 \mathrm{kPa} \mathrm{CO}\end{array}$ & $\begin{array}{c}50.7 \mathrm{kPa} \mathrm{H} \text {, } \\
2 \mathrm{kPa} \mathrm{CO} \text { and } \\
33.5 \mathrm{kPa} \mathrm{H} \mathrm{H}_{2} \mathrm{O}\end{array}$ \\
\hline \multicolumn{4}{|l|}{ 30wt\% Pt/oxMWCNT-Py } \\
\hline $\begin{array}{ccc}\text { MEA } & 1 & 2 \mathrm{gPA} / \mathrm{gPt} \\
\mathrm{MEA} & 6 & 0.5 \mathrm{gPA} / \mathrm{gPt}\end{array}$ & $\begin{array}{c}0.0928 \\
0.906\end{array}$ & $\begin{array}{l}0.124 \\
0.113\end{array}$ & $\begin{array}{l}0.144 \\
0.135\end{array}$ \\
\hline \multicolumn{4}{|l|}{$30 w t \% \mathrm{Pt} / \mathrm{oxMWCNT}$} \\
\hline $\begin{array}{c}\text { MEA } 7 \quad 2 \mathrm{gPA} / \mathrm{gPt} \\
\text { MEA } 90.5 \mathrm{gPA} / \mathrm{gPt}\end{array}$ & $\begin{array}{l}0.0828 \\
0.0889\end{array}$ & $\begin{array}{c}0.1804 \\
0.176\end{array}$ & $\begin{array}{l}0.457 \\
0.257\end{array}$ \\
\hline \multicolumn{4}{|l|}{$30 \mathrm{wt} \% \mathrm{Pt} / \mathrm{C}$} \\
\hline $\begin{array}{ccc}\text { MEA } & 11 & 2 \mathrm{gPA} / \mathrm{gPt} \\
\mathrm{MEA} & 13 & 0.5 \mathrm{gPA} / \mathrm{gPt}\end{array}$ & $\begin{array}{l}0.0873 \\
0.0824\end{array}$ & $\begin{array}{l}0.176 \\
0.168\end{array}$ & $\begin{array}{l}0.476 \\
0.279\end{array}$ \\
\hline
\end{tabular}


The polarization resistance of the high frequency arc of MEA 1 (30\% Pt/oxMWCNT-Py), undergoes very small change as $2 \mathrm{kPa} \mathrm{CO}$ is added to the fuel feed $(50.7 \mathrm{kPa} \mathrm{H}$ balanced with Ar), 0.0928 and $0.124 \mathrm{Ohm}^{*} \mathrm{~cm}^{2}$, respectively(fig. 6-11a). For the case of $30 \%$ Pt/ox.MWCNT (MEA 7), a substantial increase in the polarization resistance is observed. In the absence of $\mathrm{CO}$ a value of $0.0828 \mathrm{Ohm}^{*} \mathrm{~cm}^{2}$ was determined, whereas 0.1804 Ohm* $\mathrm{cm}^{2}$ for $2 \mathrm{kPa} \mathrm{CO}$ (fig. 6-12a). It has to be noted that both MEAs had almost identical specta under $50.7 \mathrm{kPa} \mathrm{H}$. The difference in the anode's charge transfer resistance in the presence of $\mathrm{CO}$ between the two carbon nanotube supported electrocatlayst, indicates that the $\mathrm{H}_{2}$ oxidation rate has been reduced due to $\mathrm{CO}$ adsorption and blockage of $\mathrm{Pt}$ active sites. It is expected that the $30 \% \mathrm{Pt} / \mathrm{ox}$.MWCNT would present a higher polarization resistance, as the ECSA was smaller than in the case of the 30\%Pt/ox.MWNT-Py. Similar polarization resistance values with MEA 7, were obtained for MEA11 employing 30wt\% $\mathrm{Pt} / \mathrm{C}$. where the anodic polarization resistance increased in the presence of $2 \mathrm{kPa} \mathrm{CO}$. Additionally, since only the first arc suffers any changes in the presence of $\mathrm{CO}$, indicates that the charge transfer process originates only from the anodic electrode.

In all cases, the resistance of the membrane, Rel, shifts to lower values under synthetic reformate gas fed. This is to be expected, as the water hydrates the membrane, thus increasing the proton conductivity. Water is reported to improve proton conductivity both in the membrane and in the electrodes due to its effect on acid equilibrium dehydration reaction [27,28,29,30,31,32]. The charge transfer resistance of MEA 1, employing $30 \% \mathrm{Pt} / \mathrm{oxMWNT}-\mathrm{Py}$, reached a value of $0.144 \mathrm{Ohm}^{*} \mathrm{~cm}^{2}$ (figure $6-11 \mathrm{a}$ ). Whereas for the case of $30 \% \mathrm{Pt} / \mathrm{oxMWNT}, 0.456 \mathrm{Ohm}^{*} \mathrm{~cm}^{2}$, figure 6-12a. MEA 11, employing $30 \% \mathrm{Pt} / \mathrm{C}$, exhibited similar values with MEA 7. The increase in the polarization resistance, indicates that the charge transfer rate due to $\mathrm{H}_{2}$ oxidation rate has been reduced by both $\mathrm{CO}$ and steam, leading to the reduction of the available active free Pt sites. 

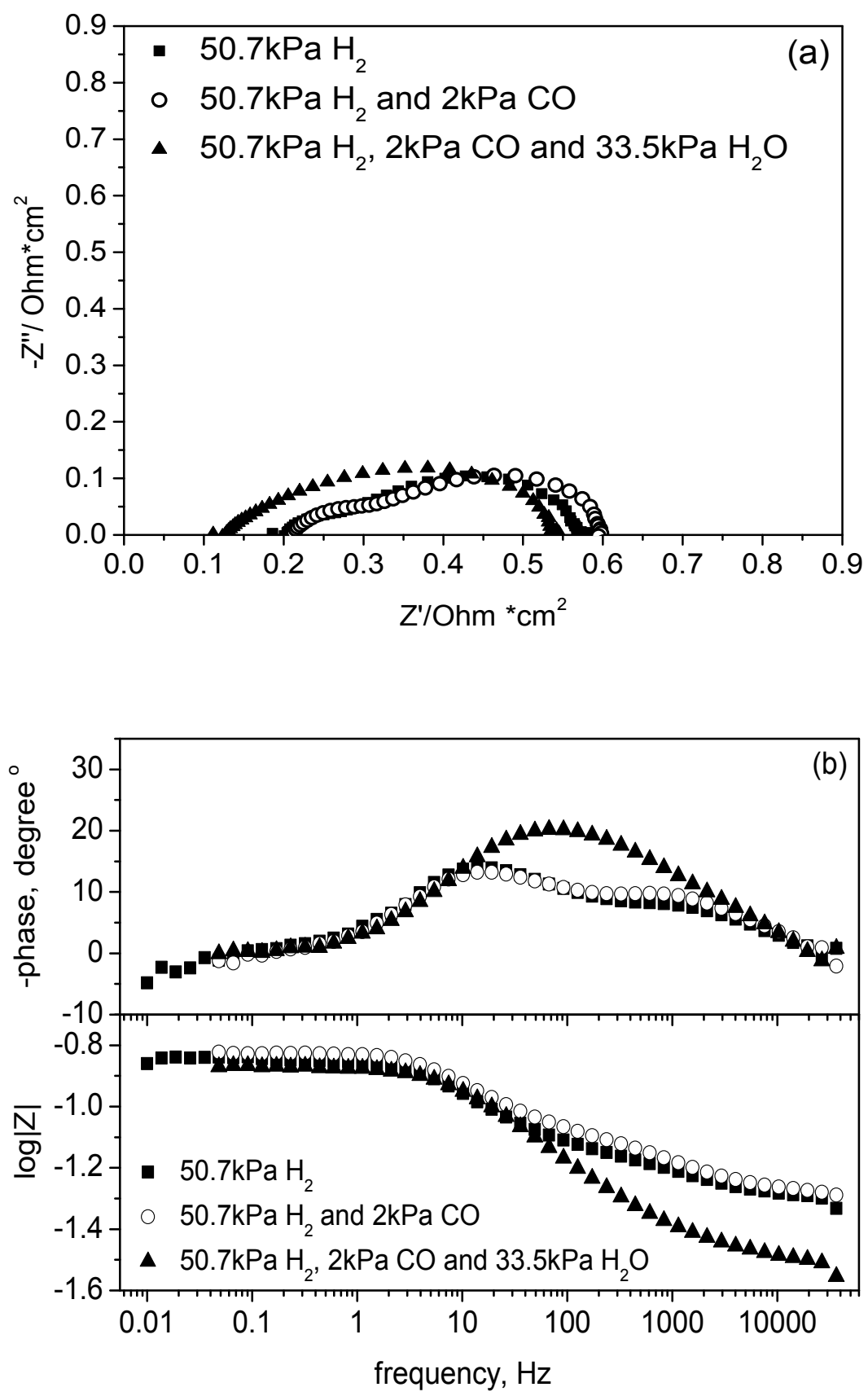

Figure 6-11. AC impedance plots a. Nyquist and b. Bode, obtained at a current of $0.2 \mathrm{~A} / \mathrm{cm}^{2}$ at $180^{\circ} \mathrm{C}$, under various fuel compositions and pure $\mathrm{O}_{2}$ flows $\left(200 \mathrm{cc} / \mathrm{min}\right.$ fuel mixture $\left./ \mathrm{O}_{2}\right)$. Black square: $50.7 \mathrm{kPa} \mathrm{H} \mathrm{H}_{2}$ balanced with $\mathrm{Ar}$, open circle: $50.7 \mathrm{kPaH}_{2}$ and $2 \mathrm{kPa} \mathrm{CO}$ balanced with Ar, and black triangle: $50.7 \mathrm{kPa} \mathrm{H}_{2}, 2 \mathrm{kPa} \mathrm{CO}$ and $33.5 \mathrm{kPa} \mathrm{H}_{2} \mathrm{O}$ balanced with Ar. MEA 1 employing 30wt\% Pt/(ox.MWCNT)-Py: $0.77 \mathrm{mgPt} / \mathrm{cm}^{2}$ and $2 \mathrm{gPA} / \mathrm{gPt}$. 

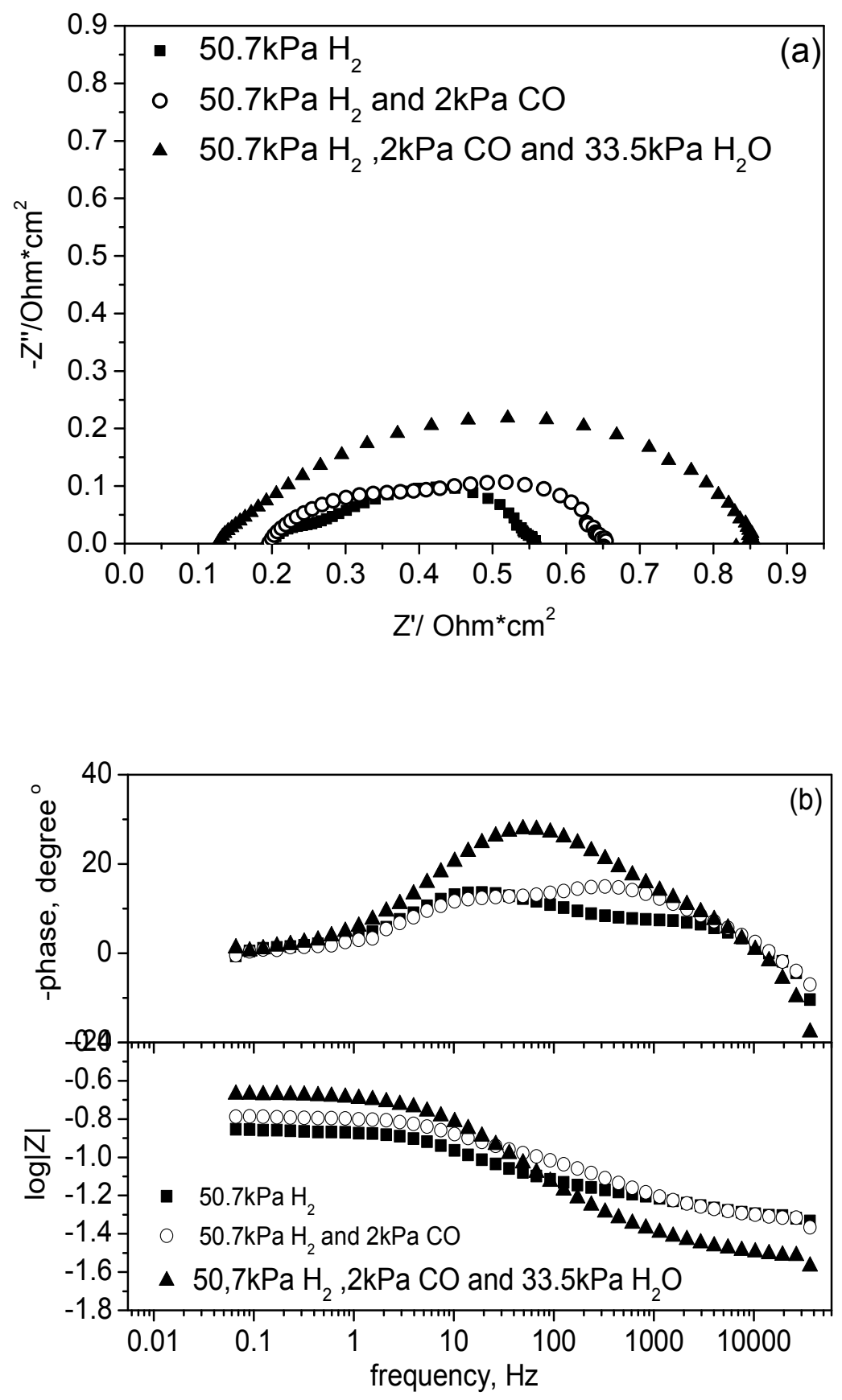

Figure 6-12. AC impedance plots a. Nyquist and b. Bode, obtained at a current of $0.2 \mathrm{~A} / \mathrm{cm}^{2}$ at $180^{\circ} \mathrm{C}$, under various fuel compositions and pure $\mathrm{O}_{2}$ flows $\left(200 \mathrm{cc} / \mathrm{min}\right.$ fuel mixture $\left./ \mathrm{O}_{2}\right)$. Black square: $50.7 \mathrm{kPa} \mathrm{H}$ balanced with $\mathrm{Ar}$, open circle: $50.7 \mathrm{kPaH}_{2}$ and $2 \mathrm{kPa} \mathrm{CO}$ balanced with $\mathrm{Ar}$, and black triangle: $50.7 \mathrm{kPa} \mathrm{H}, 2 \mathrm{kPa} \mathrm{CO}$ and $33.5 \mathrm{kPa} \mathrm{H} \mathrm{H}_{2} \mathrm{O}$ balanced with Ar. MEA 7 employing30wt\% Pt/oxMWNT: $0.77 \mathrm{mgPt} / \mathrm{cm}^{2}$ and $2 \mathrm{gPA} / \mathrm{gPt}$. 

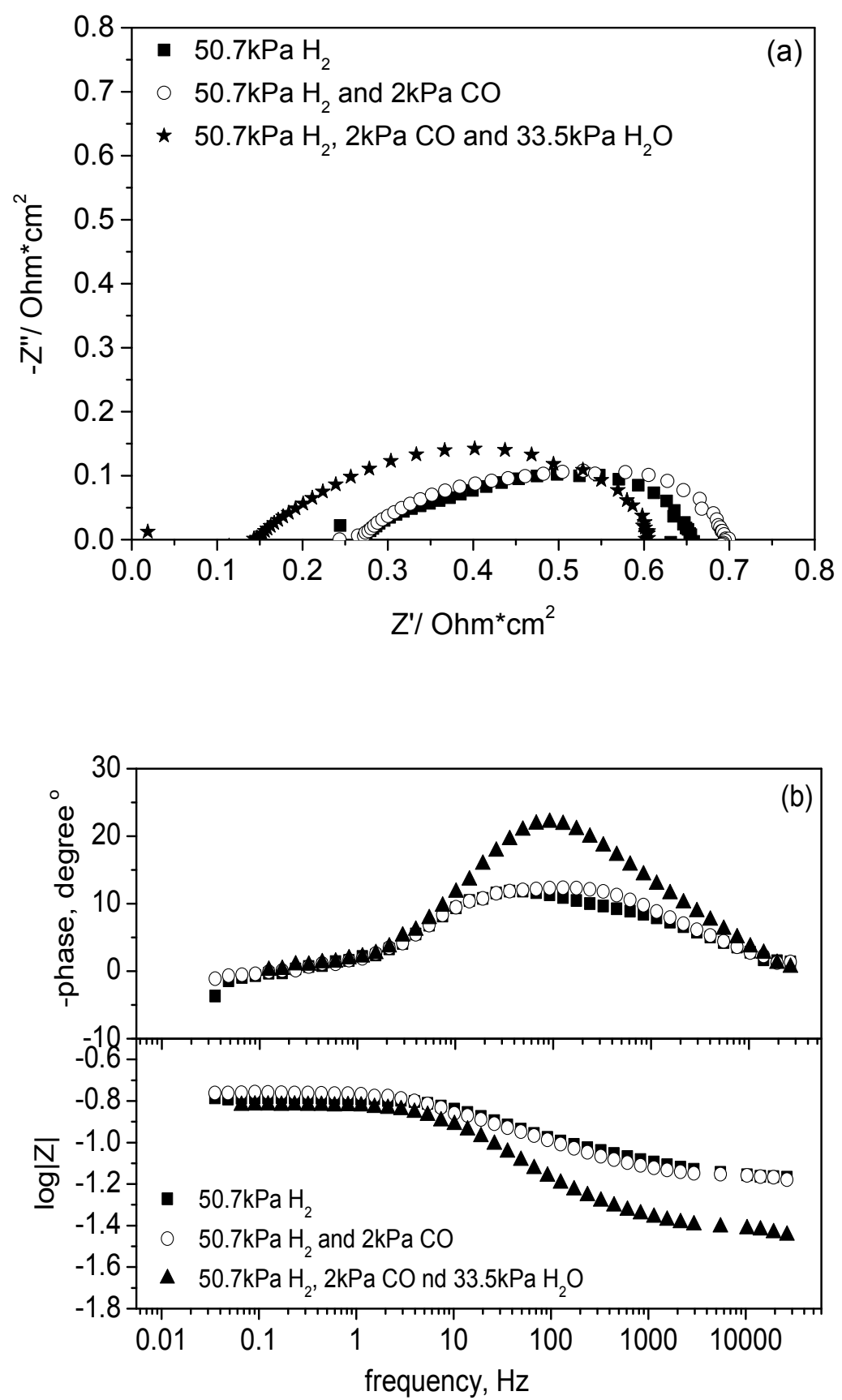

Figure 6-13. AC impedance plots a. Nyquist and b. Bode, obtained at a current of $0.2 \mathrm{~A} / \mathrm{cm}^{2}$ at $180^{\circ} \mathrm{C}$, under various fuel compositions and pure $\mathrm{O}_{2}$ flows $\left(200 \mathrm{cc} / \mathrm{min}\right.$ fuel mixture $\left./ \mathrm{O}_{2}\right)$. Black square: $50.7 \mathrm{kPa} \mathrm{H}$ balanced with $\mathrm{Ar}$, open circle: $50.7 \mathrm{kPaH}_{2}$ and $2 \mathrm{kPa} \mathrm{CO}$ balanced with $\mathrm{Ar}$, and black triangle : $50.7 \mathrm{kPa} \mathrm{H}_{2}, 2 \mathrm{kPa} \mathrm{CO}$ and $33.5 \mathrm{kPa} \mathrm{H}_{2} \mathrm{O}$ balanced with Ar. MEA 6employing30wt\% Pt/(ox.MWCNT)-Py: $0.82 \mathrm{mgPt} / \mathrm{cm}^{2}$ and $0.5 \mathrm{gPA} / \mathrm{gPt}$. 

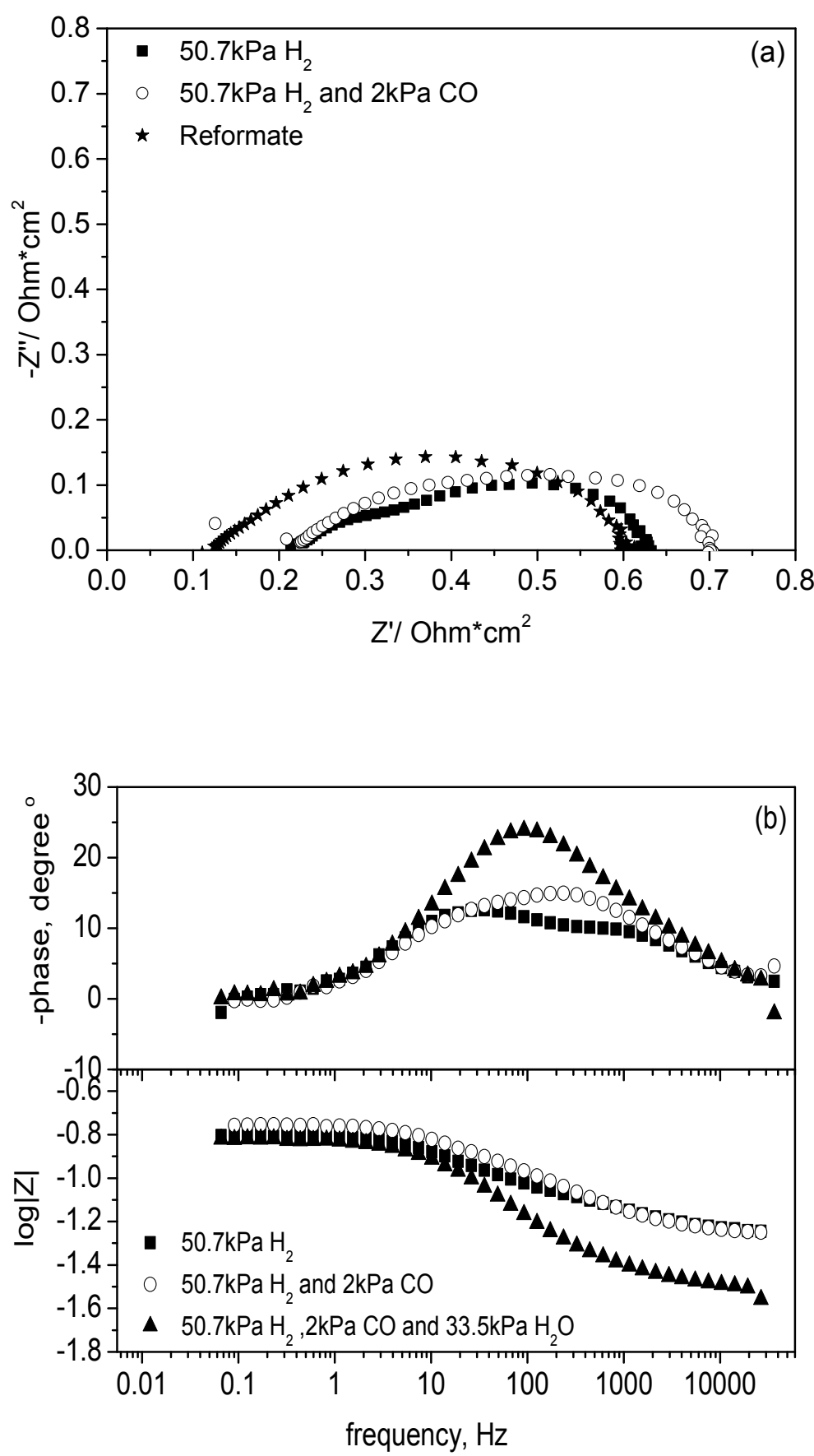

Figure 6-14. AC impedance plots a. Nyquist and b. Bode, obtained at a current of $0.2 \mathrm{~A} / \mathrm{cm}^{2}$ at $180^{\circ} \mathrm{C}$, under various fuel compositions and pure $\mathrm{O}_{2}$ flows $\left(200 \mathrm{cc} / \mathrm{min}\right.$ fuel mixture $\left./ \mathrm{O}_{2}\right)$. Black square: $50.7 \mathrm{kPa} \mathrm{H} \mathrm{H}_{2}$ balanced with $\mathrm{Ar}$, open circle: $50.7 \mathrm{kPa} \mathrm{H}_{2}$ and $2 \mathrm{kPa} \mathrm{CO}$ balanced with $\mathrm{Ar}$, and black triangle: $50.7 \mathrm{kPa} \mathrm{H}_{2}, 2 \mathrm{kPa} \mathrm{CO}$ and $33.5 \mathrm{kPa} \mathrm{H}_{2} \mathrm{O}$ balanced with $\mathrm{Ar}$. MEA9 employing 30wt $\% \mathrm{Pt} / \mathrm{oxMWNT:} 0.79 \mathrm{mgPt} / \mathrm{cm}^{2}$ and $0.5 \mathrm{gPA} / \mathrm{gPt}$. 
The difference in the polarization resistance of the anodic electrode under synthetic reformate gas among the three studied catalyst, is also related to the nature of the carbon support, table 6-2. Lowering the PA in the catalyst layer to $0.5 \mathrm{gPA} / \mathrm{gPt}$, didn't alter the pattern concerning the charge transfer resistance of the anode with respect to the fuel composition, figure 6-13 and 6-14.

\subsubsection{Stability test}

Commercially available HT-PEM MEAs require stable operation of the anode under reformate gas, especially under lean partial pressure of $\mathrm{H}_{2}$, increased $\mathrm{CO}$ and steam mole fraction. Reducing the amount of $\mathrm{Pt}$ in the CL, without sacrificing the stability and performance of the MEA is highly desired. Figure 6-15 depicts a short term stability experiment using synthetic reformate gas , consisting of $50.7 \mathrm{kPa} \mathrm{H} 2,2 \mathrm{kPa} \mathrm{CO}$ and $33.5 \mathrm{kPa}$ $\mathrm{H}_{2} \mathrm{O}$ balanced with $\mathrm{Ar}$, at $180^{\circ} \mathrm{C}$. The applied current density was set at $0.2 \mathrm{~A} / \mathrm{cm}^{2}$ and the stoichiometry of the $\mathrm{H}_{2}$ and $\mathrm{O}_{2}$ was $\lambda_{\mathrm{H} 2}=3$ and $\lambda_{\mathrm{O} 2}=5$, respectively. The platinum loading of the anodic electrodes employing MWCNT based electrocatalyst was approximately $0.8 \mathrm{mgPt} / \mathrm{cm}^{2}$ (MEA 2 and MEA 8) whereas $1.42 \mathrm{mgPt} / \mathrm{cm}^{2}$ for the case of $30 \% \mathrm{Pt} / \mathrm{C}$ (MEA 10). The amount of PA in the CL was $2 \mathrm{gPA} / \mathrm{gPt}$.

Under such harsh operating conditions the pyridine functionalized MWCNT based catalyst is remarkably stable as compared to the other two. The degradation rate for the $30 \% \mathrm{Pt} / \mathrm{oxMWCNT}$-Py was $184.6 \mu \mathrm{V} / \mathrm{h}$, whereas $12.76 \mathrm{mV} / \mathrm{h}$ for the $30 \% \mathrm{Pt} / \mathrm{oxMWCNT}$ and $182 \mathrm{mV} / \mathrm{h}$ for the $30 \% \mathrm{Pt} / \mathrm{C}$. The MEA's voltage, employing the conventional catalyst, severely declined over a period of 2 hours, reaching approximately $0.235 \mathrm{~V}$ at $0.2 \mathrm{~A} / \mathrm{cm}^{2}$. Despite the favorable gas stoichiometry and the low current density, the carbon black based electrode performed poorly, even with a Pt loading as high as $1.42 \mathrm{mgPt} / \mathrm{cm}^{2}$.

Decreasing the PA amount in the catalyst layer the stability of the cell's voltage increased under synthetic reformate conditions, figure 6-16. The tested MEAs had the same Pt loading, $0.8 \mathrm{gPt} / \mathrm{cm}^{2}$, for immediate comparison (MEA 6, 9 and 15). The fuel cells were left under continuous operation for approximately 50 hours at $0.4 \mathrm{~A} / \mathrm{cm}^{2}$ with a gas stoichiometry of $\lambda_{\mathrm{H} 2}=1.5$ and $\lambda_{\mathrm{O} 2}=2.5$. The MEA employing $30 \% \mathrm{Pt} /(\mathrm{ox} . \mathrm{MWCNT})-\mathrm{Py}$ exhibited the higher stability, where for approximately 50 hours of continuous operation did not exhibit any degradation, despite the low stoichiometry of $\mathrm{H}_{2}$. On the contrary, the catalyst supported on ox.MWCNT exhibited a degradation rate of $134.2 \mu \mathrm{V} / \mathrm{h}$. Whereas the carbon supported electrocatalyst (MEA 15) could not sustain the HOR under these conditions. When the noble metal loading was doubled for the case of $30 \% \mathrm{Pt} / \mathrm{C}$, MEA 13 $\left(1.60 \mathrm{mgPt} / \mathrm{cm}^{2}\right)$, under the same experimental conditions the fuel cell exhibited higher stability than the $30 \% \mathrm{Pt} / \mathrm{oxMWNT}$ catalyst, having a degradation rate of $8 \mathrm{uV} / \mathrm{h}$. 


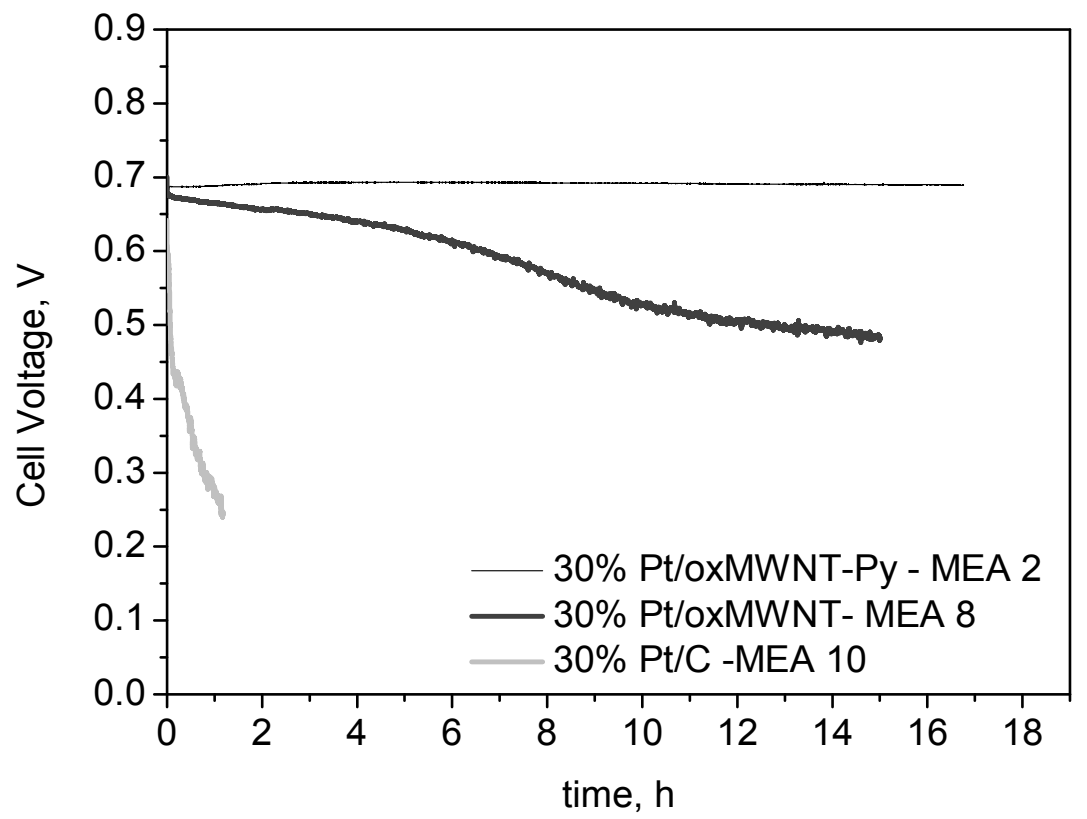

Figure 6-15. Short term stability experiment using synthetic reformate gas at $0.2 \mathrm{~A} / \mathrm{cm}^{2}$ $\left(\lambda_{\mathrm{H} 2}=3\right.$ and $\left.\lambda_{\mathrm{O} 2}=5\right)$ and $180^{\circ} \mathrm{C}$. The amount of PA in the CL was $2 \mathrm{gPA} / \mathrm{gPt}$ and the MEA that were tested: MEA 2: $30 \mathrm{wt} \% \mathrm{Pt} / \mathrm{oxMWNT}-\mathrm{Py}$ and $0.87 \mathrm{mgPt} / \mathrm{cm}^{2}$, MEA 8: $30 \mathrm{wt} \% \mathrm{Pt} / \mathrm{oxMNWT}$ and $0.875 \mathrm{mgPt} / \mathrm{cm}^{2}$ and MEA $10: 30 \mathrm{wt} \% \mathrm{Pt} / \mathrm{C}$ and $1.42 \mathrm{mgPt} / \mathrm{cm}^{2}$.

A stability test was performed using low Pt loadings $\left(\sim 0.8 \mathrm{mgPt} / \mathrm{cm}^{2}\right)$, low PA loadings $(0.5 \mathrm{gPA} / \mathrm{gPt})$ and low water content $\left(14.1 \mathrm{kPa} \mathrm{H}_{2} \mathrm{O}\right)$, figure 6-17. The gas stoichiometry was respectively $\lambda_{\mathrm{H} 2}=1.5$ and $\lambda_{\mathrm{O} 2}=2.5$ and the reformate gas consisted of $50.7 \mathrm{kPa} \mathrm{H} 2,2 \mathrm{kPa}$ $\mathrm{CO}$ and $14.1 \mathrm{kPa} \mathrm{H}_{2} \mathrm{O}$ balanced with Ar. MEA $14\left(0.7 \mathrm{mgPt} / \mathrm{cm}^{2}\right.$ and $\left.0.5 \mathrm{gPA} / \mathrm{gPt}\right)$ employing $30 \mathrm{wt} \% \mathrm{Pt} / \mathrm{C}$, was left for $140 \mathrm{~h}$ of continuous operation at $0.4 \mathrm{~A} / \mathrm{cm}^{2}$ and a cell temperature of $180^{\circ} \mathrm{C}$. Initially the cells voltage was $620 \mathrm{mV}$ and declined to $600 \mathrm{mV}$ over a period of $20 \mathrm{~h}$. The cell's voltage was then stabilized and was monitored for another $90 \mathrm{~h}$ where the degradation rate was found to be $23.6 \mu \mathrm{V} / \mathrm{h}$. MEA 5, employing $30 \mathrm{wt} \% \mathrm{Pt} / \mathrm{oxMWCNT}-\mathrm{Py}(0.68 \mathrm{mgPt} / \mathrm{cm} 2$ and $0.5 \mathrm{gPA} / \mathrm{gPt})$, was tested under the same experimental conditions. The later exhibited better performance and stability. The cell's voltage was stabilized at $614 \mathrm{mV}$ after $20 \mathrm{~h}$ and the degradation rate for the next $80 \mathrm{~h}$ was $7 \mu \mathrm{V} / \mathrm{h}$. - 


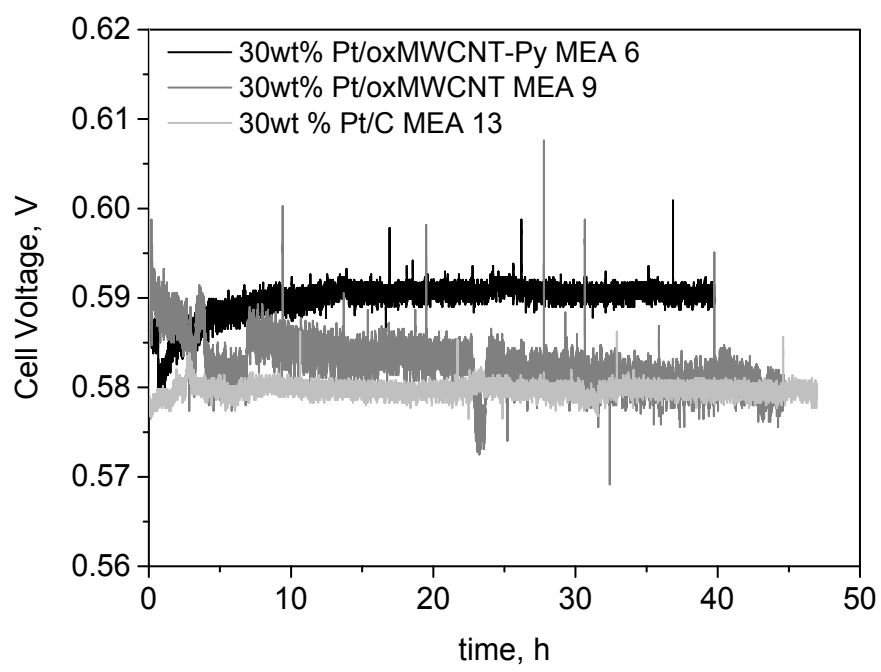

Figure 6-16. Short term stability experiment using synthetic reformate gas, consisting of $50.7 \mathrm{kPa} \mathrm{H}, 33.5 \mathrm{kPa} \mathrm{H} \mathrm{H}_{2} \mathrm{O}$ and $2 \mathrm{kPa} \mathrm{CO}$ balanced with $\mathrm{Ar}$, at $0.4 \mathrm{~A} / \mathrm{cm}^{2}\left(\lambda_{\mathrm{H} 2}=1.5\right.$ and $\lambda_{\mathrm{O} 2}=2.5$ ) and $180^{\circ} \mathrm{C}$. The amount of PA in the CL was $0.5 \mathrm{gPA} / \mathrm{gPt}$ and the MEA that were tested: MEA 6: 30wt\%Pt/oxMWNT-Py and 0.82mgPt/cm2, MEA 9: 30wt\%Pt/oxMNWT and $0.79 \mathrm{mgPt} / \mathrm{cm} 2$ and MEA $13: 30 \mathrm{wt} \% \mathrm{Pt} / \mathrm{C}$ and $1.6 \mathrm{mgPt} / \mathrm{cm}^{2}$.

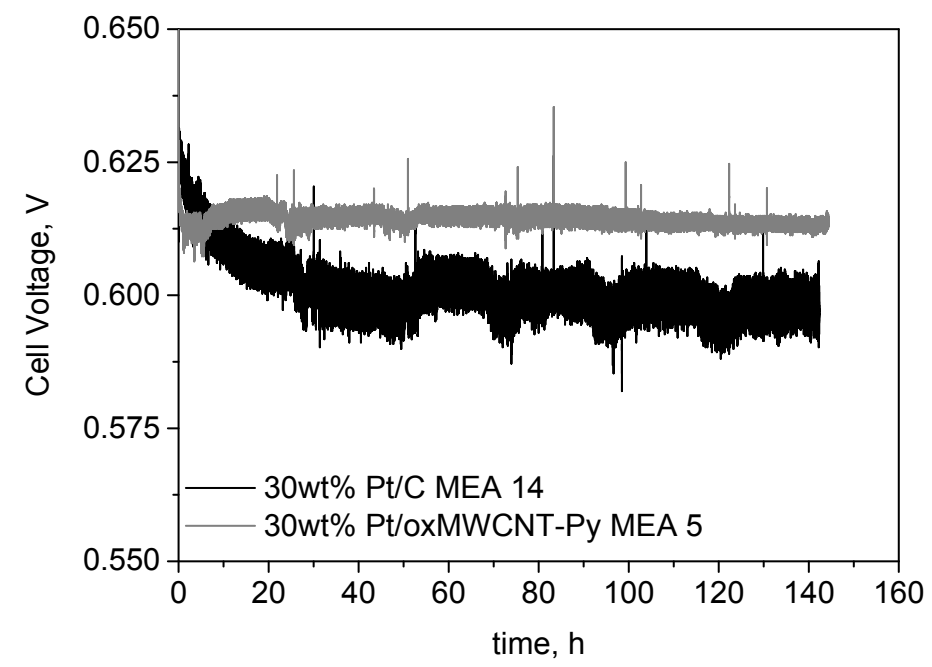

Figure 6-17. Short term stability experiment using synthetic reformate gas (consisting of $50.7 \mathrm{kPa}$ of $\mathrm{H} 2,2 \mathrm{kPa}$ of $\mathrm{CO}$ and $14.1 \mathrm{kPa}$ of $\mathrm{H}_{2} \mathrm{O}$ balanced with $\left.\mathrm{Ar}\right)$ at $0.4 \mathrm{~A} / \mathrm{cm}^{2}\left(\lambda_{\mathrm{H} 2}=1.5\right.$ and $\lambda_{\mathrm{O} 2}=2.5$ ) and $180^{\circ} \mathrm{C}$. The amount of PA in the CL was $0.5 \mathrm{gPA} / \mathrm{gPt}$ and the MEAs that were tested: MEA 5: 30wt \%Pt/oxMWNT-Py and $0.68 \mathrm{mgPt} / \mathrm{cm}^{2}$ and MEA 14: $30 \mathrm{wt} \%$ $\mathrm{Pt} / \mathrm{C}$ and $0.7 \mathrm{mgPt} / \mathrm{cm}^{2}$. 
6.3.6 Potential Oscillations under galvanostatic electrooxidation of $\mathrm{H}_{2}$ in reformate gas.

Potential oscillations were observed during the acquisition of the polarization curves under galvanostatic mode, when synthetic reformate gas was fed at the anodic compartment. These potential perturbation occurred when $2 \mathrm{gPA} / \mathrm{gPt}$ were imbedded in the anodic catalyst layer, especially above a current density of about $0.4 \mathrm{~A} / \mathrm{cm}^{2}$. The amplitude of these potential perturbations depended on the applied current density, as shown in figure 6-18. The potential oscillations had no pattern and no specific frequency and only occurred for low total reactant flow rates $\left(\lambda_{\mathrm{H} 2}=1.2\right)$.

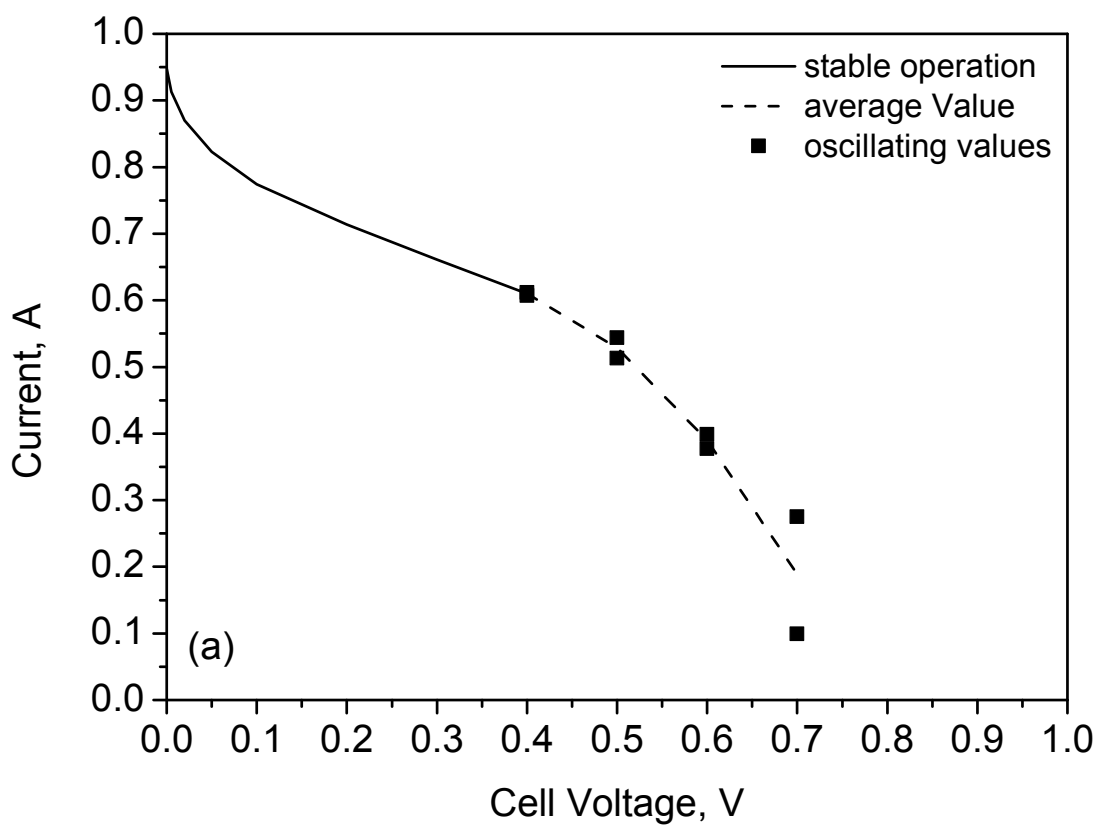

Figure 6-18. Polarization curve (MEA 1) obtained under synthetic reformate gas consisting of $50.7 \mathrm{kPa}$ of $\mathrm{H}_{2}, 33.5 \mathrm{kPa}$ of $\mathrm{H}_{2} \mathrm{O}$ and $2 \mathrm{kPa} \mathrm{CO}$ balanced with $\mathrm{Ar}$, and a stoichiometry of $\lambda_{\mathrm{H} 2}=1.2$ and $\lambda_{\mathrm{O} 2}=2$.

Kaserer et al also observed voltage oscillations by adding $\mathrm{CO}$ and water to the anode of a high temperature PEM fuel cell at high constant current densities [33]. Using a reference electrode assembly, it was confirmed that the oscillations are related to processes at the anode side. They were attributed to the cleaning of the catalyst surface by the oxidation of $\mathrm{CO}$ through adsorbed $\mathrm{OH}$. Nevertheless, they also suggested different or additional effects from other adsorbates like $\mathrm{H}_{3} \mathrm{PO}_{4}$, since the adsorption of $\mathrm{OH}$ on $\mathrm{Pt}$ surfaces takes place at 
potentials lower than $0.6 \mathrm{~V}$, far from the existing anode overpotentials under normal fuel cell operation. Thayane C.M. Nepel et al. [20] using an online mass spectrometer observed that significant $\mathrm{CO}_{2}$ production occurred at overpotential higher than $0.25 \mathrm{~V}$, using a $\mathrm{Pt} / \mathrm{C}$ electrocatalyst at $105^{\circ} \mathrm{C}$. Modestov et al [34], reported that $\mathrm{CO}$ electrooxidation can hardly play significant role in $\mathrm{CO}$ tolerance of $\mathrm{Pt}$ and $\mathrm{Pt}-\mathrm{Ru}$ anodes of PEM FC with PBI-PA membranes in a practically important regime of operation. As will be discussed in below, the oscillations were a result of the rise of the local overpotential in the anodic electrode due to the shrinkage of the electrochemical interface due to ganglia formation.

\subsection{Discussion}

\section{Kinetic of $\mathrm{H}_{2}$ electrooxidation}

To summarize, for the reader's convenience, individually water or $\mathrm{CO}$ do not affect severely the anode performance (figure 6-9 and 6-10). However, their combination in the reformate fuel mixture, lead to a severe voltage loss during operation, exhibiting a dependence on the water partial pressure in the reformate mixture and the PA amount imbedded in the CL (figure 6-4, 6-5 and 6-6, 6-7, 6-8 ). Therefore $\mathrm{H}_{2}$ co-adsorption with water and $\mathrm{CO}$ on the Pt surface cannot alone explain this dramatic loss in the cell's performance. The performance loss can be attributed to the loss of the specific electrochemically active surface area. The later will cause the severe decrease in the electrocatalytic activity, either due to the decrease in the exchange current density or due to a change of the interfacial kinetic mechanism of the electrochemical reaction. In the first case, a parallel shift of the Tafel plot is expected toward lower current densities. In the second case significant change in the Tafel slope can be observed in addition to the change in the exchange current density.

In figures 6-3 and 6-7, a change of the slope in the low performance region was obsereved, which implies a transition to a slower kinetic mechanism regarding $\mathrm{H}_{2}$ oxidation reaction. This can be attributed to the strong dependence of the HOR reaction mechanism on the partial pressure of $\mathrm{H}_{2}$. The strong dependence of the reaction kinetics on the partial pressure of $\mathrm{H}_{2}$ is shown by the $\mathrm{AC}$ impedance spectra recorded at various partial pressures of $\mathrm{H}_{2}$ and at the same current density (Figure 6-19). The spectra were recorded at high excess of the reacting gasses to ensure their uniform partial pressure distribution over the electrode's surface. The monitoring of the spectra at the same current density allows for the isolation of the effect of the partial pressure of $\mathrm{H}_{2}$ on certain features of the AC spectra that characterize the response of the anode's electrokinetic behavior. In this respect cathode remains unaffected. Thus, as it is clearly shown in Fig. 6- the variation of the polarization 
resistance of the high frequency arc is definitely attributed to the anode. Eq. 6-3, is derived from the Tafel equation and it relates the polarization resistance $R_{p}$ of the EI with the applied current I and the transfer coefficient $\alpha$ or otherwise the Tafel slope $\alpha \mathrm{F} / \mathrm{RT}$.

$$
R_{p}=\frac{1}{I} \frac{R T}{\alpha F}
$$

The variation of the polarization resistance under constant current density implies variation in the transfer coefficient $\alpha$, which depends on the reaction mechanism and the limiting step of the electrocatalytic process [35]. In the present case its variation indicates the transition in the electrochemical reaction steps that drive the $\mathrm{H}_{2}$ electrooxidation reaction. It is well known that $\mathrm{H}_{2}$ oxidation/evolution is described by the Tafel, Volmer and Heyrovski reaction steps according to the reacting scheme described by Eqs 6-4 - 6-6.

$$
\begin{array}{ll}
H_{2} \rightarrow 2 H_{a d} & \text { Tafel } \\
H_{a d} \rightarrow H^{+}+e^{-} & \text {Volmer } \\
H_{2} \rightarrow H_{a d}+H^{+}+e^{-} & \text {Heyrovski }
\end{array}
$$

Reaction 3 is fast and takes place at low overpotential being the primary charge transfer $\mathrm{H}_{2}$ electrooxidation reaction, while reaction 4 takes off at fairly higher overpotentials characterized by a larger Tafel slope [36]. Two factors corroborate for this kinetic transition. Either the low partial pressure of $\mathrm{H}_{2}$ thus affecting the rate of reaction [6-4] or the limited surface sites, thus posing restrictions on reaction [6-5] due to the low coverage of the adsorbed hydrogen $\mathrm{H}_{\mathrm{ad}}$. In this respect, the change in the slope of the I-V curves of Fig. 1 is attributed to the transition from the Volmer reaction [6-5] to the activation of the Heyrovski reaction [6-6]. This can be a combination of the lower partial pressure of $\mathrm{H}_{2}$ due to $\mathrm{H}_{2}$ utilization and the loss of active electrochemical surface area (ECSA).

Further, the poisoning effect and the role of $\mathrm{H}_{3} \mathrm{PO}_{4}(\mathrm{PA})$ at the anodic electrocatalytic layer of a high temperature polymer electrolyte membrane, has to be taken into consideration [37]. The ECSA is reduced significantly for PA lower than $3 \mathrm{gPA} / \mathrm{gPt}$, resulting in a long term gradual performance loss, under normal fuel cell operation. This is attributed to the blockage of the $\mathrm{Pt}$ surface by pyrophosphoric acid or poly-phosphates, $\mathrm{H}_{2}$ reduced polyphosphoric acid species[37,38], as well as the shrinkage of the interface due to the displacement of the $\mathrm{H}_{3} \mathrm{PO}_{4}$ by the adsorbed $\mathrm{H}_{2}$ species. Displacement of the phosphoric or pyrophosphoric acid from the Pt surface occurred also by $\mathrm{H}_{2} \mathrm{O}$ and was more enhanced in the case of CO. However as it has been explicitly discussed in chapter 5 [37], the poisoning effect of the reduced PA acid species is significantly reduced at high PA loading on the anode electrode as well as in the presence of a small partial pressure of steam not exceeding $5 \mathrm{kPa}$. Therefore the possibility of the formation of strongly adsorbed species, blocking the 
available electrocatalytic active sites, has been ruled out due to the fact that the low performance region does not appear at low PA loadings and that the effect of steam's partial pressure $>10 \mathrm{kPa}$ resulted in reduced cell performance even though anode feed was $\mathrm{CO}$ free.

\section{Distribution of PA and the Structure and stability of Electrochemical Interface}

Taking into consideration that the anode performance depends on the amount of PA introduced into the catalytic layer and the hydrophobicity of catalyst support (Figure 6-1 and 6-7), the structure of the EI plays a significant role in the anode's voltage loss. Thus, the loss of ECSA and consequently the anode's performance loss can be possibly attributed to the restructuring of the EI, as a result of the synergistic effect of the PA amount and the water solvation in the distributed PA within the catalytic layer. Taking into account that the carbon supports influenced the potential response of the anodic electrode under the same conditions and the same PA loading, indicated that the distribution of PA is a key factor in the observed phenomena.

As PA is being sprayed on the catalyst layer, uniform distribution is not guaranteed. The distribution of PA within the catalytic layer depends on the hydrophobicity of the catalyst, the pore size distribution and the porosity of the catalytic layer. Non uniform distribution of the PA, results in areas of the CL that would lack of PA and others that would be flooded. Ideally, the optimum PA distribution would be the formation of a thin film over the $\mathrm{Pt}$ surface homogeneously distributed in the CL (figure 6-20a).

The concentration of PA in the pores of the catalyst layer depends on the reactant's gas humidification (partial pressure of water) and the operation temperature. When phosphoric acid is exposed to water vapor at elevated temperature on equilibrium is established between PA and its dehydrated species like pyrophosphoric acid. The water uptake of the phosphoric acid, depends on water's partial pressure [39,40]. Both phenomena can influence the ionic conductive pathway in the CL and thus the anodic electrode's performance. It can be assumed that upon water solvation the hydrated PA swells. This may cause ganglia formation inside the pores' channels, blockage of the pores and the disruption of the continuous distribution of the PA thin film, thus resulting into the shrinkage of the electrochemical interphase as well as gas transport limitations (figure 620b). At low partial pressure of water in the reformate mixture, the equilibrium concentration of solvated water in PA is lower, thus inducing smaller changes on the EI (Figure 6-7). In the case of low PA loadings we may consider that the thinner distribution of the PA film does not swell to such degree, by its hydration under increased partial 
pressure of water, so as to result in the ganglia formation and the blockage of the pore channels or the disruption of the ionic pathway, and ultimately influences less the anodic electrode's performance.
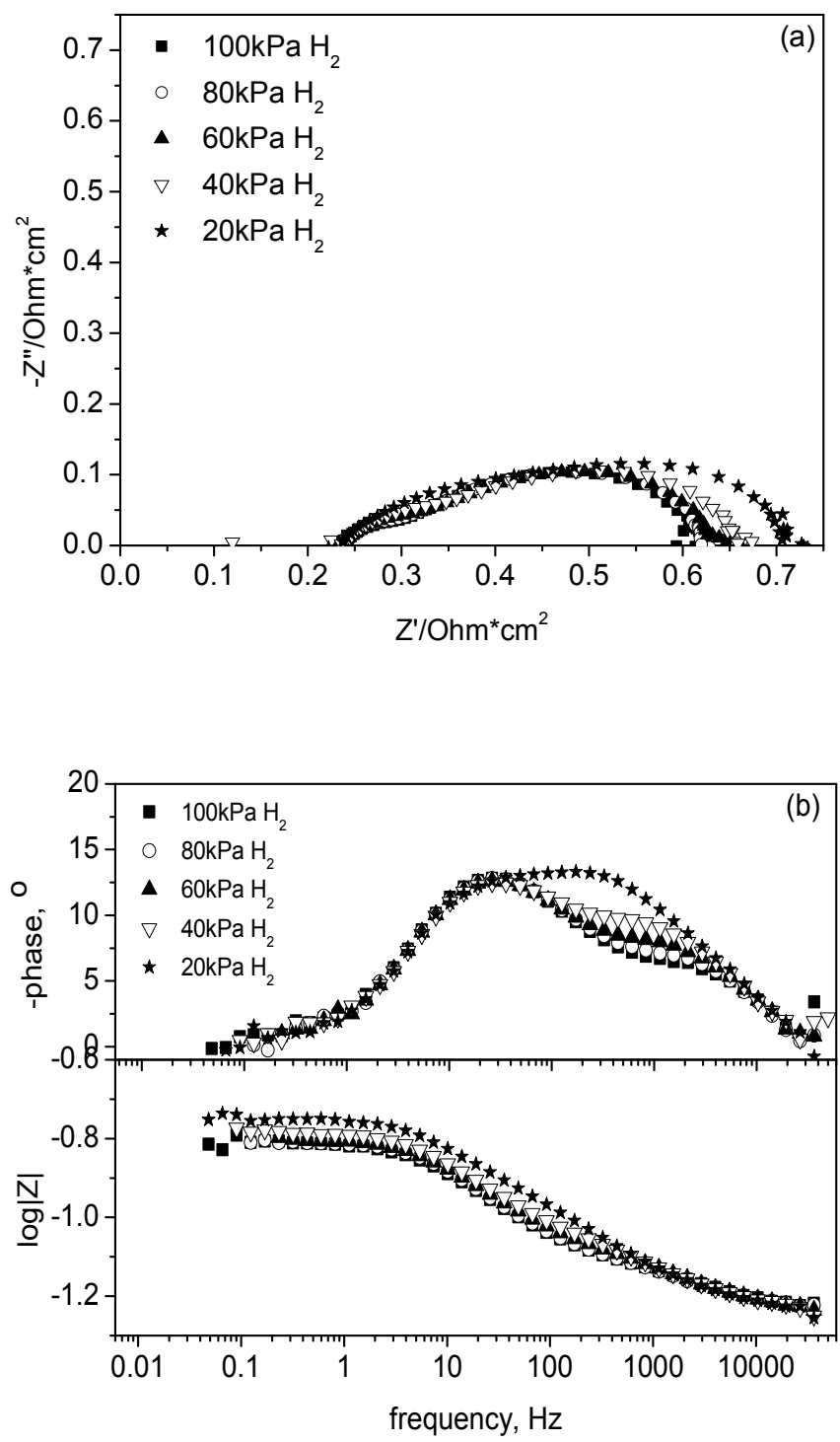

Figure 6-19. The effect of the $\mathrm{H}_{2}$ partial pressure on the polarization resistance at $0.2 \mathrm{~A} / \mathrm{cm}^{2}$. AC impedance spectra obtained at $180^{\circ} \mathrm{C}$, under various fuel compositions $\left(100 \mathrm{kPaH}_{2}-20 \mathrm{kPa} \mathrm{H}_{2}\right.$ balanced with $\left.\mathrm{Ar}\right)$. The cathodic electrode was fed with a redundant $\mathrm{O}_{2}$ flow. a. Niquist plots and b. corresponding Bode plots 
In addition as has already been shown in a previous communication [37], $\mathrm{H}_{2}$ and especially $\mathrm{CO}$ adsorption on $\mathrm{Pt}$ cause the display $\mathrm{H}_{3} \mathrm{PO}_{4}$ from the $\mathrm{Pt}$ surface and result in the shrinkage of the electrochemical interface and the reduction of the available electrochemical active sites.

Based on the above considerations it can be concluded that the control of hydrophilicity of the catalytic layer is of utmost significance for the optimum structure of the EI. This means that hydrophilic catalytic layer is preferable so that PA being at any hydration level will be able to widespread over the catalytic surface to form a thin film so as to maximize the extend of the EI and allow the fast diffusion of the reacting gases to approach the EI and electrocatalytic surface. The functionalized carbon nanotube based electrocatalyst are more hydrophilic than the carbon black based one and that makes them perfect candidates for operation under these harsh reformate conditions. The presence of pyridine groups in the catalyst layer, homogeneously distributes PA and stabilizes it inside the catalyst layer, reducing the ganglia formation. This is done on the basis of the acid-base interactions between PA and the basic pyridine moieties which are chemically grafted on the MWCNTs. The thin film of PA swells by absorbing water, which is dictated by the interfacial equilibrium between the PA and water vapors, without sever disruption of the proton conduction pathway. The created well extended 3D ionic network of PA minimizes the local flooding of the pores in the electrodes facilitating the mass transport in the catalyst layer (figure 6-21).
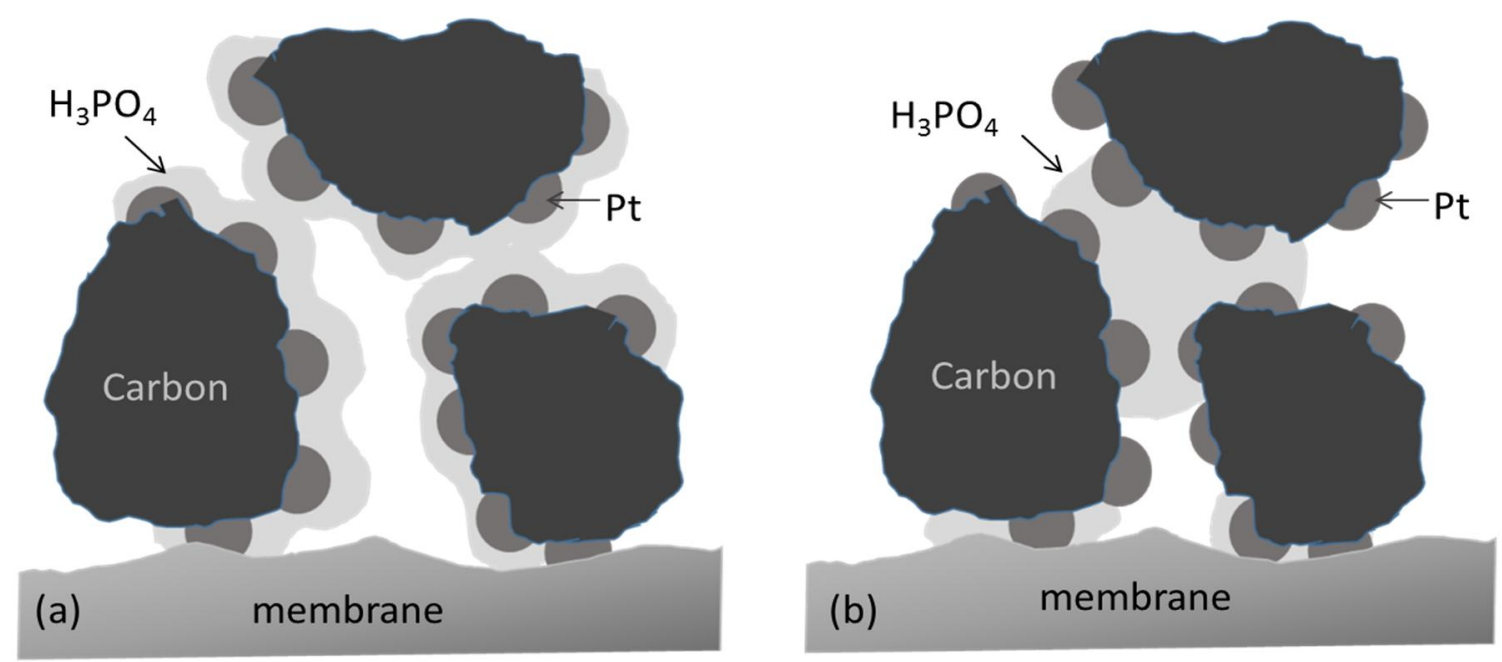

Figure 6-20. Schematic representation of the structure of the electrochemical interface for the case of $30 \mathrm{wt} \% \mathrm{Pt} / \mathrm{C}$, (a) under dry conditions (thin film formation), (b) under high humidity (ganglia formation). 


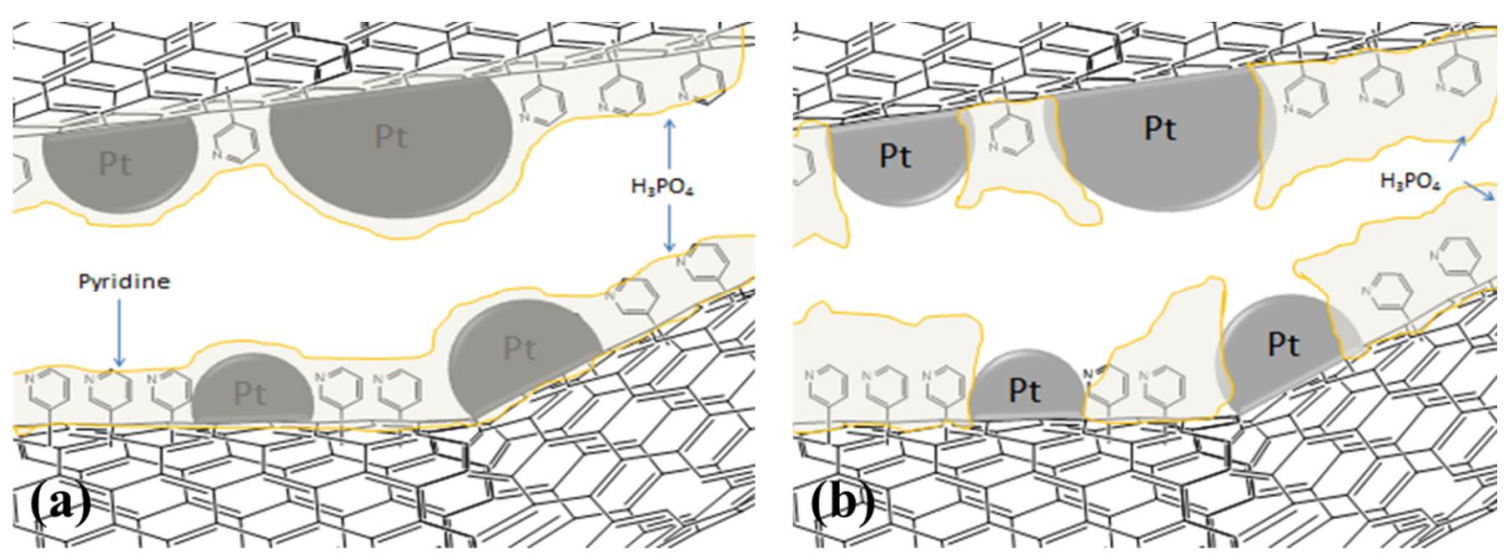

Figure 6-21. Schematic representation of the structure of the electrochemical interface, for the case of 30wt\%Pt/oxMWCNT-Py, (a) under dry conditions (thin film formation), (b) under high humidity (displacement of PA).

The 30wt\%Pt/oxMWNT-Py electrocatalyst exhibits the higher performance under reformate conditions, due to the homogeneous distribution of PA in the catalyst layer, which resulted in high ECSA and Pt utilization. This allows for the significant reduce of the Pt loading at the anodic electrode.

In conclusion the development of hydrophilic supports results in the significant decrease of Pt loading and allows for the uniform distribution of higher PA loadings within the structure of the catalytic layer. Because of the evaporation and loss of PA the latter is of utmost importance for the longtime Stability of the electrochemical performance of the anodic electrocatalytic layer operating under reformate conditions.

\subsection{Conclusions}

The observed voltage loss, in this particular system, under synthetic reformate gas rich in $\mathrm{CO}$ and steam is attributed to a multistep process. The HOR is been constrained by the remaining free $\mathrm{Pt}$ sites as a consequence of blockage due to $\mathrm{CO}$, water and phosphoric acid species adsorption on the Pt surface, as well as due to the shrinkage of the EI as a result of PA displacement from the reactions interphase. Additionally the formation of ganglia in the CL pores creates mass transport limitation hindering gas diffusivity. The amount and distribution of PA on the electrodes plays a significant role in the formation and the structure of the EI. It appears that a rather hydrophilic catalytic layer is important for the uniform distribution of PA on the electrocatalytic surface and its stability under humidified conditions. The $30 \mathrm{wt} \% \mathrm{Pt} / \mathrm{oxMWCNT}$-Py electrocatalyst are ideal candidates for operation under those harsh reformates conditions. The interaction of pyridine groups with phosphoric acid not only promotes its uniform distribution on the CL but also stabilizes the 
EI under high partial pressure of water, minimizing the ganglia formation. Additionally, the use of pyridine functionalized MWCNT based electrocatalyst gives the opportunity of lowering the Pt loading in the electrodes without sacrificing the overall cell's performance under reformate conditions, thus reducing the MEA production cost. 


\section{$\underline{\text { References }}$}

[1] G. Kohlmayr, P. Stonehart, Adsorption kinetics for carbon monoxide on platinum in hot phosphoric acid, J. Electrochim. Acta, 18 (1973) 211

[2] H.P. Dhar, L.G. Christner, A.K. Kush, H.C. Maru, Performance Study of a Fuel Cell Pt-on-C Anode in Presence of $\mathrm{CO}$ and $\mathrm{CO} 2$, and Calculation of Adsorption Parameters for CO Poisoning, J. Electrochem. Soc., 133 (1986) 1574

[3] M. Watanabe and S. Motoo, Chemisorbed $\mathrm{CO}$ on a polycrystalline platinum electrode The effect of conditioning of the surface and of partial pressure of CO, J. Electroanal. Chem. Interfacial Electrochem., 206 (1986) 197

[4] H. Igarashi, T. Fujino, and M. Watanabe, Hydrogen electro-oxidation on platinum catalysts in the presence of trace carbon monoxide, J. Electroanal. Chem 391 (1995) 119

[5]J.J. Baschuk, X.G. Li, Carbon monoxide poisoning of proton exchange membrane fuel cells, International Journal of Energy Research 25 (2001) 695.

[6] T. R. Ralph and M. P. Hogarth, Catalysis for Low Temperature Fuel Cells, Platinum Met. Rev., 46 (2002) 117.

[7] Hiroshi Igarashi, Takeshi Fujino, Yimin Zhu, Hiroyuki Uchidaand, Masahiro Watanabe, CO Tolerance of $\mathrm{Pt}$ alloy electrocatalysts for polymer electrolyte fuel cells and the detoxification mechanism, Phys. Chem. Chem. Phys. 3 (2001) 306

[8]Richard C. Urian, Andrea F. Gullá, Sanjeev Mukerjee, Electrocatalysis of reformate tolerance in proton exchange membranes fuel cells: Part I, Journal of Electroanalytical Chemistry, 554-555 (2003) 307

[9] L. W. Niedrach, D. W. McKee, J. Paynter, and I. F. Danzig, Electrochem. Technol., 5 (1967) 138

[10] A. Pitois , A. Pilenga, G. Tsotridis, CO desorption kinetics at concentrations and temperatures relevant to PEM fuel cells operating with reformate gas and $\mathrm{PtRu} / \mathrm{C}$ anodes Applied Catalysis A: General 374 (2010) 95-102

[11]A.D. Modestov, M.R. Tarasevich, V.Ya. Filimonov, E.S. Davydova, CO tolerance and CO oxidation at $\mathrm{Pt}$ and $\mathrm{Pt}-\mathrm{Ru}$ anode catalysts in fuel cell with polybenzimidazole-H 3PO 4 membrane, j Electrochimica Acta 55 (2010) 6073

[12] Jianlu Zhang, High temperature PEM fuel cells, Journal of Power Sources 160 (2006) 872

[13]Anders R. Korsgaard, Rasmus Refshauge, Mads P. Nielsen, Mads Bang, Søren K. Kær, Experimental characterization and modeling of commercial polybenzimidazole-based MEA performance, J. Power Sources, 162 (2006) 239

[14]Kyungjung Kwon, Duck Young Yoo, Jung Ock Park, Experimental factors that influence carbon monoxide tolerance of high-temperature proton-exchange membrane fuel cells,J. Power Sources, 185 (2008) 202 
[15] M. Geormezi, F. Paloukis, A. Orfanidi, M.K. Daletou, S.G. Neophytides, The structure and stability of the anodic electrochemical interface in a high temperature Polymer Electrolyte Membrane Fuel Cell under reformate feed, (submitted to Journal of Power Sources)

[16] A.Orfanidi, M.K.Daletou and S.G.Neophytides Preparation and characterization of Pt on modified multi-wall carbon nanotubes to be used as electrocatalyst in high temperature fuel cells applications, Appl. Catalysis B: Environmental, 106 (2011) 379

[17] Jose' J. Linares, Cassandra Sanches, Valdecir A. Paganin, Ernesto R. Gonzalez, Performance of a poly(2,5-benzimidazole)-based polymer electrolyte membrane fuel cell, Int. Journal of Hydrogen Energy 37 (2012) 7212

[18]Andreas Huth, Bastian Schaar, Torsten Oekermann, A "proton pump" concept for the investigation of proton transport and anode kinetics in proton exchange membrane fuel cells, Electrochimica Acta 54 (2009) 2774

[19]Jingxin Zhang, Tony Thampan, and Ravindra Datta, Influence of Anode Flow Rate and Cathode Oxygen Pressure on CO Poisoning of Proton Exchange Membrane Fuel Cells, J. Electrochem. Soc. 149 (2002) A765

[20] Thayane C.M. Nepel, Pietro P. Lopes, Valdecir A. Paganin, Edson A. Ticianelli, CO tolerance of proton exchange membrane fuel cells with $\mathrm{Pt} / \mathrm{C}$ and $\mathrm{PtMo} / \mathrm{C}$ anodes operating at high temperatures: A mass spectrometry investigation, Electrochimica Acta, 88 (2013) 217

[21]Chien-Ping Wang, Hsin-Sen Chu, Yi-Yie Yan, Kan-Lin Hsueh, Transient evolution of carbon monoxide poisoning effect of PBI membrane fuel cells, Journal of Power Sources 170 (2007) 235

[22] Andreasen SJ, Jespersen JL, Schaltz E, Kær SK., Characterisation and modelling of a high temperature PEM fuel cell stack using electrochemical impedance spectroscopy, Fuel Cells 9 (2009) 463

[23] ZhuWH, Payne RU, Tatarchuk BJ. PEM stack test and analysis in a power system at operational load via AC impedance, Journal of Power Sources 168 (2007) 211

[24] Yuan X, Wang H, Sun JC, Zhang J. AC impedance technique in PEM fuel cell diagnosis: A review. International Journal of Hydrogen Energy 32 (2007) 4365

[25] Zhang J, Tang Y, Song C, Zhang J. Polybenzimidazole-membrane-based PEM fuel cell in the temperature range of 120-200 oC, Journal of Power Sources 172 (2007) 163

[26] Jespersen JL, Schaltz E, Kær SK. Electrochemical characterization of a polybenzimidazolebased high temperature proton exchange membrane unit cell, J. of Power Sources 191 (2009) 289

[27] Li Q, He R, Jensen JO, Bjerrum NJ., PBI-Based polymer membranes for high temperature fuel cells -preparation, characterization and fuel cell demonstration, Fuel Cells 4 (2004) 3

[28] Schechter A, Savinell RF,Wainright JS, Ray D., NMR study of phosphoric acid-doped polybenzimidazole under controlled water activity. Journal of the Electrochemical Society 156 (2009) B283 
[29] Daletou MK, Kallitsis JK, Voyatzis G, Neophytides SG. The interaction of water vapors with H3PO4 imbibed electrolyte based on PBI/polysulfone copolymer blends., J Memb Sci 326 (2009) 76

[30] Liu Z, Wainright JS, Litt MH, Savinell RF. Study of the oxygen reduction reaction (ORR) at Pt interfaced with phosphoric acid doped polybenzimidazole at elevated temperature and low relative humidity, Electrochimica Acta, 51 (2006) 3914

[31] Wippermann K, Wannek C, Oetjen HF, Mergel J, Lehnert W. Cell resistances of poly(2,5benzimidazole)-based hightemperature polymer membrane fuel cell membrane electrode assemblies: time dependence and influence of operating parameters. Journal of Power Sources 195 (2010) 2806

[32] Bonaventura M, Mendes A., Activation procedures characterization of MEA based on phosphoric acid doped PBI membranes., I J Hydrogen Energy 35 (2010) 11649

[33] S. Kaserer, C. Rakousky, J. Melke, C. Roth, Design of a reference electrode for hightemperature PEM fuel cells, J. Appl. Electrochem. 43 (2013) 1069.

[34] A.D. Modestov, M.R. Tarasevich, A.Y. Leykin, CO electrooxidation study on Pt and Pt-Ru in H3PO4 using MEA with PBI-H3PO4 membrane, J. Power Sources 196 (2011) 2994

[35] J. O’M. Bockris, A.K.N. Reddy, M. Gamboa-Aldeco, Modern Electrochemistry, Vol. 2A, Fundamentals of electrodics, Kluwer Academic/Plenum Publishers, New York ISBN: 0-30646166-8 Z, 2nd ed., (2000), 1166.

[36] P.M. Quaino, J.L. Fernández, M.R. Gennero de Chialvo, A.C. Chialvo, Hydrogen oxidation reaction on microelectrodes: Analysis of the contribution of the kinetic routes, J. Mol. Catal. A: Chem. 252 (2006) 156.

[37] A. Orfanidi, M. K. Daletou, L. Sygellou, S. G. Neophytides, The role of phosphoric acid in the anodic electrocatalytic layer in high temperature PEM fuel cells, J. Appl. Electrochem. 43 (2013) 11.

[38] W. Doh, L. Gregoratti, M. Amati, S. Zafeiratos, Y.T. Law, S. Neophytides, A. Orfanidi, M. Kiskinova, E.R. Savinova, Scanning Photoelectron Microscopy Study of the Pt/Phosphoric-AcidImbibed Membrane Interface under Polarization, ChemElectroChem. 1 (2014) 180.

[39] Maria K. Daletou, Maria Geormezi, Effrosyni Vogli, George A. Voyiatzis, Stylianos G. Neophytides, The interaction of H3PO4 and steam with PBI and TPS polymeric membranes. A TGA and Raman study, J. Mater. Chem. A (2014) 2, 1117-1127 


\section{Chapter 7}

\section{Determination of the cathodic electrode's ECSA using $\mathrm{CO}$ as a probe molecule}

\subsection{Introduction}

Towards the commercialization of low cost and high efficiency PEM fuel cells, optimization of the catalyst layer structure and evaluation of the catalytic performance, stability and degradation are essential. In order to be able to assess the electrocatalytic performance electrocatalysis metrics such as surface specific and Pt mass activity, catalyst utilization and electrochemically active surface area (ECSA) have become essential tools for activity and durability description. Determination of the ECSA is important in order to be able to normalize activities of different electrocatalysts to the same number of reactive sites and to check catalyst durability by assessing the loss of catalyst surface area during fuel cell operation.

The most popular ECSA evaluation methods, in low temperature fuel cell, are the hydrogen underpotential deposition (HUPD) and CO stripping voltammetry $[1,2,3,4]$. However for the 
case of high temperature PEM fuel cells using the HUPD region is not plausible [5] due to strong phosphoric acid adsorption which inhibits hydrogen adsorption [6] and high faradaic HER currents which superimpose the pseudocapacitive currents of the HUPD layer [7]. Also HUPD formation is temperature depended [8] and at those elevated temperature (above $140^{\circ} \mathrm{C}$ ) its saturation coverage is not known. Therefore, $\mathrm{CO}$ is used as a probe molecule for the ECSA evaluation in HTPEM fuel cells [7].

As discussed in Chapter 4, for the evaluation of the ECSA of the anodic electrode, CO adsorption took place at open circuit potential. No difference in the acquired charge (Qco) was observed when $\mathrm{CO}$ was adsorbed under constant polarization of the working electrode at $0.075 \mathrm{~V}$. This is due to the reduced Pt surface. In the case of cathodic electrodes, the Pt surface in partially covered by oxides thus inhibiting $\mathrm{CO}$ adsorption at OCP conditions [9]. A typical procedure for the ECSA evaluation of cathodic electrodes involves polarization of the working electrode at low potentials (below 0.150V) during CO adsorption [10,7]. Polarization of the electrode at such low values is needed for the removal of surface oxides [11]. This chapter focuses on optimizing the ECSA evaluation methodology with the minimum possible disruption of the Pt particle size distribution of the cathodic catalyst layer.

\subsection{Experimental}

\subsubsection{Materials}

Phosphoric acid doped polymer electrolyte membranes were provided from Advent Technologies S.A.. Catalyst powder 30wt \% Pt/Vulcan XC-72R was purchased from Tanaka Kikinzoku International. For the construction of the electrodes, carbon cloth supporting layer was purchased from Fuel Cell Earth LLC, Vulcan XC-72R powder from RawChem Ltd and the PTFE dispersion in water from Sigma-Aldrich. 2-propanol 99.8 $\leq$ and $\mathrm{H}_{3} \mathrm{PO}_{4}$ purum p.a. $\geq 85 \%$ were purchased from Merck or Sigma Aldrich. All chemicals were used as received unless otherwise noted.

\subsubsection{Instrumentation}

The electrochemical measurements were performed using a single cell purchased from Fuel Cell Technologies Inc. Both graphite bipolar plates of the single cell had the same single serpentine flow field for the distribution of reacting gases. The active area of the electrodes was $2 \mathrm{~cm} \times 2 \mathrm{~cm}$. The assembling torque applied for the single cell was $4.8 \mathrm{Nm}$. The cell was installed in a fuel cell test station which was built in-house and had provisions for 
controlling temperature, humidification, and flow of reacting gases. The measurements were made in a two-electrode arrangement using an Autolab potentiostat-galvanostat PGSTAT-302. The size of the Pt nanoparticles was characterized by transmission electron microscopy (TEM) on JEOL JEM2100 operating at $200 \mathrm{kV}$. Sample preparation for TEM examination involved the ultrasonic dispersion of the sample in water and placing a drop of the suspension on $3 \mathrm{~mm}$ carbon coated copper grids (Electron Microscopy Sciences). The crystalline structure of the catalysts was analyzed with an Xray powder diffractometer (Bruker D8 Advance) using $\mathrm{Cu} \mathrm{K} \alpha$ radiation $(\lambda=0.15418 \mathrm{~nm})$. The XRD measurements were carried out in the $2 \theta$ angle with the range of 20-90。. The mean particle diameter of $\mathrm{Pt}$ was calculated from the X-ray line broadening of the $\operatorname{Pt}(111)$ diffraction peak according to Scherrer's equation.

\subsubsection{Electrode Preparation and MEA assembly}

Electrodes' fabrication: The gas diffusion layer, GDL, used in this study was prepared by spraying a slurry made of carbon Vulcan XC-72R and PTFE solution (50 wt\% PTFE/C) on carbon cloth, followed by heat treatment at $300^{\circ} \mathrm{C}$ under static air for $40 \mathrm{~min}$. The electrodes containing 30wt \% Pt/C were prepared by spraying 2-propanol-Pt/C ink on the GDL. The electrodes were then treated for 12 hours at $80^{\circ} \mathrm{C}$ and 3 days at $190^{\circ} \mathrm{C}$ under vacuum, in order to remove all traces of the organic solvent. Both anodic and cathodic electrodes had the same metal loading approximately $1.3 \sim 1.5 \mathrm{mgPt} / \mathrm{cm}^{2}$.

Membrane Electrode Assembly, MEA: Before the MEA assembly, a certain amount of phosphoric acid, PA, was sprayed onto the electrodes. The both anodic and cathodic electrodes were sprayed with $2 \mathrm{gPA} / \mathrm{gPt}$. The electrode active area was $2 \mathrm{~cm}$ x $2 \mathrm{~cm}$. The

MEAs were fabricated by hot pressing, the phosphoric acid doped membranes (doping level $\sim 200 \mathrm{wt} \%$ ) between the two electrodes at $150^{\circ} \mathrm{C}$ for $5 \mathrm{~min}$.

\subsubsection{Electrochemical Characterization}

All MEAs were activated under galvanostatic mode. Initially the cell's temperature was increased from room temperature to $100^{\circ} \mathrm{C}$ while $\mathrm{Ar}$ flushed both compartments. Subsequently, the gases were switched to pure $\mathrm{H}_{2}$ and $\mathrm{O}_{2}$ and the temperature was set at $140^{\circ} \mathrm{C}$. Once the temperature was reached, a current of $0.2 \mathrm{~A} / \mathrm{cm}^{2}$ was applied to the fuel cell. The temperature was then set at $180^{\circ} \mathrm{C}$. The break in period was found to be approximately 48 hours. Details concerning the activation of the MEAs are provided in detail in the appendix A1. 


\subsubsection{Cyclic Voltammetry}

The pretreatment of the cathodic electrode, before CO stripping voltammetry measurements, were conducted via linear sweep voltammetry. The potential was cycled between 0.075 and $0.9 \mathrm{~V}$ with a scan rate of $10 \mathrm{mV} / \mathrm{s}$. The cell temperature was $140^{\circ} \mathrm{C}$. One compartment was supplied with a constant flow of $200 \mathrm{cc} / \mathrm{min}$ of $10 \% \mathrm{H}_{2}$ in argon and the corresponding electrode acted as the reference electrode. The compartment with the electrode under investigation (working electrode) was fed with $200 \mathrm{cc} / \mathrm{min}$ of Ar flow, which was prior humidified by passing through a thermostated humidifier and the partial pressure of water was adjusted at $9.56 \mathrm{kPa}$ by controlling the operating temperature of the humidifier at $45^{\circ} \mathrm{C}$.

\subsubsection{CO stripping voltammetry}

The electrochemically active surface area, ECSA, of the Pt based catalyst was determined via $\mathrm{CO}$ stripping voltammetry. The cell temperature was kept constant at $140^{\circ} \mathrm{C}$. One compartment was supplied with a constant flow of $200 \mathrm{cc} / \mathrm{min}$ of $10 \% \mathrm{H}_{2}$ in argon and the corresponding electrode acted as the reference electrode. The compartment with the electrode under investigation (working electrode) was fed with $1.8 \% \mathrm{CO}$ in argon (air liquide) at a flow rate of $100 \mathrm{cc} / \mathrm{min}$. The $\mathrm{CO}$ adsorption conditions varied (under open circuit potential or polarization $0.1 \mathrm{~V}$ and $\mathrm{CO}$ adsorption in the presence or absence of $\mathrm{H}_{2} \mathrm{O}$ ). It was found that the maximum coverage with $\mathrm{CO}$ was achieved in approximately 20 minutes. Subsequently, the compartment was flushed with $200 \mathrm{cc} / \mathrm{min}$ argon in order to remove all the traces of $\mathrm{CO}$ in the gas phase. Prior to its introduction to the cell, the argon flow was humidified by passing through a thermostated humidifier kept at a constant temperature of $45^{\circ} \mathrm{C}$. The potential was then cycled between 0.075 and $0.9 \mathrm{~V}$ with a scan rate $10 \mathrm{mV} / \mathrm{s}$. To calculate the CO stripping charge, $\mathrm{Q}_{\mathrm{CO}}$ (in C), the area under the peak that corresponds to the $\mathrm{CO}$ electrooxidation was integrated. The second cycle in the same experiment was used for baseline subtraction, considering that during the second cycle $\mathrm{CO}$ was absent from the Pt surface. The ECSA, in $\mathrm{m}^{2}$, was determined considering a charge of $420 \mu \mathrm{C} \mathrm{cm}^{-2}$ for $\mathrm{CO}$ oxidation that corresponds to a monolayer of adsorbed $\mathrm{CO}$ on polycrystalline $\mathrm{Pt}$ as follows:

$$
\operatorname{ECSA}\left(m^{2}\right)=\operatorname{Qco}(C) / 4.20 \mathrm{C} \mathrm{m}^{-2}
$$

\subsubsection{CO bulk electroxidation (CO annealing)}

Preconditioning of the Pt surface was also conducted via $\mathrm{CO}$ bulk electroxidation. The cell 
temperature was kept constant at $140^{\circ} \mathrm{C}$. One compartment was supplied with a constant flow of $200 \mathrm{cc} / \mathrm{min}$ of $10 \% \mathrm{H}_{2}$ in argon and the corresponding electrode acted as the reference electrode. The compartment with the electrode under investigation (working electrode) was fed with a gas mixture of $1.8 \mathrm{kPa}$ of $\mathrm{CO}$ balanced with argon (air liquide). The gas mixture was then humidified by passing through a thermostated humidifier kept at a constant temperature of $45^{\circ} \mathrm{C}$, prior to its introduction to the cell. The potential was then cycled between 0.075 and $0.9 \mathrm{~V}$, with a scan rate of $40 \mathrm{mV} / \mathrm{s}$.

\subsubsection{TEM}

Post mortem analysis of the catalytic layer of used membrane electrode assemblies (MEA) was carried out by using transmission electron microscopy (TEM) .The electrodes were separated from the membrane after approximately 50 hours of continuous fuel cell operation for the evaluation of the Pt particle size distribution and morphology of the electrocatalyst. Adequate amounts of catalysts were scrapped off the used electrodes and were ultrasonically dispersed in water. A drop of the suspension was placed on a $3 \mathrm{~mm}$ carbon coated copper grids (Electron Microscopy Sciences). The Pt particle size distributions were obtained by manually measuring over $1000 \mathrm{Pt}$ particles from the TEM pictures. The mean $\mathrm{Pt}$ particle size was then calculated using the following equation:

$$
\mathrm{D}_{\mathrm{APt}}=\Sigma \mathrm{D}_{\mathrm{Pt}} \mathrm{N}_{\mathrm{D}} / \mathrm{n} \quad(\mathrm{nm})
$$

where $\mathrm{N}_{\mathrm{D}}$ is the number of particles corresponding to a Pt particle size with diameter $\mathrm{D}_{\mathrm{Pt}}$ $(\mathrm{nm})$ and $\mathrm{n}$ is the total number of measured particles.

\subsection{Results and discussion}

All MEAs used in this section employed 30wt $\% \mathrm{Pt} / \mathrm{C}$ and each electrode had a $\mathrm{Pt}$ loading of approximately $1.4 \mathrm{mgPt} / \mathrm{cm}^{2}$ and PA loading equal to $2 \mathrm{gPA} / \mathrm{gPt}$. All measurements were conducted after 48 hours of continuous operation at $0.2 \mathrm{~A} / \mathrm{cm}^{2}$ and $180^{\circ} \mathrm{C}$. Initially, the determination the ECSA at the cathodic electrode was conducted via the most popular and standard procedure, which involves polarizing the working electrode during $\mathrm{CO}$ adsorption. The procedure that was followed, was based on the recent published work by Tom Engl et al [7]. For our case it was considered more suitable to polarize the electrode at $0.1 \mathrm{~V}$ during $\mathrm{CO}$ adsorption while keeping the cell's temperature at $140^{\circ} \mathrm{C}$. A gas flow consisting of $1.8 \% \mathrm{CO}(100 \mathrm{cc} / \mathrm{min})$ was fed for $20 \mathrm{~min}$ at the cathodic compartment. Thereafter, the working compartment was flushed with $\operatorname{Ar}(200 \mathrm{cc} / \mathrm{min})$ while keeping the electrode at $0.1 \mathrm{~V}$, and additional $5 \mathrm{~min}$ at $\mathrm{OCP}$, to remove all traces of $\mathrm{H}_{2}$ originating from 
the $\mathrm{H}_{2}$ evolution at $0.1 \mathrm{~V}$. CO stripping procedure that was then followed, is described in detail in the experimental section. The obtained CO stripping voltammogram is depicted in figure $7-1$.

Two sequential CO stripping experiments were recorded, in order to ensure the reproducibility of the $\mathrm{CO}$ electrooxidation peak's area. As also discussed in chapter 4 and reported by T.Eng et al [7] side reactions that occur on the Pt surface during polarization of the electrode at $0.1 \mathrm{~V}$, influence the ECSA determination. When HER is initiated at $0.1 \mathrm{~V}$ on the working electrode, catalytic and electrocatalytic phosphoric acid reduction takes place in the presence of $\mathrm{H}_{2}$ (chapter 4). Thus the second potential sweep of the same $\mathrm{CO}$ stripping experiment is not used as a baseline. Measurement of an additional cyclic voltamogram is required and used as a baseline to determine the CO oxidation charge, and is referred as a reference baseline. The latter is obtained after polarizing of the working electrode at $0.1 \mathrm{~V}$ for $20 \mathrm{~min}$ in the absence of $\mathrm{CO}$. This is necessary in order to subtract the contributions from the oxidation of the reduced PA species from the overall stripping charge [7]. Figure 7-1b depicts the first and second cycle of the reference cyclic voltamograms. It is clear that after polarization of the electrode at $0.1 \mathrm{~V}$ there is a small contribution in the oxidative current from the aforementioned adsorbed reduced PA species electrooxidation, which are removed from the Pt surface, as confirmed by the second cycle of the same CV.

The ECSA, as calculated based on the above, was found to be equal to $19.1 \mathrm{~m}^{2} \mathrm{Pt} / \mathrm{gPt}$. After the above mention ECSA evaluation procedure, the MEA was subject to postmortem analysis using TEM, in order to evaluate the catalyst morphology and Pt distribution. For comparison reasons, a MEA with the same specifications operated under the same conditions and time (without ECSA evaluation), was also subjected to TEM postmortem characterization. The obtained TEM micrographs are shown in figure 7-2 and the average Pt particle size was determined as described in the experimental section. The average Pt particle size was found to be $3.8 \mathrm{~nm}$ for the MEA that was not subjected to the ECSA evaluation procedure as opposed to $5.5 \mathrm{~nm}$ for the one that was. Such catalyst degradation can occur through surface migration of platinum and platinum dissolution-redeposition with the driving force being the high free energy of the small platinum particles. The decrease in the electrochemically active surface area is linked to an increase in the platinum particle size. It is clear that the even two repetition of the ECSA procedure causes a deterioration of the Pt surface, through sintering and agglomeration.

From the above, it has been made clear that this ECSA evaluation procedure raises many questions concerning its accuracy. Since the Pt particle size increases during the ECSA evaluation procedure, the obtained CO uptake value is lower than the actual ECSA of the cathodic electrode. The loss of platinum surface area through sintering or recrystallization 

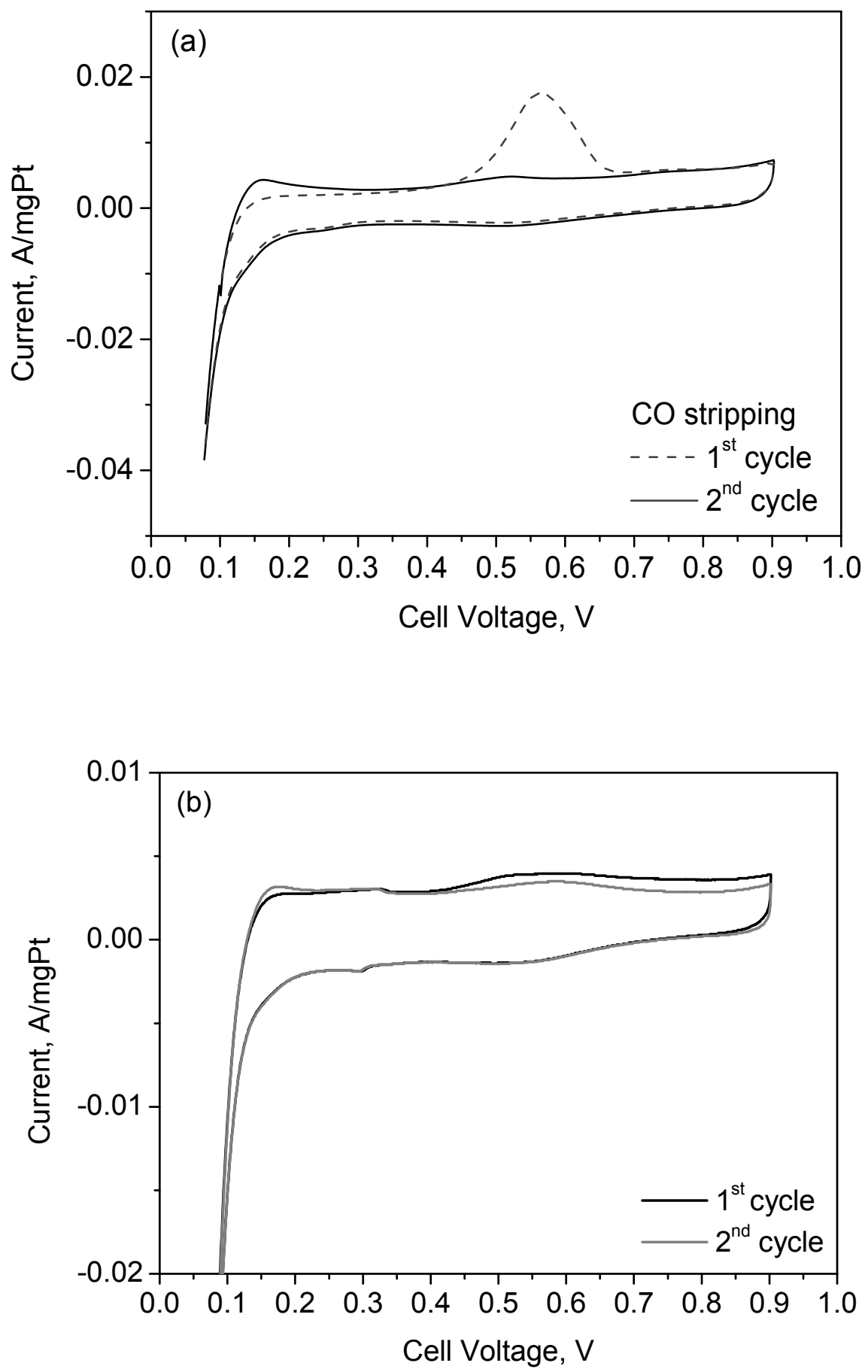

Figure 7-1. Cyclic voltamograms a. first and second $\mathrm{CO}$ adsorption under $0.1 \mathrm{~V}$ for $20 \mathrm{~min}$ and $b$. the reference baseline where the electrode was polarized at $0.1 \mathrm{~V}$ for $20 \mathrm{~min}$ in the absence of CO. 
within the porous cathode has been proposed to be the principal reason for the decline of activity in phosphoric acid fuel cells [12]. The reduced catalyst surface, results in the diminution of the cathodic electrode's performance, especially for low Pt loading as shown in figure 7-3. MEA employing $0.6 \mathrm{mgPt} / \mathrm{cm}^{2}$ and $2 \mathrm{gPA} / \mathrm{gPt}$ was subjected to the exact same ECSA evaluation procedure, as described above, where polarization curves were obtained before and after the $\mathrm{CO}$ stripping procedure. The performance of the fuel cell after $\mathrm{CO}$ stripping, was significantly reduced due to the reduction of the available ECSA, as shown in figure $7-3$.
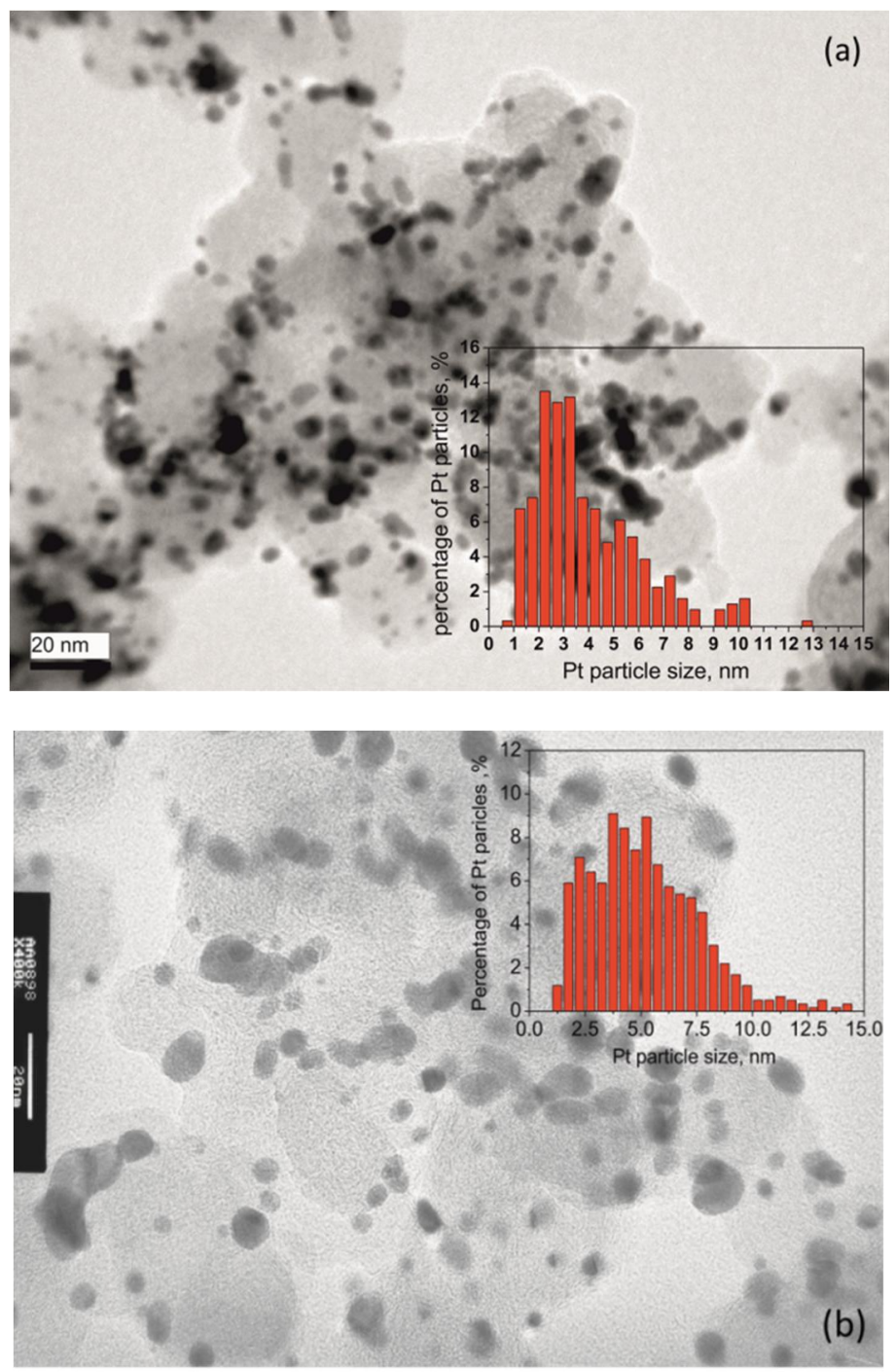

Figure 7-2. TEM micrograph and the corresponding Pt size distribution histogram of $30 \mathrm{wt} \% \mathrm{Pt} / \mathrm{C}$ obtained after the activation period: a. before $\mathrm{CO}$ stripping and $\mathrm{b}$. after 2 sequential $\mathrm{CO}$ stripping experiments, where $\mathrm{CO}$ was adsorbed under polarization $0.1 \mathrm{~V}$. The Pt particle size distribution is included as an inset. 
Although the Pt particle size increase in not so big, as shown in figure 7-2, nonetheless it is clear that it affects the performance of the fuel cell, when low Pt loading are employed at the cathodic electrode. On the other hand, for Pt loadings higher than $1 \mathrm{mgPt} / \mathrm{cm}^{2}$ at the cathodic electrode no significant difference in the performance was observed, before and after the ECSA evaluation procedure, as expected, as there is a larger number of available active sites. It has to be mentioned though, that in low temperature PEM fuel cell, during the $\mathrm{CO}$ adsorption and electrooxidation processes, no degradation in the MEA performance or increase of the Pt particle size has been reported [13]. The latter may suggest that the operation temperature [14] and the strong acidic environment [15] contribute to the degradation of the catalyst layer of the MEA during the aforementioned CO stripping procedure.

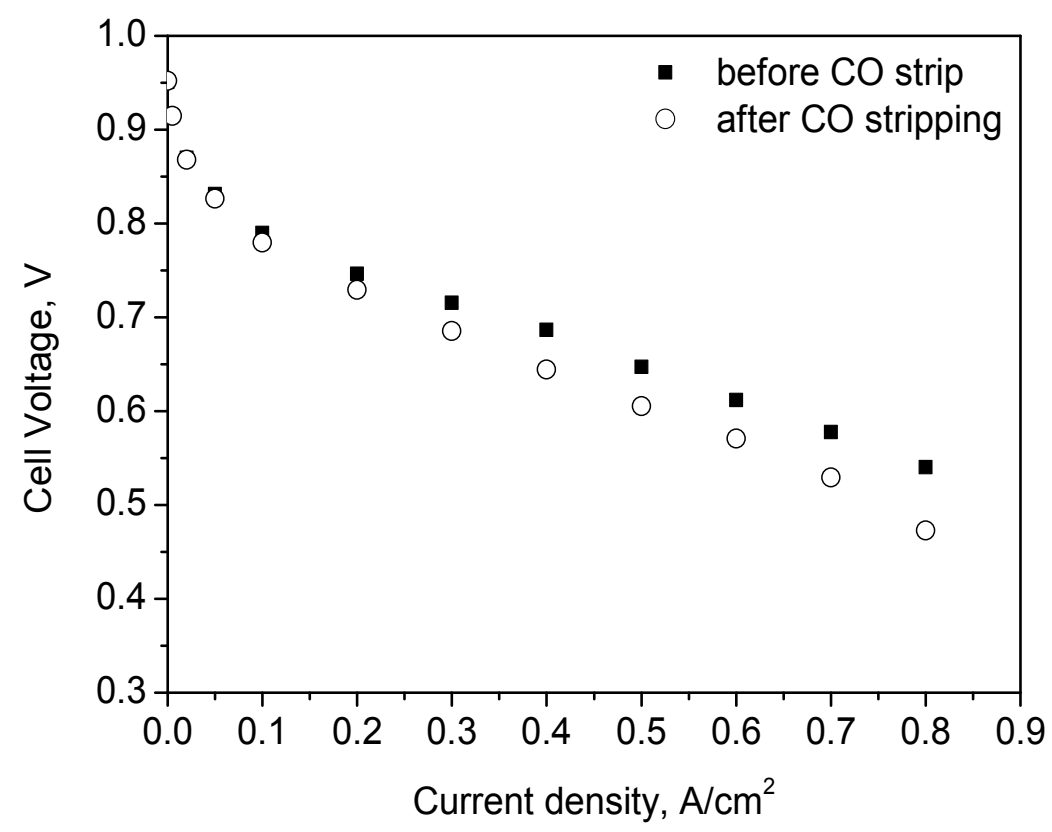

Figure 7-3. The effect of ECSA evaluation procedure on cathodic electrode performance. IR drop corrected polarization curve obtained at $180^{\circ} \mathrm{C}$ and under pure $\mathrm{H}_{2}$ and $\mathrm{O}_{2}$ (stoichiometry $\lambda_{\mathrm{H} 2}=1.2$ and $\lambda_{\mathrm{O} 2}=2$ ). MEA: $30 \mathrm{wt} \% \mathrm{Pt} / \mathrm{C} 0.6 \mathrm{mgPt} / \mathrm{cm}^{2}$ and $2 \mathrm{gPA} / \mathrm{gPt}$.

In an attempt to find a less destructive ECSA evaluation procedure, the following procedure was employed to a new MEA, with similar specifications as the one used above. The cathodic compartment, after 48 hours of continuous fuel cell operation, was flushed with Ar until complete removal of $\mathrm{O}_{2}$ traces in the gas phase and the cell's temperature was set at $140^{\circ} \mathrm{C}$. Sequentially, $100 \mathrm{cc} / \mathrm{min}_{2}$ was fed to the cathodic compartment for $5 \mathrm{~min}$, 
in order to obtain a reduced and complete oxide-free Pt surface. $\mathrm{CO}$ was then adsorbed at OCP conditions under constant flow of $1.8 \mathrm{kPa} \mathrm{CO}$ balanced in $\mathrm{Ar}(100 \mathrm{cc} / \mathrm{min})$ for $20 \mathrm{~min}$. $\mathrm{CO}$ stripping procedure was then followed, as described in detail in the experimental section, and repeated twice. It has to be mentioned that the first and second CO stripping experiment were identical, which means that only one repetition of the above procedure is needed for the accurate ECSA evaluation. As a reference cycle-baseline, the second cycle of the same CO stripping procedure was used, as no reduced PA species were formed during this short 5 min of $\mathrm{H}_{2}$ exposure. Figure 7-4 shows the obtained $\mathrm{CO}$ stripping voltammogram and is compared with the previous $\mathrm{CO}$ stripping procedure, where the working electrode was polarized at $0.1 \mathrm{~V}$ during $\mathrm{CO}$ adsorption.

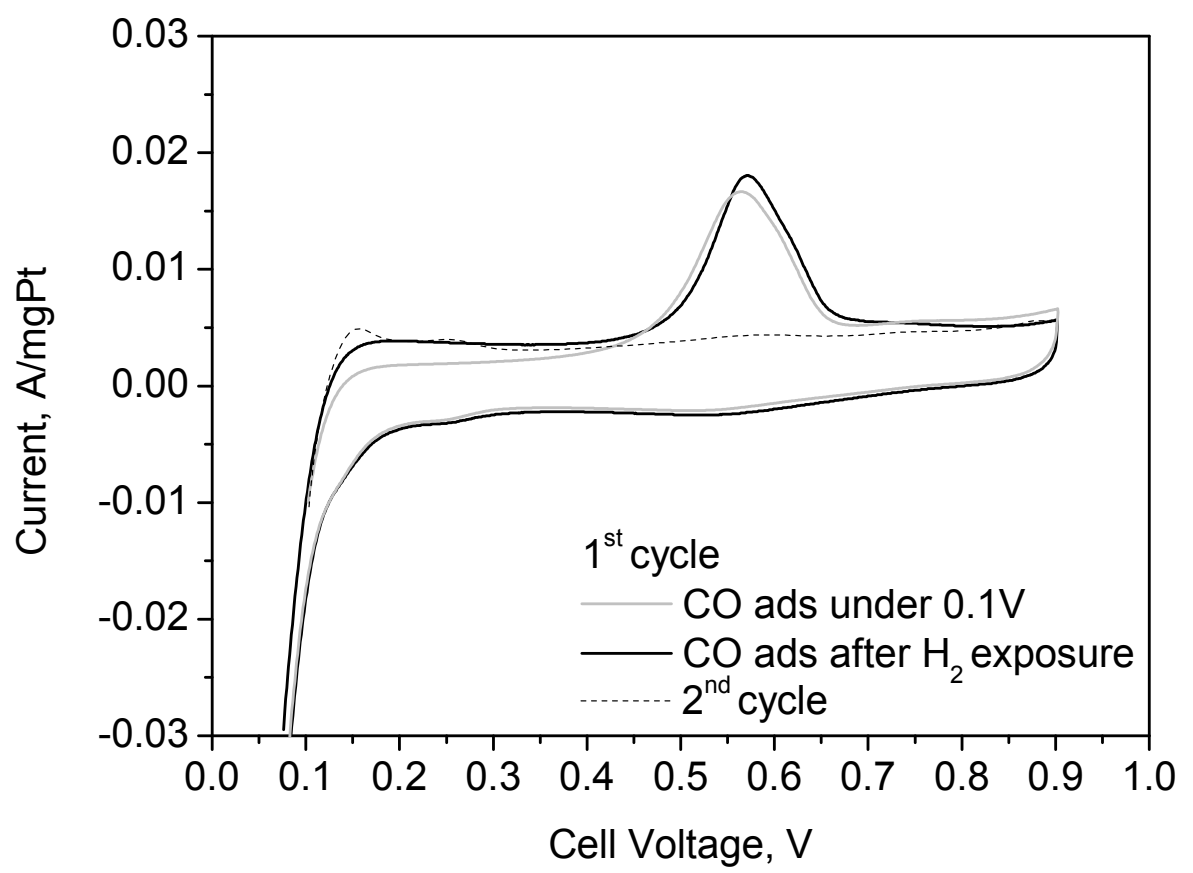

Figure 7-4. CO stripping voltammograms (first and second cycle) obtained after different $\mathrm{CO}$ adsorption procedure at $140^{\circ} \mathrm{C}$. The scan rate was $10 \mathrm{mV} / \mathrm{s}$. Both MEAs had a Pt loading of $1.4 \mathrm{mgPt} / \mathrm{cm} 2$ and $2 \mathrm{gPA} / \mathrm{gPt}$ was present in the CL.

The ECSA value was found to be $20.6 \mathrm{~m}^{2} \mathrm{Pt} / \mathrm{gPt}$, similar to the previous procedure. Although the Pt particle size, as calculated from TEM imaging, was found lower and equal to $4.7 \mathrm{~nm}$, as opposed to $5.5 \mathrm{~nm}$ when then working electrode is subjected to HER potentials during $\mathrm{CO}$ adsorption. The particle size distribution shows a clear log-normal distribution, as opposed to the previous procedure, which showed a broad distribution. 


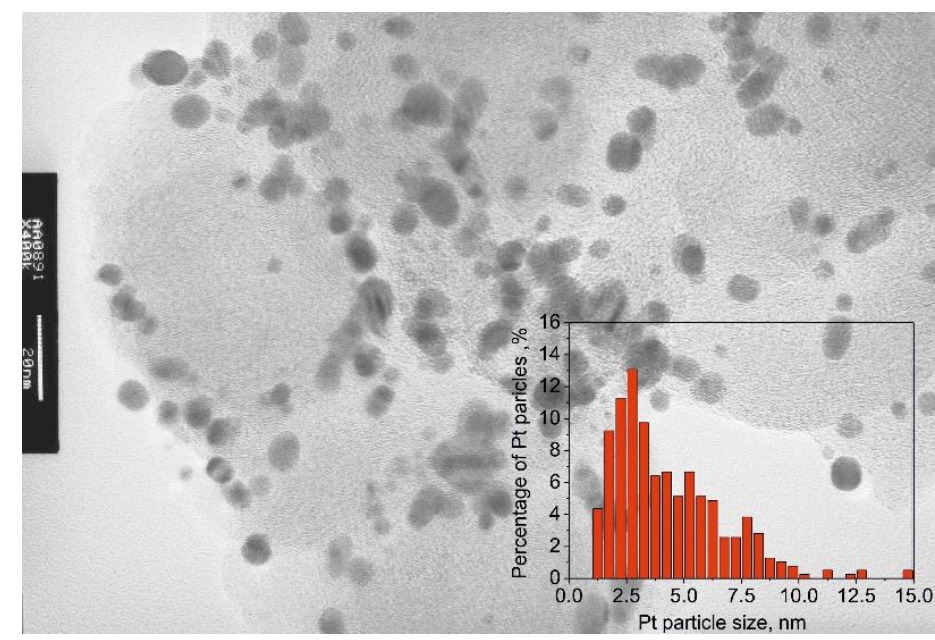

Figure 7-5. TEM micrograph and the corresponding $\mathrm{Pt}$ size distribution histogram of $30 \mathrm{wt} \% \mathrm{Pt} / \mathrm{C}$ after exposure for $5 \mathrm{~min}$ in $\mathrm{H}_{2}$ and 2 sequential $\mathrm{CO}$ stripping experiments. The $\mathrm{Pt}$ particle size distribution is included as an inset and the average Pt particle size was estimated $4.7 \mathrm{~nm}$.

It has to be noted though, that the Pt particles size after both procedures, exceeds the Pt particle size increase even after 50 hours of continuous operation at the anodic electrode, chapter 5 , where it was found to be $4.1 \mathrm{~nm}$. It is very clear that for both procedures the diminution of the catalyst surface area is not only a result of the elevated temperature and the reductive atmosphere.

To shed more light in to the matter concerning the origin of the rapid Pt particles sintering, within the short period of the experiment, ex-situ measurements were performed using an oven placed in an XRD apparatus. Commercial catalyst $30 \mathrm{wt} \% \mathrm{Pt} / \mathrm{C}$ was placed in a special oven in the XRD, the temperature was kept constant at $180^{\circ} \mathrm{C}$ and a constant gas flow was used during the whole experiment. Every hour approximately, a spectrum was obtained in order to estimate the particle size with respect to operation time and gas atmosphere, where the acquisition time of the spectrum was $20 \mathrm{~min}$. Two sets of experiments were conducted. During the first one, the oven chamber was flushed with Ar to remove oxygen traces form the gas phase and the lines, while the oven chamber was still at room temperature. In continuance the temperature was increased to $180^{\circ} \mathrm{C}$ and the gas was switched to $5 \% \mathrm{H}_{2}$ in Ar for several hours. In the second case, the catalyst was exposed to a constant flow of air while keeping the temperature at $180^{\circ} \mathrm{C}$ for several hours. The gas was then switched to pure Ar in order to remove any traces of air in the gas phase. Thereafter the chamber was supplied with $5 \% \mathrm{H}_{2}$ in Ar for several hours. For both cases the increase of Pt particle size as a function of time was monitored, and the results are depicted in figure 7-6. 
When the as received electrocatalyst is being exposed directly to $\mathrm{H}_{2}$ atmosphere the particle size reaches a value of $3.7 \mathrm{~nm}$. Whereas, when the Pt surface having a high oxide coverage is then exposed to $\mathrm{H}_{2}$ atmosphere the sintering/agglomeration process is more enhanced, resulting in a higher value of Pt average particle size reaching $4.3 \mathrm{~nm}$. The exact mechanism behind it is still unclear. Nonetheless all of the above results suggest that the sintering/agglomeration mechanism during the aforementioned ECSA evaluation procedure is highly affected by the PtOx species during the polarization of the working electrode at $0.1 \mathrm{~V}$ or $\mathrm{H}_{2}$ exposure.

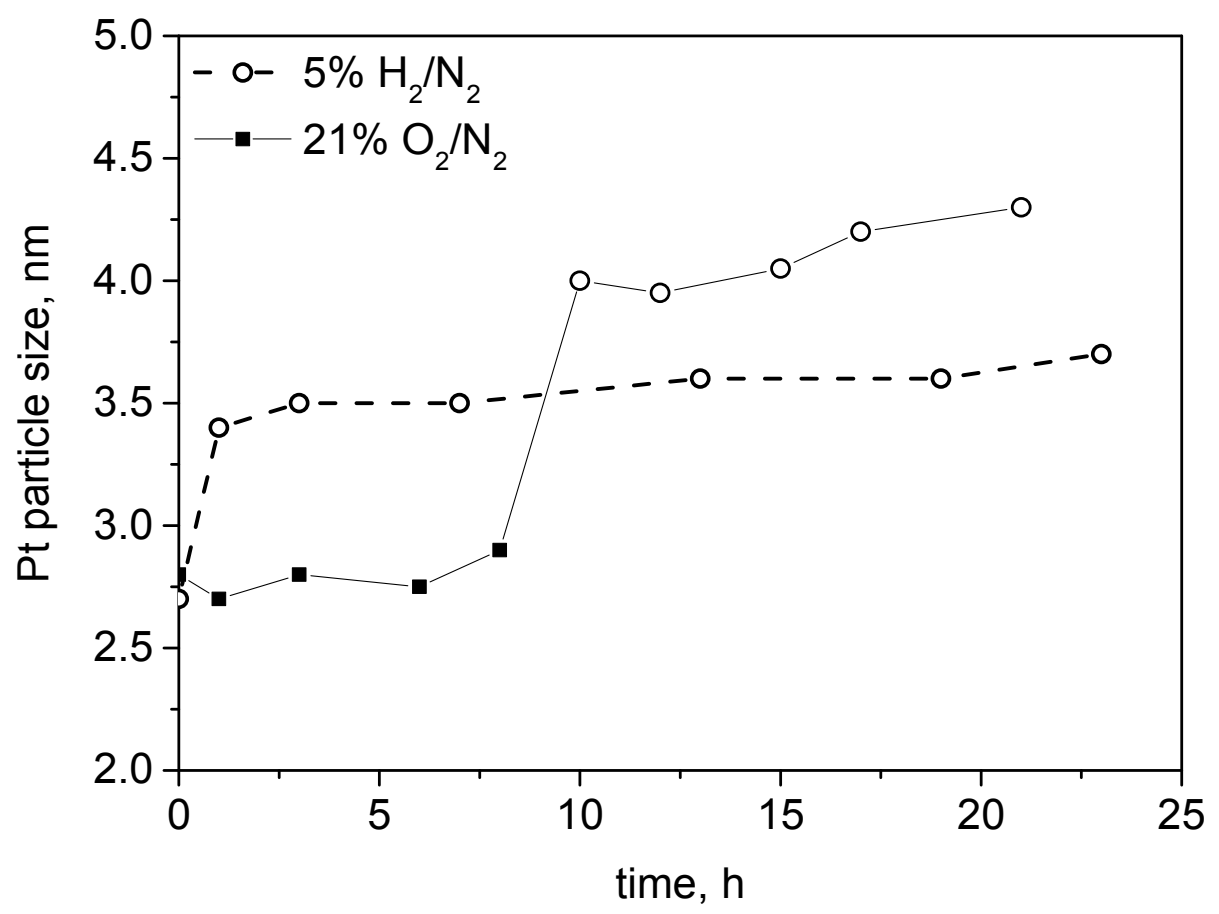

Figure 7-6. The effect of reducing atmosphere $5 \% \mathrm{H} 2 / \mathrm{Ar}$ on the Pt particle size as a function of time. The XRD diffractograms of $30 \mathrm{wt} \% \mathrm{Pt} / \mathrm{C}$ were acquired at $180^{\circ} \mathrm{C}$, with $20 \mathrm{~min}$ acquisition time.

Since the previous two methods resulted in an increase of the Pt particle size during the measurement of ECSA of the cathodic electrode, an alternative way to reduce the Pt surface was evaluated based on $\mathrm{CO}$ electrochemical annealing. Surface structuring effects due to potential cycling is termed electrochemical annealing and in the specific case of cycling in $\mathrm{CO}$ saturated solution, $\mathrm{CO}$ annealing [16,17]. CO annealing of a Pt surface is commonly used for the cleaning and preconditioning of $\mathrm{Pt}$ surface. In our case, the working electrode potential was cycled between $0.075 \mathrm{~V}$ up to $0.9 \mathrm{~V}$ in the presence of $1.62 \mathrm{kPa} \mathrm{CO}$ and $9.46 \mathrm{kPa}$ of $\mathrm{H}_{2} \mathrm{O}$ balance with Ar, with a scan rate of $40 \mathrm{mV} / \mathrm{s}$. The cell's temperature was 
kept at $140^{\circ} \mathrm{C}$. Sequentially $\mathrm{CO}$ was adsorbed at OCP under constant gas flow consisting of $1.8 \mathrm{kPa} \mathrm{CO}$ balanced in $\mathrm{Ar}(100 \mathrm{cc} / \mathrm{min})$ for $20 \mathrm{~min}$. The cathodic compartment was then flushed with $200 \mathrm{cc} / \mathrm{min}$ of Ar to remove all CO traces from the gas phase before potential cycling. The CO stripping procedure that was then followed is described in detail in the experimental section.

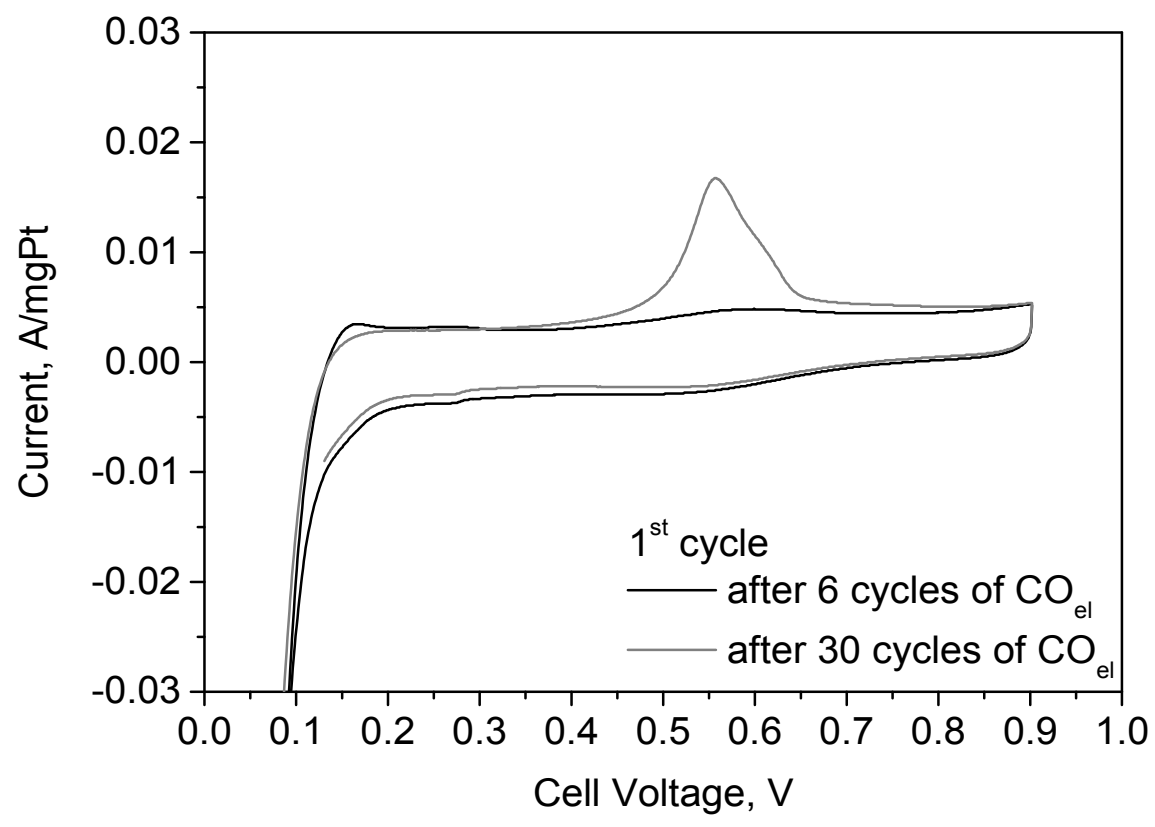

Figure 7-7. First cycle of CO stripping voltamograms, obtained after several cycles of $\mathrm{CO}$ bulk electroxiadation. $\mathrm{CO}$ was adsorbed at open circuit potential for $20 \mathrm{~min}$ at Tcell $=140^{\circ} \mathrm{C}$.

A certain number of potential cycles were required for the complete reduction of the $\mathrm{Pt}$ surface. As shown in figure 7-7, 6 potential cycles of $\mathrm{CO}$ bulk electroxidation were not sufficient for the removal of Pt oxides, as no $\mathrm{CO}$ electroxidation peak was observed. The necessary potential $\mathrm{CO}$ electroxidation cycles required for the complete reduction of the $\mathrm{Pt}$ surface was found to be 30 cycles. The second potential sweep of the same CO stripping was used as a baseline, for the determination for the ECSA. The calculated value was found to be equal to $16.2 \mathrm{~m}^{2} \mathrm{Pt} / \mathrm{gPt}$, which is significantly lower compared to the previous two ECSA evaluation procedures.

The reason for the latter was revealed after characterizing the cathodic catalyst layer via TEM imaging (figure 7-8). The Pt particle size suffered large increase in the particle size, resulting in an average value of $5.5 \mathrm{~nm}$. The Pt degradation mechanism that is believed to 
take place under these conditions is the following. During $\mathrm{CO}$ bulk electroxiation, the amount of dissolved Pt significantly increases, in the cathodic potential sweep of the $\mathrm{CV}$ [18]. After the beginning of CO electroxidation, CO strongly adsorbs on reduced Pt sites again and begins to block further the bulk $\mathrm{CO}$ oxidation. The adsorbed $\mathrm{CO}$ prevents the redeposition of dissolved Pt-ions and leads to Pt dissolution [17]. Full CO coverage of the Pt surface is not achieved within the time elapsed of one potential sweep, due to the strong dependence of the coverage on the partial pressure of $\mathrm{CO}$ in the gas phase at high temperatures. As mentioned in the experimental section $20 \mathrm{~min}$ are required to achieve full coverage when $1.8 \% \mathrm{CO}$ in Argon is used. Thus it is believed that the $\mathrm{Pt}$-ions re-deposit on the remaining $\mathrm{CO}$-free $\mathrm{Pt}$ active sites, resulting in an increase in the average $\mathrm{Pt}$ diameter.

Similar experiments using Pt carbon based catalyst where performed in a RDE at room temperature and it was reported that electrochemical annealing in $\mathrm{CO}$ saturated solution of the catalyst has only minor influence on its structure or the particle shape and size[19]. The later further enhances supports that the high operation temperature is enhancing and promoting the particle agglomeration and sintering.

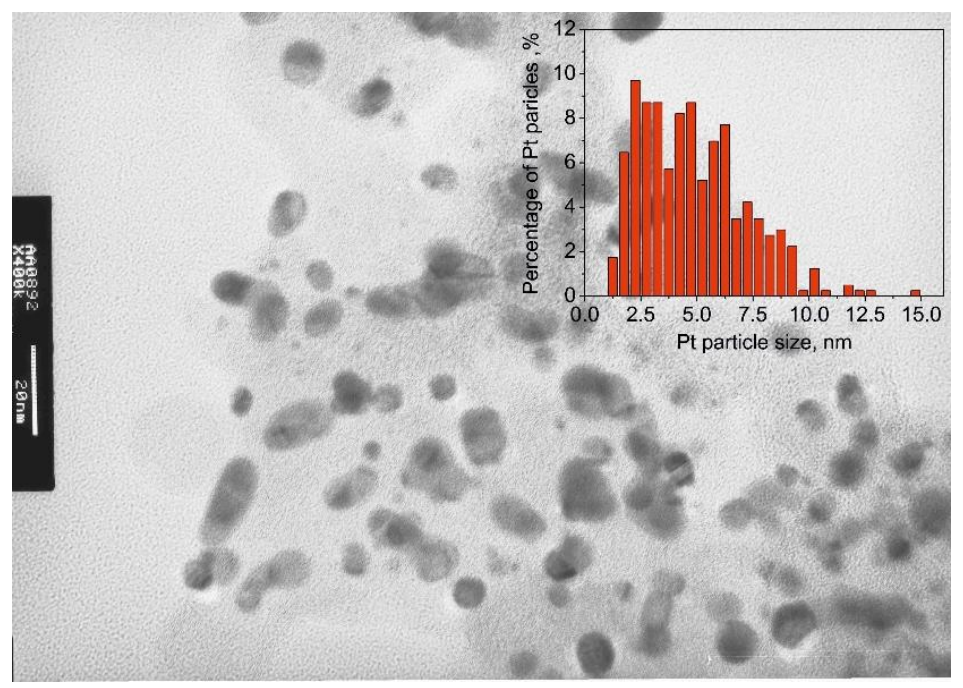

Figure 7-8. TEM micrograph and the corresponding Pt size distribution histogram of $30 \mathrm{wt} \% \mathrm{Pt} / \mathrm{C}$ after 30 cycles of $\mathrm{CO}$ bulk electroxidation. The Pt particle size distribution is included as an inset and the average Pt particle size was estimated $5.5 \mathrm{~nm}$.

The last approach used for the evaluation of ECSA is based on sequential CO adsorption and desorption. It has been reported that exposing $\mathrm{CO}$ on $\mathrm{Pt}$ and then subsequently removing the adsorbed $\mathrm{CO}$ by electrochemical stripping is an excellent method of cleaning and activating Pt [20]. During the CO adsorption and desorption processes, no degradation in the MEA performance or the ECSA of the Pt was noted for low temperatures fuel cells. 
Therefore, a similar procedure was followed for our case. More specifically, after the activation of the MEA, the fuel cell's temperature was lowered at $140^{\circ} \mathrm{C}$ and the cathodic compartment was flushed with Ar. Thereafter, $\mathrm{CO}$ was adsorbed at OCP under constant gas flow of $1.8 \mathrm{kPa} \mathrm{CO}$ balanced in $\mathrm{Ar}(100 \mathrm{cc} / \mathrm{min})$ for $20 \mathrm{~min}$. The $\mathrm{CO}$ adsorption was repeated several times. It has to be noted that the OCP changed after each $\mathrm{CO}$ adsorption.

More specifically, after fuel cell operation and sequential flushing of the cathodic compartment with Ar, the OCP is initially significantly high, around $640 \mathrm{mV}$ in the presence of CO. After the first CO stripping experiment the OCP drops around $400 \mathrm{mV}$, whereas after the third CO stripping experiment reaches $150 \mathrm{mV}$, in the presence of CO. The OCP drop to less positive potential might indicate gradual removal after each procedure of adsorbed oxide species on the Pt surface.

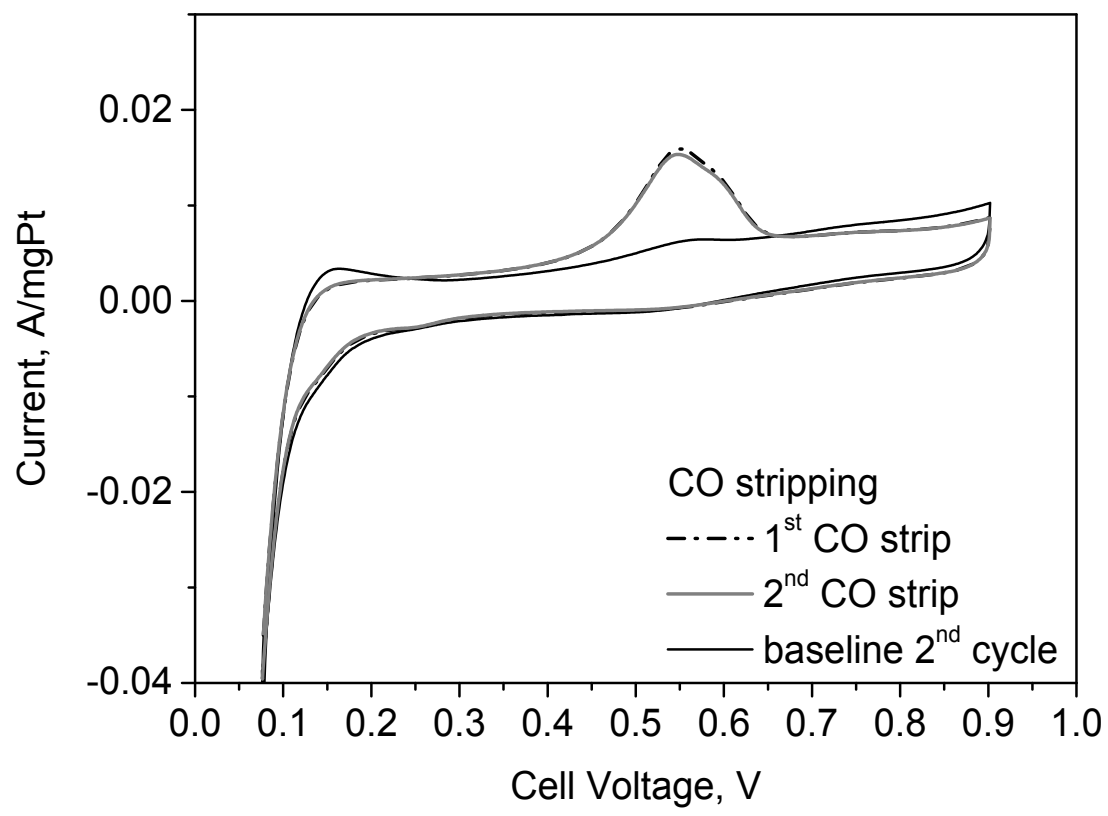

Figure 7-9. First cycle of sequential CO stripping experiments, obtained after normal fuel cell operation at the cathodic electrode. $\mathrm{CO}$ was adsorbed at open circuit potential for 20 $\min$ at $\mathrm{T}_{\text {cell }}=140^{\circ} \mathrm{C}$.

Figure 7-9, presents the obtained CO stripping voltammograms, after a few sequential CO stripping. The area of the $\mathrm{CO}$ electroxidation peak of the $1^{\text {st }}$ and $2^{\text {nd }} \mathrm{CO}$ stripping experiment were identical, therefore the measured charge corresponds to the ECSA. Therefore sequential $\mathrm{CO}$ stripping experiments was found to remove the surface oxides and calculate the ECSA of the cathodic electrode. 
The calculated ECSA was found to be equal to $20.4 \mathrm{~m}^{2} \mathrm{Pt} / \mathrm{gPt}$. TEM micrographs where obtained after the above mentioned procedure, figure 7-10. The average Pt particle size was found to be $4.3 \mathrm{~nm}$. Sequential CO stripping cyclic voltammetry induces less $\mathrm{Pt}$ dissolution [17], and therefore less particle agglomeration and sintering is observed.

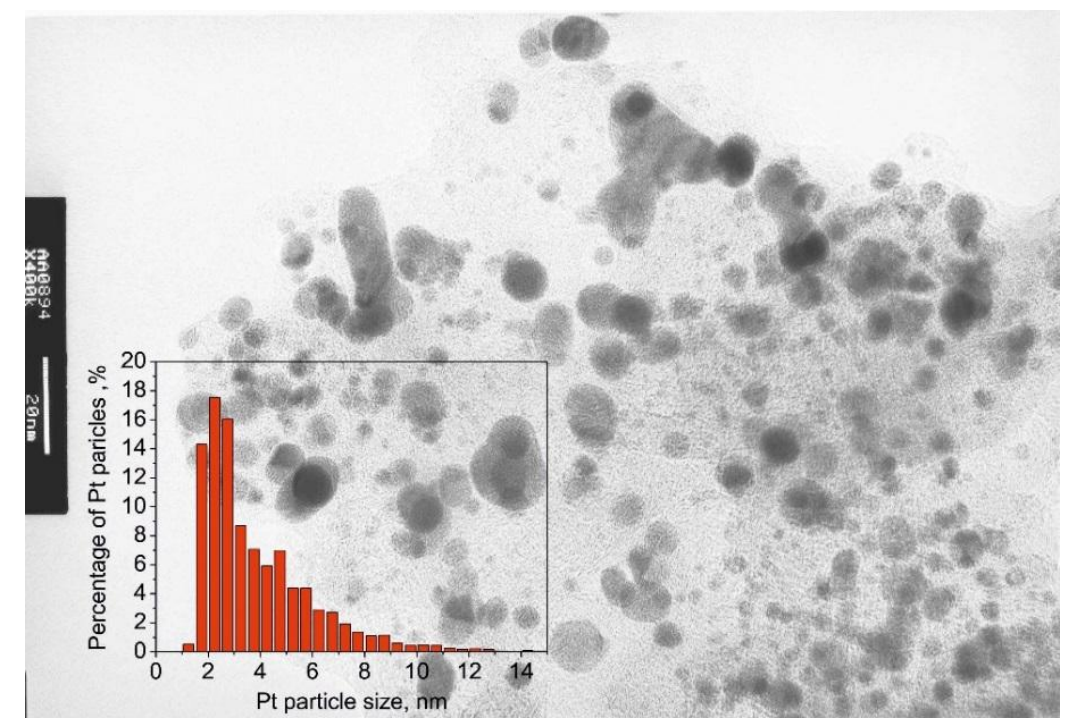

Figure 7-10. TEM micrograph and the corresponding Pt size distribution histogram of $30 \mathrm{wt} \% \mathrm{Pt} / \mathrm{C}$ after 5 sequential $\mathrm{CO}$ stripping experiments. The average Pt particle size was found to be $4.3 \mathrm{~nm}$.

The above procedure is ideal for the determination of ECSA in HTPEM fuel cells, as the minimum possible alteration on the Pt shape and size is observed. Although, the difference between the Pt particle size when no CO striping experiment is conducted, which is approximately $1 \mathrm{~nm}$, rises practical issues concerning the stability of the Pt catalyst during the evaluation of the ECSA using CO in general in PEM fuel cells, especially when operating in high temperature range.

\subsection{Conclusion}

CO stripping voltammetry based procedures were evaluated and the optimum one was chosen for our system, in order to determine the ECSA of a cathodic catalyst layer without severely affecting the Pt particle size distribution. It was found that sequential CO adsorption until complete reduction of the Pt surface is the more appropriate ECSA evaluation procedure causing the minimum damage to the Pt particle size. 


\section{$\underline{\text { References }}$}

[1] Tanja Vidakovic, Mihai Christov, Kai Sundmacher, The use of CO stripping for in situ fuel cell catalyst characterization, Electrochimica Acta 52 (2007) 5606

[2] A. Taniguchi, T. Akita, K. Yasuda, Y. Miyazaki, Analysis of electrocatalyst degradation in PEMFC caused by cell reversal during fuel starvation, J. Power Sources 130 (2004) 42

[3] H.N. Dinh, X. Ren, F.H. Garzon, P. Zelenay, S. Gottesfeld, Electrocatalysis in direct methanol fuel cells: In-situ probing of PtRu anode catalyst surfaces, J. Electroanal.Chem. 491 (2000) 222

[4] S.Q. Song, Z.X. Liang, W.J. Zhou, G.Q. Sun, Q. Xin, V. Stergiopoulos, P.Tsiakaras, Direct methanol fuel cells: The effect of electrode fabrication procedure on MEAs structural properties and cell performance, J. Power Sources 145 (2005) 495

[5] P.Stonehart, J. Hochmuth, Survey on ageing on electrodes and electrocatalysts in phosphoric acid fuel cells, Task 10 of Contract DEN-3-176, Progress Report Stonehart Associates, Inc. Madison, CT (1981)

[6] P.N.Ross, P.Andriacacos, J.Electroanal. Chem. Interfacial Electrochem., 154 (1983) 205

[7] T. Engl , K.E. Waltar, L. Gubler, T.J. Schmidt, Second cycle is dead: Adavanced Electrdoe Diagnostics for High-Temperature Polymer Electrolyte Fuel Cells, J. Electroch. Soc., 161 (2004) F500

[8] N.M. Marcovic, T.J. Schmidt, B.N. Grgur, H.A. Gasteiger, R.J Behm and P.N.Ross, Effect of Temperature on Surface Processes at the Pt(111)-Liquid Interface: Hydrogen Adsorption, Oxide Formation, and CO Oxidation, J. Phys. Chem B,103, 8568 (1999)

[9] K.J.J. Mayrhofer, M. Arenz 1, B.B. Blizanac, V. Stamenkovic,P.N. Ross, N.M. Markovic, CO surface electrochemistry on Pt-nanoparticles: A selective review, Electrochimica Acta 50 (2005) 5144

[10] Evaluation of electrochemical performance for surface-modified carbons as catalyst support in polymer electrolyte membrane (PEM) fuel cells, Abhishek Guha. Thomas A. Zawodzinski Jr.,David A. Schiraldi, Journal of Power Sources 172 (2007) 530

[11] A.M. Chaparro, R.Benitez, L. Gubler, G.G. Scherer, L. Daza, Study of membrane electrode assemblies for PEMFC, with cathodes prepared by the electrospray method, Journal of Power Sources 169 (2007) 77

[12] P. Bindra, S.J. Clouser, E. Yeager, Pt dissolution in concentrated phosphoric acid, J. Electrochem. Soc. 126 (1979) 1631.

[13] PEM FUEL CELL DURABILITY HANDBOOK :PEM Fuel Cell Diagnostic Tools Edited by Haijiang Wang, Xiao-Zi Yuan, Hui Li, CRC Press 2012, International Standard Book Number13: 978-1-4398-3920-1, page 82

[14] S. Brimaud, C. Coutanceau, E. Garnier, J.-M. Le ger,F. Gerard, S. Pronier, M. Leoni, Influence of surfactant removal by chemical or thermal methods on structure and electroactivity of $\mathrm{Pt} / \mathrm{C}$

210

Department of Chemical Engineering, University of Patras 
catalysts prepared by water-in-oil microemulsion, Journal of Electroanalytical Chemistry 602 (2007) 226

[15] P. Stonehart, P.A. Zucks, Sintering and recrystallization of small metal particles. Loss of surface area by platinum-black fuel-cell electrocatalysts, Electrochim. Acta 17 (1972) 2333.

[16] K.J.J. Mayrhofer, M. Arenz, B.B. Blizanac, V. Stamenkovic, P.N. Ross, N.M. Markovic, CO surface electrochemistry on Pt-nanoparticles: A selective review, Electrochim. Acta 50 (2005) 5144.

[17] M. Arenz, K.J.J.Mayrhofer, V. Stamenkovic, B.B. Blizanac, T. Tomoyuki, P.N. Ross, N.M. Markovic, The effect of the particle size on the kinetics of $\mathrm{CO}$ electrooxidation on high surface area Pt catalysts, J. Am. Chem. Soc. 127 (2005) 6819

[18] Angel A. Topalov, Aleksandar R. Zeradjanin, Serhiy Cherevko, Karl J.J. Mayrhofer, The impact of dissolved reactive gases on platinum dissolution in acidic media, Electrochemistry Communications 40 (2014) 49

[19] Karl J.J. Mayrhofer, Marianne Hanzlik, Matthias Arenz, The influence of electrochemical annealing in CO saturated solution on the catalytic activity of Pt nanoparticles, Electrochimica Acta 54 (2009) 5018

[20] Zhiqiang Xu , Zhigang Qi, Arthur Kaufman, Activation of proton-exchange membrane fuel cells via CO oxidative stripping, Journal of Power Sources 156 (2006) 281 


\section{Chapter 8}

\section{Parametric Analysis: Optimization of cathodic catalyst layer of HTPEM fuel cells employing Pt nanoparticles decorated on functionalized MWCNT}

\subsection{Introduction}

Up to date, PEMFCs commercialization is still significantly restricted by its high noble metal content and low cathode performance. Reducing cost via reduction of the Pt content, arising from high catalyst utilization and stability, is highly desired. The design and optimization of electrocatalytic layer, towards the increase of the electrocatalytic active surface area, has been the focus of many studies $[1,2,3,4]$.

The porous electrode's structure consists of the catalyst, usually Pt dispersed on carbon, mixed with a binder-ionomer and an ionic carrier, in order to establish a 3-D ionic link between the Pt catalyst active sites and the polymer electrolyte. In HTPEM fuel cell's, PBI is commonly used as the ionomer [5,6,7], and phosphoric acid as the ionic-proton carrier. The presence of PA possess limitations; slow kinetic of the oxygen reduction reaction [8] 
and reduced oxygen solubility in hot phosphoric acid [9] restricts the performance of the cathodic electrode. The limiting effect of phosphoric acid on the kinetic of the ORR is clearly shown when comparing a typical Tafel slope of PAFC, having values around 110$120 \mathrm{mV} /$ dec $[10,11,12,13]$, with the case of traditional Nafion-based PEMFC, exhibiting values of $60 \mathrm{mV} / \mathrm{dec}[14,15]$. In addition, due to the liquid state of PA and its mobility, maximization of the triple-phase boundary is difficult to achieve. Thus, a certain amount of ionomer is required, that will bind the free PA in the catalyst layer, providing adequate contact with the Pt particles, in order to increase the catalyst utilization. The addition of binder also enhances the kinetic of the oxygen reduction reaction, due to the increased oxygen solubility and suppresses the PA adsorption on the Pt active sites [16,17]. Therefore, a delicate balance must be achieved between the binder and the ionic carrier for the efficient transport of gases and conductance of electrons and protons to the electrochemical interface.

The amount of ionomer and $\mathrm{H}_{3} \mathrm{PO}_{4}$ added to the catalytic layer has been one of the most studied parameters, in order to optimize fuel cell's performance. The amount of phosphoric acid added to the electrode depends on the PBI content for PBI based fuel cells. The influence of the amount of polybenzimidazole $\mathrm{PBI}_{-} \mathrm{H}_{3} \mathrm{PO}_{4}$ (normalized with respect to the PBI loading, expressed as C/PBI weight ratio) content in both the anode and the cathode has been extensively studied for a PBI-based high temperature proton exchange membrane (PEM) fuel cells $[18,5,19]$. It was observed that as the $\mathrm{C} / \mathrm{PBI}$ weight ratio increases the specific pore volume also increases. These differences in the pore volume are mainly located in the micropores and mesopores [20,21], which corresponds to the spaces between the agglomerates of catalyst particles and the spaces within the own agglomerate. For that reason during the preparation of the catalytic ink the ionomer is in solution state in order to penetrate into both types of space [23]. Thus, low PBI loading in the catalytic layer results in high pore volumes and are highly favourable. Parrondo et al. prepared membrane electrode assemblies (MEAs) with various contents of PBI (1-30wt.\%) in the gas diffusion electrode (GDE). The MEAs were tested in the temperature range of $140{ }^{\circ} \mathrm{C}-200^{\circ} \mathrm{C}$ and found that 5wt.\% PBI-based MEA showed the best performance [22]. Also, Kim et al. using phosphoric acid-doped ABPBI membrane reported that with a weight ratio of ionomer to $\mathrm{Pt} / \mathrm{C}$ of $1: 4$ (20wt.\% ionomer in cathodic catalyst layer), the fuel cell shows the lowest ohmic resistance [23].

Uniform mixing and distribution of these components is hard and is not guaranteed, as a result part of the electrocatalyst is not used. Thus, many studies have been focused on another approach using carbon MWNT or nanofiber supported Pt catalyst, aiming the increase of Pt utilization in fuel cells. To this respect, many groups have successfully functionalized MWNT using polymers or monomers in an attempt to increase the threephase boundary (TPB). Nevertheless, to the author's knowledge, few papers or reviews are 
published on their application and testing in fuel cell. Hyung-Suk Oh et al.[24] functionalized CNTs with a thin layer of polypyrrole (PPy) to serve as a catalyst support in PEM fuel cells. They found that the performance of PEM fuel cell when Pt/PPy-CNT catalyst was employed at the cathodic electrode, was significantly enhanced, compared to the Pt/CNT without PPy functionalization. Kazuya Matsumoto et al. [25] wrapped MWNT individually with pyridine based PBI and immobilized Pt particles on the polymer. They demonstrated that the use of the MWNT/PyPBI/Pt catalyst for the PBI-based PEFCs operating at high temperatures in non-humid conditions $\left(120^{\circ} \mathrm{C}\right)$ exhibited higher performance as compared with the non-functionalized nanotubes catalyst, resulting in a good ORR activity.

In a previous work, a new functionalized MWCNT Pt based electrocatalyst was developed in an attempt to increased catalyst utilization in high temperature PEMFCs [26]. Pyridine groups were covalently attached at the outer wall of the MWCNT and were used as the catalyst support. The aim was to achieve a uniform distribution of polar pyridine groups in the catalyst layer. The pyridine groups are known to bind phosphoric acid molecules and was expected that a good proton conductive pathways would be created throughout the catalytic layer, which would result in the increase of the three phase boundary and ultimately the catalyst utilization. The obtained Pt/(ox.MWCNT)-Py electrocatalyst had finely and homogeneously distributed Pt particles, with size ranging between $2-4 \mathrm{~nm}$. In continuance to this work, as presented in chapter 5 and 6, CL were fabricated employing this newly synthesized electrocatalyst and were tested as anodic electrocatalysts. It was found that the Pt utilization reached values higher than $80 \%$, compared to the commercial $30 \mathrm{wt} \% \mathrm{Pt} / \mathrm{C}$ which reached approximately $50 \%$, establishing the role of pyridine groups in the creation of high TPB.

The present chapter focuses on enhancing the cathodic electrode's performance through improvement of the platinum utilization and PA distribution, which would ultimately result in the reduction of the Pt loading without sacrificing the overall cell's performance. A full investigation of the effect of PA loading and its distribution as well as the catalyst type, on the cathode's catalyst layer performance is being presented.

\subsection{Experimental}

\subsubsection{Materials}

Phosphoric acid doped polymer electrolyte membranes were provided from Advent Technologies S.A..The in-house synthesized 30wt\% Pt/(ox.MWCNT)-Py catalyst used in this study was synthesized according to literature [26]. Catalyst powder 30wt \% Pt/Vulcan $\mathrm{XC}-72 \mathrm{R}$ was purchased from Tanaka Kikinzoku International. For the construction of the

214 
electrodes, carbon cloth supporting layer was purchased from Fuel Cell Earth LLC, Vulcan $\mathrm{XC}-72 \mathrm{R}$ powder from RawChem Ltd and the PTFE dispersion in water from SigmaAldrich. 2-propanol $99.8 \leq$ and $\mathrm{H}_{3} \mathrm{PO}_{4}$ purum p.a. $\geq 85 \%$ were purchased from Merck or Sigma Aldrich. All chemicals were used as received unless otherwise noted.

\subsubsection{Instrumentation}

The electrochemical measurements were performed using a single cell purchased from Fuel Cell Technologies Inc. Both graphite bipolar plates of the single cell had the same single serpentine flow field for the distribution of reacting gases. The active area of the electrodes was $2 \mathrm{~cm} \times 2 \mathrm{~cm}$. The assembling torque applied for the single cell was $4.8 \mathrm{Nm}$. The cell was installed in a fuel cell test station which was built in-house and had provisions for controlling temperature, humidification, and flow of reacting gases. The measurements were made in a two-electrode arrangement using an Autolab potentiostat-galvanostat PGSTAT-302. The size of the Pt nanoparticles of the MWCNT and carbon based electrocatalysts, after short term operation, was characterized by transmission electron microscopy (TEM) on JEOL JEM 2100 operating at $200 \mathrm{kV}$. The photoemission experiments were carried out in a ultrahigh vacuum system equipped with a SPECS LHS10 hemispherical electron analyzer, which additionally comprises a water cooled high pressure cell $\left(10^{-3}\right.$ mbar- 2 bar) connected with the vacuum chambers through a fast entry system, a sample preparation chamber (base pressure $5 \times 10^{-9}$ ) and an analysis chamber with a base pressure $\sim 10^{-10}$ mbar where the XPS measurements took place. The XPS measurements were performed at room temperature using unmonochromatized $\mathrm{AlKa}$ radiation under conditions optimized for maximum signal (constant $\Delta \mathrm{E}$ mode with pass energy of $97 \mathrm{eV}$ giving a full width at half maximum (FWHM) of $1.7 \mathrm{eV}$ for the $\mathrm{Au} 4 \mathrm{f}_{7 / 2}$ peak). The measurements were recorded at $0^{\circ}$ with respect to the sample surface normal. The analyzed area was a rectangle with dimensions $2.5 \times 4.5 \mathrm{~mm}^{2}$.

\subsubsection{Electrode Preparation and MEA assembly}

Electrodes' fabrication: The gas diffusion layer, GDL, used in this study was prepared by spraying a slurry made of carbon Vulcan XC-72R and PTFE solution (50 wt\% PTFE/C) on carbon cloth, followed by heat treatment at $300^{\circ} \mathrm{C}$ under static air for $40 \mathrm{~min}$. The electrodes were prepared using two different optimized methods, depending on the catalyst type. Two types of electrocatalysts were used, Pt supported on carbon black (30wt \% Pt/C) and Pt supported on MWCNTs (30wt \%t Pt/(ox.MWCNT)-Py). The electrodes containing $30 \mathrm{wt} \% \mathrm{Pt} / \mathrm{C}$ were prepared by spraying 2-propanol-Pt/C ink on the GDL. The electrodes were then treated for 12 hours at $80^{\circ} \mathrm{C}$ and 3 days at $190^{\circ} \mathrm{C}$ under vacuum, in order to 
remove all traces of the organic solvent. The electrodes containing MWCNT based catalysts were prepared by dispersing the catalyst powder in a 2-propanol/3- $\mathrm{D} \mathrm{H}_{2} \mathrm{O} 2 / 1$ (v/v) solution. The catalyst slurry was applied on the GDL by a spatula and the electrodes were left over night to dry at room temperature prior heating at $100^{\circ} \mathrm{C}$ in an oven for 1 hour. The Pt loading of the constructed cathodic electrodes varied from 0.43 to $2.20 \mathrm{mgPt} / \mathrm{cm}^{2}$. The anode's electrode specifications were kept constant for all MEAs. The carbon black based electrocatalyst (30wt\% Pt/C) was used as counter electrode and the Pt loading was around $1.5 \mathrm{mgPt} / \mathrm{cm}^{2}$ in all cases.

Membrane Electrode Assembly, MEA: Before the MEA assembly, a certain amount of phosphoric acid, PA, was sprayed onto the electrodes. The anodic electrodes were sprayed with $2 \mathrm{gPA} / \mathrm{gPt}$, whereas in the case of the cathodic electrodes the ratio of $\mathrm{gPA} / \mathrm{gPt}$ varied from 2 to 8 . The electrode active area was $2 \mathrm{~cm} \times 2 \mathrm{~cm}$. The specifications of the cathodic electrodes employing either $30 \mathrm{wt} \% \mathrm{Pt} /(\mathrm{ox} . \mathrm{MWCNT})-\mathrm{Py}$ (MEA 1-12) or 30wt \% Pt/C (MEA 13-20) are presented in Table 8-1. The MEAs were fabricated by hot pressing, the phosphoric acid doped membranes (doping level $\sim 200 \mathrm{wt} \%$ ) between the two electrodes at $150^{\circ} \mathrm{C}$ for $5 \mathrm{~min}$.

\subsubsection{Electrochemical Characterization and Catalyst layer characterization}

\subsubsection{Activation of MEA}

All MEAs were activated under galvanostatic mode. Initially the cell's temperature was increased from room temperature to $100^{\circ} \mathrm{C}$ while $\mathrm{Ar}$ flushed both compartments. Subsequently, the gases were switched to pure $\mathrm{H}_{2}$ and $\mathrm{O}_{2}$ and the temperature was set at $140^{\circ} \mathrm{C}$. Once the temperature was reached, a current of $0.2 \mathrm{~A} / \mathrm{cm}^{2}$ was applied to the fuel cell. The temperature was then set at $180^{\circ} \mathrm{C}$. The break in period was found to be approximately 48 hours. Details concerning the activation of the MEAs are provided in detail in the appendix A1.

\subsubsection{Electrochemical Impedance Spectroscopy measurements}

All electrochemical impedance spectroscopy measurements were conducted in galvanostatic mode using a sinusoidal current wave with an amplitude of $5 \mathrm{~mA} / \mathrm{cm}^{2}$ while sweeping the frequency from $50 \mathrm{kHz}$ up to $10 \mathrm{mHz}$ with a logarithmic distribution of 50 points. The measurements were conducted without interrupting the cell's operation. 
Table 8-1. Specifications of the cathodic electrode of the tested MEAs (noble metal loading and phosphoric acid, PA, sprayed on the electrodes prior to the MEA assembly)

\begin{tabular}{|c|c|c|c|}
\hline Catalyst used & MEA No & $\begin{array}{l}\text { Pt loading } \\
\left(\mathrm{mgPt} / \mathrm{cm}^{2}\right)\end{array}$ & $\begin{array}{l}\text { PA amount } \\
\text { (gPA/gPt) }\end{array}$ \\
\hline \multirow{12}{*}{ 30\% Pt/(ox.MWCNT)-Py } & 1 & 0.43 & 2 \\
\hline & 2 & 1.25 & 2 \\
\hline & 3 & 1.76 & 2 \\
\hline & 4 & 2.20 & 2 \\
\hline & 5 & 0.45 & 3 \\
\hline & 6 & 0.50 & 3 \\
\hline & 7 & 0.65 & 3 \\
\hline & 8 & 0.76 & 3 \\
\hline & 9 & 1.29 & 3 \\
\hline & 10 & 1.34 & 4 \\
\hline & 11 & 1.25 & 5 \\
\hline & 12 & 1.31 & 8 \\
\hline \multirow{8}{*}{$30 \% \mathrm{Pt} / \mathrm{C}$} & 13 & 0.48 & 2 \\
\hline & 14 & 0.62 & 2 \\
\hline & 15 & 1.55 & 2 \\
\hline & 16 & 2.14 & 2 \\
\hline & 17 & 1.53 & 3 \\
\hline & 18 & 1.61 & 4 \\
\hline & 19 & 1.62 & 6 \\
\hline & 20 & 1.62 & 8 \\
\hline
\end{tabular}




\subsubsection{CO stripping voltammetry}

The electrochemically active surface area (ECSA) of the cathodic CL employing Pt based catalyst was determined via CO stripping voltammetry, according to an optimized procedure presented in Chapter 7. More specifically sequential CO adsorption was used until full reduction of the Pt surface. The cell temperature was kept constant at $140^{\circ} \mathrm{C}$. One compartment was supplied with a constant flow of $200 \mathrm{cc} / \mathrm{min}$ of $10 \% \mathrm{H}_{2}$ in argon and the corresponding electrode acted as the reference electrode. The compartment with the electrode under investigation (working electrode) was fed with $1.8 \% \mathrm{CO}$ in argon (air liquide) at a flow rate of $100 \mathrm{cc} / \mathrm{min}$. $\mathrm{CO}$ was adsorbed at open circuit potential. It was found that the maximum $\mathrm{CO}$ coverage was achieved in approximately 20 minutes. Subsequently, the compartment was flushed with $200 \mathrm{cc} / \mathrm{min}$ argon in order to remove all the traces of $\mathrm{CO}$ in the gas phase. The argon flow was humidified by passing through a thermostated humidifier kept at a constant temperature of $45^{\circ} \mathrm{C}$, prior to its introduction to the cell. The potential was then cycled between 0.075 and $0.9 \mathrm{~V}$ with a scan rate 10 $\mathrm{mV} / \mathrm{s}$. The above procedure was repeated until the maximum $\mathrm{CO}$ electroxidation peak was obtained. To calculate the CO stripping charge, $\mathrm{Q}_{\mathrm{CO}}$ (in $\mathrm{C}$ ), the area under the peak that corresponds to the $\mathrm{CO}$ electrooxidation was integrated. The second cycle in the same experiment was used for baseline subtraction, considering that during the second cycle $\mathrm{CO}$ was absent from the Pt surface. The ECSA, in $\mathrm{m}^{2}$ was determined considering a charge of $420 \mu \mathrm{C} \mathrm{cm}^{-2}$ for $\mathrm{CO}$ oxidation that corresponds to a monolayer of adsorbed $\mathrm{CO}$ on polycrystalline $\mathrm{Pt}$ as follows:

$$
\operatorname{ECSA}\left(\mathrm{m}^{2}\right)=\mathrm{Q}_{\mathrm{CO}}(\mathrm{C}) / 4.20 \mathrm{C} \mathrm{m}^{-2}
$$

\subsubsection{TEM analysis}

Post mortem analysis of the cathodic catalytic layer of used membrane electrode assemblies (MEA) was carried out by using transmission electron microscopy (TEM). The electrodes were separated from the membrane for the evaluation of the Pt particle size distribution and morphology of the electrocatalysts. Adequate amounts of catalysts were scrapped off the used electrodes and were ultrasonically dispersed in water. A drop of the suspension was placed on a $3 \mathrm{~mm}$ carbon coated copper grids (Electron Microscopy Sciences). The Pt particle size distributions were obtained by manually measuring over $1200 \mathrm{Pt}$ particles from the TEM pictures. The mean Pt particle size was then calculated using the following equation: 


$$
\mathrm{D}_{\mathrm{APt}}=\Sigma \mathrm{D}_{\mathrm{Pt}} \mathrm{N}_{\mathrm{D}} / \mathrm{n} \quad(\mathrm{nm})
$$

where $\mathrm{N}_{\mathrm{D}}$ is the number of particles corresponding to a Pt particle size with diameter $\mathrm{D}_{\mathrm{Pt}}$ $(\mathrm{nm})$ and $\mathrm{n}$ is the total number of measured particles. It has to be noted that the MEAs that were subjected to ECSA evaluation were not used for postmortem analysis; as the CO stripping procedure at the cathodic electrode was proven to damage the Pt distribution and increase the average Pt size, as discussed in detail in Chapter 7.

\subsection{Results and discussion}

\subsubsection{Influence of PA amount in the CL on the fuel cell's performance}

In phosphoric acid fuel cell there is an optimum acid loading for a given catalyst layer thickness, catalyst type or amount of binder in the catalyst layer. The distribution of PA within the catalytic layer depends on the hydrophobicity of the catalyst, the pore size distribution and the porosity of the catalytic layer (CL). Whereas the concentration of PA in the pores of the catalyst layer depends on the partial pressure of water and the operation temperature. Therefore, it was necessary to investigate the effect of the PA amount imbedded in the cathodic CL on the fuel cell's performance, for the newly synthesized catalyst 30wt\%Pt/oxMWCNT-Py, as well as for the commercial 30wt $\% \mathrm{Pt} / \mathrm{C}$. The $\mathrm{H}_{3} \mathrm{PO}_{4}$ amount in the catalyst layer was altered between $2 \mathrm{gPA} / \mathrm{gPt}$ and $8 \mathrm{gPA} / \mathrm{gPt}$. The Pt loading was kept constant around $1.3 \mathrm{mgPt} / \mathrm{cm}^{2}$ for the catalyst layer employing $30 \mathrm{wt} \%$ Pt/oxMWCNT-Py (MEA 2, 9-12), whereas approximately $1.5 \mathrm{mgPt} / \mathrm{cm}^{2}$ for the case of $30 \mathrm{wt} \% \mathrm{Pt} / \mathrm{C}$ (MEA 17-20). Polarization curves were obtained at $180^{\circ} \mathrm{C}$ under pure $\mathrm{H}_{2}$ and $\mathrm{O}_{2}$ flow (gas stoichiometry $\lambda_{\mathrm{H} 2}=1.2$ and $\lambda_{\mathrm{O} 2}=2$ ) and the results are depicted in figure 8-1. Increasing the PA amount present in the cathodic catalyst layer, as shown in figure 8-1, results in decrease of the overall fuel cell's performance. This is to be expected, as excessive PA amount in the cathodic electrode, floods the pores of the catalyst layer, increasing the mass transport resistance due to low solubility of oxygen and its slow diffusion in phosphoric acid [27]. Nonetheless the observed voltage loss depends on the catalyst type. The MEAs employing the commercial catalyst $30 \mathrm{wt} \% \mathrm{Pt} / \mathrm{C}$, exhibit a gradual voltage loss as the ionic carrier amount was increased in the electrode, whereas for the case of the $30 \mathrm{wt} \% \mathrm{Pt} / \mathrm{oxMWCNT}-\mathrm{Py}$, up to $4 \mathrm{gPA} / \mathrm{gPt}$, no significant voltage loss was observed. Comparing the performance between the two electrocatalysts for the same PA content, the $30 \mathrm{wt} \% \mathrm{Pt} / \mathrm{oxMWCNT}$-Py exhibits smaller voltage losses, under the same experimental conditions. The latter is clearly depicted in figure 8-2, where the acquired voltage, IR drop 

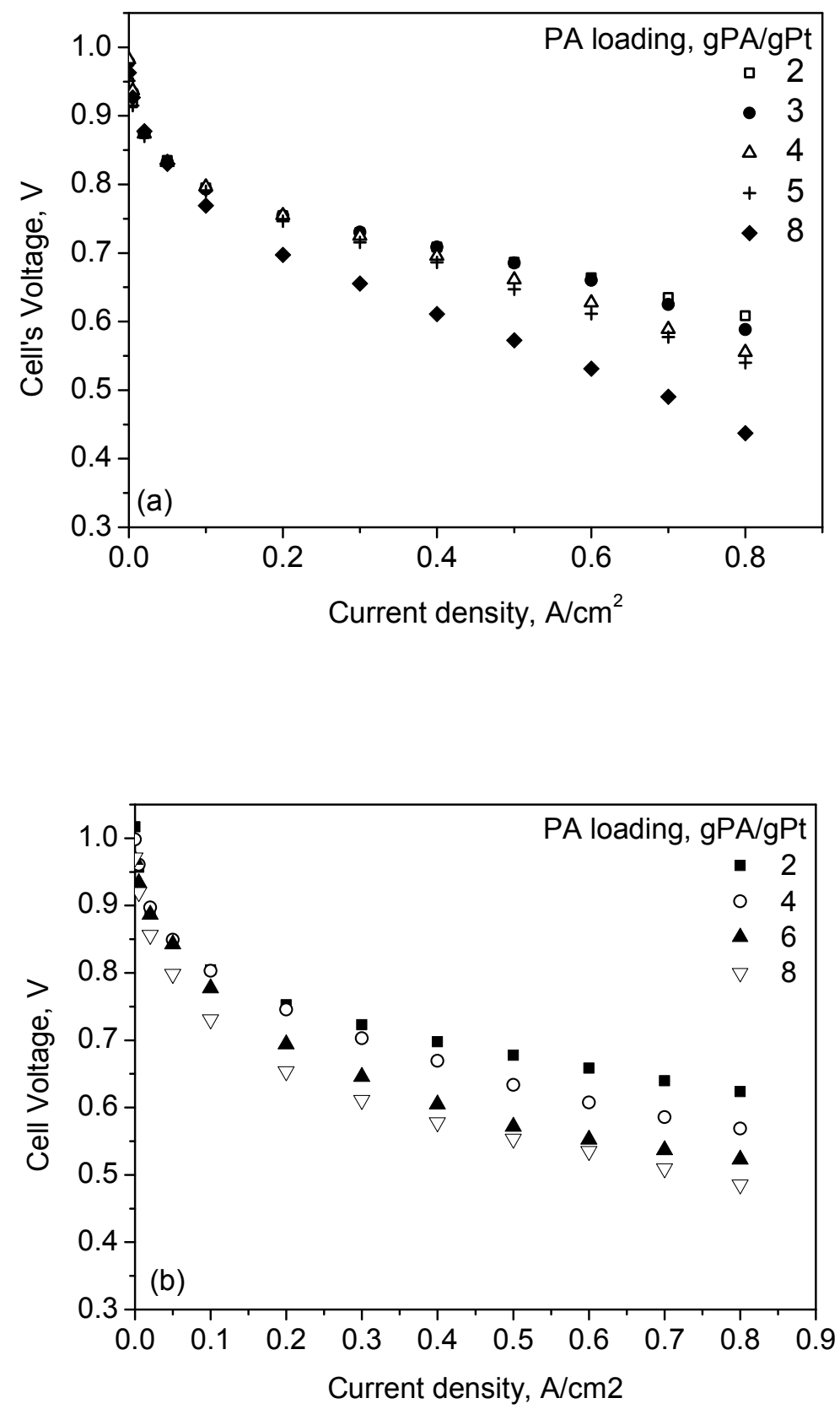

Figure 8-1. The effect of PA amount imbedded in the cathodic electrode, on the overall fuel cell's performance. The polarzation curves, IR drop corrected, were obtained at $180^{\circ} \mathrm{C}$ and gas stoichiometry of 1.2 and 2 of pure $\mathrm{H}_{2}$ and $\mathrm{O}_{2}$, respectively. The PA amount varied between 2 and $8 \mathrm{gPA} / \mathrm{gPt}$. a. MEAs 2,9-12 employing 30wt\% Pt/oxMWCNT-Py and b. MEAs 17-20 employing 30wt\% Pt/C. 

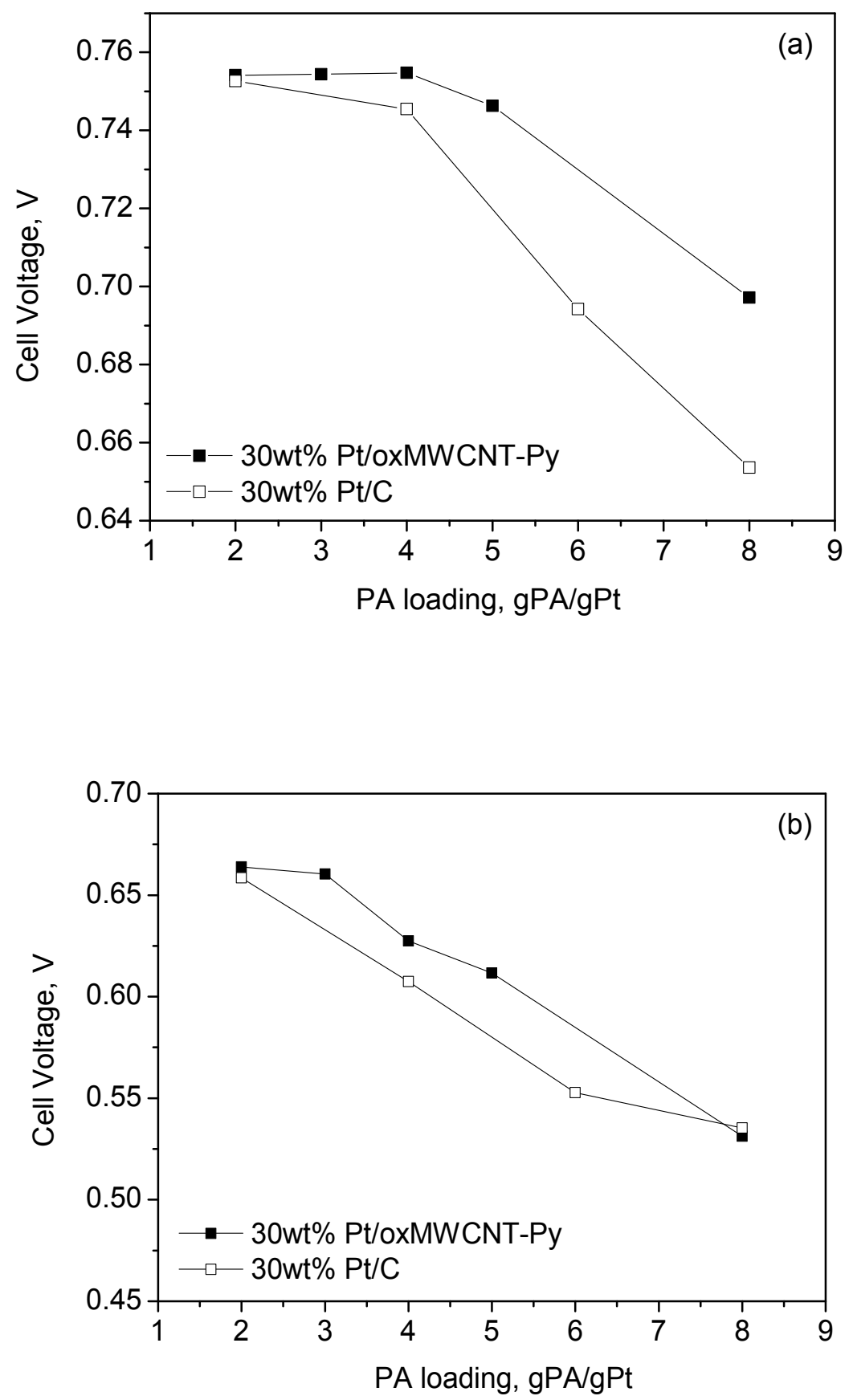

Figure 8-2. The effect of PA amount on the fuel cell's voltage (IR drop corrected) under pure $\mathrm{H}_{2}$ and $\mathrm{O}_{2}$ (gas stoichiometry $\lambda_{\mathrm{H} 2}=1.2$ and $\lambda_{\mathrm{O} 2}=2$ ) at $180^{\circ} \mathrm{C}$ at a. $0.2 \mathrm{~A} / \mathrm{cm}^{2}$ and b. 0.6 $\mathrm{A} / \mathrm{cm}^{2}$ 
corrected, is plotted versus the PA amount in the catalyst layer for specific current densities. When phosphoric acid is exposed to elevated temperature two simultaneous phenomena are initiated: dehydration due to elevated temperature and formation of pyrophosphoric acid, and hydrolysis of the later from the produced water, as a result of its hydroscopic nature. The water uptake of the phosphoric acid, depends on the water's partial pressure $[28,29]$. Both phenomena can influence the ionic conductive pathway in the CL and thus the cathodic electrode's performance. During normal fuel cell operation, the distributed PA within the catalytic layer is being hydrated by the produced steam. It can be assumed that upon water solvation the hydrated PA swells. This may cause ganglia formation inside the pores' channels, blockage of the pores and the disruption of the distributed PA thin film, thus resulting into the shrinkage of the electrochemical interphase as well as additional gas transport limitations besides the ones originating from low solubility of oxygen and slow diffusion in phosphoric acid. In the case of low PA loadings we may consider that the thinner distribution of the PA film does not swell to such degree so as to result in the ganglia formation and the blockage of the pore channels or the disruption of the ionic pathway. The presence of pyridine groups in the catalyst layer, homogeneously distributes PA and stabilizes it inside the catalyst layer, reducing the ganglia formation. Thus the thin film of PA swells by the produced water without severe disruption of the proton conduction pathway and blockage of the pores, due to positive effect of pyridine-phosphoric acid interaction. The created well extended 3D ionic network of PA minimizes the local flooding of the pores in the electrodes facilitating the mass transport in the catalyst layer.

In order to further support the above, Nyquist and Bode plots were acquired from the above MEAs, under the same experimental conditions, at a current density of $0.2 \mathrm{~A} / \mathrm{cm}^{2}$. Figure 8-3 and 8-4 shows the change in the polarization resistance with respect to the PA amount in the catalyst layer, for both electrocatalysts. Two arcs are observed at the Nyquist plot for high and intermediate frequencies, fig 8-3a and 8-4a. The first one, which appears at high frequencies corresponds to the impedance of the $\mathrm{H}_{2}$ oxidation reaction at the anode side [30,31], as also shown in chapter 5. Although, other claim that the high frequency arc is related to the proton conduction in the catalyst layer [32]. Whereas for the second arc, appearing in the intermediate frequency, the general consensus is that it originates from activation losses and from dominated cathode effects. Some research groups claim that it originates from the combination of anode and cathode activation [33,34]. Whereas in the low frequency range, two different contributions are observed, related to the PA amount present in the CL. The Nernst impedance (finite diffusion) as an additional loop (figure 8$4 \mathrm{a}$ for $2 \mathrm{gPA} / \mathrm{gPt}$ ), and the Warburg impedance (infinite diffusion) as a straight line with a slope of 1 (real part = imaginary part). 

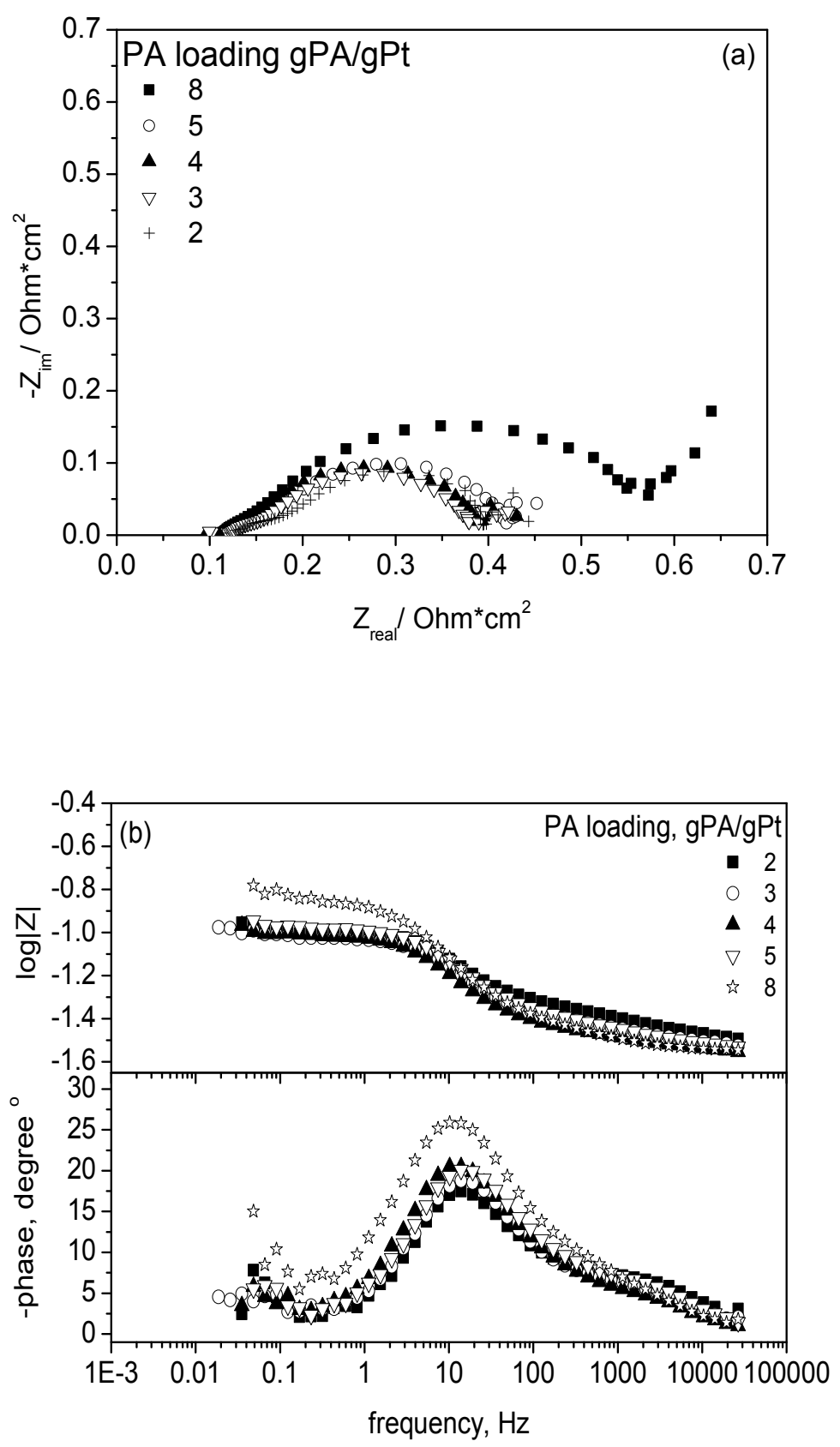

Figure 8-3. The effect of the PA amount imbedded in the cathodic catalyst layer on the charge transfer resistance a. Nyquist and b. bode plots. The data were obtained at $180^{\circ} \mathrm{C}$ and at a gas stoichiometry of 1.2 and 2 of pure $\mathrm{H}_{2}$ and $\mathrm{O}_{2}$, respectively. The current was set at $0.2 \mathrm{~A} / \mathrm{cm}^{2}$. The MEAs (2,9-12) employing 30wt\% Pt/oxMWCNT-Py MEA. The Pt loading was similar for all MEAs, whereas the amount of PA was altered from 2 to 8 $\mathrm{gPA} / \mathrm{gPt}$. 

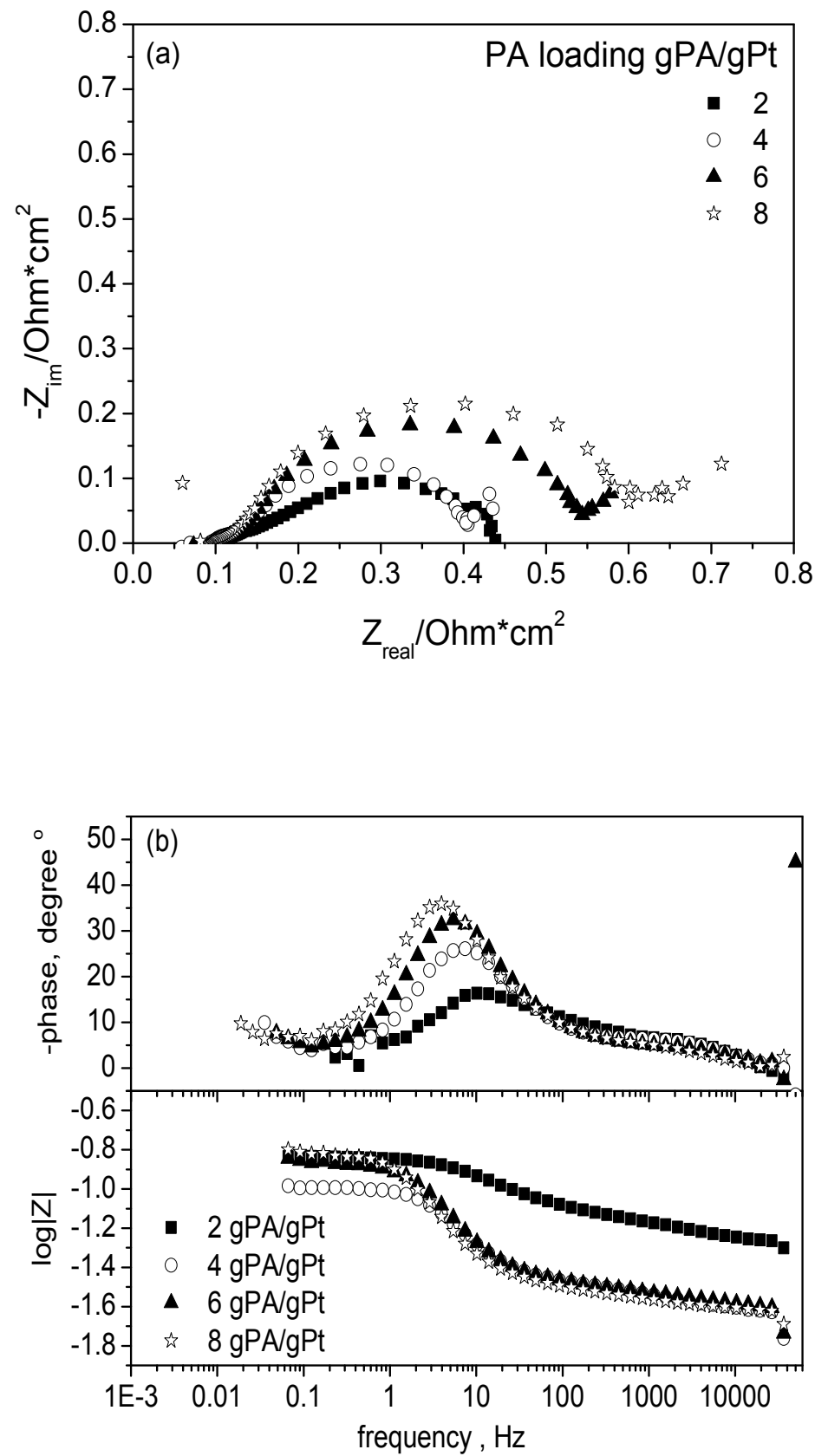

Figure 8-4. The effect of the PA amount imbedded in the cathodic catalyst layer on the charge transfer resistance a. Nyquist and $\mathrm{b}$. bode plots. The data were obtained at $180^{\circ} \mathrm{C}$ and at a gas stoichiometry of 1.2 and 2 of pure $\mathrm{H}_{2}$ and $\mathrm{O}_{2}$, respectively. The current was set at $0.2 \mathrm{~A} / \mathrm{cm}^{2}$. The MEAs (17-20) employed $30 \mathrm{wt} \% \mathrm{Pt} / \mathrm{C}$. The Pt loading was similar for all MEAs, whereas the amount of PA was altered from 2 to $8 \mathrm{gPA} / \mathrm{gPt}$. 
In the Bode diagram due to the logarithmic scale of the impedance the difference of the two kinds of diffusion cannot be discerned so clearly (fig 8-3b and 8-4b). Taking into consideration the thin film flooded agglomerate model, two types of diffusion take place in the CL [35]: agglomerate diffusion, related to the diffusion of the reactants through the open pores of the agglomerate spaces; and thin-film diffusion within the flooded pores (filled with the electrolyte). These two processes are believed to be affected by the $\mathrm{H}_{3} \mathrm{PO}_{4}$ amount imbedded in the CL and its distribution and are reflected under low gas stoichiometry through the potential drop, as well as through the AC impedance characteristics at the low frequency range. The acid doping level or acid film thickness over the catalyst site plays an important role in the overall performance. Increasing PA film thickness would lead to better proton conductivity as the volume fraction of the acid in the catalyst layer would increase. On the other hand, increasing thickness will lead to a smaller oxygen flux across the thin film, which will impose further mass transport limitations, as defined from Fick's law.

For both electrocatalysts, high PA amount in the cathodic catalyst layer, resulted in the increase of mass transport limitations leading to an increase of the cathodic polarization resistance and the appearance of a finite or infinite diffusion element. The minimum polarization resistance of the cathodic electrode was achieved for $2-3 \mathrm{gPA} / \mathrm{gPt}$, which means that the oxygen reduction reaction was carried out under the optimum kinetic and mass transport conditions. More specifically, for the case of $30 \mathrm{wt} \% \mathrm{Pt} / \mathrm{C}$ and for $2 \mathrm{gPA} / \mathrm{gPt}$, the electrode presents a finite diffusion element, which turns to an infinite diffusion element as the PA amount increases in the CL. For the case of the catalyst layer employing $30 \mathrm{wt} \% \mathrm{Pt} / \mathrm{oxMWCNT}-\mathrm{Py}$, the infinite diffusion element is only present at higher PA loadings. In addition, the polarization resistance of the latter is slightly influenced by the PA amount up to $5 \mathrm{gPA} / \mathrm{gPt}$, as opposed to the $30 \mathrm{w} \% \mathrm{Pt} / \mathrm{C}$ catalyst layer (fig. 8-3a and 84a). This is a clear indication of the homogeneous PA distribution, due to the presence of pyridine groups in the catalyst layer, which minimizes the mass transport limitations.

When the performance of the fuel cell exhibits a substantial increase as the gas stoichiometry increases, especially at the cathodic electrode, it indicates mass transport limitation [36]. Therefore to shed more light into the matter, the gas stoichiometry was increased by supplying the anodic and cathodic compartment with a gas flow of $200 \mathrm{cc} / \mathrm{min}$ $\mathrm{H}_{2}$ and $\mathrm{O}_{2}$ respectively, corresponding to a nominal current value of $6.6 \mathrm{~A} / \mathrm{cm}^{2}$. As shown in figure 8-5, a substantial improvement in the performance was observed when the volumetric flow is increased, as compared to the results depicted in figure 8-1 under normal fuel cell operation (gas stoichiometry of 1.2 and 2 of pure $\mathrm{H}_{2}$ and $\mathrm{O}_{2}$ respectively). Under this high stoichiometry, the MEAs employing 30\% Pt/oxMWCNT-Py exhibit similar 
performance, regardless of the PA amount in the cathodic catalyst layer, as expected. The same behavior was observed for the MEAs employing $30 \mathrm{wt} \% \mathrm{Pt} / \mathrm{C}$.

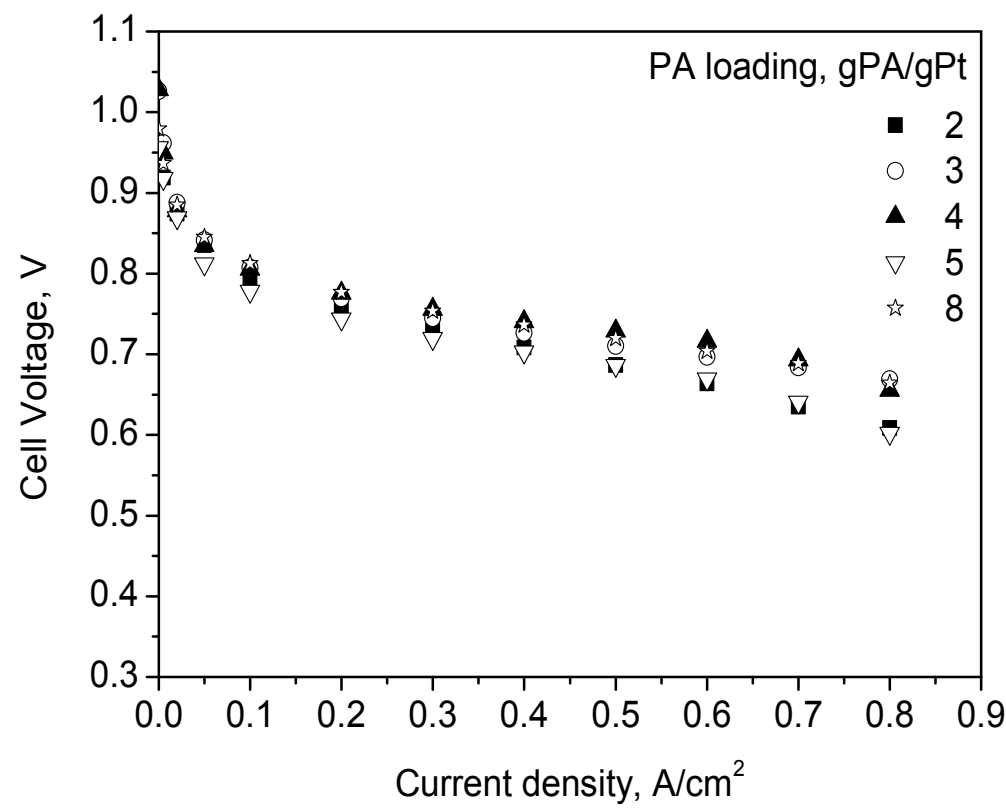

Figure 8-5 Polarization curves, IR drop corrected, at $180^{\circ} \mathrm{C}$ of MEAs 2 and 9-12, employing 30wt\% Pt/oxMWCNT-Py with different amount of sprayed PA at the cathodic electrode (2 to $8 \mathrm{gPA} / \mathrm{gPt}$ ) under high $\mathrm{H}_{2}$ and $\mathrm{O}_{2}$ flows $(200 \mathrm{cc} / \mathrm{min}$ respectively).

As shown from comparison of figure 8-4 and 8-5, the gas stoichiometry has a larger effect on the fuel cell's performance for high PA loadings in the catalyst layer. A small increase on the fuel cell's performance when increasing the gas stoichiometry is expected as the partial pressure of $\mathrm{O}_{2}$ is higher along the channel and at the exit of the fuel cell. Nonetheless, a performance increase above $50 \mathrm{mV}$ suggests mass transport limitation at the cathodic electrode due to excessive PA. Due to water production at the cathodic electrode PA upon hydration swells, causing blockage of the pores. The PA's water uptake largely depends on the water's partial pressure. Therefore, as the gas stoichiometry increases the water partial pressure at the cathodic electrode significantly decreases, shifting the equilibrium towards the formation of dehydrated PA, which results in shrinkage of the PA thin film, increasing the free volume of the catalyst layer's pores. Electrochemical 
Impedance spectra were acquired at the above high volumetric flows and at current density of $0.2 \mathrm{~A} / \mathrm{cm}^{2}$, the results are summarized in figure 8-6. The cathode and anode stoichiometry has a clear impact on the polarization resistance $(\mathrm{Rp})$ but also on the proton conduction resistance, Rel. The ohmic resistance, which is the sum of membrane resistance, the ohmic resistance of the electrodes as well as contact resistances [37], shifts to higher values when the volumetric flow is shifted from $\lambda_{\mathrm{H} 2}=2$ and $\lambda_{\mathrm{O} 2}=2$ to $\lambda_{\mathrm{H} 2}=33.3$ and $\lambda_{\mathrm{O} 2}=66.6$ for $0.2 \mathrm{~A} / \mathrm{cm}^{2}$. This is to be expected as the ohmic resistance of a fuel cell depends on the applied current density, the gas flow and the operating temperature [38].

Reduction of the flow rate, especially of the cathodic compartment, results in higher proton/membrane conductivity due to changes in the water molar fraction. As O2 volumetric flow increases the water molar fraction decreases in the fuel cell, resulting in a shift of the reaction equilibrium towards the formation of pyrophosphoric acid, as also mentioned in detail in chapter 5. This causes a decrease in the proton conductivity of the MEA and the catalytic activity. The latter is more enhanced as the PA amount decreases in the catalyst layer, figure 8-6.

When the cell is supplied with a sufficient flow of reactants the gas transfer resistance becomes negligible or at least constant if the flow remains unchanged. No contribution of either Nernst or Warburg diffusion is observed. The elimination of the low frequency component is believed to be due to the mass flow being forced through the GDL by convection, as the pressure drop is higher along the flow channels, as opposed to the case of low stoichiometry, $\lambda \mathrm{O} 2=2$, The later depends on the GDL structure and the flow field geometry $[39,40]$. As well as due to the higher free volume in the porous structure of the electrodes due to dehydration of the PA and shrinkage of the PA thin film.

The polarization resistance of the cathodic electrode was calculated, after fitting of the Impedance data, using the appropriate equivalent circuit and the Autolab software. The polarization resistance's value were found to be similar regardless of the PA imbedded in the catalyst layer at the above conditions. The cathodic polarization resistance were found to be in the range of $216 \mathrm{mOhm} * \mathrm{~cm} 2$ and $204 \mathrm{mOhm} * \mathrm{~cm} 2$. . 

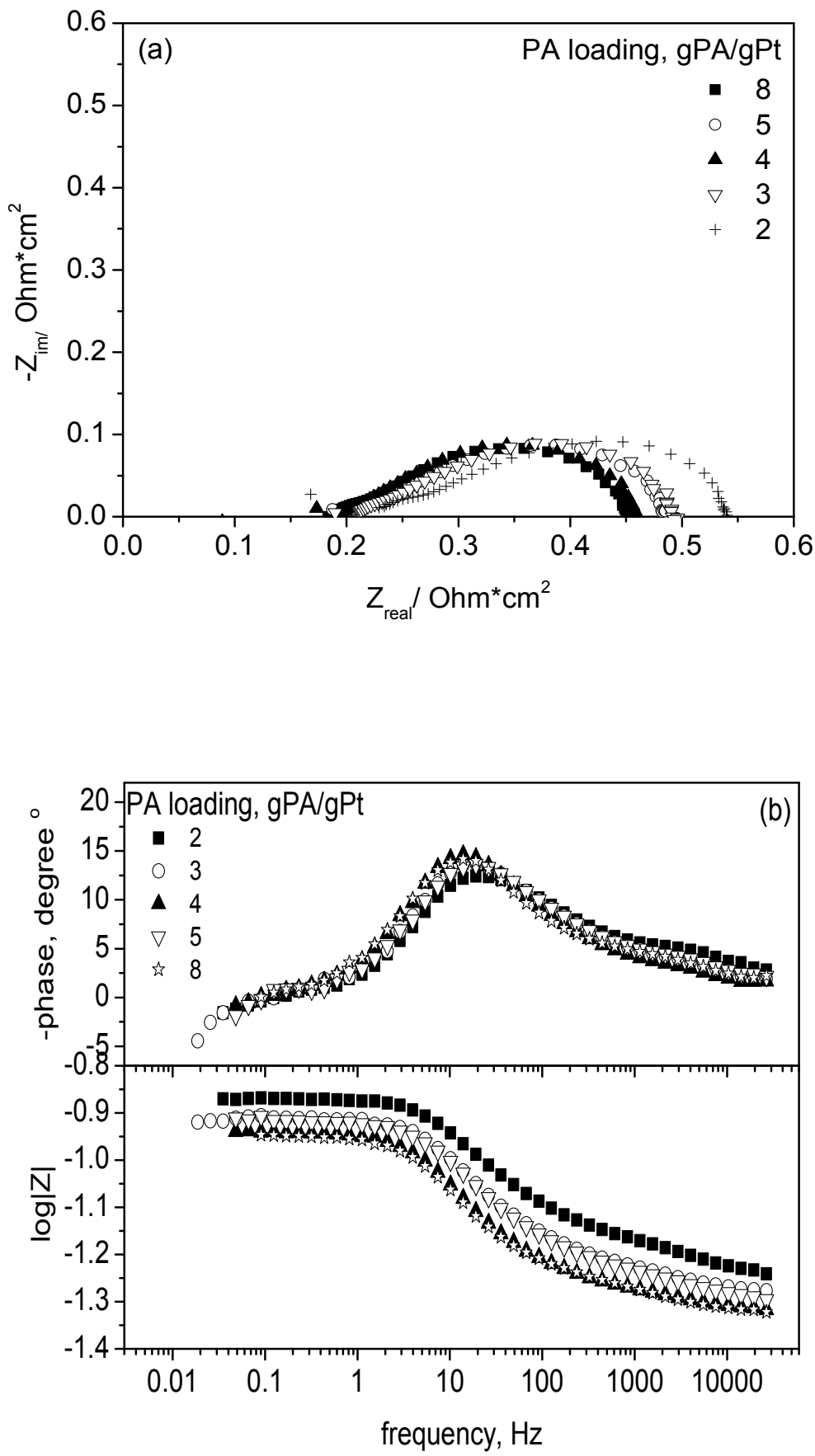

Figure 8-6 a. Nyquist and b. bode plots of MEA - employing 30wt\% Pt/oxMWCNT-Py. The data were obtained at $0.2 \mathrm{~A} / \mathrm{cm}^{2}$ and at $180^{\circ} \mathrm{C}$ under constant high gas flow of pure $\mathrm{H}_{2}$ and $\mathrm{O}_{2}(200 \mathrm{cc} / \mathrm{min}$ respectively). The Pt loading was similar for all MEAs (2, 9-12), whereas the amount of PA was altered from 2 to $8 \mathrm{gPA} / \mathrm{gPt}$. 


\subsubsection{Influence of PA amount on the ECSA}

Determination of the ECSA of the cathodic catalyst layers employing 30wt $\%$ $\mathrm{Pt} /$ oxMWCNT-Py and 30wt\% $\mathrm{Pt} / \mathrm{C}$ with respect to the PA loading was performed via $\mathrm{CO}$ stripping voltammetry. The results are depicted in figure 8-7a. It is clear that the catalyst bearing pyridine groups exhibits higher ECSA values compared to the commercial catalyst. The ECSA is found to be independent of the PA amount in the CL for values higher than $3 \mathrm{gPA} / \mathrm{gPt}$ for the case of the $30 \mathrm{wt} \% \mathrm{Pt} / \mathrm{oxMWCNT}-\mathrm{Py}$, as opposed for the case of $30 \mathrm{wt} \% \mathrm{Pt} / \mathrm{C}$, where there is a linear reliance between the PA amount present in the CL. The values of the measured ECSA were lower than expected in both cases, especially for low PA loadings. The cathodic catalyst layer's ECSA was found to be between 17 and 30 $\mathrm{m}^{2} \mathrm{Pt} / \mathrm{gPt}$ for the $30 \mathrm{wt} \% \mathrm{Pt} / \mathrm{C}$ and 27 to $35 \mathrm{~m}^{2} \mathrm{Pt} / \mathrm{gPt}$ for the case of $30 \mathrm{wt} \% \mathrm{Pt} / \mathrm{oxMWCNT}$ Py.

As mentioned in chapter 5, the ECSA for both electrocatalysts, 30wt $\% \mathrm{Pt} / \mathrm{C}$ and $30 \mathrm{wt} \% \mathrm{Pt} / \mathrm{oxMWCNT}-\mathrm{Py}$, when used at the anodic electrode had values of 35 and 40 $\mathrm{m}^{2} \mathrm{Pt} / \mathrm{gPt}$ for PA loading of $2 \mathrm{gPA} / \mathrm{gPt}$, respectively. This values are higher than the one obtained when used at the cathodic electrode for the same PA loading, $2 \mathrm{gPA} / \mathrm{gPt}$, especially for the case of $30 \mathrm{wt} \% \mathrm{Pt} / \mathrm{C}$. More specifically for $2 \mathrm{gPA} / \mathrm{gPt}$ when $30 \mathrm{wt} \% \mathrm{Pt} / \mathrm{C}$ is used at the cathode has a ECSA value of $17 \mathrm{~m}^{2} \mathrm{Pt} / \mathrm{gPt}$ as opposed to $35 \mathrm{~m}^{2} \mathrm{Pt} / \mathrm{gPt}$ when used at the anode. One could claim that the later could be a difference in the Pt particle size, which would influence the available Pt surface. Table 8-2 summarizes the Pt average size of both electrocatalyst after the activation of the fuel cell when employed either at the cathode or the anode. Interestingly the Pt particle size for both electrocatalyst is higher after the activation process at the anodic electrode, compared to the cathode. Therefore, based on the latter, the ECSA of the cathodic electrode should be higher than the anodic electrodes, since the Pt particle is smaller compared to the anode's after the activation period. Although this was not the case, as the electrochemical active surface area is higher for the case of the anodic electrode. This suggest that the lower ECSA values obtained from the cathodic electrode are linked to the distribution of the PA and the three phase boundary length.

Table 8-2. The average Pt particle size of the $30 \mathrm{wt} \% \mathrm{Pt} / \mathrm{oxMWCNT}-\mathrm{Py}$ and $30 \mathrm{wt} \% \mathrm{Pt} / \mathrm{C}$ when employed at the anode and cathode. The measurement of the Pt sizes were conducted after the activation period.

\begin{tabular}{ccc}
\hline Operation condition & $\begin{array}{c}\text { Pt average particle size } \\
\text { 30\%Pt/ox.MWCNT-Py }\end{array}$ & $\begin{array}{c}(\mathrm{nm}) \\
30 \% \mathrm{Pt} / \mathrm{C}\end{array}$ \\
\hline Cathode & 4.2 & 3.8 \\
Anode & 5.6 & 4.1 \\
\hline
\end{tabular}



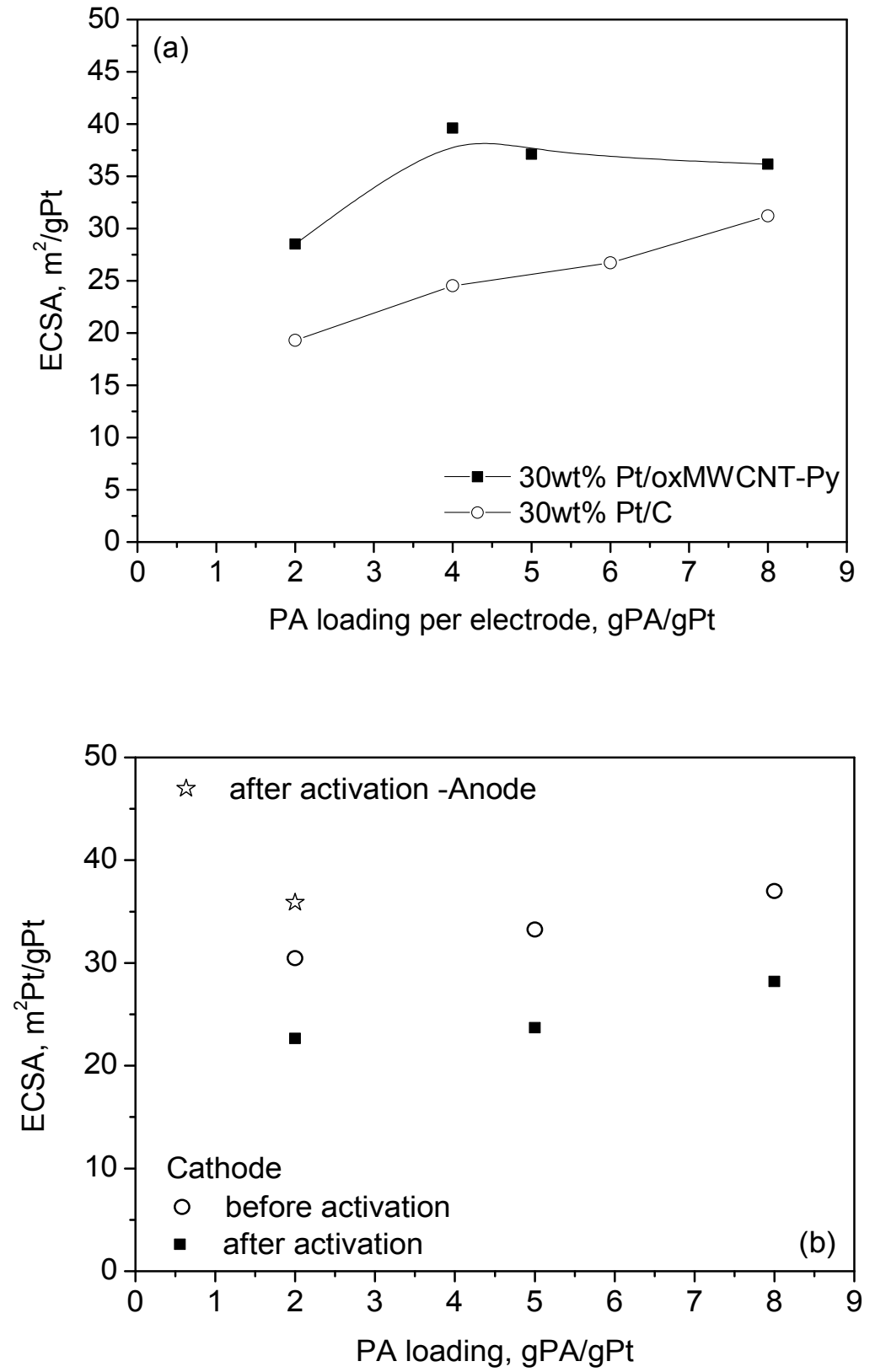

Figure 8-7. ECSA as measured via CO stripping, with respect to the $\mathrm{PA}$ amount in the CL a. after the activation of MEA employing 30wt\%Pt/oxMWCNT-Py and $30 \mathrm{wt} \% \mathrm{Pt} / \mathrm{C}$. b. before and after activation for MEAs employing 30wt\% Pt/C 
To shed more light into the matter, the ECSA of the cathodic electrode was evaluated before and after the activation of the MEA. The MEAs that were used had the same Pt loading employing $30 \mathrm{wt} \% \mathrm{Pt} / \mathrm{C}, 1.55 \mathrm{mgPt} / \mathrm{cm}^{2}$, while the $\mathrm{PA}$ loading varied from $2 \mathrm{gPA} / \mathrm{gPt}$ to $8 \mathrm{gPA} / \mathrm{gPt}$. The results are shown in figure $8-7 \mathrm{~b}$. The ECSA before activation of the MEA for all cases was higher by approximately $9 \mathrm{~m}^{2} / \mathrm{gPt}$ compared to after the activation. The latter is in contrast to the literature, as after the activation due to the redistribution of PA the ECSA is higher. Lobato et al. using a PBI based fuel cell, studied the influence of the ionomer/H3PO4 to the electrochemical interface of both the anode and the cathode. They report values of ECSA as high as $50 \mathrm{~m}^{2} \mathrm{Pt} / \mathrm{gPt}$ for an ionomer to carbon ration between the range of 8 and 20 at the cathodic electrode [41]. This values are significantly higher than in our case for both electrocatalysts. The deviation from the reported values of ECSA as well as the reduction of the ECSA after activation, is due to the lack of binder in our catalyst layer, which cannot retain the free PA at the electrochemical interface. During the activation period, as water is being produced at the cathodic compartment, PA is being hydrated and displaced from the electrochemical interface. Similar mechanism was reported in chapter 6 , when the supplied gas feed was humidified, and lead to the displacement of the PA form the electrochemical interface of the anodic electrdoe. As a result the electrochemical interface (EI) shrinks. Therefore when the PA increases in the cathodic catalyst layer, due to the increase in the available ionic carrier despite the shrinkage of the EI, the measured ECSA is higher. The fact that the $30 \mathrm{wt} \% \mathrm{Pt} / \mathrm{oxMWCNT}$-Py catalyst exhibits higher ECSA values is simply because the displacement is being minimized due to the presence of pyridine groups, which act as a binder.

\subsubsection{Catalyst loading effect on MEA performance}

High temperature PEM fuel cell using phosphoric acid imbibed polymer electrolyte require higher catalyst loading compared to the perfluorosulfonic-acid-type membrane based PEM fuel cell. Besides low solubility of oxygen and slow diffusion in phosphoric acid, phosphoric acid adsorbs and poisons the Pt surface. The latter is responsible for the higher demand in Pt loading, compared to the Nafion based fuel cells. It has been reported that PBI based fuel cell are able to operate without significant loss in their performance utilizing Pt loading as low as $0.6 \mathrm{mgPt} / \mathrm{cm} 2$ for the cathodic electrode, for specific PBI loading in the CL $[18,19]$. The effect of the Pt loading on the fuel cell's performance is widely reported. 
In the present study, the cathodic performance was investigated with respect to the noble metal loading for the newly synthesized catalyst 30wt\%Pt/oxMWCNT-Py as well as for the commercial catalyst $30 \mathrm{wt} \% \mathrm{Pt} / \mathrm{C}$. The noble metal loading varied from approximately $0.4 \mathrm{mgPt} / \mathrm{cm} 2$ to $2.2 \mathrm{mgPt} / \mathrm{cm}^{2}$ whereas the amount of PA sprayed on the cathodic electrode was kept constant for all cases at $2 \mathrm{gPA} / \mathrm{gPt}$. Polarization curves were obtained at $180^{\circ} \mathrm{C}$ under pure $\mathrm{H}_{2}$ and $\mathrm{O}_{2}$ flow $\left(\lambda_{\mathrm{H} 2}=1.2\right.$ and $\left.\lambda_{\mathrm{O}_{2}}=2\right)$ and were IR drop corrected. The results are depicted in figure 8-8a. As shown in figure 8-8a, increasing the Pt loading of the catalyst layer did not improve the oxygen reduction reaction or the cell performance for noble metal loadings higher than $1.3 \mathrm{mgPt} / \mathrm{cm}^{2}$. This is attributed to the reduction of gas permeability of the catalytic layer, due to excessive thickness of catalyst layer. Carbon materials which have filamentous morphology, like carbon nanotubes and carbon nanofibers, exhibit increased porosity compared to conventional carbon blacks when used for the construction of electrodes [42,43]. However, despite the high porosity of the catalyst layer, at high catalyst loading the catalyst layer is so thick as to cause significant mass transfer limitation [44]. In our case, the higher performance was achieved for Pt loadings between 0.7 and 1.3 $\mathrm{mgPt} / \mathrm{cm}^{2}$. That was not the case for the commercial catalyst. On the contrary, the performance was enhanced as the noble metal loading increased without exhibiting any restrictions concerning gas diffusion limitations through the CL. As depicted in figure 8$8 \mathrm{a}$, the commercial $30 \mathrm{wt} \% \mathrm{Pt} / \mathrm{C}$ with $2.2 \mathrm{mgPt} / \mathrm{cm}^{2}$ exhibits higher performance under the same conditions as compared to the $30 \mathrm{wt} \% \mathrm{Pt} / \mathrm{oxMWCNT}-\mathrm{Py}$.

Although, excessive platinum does not always imply effective use of Pt-catalyst. C.Wannec et al. [45] using a AB-PBI based PEM fuel cell did not observe any significant improvement/ difference in the fuel cells performance for Pt loadings above $0.6 \mathrm{mgPt} / \mathrm{cm}^{2}$, whereas lower Pt loading performed less satisfactory. Although when the PA loading was increased to $3 \mathrm{gPA} / \mathrm{gPt}$, the performance of the cathodic electrode improved significantly even for low Pt loadings and did not exhibit major differences in the fuel cell performance with respect to the noble metal loading, figure $8-8 \mathrm{~b}$. Therefore, based on the results from figure $8-1$ and $8-8 \mathrm{~b}$, the PA loading was set at $3 \mathrm{gPA} / \mathrm{gPt}$ as a benchmark, for low $\mathrm{Pt}$ loadings for the $30 \mathrm{wt} \% \mathrm{Pt} / \mathrm{oxMWCNT}-\mathrm{Py}$. 

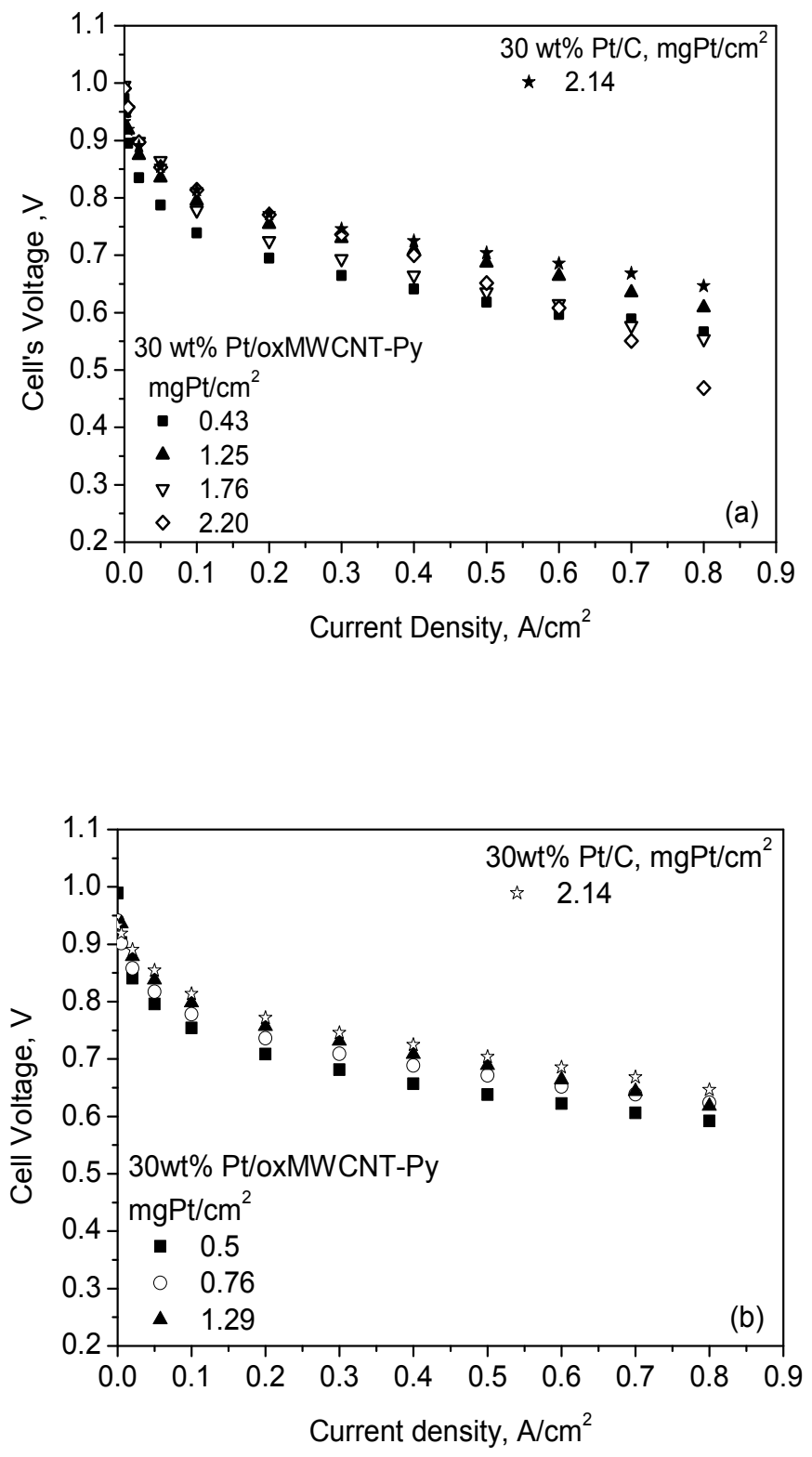

Figure 8-8. The effect of the Pt loading of the cathodic electrode on the MEA's performance. Polarization curves, IR drop corrected, at $180^{\circ} \mathrm{C}$ of MEAs employing $30 \mathrm{wt} \%$ Pt/oxMWCNT-Py. The gas stoichiometry was 1.2 and 2 for pure $\mathrm{H}_{2}$ and $\mathrm{O}_{2}$, respectively. The Pt loading varied between 0.43 and $2.2 \mathrm{mgPt} / \mathrm{cm}^{2}$ and the PA loading was a. $2 \mathrm{gPA} / \mathrm{gPt}$ (MEA 1-4) and b. 3gPA/gPt (MEA 6,8 and 9). For comparison reasons MEA 16, employing 30wt $\% \mathrm{Pt} / \mathrm{C}$, was used. 

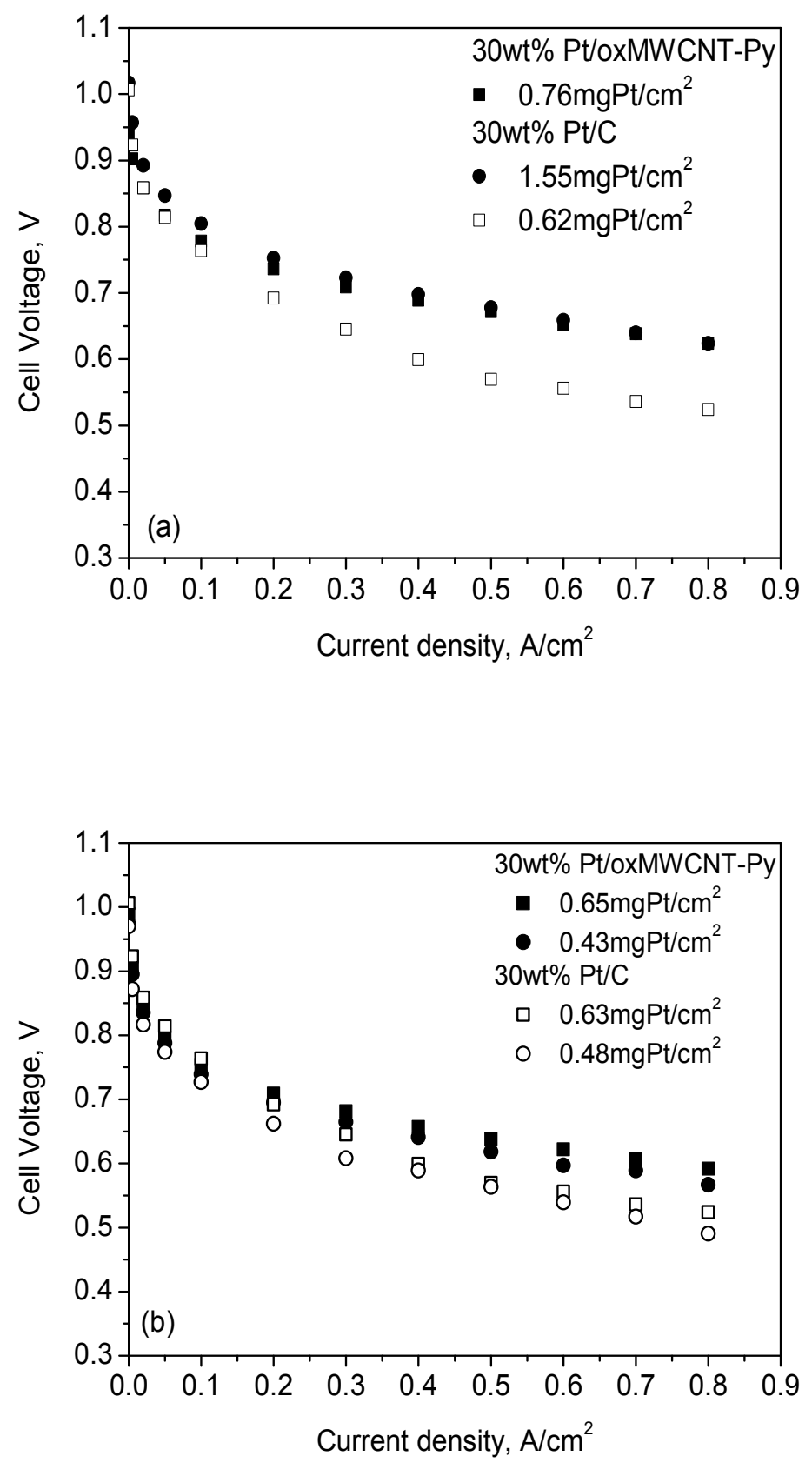

Figure 8-9. The effect of low Pt loadings cathode on the fuel cell's performance. Polarization curves, IR drop corrected, at $180^{\circ} \mathrm{C}$ of MEAs employing $30 \mathrm{wt} \%$ Pt/oxMWCNT-Py and 30wt \% Pt/C . The gas stoichiometry was 1.2 and 2 for pure $\mathrm{H}_{2}$ and $\mathrm{O}_{2}$, respectively. The PA amount imbedded in the cathodic catalyst layer was $3 \mathrm{gPA} / \mathrm{gPt}$ and $2 \mathrm{gPA} / \mathrm{gPt}$ for the $30 \mathrm{wtPt} / \mathrm{oxMWCNT}$-py and $30 \mathrm{wt} \% \mathrm{Pt} / \mathrm{C}$, respectively. a. MEA:,8, 14 and 15 and $\mathrm{b}$. MEA: 5,7,13 and 14 
Since the goal is the operation of a fuel cell with the lowest possible Pt content in the cathodic catalyst layer, the performance between the two electrocatalysts was compared and is depicted in figure 8-9. The optimum PA amount imbedded in the cathodic catalyst layer for each electrocatalyst was chosen according to the above results. For the case of $30 \mathrm{wt} \% \mathrm{Pt} / \mathrm{oxMWCNT}$-Py the PA loading was set at $3 \mathrm{gPA} / \mathrm{gPt}$ whereas for the case of $30 \mathrm{wt} \% \mathrm{Pt} / \mathrm{C} 2 \mathrm{gPA} / \mathrm{gPt}$. As in figure 8-9, the MEA employing 30\%Pt/oxMWCNT-Py exhibited the same performance with half the noble metal loading of the MEA 15 employing the commercial catalyst $30 \mathrm{wt} \% \mathrm{Pt} / \mathrm{C}$. Reduction of the $\mathrm{Pt}$ loading of the commercial electrocatalyst in the cathodic electrode, to similar values as the one for $30 \mathrm{wt} \% \mathrm{Pt} / \mathrm{oxMWCNT}-\mathrm{Py}$, results in significant voltage loss. It is clear from figure $8-9 \mathrm{a}$, that the functionalized carbon nanotube based electrocatalyst exhibits higher performance under the same experimental conditions for low Pt loadings. The latter is attributed to the presence of pyridine groups which increased the ionic pathway through the catalyst layer, thus increasing the three phase boundary. Further reduction of the noble metal loading below $0.7 \mathrm{mgPt} / \mathrm{cm}^{2}$ resulted in a diminution of the cell's performance for both electrocatalyst, 30\% Pt/C and 30\% Pt/oxMWCNT-Py (figure 8-9b). Nonetheless, the catalyst bearing pyridine groups exhibits higher performance.

\subsubsection{Kinetics of $\mathrm{O}_{2}$ reduction reaction}

In this section the dependence of the reaction kinetics on the partial pressure of $\mathrm{O}_{2}$ is shown for both electrocatlaysts, using polarization and Impedance spectroscopy data. Initially the kinetic of $\mathrm{O}_{2}$ reduction was evaluated by $\mathrm{AC}$ impedance spectra, which were recorded at the same current density for MEA 15 employing 30wt\%Pt/C (Figure 8-10) and MEA 9 employing 30wt\%Pt/oxMWCNT-Py (figure 8-11). The spectra were recorded at high excess of the reacting gasses to ensure their uniform partial pressure distribution over the electrode's surface. The monitoring of the spectra at the same current density allows for the isolation of the effect of the partial pressure of $\mathrm{O}_{2}$ on certain features of the $\mathrm{AC}$ spectra that characterize the response of the cathode's electrokinetic behavior. In this respect anode remains unaffected. As mentioned in chapter 5 and 6, it was clear that the change in partial pressure of $\mathrm{H}_{2}$ resulted in the variation of the polarization resistance of high frequency arc and was definitely attributed to the anode. On the contrary variation of the oxygen partial pressure did not result in any significant change in the polarization resistance of the medium/low frequency arc, which corresponds to the polarization resistance of the cathodic electrode, as clearly shown in figure 8-10 and 8-11. Equation 83 , which is derived from the Tafel equation, relates the polarization resistance $R_{p}$ of the EI with the applied current I and the transfer coefficient $\alpha$ or otherwise the Tafel slope $\alpha \mathrm{F} / \mathrm{RT}$, 


$$
R_{p}=\frac{1}{I} \frac{R T}{\alpha F}
$$

The charge transfer coefficient, with respect to the partial pressure of $\mathrm{O}_{2}$, was calculated using equation 8-3 and after deconvolution and fitting of the AC impedance spectra with the appropriate equivalent circuit, in order to acquire the $\mathrm{Rp}$ polarization resistance of the cathodic electrode for both MEAs. The as calculated transfer coefficient values are summarized in table 8-3, where both electrocatalysts exhibited similar values. Although it has to be mentioned that at $20 \mathrm{kPa} \mathrm{O}_{2}$ for both cases the transfer coefficient values were deviating from the other average values acquired for higher partial pressures of $\mathrm{O}_{2}$. This is attributed mainly to the contribution of the mass transport resistance at such low concentrations of $\mathrm{O}_{2}$ to the overall polarization resistance of the cathodic electrode. Since the polarization resistance remains unaffected by the $\mathrm{O}_{2}$ partial pressure under constant current density, according to eq. 8-3 the transfer coefficient $\alpha$ remains constant. It is widely known that the transfer coefficient depends on the reaction mechanism and the limiting step of the electrocatalytic process [46]. Therefore, in the present case it indicates that the reaction kinetics of ORR does not change with respect to the $\mathrm{O}_{2}$ partial pressure. Also since similar values were acquired for the case of 30wt\%Pt/oxMWCNT-Py, it is clear that the presence of pyridine groups did not affect the electrochemical reactions steps.

Table 8-3. The as calculated Tafel slopes, exchange current densities and charge transfer resistance from MEA 9 and MEA 15 with respect to the partial pressure of $\mathrm{O}_{2}$.

\begin{tabular}{ccccc}
\hline $\mathrm{kPa}_{\mathrm{O} 2}$ & $\begin{array}{c}\text { Tafel slope } \\
\mathrm{mV} / \mathrm{dec}\end{array}$ & $\begin{array}{c}\text { io } \\
\mathrm{A} / \mathrm{m}^{2} \mathrm{Pt}\end{array}$ & $\begin{array}{c}\text { Charge transfer } \\
\text { coefficient from Tafel }\end{array}$ & $\begin{array}{c}\text { Charge transfer } \\
\text { from AC }\end{array}$ \\
\hline MEA 9 & & & & \\
100 & 102.2 & 0.0216 & 0.880 & 0.916 \\
80 & 103.1 & 0.0170 & 0.873 & 0.864 \\
60 & 100.7 & 0.0117 & 0.893 & 0.852 \\
40 & 103.9 & 0.0097 & 0.865 & 0.849 \\
20 & 103.8 & 0.0092 & 0.854 & 0.713 \\
MEA 15 & & & & \\
100 & & & & \\
80 & 99.67 & 0.0159 & 0.902 & 0.927 \\
60 & 104.4 & 0.0147 & 0.861 & 0.878 \\
40 & 103.3 & 0.0128 & 0.870 & 0.858 \\
20 & 103.1 & 0.0114 & 0.872 & 0.786 \\
\hline
\end{tabular}


The effect of the PA distribution in the CL and the catalyst substrate on the reaction kinetic mechanism (Tafel slope and transfer coefficient) was also evaluated for the oxMWCNTPy based catalyst and compared with the $30 \mathrm{wt} \% \mathrm{Pt} / \mathrm{C}$ using equation $8-4$. The Tafel slopes were estimated by fitting the experimental data (figure 8-12)-polarization curves with the equation, from the region governed by the kinetic of the ORR :

$$
\mathrm{E}=\mathrm{E} o+\mathrm{a}-\mathrm{blogi}-\mathrm{RI}
$$

where $\mathrm{E}$ is the cell voltage, Eo is the open circuit voltage, $\mathrm{b}$ is the Tafel slope, and $\mathrm{R}$ accounts for the ohmic resistance, the polarization resistance of the hydrogen oxidation, and mass transfer limitation both in the electrode and in the gas diffusion layer. The results are summarized in table 8-3.

It is clear from table 8-3, that the transfer coefficient are similar in values for both electrocatalyst. Also the values are in good agreement with the ones derived from the AC impedance measurements. In all the cases the Tafel slope were in the range of 100$107 \mathrm{mV} / \mathrm{dec}$.This shows that the $\mathrm{H}_{3} \mathrm{PO}_{4}$ distribution and the presence of pyridine groups in the electrode structure does not change the kinetic of the ORR. The Tafel slopes values were in accordance to values reported in the literature [18]. 

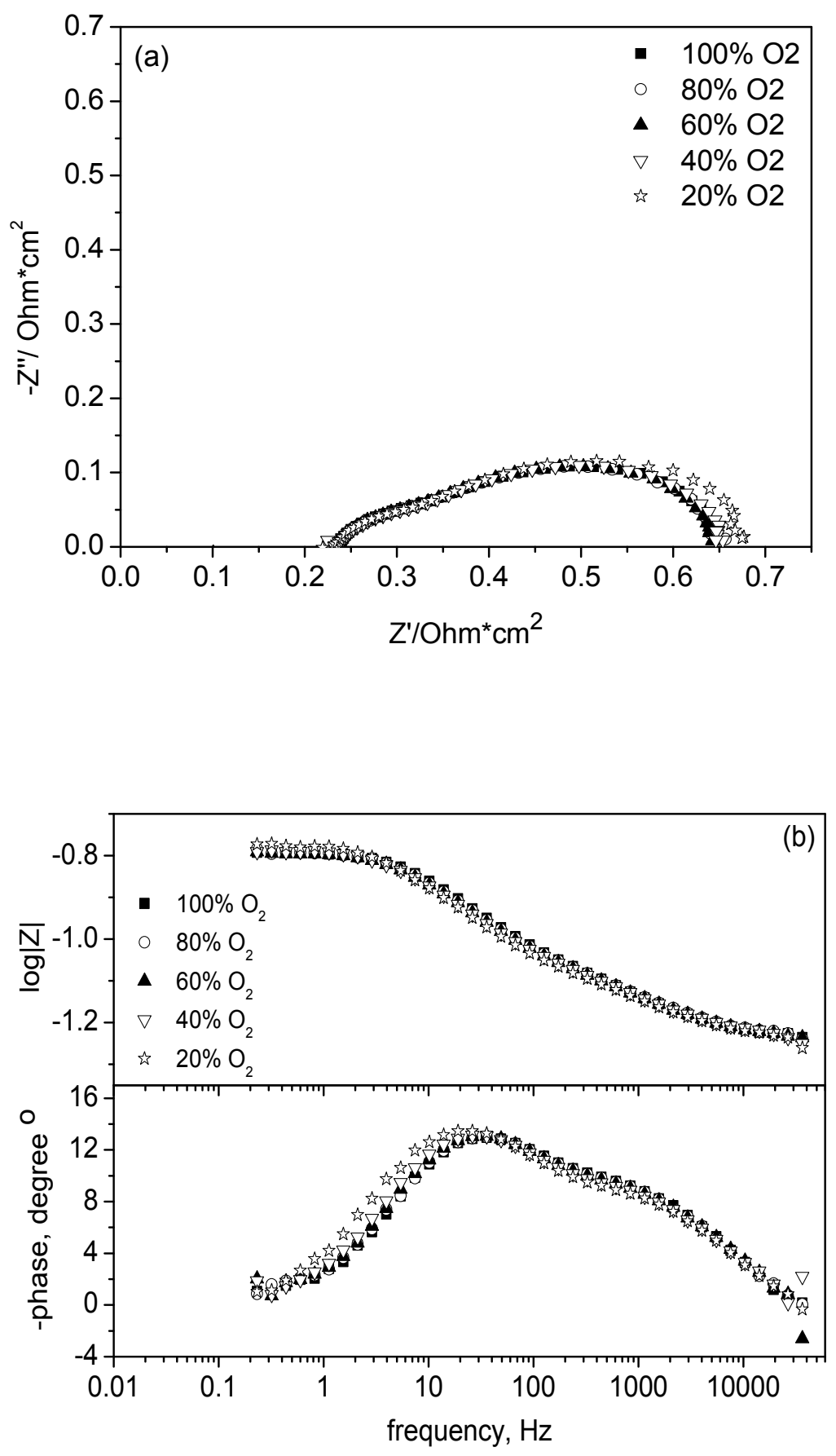

Figure 8-10 The effect of $\mathrm{O}_{2}$ partial pressure on the polarization resistance of the cathodic electrode of MEA 15. AC impedance spectra obtained at a current of $0.2 \mathrm{~A} / \mathrm{cm}^{2}$ at $180^{\circ} \mathrm{C}$, under various partial pressure of $\mathrm{O}_{2}$ balanced with Ar. The anodic electrode was fed with redundant $\mathrm{H}_{2}$ flow. a. Nyquist plots and b. corresponding Bode plots 

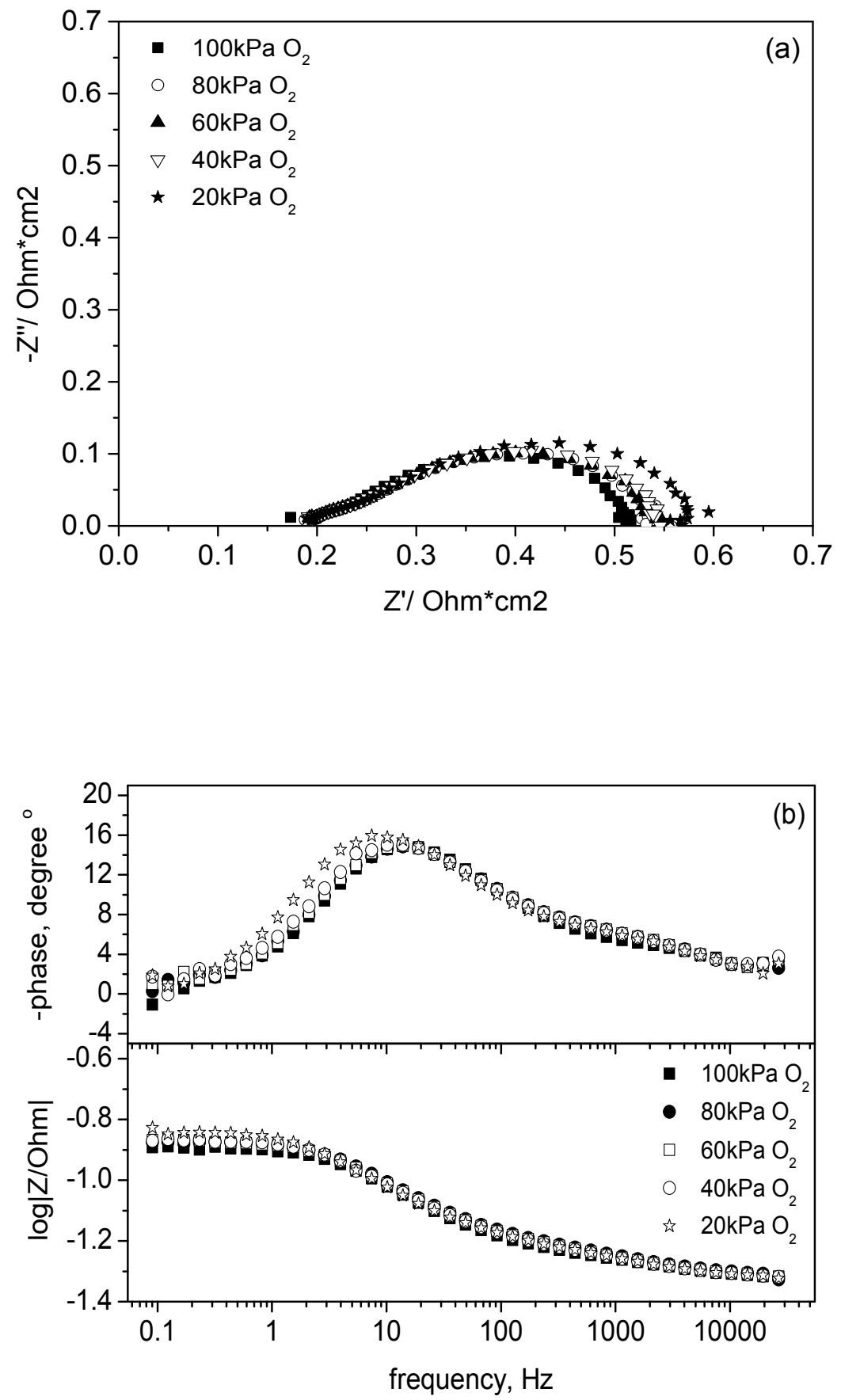

Figure 8-11. The effect of $\mathrm{O}_{2}$ partial pressure on the polarization resistance of the cathodic electrode of MEA 9. AC impedance spectra obtained at a current of $0.2 \mathrm{~A} / \mathrm{cm}^{2}$ at $180^{\circ} \mathrm{C}$, under various partial pressure of $\mathrm{O}_{2}$ balanced with Ar. The anodic electrode was fed with redundant $\mathrm{H}_{2}$ flow. a. Nyquist plots and b. corresponding Bode plots 

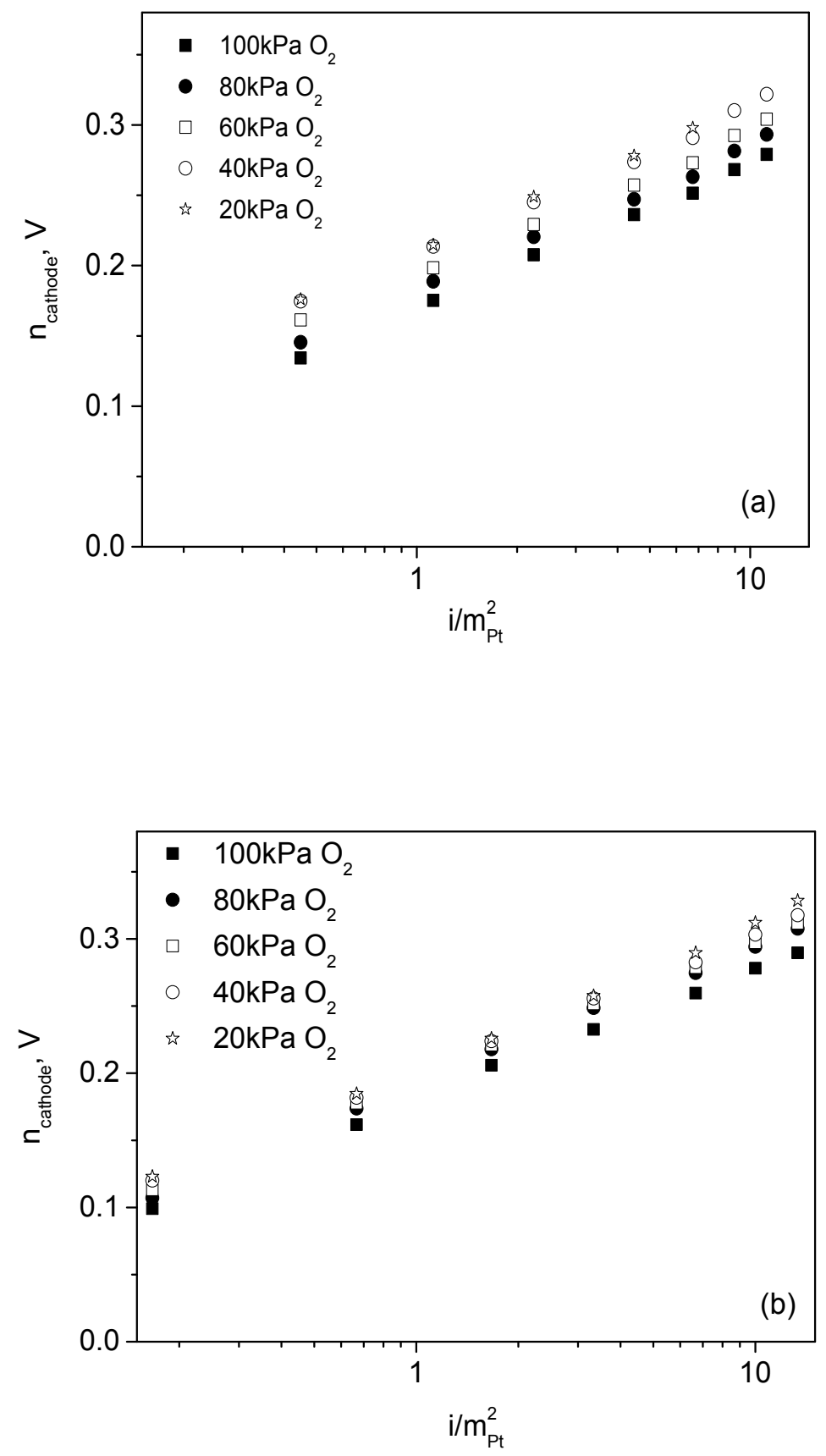

Figure 8-12. The effect of the catalyst support and the PA distribution on the Tafel slopes under various $\mathrm{O}_{2}$ partial pressures. The measurements were conducted under $200 \mathrm{cc} / \mathrm{min}$ of pure $\mathrm{H}_{2}$ and $\mathrm{O}_{2}$ balanced with $\mathrm{Ar}$, respectively, at $180^{\circ} \mathrm{C}$ : a. MEA 9 employing 30wt\%Pt/ox.MWCNT-Py and b. MEA 15 employing 30wt\%Pt/C . 


\subsubsection{XPS post mortem analysis}

Pyridine groups are an essential part of the newly synthesized catalyst, as they interact with the free phosphoric acid, homogeneously distributing it in the catalyst layer. Although thermogravimetric analysis showed that the pyridine groups are thermally stable below $200^{\circ} \mathrm{C}$ under air [47], due to the harsh operating conditions of a HT -PEM fuel cell it was necessary to investigate the stability of the pyridine groups after various operation periods. For that reason, MEAs were formulated with the same specifications. The cathodic electrode, which employed 30\%Pt/oxMWCNT-Py, had a Pt loading of approximately $1 \mathrm{mgPt} / \mathrm{cm}^{2}$ and an acid loading of $4 \mathrm{gPA} / \mathrm{gPt}$. The acid loading was chosen to be higher than the optimum $(3 \mathrm{gPA} / \mathrm{gPt})$ in order to add further stress on the cathodic electrode during operation.

The MEAs were left at $180^{\circ} \mathrm{C}$ and operated for various periods (24 hours to 6 days) at a constant current density of $0.2 \mathrm{~A} / \mathrm{cm}^{2}$ using pure $\mathrm{H}_{2}$ and $\mathrm{O}_{2}$ (gas stoichiometry $\lambda_{\mathrm{H} 2}=1.2$ and $\lambda_{\mathrm{O} 2}=2$ ). Moreover, an amount of the catalyst powder was placed in a furnace at $180^{\circ} \mathrm{C}$ and left under air atmosphere for almost 24 hours and an MEA was left at OCP conditions at $180^{\circ} \mathrm{C}$ for $24 \mathrm{~h}$. Thereafter, the MWCNT based catalyst was characterized using X-ray photoelectron spectroscopy in order to determine and verify the functionalization degree of the carbon nanotube based catalyst.

Table 8-4. N/Pt atomic ratio after certain operation time of the cathodic electrode of an MEA employing 30wt\% Pt/(ox.MWCNT)-Py.

\begin{tabular}{cc}
\hline Operations Conditions & $\mathrm{N} / \mathrm{Pt}$ atomic ratio \\
\hline Oven at $180 \mathrm{C}$ & 0.325 \\
OCV for $24 \mathrm{~h}$ & 0.230 \\
$0.2 \mathrm{~A} / \mathrm{cm}^{2}$ for $24 \mathrm{~h}$ & 0.220 \\
$0.2 \mathrm{~A} / \mathrm{cm}^{2}$ for $50 \mathrm{~h}$ & 0.253 \\
$0.2 \mathrm{~A} / \mathrm{cm}^{2}$ for 6 days & 0.179 \\
\hline
\end{tabular}


Table 8-4 depicts the results of the XPS characterization. Due to the structure of the electrode, after fuel cell operation, it is very difficult to scratch off the catalytic layer without any trace of the gas diffusion layer. Therefore, the functionalization degree of the carbon nanotube could not be estimated through the atomic ratio of N/C. For that reason it was considered more appropriate to use the atomic ratio of $\mathrm{N} / \mathrm{Pt}$. Also one has to take into consideration that XPS is a surface sensitive technique and that any changes on the $\mathrm{Pt}$ particle size on the catalyst would influence and change the atomic ratio of N/Pt. Therefore only an estimation concerning the pyridine groups' stability can be made. From the results shown in table 8-4, it seems that the pyridine groups are stable for at least short term fuel cell operation.

\subsubsection{Investigation of Pt particle size and distribution via TEM Imaging after short term operation}

The reduction of the electrochemical active surface area during operation of a PEM fuel cell is attributed to several factors, such as Pt sintering/agglomeration or Pt migration and loss. In order to investigate the stability of the newly synthesized electrocatalyst, after short term operation, used membrane electrode assemblies (MEA) were analyzed using transmission electron microscopy (TEM).

MEAs were left to operate for various periods at $180^{\circ} \mathrm{C}$ at constant current density of 0.2 $\mathrm{A} / \mathrm{cm}^{2}$ using pure $\mathrm{H}_{2}$ and $\mathrm{O}_{2}$ (gas stoichiometry $\lambda_{\mathrm{H} 2}=1.2$ and $\lambda_{\mathrm{O} 2}=2$ ). The same MEAs as the one mentioned in section 8.3.4, were used for the determination of the Pt particle size with respect to operation time. For comparison reasons the same experiments were performed for MEAs employing at the cathodic electrode $30 \mathrm{wt} \% \mathrm{Pt} / \mathrm{C}$, which had the same specifications as the ones employing 30wt\% Pt/oxMWCNT-Py. Moreover, an amount of each catalyst powder was placed in a furnace at $180^{\circ} \mathrm{C}$ and left under air atmosphere for almost 24 hours and an MEA was left at OCP conditions at $180^{\circ} \mathrm{C}$ for $24 \mathrm{~h}$. The results are summarized in table 8-5.

The as synthesized 30wt\% Pt/oxMWNT-Py electrocatalyst has an average Pt particle size of $3.2 \mathrm{~nm}$, whereas the as received $30 \mathrm{wt} \% \mathrm{Pt} / \mathrm{C} 2.9 \mathrm{~nm}$. As shown in table 8-5, the $30 \mathrm{wt} \% \mathrm{Pt} / \mathrm{oxMWCNT}$-Py catalyst when left in an oven at $180^{\circ} \mathrm{C}$ under constant air flow appears with an average diameter $3.7 \mathrm{~nm}$, similar values were obtained for the case of $30 \mathrm{wt} \% \mathrm{Pt} / \mathrm{C}$. Whereas, it is clearly shown that after 144 hours of constant operation at $0.2 \mathrm{~A} / \mathrm{cm}^{2}$, the $30 \% \mathrm{Pt} /$ ox.MWCNT-Py electrocatalyst undergoes the similar Pt particle growth as the commercial electrocatalyst. These results are encouraging since at least for short term operation the newly synthesized electrocatalyst exhibits the same stability as the commercial catalyst. In addition in both cases the Pt particle size distribution was not severely affected, as shown in figure 8-13. 

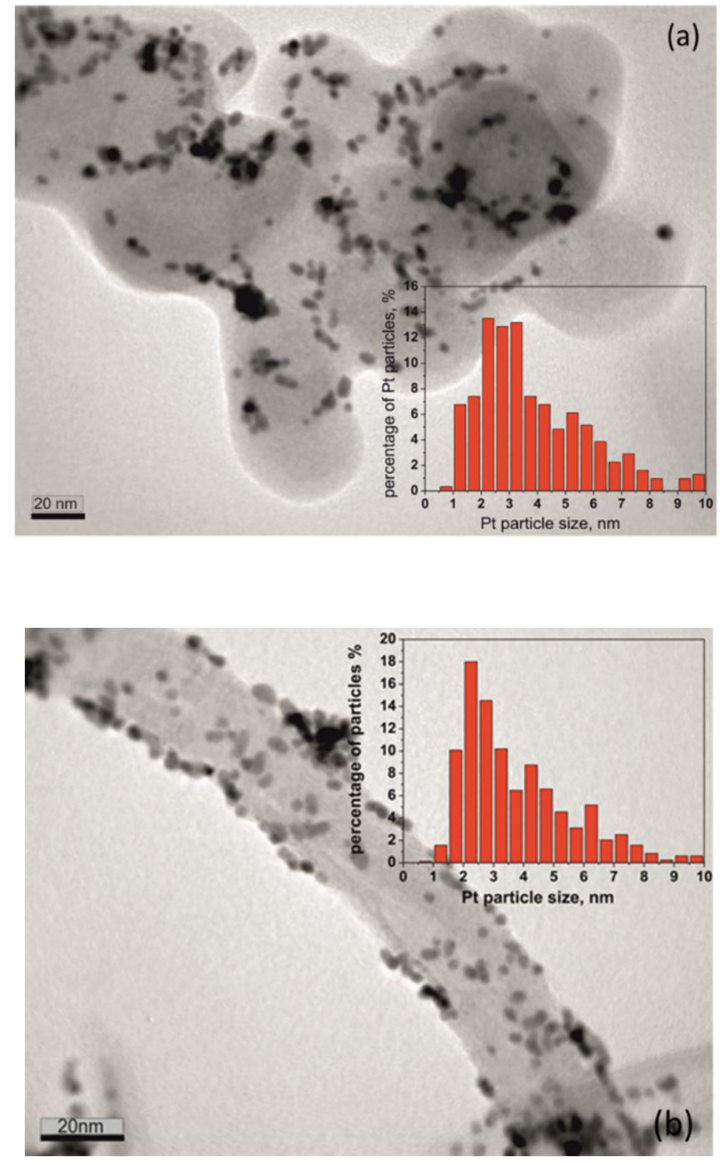

Figure 8-13. TEM micrographs after 144 hours of continuous operation of cathodic electrode employing a. 30wt\% Pt/C and b. 30\% Pt/oxMWCNT-Py. The Pt particle size distribution is included as an inset.

Table 8-5. Pt particle size average diameter (nm) with respect to operation time as estimated by TEM imaging for cathodic electrodes employing 30wt $\%$ Pt/ox.MWCNT-Py and $30 \mathrm{wt} \% \mathrm{Pt} / \mathrm{C}$.

\begin{tabular}{ccc}
\hline Operation conditions & $\begin{array}{c}\text { Pt average particle size } \\
\text { 30\%Pt/ox.MWCNT-Py }\end{array}$ & $\begin{array}{c}(\mathrm{nm}) \\
30 \% \mathrm{Pt} / \mathrm{C}\end{array}$ \\
\hline Oven at 180C for 24 h & 3.7 & 3.5 \\
OCV for 24h & 4.2 & N/A \\
$0.2 \mathrm{~A} / \mathrm{cm}^{2}, 24$ hours & 3.8 & 3.5 \\
$0.2 \mathrm{~A} / \mathrm{cm}^{2}, 50$ hours & 4.2 & 3.8 \\
$0.2 \mathrm{~A} / \mathrm{cm}^{2}, 144$ hours & 4.3 & 4.0 \\
\hline
\end{tabular}




\subsection{Conclusions}

The catalyst efficiency largely depends on the catalyst morphology and its interface with the proton conductor, thus this study opens new roads towards the creation of high efficiency catalyst layer employing very low Pt loadings. The newly synthesized electrocatalyst 30wt\% Pt/oxMWCNT-Py was evaluated as a cathodic electrocatalyst in

HTPEM fuel cell. The presence of pyridine groups was found to homogeneously distribute PA in the catalyst layer minimizing the blockage of the pores of the catalyst layer as well as increase the three phase boundary. As a result the MEA employing $30 \mathrm{wt} \% \mathrm{Pt}$ oxMWCNT-Py showed the same performance as the $30 \% \mathrm{Pt} / \mathrm{C}$ for half the $\mathrm{Pt}$ loading. Despite the hard operating conditions the Pt particles attached to the ox.MWCNT-Py substrate exhibit the same stability as the commercial catalyst and the pyridine groups were found to be stable, at least for short term operation.

The advantage of this approach towards the increase of catalyst utilization lies in the lack of need for the use of a polymer-binder inside the catalytic layer, thus avoiding problems of inhomogeneous binder distribution and/or electronic insulation of catalyst nanoparticles. Using this newly synthesized electrocatalyst opens the possibility of significant reduction of the amount of $\mathrm{Pt}$ in the cathodic electrodes without sacrificing the performance and stability of the fuel cell. 


\section{References}

[1] J.M. Song, S. Suzuki, H. Uchida, M. Watanabe, Preparation of High Catalyst Utilization Electrodes for Polymer Electrolyte Fuel Cells, Langmuir 22 (2006) 6428.

[2] Z. Xu, Z. Qi, A. Kaufman, Superior Catalysts for Proton Exchange Membrane Fuel Cells: Sulfonation of Carbon-Supported Catalysts Using Sulfate Salts, J Electrochem. Solid-State Lett. 8 (2005) A313

[3] H. Kim, W. Lee, D. Yoo, Functionalized carbon support with sulfonated polymer for direct methanol fuel cells, J Electrochim. Acta 52 (2007) 2620

[4] C.Y. Du, T.S. Zhao, Z.X. Liang, Sulfonation of carbon-nanotube supported platinum catalysts for polymer electrolyte fuel cells, J. Power Sources 176 (2008) 9

[5] Li Q, Jensen JO, Pan C, Bandur V, Nilsson MS, Schonberger F, et al., Partially fluorinated aarylene polyethers and their ternary blends with PBI and H3PO4. Part II. Characterisation and fuel cell test of the ternary membranes. Fuel Cells 3-4 (2008) 188

[6] Kim J-H, Kim H-J, Lim H-T., Dependence of the performance of a high-temperature polymer electrolyte fuel cell on phosphoric acid-doped polybenzimidazole ionomer content in cathode catalyst layer, J Power Sources 170 (2007) 275

[7] Pan C, Li Q, Jensen JO, He R, Cleemann LN, Nilsson MS,Preparation and operation of gas diffusion electrodes for high-temperature proton exchange membrane fuel cells, J Power Sources 172 (2007) 278

[8] Qinggang He,Xiaofang Yang,Wei Chen, Sanjeev Mukerjee,Bruce Koel and Shaowei, Influence of phosphate anion adsorption on the kinetics of oxygen electroreduction on low index Pt(hkl) single crystals, Chen, Phys. Chem. Chem. Phys. 12 (2010) 12544

[9] F. Gan, D.-T. Chin, Determination of diffusivity and solubility of oxygen in phosphoric acid using a transit time on a rotating ring-disc electrode, J. Applied Electrochemistry 23 (1993) 452

[10] Park JO, Hong SG, Kim TY, Kwon KJ, Suh SH, Cho MD, Role of binders in high temperature PEMFC electrodes, ECS Trans 3 (2006) 447

[11] Chu D., $\mathrm{O}_{2}$ reduction at the Pt/Nafion interface in 85\%concentrated H3PO4., J Electrochim Acta 43 (1998) 3711

[12] Giordano N, Passalacqua E, Antonucci PL, Pino L, Vivaldi M, Patti A, Influence of physicochemical properties on the performance of $\mathrm{Pt} / \mathrm{C}$ porous electrodes for oxygen reduction in phosphoric acid, J Electrochim Acta 38 (1993) 913

[13] Roy Choudhury S, Deshmukh MB, Rengaswamy R., A two-dimensional steady-state model for phosphoric acid fuel cells (PAFC), J Power Sources 112 (2002) 135.

[14] Zhang X, Shi P., Nafion effect on dual-bonded structure cathode of PEMFC, J Electrochem Commun 8 (2006) 1615. 
[15] Lee SJ, Mukerjee S, McBreen J, Rho YW, Kho YT, Lee TH., Effects of Nafion impregnation on performances of PEMFC electrodes., J Electrochim Acta 43 (1998) 3693

[16] Liu Z, Wainright JS, Litt MH, Savinell RF., Study of the oxygen reduction reaction (ORR) at Pt interfaced with phosphoric acid doped polybenzimidazole at elevated temperature and low relative humidity, J Electrochim Acta 51 (2006) 3914

[17] Li Q, Hjuler HA, Bjerrum NJ. Oxygen reduction on carbon supported platinum catalyst in high temperature polymer electrolytes, J Electrochim Acta 45 (2000) 4219

[18] Justo Lobato, Pablo Canizares, Manuel A. Rodrigo, Jose J. Linares, F. Javier Pinar, Study of the influence of the amount of PBI-H3PO4 in the catalytic layer of a high temperature PEMFC, International journal of Hydrogen energy 35 (2010) 1347

[19] F. Seland, T. Berning, B. Børresen, R. Tunold, Improving the performance of high-temperature PEM fuel cells based on PBI electrolyte, Journal of Power Sources 160 (2006) 27

[20] Kong C-S, Kim D-Y, Lee H-K, Shul Y-G, Lee T-H. Influence of pore-size distribution of diffusion layer on mass-transport problems of proton exchange membrane fuel cells. J Power Sources 108 (2002) 185

[21] Lee H-K, Park J-H, Kim D-Y, Lee T-H. A study on the characteristics of the diffusion layer thickness and porosity of the PEMFC. J Power Sources 131 (2004) 200

[22] Javier Parrondo, Chitturi Venkateswara Rao, Sundara L. Ghatty, and B. Rambabu, Electrochemical Performance Measurements of PBI-Based High-Temperature PEMFCs, Research International Journal of Electrochemistry (2011), Article ID 261065, doi: 10.4061/2011/261065

[23] Jeong-Hi Kim, Hyoung-Juhn Kim, Tae-Hoon Lim, Ho-In Lee, Dependence of the performance of a high-temperature polymer electrolyte fuel cell on phosphoric acid-doped polybenzimidazole ionomer content in cathode catalyst layer, Journal of Power Sources 170 (2007) 275

[24] Hyung-Suk Oh, Kwanghyun Kim, Hansung Kim, Polypyrrole-modified hydrophobic carbon nanotubes as promising electrocatalyst supports in polymer electrolyte membrane fuel cells, International journal of Hydrogen energy 36 (2011) 11564

[25] Kazuya Matsumoto,Tsuyohiko Fujigaya, Kazunari Sasaki and Naotoshi Nakashima, Bottomup design of carbon nanotube-based electrocatalysts and their application in high temperature operating polymer electrolyte fuel cells, J. Mater. Chem. 21 (2011) 1187

[26] A. Orfanidi, M.Daletou, S.G. Neophytides, Preparation and characterization of Pt on modified multi-wall carbon nanotubes to be used as electrocatalysts for high temperature fuel cell applications, J Applied Catalysis B: Environmental 106 (2011) 379

[27] Kyungjung Kwon, Tae Young Kim, Duck Young Yoo, Suk-Gi Hong, Jung Ock Park, Maximization of high-temperature proton exchange membrane fuel cell performance with the optimum distribution of phosphoric acid, Journal of Power Sources 188 (2009) 463

[28]Maria K. Daletou, Maria Geormezi, Effrosyni Vogli, George A. Voyiatzis, Stylianos G. Neophytides, The interaction of H3PO4 and steam with PBI and TPS polymeric membranes. A TGA and Raman study, J. Mater. Chem. A, 2 (2014) 1117 
[29] M. K. Daletou, J. K. Kallitsis, G. Voyiatzis , S. G. Neophytides, The interaction of water vapors with H3PO4 imbibed PBI/polysulfone copolymer blends, J. Membr. Sci., 326 (2009) 76

[30] Andreasen SJ, Jespersen JL, Schaltz E, Kær SK., Characterisation and modelling of a high temperature PEM fuel cell stack using electrochemical impedance spectroscopy. Fuel Cells 9 (2009) 463

[31] ZhuWH, Payne RU, Tatarchuk BJ. , PEM stack test and analysis in a power system at operational load via AC impedance., Journal of Power Sources 168 (2007) 211

[32] Yuan X, Wang H, Sun JC, Zhang J. ,AC impedance technique in PEM fuel cell diagnosis e A review. International Journal of Hydrogen Energy 32 (2007) 4365

[33] Zhang J, Tang Y, Song C, Zhang J. Polybenzimidazole-membrane-based PEM fuel cell in the temperature range of 120-200 oC, Journal of Power Sources 172 (2007) 163

[34] Jespersen JL, Schaltz E, Kær SK., Electrochemical characterization of a polybenzimidazolebased high temperature proton exchange membrane unit cell, Journal of Power Sources 191 (2009) 289

[35] Springer TE, Raistrick ID. Electrical impedance of a pore wall for the flooded-agglomerate model of porous gas-diffusion electrodes, J Electrochem Soc 136 (1989) 1594

[36] Nikhil H. Jalani, Manikandan Ramani, Kristina Ohlsson, Steve Buelte, Greg Pacifico, Richard Pollard, Rhonda Staudt, Ravindra Datta, Performance analysis and impedance spectral signatures of high temperature PBI-phosphoric acid gel membrane fuel cells, Journal of Power Sources 160 (2006) 1096

[37] M. Mamlouk, K. Scott, Analysis of high temperature polymer electrolyte membrane fuel cell electrodes using electrochemical impedance spectroscopy, Electrochimica Acta 56 (2011) 5493

[38] K. Wippermann, C. Wannek, H.-F. Oetjen, J. Mergel, W. Lehnert, Cell resistances of poly(2,5benzimidazole)-based high temperature polymer membrane fuel cell membrane electrode assemblies: Time dependence and influence of operating parameters, Journal of Power Sources 195 (2010) 2806-2809

[39] I.A. Schneider, S.A. Freunberger, D. Kramer, A. Wokaun, G.G. Scherer,Oscillations in Gas Channels Part I. The Forgotten Player in Impedance Spectroscopy in PEFCs, J. Electrochem. Soc. 154 (2007) B383

[40] I.A. Schneider, D. Kramer, A.Wokaun, G.G. Scherer, Oscillations in Gas Channels II. Unraveling the Characteristics of the Low Frequency Loop in Air-Fed PEFC Impedance Spectra, J. Electrochem. Soc. 154 (2007) B770

[41]Justo Lobato, Pablo Can izares, Manuel A. Rodrigo, Jose' J. Linares, F. Javier Pinar Study of the influence of the amount of PBI-H3PO4 in the catalytic layer of a high temperature PEMFC,International journal of H2 energy, 35 (2010) 1347

[42] K. Fukuda, I. Tanaka, M. Tani, J. Matsuo, EP Patent 1,429,403, 2004.

[43] R.I. Masel, C.A. Rice, SU Patent 7,108,773, 2006. 
[44] Tatyana V. Reshetenko, Hee-Tak Kim, Ho-Jin Kweon, Modification of cathode structure by introduction of CNT for air-breathing DMFC, Electrochimica Acta 53 (2008) 3043

[45]ChristophWannek, Werner Lehnert, Jürgen Mergel, Membrane electrode assemblies for hightemperature polymer electrolyte fuel cells based on poly(2,5-benzimidazole) membranes with phosphoric acid impregnation via the catalyst layers, Journal of Power Sources 192 (2009) 258

[46] J. O’M. Bockris, A.K.N. Reddy, M. Gamboa-Aldeco, Modern Electrochemistry, Vol. 2A, Fundamentals of electrodics, Kluwer Academic/Plenum Publishers, New York ISBN: 0-30646166-8 Z, 2nd ed., 2000, p. 1166.

[47] Alin Orfanidi, Master thesis, Synthesis and characterization of Pt /modified MWNT as electrocatalysts for application in high temperature PEMFCs', University of Patras, http://nemertes.lis.upatras.gr/jspui/bitstream/10889/5072/3/Nimertis_Orfanidi 


\section{Chapter 9}

\section{Summary and Future Work}

\subsection{Summary}

The scope of this thesis is to understand and improve the electrochemical interface of HTPEM fuel cells and to optimize the catalyst layer structure in order to achieve higher efficiency. For that reason the effect of the PA amount in the catalyst layer and the effect of the catalyst's substrate on the fuel cell's performance were investigated.

Although PA is an essential part of the catalyst layer structure, it causes limitations on the cathodic electrode's performance, which has been thoroughly discussed and reported in the literature. For the first time, the poisoning effect of PA on the anodic electrode was investigated during this thesis. It was observed that the reversible performance loss of the anodic electrode was a function of the PA amount in the catalyst layer. More specifically, under low PA loading $(<3 \mathrm{gPA} / \mathrm{gPt})$ on the anodic electrode, $<10 \%$ of the Pt active surface is electrochemically active under fuel cell operating conditions. This was attributed to the blockage of the Pt surface by pyrophosphoric acid or poly-phosphates, $\mathrm{H}_{2}$ reduced polyphosphoric acid species and the shrinkage of the interface due to the displacement of the $\mathrm{H}_{3} \mathrm{PO}_{4}$ by the adsorbed $\mathrm{H}_{2}$ species. The results are dramatically different for high PA loadings, where it was found that the Pt surface utilization was very high $(>80 \%)$ and has been attributed to the enhanced degree of the hydration of the electrochemical interface that accompanies the more uniform distribution of the $\mathrm{H}_{3} \mathrm{PO}_{4}$ within the catalytic layer. The controlled and increased PA content within the catalytic layer can result even up to the tenfold decrease in the Pt loading when the anode operates under $\mathrm{H}_{2}$ rich conditions. 
The catalyst efficiency largely depends on the catalyst morphology and its interface with the proton conductor. Thus a new catalyst support based on pyridine functionalized carbon nanotubes was synthesized in order to increase the TPB and lower the Pt loading. The newly synthesized electrocatalyst 30wt\% Pt/oxMWCNT-Py was evaluated as an anodic electrocatalyst in a HTPEM fuel cell. The presence of pyridine groups was found to homogeneously distribute PA in the catalyst layer, resulting in high ECSA values, $40 \mathrm{~m}^{2} / \mathrm{gPt}$ (Chapter 5). As a result the MEA employing 30wt\% Pt oxMWCNT-Py showed the same performance as the $30 \% \mathrm{Pt} / \mathrm{C}$ (having $1.3 \mathrm{mgPt} / \mathrm{cm}^{2}$ ), for Pt loading loadings as low as $0.2 \mathrm{mgPt} / \mathrm{cm}^{2}$. The performance of the anodic electrode was also found to be largely depended on the PA amount imbedded in the CL, when low Pt loading were used. The latter was an effect of the shrinkage of the ECSA as a result of the formation of PA poisoning species, as also mentioned in the previous paragraph. Post mortem analysis of the $30 \mathrm{wt} \% \mathrm{Pt} / \mathrm{oxMWCNT}-\mathrm{Py}$ catalyst, showed that despite the hard operating conditions the Pt particles attached to the ox.MWCNT-Py substrate exhibit similar stability as the commercial catalyst and the pyridine groups were found to be stable as well, at least for short term operation.

Operating a fuel cell under pure $\mathrm{H}_{2}$ ensures the anode's optimum performance. However the production of pure $\mathrm{H}_{2}$ presents many difficulties, as opposed to a typical reformate mixture rich in $\mathrm{H}_{2}$. Typically, the composition of reformate gas is a mixture of approximately $50-60 \% \mathrm{H}_{2}, 1-2 \% \mathrm{CO}, 10-15 \% \mathrm{CO}_{2}$ and $20-33 \% \mathrm{H}_{2} \mathrm{O}$. It is a real challenge to be able to operate a fuel cell under reformate conditions. Since $30 \mathrm{wt} \% \mathrm{Pt} / \mathrm{oxMWCNT}$ Py exhibited very promising results with respect to the high ECSA, its performance under synthetic reformate gas was evaluated. The synthetic reformate gas that was used comprised of $50.7 \mathrm{kPa}$ of $\mathrm{H}_{2}, 2 \mathrm{kPa}$ of $\mathrm{CO}$ and $33.5 \mathrm{kPa}$ of $\mathrm{H}_{2} \mathrm{O}$ balanced with Ar. It was found that the observed voltage loss, in this particular system, under synthetic reformate gas rich in $\mathrm{CO}$ and steam, is attributed to a multistep process. The HOR is been constrained by the remaining free $\mathrm{Pt}$ sites as a consequence of blockage due to $\mathrm{CO}$, water and phosphoric acid species adsorption on the Pt surface, as well as due to the shrinkage of the EI as a result of PA displacement from the reactions interphase. Additionally the formation of ganglia in the CL pores, due to hydration and swelling of PA, creates mass transport limitation hindering gas diffusivity. The amount and distribution of PA on the electrodes plays a significant role in the formation and the structure of the EI. It appears that a rather hydrophilic catalytic layer is important for the uniform distribution of PA on the electrocatalytic surface and its stability under humidified conditions. The $30 \mathrm{wt} \% \mathrm{Pt} / \mathrm{oxMWCNT}$-Py electrocatalyst are ideal candidates for operation under those harsh reformates conditions, as they exhibited smaller voltage losses and higher stability under these conditions. The interaction of pyridine groups with phosphoric acid not only promotes its uniform distribution on the CL but also stabilizes the EI under high partial pressure of water, minimizing the ganglia formation. Additionally, the use of pyridine functionalized MWCNT based electrocatalyst gives the opportunity of lowering the $\mathrm{Pt}$ 
loading in the electrodes without sacrificing the overall cell's performance under reformate conditions, thus reducing the MEA production cost.

Up to date, PEMFCs commercialization is still significantly restricted by its high noble metal content and low cathode performance. The presence of PA possess limitations; slow kinetic of the oxygen reduction reaction and reduced oxygen solubility in hot phosphoric acid restricts the performance of the cathodic electrode. In an attempt to lower the $\mathrm{Pt}$ loading at the cathodic electrode the newly synthesized electrocatalyst $30 \mathrm{wt} \%$ Pt/oxMWCNT-Py was evaluated wit hrespect tot the PA loading and the Pt loading in the catalyst layer. The presence of pyridine groups was found to homogeneously distribute PA in the catalyst layer minimizing the blockage of the pores of the catalyst layer and increase the three phase boundary. As a result the MEA employing 30wt\% Pt oxMWCNT-Py showed the same performance as the $30 \% \mathrm{Pt} / \mathrm{C}$ for half the Pt loading. Despite the hard operating conditions the Pt particles attached to the ox.MWCNT-Py substrate exhibit the same stability as the commercial catalyst and the pyridine groups were found to be stable, at least for short term operation at the cathodic electrode. It has to be mentioned that for the determination of the ECSA CO stripping voltammetry based procedures were evaluated and the optimum one was chosen for our system, in order to determine the ECSA of a cathodic catalyst layer without severely affecting the Pt particle size distribution. It was found that sequential $\mathrm{CO}$ adsorption until complete reduction of the Pt surface is the more appropriate ECSA evaluation procedure causing the minimum damage to the Pt particle size.

It is clear that the use of this newly synthesized electrocatalyts $30 \mathrm{wt} \% \mathrm{Pt} / \mathrm{oxMWCNT}-\mathrm{Py}$, at both electrodes, has major advantages as it increase the catalyst utilization and there is no need to use a polymer-binder inside the catalytic layer. Thus avoiding problems of inhomogeneous binder distribution and/or electronic insulation of catalyst nanoparticles. Using this newly synthesized electrocatalyst opens the possibility of significant reduction of the amount of $\mathrm{Pt}$ on both anodic and cathodic electrodes, under various operation conditions, without sacrificing the performance and stability of the fuel cell.

\subsection{Proposal for future work}

The results obtained from the newly synthesized electrocatalyst, 30wt\% Pt/oxMWCNTPy, are very promising for future applications at both the anodic and cathodic electrode. Optimizing the fabrication procedure of the catalyst layer, in order to increase the porosity and the three-phase boundary, would be the next step. Decal CCM MEA fabrication procedure is one of the most popular and industrially used procedures, giving high quality catalyst coatings and reproducible fuel cell performances. Therefore, using the latter method may lead to further reduction of the Pt loading of the CL, resulting in further reduction of the production cost without sacrificing the fuel cell's performance.Also, since the pyridine groups are an essential part of the newly synthesized electrocatalyst, the effect 
of the functionalization degree on both the anode's and cathode's electrode performance should also be investigated. The atomic ratio of pyridine to carbon, needs to be optimized, as based on the results presented in this thesis, it is suspected to influences the overall fuel cell performance. 


\section{$\underline{\text { Appendix }}$}

\section{A1. Activation process of TPS ${ }^{\circledR}$ based MEAs}

\section{A.1.1 Introduction}

The activation period of a fuel cell is a specific period of fuel cell operation, during which the cell's performance is increased and stabilized. The break in period of the fuel cell is critical before normal operation in order to obtain maximum performance and reproducibility of results. The activation procedure and mechanism for the low temperature fuel cell have been widely and fully studied [1-6], as opposed to high temperature fuel cells. In Nafion-based fuel cells the activation mechanism has been identified as a two-step procedure occurring simultaneously: hydration of the membrane during the first hours of operation, which reaches equilibrium state resulting in an increase in the proton conductivity [7] and activation of the sulfonic groups of the binder and the establishment of ionic conductive pathway for the good migration of the hydrated protons [8]. To the authors knowledge, there are limited published work concerning the activation protocols and mechanisms of PBI based fuel cells. Thomas Tingelof et al. [8] investigated different types of break-in procedures using PBI based fuel cells. They focused on methods that can rapidly and reproducibly ensure stable cell behavior conserving the performance of the cell. They found that galvanostatic operation clearly enhanced PBI fuel cell performance. More specifically they proposed a galvanostatic operation for 24 hours at $200 \mathrm{oC}$ and then a 48 hour relaxation at $160^{\circ} \mathrm{C}$. M. Boaventura et al. [9] reported also that galvanostatic preconditioning of the MEA resulted in higher performance. They claimed that the catalyst activity increased due to the migration of phosphoric acid from the membrane to the catalyst layer, increasing the three-phase boundary, and by the removal of impurities from catalyst or redistribution of electrolyte in the case of commercial MEA. Therefore, depending on the activation procedure one may use, the required break in period vary [10].

The aim of this section is to present the activation process of a phosphoric acid doped membrane based fuel cell, employing a TPS ${ }^{\circledR}$ type of membrane, for the reader's convenience. 
A.1.2 Results

All MEAs were activated under galvanostatic mode. Initially the cell's temperature was increased from room temperature to $100^{\circ} \mathrm{C}$ while $\mathrm{Ar}$ flushed both compartments. Subsequently, the gases were switched to pure $\mathrm{H}_{2}$ and air and the temperature was set at $140^{\circ} \mathrm{C}$. Once the temperature was reached, a current of $0.2 \mathrm{~A} / \mathrm{cm}^{2}$ was applied to the fuel cell. The temperature was then set at $180^{\circ} \mathrm{C}$. The break in period was found to be approximately 48 hours. The polarization measurements were obtained under galvanostatic mode during various interval time of the activation period. High flows were employed for both compartments in order to minimize any mass transport resistances during the polarization curves acquisition, 200cc/min of pure $\mathrm{H}_{2}$ and $\mathrm{O}_{2}$, which corresponds to a nominal current density of $6.6 \mathrm{~A} / \mathrm{cm}^{2}$. All MEAs used during the fulfillment of this $\mathrm{PhD}$ thesis, presented the exact same pattern during activation procedure. The results presented in this section are a characteristic example, obtained from an MEA employing at both electrodes $30 \mathrm{wt} \% \mathrm{Pt} / \mathrm{C}$ and having a noble metal loading of $1.5 \mathrm{mgPt} / \mathrm{cm}^{2}$.

As shown in figure A-1, the cell's performance increases during the first hours of break inperiod. The performance in the ohmic region of the polarization curve exhibits a small increase, whereas in the limiting current region shifts significantly to higher current densities values during the activation period.

The open circuit is a thermodynamic parameter and it's widely known that its value depends on the concentration of the adsorbed species on the Pt surface and the operation temperature of the fuel cell [11]. During the activation process, it was observed that the open circuit potential increased, from an average value of $0.930-0.945 \mathrm{~V}$ to $1.030 \mathrm{~V}$. The increase of the OCP might be an indication of the desorbtion of impurities form the $\mathrm{Pt}$ surface or reorganization of the PA species near the Pt interface.

The resistance of the proton conduction during the activation period was measured via AC impedance and the values were obtained with respect to the applied current density, Figure A-2. All electrochemical impedance spectroscopy measurements were conducted in galvanostatic mode using a sinusoidal current wave with an amplitude of $5 \mathrm{~mA} / \mathrm{cm}^{2}$ while sweeping the frequency from $50 \mathrm{kHz}$ up to $10 \mathrm{mHz}$ with a logarithmic distribution and 50 points. The measurements were conducted without interrupting the cell's operation. 


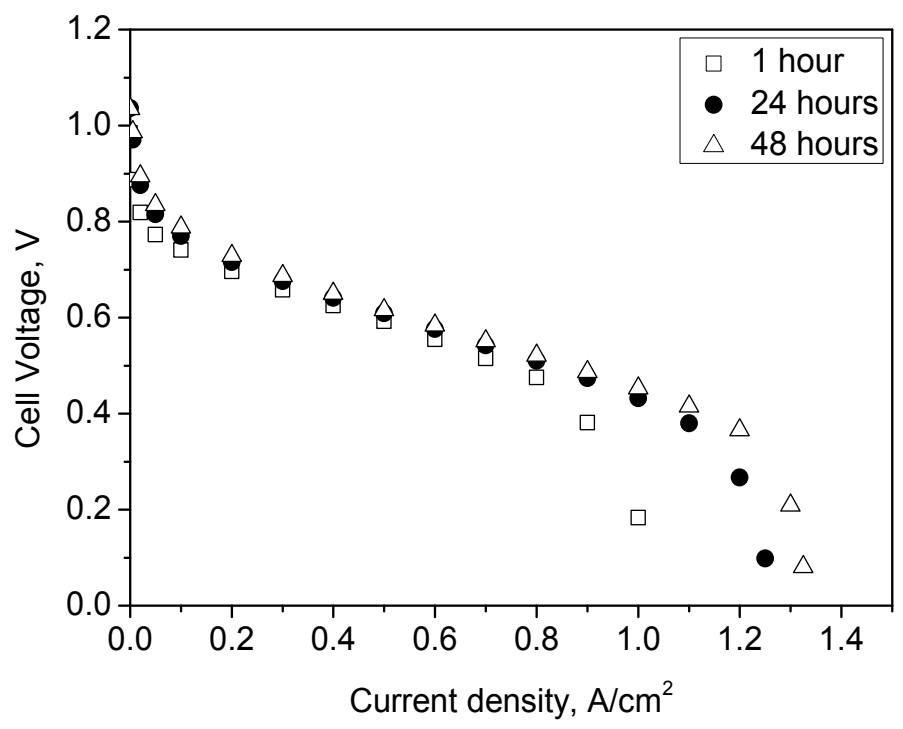

Figure A-1. The effect of the activation time on the fuel cell's performance. Polarization curves were obtained at $180 \mathrm{oC}$ and constant flow of pureH2 and $\mathrm{O} 2(200 \mathrm{cc} / \mathrm{min})$. The voltage values are IR drop corrected.

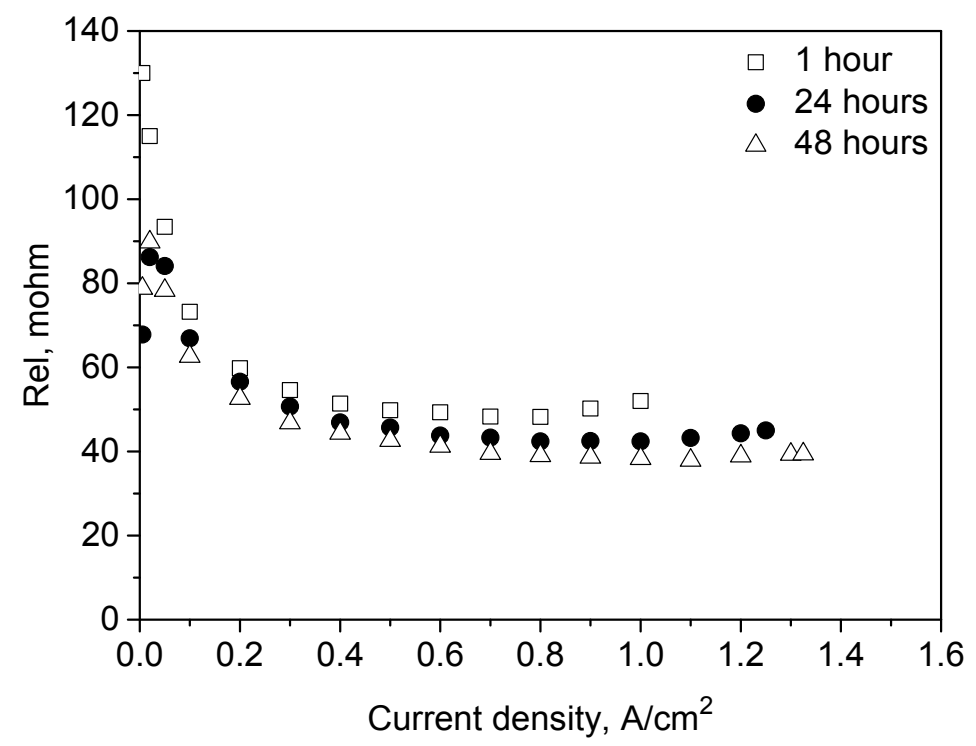

Figure A-2. The change of the proton conduction resistance with respect to the applied current density at various time during the activation period. 
In a typical Nyquist plot both real and imaginary components of the Impedance $\mathrm{Z}$ are plotted. The intersept at high frequencies with the $\mathrm{x}$ axis -real component is considered to be dominated by the ohmic resistance of the membrane/ proton conduction resistance and the total electrical resistance. The total electric resistance which is included in the intersept value is a combination of the resistanses of the bipolar plates, the graphine flow plates, the gass diffusion layer, the electrolyte membrane and the contact resistances included in between. Since the bipolar and graphite plates and the gas diffusion layers have a very high electric conductivity, the high frequency resistance value comprises mainly the ionic resistance.

As shown in figure A-2, during the activation process the membrane resistance is decreasing as expected. At low current densities the resistance of the membrane is high due to the low water production at the cathodic electrode and the high flow of the reactant gasses, which is expected. As the current increases the membrane resistance decreases and remains constant before reaching the limiting current region. At high current densities, at the limiting current region, it was found that the resistance of the membrane is increasing prior to activation. The increase in the Rel in the limiting region means that the proton conduction mechanism is the one of the limiting steps in the performance of the cell. As the activation continuous, the resistance of the membrane in the limiting current region diminishes and stabilizes. During the activation procedure of very important step is the hydration of the electrolyte. Although HTPEM fuel cell don't require external hydration during operation, it has been reported that the produced water from the cathode or even external addition of water can increase the proton conduction of the electrolyte membrane. This is to be expected, as the water hydrates the membrane, thus increasing the proton conductivity. Water is reported to improve proton conductivity both in the membrane and in the electrodes due to its effect on acid equilibrium [12-18].

The performance of the cell is increasing during the activation procedure and the measured ohmic resistance is constantly decreasing as well. One cannot imply that this is a result of water adsorption from the PA in the membrane or the CL as it was found from Daletou et al. that a $\mathrm{H}_{3} \mathrm{PO}_{4}$ doped membrane reaches full hydration after $60 \mathrm{~min}\left[{ }^{19}\right]$. Nevertheless the observed gain in the cell's voltage cannot be explain solely by the gain in the decreased resistance of the cell, as has been seen from the IR drop corrected IV plots. Based on the above facts one cannot directly correlate the dramatic increase in the cell's performance in the limiting current region only to the decrease in the ionic resistance. 
Figure A-3. shows the results form Nyquist plots acquired during different operation hours of the activation procedure form the above MEA. Two arcs are observed at the Nyquist plot. The first one, which appears at high frequencies corresponds to the impedance of the $\mathrm{H} 2$ oxidation reaction at the anode side $[20,21]$ and as thoroughly discussed in chapter 5 . Although, other claims that the high frequency arc is related to the proton conduction in the catalyst layer [22]. Whereas for the second arc ,appearing in the intermediate and low frequency, the general consensus is that it originates from activation losses and from dominated cathode effects. Some research groups claim that it originates from the combination of anode and cathode activation [23,24].

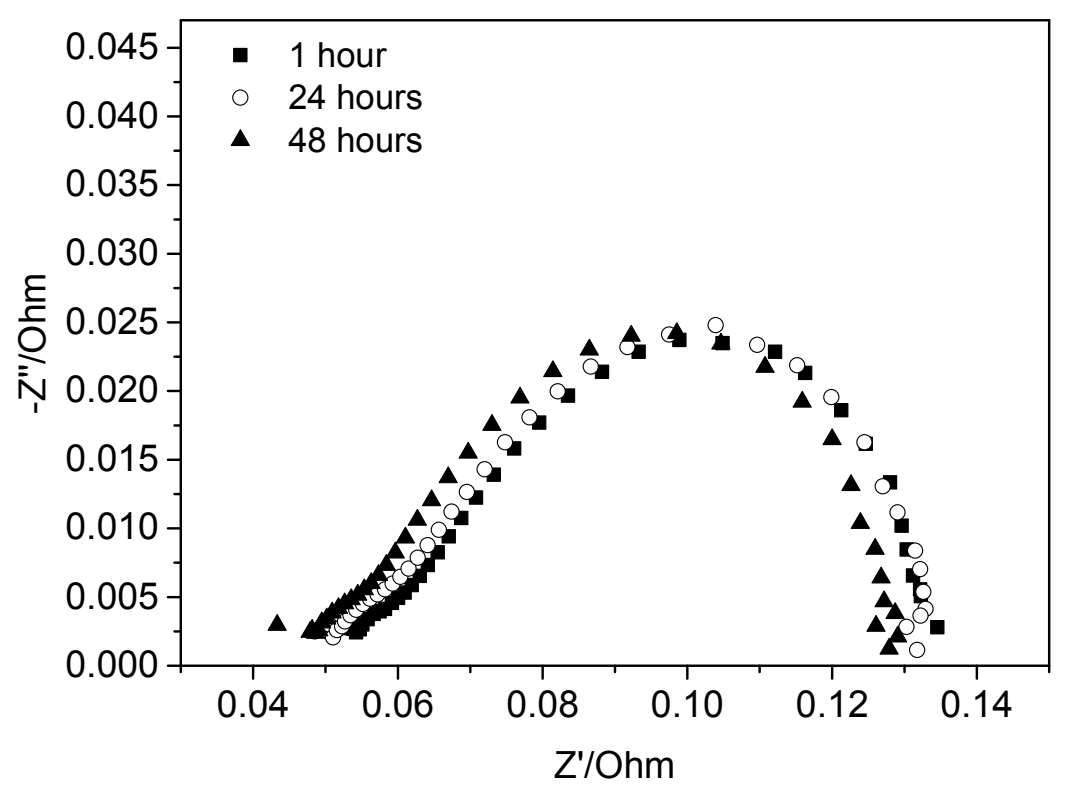

Figure A-3. Impedance spectra acquired at different times of the activation period at $0.2 \mathrm{~A} / \mathrm{cm}^{2}$ and $180^{\circ} \mathrm{C}$. The total flow was kept constant at $200 \mathrm{cc} / \mathrm{min}$ of pure $\mathrm{H}_{2}$ and $\mathrm{O}_{2}$.

As also shown in figure A-3, the high frequency resistance decreases during the activation procedure, whereas the total charge transfer resistance is kept constant. 


\section{B1. Polarizations curves under different fuel compositions -supporting information}

The voltage loss of the anodic electrode under various fuel compositions, as described in detail in chapter 6 , were calculated using the following polarizations curves. The voltage is IR drop corrected and the measurements were acquired under excess of reacting gasses in order to minimize mass transport limitations. The polarization curves were acquired under various fuel compositions and pure $\mathrm{O}_{2}$ flows (200cc/min respectively): Black square: $100 \mathrm{kPa}$ of $\mathrm{H} 2$, black circle: $50.7 \mathrm{kPaH}_{2}$ balanced with $\mathrm{Ar}$, open square: $50.7 \mathrm{kPa} \mathrm{H}$ and $2 \mathrm{kPa} \mathrm{CO}$ balanced with $\mathrm{Ar}$, and open circle: $50.7 \mathrm{kPa} \mathrm{H} 2,2 \mathrm{kPa} \mathrm{CO}$ and $33.5 \mathrm{kPa} \mathrm{H}_{2} \mathrm{O}$ balanced with Ar. The PA loading, Pt loading and catalyst type (MEA number) is being mention in the inset of each graph.

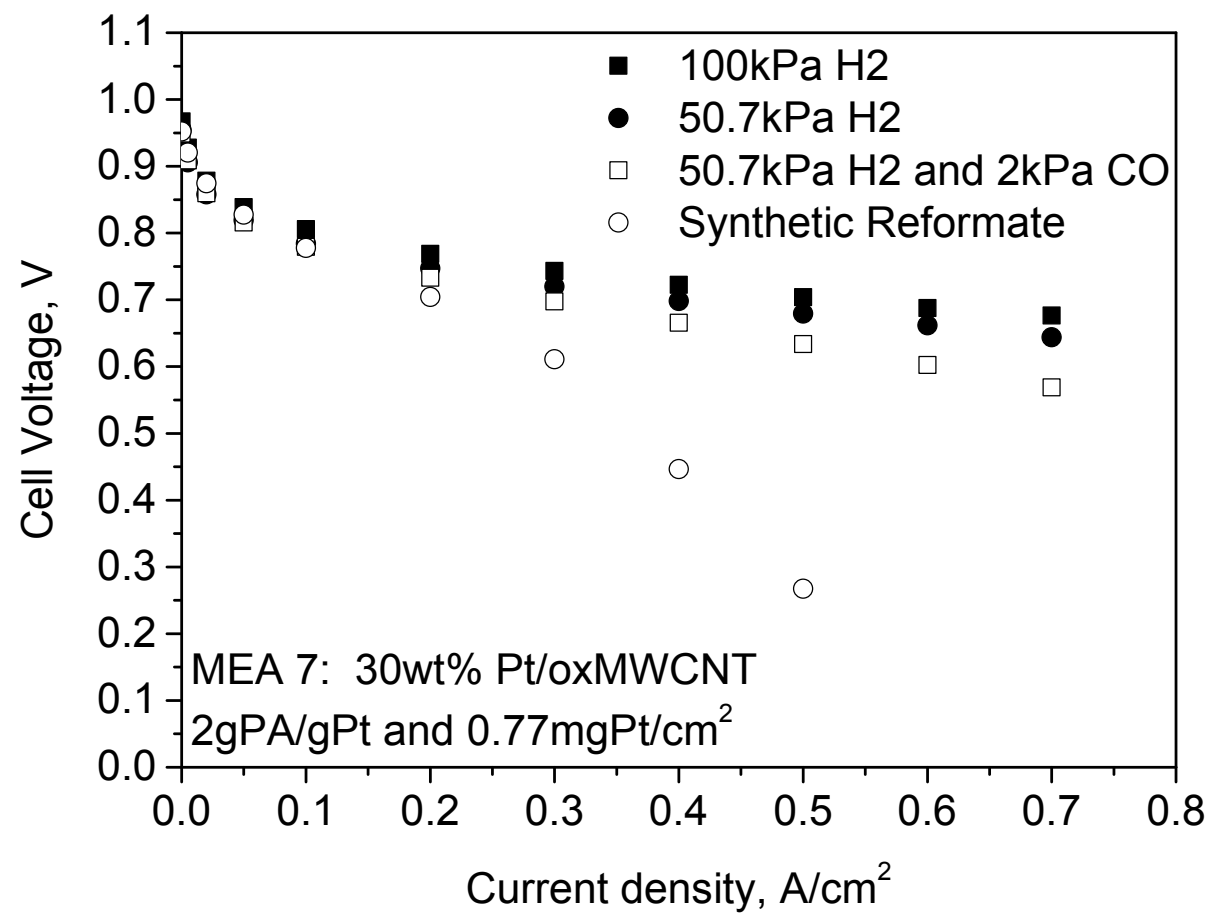



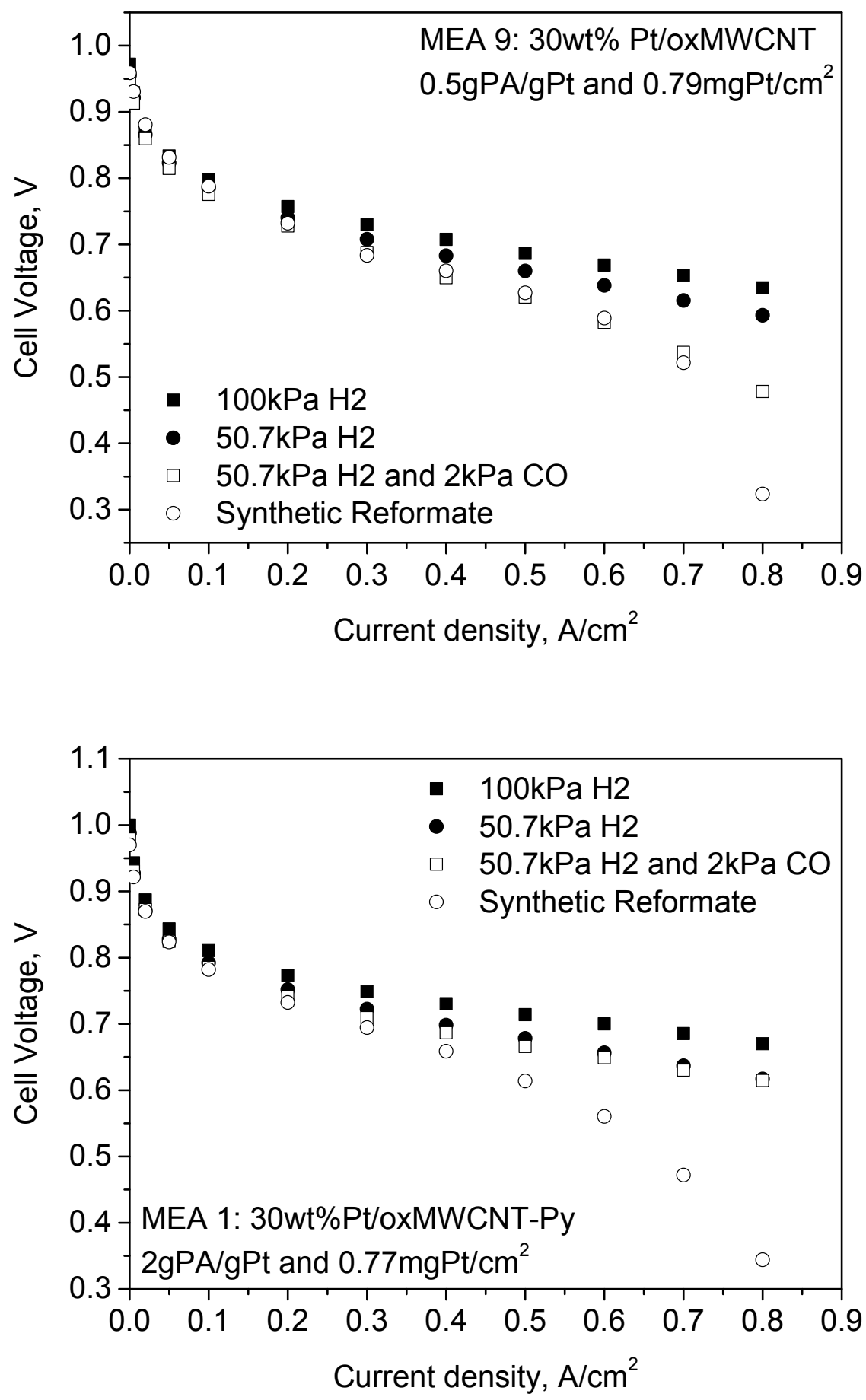

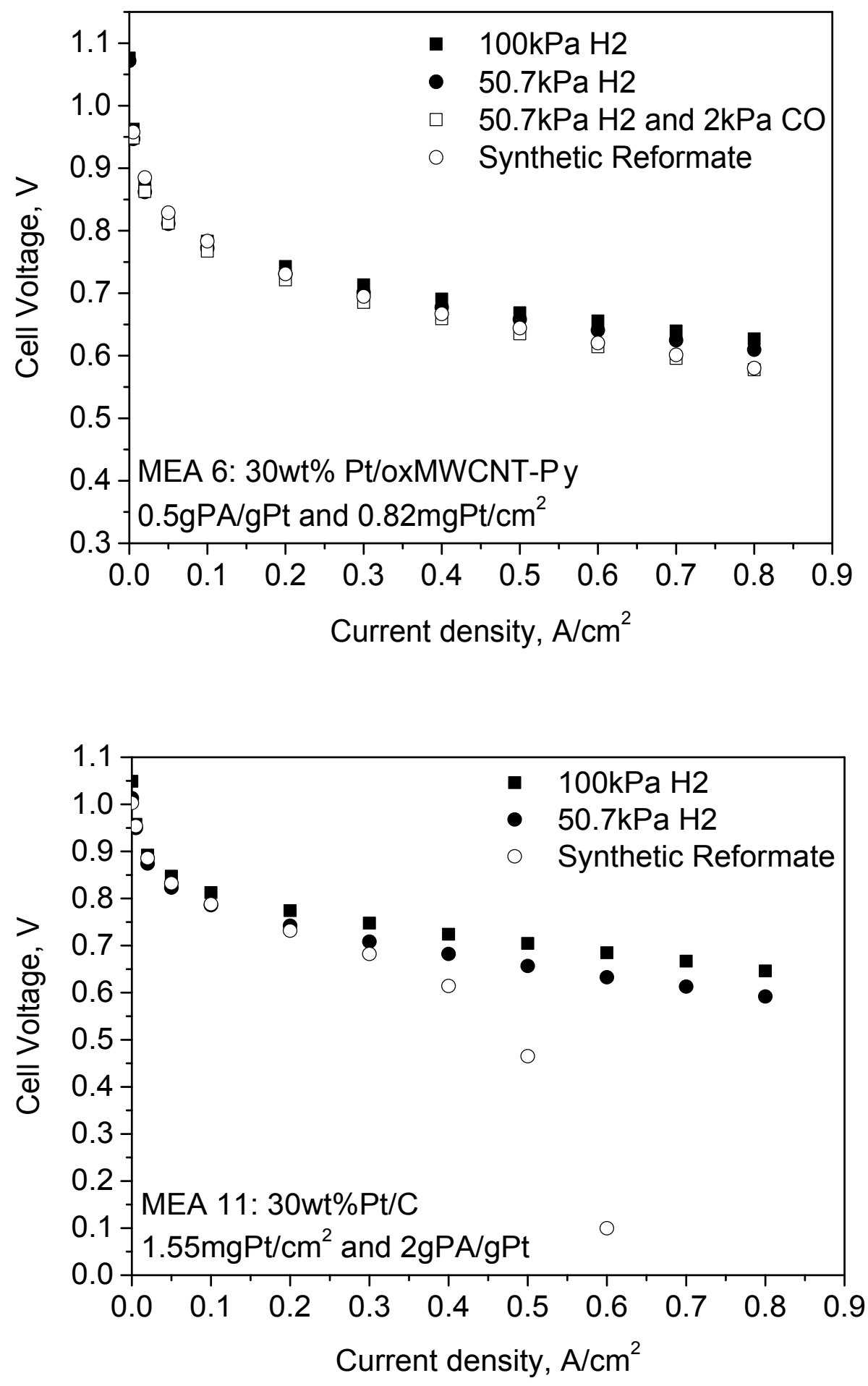


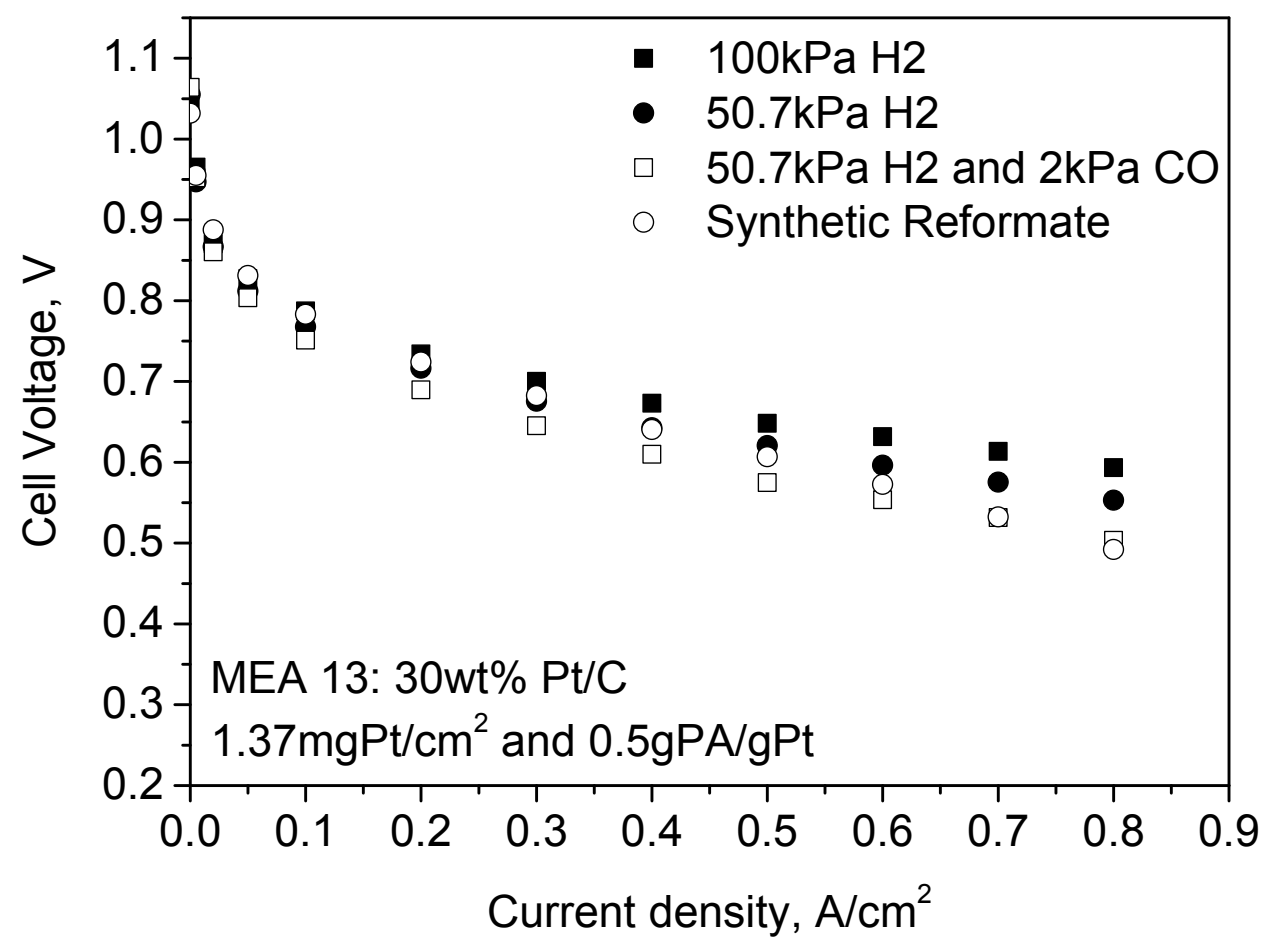

\section{$\underline{\text { References }}$}

[1] Qi ZG, Kaufman A. Activation of low temperature PEM fuel cells. Journal of Power Sources $111(2002) 181$

[2] Xu ZQ, Qi ZG, He CZ, Kaufman A. Combined activation methods for proton-exchange membrane fuel cells. Journal of Power Sources 156 (2006) 315.

[3] Xu ZQ, Qi ZG, Kaufman A. Activation of proton-exchange membrane fuel cells via CO oxidative stripping. Journal of Power Sources 156 (2006) 281

[4] Qi Z, Kaufman A. Enhancement of PEM fuel cell performance by steaming or boiling the electrode. Journal of Power Sources 109 (2002) 227

[5] Cho KY, Lee KS, Cho IH. Method of activating membrane electrode assembly (PEM) of polymer electrolyte membrane fuel cell (PEMFC) using cyclic voltammetry (CV). Patent application number: 20090155635. IPC8 class: AH01M800FI; USPC class:429,13.

[6] Qi ZG, Kaufman A. Quick and effective activation of proton exchange membrane fuel cells. Journal of Power Sources 114 (2003) 21 
[7] Morris DR, Sun X. Water-sorption and transport properties of Nafion 117 H., Journal of Applied Polymer Science 50 (1993) 1445

[8] Choi P, Jalani NH, Datta R. Thermodynamics and proton transport in Nafion II. Proton diffusion mechanisms and conductivity, Journal of the Electrochemical Society 152 (2005) E123

[9] Thomas Tingelöf, Jari K. Ihonen, A rapid break-in procedure for PBI fuel cells, International journal of hydrogen energy 34 (2009) 6452-6456

[10] M. Boaventura, A. Mendes, Activation procedures characterization of MEA based on phosphoric acid doped PBI membranes, International journal of hydrogen energy 35 (2010) 11649

[11] Samuele Galbiati, Andrea Baricci, Andrea Casalegno, Giulia Carcassola, Renzo Marchesi, On the activation of polybenzimidazole-based membrane electrode assemblies doped with phosphoric acid, International journal of hydrogen energy 37 (2012) 14475-1448

[12] Jianlu Zhang, Zhong Xie, Jiujun Zhang, Yanghua Tang, Chaojie Song, Titichai Navessin, Zhiqing Shi, Datong Song, Haijiang Wang, David P. Wilkinson, Zhong-Sheng Liu, Steven Holdcroft, High temperature PEM fuel cells, Journal of Power Sources 160 (2006) 872

[13] Li Q, He R, Jensen JO, Bjerrum NJ. PBI-Based polymer membranes for high temperature fuel cells -preparation,characterization and fuel cell demonstration. Fuel Cells 4 (2004) 3

[14] Schechter A, Savinell RF, Wainright JS, Ray D. 1H and 31P NMR study of phosphoric aciddoped polybenzimidazole under controlled water activity. Journal of the Electrochemical Society 156 (2009) B283

[15] Daletou MK, Kallitsis JK, Voyatzis G, Neophytides SG. The interaction of water vapors with H3PO4 imbibed electrolyte based on PBI/polysulfone copolymer blends. J Memb Sci 326 (2009) 76

[16] Liu Z, Wainright JS, Litt MH, Savinell RF. Study of the oxygen reduction reaction (ORR) at Pt interfaced with phosphoric acid doped polybenzimidazole at elevated temperature and low relative humidity. Electrochimica Acta 51 (2006) 3914

[17] Wippermann K, Wannek C, Oetjen HF, Mergel J, Lehnert W. Cell resistances of poly(2,5benzimidazole)-based high temperature polymer membrane fuel cell membrane electrode assemblies: time dependence and influence of operating parameters. Journal of Power Sources 195 (2010) 2806.

[18] Bonaventura M, Mendes A. Activation procedures characterization of MEA based on phosphoric acid doped PBI membranes. I J Hydrogen Energy 35 (2010) 11649.

[19] Maria K. Daletou, Maria Geormezi, Effrosyni Vogli, George A. Voyiatzis, Stylianos G. Neophytides, The interaction of H3PO4 and steam with PBI and TPS polymeric membranes. A TGA and Raman study, J. Mater. Chem. A, 2 (2014) 1117

[20] Andreasen SJ, Jespersen JL, Schaltz E, Kær SK. Characterisation and modelling of a high temperature PEM fuel cell stack using electrochemical impedance spectroscopy. Fuel Cells 9 (2009) 463. 
[21] ZhuWH, Payne RU, Tatarchuk BJ. PEM stack test and analysis in a power system at operational load via AC impedance. Journal of Power Sources 168 (2007) 211

[22] Yuan X, Wang H, Sun JC, Zhang J. AC impedance technique in PEM fuel cell diagnosis e A review, International Journal of Hydrogen Energy 32 (2007) 4365

[23] Zhang J, Tang Y, Song C, Zhang J. Polybenzimidazole-membrane-based PEM fuel cell in the temperature range of $120-200{ }^{\circ} \mathrm{C}$. Journal of Power Sources 172 (2007) 163.

[24] Jespersen JL, Schaltz E, Kær SK. Electrochemical characterization of a polybenzimidazolebased high temperature proton exchange membrane unit cell., Journal of Power Sources 191 (2009) 289 


\section{C1. H $\Lambda$ EKTPOXHMIKO $\Sigma$ XAPAKTHPI $\Sigma M O \Sigma$ KE $\Lambda$ I $\Omega$ N KAY $\Sigma I M O Y$

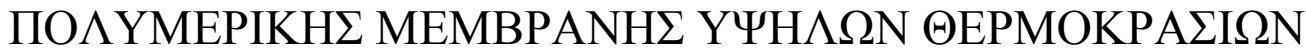

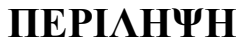

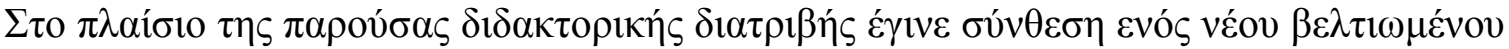

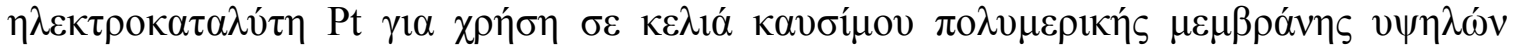

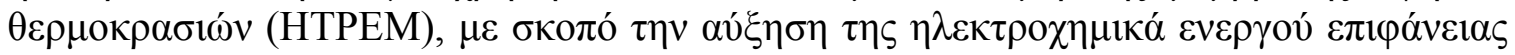

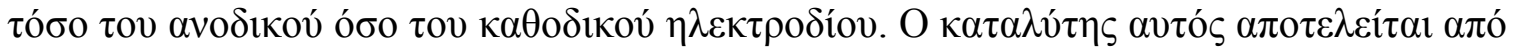

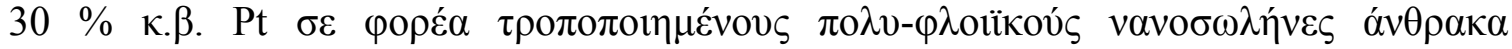

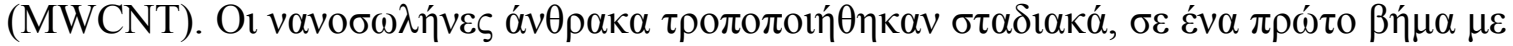

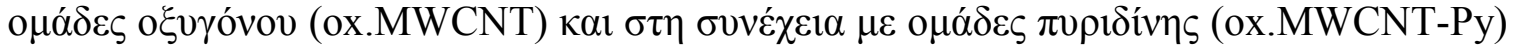

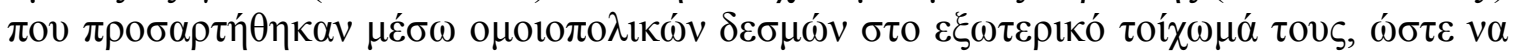

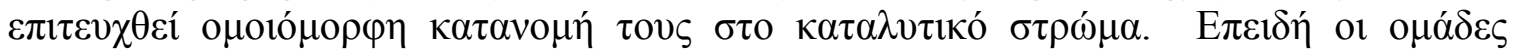

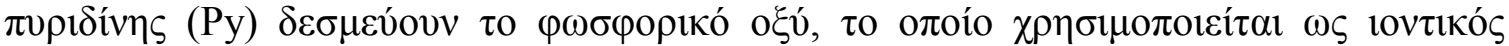

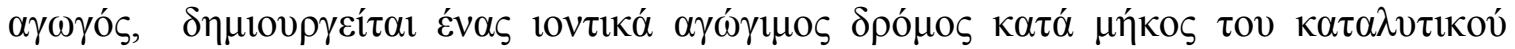

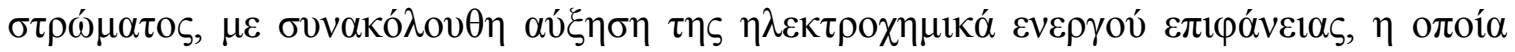

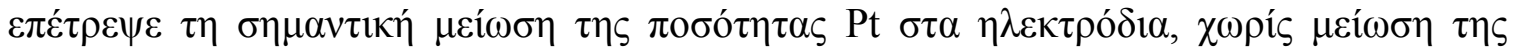

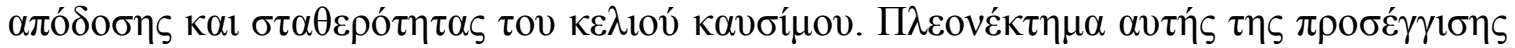

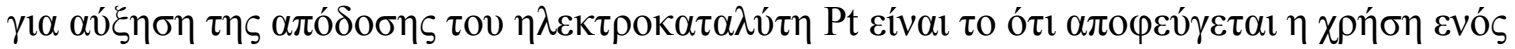

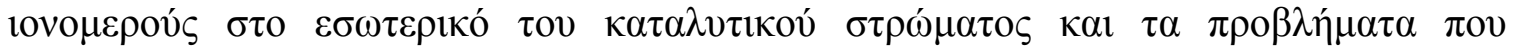

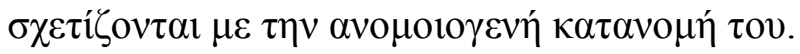

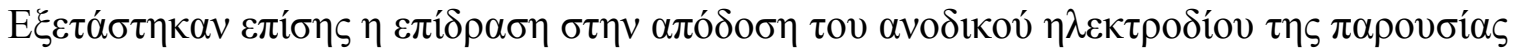

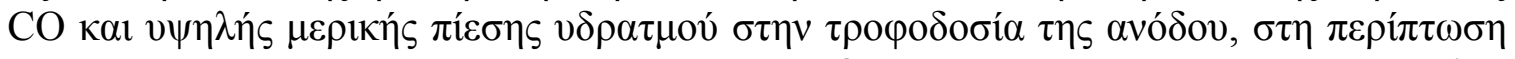

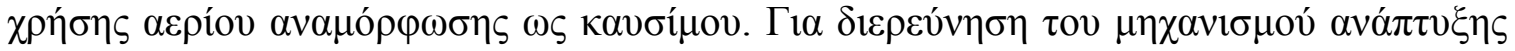

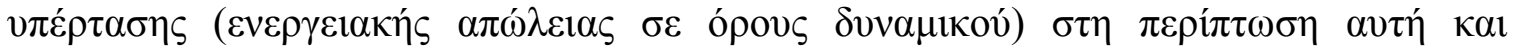

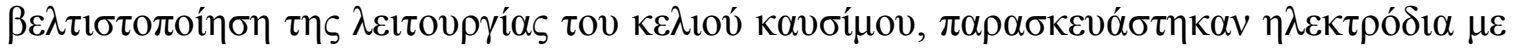

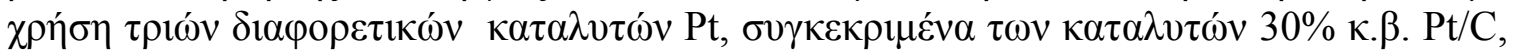

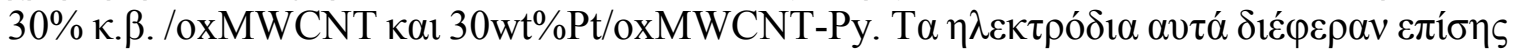

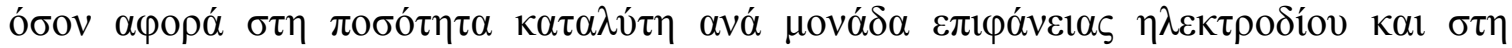

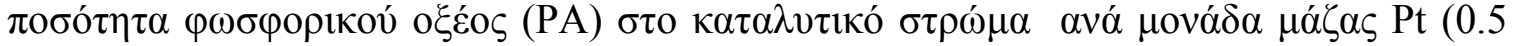

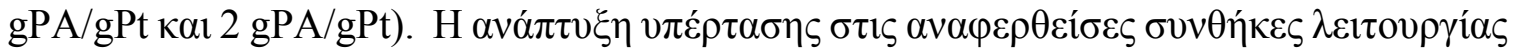

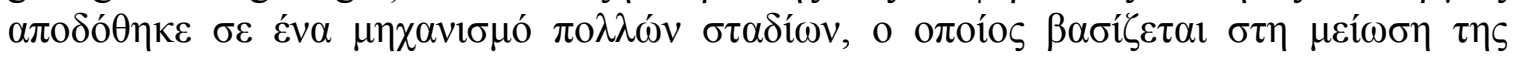

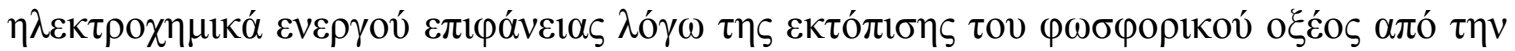

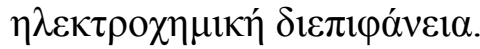

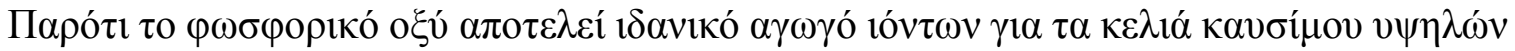

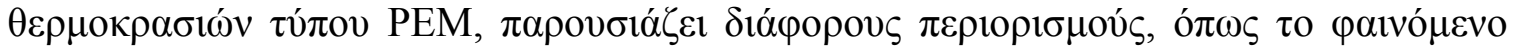

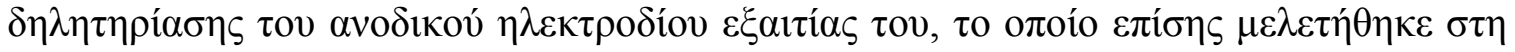

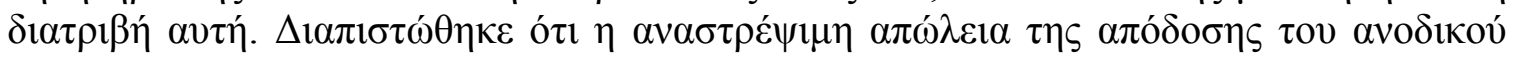

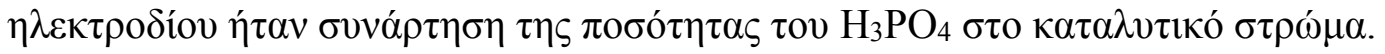

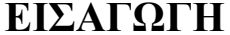

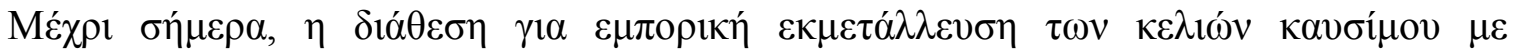

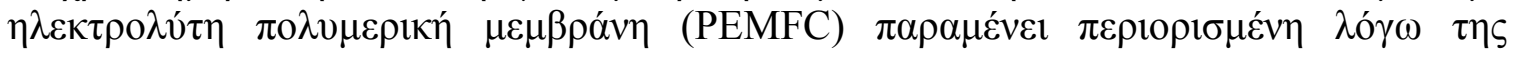




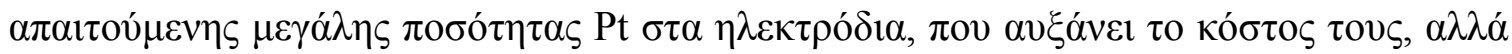

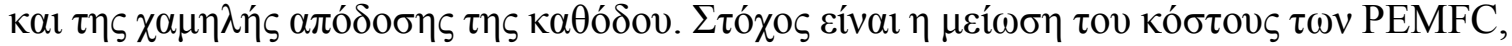

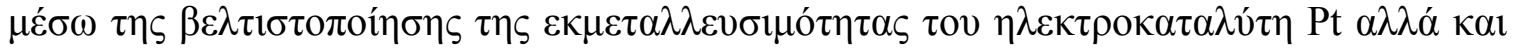

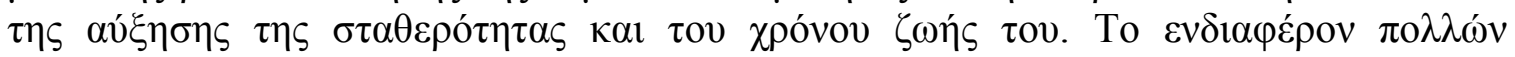

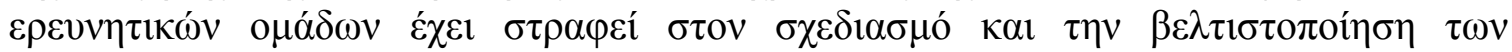

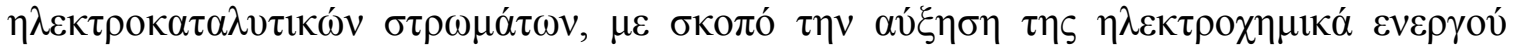

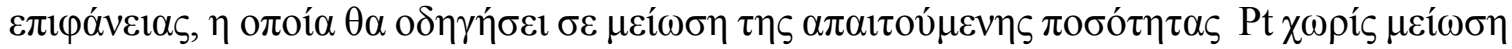

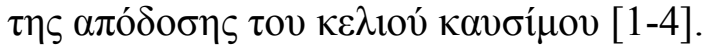

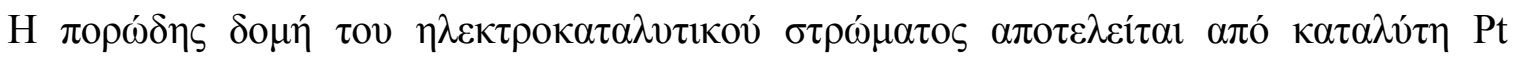

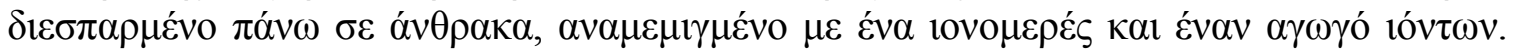

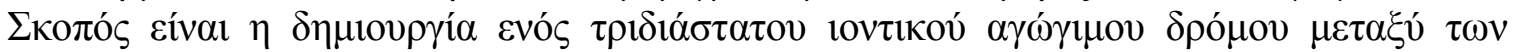

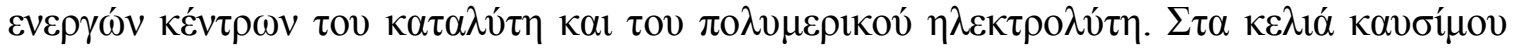

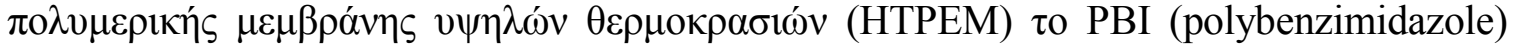

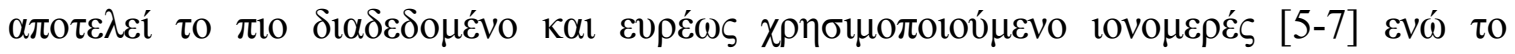

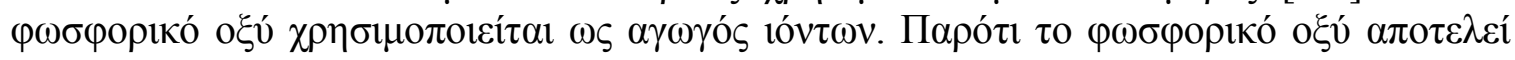

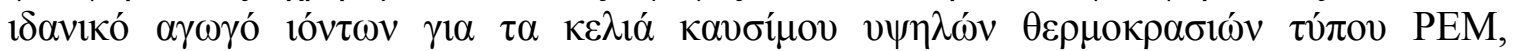

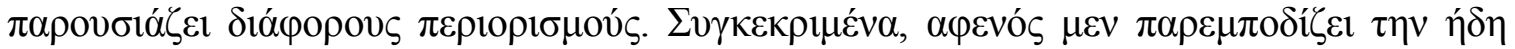

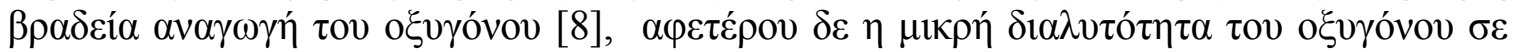

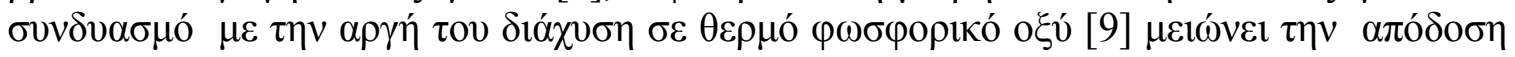

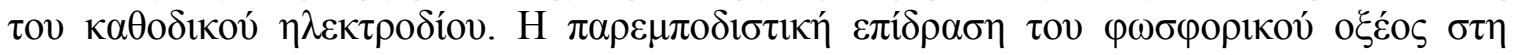

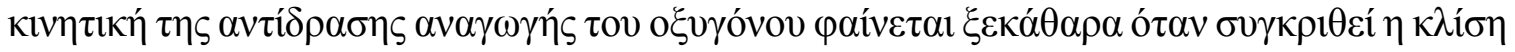

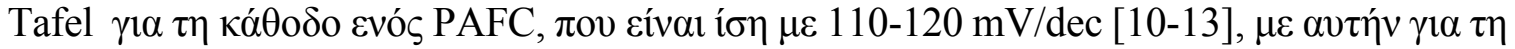

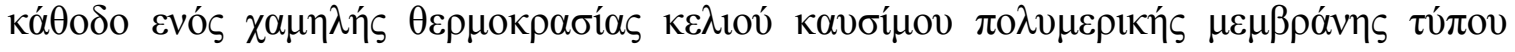

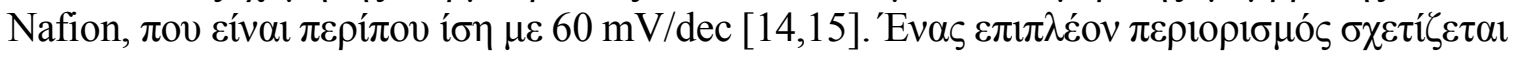

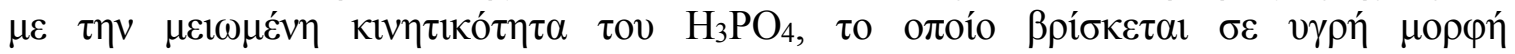

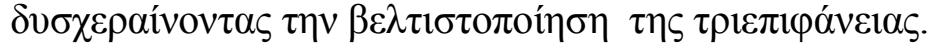

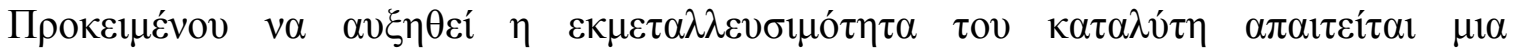

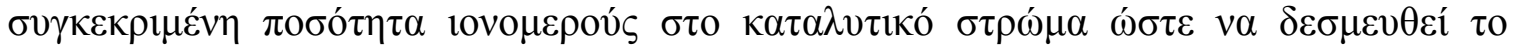

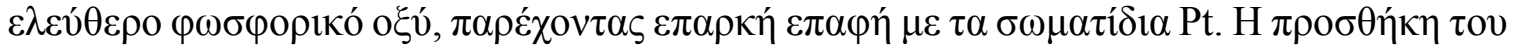

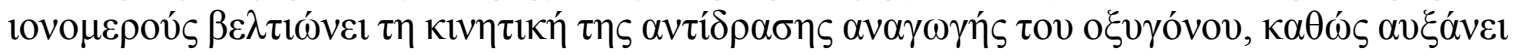

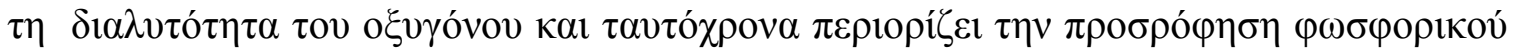

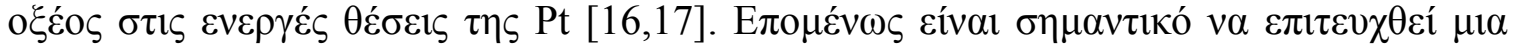

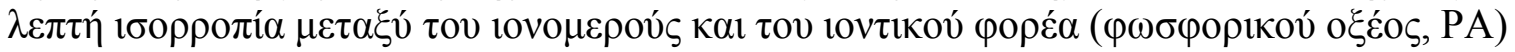

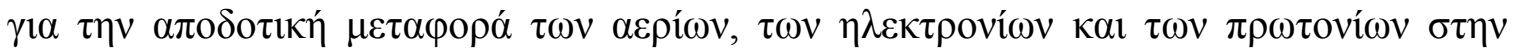

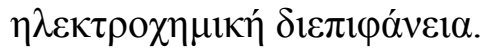

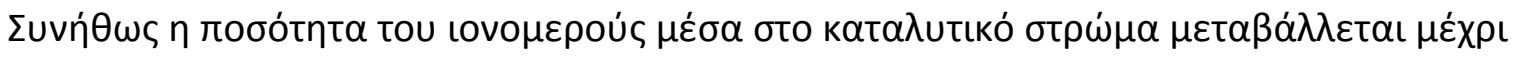

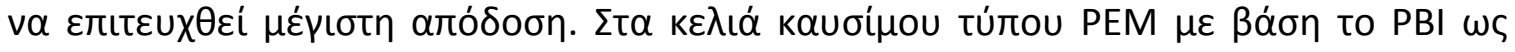

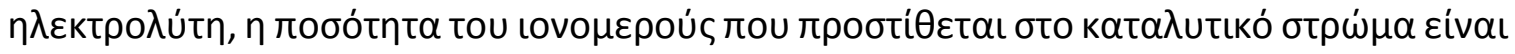

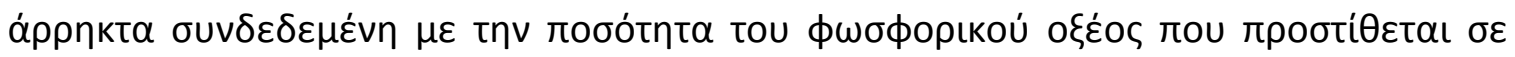

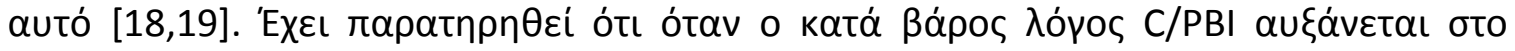

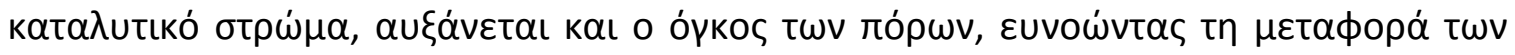

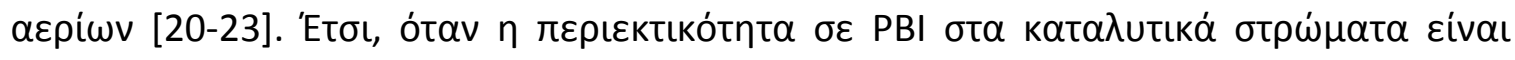

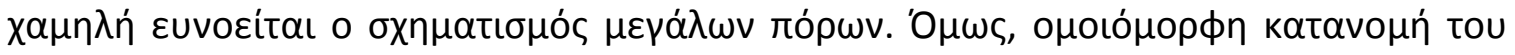

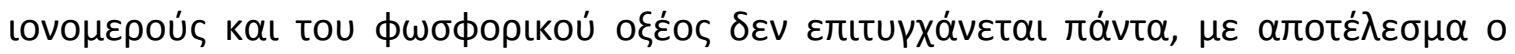




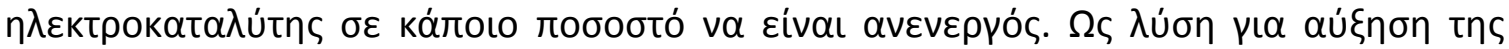

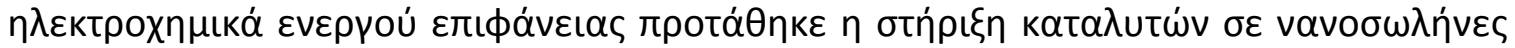

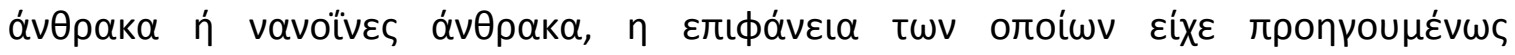

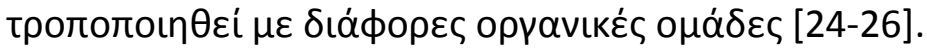

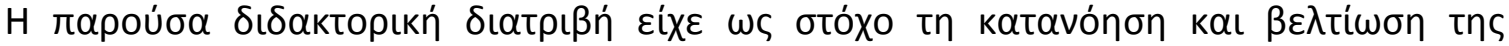

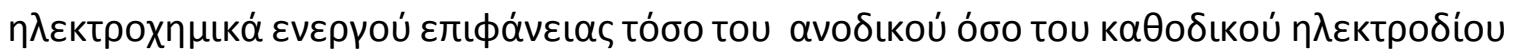

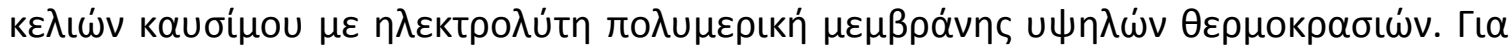

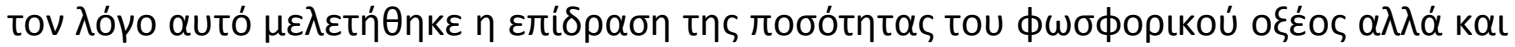

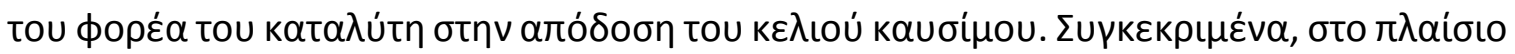

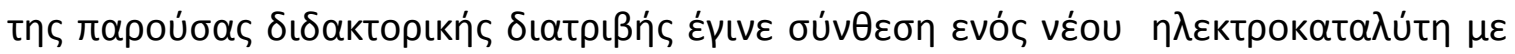

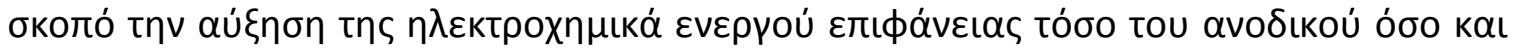

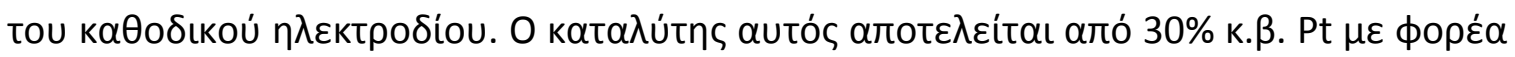

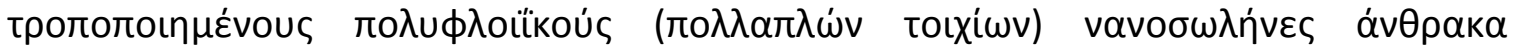

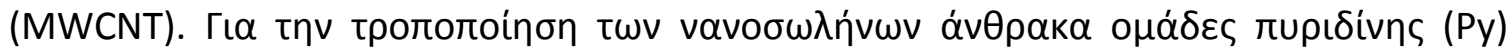

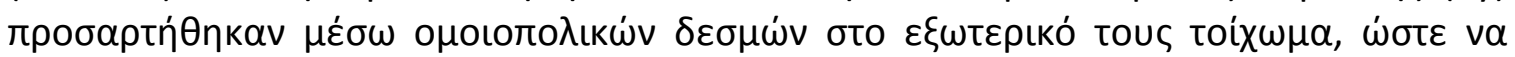

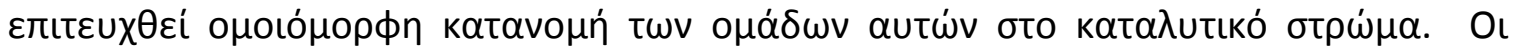

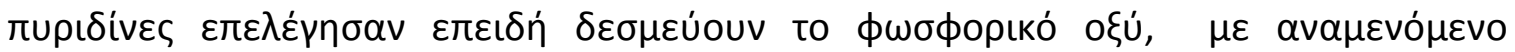

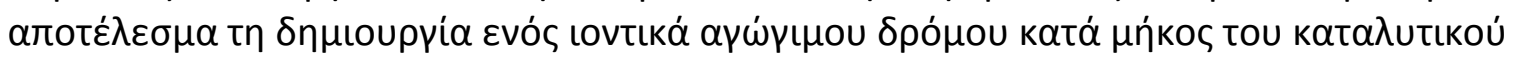

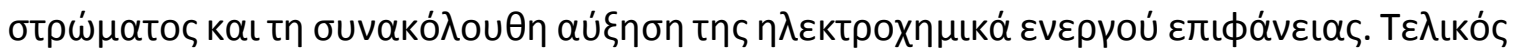

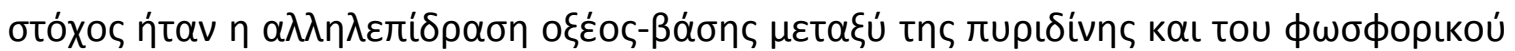

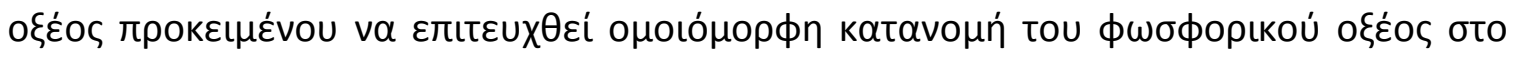

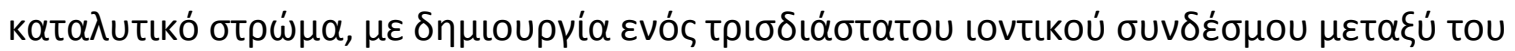

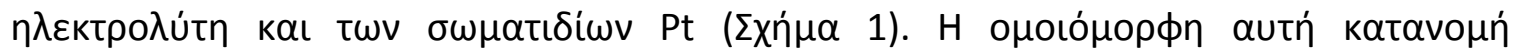

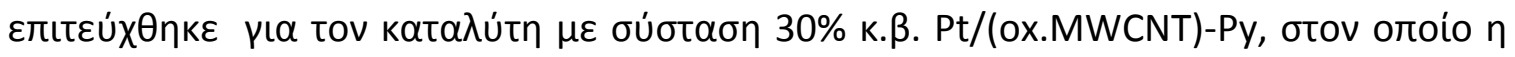

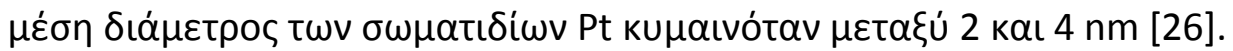
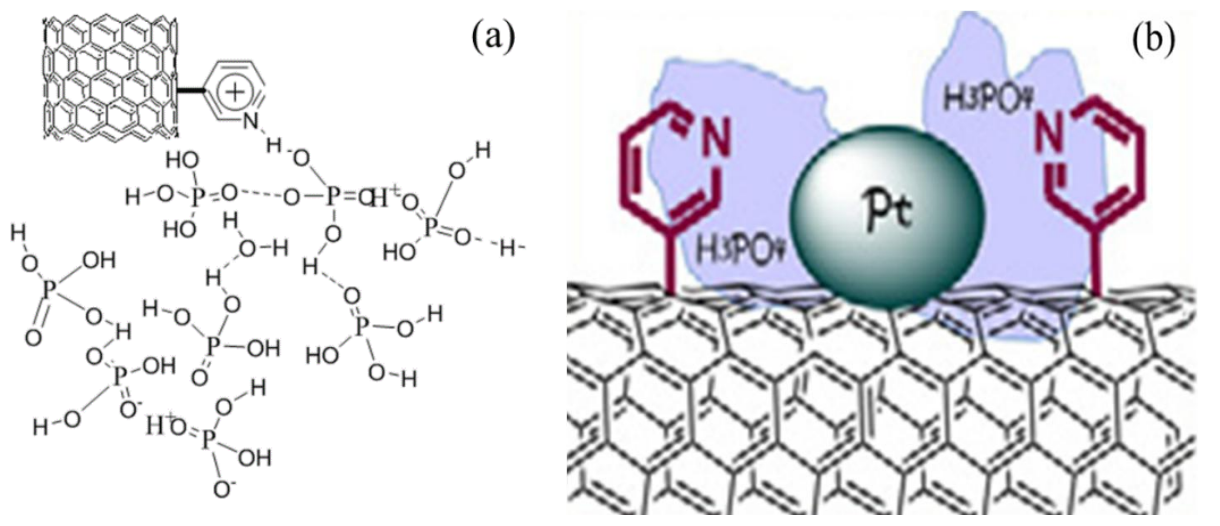

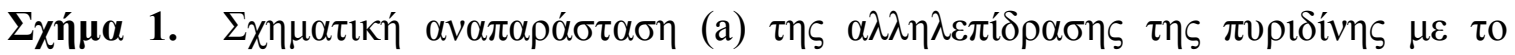

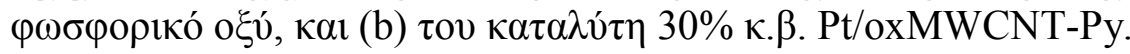




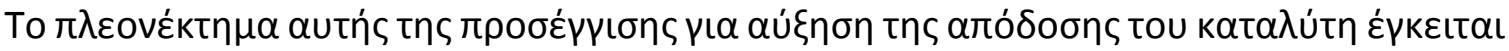

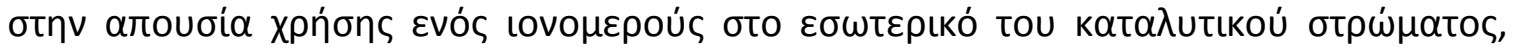

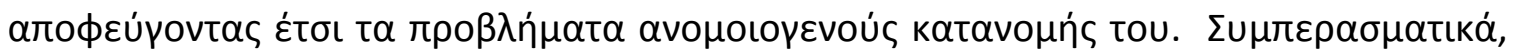

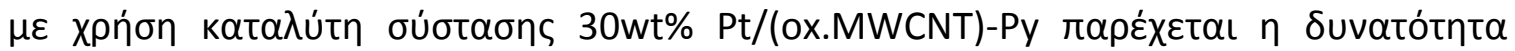

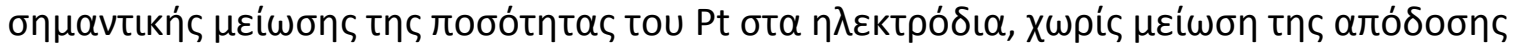

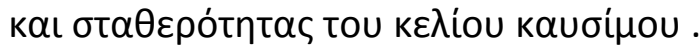

\section{ПЕIPAМАTIKO MEPOऽ}

\section{Y入ıко́}

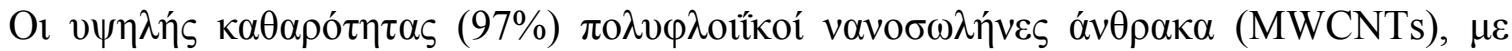

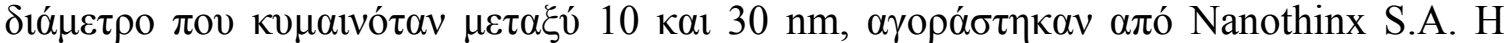

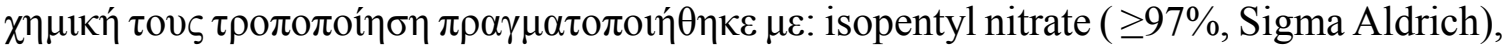

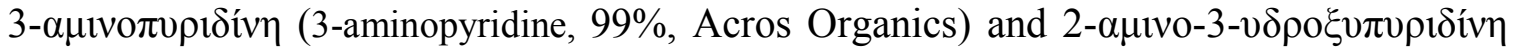

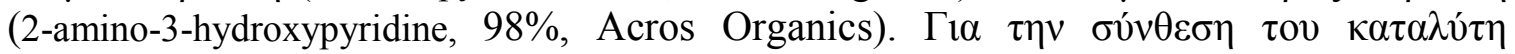

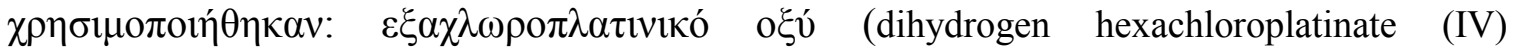

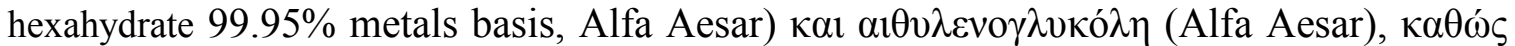

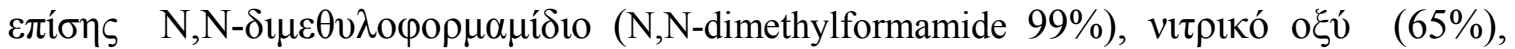

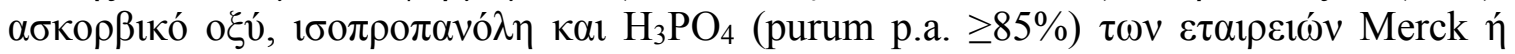
Sigma Aldrich.

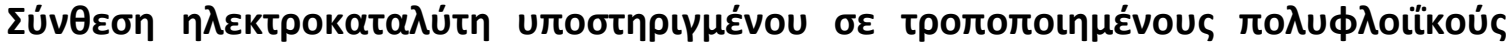

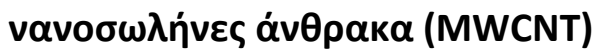

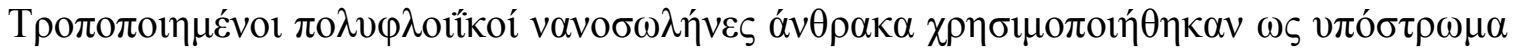

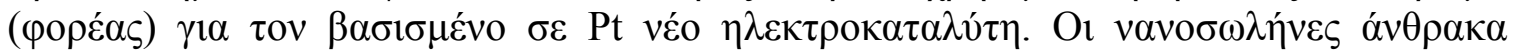

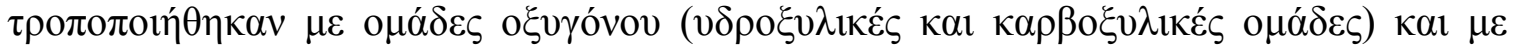

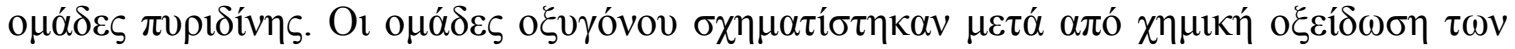

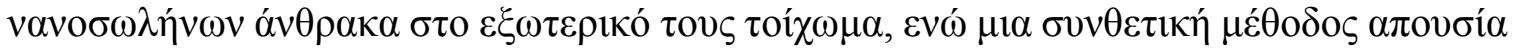

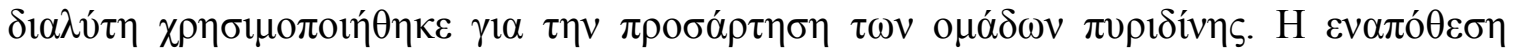

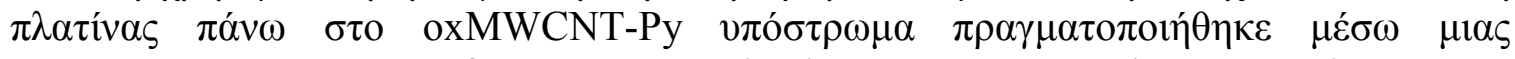

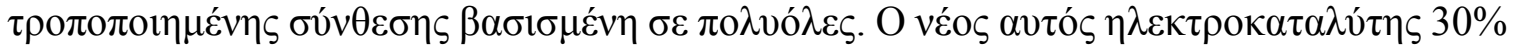

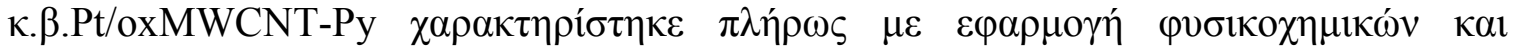

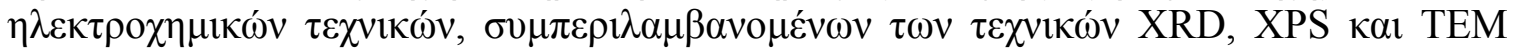

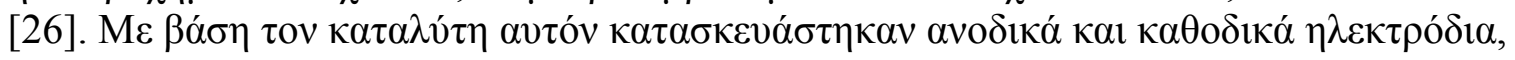

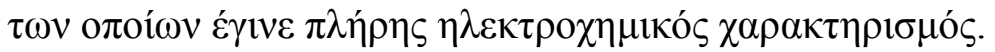

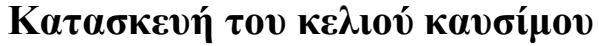

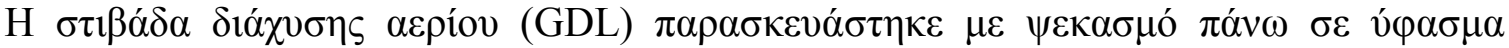

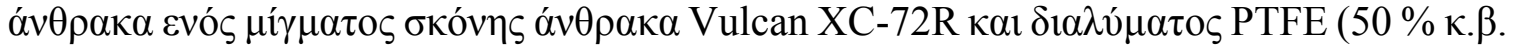

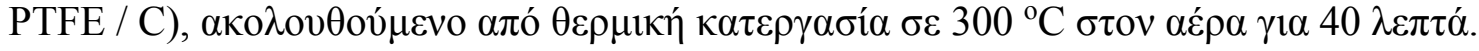

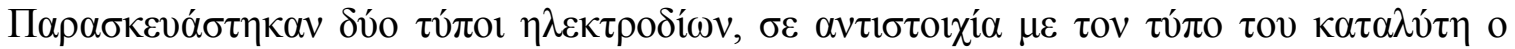

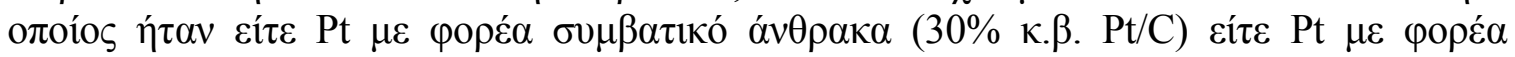




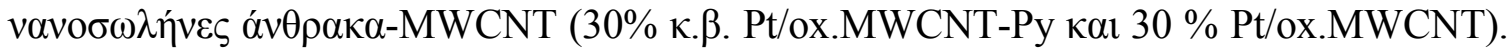

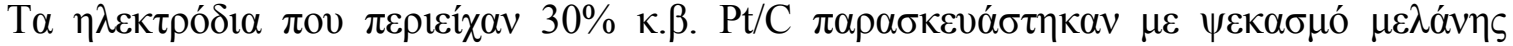

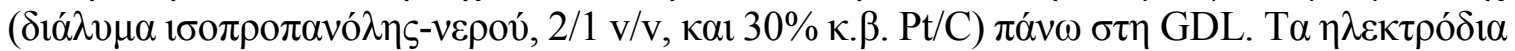

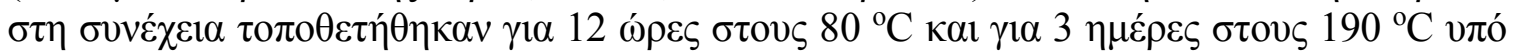

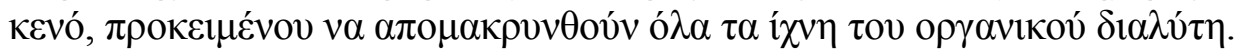

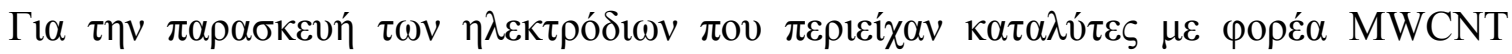

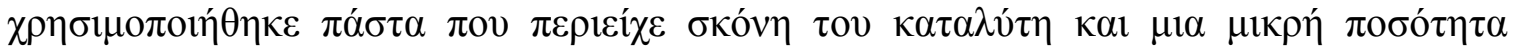

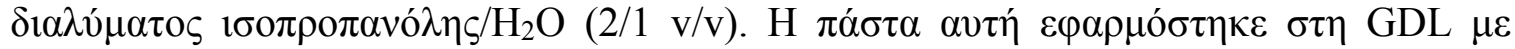

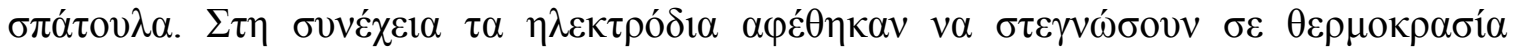

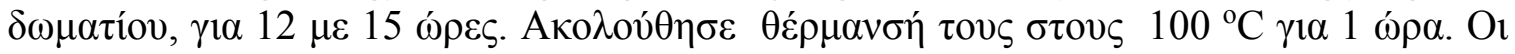

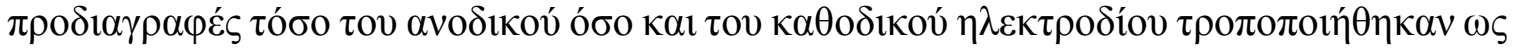

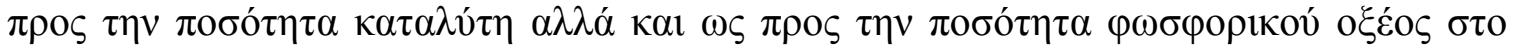

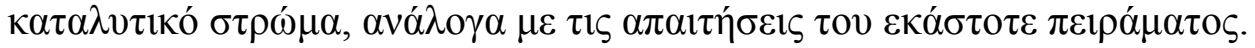

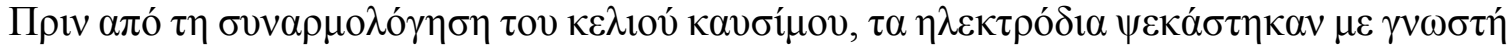

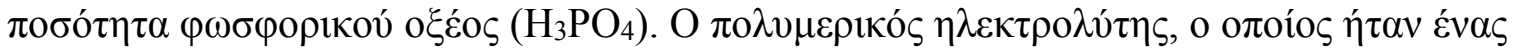

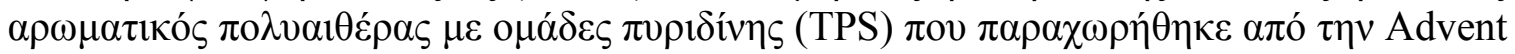

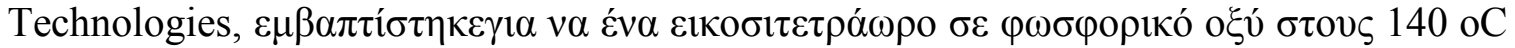
$\mu \varepsilon ́ \chi \rho ı ~ v \alpha$ a

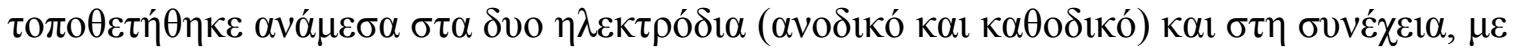

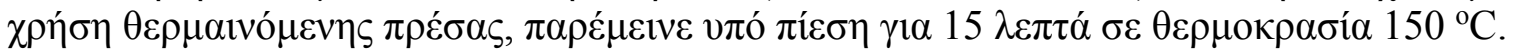

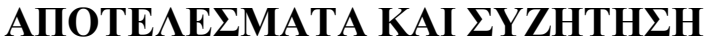

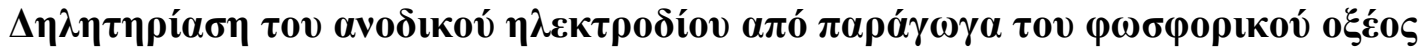

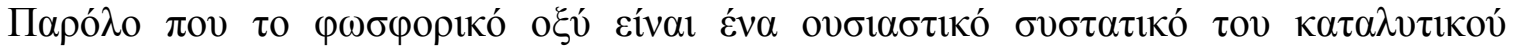

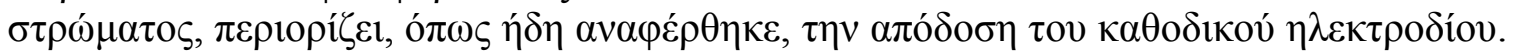

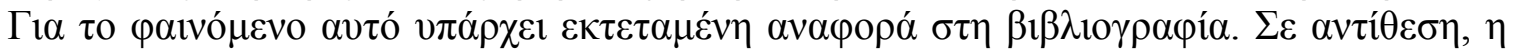
$\delta \eta \lambda \eta \tau \eta \rho i ́ \alpha \sigma \eta \tau$

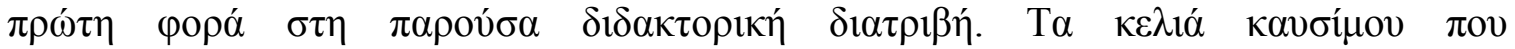

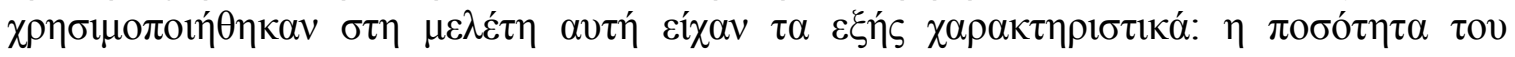

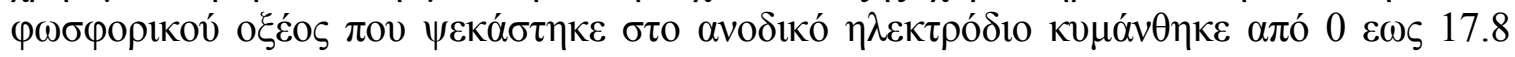

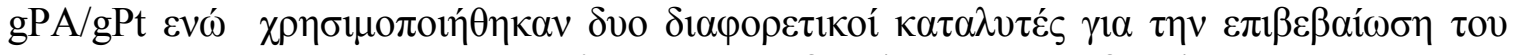

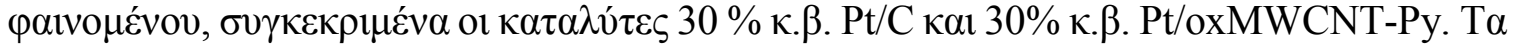

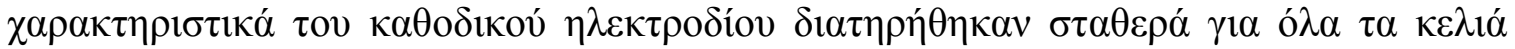

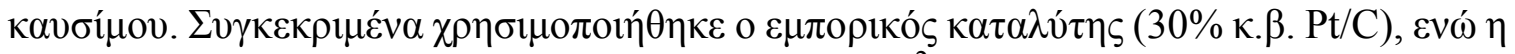

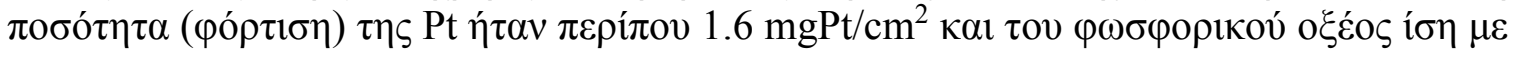
$2 \mathrm{gPA} / \mathrm{gPt} . \Sigma \tau \eta \quad \mu \varepsilon \lambda \varepsilon \dot{\tau} \tau \eta$

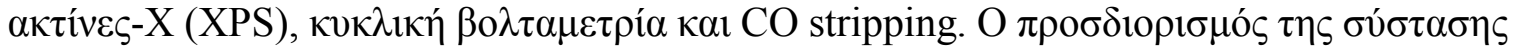

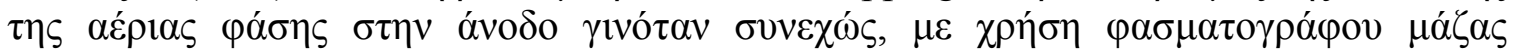

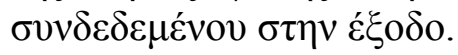



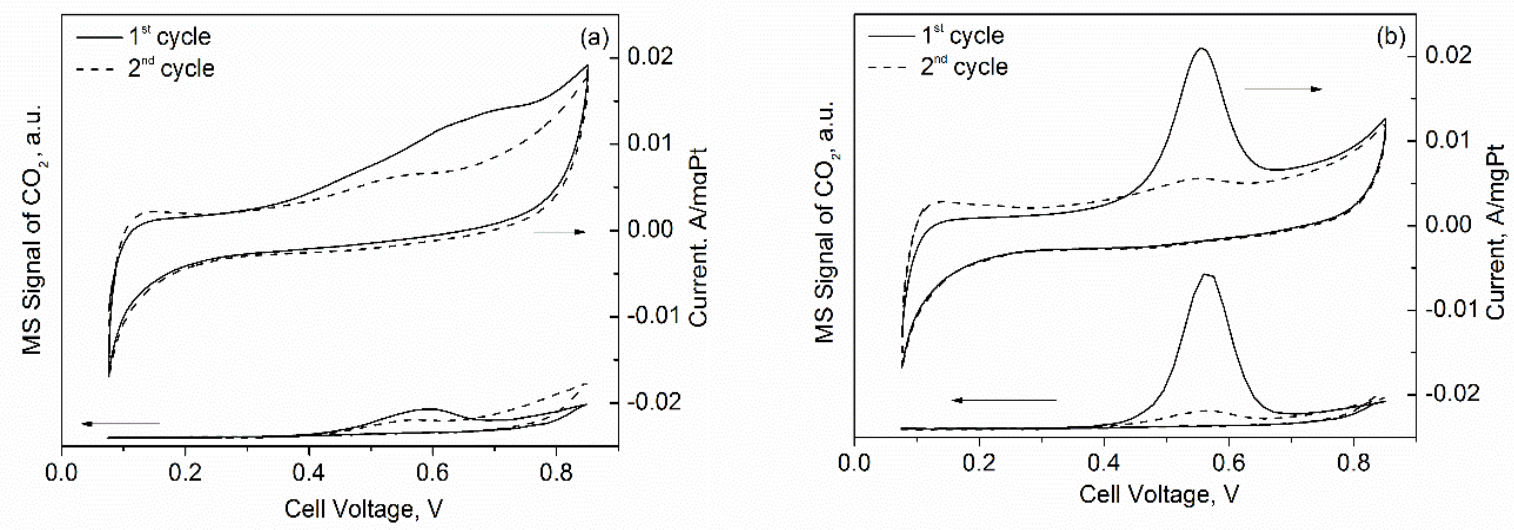

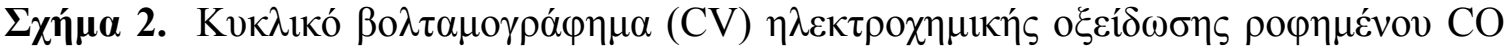

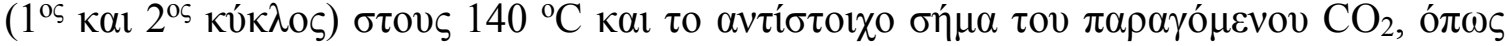

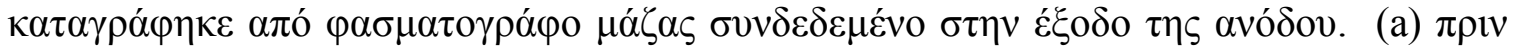

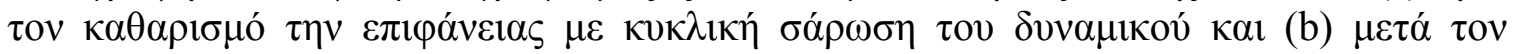

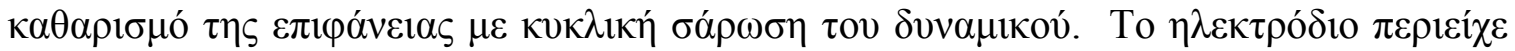
$<3 \mathrm{gPA} / \mathrm{gPt}$.

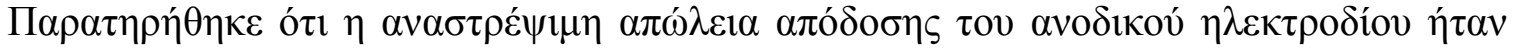

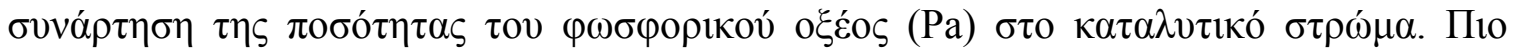

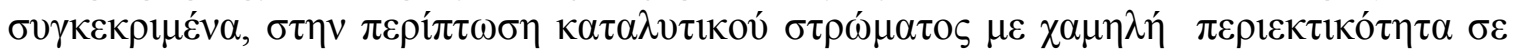

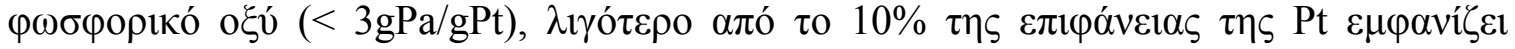

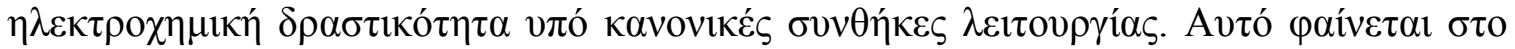

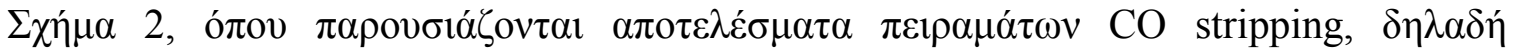

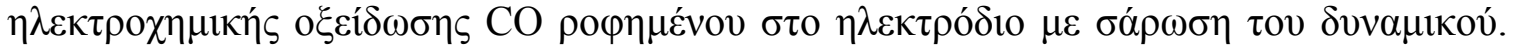

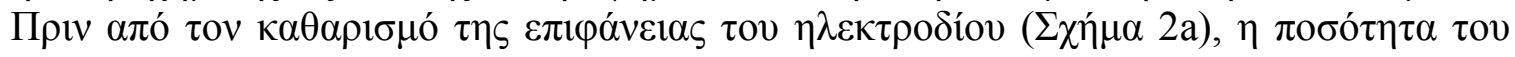

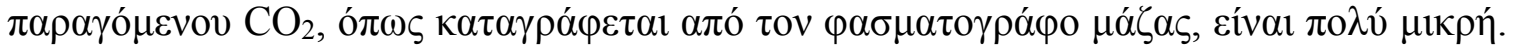

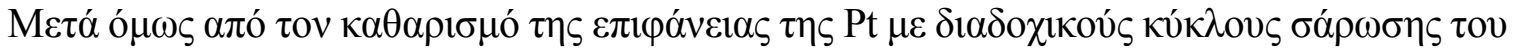

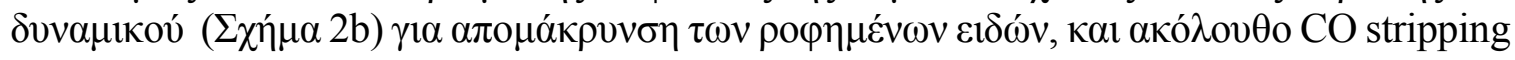

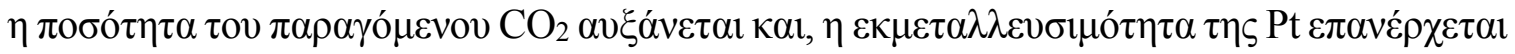

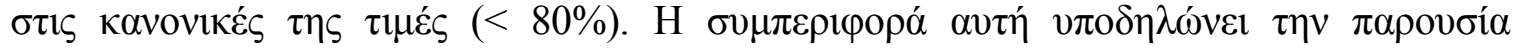

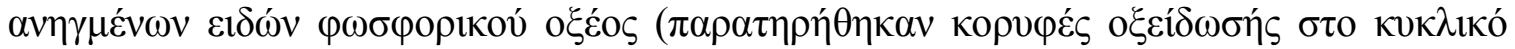

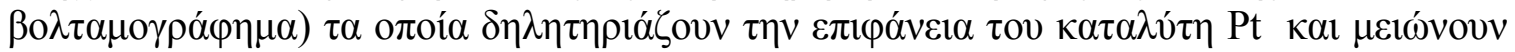

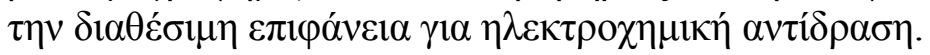

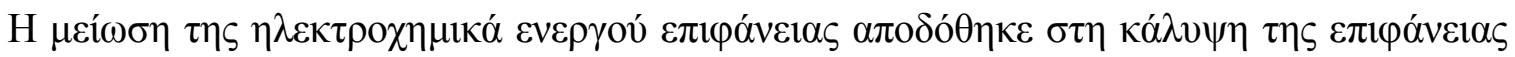

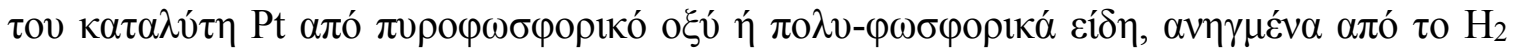

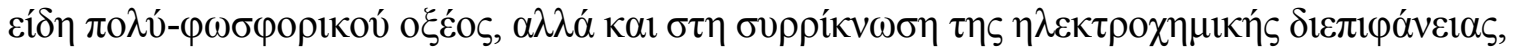

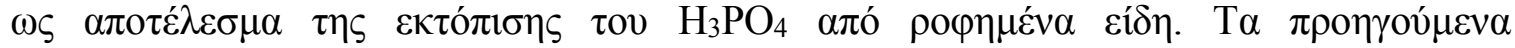

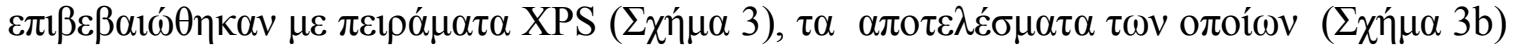

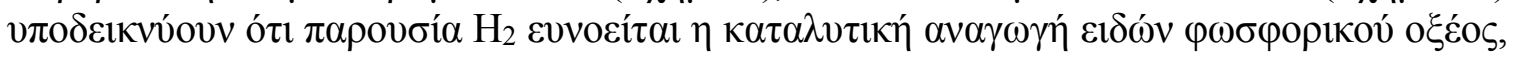

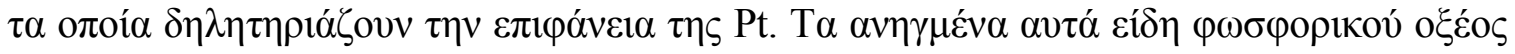

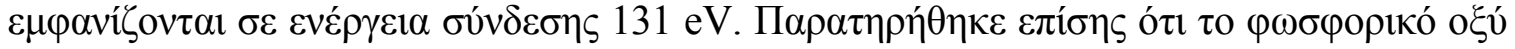

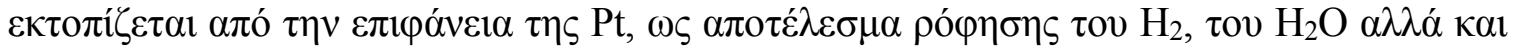

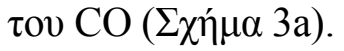




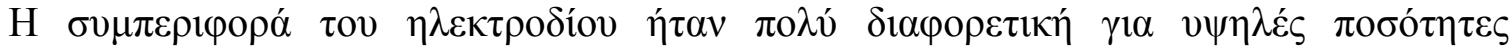

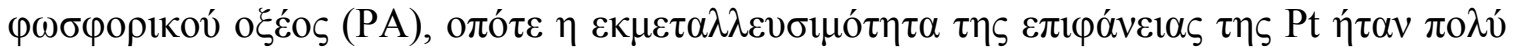

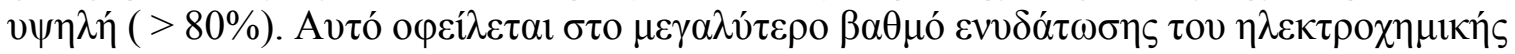

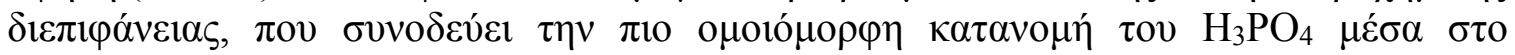

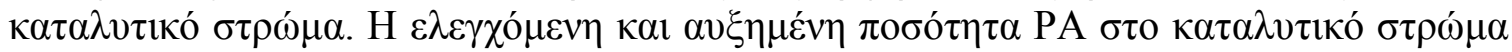

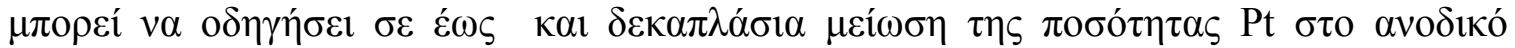

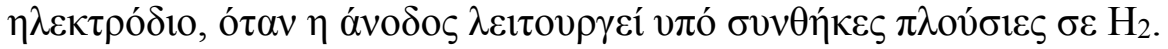
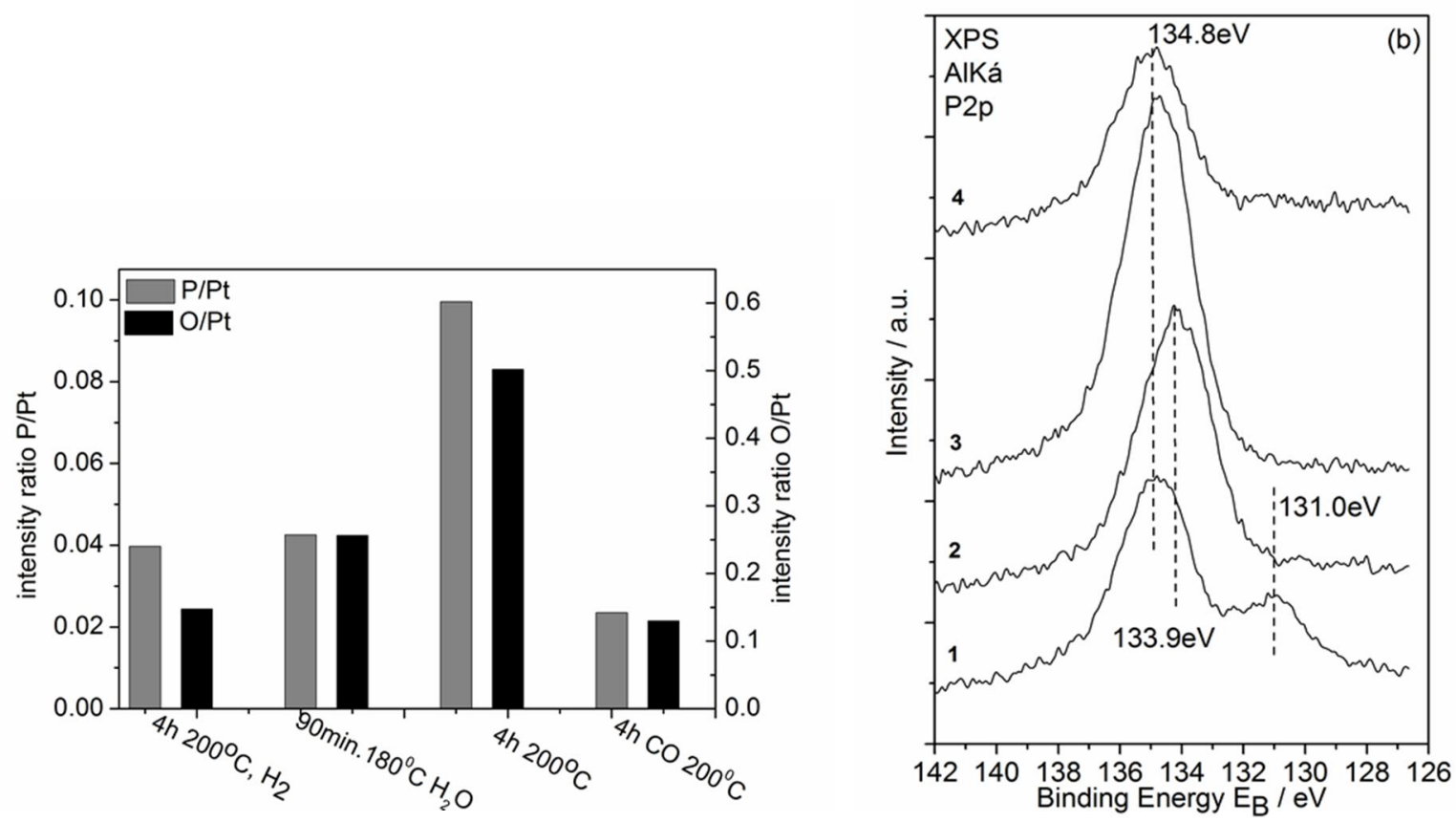

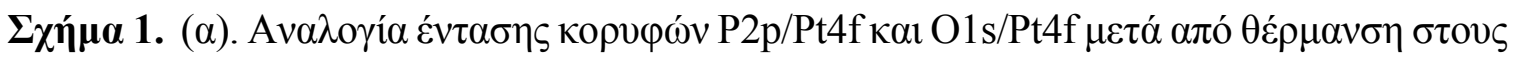

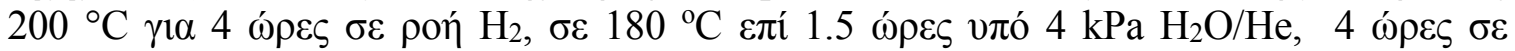

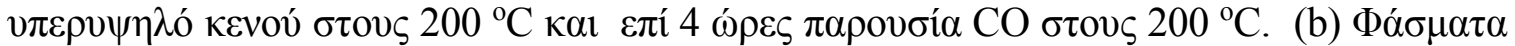

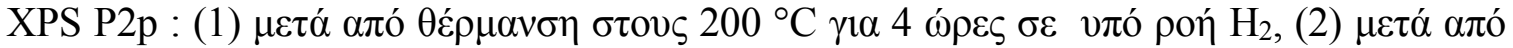

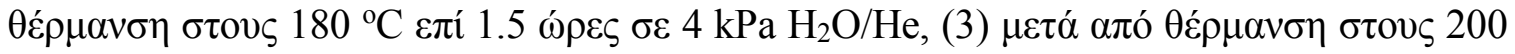

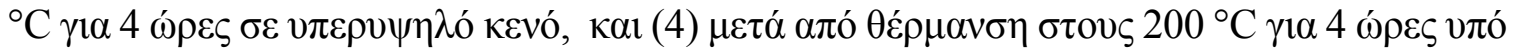
роฑ́ CO.

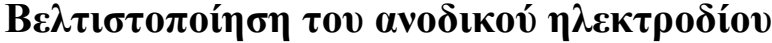

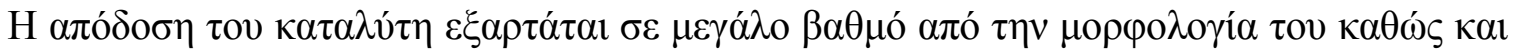

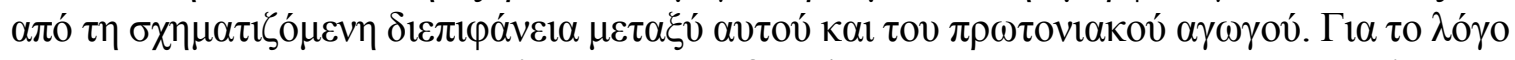

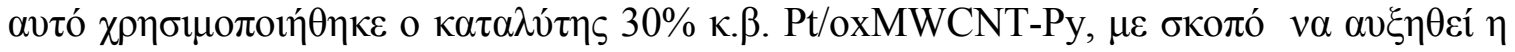

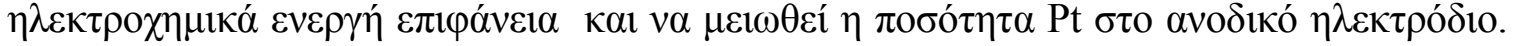

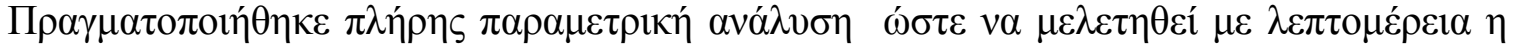

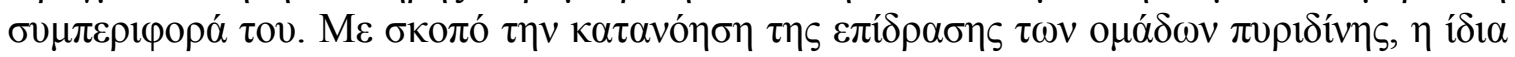

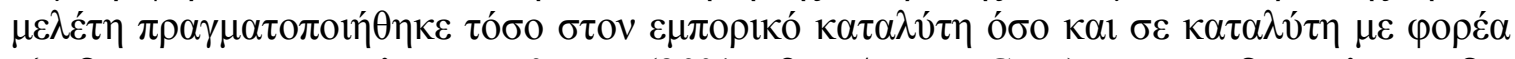

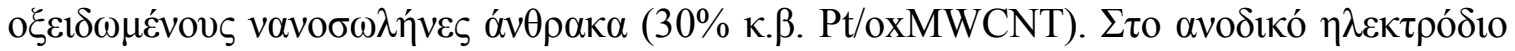




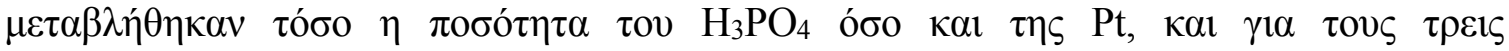

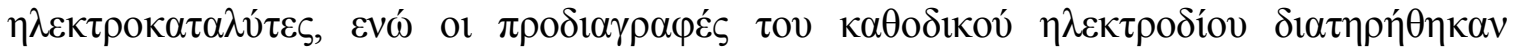

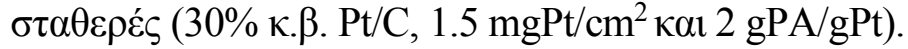

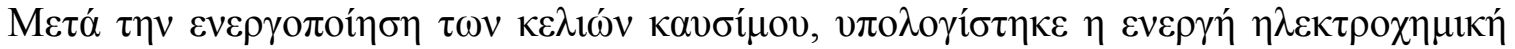

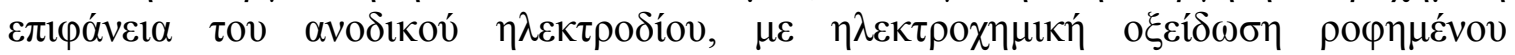

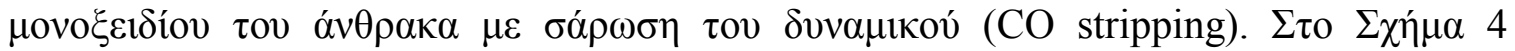

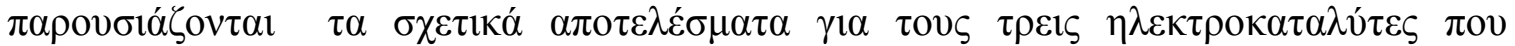

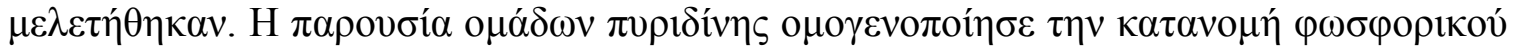

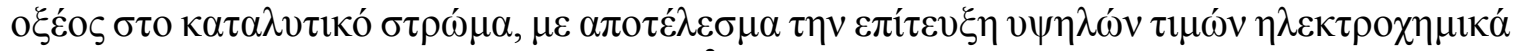

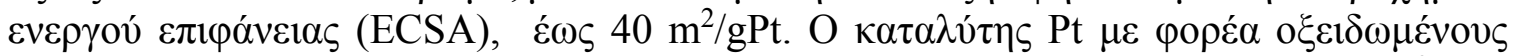

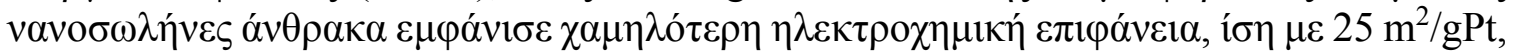

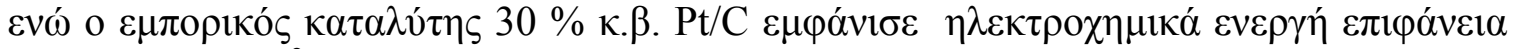
$\varepsilon \dot{\epsilon} \omega$ í $\sigma \eta \mu 35 \mathrm{~m}^{2} / \mathrm{gPt}$.

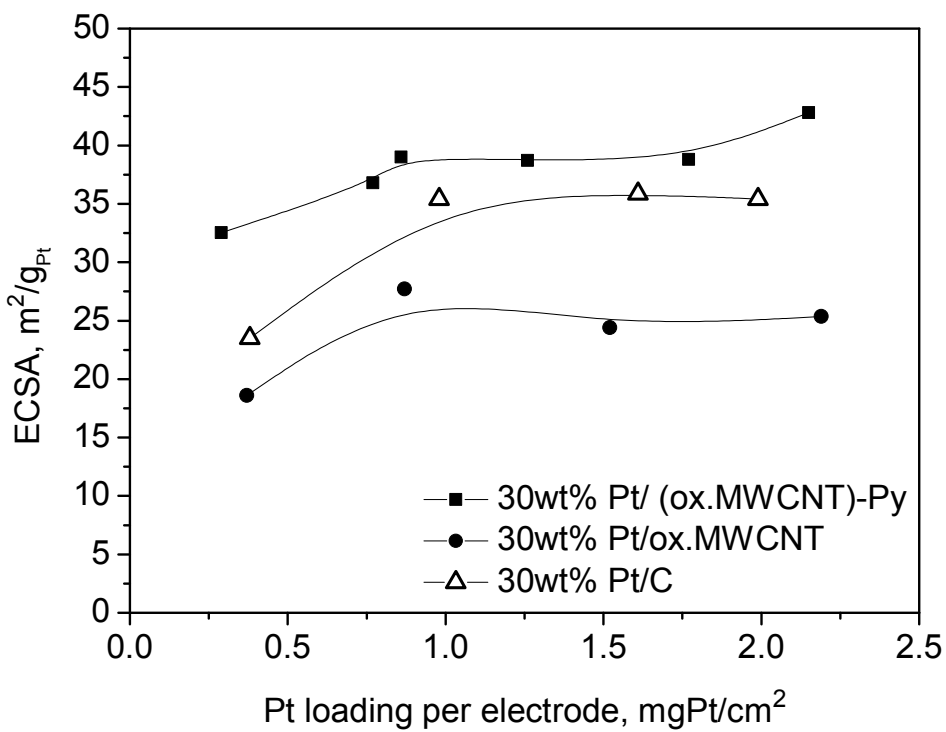

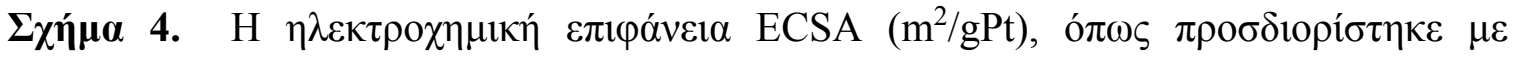

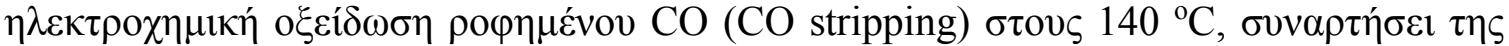

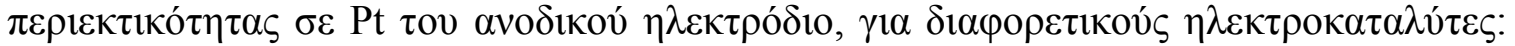

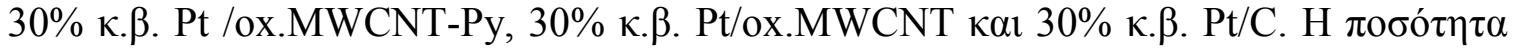

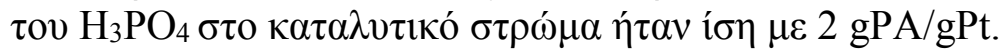

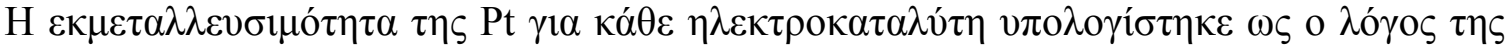

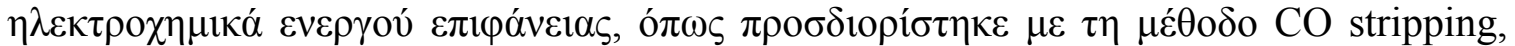

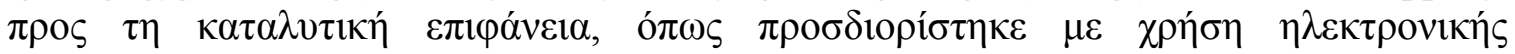

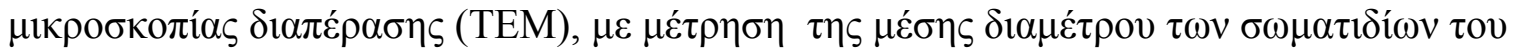

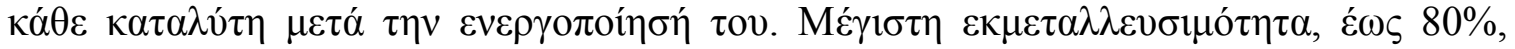

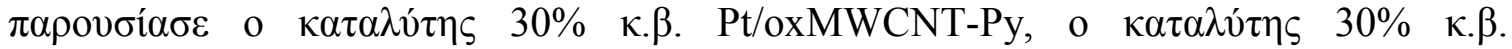

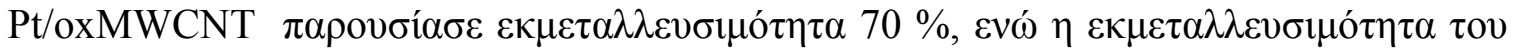

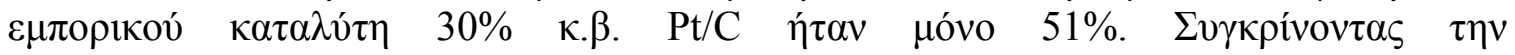

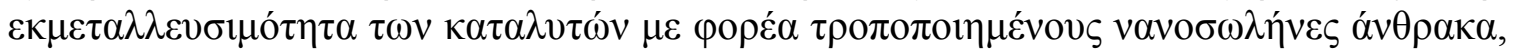




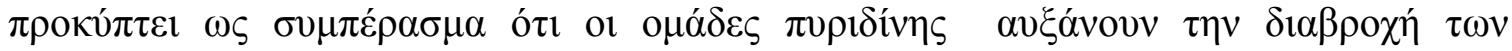

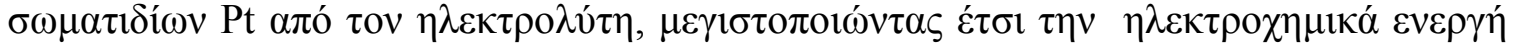

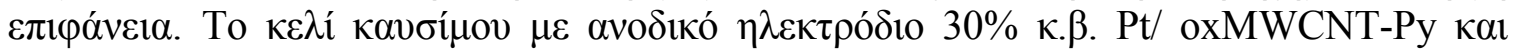

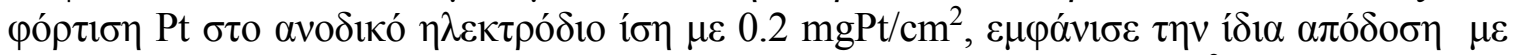

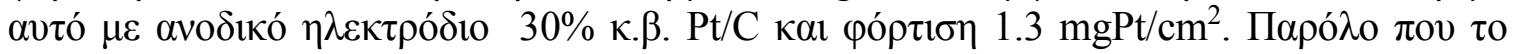

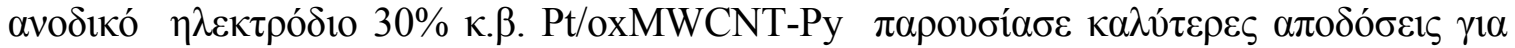

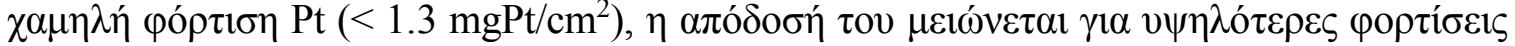

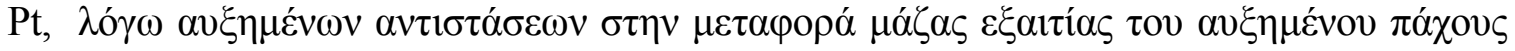

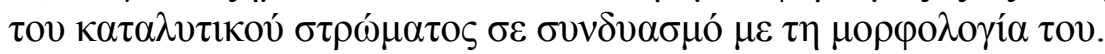

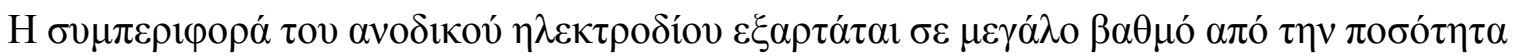

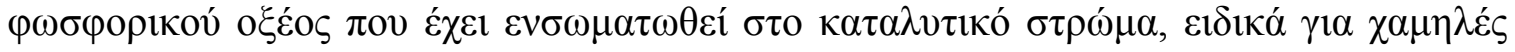

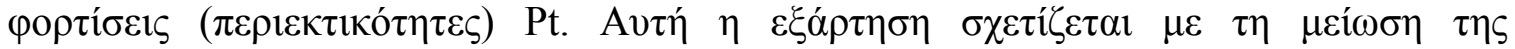

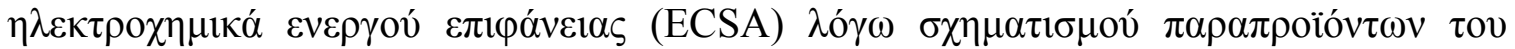

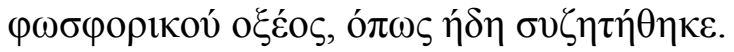

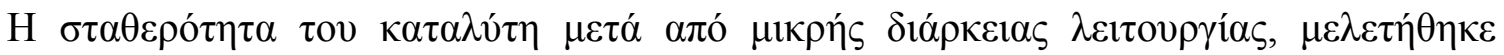

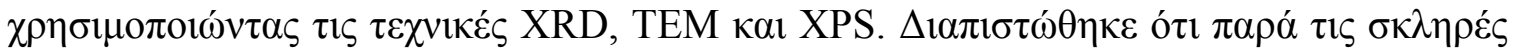

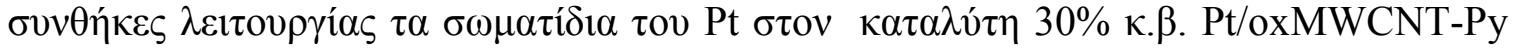

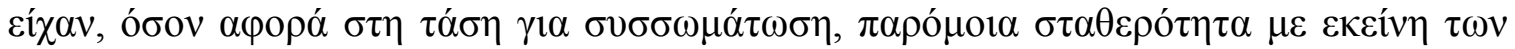

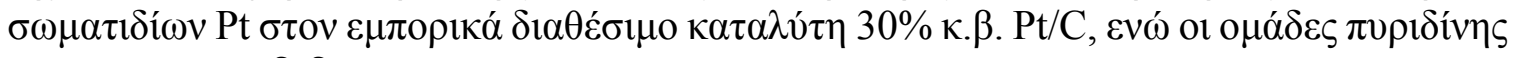

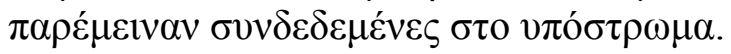

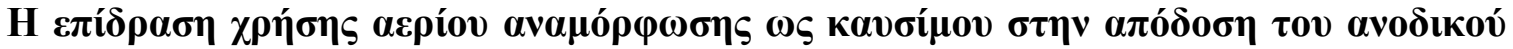

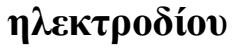

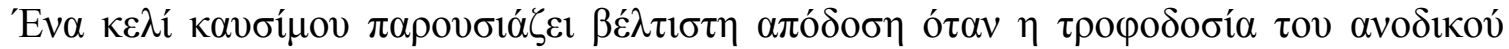

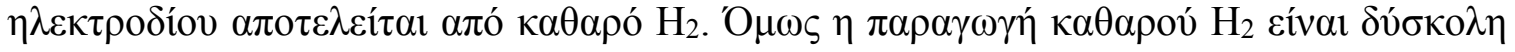

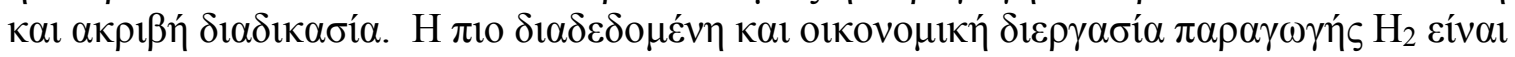

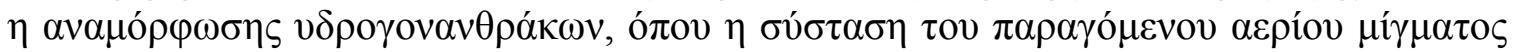

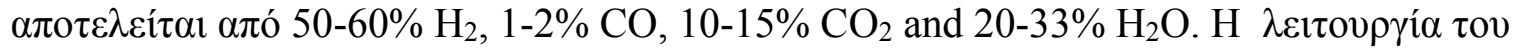

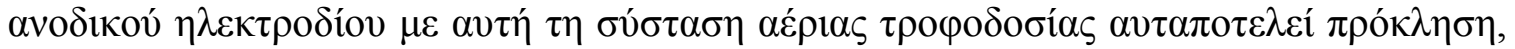

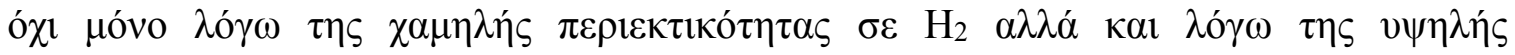

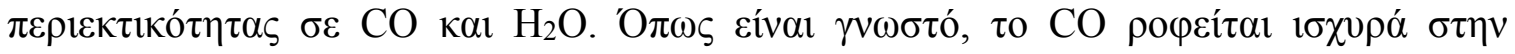

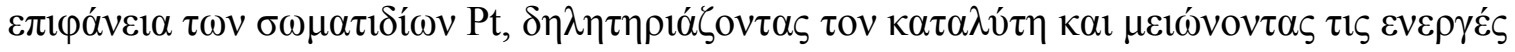

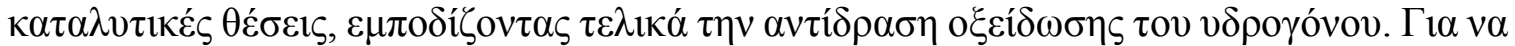

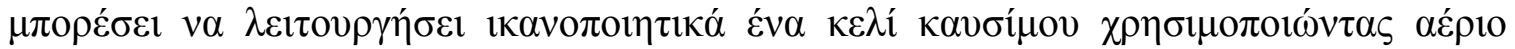

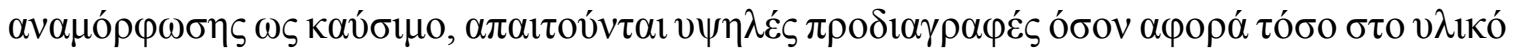

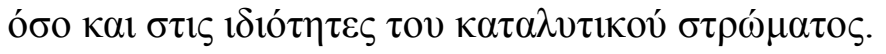

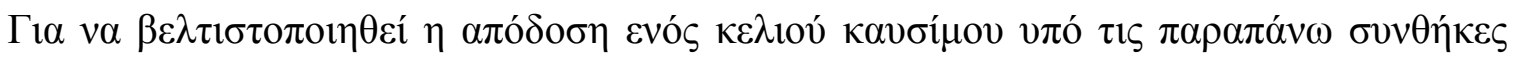

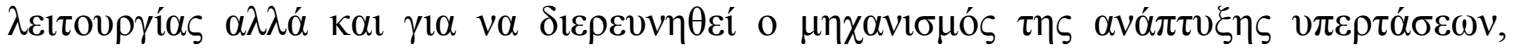

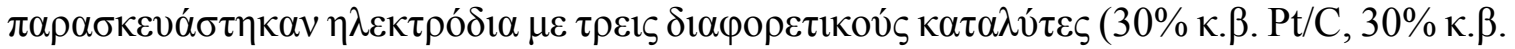

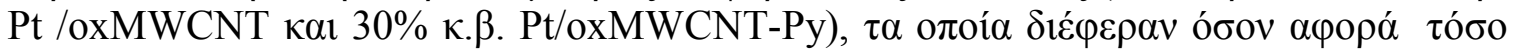

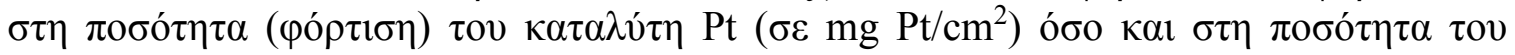

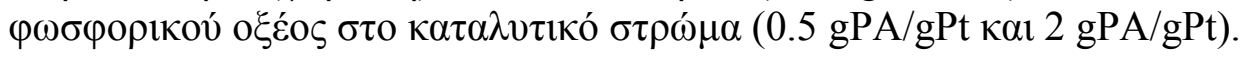



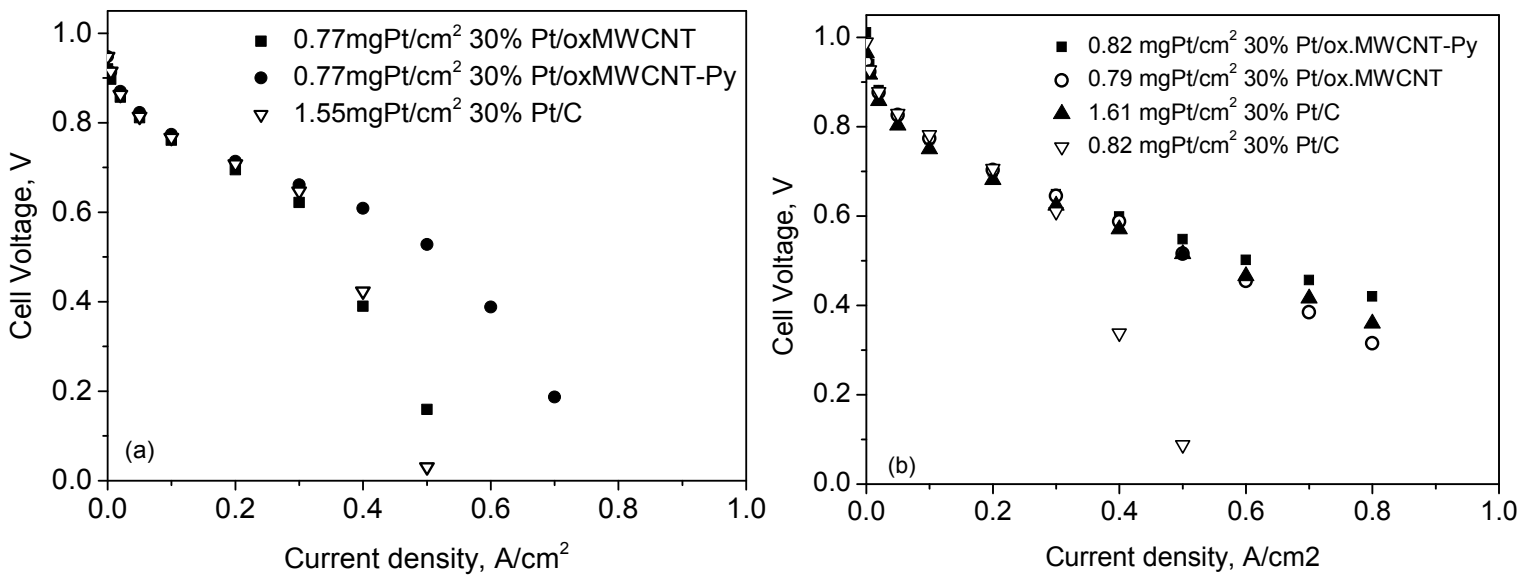

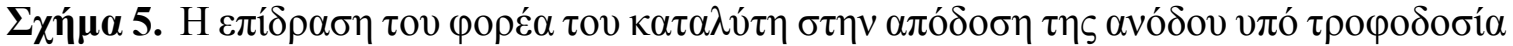

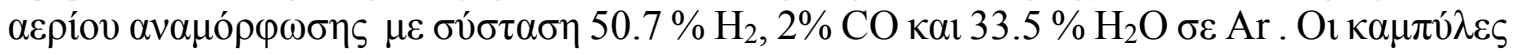

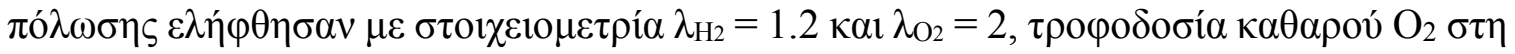

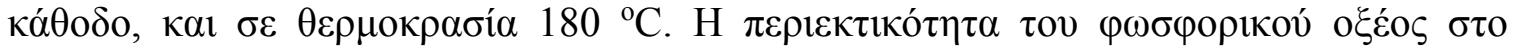
$\kappa \alpha \tau \alpha \lambda v \tau \iota \kappa o ́ ~ \sigma \tau \rho \omega ́ \mu \alpha ~ \eta ́ \tau \alpha \nu ~(a) ~ 2 \mathrm{~g} \mathrm{PA/gPt} \mathrm{\kappa \alpha ı} \mathrm{(b)} 0.5 \mathrm{~g} \mathrm{PA} / \mathrm{gPt}$.

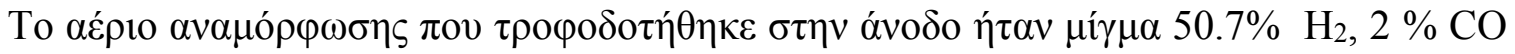

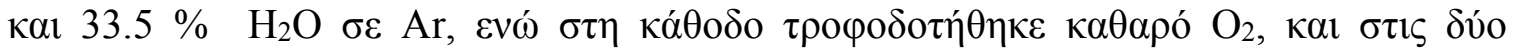

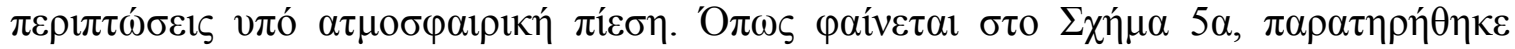

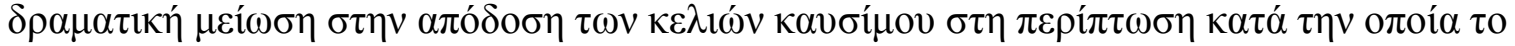

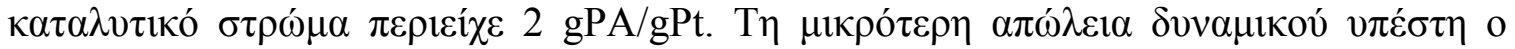

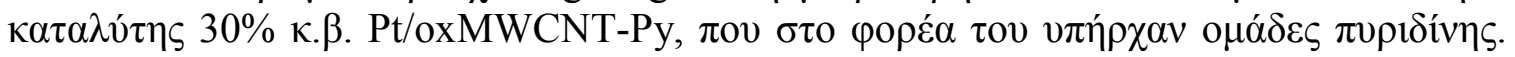

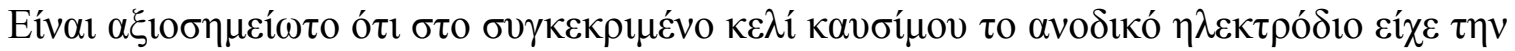

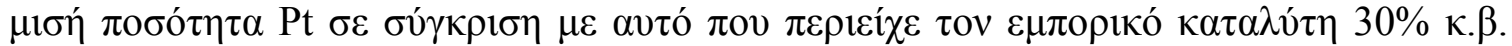

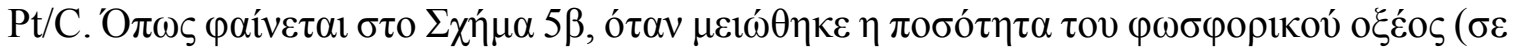

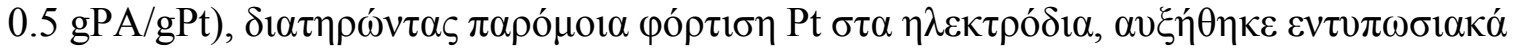

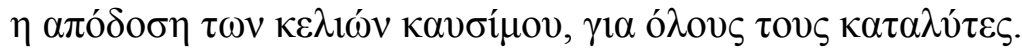

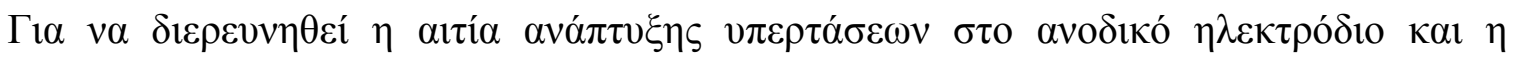

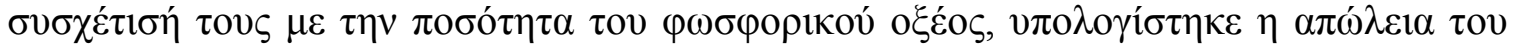

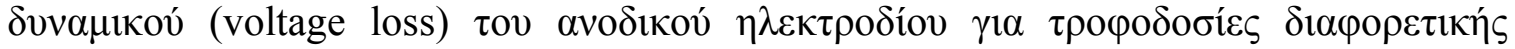

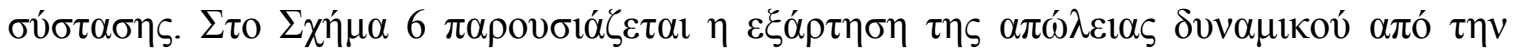

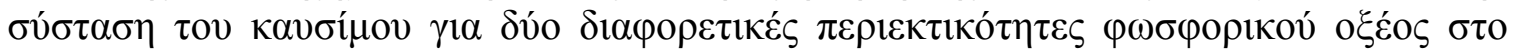

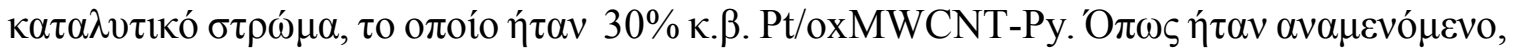

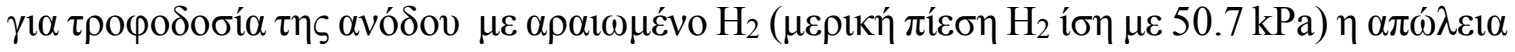

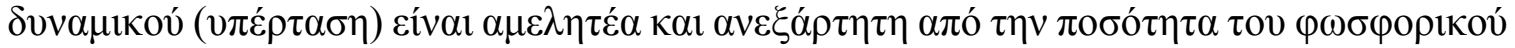

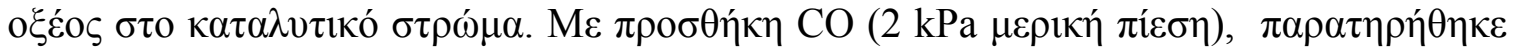

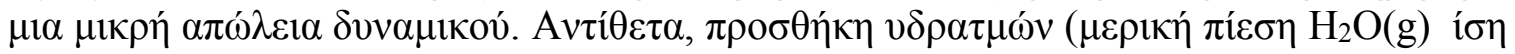

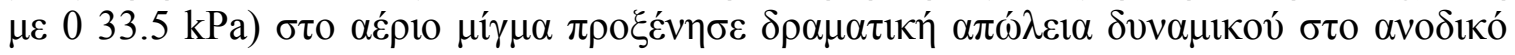

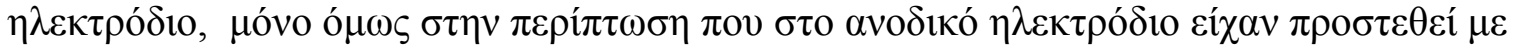

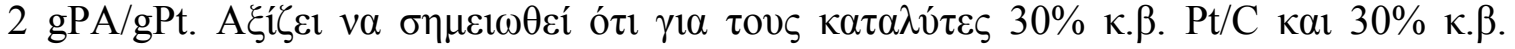

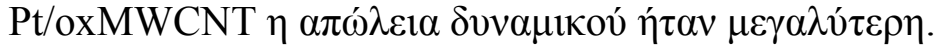



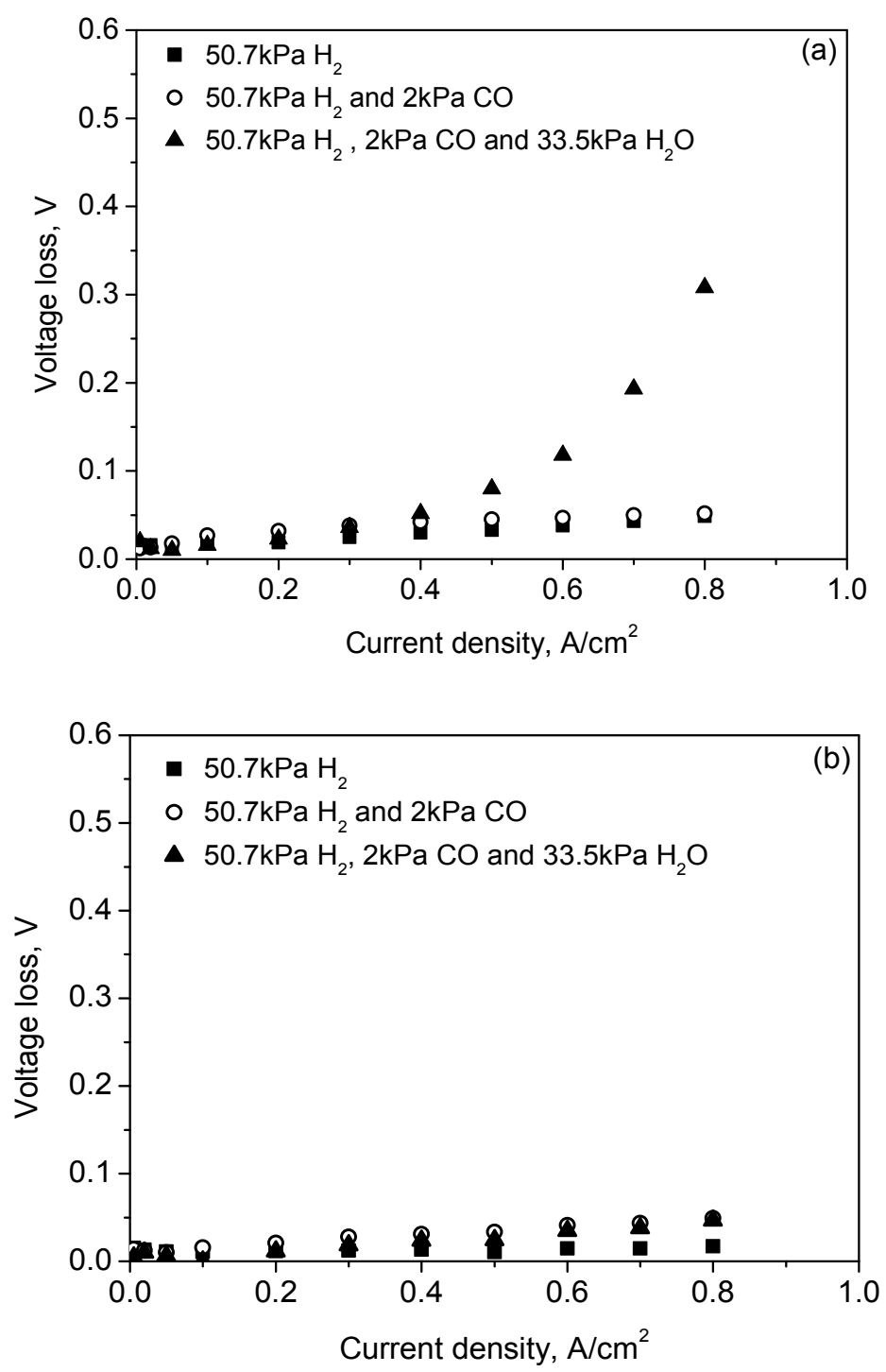

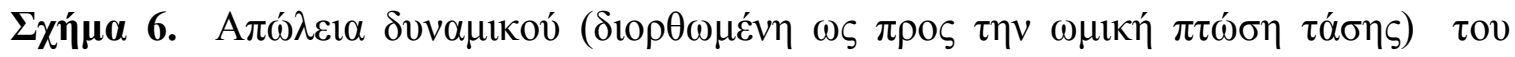

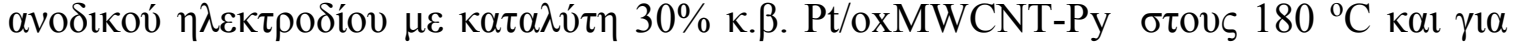

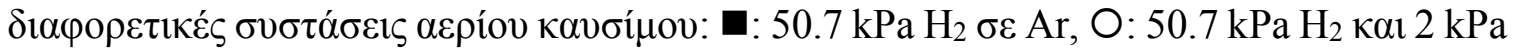

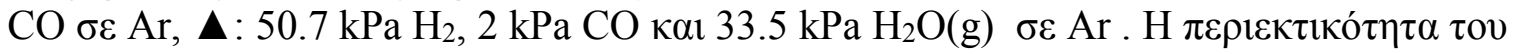

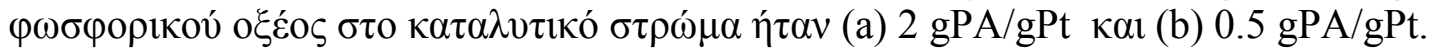

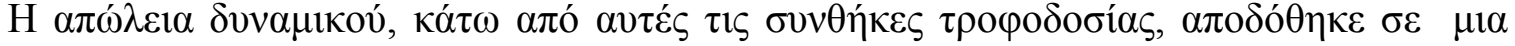

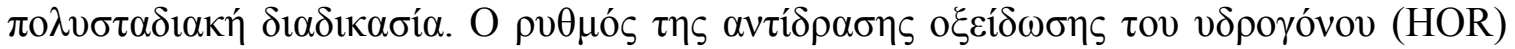

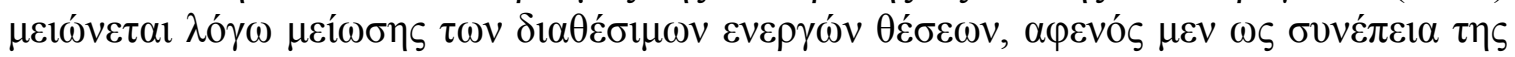

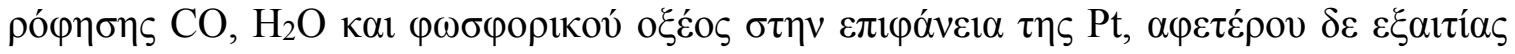

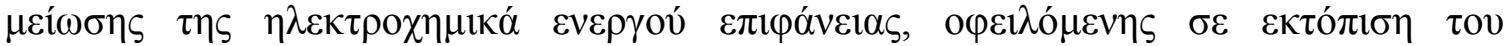

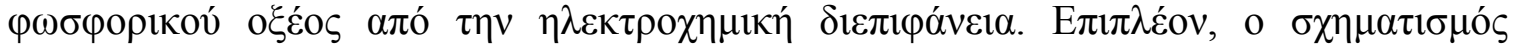




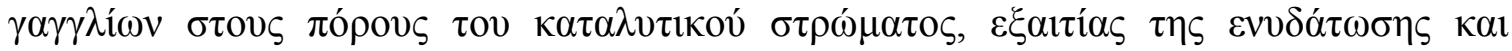

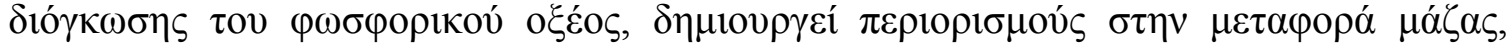

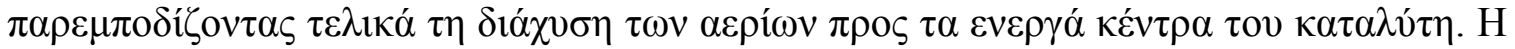

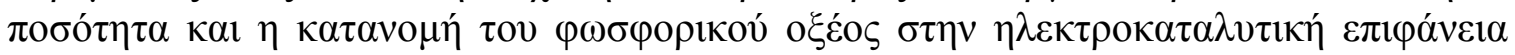

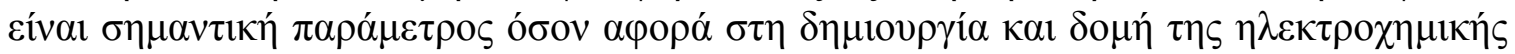

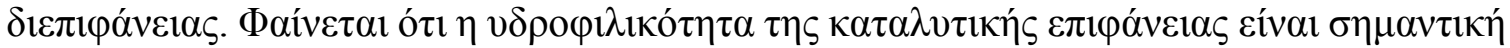

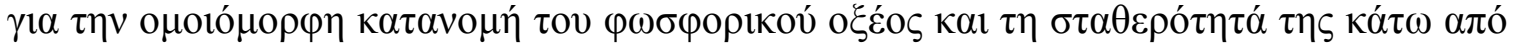

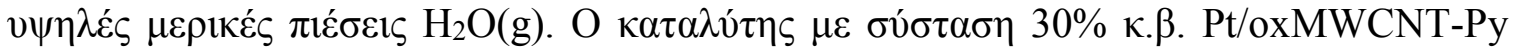

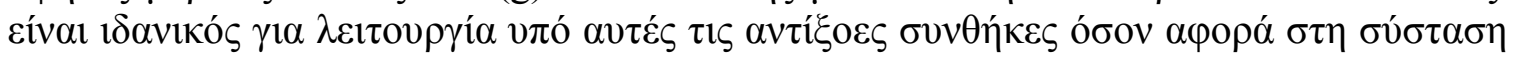

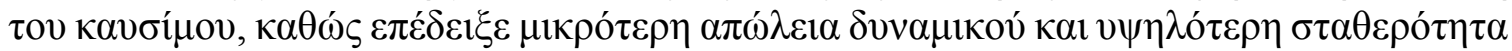

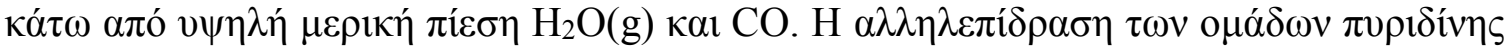

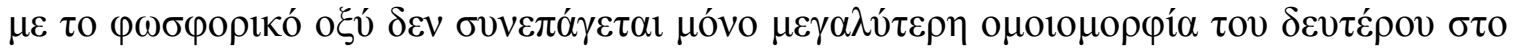

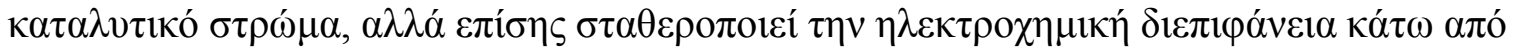

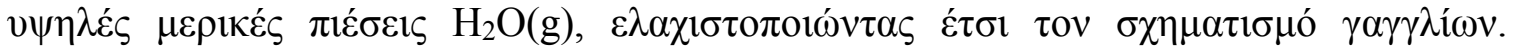

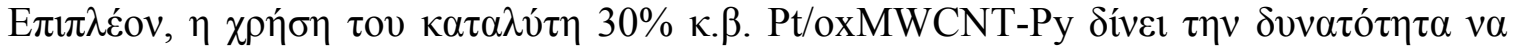

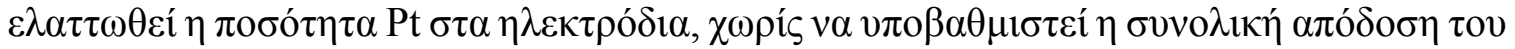

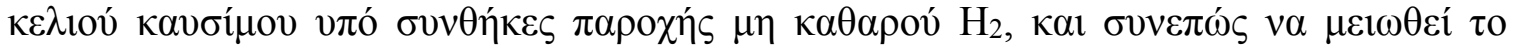

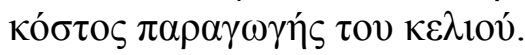

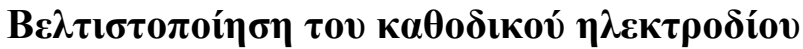

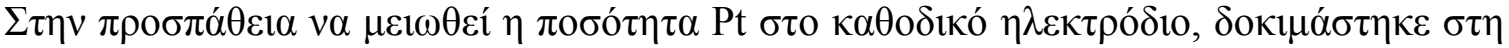

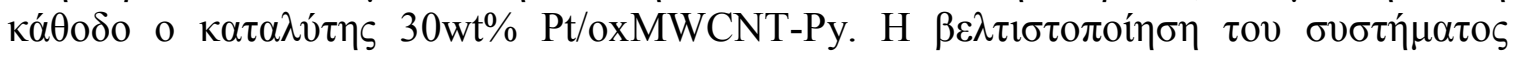

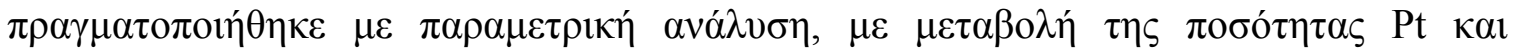

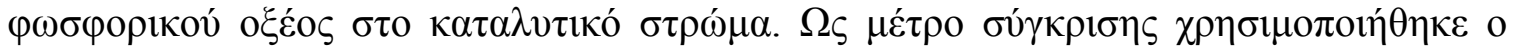

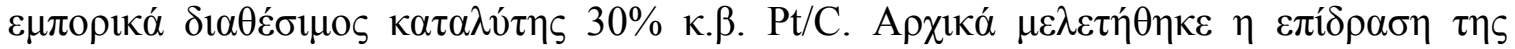

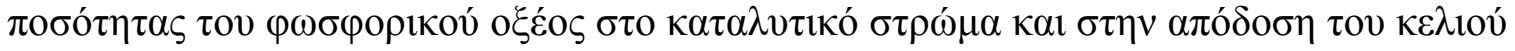

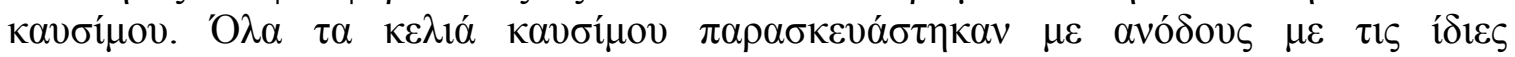

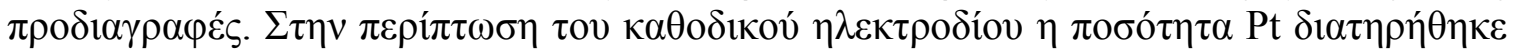

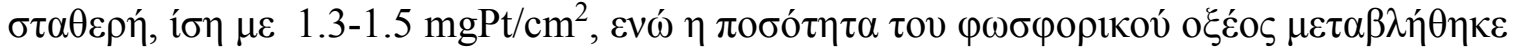

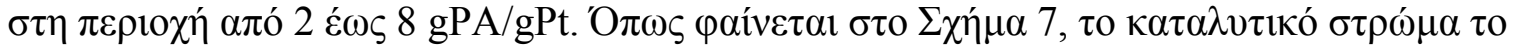

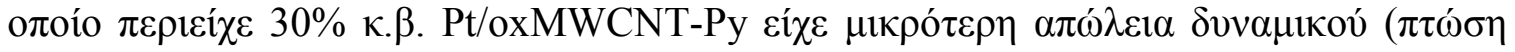

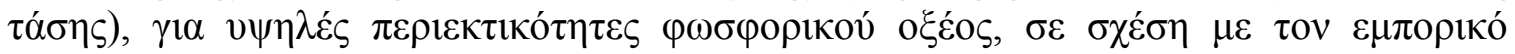

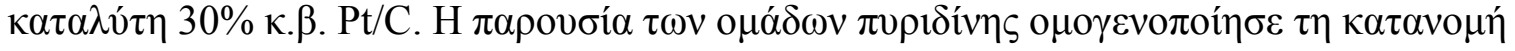

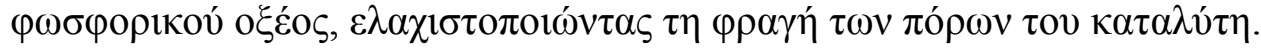

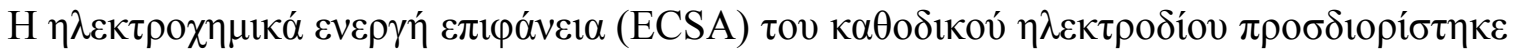

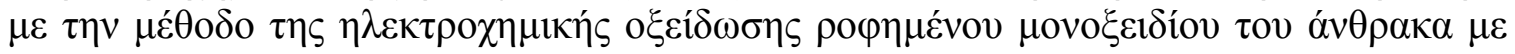

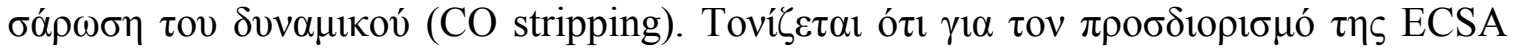

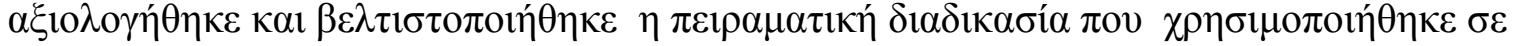

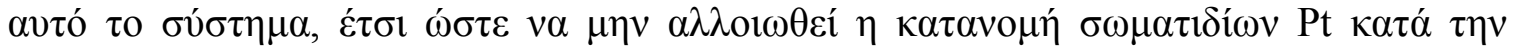

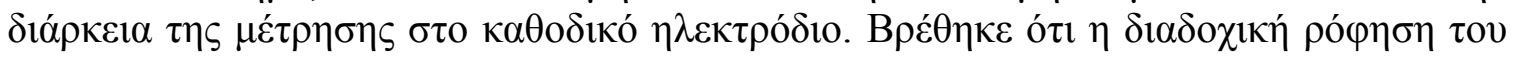

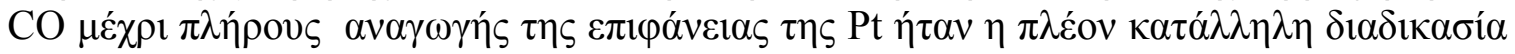

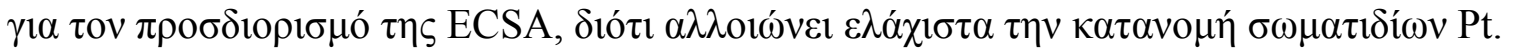

275

Department of Chemical Engineering, University of Patras 


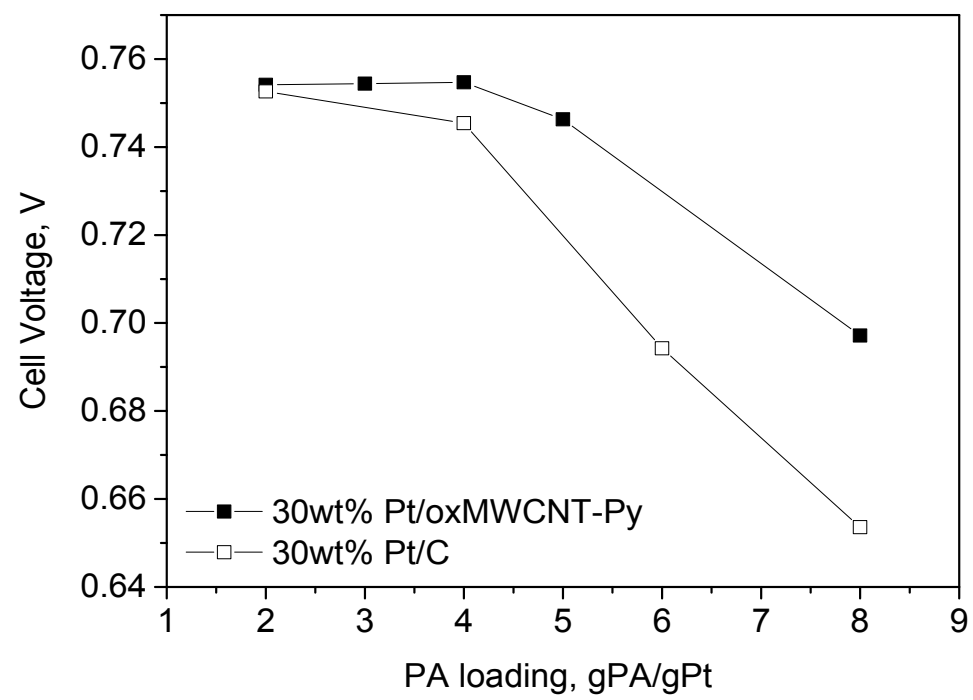

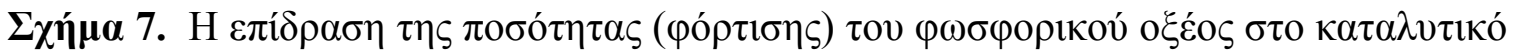

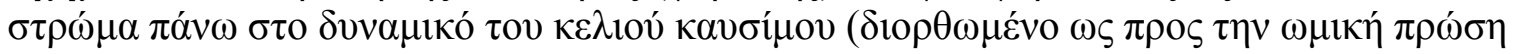

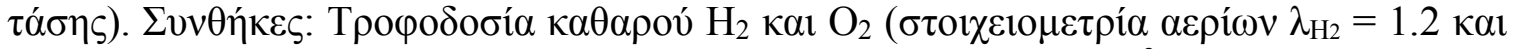

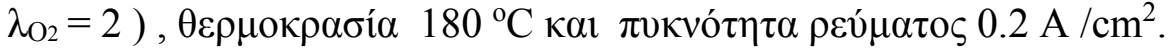

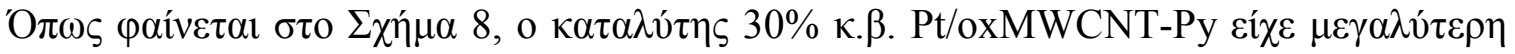

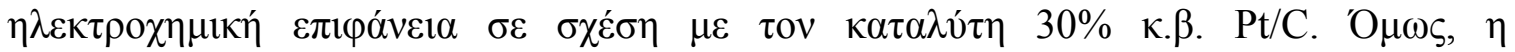

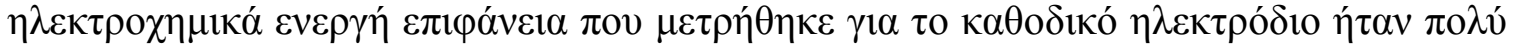

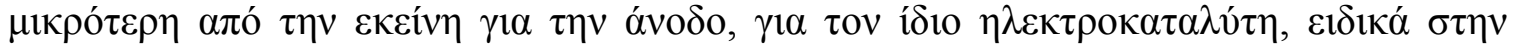

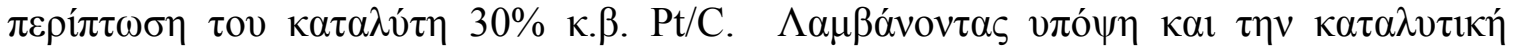

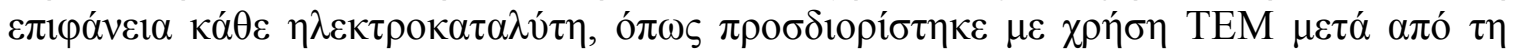

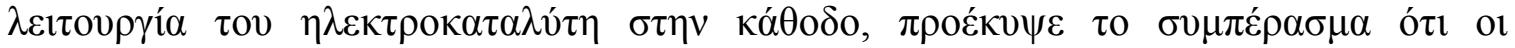

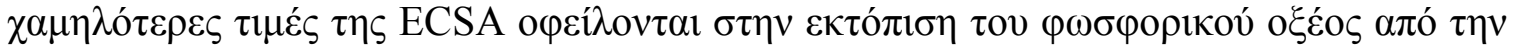

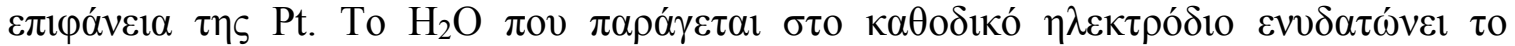

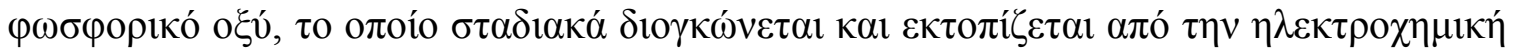

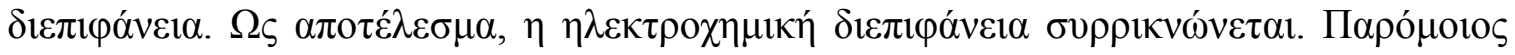

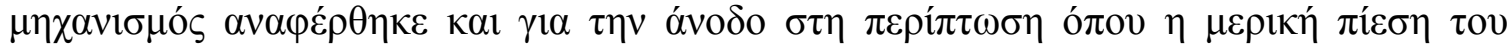

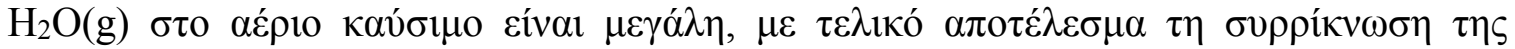

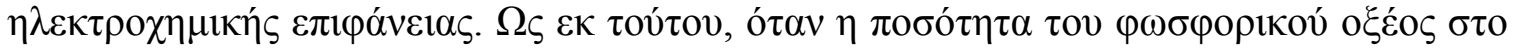

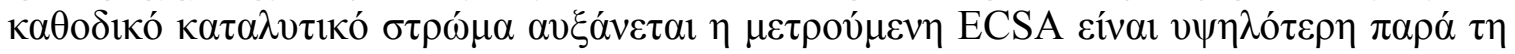

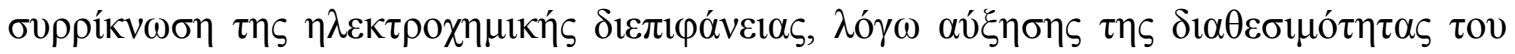

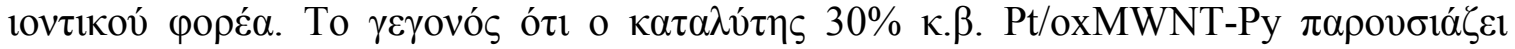

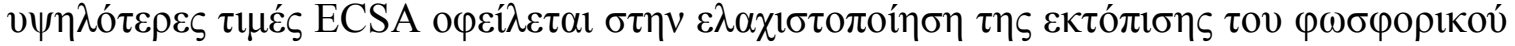

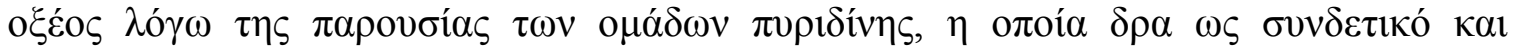

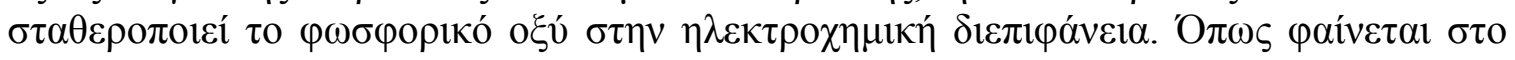

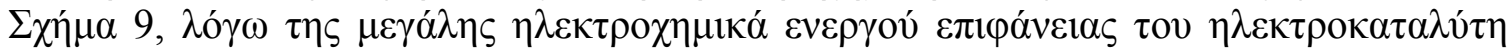

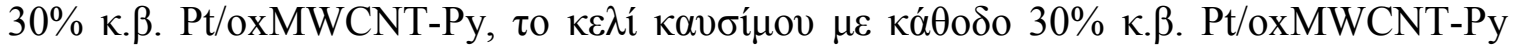

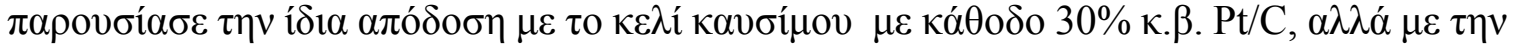

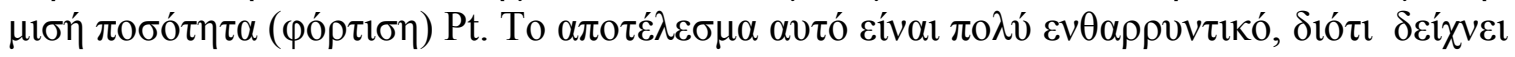

276

Department of Chemical Engineering, University of Patras 


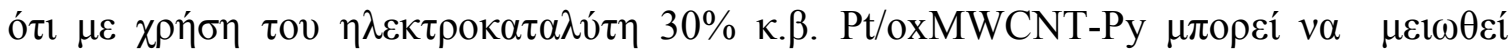

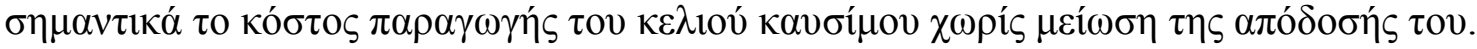

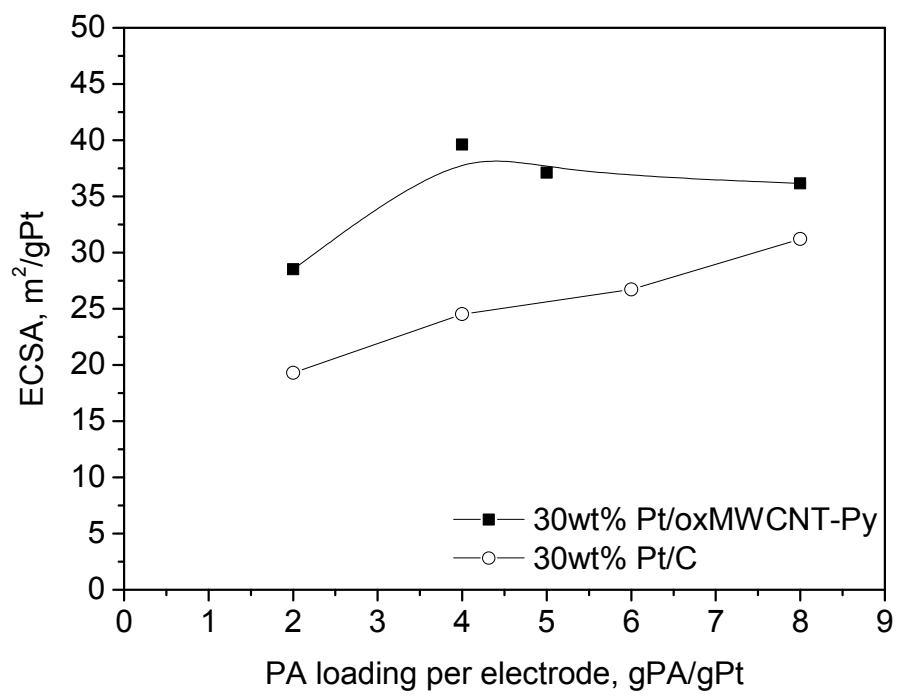

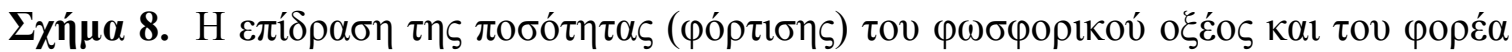

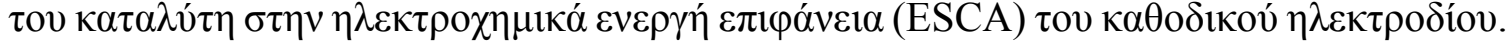

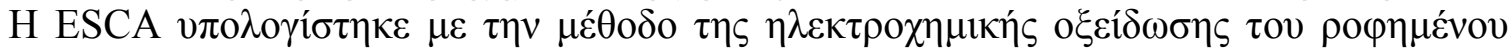

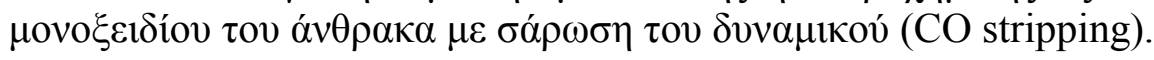

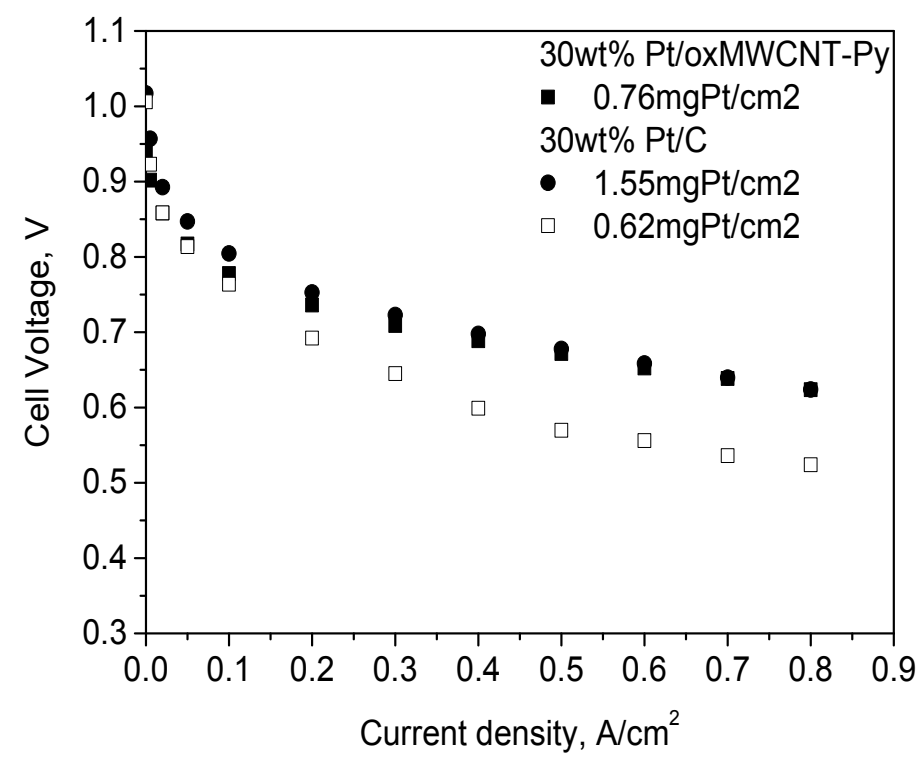

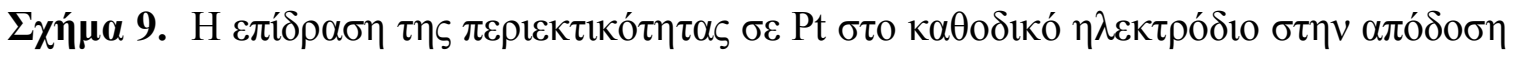

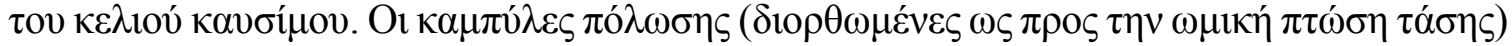

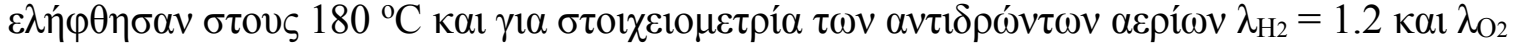

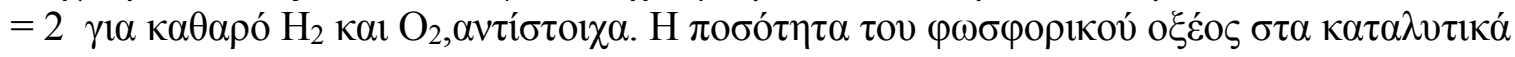




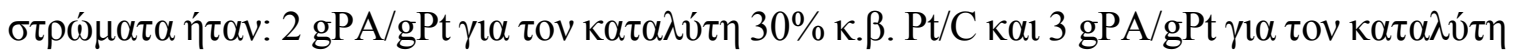
$30 \%$ к. $\beta$. Pt/oxMWCNT-Py.
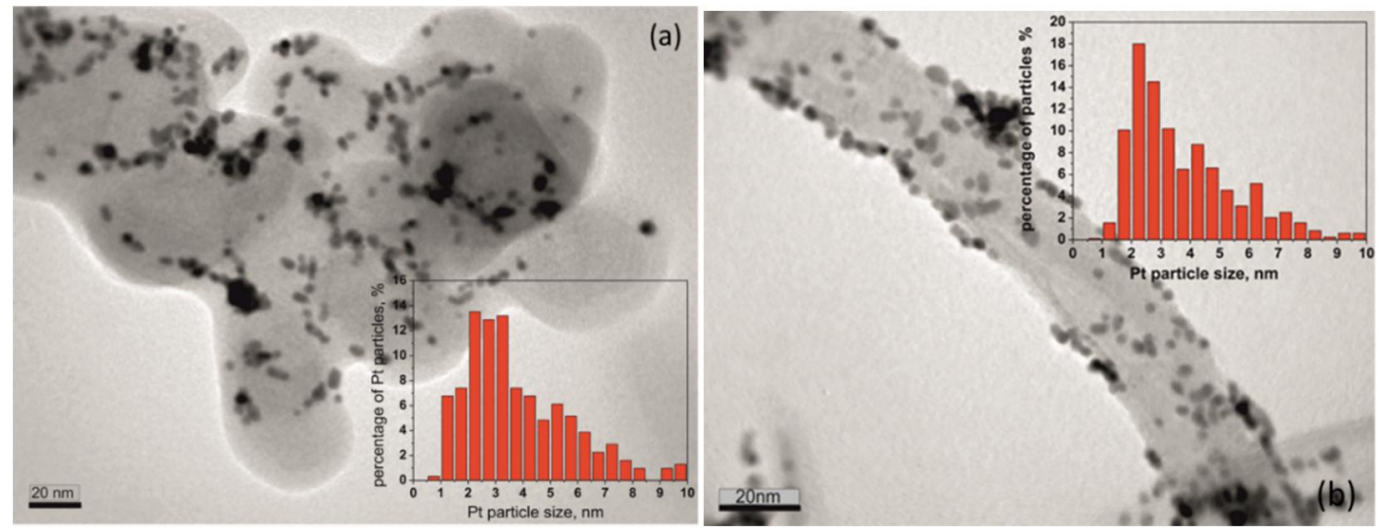

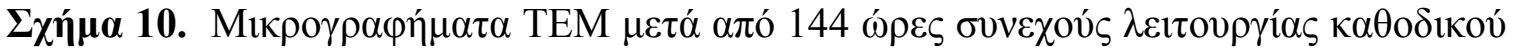

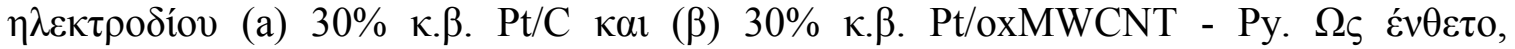

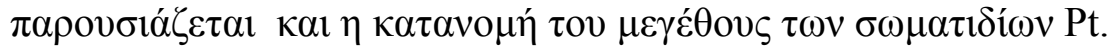

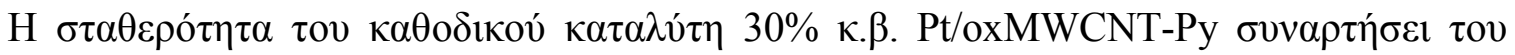

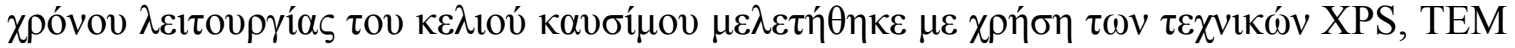

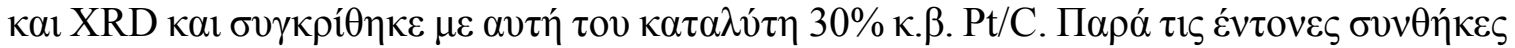

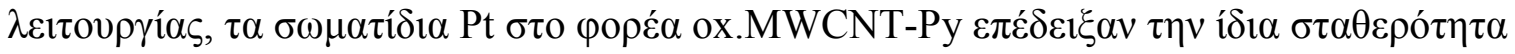

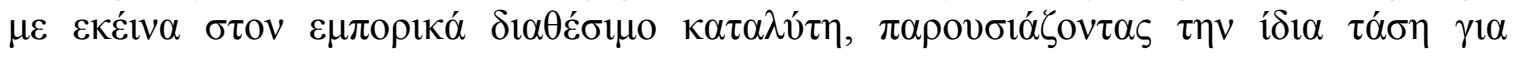

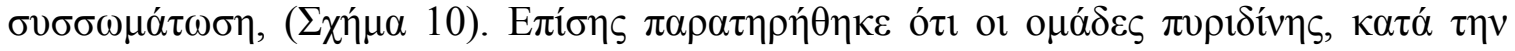

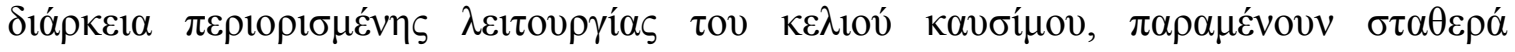

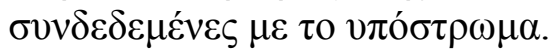

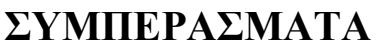

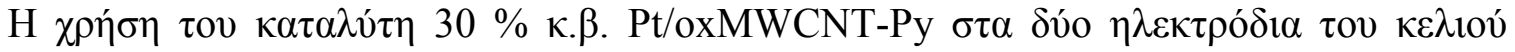

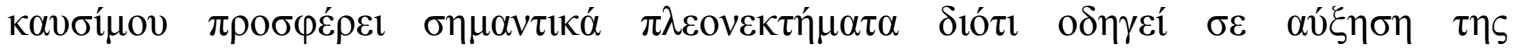

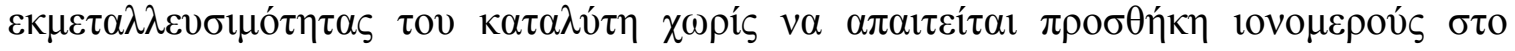

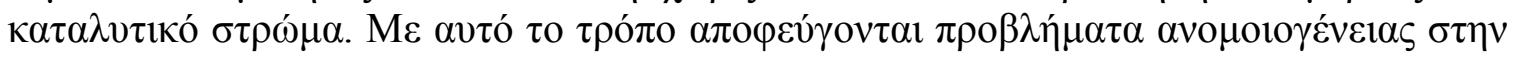

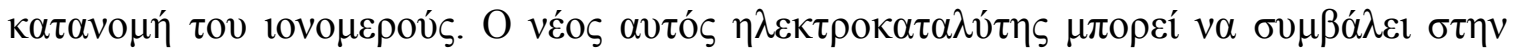

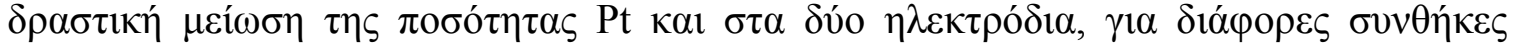

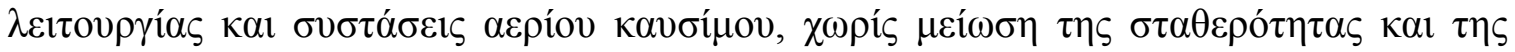

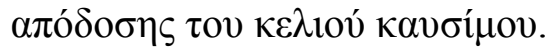

\section{ВIBАIOГРАФIA}

[1] Song JM, Suzuki S, Uchida H, Watanabe M (2006) Preparation of high catalyst utilization electrodes for polymer electrolyte fuel cells. Langmuir 22(14): 6422-6428. doi: 10.1021/la060671

[2] Xu Z, Qi Z, Kaufman A (2005) Superior catalysts for proton exchange membrane fuel cells. Electrochem Solid-State Lett 8(6):A313-315. doi: 10.1149/1.1912018 
[3] Kim H, Lee W, Yoo D (2007) Functionalized carbon support with sulfonated polymer for direct methanol fuel cells. Electrochim Acta 52(7):2620-2624. Doi: 10.1016/j.electacta.2006.09.017

[4] Du CY, Zhao TS, Liang ZX (2008) Sulfonation of carbon-nanotube supported platinum catalysts for polymer electrolyte fuel cells. J Power Sources 176(1):9-15. doi: 10.1016/j.jpowsour.2007.10.016

[5] Li Q, Jensen JO, Pan C, Bandur V, Nilsson MS, Schonberger F, et al., Partially fluorinated aarylene polyethers and their ternary blends with PBI and H3PO4. Part II. Characterisation and fuel cell test of the ternary membranes. Fuel Cells (2008) 34, p188-199

[6] Kim J-H, Kim H-J, Lim H-T., Dependence of the performance of a high-temperature polymer electrolyte fuel cell on phosphoric acid-doped polybenzimidazole ionomer content in cathode catalyst layer, J Power Sources (2007) 170, p275-80

[7] Pan C, Li Q, Jensen JO, He R, Cleemann LN, Nilsson MS,Preparation and operation of gas diffusion electrodes for high-temperature proton exchange membrane fuel cells, J Power Sources (2007)172, p278-86.

[8] Qinggang He,Xiaofang Yang,Wei Chen, Sanjeev Mukerjee,Bruce Koel and Shaowei, Influence of phosphate anion adsorption on the kinetics of oxygen electroreduction on low index Pt(hkl) single crystals, Chen, Phys. Chem. Chem. Phys. (2010) 12, p 12544

[9] F. Gan, D.-T. Chin, Determination of diffusivity and solubility of oxygen in phosphoric acid using a transit time on a rotating ring-disc electrode, J. Applied Electrochemistry 23 (1993) 452-455

[10] Park JO, Hong SG, Kim TY, Kwon KJ, Suh SH, Cho MD, et al. Role of binders in high temperature PEMFC electrodes. ECS Trans (2006) 3(1), p447-51.

[11] Chu D., O2 reduction at the Pt/Nafion interface in $85 \%$ concentrated H3PO4., J Electrochim Acta (1998) 43, p 3711-8.

[12] Giordano N, Passalacqua E, Antonucci PL, Pino L, Vivaldi M, Patti A, Influence of physicochemical properties on the performance of $\mathrm{Pt} / \mathrm{C}$ porous electrodes for oxygen reduction in phosphoric acid, J Electrochim Acta (1993) 38, p913-8.

[13] Roy Choudhury S, Deshmukh MB, Rengaswamy R., A two-dimensional steady-state model for phosphoric acid fuel cells (PAFC), J Power Sources (2002) 112, p137-52.

[14] Zhang X, Shi P., Nafion effect on dual-bonded structure cathode of PEMFC, J Electrochem Commun (2006) 8, p1615-20.

[15] Lee SJ, Mukerjee S, McBreen J, Rho YW, Kho YT, Lee TH., Effects of Nafion impregnation on performances of PEMFC electrodes., J Electrochim Acta (1998) 43 , p3693-701.

[16] Liu Z, Wainright JS, Litt MH, Savinell RF., Study of the oxygen reduction reaction (ORR) at Pt interfaced with phosphoric acid doped polybenzimidazole at elevated temperature and low relative humidity, J Electrochim Acta (2006) 51, p3914-23

[17] Li Q, Hjuler HA, Bjerrum NJ. Oxygen reduction on carbon supported platinum catalyst in high temperature polymer electrolytes, J Electrochim Acta (2000),45, p4219

[18] Justo Lobato, Pablo Canizares, Manuel A. Rodrigo, Jose J. Linares, F. Javier Pinar, Study of the influence of the amount of PBI-H3PO4 in the catalytic layer of a high 
temperature PEMFC, International journal of Hydrogen energy 35 (2010) 13471355

[19] F. Seland, T. Berning, B. Børresen, R. Tunold, Improving the performance of hightemperature PEM fuel cells based on PBI electrolyte, Journal of Power Sources (2006) 160, p27-36

[20] Kong C-S, Kim D-Y, Lee H-K, Shul Y-G, Lee T-H. Influence of pore-size distribution of diffusion layer on mass-transport problems of proton exchange membrane fuel cells. J Power Sources (2002)108, p 185-91.

[21] Lee H-K, Park J-H, Kim D-Y, Lee T-H. A study on the characteristics of the diffusion layer thickness and porosity of the PEMFC. J Power Sources (2004) 131, p200

[22] Javier Parrondo, Chitturi Venkateswara Rao, Sundara L. Ghatty, and B. Rambabu, Electrochemical Performance Measurements of PBI-Based High-Temperature PEMFCs, Research International Journal of Electrochemistry (2011), Article ID 261065, doi: 10.4061/2011/261065

[23] Jeong-Hi Kim, Hyoung-Juhn Kim, Tae-Hoon Lim, Ho-In Lee, Dependence of the performance of a high-temperature polymer electrolyte fuel cell on phosphoric aciddoped polybenzimidazole ionomer content in cathode catalyst layer, Journal of Power Sources 170 (2007) 275-280

[24] Hyung-Suk Oh, Kwanghyun Kim, Hansung Kim, Polypyrrole-modified hydrophobic carbon nanotubes as promising electrocatalyst supports in polymer electrolyte membrane fuel cells, International journal of Hydrogen energy 36 (2011) p. 1156411571

[25] Kazuya Matsumoto,Tsuyohiko Fujigaya, Kazunari Sasaki and Naotoshi Nakashima, Bottom-up design of carbon nanotube-based electrocatalysts and their application in high temperature operating polymer electrolyte fuel cells, J. Mater. Chem. (2011), 21, p1187-1190

[26] A. Orfanidi, M.Daletou, S.G. Neophytides, Preparation and characterization of Pt on modified multi-wall carbon nanotubes to be used as electrocatalysts for high temperature fuel cell applications, J Applied Catalysis B: Environmental 106 (2011) 379- 389 
Dr. Alin Orfanidi
Birth Date: 27 June 1985

Birth Place: Anderlecht, Belgium

Current Address: Furstenbergstrass 26, Munich, Germany

Nationality : Greek and Belgium

Email: alin.orfanidi@tum.de

\section{Scientific Interests \\ Employment \\ Education \\ Participation \\ in Research \\ Projects \\ Publications \\ Laboratory Experience \\ 01.09.2014-on going \\ $01.11 .2011-$ \\ 20.01.2015 \\ 01.09.2009- \\ 21.10.2011 \\ 22.09.2003- \\ 20.07.2009 \\ 2010-2012 \\ 2013-2014 \\ 2009-up to now \\ $01.06 .2007-$ \\ 27.08.2014 \\ $0.1-31.07 .2012$}

$13-19.05 .2012$
Applied Electrochemistry, Fuel Cells, Electrolysis, Electrocatalysis, Synthesis of Nanoparticles and Alloys

Postdoc Fellow in Electrochemistry, Chair of Technical Electrochemistry, Prof. Dr. H.Gasteiger's Group, Technical University of Munich.

Ph.D. in Electrochemistry-University of Patras, Greece PhD Thesis: Electrocatalytic Investigation of High Temperature PEMFCs. Advised by Dr. S.Neophytides. Defence Date: 15.12.2014

M.Sc. in Chemical Engineering - University of Patras, Greece

Master Thesis: Synthesis and Characterization of Pt/functionalized MWNT as Electrocatalysts for Applications in High Temperature PEMFCs. Advised by Dr. S.Neophytides.

Diploma in Chemical Engineering- University of Patras, Greece

Diploma Thesis: Study of Endurance Towards Carbon Deposition using Dual-Metal Electrocatalysts Based on $\mathrm{Ni}$ Under Internal Steam Reforming of $\mathrm{CH}_{4}$ in SOFCs.

DEMMEA: Understanding the Degradation Mechanisms of Membrane electrode assembly for High Temperature PEMFCs and Optimization of the Individual Components, Project Founded by the European Commission.

CATHCAT: Novel Catalyst Materials for the Cathode Side of MEAs Suitable for Transportation Applications, Project Founded by the European Commission.

Published Peer Review Process: 6 (2 First Author)

Submitted with Peer Review Process: 1

Published without Peer Reviews: 1

In Preparation: 3 (2 First Authors)

Laboratory of Electrochemical Processes and Hydrogen Technology, ICE-HT/FORTH, Patras, Greece CNRS, Laboratory of Electrochemistry, Strasbourg, France, Group of Prof. Dr. E.R.Savinova

Spectro-Microscopy ,Electra Synchrotron Trieste, Italy 


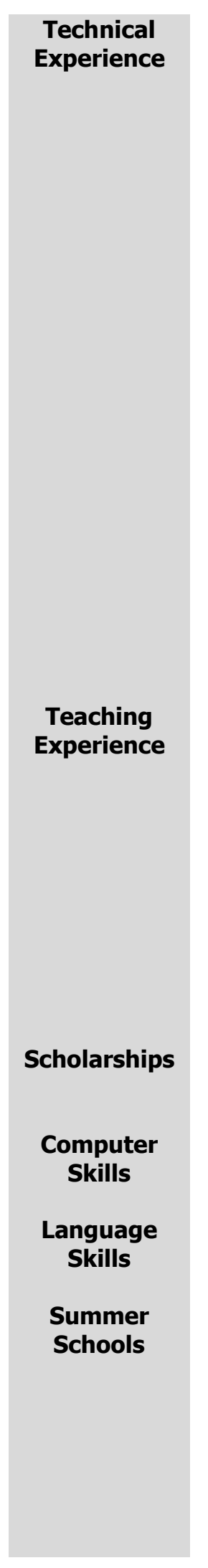

Experienced in

Familiar with

01.03.2011-

31.06.2011

01.03.2012-

31.06 .2012

01.10.2012-

31.01 .2013

2011-2014

06.20113

10.2010

03.2007
- Modification of Electrochemical Test Bench.

- Manufacturing of MEAs

- Surface Area Measurement Using the BET Method

- Catalytic Surface Area Measurement by $\mathrm{H}_{2}$ Chemisorption

- X-Ray Diffraction (XRD)

- Thermo-Gravimetric Analysis (TGA)

- Secondary Ion Mass Spectrometry(miniSIMS)

- Mass Spectrometry (MS)

- $\quad$ Rotating Disc Electrode (RDE)

- Preparation, Activation and Characterization of Electro-catalysts and Nanoparticles.

- Electrochemical Characterization Techniques: Cyclic Volta-metry, CO Stripping and Electrochemical Impedance

- Spectroscopy X-Ray Photoelectron Spectroscopy(XPS)

- Scanning Electron Microscopy (SEM/EDX)

- Transmission Electron Microscopy (TEM)

- Manufacture and Repair of Glassware for Experimental Applications

\section{Teaching Assistance}

Chemical Process 2- Heterogenous Catalysis, Prof. Verykios, Department of Chemical Engineering, University of Patras, Greece

Laboratory of Inorganic Chemistry, Prof. Staikos, Department of Chemical Engineering, University of Patras ,Greece

Laboratory of Organic Chemistry, Prof. Staikos, Department of Chemical Engineering, University of Patras ,Greece

Participation in supervision and conduct of 4 diploma thesis

From Project Founded by the European Commission: TCELL, CATHCAT, IRAFC, REALSOFC

Windows7-8.1, Microsoft Office 2010, Hysys, Origin8.5, Fortran, Autolab, Corel, ZSimpWin

Greek (Native Speaker), English (Fluent), French (Fluent) Flemish (Beginner)

Summer School of Graphene: Properties and Applications, ICE-HT/FORTH, Patras ,Greece

SUSHGEN Summer School: Electrolytes for PEM water Electrolysis, ICE-HT/FORTH, Patra , Greece

2nd Greek Symposium of Green Chemistry and sustainable development, University of Patras, Greece 
Conference Presentation
$19-23.06 .2011$

26-28.05.2011
A.Orfanidi, M.K.Daletou, S.G.Neophytides, Preparation and Characterization of Pt/Modified MWCNT as Electrocatalysts for High Temperature PEMFCs, 9th European Symposium on Electrochemical Engineering, Chania ,Crete, Creece

A.Orfanidi, MK.Daletou and S.G.Neophytides, Preparation and Characterization of Pt/modified MWCNT as Electrocatalysts for High Temperature PEMFCs, 8th Greek Conference of Chemical Engineering, Thessaloniki, Greece

\section{List of Publications}

\section{A) Publications with peer review process}

1. A. Bonnefont, P. Ruvinskiy, M. Rouhet, A. Orfanidi, S .Neophytides, E. Savinova, Advanced catalytic layer architectures for polymer electrolyte membrane fuel cells, WIREs: Energy and Environ., 3 (2014) 505-521

2. W. Doh, L. Gregoratti, M. Amati, S. Zafeiratos, Y.T. Law, S. Neophytides, A. Orfanidi, M. Kiskinova, E.R. Savinova, Scanning Photoelectron Microscopy Study of the Pt/Phosphoric-Acid-Imbibed Membrane Interface under Polarization, ChemElectroChem. 1 (2014) 180-186

3. A. Orfanidi, M.K. Daletou, L. Sygellou, S.G. Neophytides, The role of phosphoric acid in the anodic electrocatalytic layer in high temperature PEM fuel cells, J. Appl. Electrochem. 43 (2013) 1101-1116

4. G. Neophytides, L. Quaroni, F.N. Büchi, A. Orfanidi, S.G. Neophytides, T.J. Schmidt, A new in-situ spectroelectrochemical setup for FTIR measurements in operating high temperature polymer electrolyte fuel cells, Electrochemistry Communications 34 (2013) 200-203

5. A. Orfanidi, M.K. Daletou, S.G. Neophytides, Preparation and characterization of Pt on modified multi-wall carbon nanotubes to be used as electrocatalyst in high temperature fuel cells applications, J Appl. Catal. B: Environmental, 106 (2011) 379-389

6. I. Gavrielatos, D. Montinaro, A. Orfanidi, S.G. Neophytides, Thermogravimetric and electroca-talytic study of carbon deposition of Ag-doped Ni/YSZ electrodes under internal $\mathrm{CH}_{4}$ steam reforming conditions, Fuel Cell ,9 (2009) 883-890

\section{B) Submitted publications with peer review process}

1. M. Geormezi, F. Paloukis, A. Orfanidi, M.K. Daletou, S.G. Neophytides, The structure and stability of the anodic electrochemical interface in a high temperature Polymer Electrolyte Membrane Fuel Cell under reformate feed, (publisher's acknowledgement of receipt is enclosed, Journal of Power Sources)

C) Published without peer review process 
1. Y.T. Law, S. Zafeiratos, S.G. Neophytides, A. Orfanidi, R. Arrigo, A. Knop-Gericke, R. Schloegl, E.R. Savinova, An Ambient Pressure Photoelectron Spectroscopy Investigation of a Membrane-Electrode Assembly of a High Temperature PEM Under Potential Control, 224th ECS Meeting; 10/2013 